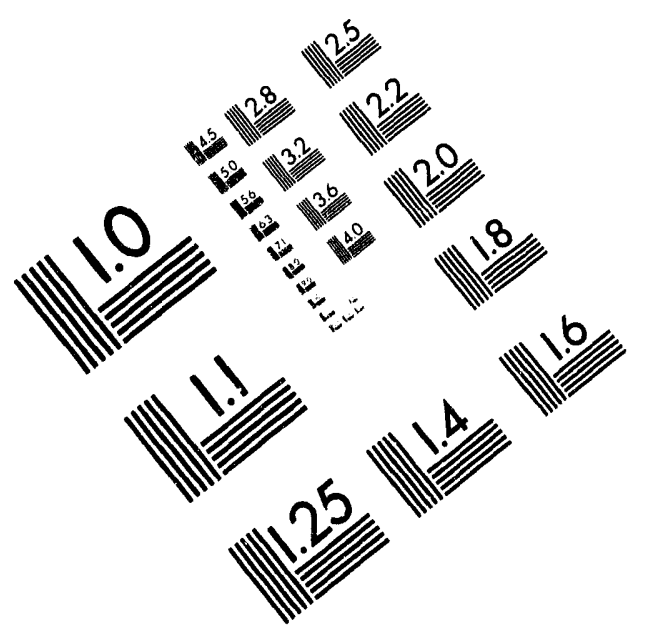

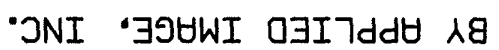

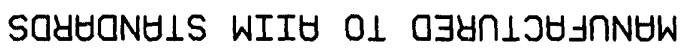
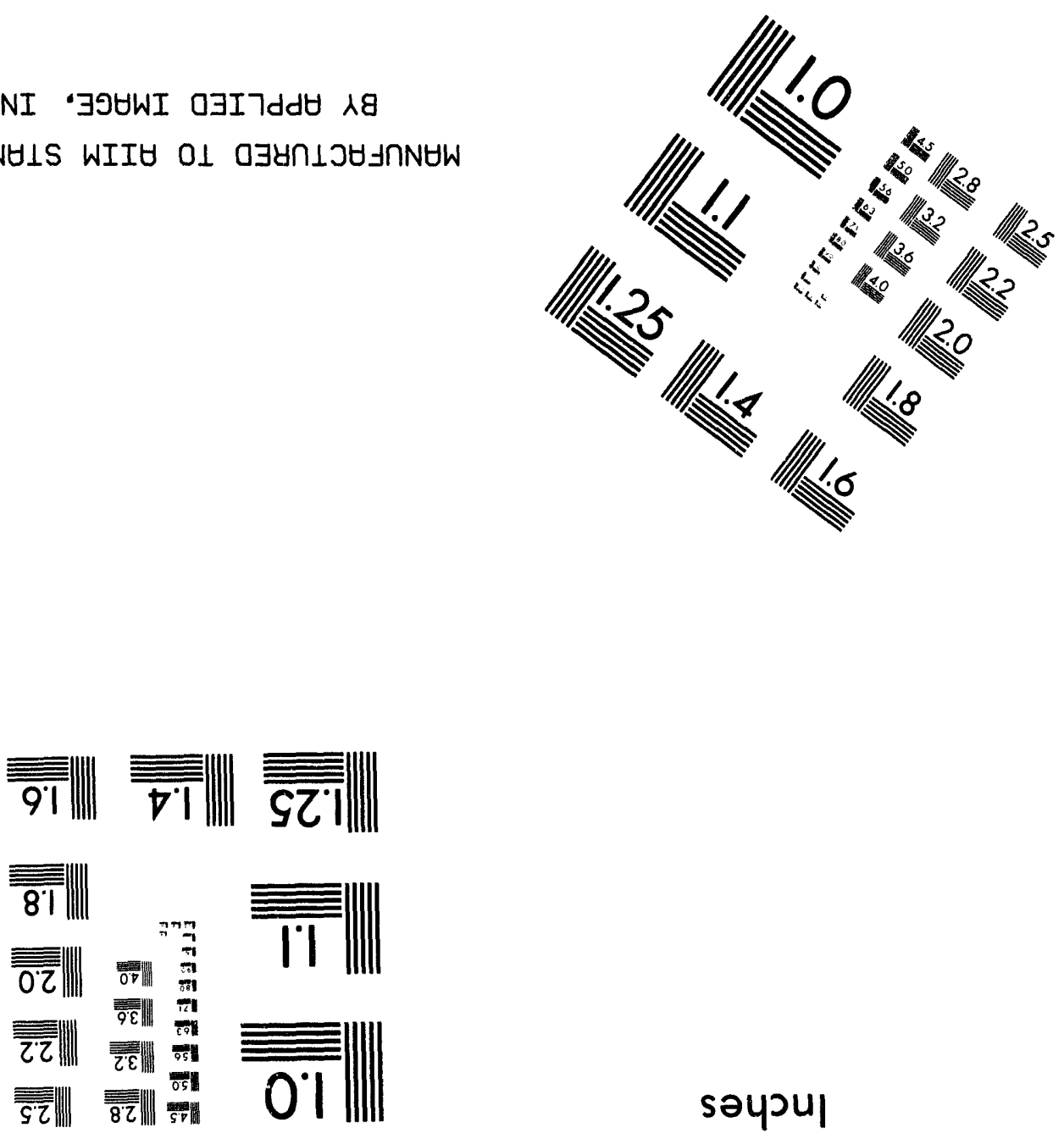

sәчगи
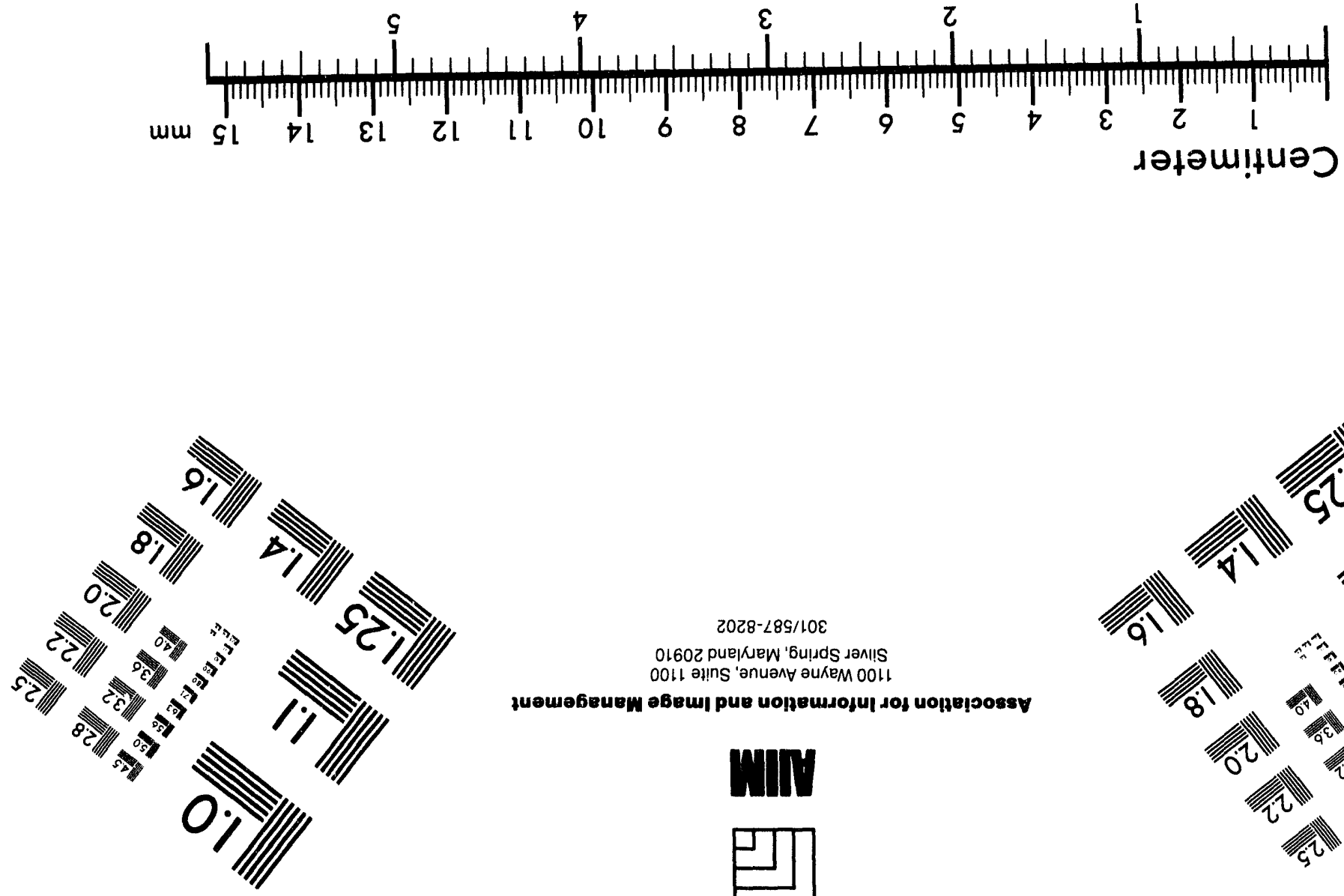

2028- $\angle 89 / 10 \varepsilon$

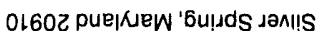

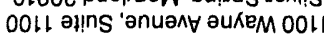

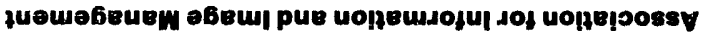
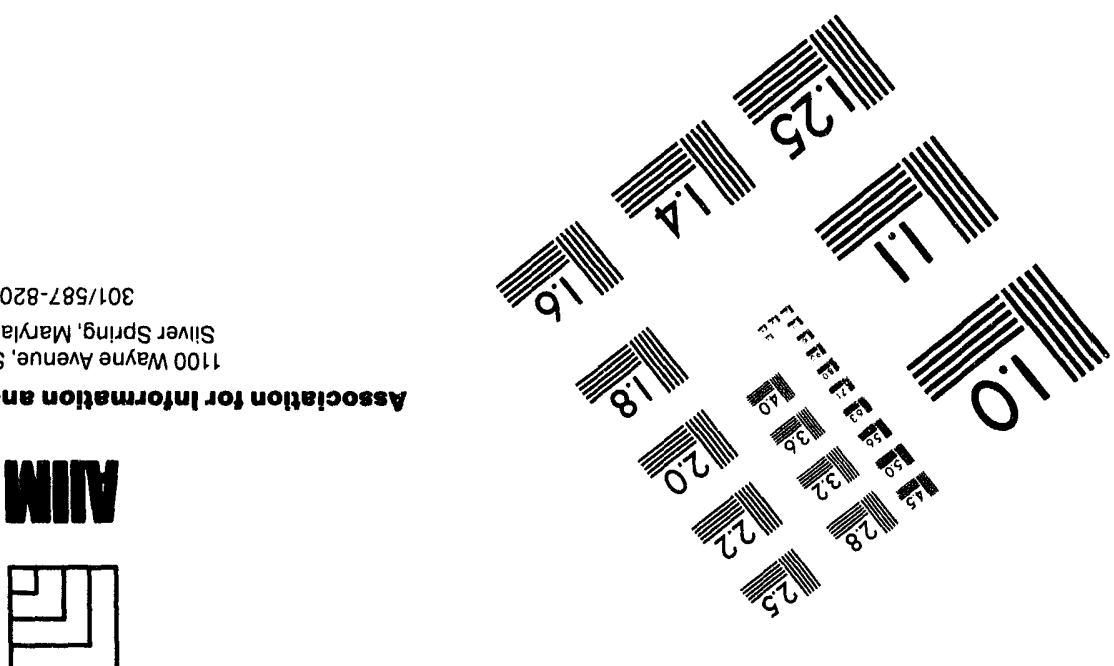

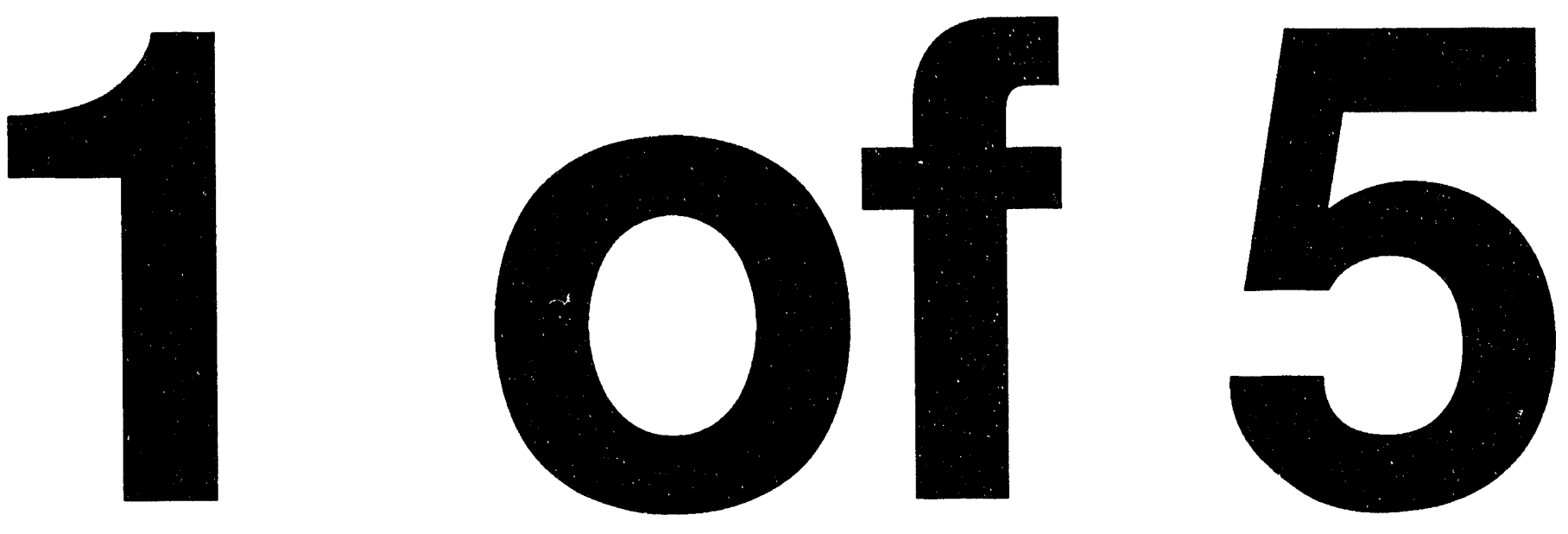


\title{
RESEARCH AND DEVELOPMENT OF METHODS AND TOOLS FOR ACHIEVING AND MAINTAINING CONSENSUS PROCESSES IN THE FACE OF CHANGE WITHIN AND AMONG GOVERNMENT OVERSIGHT AGENCIES
}

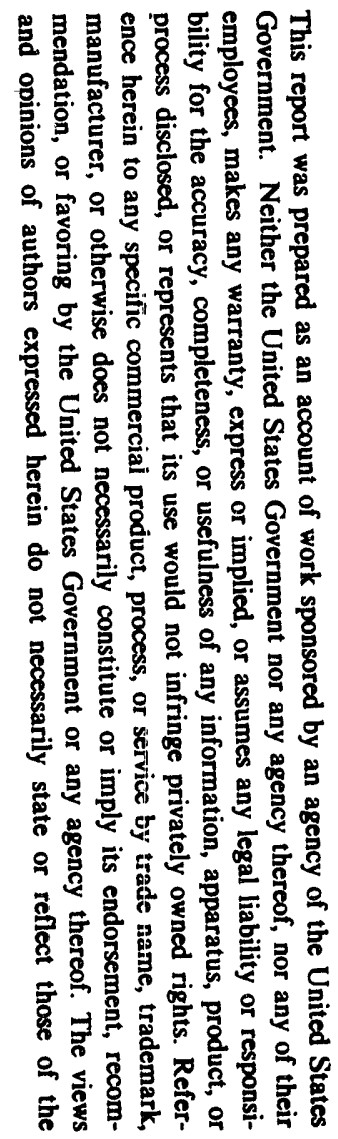

\author{
Volume II \\ Papers, Presentations, and Conferences \\ for Period October 1, 1992 - March 31, 1994 \\ Management Systems Laboratories \\ Virginia Polytechnic Institute and State University \\ Blacksburg, Virginia 24060 \\ June 1994 \\ Prepared for . \\ The U. S. Department of Energy \\ Agreement No. DE-FG02-88DP48058
}

量 


\section{NOTICE}

This report was prepared as an account of work sponsored by the United States Government. Neither the United States nor the Department of Energy, nor any of their employees, nor any of their contractors, subcontractors, or their employees, makes any warranty, expressed or implied, or assumes any legal liability or responsibility for the accuracy, completeness, or usefulness of any information, apparatus, product or process disclosed or represents that its use would not infringe privatelyowned rights. 


\section{Hert crint rour}

The lleted paphre and propontatione wore propared as a part of Hantgohoht

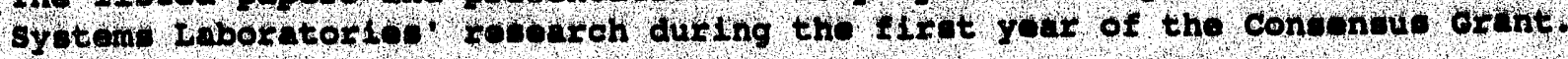

The papore wore both prosented tt varlous conferences and publilhed in the conference prodtodings. The probontations are indopendent of the pupere, aro not publibhed, and were given in dadtion to the paper prosontatelono. Coples of the papere follow.

\section{theth}

nets

Dow th

Papors:

Achieving consensus in

Environmentel Programe.

waste Management 89 symposlum.

\section{Prosentations:}

Waste Management Studies

at Ust. National

Proceses

Communications council.

Ueing A coneensus-Based

$4 / 89$

Consensus

Participative Decision Procese To Solve A Multi-jurlisaletional

Process

Emergency Management Problem.

Aspa National conference. 


\title{
ACHIEVING CONSENSUS IN ENVIRONMENTAL PROGRAMS
}

\author{
Harold A. Kurstedt, Jr., R. Martin Jones, J. Andrew Walker, \\ and Louis I. Middleman \\ Management Systems Laboratories \\ Virginia Polytechnic linstitute and State University \\ 1900 Kraft Drive, Blacksburg, VA 24060
}

\begin{abstract}
In this paper, we describe a new research effort on consensus tied to the Environmental Restoration Program (ERP) within the U.S. Department of Energy's Office of Defense Waste and Transportation Management (DWTM). We define consensus and explain why consensus decisions are not merely desirable but necessary in furthering ERP activities. As examples of our planned applied research, we first discuss Nominal Group Technique as a representative consensus-generating tool, and we conclude by describing the consensus-related mission of the Waste Management Review Group, established at Virginia Tech to conduct independent, third-party review of DWTM/ERP plans and activities.
\end{abstract}

\section{INTRODUCTION}

The key defining feature of the organizations responsible for ERP activities isn't autonomy but "polyonomy," a term we've invented to signify the diffusion of responsibility and power among many agencies and sub-agencies. The U.S. Department of Energy (DOE) is what we call a "Government Oversight Agency" (GOA); GOA's at all levels of government (federal, state, and local) must implement laws made by the Congress and by state legislatures--must coordinate their overlapping roles and responsibilities. Further, they must learn to cooperate in an arena that rewards adversariality, and they must learn to maintain this cooperation over long periods, to adapt to inevitable change.

\section{RESEARCH CONTEXT}

The popularity of books and articles on the "new" management and manager, emphasizing consensus rather than edict, is in part the result of a drastic increase in the number of organizations whose authority isn't complete and autonomous but partial and polyonomous--literally, "many-portioned" or "many-ruled." Even the few autonomous organizations left today are undergoing changes to be or remain competitive. Although many people are "only vaguely aware" of it, "modern society is a complex of interdependent groups or teams" (1). Autocratic management may work in small, centralized, independent, isolated organizations where one person's word is law and where formal and informal public scrutiny is low. Nothing could be further from describing the characteristics of GOA's: large, decentralized, regulated, dependent, and in a veritable fishbowl of public scrutiny. Getting things done within and among GOA's means implementing national policy derived from Congressional legislation. A GOA manager's first step toward such implementation normally meets with resistance from outside agencies and from various levels of parallel sub-organizations within the manager's own organization. That's no accident. Indeed, in principle it's a tine setup: a multitude of mandated checks and balances on everything actually or potentially affecting the public good.

Consensus within such a polyonomous system requires "acceptance-level decisions" (2) in addition to and as distinguished from "technical-level decisions." The latter refer to 
decisions about what will or won't "work"--for example, what kind of treatment will or won't reduce PCB contamination at a certain waste disposal site to an acceptable amount. The point is, whether there be only one or a number of equally effective and efficient technical solutions to such a problem, none will be implemented unless all affected parties agree (or are compelled).

The consensus necessary to achieve acceptance-level decisions within, between, and among organizations is often the result of what are called "informal" and "lateral" processes, as contrasted with those of the formal hierarchy. Galbraith (3) stresses the importance of not leaving informal stru ctures to chance. "These informal processes," Galbraith writes, "are thought to arise spontaneously and are the processes through which most organizations accomplish their wcirk despite the formally designed structure. A typical point of view is, 'If we had to go through channels, we would never get anything done.' The point of view being taken here is that these informal processes are necessary as well as inevitable, but their use can be substantially improved by designing them into the formal onganization.... [A] more important reason for formalization is that these processes do not always arise spontaneously from the task requirements, especially in highly differentiated organizations. When the relevant participants have different and sometimes antagonistic attitudes... and are separated geographically, the effective use of joint decision making requires formally designed processes" (italics added).

\section{DEFINING CONSENSUS}

Consensus denotes "collective opinion or concord; general agreement or accord"(4). Further elucidation comes through the word's origin in the Indo-European root sent, meaning "to head for, go" following the prefix con-meaning "along with" or "together." This etymology suggests why consensus is necessary to the effective and efficient work of a system (like a GOA) with many parts. A system is a collection of entities, related by structure or communication, such that a perceptible or measurable change in one part causes a perceptible or measurable change in all the other parts. And work is the application of a force through a distance. For efficient work, the sum of the movement of the system's interdependent parts must point toward the objective and exert maximum energy in that direction. GOA's need consensus about these two things: the objective and the movement--the ends and the means. If you have the objective but not the ability to move as a unit, you won't get there (zero or low efficiency). If you have the ability to move but don't know where to go, your energy is pointless and will be wasted (zero or low effectiveness).

Defined in this way, consensus is pragmatically and morally neutral. Consensus on means and ends is a necessary condition of progress toward an objective, but it isn't sufficient. For, as a "collective opinion," consensus by itself implies neither accuracy nor morality nor even feasibility. Opinion is, in Plato's words, "something between ignorance and knowledge." The better opinions are those closer to knowledge, and the way you show this closeness is through adequate supporting information. An opinion, though collective among a certain group, can still be bad (evil); wrong (incorrect); and difficult, perhaps even impossible to implement if it has to tight against a strong or stronger opposition. Consider the ancient consensus achieved by Pontius Pilate and his lieutenants, the ongoing consensus among the membership of the Flat Earth Society, and the 1982 Congressional consensus that high-level radioactive waste be permanently disposed of in a deep geologic repository. 


\section{Consensus as a State Variable}

Consensus, then, is a parameter, a variable that must always be taken into account in describing the state of a system. Our research focuses on interactions within and among systems of people (GOA managers)--and between these systems and the population they serve; we're using the term as a gross or macro-level "state variable" characterizing the degree to which a group of people behaves as one person. It's the measure of a group's tendency, as it moves through time, to behave as a unito-irrespective of the force behind this tendency (reward/punishment), the source of the movement (internal/external), or the level of the source (horizontal/vertical/mixed).

\section{Consensus Distinguished from and Favored over Compulsion}

When consensus results from a directive that doesn't fit what the group would have come up with had it been asked, we call it compulsion, meaning that the environment (i.e., one or more people coupled with one or more conditions) forces the group to behave in a certain way and prevents the group from behaving in any other way.

No group is ever completely free of compulsion. The environment always more or less constrains this freedom, making certain behaviors undesirable or impossible and requiring certain behaviors nobody likes. Galbraith (3) calls compulsion "forcing," which he defines as "power or position or knowledge being used to force a preferred alternativ on the rest of the group." Forcing, says Galbraith, will lead to ineffective decisions if it is the dominant mode. If one function or dominant department always forces, then there is no need for a group effort, since information from other departments is ignored.

Suboptimal decisions and poor implementation result when a forced solution is based on local information in the presence of interdependence. The preferred approach to conflict resolution therefore is to use confrontation and problem solving backed up by occasional forcing when lack of agreement stymies the group" (italics added).

\section{ACTUAL VERSUS PERCEIVED CONSENSUS}

When we look at consensus as common or unified behavior, we see consensus if the behavior shows it. The observable behavior, however, may or may not be congruent with group members' unobservable internal states. We can define the degree of actual as opposed to perceived consensus as the difference, if any, between the assumptions an external observer or a group member would make about a group's attitudes based on the group's objective (visible) behavior, and the group's subjective (invisible) thoughts and feelings about this behavior. Consensus can be more perceived than actual, or vice versa, both from the perspective of a group member (including the leader or the person who called the group together) and from the perspective of someone outside. The importance of the distinction between actual and perceived consensus may be seen in the following example.

\section{Avoiding the Trip to Abilene}

Unhealthy agreement-the false pretense of consensus born of fear of selfdisclosure--can move a group to unwanted behavior as much as, perbaps even more than, an excess of explicit conflict can stymie them. The "Abilene Paradox" (5) is the cautionary tale of a Texas family that reaches a false but powerful consensus and ends up doing something none of them actually wants to do. They somebow find themselves driving a dusty 106 miles in an un-air-conditioned car on a 104-degree summer afternoon from Coleman to Abilene to eat unpalatable food in a fourth-rate cafeteria, instead of doing what they all really want--to stay out on the electric fan-equipped screened-in porch, play 
dominos, listen to the radio, drink lemonade, and chat. Having returned from Abilene thoroughly disgruntled, they reveal their true thoughts and feelings--and their bewilderment at how they ever decided on that stupid trip.

Consensus like that which motivated this family is false to the group members' thoughts and desires. In its effects, however, their consensus is actual, and therein lies the danger. We humans are skilled at hiding thoughts and feelings from others, but we forget others are just as good at hiding theirs from us. To avoid and prevent trips to Abilene, group members (meaning, one time or another, everyone) must learn the skills of selfdisclosure and learn to overcome the fear of risking this disclosure.

\section{HOW MUCH CONSENSUS IS ENOUGH?}

Our behavior-based definition of consensus provides an answer. A group member's behavior, either toward or away from the group's ostensible objective (what an observer would predict to be the effect of the group's decision) is analogous to a vector. If the vector sum of all members' behavior is toward the ostensible objective, then there's enough consensus. And "enough" isn't strictly a matter of numbers, of how many participants' vectors point the same way, but of their relative magnitudes, and moreover, the changing magnitude of their vector sum over time. Over time, the objective will be reached if and only if the sum of all consensus vectors is greater than the sum of all antagonistic compulsion vectors.

There's no necessary one-to-one correlation between a group's achievement of consensus on an objective and the reaching of that objective. Consensus by itself, though necessary to reaching the objective, is not sufficient. The consensus reached in a football huddle may be total, yet the play may fail because, literally and figuratively, it bumps into another and stronger consensus. In terms of the effects of GOA group decision-making, we must measure consensus by discovering how to measure the extent to which the actions and conditions (including costs and schedules) that should follow from the group decision actually do follow, and by how long they remain in effect in comparison to how long the decision-makers expected them to remain in effect.

\section{WHY CONSENSUS IS NECESSARY FOR GOA'S}

Nowhere is interdependency more apparent than in and among GOA's and in interdependent programs like the ERP. These organizations differ from product and service organizations in the private sector and from other government field offices and public works units. Whereas the latter are close to the provision of services to the public, GOA's are close to the legislative bodies from which they take their mandates. As such they are the interface points between public policy-making and implementation and must participatively interact with legislatures, other GOA's, and other elements within their own organizations. However, when called upon to solve problems GOA's don't have tools, methods, or processes uniquely tailored to fit their participative environment. Rather they have tools, methods, and processes designed for hierarchically structured environments.

Given so much to do with such a (relative) paucity of resources, those people responsible for managing and implementing the ERP must invite consensus on task and funding prioritization. Numerous organizations at all levels of government, as well as private interest groups and individual citizens, are bound to be dissatisfied no matter how the funds are spent, unless there's consensus on the prioritics. But what--this is the key question--nationally constitutes the greatest overall benefit to be realized from the available dollars? It's one thing to prioritize activities vertically at each DWTM field site 
(and that's no small matter in itself, for the potential hazards of many waste sites may take years to estimate accurately). It's quite another to prioritize activities horizontally across all field sites. There are simply too many squeaky wheels and too little grease. The first formal activity of the Waste Management Review Group, discussed at the end of this paper, is to review a prioritization model, the Program Optimization System (POS), and recommend ways to assess the degree of consensus likely to result among stakeholders nationally.

If short-term consensus about ERP priorities is hard to achieve at all, it's surely harder to maintain in the moderate run, and it's exponentially more difficult in the long run. This is partly because, like any organization, this DWTM and ERP will see changes in leadership and personnel (nobody working in DWTM will be working there sixty years from now). It's also because GOA managers are, as Kotter (6) says, "rewarded almost entirely for short-run performance." Though DWTM's programs are all long-term effor. funding comes just once a year, and it's impossible with any confidence to allocate dollars you don't yet have. As a result of this short-run focus, "they spend far too much... of their discretionary resources trying to keep current processes effective and efficient, far too little of their time and other resources trying to creste or maintain a coalignment, and far too little effort trying to create adaptive element stittes."

\section{HOW WE CAN APPLY THEORY TO UNDERSTAND ERP PROBLEMS AND FIND SOLUTIONS}

We're at the very beginning of our study, with more questions than answers--and probably with not enough questions. How can we move forward? In this section we give two examples of our research approach. In the first example, we start with a popular method for getting consensus, analyze this method, and consider its usefulness both as a vehicle for learning about consensus and as an aid to ERP success. In the second example, we start not with a method but with a group-with the assembling of an expert review group-so we can observe and understand how that group's recommendations can affect consensus beyond the group, in the wider population of which the members constitute we hope, a representative sample. In short, our research involves intra-group consensus, inter-group consensus, and (most importantly), extra-group consensus.

\section{Examining the Nominal Group Technique (NGT)}

We want to find out what works and why it works. At first we'll find out more of what works (and doesn't). We'll apply tools and methods people have tried to use for generating consensus, and see what happens. We assume no one method will work all the time. What works will depend on the group's characteristics and the problem to be solved or the decision to be made. When we find something that works, what we'll be finding is something that works in a particular, constrained situation. If we understand the constraints, we ought to be able to make it work in a similarly constrained situation. And if we get things to work enough times in enough differently constrained situations, we can begin to generalize about why things work.

The Nominal Group Technique (NGT) was first developed by Van de Ven and Delbecq (7). NGT is a method--a series of tools applied in sequence--to provide free and equal expression and ranking of opinions in groups which otherwise might be dominated by certain individuals or certain paths of thought. Both the tools for idea proposal and the tools for ranking limit the potential for conflict between group members. The four steps of the NGT proposed by Van de Ven and Delbeng are as follows: a) Individual members first silently and independently generate their ideas on a problem or task in writing; b) This period of silent writing is followed by a recorded round-robin procedure 
in which each group member (one at a time, in turn, around the table) presents one of his or her ideas to the group without discussion. The ideas are summarized in a terse phrase and written on a blackboard or sheet of paper on the wall; $c$ ) After all individuals have presented their ideas, there is a discussion of the recorded ideas for the purposes of clarification and evaluation; d) The meeting concludes with a silent independent voting on priorities by individuals through a rank ordering or rating procedure, depending upon the group's decision vote. Other practitioners expand NGT to six steps $(8,9)$, affording more interaction among group members: e) After the voting and ranking, participants consider the aggregated results to measure the extent to which they've supported their true positions or taken a trip to Abilene. At this point they may revise their rank ordering of ideas; f) Finally they consider the resources available to implement their high-priority action items, choose a set of these items to scope for implementation, and divide into smaller groups to begin the scoping process, which involves measuring the feasible application of available human, funding, and material resources within known or estimated time constraints.

"Nominal" is the key word describing this method; the group that meets for an NGT session isn't (or isn't necessarily) a regular work group within one organization. It's not a real group (a group sharing common aims and values and therefore predisposed to cooperate) but a group in name only (a nominal group). The question arises whether consensus is possible within a nominal group; if not, then you have to take a nominal group and somehow transform it into a real group. Can NGT help in this transformation? We need to ask such questions because the set of ERP stakeholders would have to be called a nominal group.

At least one NGT study, Mahler's (10), suggests group dissatisfaction with the resulting decision is high; however, the results come from 45-minute applications with student groups working on unsolvable problems in which they had no stake. Studies made on day-long applications outside classroom situations and with real, pressing, solvable problems have found more favorable results. Mahler is correct in linking the use of pure NGT primarily to the generation rather than to the evaluation of ideas. Indeed, in its original formulation, since ranking is numerical and done by straw vote, a "pure" NGT application gives little chance for conflict between members and therefore little opportunity for persuasion, bargaining, compromise, or what can loosely be called the politics of consensus. In the generative and in portions of the evaluative stage of consensus reaching, this is an advantage; later, however, a group would probably wish to tailor the technique to provide for surfacing enough essential conflicts (ad hoc rather than ad hominem) to increase the probability of results participants won't torpedo once they leave the meeting.

NGT is a popular technique. Though until just recently we never felt the compulsion to research it systematically and longitudinally, at Management Systems Laboratories we've collectively had more than 40 person-years experience conducting, observing, and participating in a wide variety of NGT-type sessions, several recently within the ERP itself. We know what works in one circumstance will fail in another. We've seen an apparent deadlock become a consensus as if by magic, not in the meeting itself but in the hallway out to the parking lot. We've seen an apparent consensus fall to pieces when participants realized some were using a term to mean one thing and others something different. We see NGT as a group of tools and we believe there must be a fit between the users and the problem to be solved. Just as with computers, you don't simply accept NGT because you heard it worked somewhere else.

We can hypothesize an ideal group on which NGT should work. Such a group, of about eight to 12 people, would likely share interest in common issues and be all at about 
the same level in their organizations. They'd probably be more divergent than convergent in their information gathering styles, preferring open-endedness to closure. NGT would both satisfy their need to brainstorm--it's a great idea-generating technique--and constrain their divergence through the requirement to vote and rank.

Okay. Say (ho simple matter) we find the tools that work for our ideal nominal group. We're likely to encounter an actual ERP nominal group that's "all wrong"-convergent if we want divergent; at levels all over the hierarchies of their respective organizations, larger than we think any group should be to function well together but needing to be that size or else we lose legitimate stakeholders who if not included will vitiate the efforts of the rest. Well, we don't have a perfect tool, so we try to combine or modify tools we know to get as close as possible. In our research we must ask, What are the important characteristics of groups? and What are the important characteristics of tools? Then we can hope to synthesize an answer to the question of the relationships between these sets of characteristics and why they hold.

We must identify all tools, methods, techniques that might apply to our real-world nominal groups and keep a running list, a living list. We'll do test runs, we'll discover that a certain tool works nine times out of 10 and use that fact as a reference point. We'll ask, if you change the group in such-and-such a way, what must you do to the NGT to make it work? We'll start to understand what parts of NGT work in different situations and begin to generalize and to understand why. In our attempt to understand why and confirm that understanding, we'll first generalize tool by tool; then group of tools by group of tools. Finally, we'll hope to generalize in terms of all consensus :00ls. When we can do this, we can predict outcomes and prescribe the sets of tools likeliest to facilitate consensus within various given situations.

\section{The Waste Management Review Group (WMRG): A Forum for Consensus}

Whereas with NGT we start with a tool or a group of tools (a method) and try to figure out how that method yields or coesn't yield consensus, with WMRG we start with a group, a group specifically selected for the necessity of its members' knowledge and experience to the furthering of the ERP. The ides for such a group came from the realization that another group, the Technical Review Group (IRG) convened by Martin Marietta Energy Systems, Inc. was increasingly spending its time on policy issues rather than on purely technical issues. Since both kinds of issues are vital to the ERP, it was decided to form a second group devoted entirely to policy. The WMRG's chartered scope is to provide 1) objective reviews, evaluations, and assessments of current plans, projects, and activities related to DWTM policies and actions mandated by Congress, the Executive Branch, and the DOE; and 2) research, analysis, and communication necessary to substantiate the reviews, evaluations, and assessments and provide useful information, conclusions, and recommendations to a new organization, the Waste Policy Institute (WPI).

The WMRG was chartered and convened under the auspices of WPI, a not-forprofit university-related corporation at Virginia Tech . The principles for assembling the WMRG were instinctive, intuitive. It had to be done that way because we don't know enough about GOA team building processes to have put it together scientifically. What we tried to do was exhaust the categories representing those constituencies in the general population which must form part of the consensus necessary ensure ERP success. The five categories from which we chose members are universities, industry, States and Indian tribes, the Federal government, and public interest groups and professional societies. 
Though not all members know each other, the WMRG starts out probably closer to a real group than a nominal group. But though for this reason it should find it easier to achieve consensus on prioritization in its review of the POS, what DWTM needs is far beyond the consensus of that group. What DWTM needs us to do in our research is figure out how to configure WMRG so it's a faithful microcosm of the population it needs to represent. To put it another way, our job wasn't to set up the group. Setring up the group was a necessary precondition, like setting up experimental apparatus. Our research mission is to discover the extent to which our partitioning of the ERP-related world has yielded the right constituencies--all the right ones--so this wider world will look at the WMRG's conclusions and adopt them because the WMRG's consensus is representative; because the WMRG sample represents the relevant population.

The convener of the TRG attends.but is not a member of the WMRG. The convener of the Ad Hoc Waste Contractors Group attends but is not a member of the WMRG. And I, the convener of the WMRG attend but am not a member of the TRG and the Ad Hoc Waste Contractors Group. We call it "interdigitation." We watch the extent to which consensus on technical issues and consensus on policy issues combines to yield a larger, synergistic consensus. The WMRG and related activities thus provide an ideal real-world laboratory for our research, a perfect opportunity to study intra-group consensus, inter-group consensus, and extra-group consensus.

\section{ACKNOWLEDGEMENTS}

The preparation of this paper is funded by U.S. Department of Energy (DOE) Grant No. DE-FG02-88DP48058. Management Systems Laboratories thanks the DOE's Office of Defense Waste and Transportation Management for providing us a real-world laboratory for the research, development, and testing of state-of-the-art management tools and the frameworks for understanding how to make them successful.

\section{REFERENCES}

1. Dyer, William G. Team Building: Issues and Alternatives, 2nd edition. Reading, Massachusetts: Addison-Wesley Publishing Company, 1987.

2. Vroom, Victor H. and Phillip W. Yetton. Leadership and Decision-Making. University of Pittsburgh Press, 1973.

3. Galbraith, Jay. Designing Complex Organizations. Reading, Massachusetts: AddisonWesley Publishing Company, 1973.

4. William Morris, ed. The American Heritage Dictionary of the English Language. Boston: Houghton Mifflin Company, 1976.

5. Harvey, J. "Managing Agreement in Organizations: The Abilene Paradox." Organizational Dynamics, Summer 1974, pp. 63-80.

6. Kotter, John P. Organizational Dymamics: Diagnasis and Intervention. Reading, Massachusetts: Addison-Wesley Publishing Coriapany, 1978.

7. Van de Ven, Andrew H. and Andre Delbecq. The Effectiveness of Nominal, Delphi, and Interacting Group Decision Making Processes." Academy of Management Joumal, vol. 17, no. 4, 1977, pp. 605-621. 
8. Rohrbaugh, John. "Improving the Quality of Group Judgment: Social Judgment Analysis and the Nominal Group Technique." Organizational Behavior and Human Performance, vol. 28, 1981, pp. 272-288.

9. Sink, D. Scott. "Using the Nominal Group Technique Effectively." National Productivity Review, Spring 1983, pp. 173-184.

10. Mahler, Julianne G. "Structured Decision Making in Public Organizations." Public Administration Review, July/August 1987.

9 


\section{socond Grant reht}

The libted papere and presentations were prepared ts a part of yanagement systems Laborator 1 od rowearch durling the decond year of the Consenswe Grant.

The Papers were both presented at various conforences and published In the conference proceedings. The presentations are lindependent of the pepere, are not published, and wore given in addition to the paper preantations. The Journals represent papere which weren't presented at conferences but were sent directly for publication in a journal. coples of the papers and journal articles follow.

Iitse

Papers:

Measuring Consensus. Southeastern Chapter of the Institute of

Management sciences.

A Framework for Data and Information. southeastern chapter of the Institute of Management sciences.

Radioactive and Hazardous Materials Transportation: What Local officials Are Telling Us. Weate Kanagement 90.

Tabletop Exercises: Cooperathe Training for Tranoportation Incidente. waste Management 90 .

Programmatic Issues Affecting the Implementation of the DOE Five-Year Plan. Waste Kanagement 90 .

Institutional Factors Affecting DOE Waste Management and Environmental Restoration Planning. Waste Management $\cdot 90$.

Automation Through the PIP Concurrence syetem Improves Information sharing Among DOE Managers. Waste Management 90 .

The Effects of Information Availability on the Group Consensus Process. 12th conference on Computers and Induetrial Engineering.

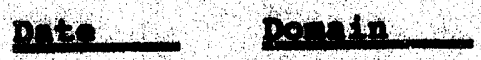

$10 / 89$

Fundamental

$10 / 89$

$2 / 90$

Consensus

Process

$2 / 90$

Consensus

Process

$2 / 90$

Consensus

Procese

$2 / 90$

Consentus Process

$2 / 90$

Information syotems

$3 / 90$

Fundamental 
Apg 1 lapl1 ity of the integrat ive Bhroulning prooese for fogotlat lng and Inplomontelng roderal pact 1 lty thvltomidnt al compllineo ngrowonte. Internat tonal a woelat on for cons1 lot whogomnt.

Exportono in bostoning and ushng a ilt structure In a Mit 1-project Roparoh orguntetelon. 10th IWHetwer Worla congrese on project yinagunt.

Prosentations:

Alourling the Integrlty of the Implementation proces of bos'a Ave-roar warto plan. Wabhington Internatlonal Bnorgy Group.

yentgement rools, culture, and the Manugomont proeere. Hoet lnghouee Nuclear sefoty and gnvi ronmental oyerulght comilttee for coco operation.

Journala:

slmulation por Bmergency Manegement: Taking Advantago of automntion in Emergenoy Rreparedness. Slinilation.
$6 / 0$ oononous procous

6190

Intorination syotom.

$10 / 89$

consensus Proeses

$2 / 90$

Coneoneus Procese 


\title{
MEASURING CONSENSUS
}

\author{
Harold A. Kurstedt, Jr., Va. Tech \\ D. Mark Brubaker, Va. Tech \\ Anne R. Doss, Va. Tech \\ C. Patrick Koolling, Va. Tech
}

\begin{abstract}
For this paper, I wanted to compare mathematical techniques against group interaction in generating consensus for a ranking decision. I convened a group to come to consensus on ranking items needed for survival on the moon. $i$ chose this problem because NASA has an approved solution. I solicited the group's individual rankings before and after discussion. I used Kendall's coefficient of concordance to measure the level of consensus before and after discussion and compared the results against individual qualitative responses to a questionnaire designed to also measure consensus. The approved solution allowed me to see if the group felt more or less agreement as the moved closer or further from the approved solution.
\end{abstract}

As background for this experiment, I researched the existing knowledge on measuring consensus. I make a distinction between consensus and successful consensus, define them, and operationalize them for the purposes of this study. I define different levels of consensus which can be reached regardless of the success of the consensus. In this experiment, I determined the interactive discussion produced consensus, but not successful consensus. The mathematical technique produced a ranking closer to the accepted answer than the group discussion did.

\section{BACKGROUTD}

What is consensus? Everyone probably has "feel" for what consensus is but can't put it into words. The many different definitions and operationalizations demonstrate there is no consensus on consensus.

The best way to characterize consensus is as an "hypothetical corstruct." We often presume consensus exists at some point when everyone's ideas have been combined into one complex whole. We may not be able to measure consensus directly. Most measurements in the 11terature have been measurements of simpler ideas from which the presence or non-presence of consensus has been inferred. Some ways consensus has been measured are by degree of agreement [1][2], Individual feelings about the probability of an event occurring [10], interaction patterns within a group [2], and individual preferences [15].

Adding to this confusion is the fact that consensus refers to both 1 ) an ideal situation in groups and 2) the ideal mean to achieve group goals [12]. Consensus in a small group setting can apply to a decision making process or the result of the process. Jay Hall [4], defines consensus as "a decision process for making full use of avallable resources and for resolving conflicts creatively." If we look at consensus as the ldeal goal in groups instead of a process, we are still faced with different meanings. Definitions used in the literature for consensus range from the degree to which the most influential people in a group agree (9) to perfect unanimity among all group members (5). 
Some people refer to consensus as a state of mind, a "we" feeling among the participants in a group [3], Irving Janis emphasizes the negative aspects of this in his book Groupthink [8]. He defines groupthink as "... the desperate drive for consensus at any cost that suppresses dissent..."

\section{Levels of Consensus}

Consensus, as a process or a group feeling, is a means to an end. A successful consensus decision can be measured by the end result of the decision; i.e., the actions taken as a result of the group's interaction. It is important to recognize that a group can form consensus but not have it be a successful consensus due to circumstances beyond their control. In other words, consensus can be present but unsuccessful if the agreed upon actions don't produce the desired results. If I measure the success of consensus in this way, I can then characterize the consensus achieved within a group on a continuum based on the way the actions were decided upon. My continuum ranges from coercion to unanimity (see Figure 1). I belleve, with all other variables held constant, decisions have a higher probability of becoming successful actions as the group interaction approaches unanimity.

\section{Figure 1.}

Consensus can be rleved as a continuum.

coercion compulsion acceptance agreement unanimity

CONSENSUS

I define these terms this way:

coercion - one person defines the behavior (idea, plan, decision) and has the power, either real or perceived, to sway the other group members to accept the behavior.

compulsion - one or more people (the total being a minority in the group) are the main drivers of the bohavior while the others "go along".

acceptance - one or more members find the behavior undesirable but will go along with it.

agreement - the behavior has the support of the entire group although it is not the ideal behavior for some or tost of the group members.

unanimity - all members unanimously and enthusiastically agree on the behavior and support it.

Considerable research suggests that groups typically make decisions superior to those made by individuals [4][13]. Processes used for group decisions however, aren't equally effective [6]. Van de Ven and Delbecq found nominal and delphi techniques superior to interacting methods in the quantity of ideas 
generated on an unstructured problem [14] Tjosvold and Field found consensus methods were superior to majority voting in establishing decision acceptance but found no effect on decision quality [13]. What needs to be done now is to research all the different situations in which consensus is used and identify key variables which help facilitate or hinder a successful consensus. The study done for this paper is a small step in this direction which I hope to build upon in the future.

SETHOD

\section{Subjects}

The participants were a group of graduate students and employees of Management Systems Laboratories (MSL) at Virginia Tech. The group consisted of three master's students, one doctoral student; two professors of Industrial Engineering, and four professional research managers from MSL. I consider the subjects used as a real group. I haven't completely defined a real group; but, when compared to a nominal group, I expect developed roles, mutual trust and expectations, mutual understanding, etc.

The group members were familiar with each other. They work iogether and usually meet twice a week. Because of this, I expected the group to be cooperative and to reach consensus easily.

\section{Procedure}

The experiment consisted of three phases. In phase one, the subjects were asked to individually rank order 15 items based on importance for survival in the "Lost on the Moon" exercise [4]. In phase two, they were instructed to discuss and arrive at a consensus ranking of the items. In phase three, the subjects individually ranked the items again and completed a questionnaire [13][2]. I designed the questionnaire to qualitatively measure the consensus and subject attitudes toward their group.

\section{Measures}

I used the Kendall Correlation Coefflcient (W) to measure whether consensus was achieved and then to characterize its level. Consensus was achieved if the group gave me a consensus ranking and the correlation coefficient of the rankings done after the discussion increased compared to the correlation coefficient of the rankings done before the discussion. I used the correlation coefficient and I characterized my consensus levels in this way: 
0.0 - .50 In this range I expect to observe characteristics of coercion or compulsion. Consensus is achieved but there is little correlation among the individual group member opinions.

$.50-.75$ In this range I expect to observe a few group members who don't agree with the consensus but decide to accept it after voicing their disapproval. This is characteristic of acceptance.

$.75 \cdot 1.0$ In this range I expect to observe agreement. All group members support the consensus decision but still have slightly different individual opinions.

1.0 Perfect unanimity. If this is achieved, every individual ranking will be the same as the group consensus.

After discussion, the subjects indicated on the questionnaire (see Table 1 for questions) their attitudes toward the group and their opinion as to whether consensus was achieved or not. I used a 7-point scale with 1 labeled "very little" and 7 labeled "a great deal" for the first four questions. The fifth question was labeled "competitive" for 1 , "neither competitive nor cooperative" for 4, and "cooperative" for 7. The closer the average of the first three questions to 7 , the stronger the group feels they achieved consensus. The closer the average response on the last two questions to 1 the more competitive the group and the closer to 7 , the more cooperative.

For this experiment, I define consensus as being successful if the group consensus ranking is closer to the accepted solution than the mathematical combination of the individual rankings. This definition is based on research which has shown "the quality of a group decision cannot be accounced for only by the sum of its constituent individual decisions: the whole appears to be greater then the sum of its parts" [6]. Very seldom will we ever have an "accepted" solution to a problem we need consensus on in the real world. For this study however, we can contrive a correct solution and check the success of the consensus by comparing the consensus ranking to the ranking supplied by NASA [4] and to the ranking methematically generated from the initial individual rankings. The algorithn used to generate the mathematical consensus was taken from Cook and seiford [1]. To be successful, the consensus must be better than the mathematical combination of the initial rankings.

I computed the absolute difference between the group's ranking and the correct ranking to measure the quality of the consensus. I used the same method to measure the quality of the mathematically derived ranking. The lower the score, the higher the quality of the decision.

Results

The correlation coefficient ( $p, .001$ ) of the rankings before discussion was .509. Because group interaction on sone level is necessary for true consensus, this correlation can't be used to characterize any level of consensus present in the group before discussion. The correlation coefficient after group discussion was .895 . Therefore, consensus was achieved at the 
agreement level. The observations of the group were what I expected for this level of consensus. The group members, while having minor differences of opinion, eventually decided that their ranking was the best decision. No one remained opposed to the decision at the end of the discussion. Part of the reason for this might have been the cohesiveness of the group going into the exercise.

The consensus decision reached was not successful. The mathematical combination of the individual rankings produced a mathematicai consensus ranking which scored a 30 when compared to the correct ranking. The group consensus ranking scored a 44 (the closer the ranking is to the accepted solution, the lower the score). Interestingly, the average of the individual scores before discussion was 40 . Other studies have shown that over $80 \mathrm{z}$ of groups usually come to a consensis decision better than the average of their indivijual scores.

The questionnaire supported the finding that consensus was achieved and showed the group viewed themselves as cooperative. All the questions used a 7 point scale where 7 represented a strong consensus and a cooperative situation. The average response for the first three questions designed to measure consensus was 5.87 with a standard deviation of .86 . The average on the last two questions designed to characterize the group as competitive or cooperative was 5.75 with a standard deviation of 1.37 .

\section{Tabie 1. \\ Results of Questionnaire}

Question

1. To what degree do you support the group's solution to the avg. $\underline{s}$ problem you investigated?

2. In general, how satisfied are you with your group right $6.2 \quad .92$ now?

3. How effective do you think your group was in reaching a $5.5 \quad .97$ decision?

4. If you had a choice, would you work with this group again? $6.1 \quad .99$

5. How would you characterize this group, where 1 is very $5.4 \quad 1.65$ competitive and 7 is very cooperative?

\section{DIscussion}

The group achieved a strong level of consensus. This was expected. The group members all knew each other for a significant period of time and had worked together before. This may have contributed to an unsuccessful consensus. Assumptions were made by individuals during the discussion which caused the group to make incorrect choices. These assunptions woron't challenged like they might have been if the group hadn't known each other. Another factor which may have contributed was a percelved time constraint. Although the group had as much time as they needed, many mombers either wanted to get back 
to work or go to lunch. These members pushed the group to finish the discussion quickly. This may have hindered some people from questioning assumptions.

The most significant contributor to the unsuccessful consensus was a lack of information. No one in the group was familiar with the moon or the uses of the items they were ranking. This caused them to make many assumptions in order to arrive at a consensus. Some of these assumptions were wrong. If the group had access to more information about the moon and the uses of the fifteen items, they wouldn't have made some of the assumptions they did and their consensus would have had a much higher probability of being successful. An area for future research would be to give the group information after they've reached their consensus and allow them to change their ranking. I would hypothesize that the quality of the ranking would greatly improve. This exercise could also be done with different groups and different facilitation methods to see which work better to achieve a successful consensus decision. Finally, the questionnaire used in this study can be greatly improved. The questionnaire I used was a rough first attempt to qualitatively measure consensus. It can be improved as I learn more.

\section{ACKNORLEDGECENS}

The ideas presented in this paper represent the cumulative work of hundreds of people over twelve years at Virginia Tech's Management Systems Laboratories. Professional managers from business and industry, and faculey, graduate students, and undergraduates from a wide variety of engineering and other disciplines have participated in the research and development of these tools and techniques. the authors thank them for their good work. The pronoun "I" is used throughout to separate all of us using management systems (we) from the authors (I). However, please recognize "I" is more than one contributor.

The preparation of this paper is funded by U.S. Department of Energy Special Research Grant No. DE-FG02-88DP48058. Management Systems Laboratories thanks the Department of Energy's Office of Nuclear Materials for providing us a real-world laboratory for the research, development, and testing of state-ofthe-art management tools and the franoworks for understanding how to make them successful.

\section{REFERECES}

(1) Cook, W.D. and Selford, L.M., "Priority Ranking and Consensus Formation." Manarement Science, vol. 24(16), 1978.

[2] Destephen, R.S., "HIgh and Low Consensus Groups - A Content and Relational Interaction Analysis." Small Group Behavior, May, 1983.

(3) Drake, M.C., Qunker Consensug: Heloting Leneners Understand and Particlpate in the Ounker Wey of Renching a Group Decision. (Doctoral Dissertation: Ohio State University, 1973.)

(4] Hall, J., "Decisions, Decisions, Decisions." Esychology Today, November, 1971. 
(5) Hirokawa, R.Y., Emergent Issues in Human Decision Making, (Phillips, G.M. and Wood, J.T., eds. Carbondale, IL: Southern Illinois Press, 1985.)

[6] Herbert, T. and Yost, E., "A Comparison of Decision Quality Under Nominal and Interacting Consensus Group Formats: The Case of the Structured Problem." Dectsion Sciences, vol. 10, 1979.

(7) Human Synergistics, The Desere Survivel Sifuation. Lafferty, J. C., consultant, 1974.

[8] Jan1s, I.L., Groupthink. (Boston, MA: Houghton Miffiin Company, 1982.)

[9] Kacprzyk, J. and Fedr1zz1, H., "A 'Soft' Measure of Consensus in the Setting of Partial (Fuzzy) Preferonces." European Journal of Operational Research, vol, 34, 1988.

[10] Lehrer, K., "Consensus and the Ideal Observer." Synthese, vol. 62(1), 1985 .

[11] Price, J.L., Handbook of Organizational Measurement. (Lexington, MA: D.C. Heath and Company, 1972.)

[12] Rawlins, W.K., Emergent Issues in Human Decteien Making, (PhIllips, G.M. and Wood, J.T., eds. Carbondale, IL: Southern Illinols University Press, 1984.)

(13) Tjosvold, D. and Field, R., "Effects of Social Contoxt on Consensus and Majority Vote Decision Making." Acadnny of Management Journal, vol. $26(3), 1983$.

[14] Van de Ven, A.H. and Delbecq, A.I., "The Effectiveness of Nominal, Delphi, and Interacting Group Decision Making Processes." The Journal of Soctal Psychology, vol 125(3), 1985.

[15] Zahed1, R., "Group Consensus Function Estimation When Preferences Are Uncertain." Operations Resenech, vol. 34(6), 1986. 
D. Scuvali Buruba, Vlisisu

llarold A. Kurstedt, Jr., VPI\&SU

R. Mililli Jollin, Virissu

\section{ABSTRACT}

The design of information systems is scill very much an art. We design information systems by looking at the characteristics of information, decistons, and the manager. For information system design to move from an art to a science, wo need a detalled understanding of data, information, and the process by which data become information. Information systems are falling because system designers and operators don't have enough detailed understanding of data, information, and the data-to-information process to satisfy managers' information needs.

There currently are no comprehensive frameworks that define data and information in enough detall to describe the data-to-information process. Worse, there's generally confusion about the terms "data" and "information", and the two terms are often used interchangeably [1]. Upon close examination of the literature, I've found definitions and relationships that each serve to describe a portion of the data-to. information process. By assembiling these definletions and relationships, I'Il dovelop a framework to describe data, information, and the relationships between daca and information.

I'll define data, and show the components of a datum and how they work together. I'll describe the fundamental types of data, and how these types combine to produce quantitative information. I'li show how quantitative data are converted to qualltative data, which can be used for decision-making. I'll describe the role of the manager and his or her cools in the data-to-information process, and the Interface between the manager and the tools. I'll then discuss the implications of this data and information framework for information systems, and describe areas for future research.

\section{DATA AND DATA COMPONENTS}

Data are what are commonly referred to as facts. They serve as a surrogate for the things we manage by identifying the attributes of the things we manage. Bender (2) defines data as symbollc representations of reality. These symbolic representations, or facts, describe reallty though its attributes. If we want to know the performance of a machine, we measure attributes of the machine, such as lts operating speed, its throughput, its downtime, or others. Each attribute is a symbolic representation of the machine, and ach is a datum.

There are two components of each datum: a kernel, and a set of speciflers. The kernel is the actual value of the attribute. The kernel is called a "fact" by Appleton (1) and IJiri [3]. A kernel has the pote..izal to mean something to a manager, but by itself, a kernel is meaningless. Wo have to add ingredients, called specifiers, to get meaning. Specifiers at called "meaning" by Appleton.
Appleton's "meaning" can apply to zero, one, or many "facts", but my set of specifiers uniquely defines one kernel. Recording data kernels rumoves them from context. Specifiors are used to carry the context of the kernel to the manager who uses the data. When I measure the throughput of a machine as 23 units. It's easy for me to see that the units are widgets, and the measurement was taken at 3:00 PM on July 22. When I record the number 23 on the data collection sheet, all the speciflers are lost and the kernel has no context. Specifiers must be included with the kernel to convey the context of the data to the manager. Specifiers fulfill the requirement of data by Iflri, who says data must be well-specified.

\section{TWO DATA TYPES MAKE INFORMATION}

Peterson [4] says data acquire meaning when compared to other data. Peterson uses the word "meaning" to denote something managers can use to make declsions. Comparison is the way data become information. The comparison of any two data produce information. Each datum plays a special role in producing information. One datum is selected to provide Information to the manager. This datum I call an indicator. To use this Indicator to produce information, a benchmark must be selected to compare the indicator against. This benchmark I call a reference. Kurstedt [5] shows data being compared to setpoints or references to generate information. Togethar, one indicator datum and one reference datum make an information packet. In this information packet are all the ingredients to produce information.

The Information packet is evaluated in two steps to produce information. Recall that the Indicator and the reference are both data, and each has a kernel and a set of specifiers. In the first step, the indicator kernel is compared to the reference kernel to determine the difference. This comparison may be expressed as a percentage or as the numeric difference between kernels. The second step is to compare Indicator speciflers with the reference specifiers. The specifier differences describe what the kernel differences can be attributed to. To evaluate the production of a machine thls week, I might compare it to the production of the same machine last week. The difference in the number of units prodised each week is the kernel difference. The specifier difference is last week as opposed to this week.

Quantitative information is produced by evaluating the information packet. Quantitative information describes the differences between the indicator and the reference datum. Quancitative information might be "widget production for machine $A$ is up 12 this week from last week". The comparison of the indicator and reference data through evaluation of the information packet is ovident in the quantitative information. The difference in speciflers is the week during which each datum was collected. The kernel difference is 128, so the indicator kernel was 128 greater than the reference kernel. 
The manacer now has quantfative linformation that widget production for machine $A$ is up 128 from last week. Is this good or bad? It would be good if the manager needed more widgets, but what if the manager is overstocked with widgets? Quantitative information will not support magers' decisions without further processing. The quantitative information must be converted to qualltative information to be used for decisions. Qualitative information are the triggers to action for inanagers. Qualltative inforination is the subjective incerpretation of quantitative Information, and is expressed in terms of good, bad, too much, too little, go, no go, etc.

The process of going from quantitative to qualltative informicion is complex. The amount of quantitative information required to produce qualitative information varies according to the type of decision being made. Gorry and Scote Morton [6] use Thompson's (7) two decision dimensions to describe unstructured and structured decisions. Decisions are elther certain or uncertain in two dimensions: bellefs about cause/effect relations, and preferences regarding possible outcomes. Structured decisions have certainty in both causation and preferences regarding possible outcomes, while unstructured decistons have uncertaincy in both dimensions. Semistructured decisions have uncertalnty in elther the rausation dimension or preferences regarding possible jutcomes. For a structured problem, a manager needs only a few pleces of quantitative information to make loglcal qualitative extrapolations with confidence. If the manager faces unstructured problems, where uncertainty exists in causation and preferences regarding possible outcomes, more pleces of quantitative information are needed to make logical qualitative extrapolations with confidence. These qualitative extrapolations from quantitative information are qualitative information, which can be used in decisilon-making.

\section{SUMMARY OF THE DATA-TO-INFORMATION PROCESS}

Data are representations of reality. They represent reality by describing attributes of reality. A datum consists of a kernel and a set of specifiers. The kernel is the actual value of the atcribute, and specifiers uniquely define the kernel and provide context. When a manager needs information, two data eypes are needed: an indicator and reference. The indicator datum represents what the manager wants information about. The reference datum is selected as a benchmark to evaluate the indicator. An indicator datum and a reference datum make up an information packet, which can be evaluated to produce quantitative information. The evaluation is two steps: tho indicator and reference kernels are compared, and the Indicator and reference specifiers are compared. Quantitative Information is generated by evaluating an information packet, and expresses the indicator datum in terms of the reference datum. Qualitative information is the subjective evaluation of quantitative information. As the problem becomes more unstructured, more quantitative information is needed before qualitative information can be produced. Qualltative information are triggers to action, and can be used in decision-making.
A manager converts Information to action through decisions (8). Most managers rely on informacion tools to provide them data and information. Together, the manager and the tools perform the data-toinformacion process I've described. The point in the data-to-information process at which the tools stop and the manager continues the process is the point at which portrayal occurs. Portrayal can be data, quantltative inforination, or qualleative inforination. Kurstedt (5) defines four portrayal formats: tables, graphs, checklists, and narrative. Daft and Lengal [9] define five media types: numerle, wrieten formal, written personal, celephone, and face-to-face. The purpose of portrayal is to convey the results of the data-to-information process to the manager at the approprlate step in this process. The appropriate step should be the point at which the manager is cafable of completing the process without further help from the tools.

Portrayal takes place at different steps for different managers and different situations. The portrayal interface between the manager and the tools depends on the manager's experience and knowledge, organizational level, personality type, and the complexity of the problem to be solved. Managers can perform the entire data-to-information process, as when a manager walks around his or her domain to observe the operations first-hand. Managers can also perform little of the data-to-information process, as when a manager hires a consultant to provide quelitative information for decision-making. In this case, the consultant is acting as an information tool for the manager.

In summary. each step in the data-to-information process must be completed if information is provided to managers for use in decision-making. The manager and her or his information tools perform this data-toinformation process. At the point where the tools stop and the manager picks up the data-to-information process, portrayal takes place. This portrayal should occur at the step where the manager can complete the data-to-information process without further help. The portrayal interface between the manager and the information tools takes place at different steps in the data-to-information process doponding on the manager's experlence and knowledge, personality type, organizational level, and the complexity of the problem.

\section{IMPLICATIONS FOR INFORMATION SYSTEMS}

Information systems should be built and operated with an understanding of the daca-to-information process they support. The data-to-information process serves as a guide to providing the right data and informatiun to managers by showing the data components and data and Information types, and how data become information. Further, the portrayal interface between the information tools and the manager describes the right way to provide data and information to the manager. If the tools complete more steps than needed by the manager, the information tools are inefficlent, and the manager loges the ability to use his or her personal preferences in the data-to-information process. If the information tools complete fower steps in the process than the manager needs, the portrayal will be incomplete, and the manager will be unable to produce qualitative information in a timely 
manner. The data-to-information process points the way to the effoctivonoss of the inforination tools (getting the right data and information to the inanagor) and tho corroct portrayal incorface poincs coward the efflclency of the information tools (providing data and information tho right way).

\section{AREAS FOR FUTURE RESEARCH}

More work is required on the data-to information process to more accurately describe the relationship between quantitativo and qualitative information. As task complexity increases, more quantitative information is required to produce qualitacive information. The relationship between the amount of quantitative information and task complexity should be studied to better define this relationship.

Much work remains to be done on the portrayal interface between the manager and the informacion tools. The poine at which this interface takes place should be explored to see whether it can be predecermined for managers performing certain decisions. If not, the best solution may be a flexible portrayal interface built inco the information system that allows managers to decide the point at which the information tools turn the datato-information process over to the manager.

\section{ACKNOWLEDGEMENTS}

The ideas presented in this paper represent the cumulative work of hundreds of people over twelve years at Virginia Tech's Management Systems Laboratories. Professional managers from business and industry, and faculty, graduate students, and undergraduates from a wide variety of engineering and other disciplines have particlpated in the research and development of these tools and techniques. the authors thank them for their good work. The pronoun "I" is used throughout to separate all of us using management systems (we) from the authors (I). However, please recognize "I" is more than one contributor.

The preparation of this paper is funded by U.S. Department of Energy Special Research Grant No. DE. FG02-88DP48058. Management Systems Laboratorles thanks the Department of Energy's Offlce of Nuclear Materials for providing us a real-world laboracory for the research, development, and testing of state-ofthe-art management tools and the frameworks for understanding how to make them successful.

\section{REFERENCES}

(1) Appleton, Daniel S. Morganizing and Managing Information Resources in the Data-Di:iven Encerprise." Industrial Ensineerting (June, 1986), PP. 62.73.

(2) Bender, Paul S., Resource Manazement (New York, John Wiley, 1983, pp.108-115).

(3) IjIri, Y. Theory of Accounting Measurement (Sarasota, American Accounting Assoclation, 1975, p. 36).
(4) Peterson, Richard E., "The Components of Inforination." Interfoces, Vol. 7, No. 4 (August, 1977). Pp.87.91.

(5) Kurstede, H.A., Jr. (1985). Serles of working articles doscribing the Managoinont systor Model. Article "l: "The induserlal englneer's systematic approach to management." Article \#2: "The Management System Model helps your tools work for you." Artlcle 3 : Managling a management systoin roduces to concentrating in the Interfaces between components." (Drafts avallable from Management System Laboratories, VIrginia Tech, Blacksburg, VA).

16] Gorry, G. Anthony and Michael S. Scott Morton, "A Framework for Management Information Systems." Sloan Manarement Review, Vol. 13 (Fal1, 1971), Pp. 55-70.

(7) Thompson, James D., Organtzatiens in Action (New York: McCraw-H111, 1967).

(8) Drucker, Peter F., The Effective Executive (New York: Harper and Row, 1966).

(9) Daft, Richard $L$. and Robert $H$. Le "Information Richness: A New Approach Managerial Behavior and Organizational Design." Research in Oreanlzaclonal Behavior, Vol. 6 (1984), Pp. 191-233. 


\title{
RADIOACTIVE AND HAZARDOUS MATERLALS TRANSPORTATION: WHAT LOCAL OFFICIALS ARE TELLING US
}

\author{
J. Andrew Walker \\ Management Systems Laboratories \\ Virginia Polytechnic Institute \& State Univ. \\ 1900 Kraft Drive \\ Blacksburg, VA 24060 \\ (703) 231-3501
}

\author{
George E. Ruberg \\ Management Systems Laboratories \\ Virginia Polytechnic Institute \& State Univ. \\ 1900 Kraft Drive \\ Blacksburg, VA 24060 \\ (703) 231.3501
}

\author{
Susan H. Denay \\ U.S. Department of Energy \\ Transportation Management Division \\ 19901 Germantown Road \\ Germantown, MD 20545
}

(301) $353-2498$

\begin{abstract}
This paper summarizes the results of a 1989 interactive meeting of U.S. Department of Energy (DOE) representatives with over twenty local government officials from cities and counties around the country. The DOE officials were from the Transportation Management Division and the local officials were primarily senior and mid-level managers with interests in transportation and emergency management.

Management Systems Laboratories of Virginia Tech, in coordination with the Energy Task Force Management Corporation (now called the Urban Energy \& Transportation Corporation), designed and facilitated the meeting with these goals:

- Share information that local government officials can apply to their own communities

- Exchange experiences and ideas applicable to other emergency management programs

- Identify areas of productive action for DOE and local government to address issues of mutual concern
\end{abstract}

The highlight of the meeting was a Program Planning Exercise. The participants, playing the roles of Federal managers in DOE, developed programs to address the concerns of local governments on the subjects of transportation of hazardous and nuclear materials, and emergency preparedness related to incidents involving shipments of thoce materials.

\section{BACKGROUND: DOE'S EFFORT TO NETWORK WITH LOCAL GOVERNMENTS}

DOE's Transportation Management Division (TMD) is responsible for supporting DOE programs in the movement of all materials, including the development of packaging and systems for transporting radionctive materials. TMD also coordinates DOE's program for regulatory and policy development in this area.
As part of a Transportation Management Division ongoing effort to eatablish a network of local government contacts, they sponsored four major workshops -- one of which this paper describes -. and a national conference during 1988 and 1989. The 1988 workshops focused on issue identification and prioritization, while the 1989 programs began to develop policy recommendations and action items as well as specific products that local government participants could take back bome. 
More than 100 local government participants at these programs represented professions such as law enforcement, emergency management, fure protection, environmental planning, public health, and elected and appointed officials. They came from over 50 cities and counties and were generally senior managers within their agencies: division, battalion, and depury chiefs in fire departments; heads of public bealth, public safety, and emergency management departments; environmental and emergency medical program coordinators; and city managers and council members.

\section{PROGRAM PLANNING EXERCISE METHODOLOGY}

The Program Planning Exercise used meeting techniques designed to develop ideas for a Department of Energy program plan to address local government concerns about emergency preparedness for auclear materials transportation incidents. Participants were asked to play the role of a staff member for a DOE administrator, to review a report identifying local government concerns (the "report" used was based on the actual product of an earlier workshop), and to develop a program plan for FY 90 and subsequent years to address those concerns.

We designed a structured process to accomplish our objective and maximize our limited time. The process was interactive, collectively building a consensus plan based on both individual participation and team thinking. The exercise placed the participants in the role of a Federal manager faced with a difficult job-trying to satisfy transportation concerns while staying within organizational constraints. We hoped the result of our participative exercise would be two-fold: tirst, to generate a program of iritiatives to address local concerns (and sugezestions for their implementation); and second, to fouter a better mutual appreciation of the problems facing all of us' in developing such a program.

To do this we designed an exerciee with three main parts. First we divided into teams for the purpose of designing programs to meet our objective. For the second part we reconvened to have team leaders report back to the larger group on the initiatives and implementing steps developed by each team. And for the third part we discussed the various aspects of each plan and achieved consensus on a composite program plan that might serve as a roodel fo: continued cooperation between DOE and local governments.

\section{PROGRAM INITLATIVES RECOMMENDED BY LOCAL OFFICLALS}

The teams initially came up with a list of over 50 program recommendations covering areas such as emergency response information; public education; training; planning support; regulations and enforcement; and institutional arrangements. From this list, the teams selected the nine detailed program suggestinas. For each initiative, they also listed the desired outcomes, significant milestone? resources, and other items which each team of role players identified. The key recommendations from the exercise are summarized below:

1. Develop a central database of radioactive materials transportation information with electronic access by local governments.

2. Expand the existing public information program.

3. Facilitate the development of national standards of operation and training for emergency responders.

4. Establish a Nuclear Transportation Advisory Committee made up of interested (targeted) Federal agencies, States, and local governments.

5. Develop a cooperative Federal training program for local first responders and managers, with representatives from all Federal agencies involved in nuclear materials transportation.

6. Support general public education on transportation and repository programs to reinforce credibility with targetdd audiences.

7. Develop an integrated training program to certify first responders for radioactive materials transport accidents. 
8. Provide policy guidelines and procedures for local governments to incorporate into their overall emergency plans.

9. Require States to notify local jurisdictions on some radioactive material shipments, through Federal legislation or regulations.

\section{WHAT LOCAL OFFICIALS ARE TELLING US}

Based on the Program Planning Exercise and the other agenda items at the Workshop -- including presentations by local government representatives of problems and good practices in their own jurisdictions -- local officials are delivering a consistent set of messages:

1. They view Federal programs as disjointed and uncoordinated. Local officials may be unaware of many inter-agency coordination efforts.

Nevertheless, they consistently called for greater coordination among Federal agencies, especially in the areas of training delivery, resource sharing, and research.

2. They cited many ways the Federal government could help which did not involve a direct punchase, subsidy, or transfer of funds for local governments. Unfortunately, their suggestions often require a greater time and manpower commitment, as with recommendations for more participation in emergency response planning efforts with local governments and working with local committees to resolve issues such as routing, and chain of command, mutual aid, and communications during emergencies.

Additionally, local officials feth thet Federal agencies could contribute to their profestional development, for example, by working with national standard-making and certification organizations such as the National Fire Protection Association, and by providing technical guidance on nuclear hazards and safety.

3. Local officials want to increase their capability to directly access Federal databases and decisionmakers. They want this partly because the cities and counties represented (most of them large and mid-size juriscictions) felt they had the means and the aeed and partly because they were diseatisfied with the States' role as information middlemen. Thus, they called for electronic access to DOE's transportation databases, representation on Federal advisory committees, and Federal insistence that States do more and share more with local governments, even calling for Federal regulatious requiring States to give advance shipment information to local agencies. They also want to see DOE meet with and work with their membership organizations and associations that develop policy recommendations and propose legislation related to nuclear and hazardous materials transportation.

4. In conjunction with the expanded community relations effor discussed in item 2 , they believe that $D O E$ needs 10 greatly axpand and upgrade its public educcation program. Workshop participants said that DOE needs to focus on more specific audiences and to be more diverse in its efforts to expand community awareness and distribute information, for example, by developing education programs or workshops for members of local judicial systems.

\section{EVALUATION}

We distributed a questionnaire to all workshop participants. All respousdents said the Program Planning Exercise was very useful in providing an opportunity to develop specific program ideas for DOE that would directly address their local needs.

Some other general and logistical suggestions included:

- Add more time for thorough discussion; not enough time to agree on common thought; devote a whole day to the Program Planning Exercise

- Use a pro-con presentation, then discussion, for some items

- Make the workshop more task oriented. with an even greater emphasis on outputs

- Focus on one or two specific issues at each workehop

\section{CONCLUSIONS}

The objective of our exercise was to develop a Department of Energy program plan. The initiatives address the concerns of local governments in the transportation of hazardous 
and nuclear materials and emergency preparedness related to potential incidents involving shipment of those materials.

We believe the Program Planning Exercise was successful in developing the set of recommendations discussed bere because: (1) we designed a structured method, which clearly spelled out the steps the participants should take to reach the objective; (2) we used a modified nominal group technique which gave people time to generate and list ideas before they were discussed and elaborated on; and (3) we incorporated consensus-building, teaming, and role playing techniques, which encouraged participation and creativity and even made it fun.

We believe the workshop overall was a success because of the collegial atmosphere, which emphasized what local governments could share with one another as well as what local and Federal agencies think each could do to improve their programs The workshop also had a good mix of new participants along with people who had attended previous workshops or were familiar with DOE programs or nuclear materials transportation issues.

\section{ACKNOWLEDGEMENTS}

The ideas presented in this paper represent cumulative work of bundreds of people over twetve years at Virginia Tech's Management Systems Laboratories. Professional managers from business and industry, and faculty, graduate students, and undergraduates from a wide variety of engineering and other disciplines have participated in the research and development of these tools and techniques. The aubors thank them for their good work.

The preparation of this paper is funded by U.S. Department of Energy (DOE) Grant No. DEFG02-88DF 48058 and by Energy Tack Force Management Corporation (now the Urban Energy \& Transportation Corporation) Cooperative Agreement No. DE-FCO2-87CH10343. The authors thank DOE's Transportation Management Division for providing us a real-world laboratory for the research, development, and teating of stateof-the-art management tools and the frameworks for understanding how to make them successful. 


\title{
TABLETOP EXERCISES: COOPERATIVE TRAINING FOR TRANSPORTATION INCIDENTS
}

\author{
J. Andrew Walker and George E. Ruberg \\ Management Systems Laboratories \\ Virginia Polytechnic Institute and State University \\ 1900 Kraft Drive \\ Blacksburg, Virginia 24060 \\ (703) $231-3550$
}

\begin{abstract}
Responding to transportation incidents frequently involves the cooperative efforts of local, state, and federal officials. Rarely do they get the opportunity to work together before the stress and strain of a real incident. Tabletop exercises provide part of the solution. They are an effective method for cooperative training of emergency managers and responders for transportation accidents.

Tabletop exercises assemble the right people together and mentally simulate a problem that requires their collective response to mitigate. They don't require the high resource and budget costs of full-scale exercises.

We've worked with emergency managers and responders at all levels. We understand the usefulness of tabletops and how to design them to fit specific applications. Our paper addresses why tabletops are particularly suited to the special problems of training people for transportation incidents and gives "how-to" guidance in the form of checklists for preparing and conducting tabletops.
\end{abstract}

\section{PROBLEM STATEMENT}

Two overarching assumptions permeate planning for a potential transportation emergency involving radioactive materials. First, local responders have the responsibility and jurisdiction to protect life and property. Second, selected Federal agencies and the state where an accident occurs will provide technical assistance to local governments when requested. Local emergency plans, when they exist, are based on these assumptions. Plans envision a coordinated response by local fire and police hazardous materials specialiets supported by the technical resources of state and Federal agencies. Any transportation accident involving radioactive materials will by definition be sionificant and involve multiple responders from various levels of government. As a result, the foundation of transportation emergency response for accidents involving radioactive materials is on coordinated and cooperative mobilization and use of established and tested intergovernmental resources.

Several related problems exist that separate plan from practice. First, the dependence on local agencies supported by other levels of government for emergency response makes practical sense but increases the complexities of coordinating potential responders. In many instances complexities haven't been resolved and will adversely affect the application of multiple agency resources to a transportation accident. Second, the team that doesn't practice together doesn't play well together. What's true on the basketball court is also true in emergency response. Local, state, and Federal agencies rarely get the opportunity to practice their roles and work out problems. Third, emergency management systems for the transportation sector bave different variables than fixed facilities. The wider geographical location of potential incidents and the variety of materials and modes of transportation that can be involved increase the complexity and cost of reviewing, training, and testing the emergency system. Tabletop exercises are a method that can successfully resolve problems and issues related to preparing for transportation accidents.

\section{WHAT ARE TABLETOP EXERCISES}

Tabletop exercise" refers to the process of exercising one or more elements of an emergency management system by assembling, usually around a table, the people who would be involved in one or 
more of the elements of the response to an actual incident. A scenario is presented "as if something had actually occurred. This allows observing the extent to which the system element(s) enable clear and adequate communication and mitigation. Although tabletop exercises may appear to be primarily testing the people around the table, this is not the case. The focus is on testing the system and training responders both as individuals and as a unit. Exercise play can benefit from scenario enhancing tools such as videotapes to belp disclose scenarios. Exercises designed specifically for first responder training can be enhanced with the use of props, such as model layouts and maps.

Here's how a typical tabletop exercice session goes: Exercise participants are seated together and are presented with a series of simulated problems based on a scenario. These problems are presented through questions. The exercise is moderated to control play and can be formal or informal; that is, it can be formally evaluated or used as an occasion for brainstorming to motivate informal interchange. It's designed to elicit discussion by the participants as they attempt to resolve problems based on their assigned responsibilities. What they in effect do is describe what they see as their responsibilities and how they think they'd discharge them.

\section{ADVANTAGES OF TABLETOP EXTRCISES}

There are several mutually supportive advantages of tabletop exercises. These include: establishing and maintaining relationships between people and organizations, providing interesting training, adding a flexible and adaptable tool to the manager's toolkit, and accomplishing gonls efiiciently. Each of these advantages is elaborated below.

\section{Teambuilding}

A powerful advantage of tabletop exercies is teambuilding. Considering the multiple skills and organizations that are a part of transportation accident response, tabletop exercises are particularly well suited for getting people together and stepping them through their planned responsibilities and actions. Because a tabletop is accomplished in controlled phases to allow discrete, individual answers, group attention is focused on each point of the response. A common understanding of individual roles and responsibilities and the entire response sequence is gained by all participants through group discussion. This allows participants to find the strengths and weaknesses in their individual and collective response and to suggest improvements. The assembly of all potential responders at a cisutral location with each individual and group able to observe and discuss responsibilities and actions is a unique attribute of tabletop exercises.

\section{Interestine Training}

There is little to encourage response personnel to pick up emergency plans and study them as though their lives depended on it. Emergency plans and procedures can make pretty dull reading, especially since they have no "life" except as part of the drama that includes other players actually using the' procedures in a response. Tabletops, unlike individual review, are thorough rather than perfunctory, interesting rather than dull. Systems that depead on individual review without simulating an emergency doa't syatematically collect complete and substantive data. Tabletops generate quality data because as the scenario unfolds, players discover together gape, overlaps, and inconsistencies in the response sequence and pinpoint specific needs for upgrades in training, equipment, and in the system itself.

Full scale, field exercises also have drawbacks. First, although escential for formal systems validation, they can be premature. Full-scale teating of a syatem that hasn't had interim acceptance teats conducted during development and the necesenry system modifications made along the way wastes resources, makes it more difficult to identify specific problem areas, and causes a general feeling of frustration among responders when evaluators find deficiencies in the system and their performance. Second, field exercises are highly visible. Confidence in the system must come before a craded field exercice. Teating a poorly developed syatem can result in a loss in system confidence by emergerecy responders and outside obeervers. It may also result in unwanted (and unnecesenry) bad publicity. 
Most emergency management systems rely heavily on individual review or field exercises for finding areas for improvement. Tabletops are a logical link between the extremes of individual review and major field exercises. They provide a continuing way to conduct interim acceptance testing and training during systems development and modification. Tabletops allow, and indeed encourage, participants to collectively suggest and agree on improvements, learn through participation, and build individual confidence plus confidence in the system.

\section{Elexibility and Adaptability}

As flexible tools, tabletop exercises can have a variable focus--on the whole response system, parts of the system, an isolated problem area that requires attention, or in condusting an appraisal. Exercise complexity can be adjusted to fit the exercise objectives.

The tabletop exercise process gives emergency managers an adaptable tool. As we've established, they can be used for formal validation or for informal brainstorming, problem solving, or teambuilding. The versatility of tabletops also permits their adaptation to all stages of emergency preparedness--development, teating, maintenance, and revision. The power behind this versatility-and perhaps the most overarching benefit of tabletops--is that they require response system elements to pay attention to the syctem boch as it initially develops and as it evolves. The simple fact is that mout of the people who muat perform difficult tasks in concert during an emergency have little opportunity to practice their parts, as individuals or as groupe.

\section{Efficiency}

All these benefits can be sccomplished costeffectively by using tabletop exercises. They don't require the direct participation of all system elements or the mobilization of expensive facilities and equipment, and they eliminate many safety and liability concerns.

\section{CHECKLIST FOR DESIGNING AND CONDUCTING TABLETOP EXERCISES}

Just as in emergency response, planning is the foundation of any successful exercise. Although the concept of a tabletop exercise is commonly understood, the full realization of its advantages requires planning.

Our checklist (Figure 1) provides a systematic way for planners to design and conduct a tabletop exercise. The checklist is a condensed version of a larger workbook and checklist which also provides tips for conducting a tabletop, such as, starting and finishing promptly, remembering to make appropriate introductions and review player packages before play begins, reading exercise messages out loud, and building flexibility into the schedule and script. The checklist provides a summary of the major steps in design, implementation, and follow-up.

\section{ACENOWLEDGEMENTS}

The ideas presented in this paper represent cumulative work of bundreds of people over twelve years at Virginia Tech's Management Systems Laboratories. Professional managers from business and industry, and faculty, graduate students, and undergraduates from a wide variety of engineering and other disciplines have participated in the research and development of these tools and techniques. The authors thank them for their good work.

The preparation of this paper is funded by U.S. Department of Enersy (DOE) Grant No. DEFC02-88DP48058 and by Energy Task Force Management Corporation (now the Urban Energy \& Transportation Corporatioa) Cooperative Afreement No. DE-FCO2-87CH10343. The authors thank DOE's Transportation Management Division for providing us a real-world laboratory for the research, development, and testing of stateof-the-art manapement tools and the frameworks for underatanding how to make them successful. 
DESIK.N

II DEFINE GOALS:

- derermine needs and select npe -imaroduce/dervelop new concepts - oraining

-mainicuance

- malidation

- prioritize mulaiple eonls

- recogrize hidden aquendas

(] ESTABLISH OBJECTIVES

- liss abjectives

- ensure objectives suppon goals

II SELECT PARTICIPANTS BASED ON GOALS AND OBJECTMES

II DETERMINE SCALE AND SUPPORT REQUIRED

- select night parriciparis and get them there

- select nght paricipants and get

- set specific, detailed

- Iogistics

- exercise moderabion, evaluation, and support roles

II CONSIDER DETAILS

- rime, place, and seating

- social aspects

- poll key paricipants

- destribute advance notice

- name renis and rags

- microphones

- audio and video reconding

- videorapes, slides, overheads

- maps, building lonoues.

- creative props 10 increase realism
IMPLEMENTATION

I) SELECT TABLETOP LEADERSHIP AND PARTICIPANTS

- Moderivar

D CHOOSE A CREDLLE SCENARIO

- ensure ir supporas you goals and objectives

- ensure secenorio accurreg, consider. -incident and hored rechuical den

- juriedictional invahument

- consider using hiseorical informanion

- consenter rechnical expers

\section{PHASE THE SCENARIO}

- breat scencrio inco phases for incremeneal disclosure - conver each phase invo details necessary lo stimulare plag

pevercise mexsones

- moderwor questions and expecied responses

II DEVELOP QUESTION MATRIX

- parricipane liss us. scenario phases

- beck-jit scenario as required

I DEVELOP EXERCISE MATERLAL PACKAGES

- participare package

- introduction and agenda - abjectives and nules of play

- room lajour and seating enangements

- room lagoun and seating

- initid scenario

- maining materials and documentasion

- modenitar package

- duplicases final parricipants packese

-erencise messazes

modemer questions and expected responses

- evoluator package

- modified panicipanis packase

enoluations tools (iimeline, checklists, namative

semmmany'

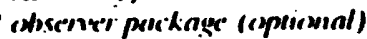

- comphossze bole fon obsements
FOUNOW-UP

CHOOSE THE METHOD(S) THAT SUPPORT(S) YOUR GOALS

D PARTICTPANT SELF-CRTIQUE METHOD

- conduct at ibe conchasion of die cablesop

- use norind robin rechinique

D RECORDER AND MODERATOR OBSERVATIONS METHOD

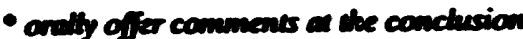

- we nocies 10 docament problemes and ponericial

solumions

- discress each observation and solumions

D FORMAL EVALUATION METHOD

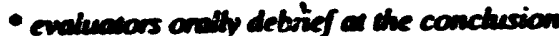
- conrect envos in obsenvaions -present obscrived strengeths and meatonesses - seek consencetive inpain

- wriver folle w-rp rapor

CHOOSE TOOL(S) TO SUPPORT METHOD OF FOLLOW-UP

I) ORAL COMMENTS AND SELF-CRTIQUE FORMS

| RECORDER AND MODERATOR NOTES

- cosel pad noves

- Madesenor Pactage

D EVALUATHON MODULES

- aimelimes

- chectlises

- nacrative summary

SELECT FOLLOW-UP ACTMTTIES AS NEEDED?

II FINAL REPORT

II ASSIGN CORRECTIE ACTIONS

II ESTAHLISII AIII.ESTONES FOR IMIULEAIENTATION

II INFORMS MIANACEMMEA 


\section{RERERENCES}

C. BATTEN, Transportation Emergency

Preparedness: Are We Ready,"

Proceedinox of the American Nuclene Socievy

Topical Meeting on Emereency Response:

Planning. Technolories, and Implemencation.

September 1988.

S.L. HERMANN, "Readiness for Radioactive Materials Transport Accidents - A State

Perspective," Proceedinne of Wrute Manneement

'89. Volume 1, ed. R.G. Yost, February 1989, 783-

786.

WJ. PETAK, "Emergency Management: A Challenge for Public Administration," Public Administration Review, Vol. 45, Special lssue (January 1985), 3-6.

U.S. DEPARTMENT OF ENERGY, August 1989.

Transporting Radioactive Material. Answers to Your Questions,"

JA. WALKER, and L.I. MIDDLEMAN, Tabletop Exercise Programs Complement Any Emergency Management System," Broceedinm of the Amerienn Nudene Socrietv Toricol Meetine on Emergency Reaponse; Plnnninc. Technolocies and Implementation September 1988. 


\title{
PROGRAMMATIC ISSUES AFTECTING THE IMPLJMENTATION OF THE DOE FIVE-YEAR PLAN
}

\author{
Harold A. Kurstedt, Jr. \\ Managemeat Systems Laboratories \\ Virginia Polytechnic Institute and State University \\ 1900 Kraft Drive, Blacksbure, VA 24060
}

\begin{abstract}
In this paper, I identify programmatic challenges the U.S. Department of Energy's Office of Eavironmental Restoration and Waste Management (EM) faces in the implementation of its five-ycar plan. I've characterized these challenges in the form of propositions, written specific challenges for these propositions, and summarized EM's situation in dealing with the propositions and related challenges. I offer suegested approaches for addressing the global propositions and meeting the more-detailed challenges, and I conclude with what I believe are the make-or-break situations for EM.
\end{abstract}

\section{INTRODUCTION}

Last fall, the United States Department of Energy (DOE) completed its five-year plan for environmental restoration and waste management. DOE reorganized and elevated its responsibilities in waste and the environment by forming the Office of Environmental Restoration and Waste Management (EM). Now EM must implement the plan. In doing so, EM faces many difficult technical challenges. But the programmatic challenges may be even more difficult. I believe EM is facing one of the greatest challenges in this nation's history. This challenge is really a number of interwoven challenges of global significance.

First, I've identified a number of programmatic challenges based on the gestalt of reviewing a large number of DOE documents and of knowledge gained from 13 years experience in studying and supporting DOE's waste managers. I've characterized the challenges in the form of propositions and sorted these propositions by whether they're controlled by EM or nor; that is, whether the proposition reflects forces internal or external to EM. Structurally, I've identified three internal and three external propositions. For each proposition, 1 intend to state the "fact of the matter." Then I've written a number of challenges each proposition poses for EM. Ive summarized EM's situation in dealing with the propositions and related challenges. I've included the propositions, challenges, and situations under the heading Propositions for Ideatifying EM's Challenges.

Second, l've sugrested approsches for addrescing the global propositions and meeting the more-detailed challenges. My surgested approsches aren't one-forone with either propositions or challenges. Rather, the approaches are general, intended, when taken together, to synergistically address all challenges. I've included the suggested approaches under the heading Suggested Approaches to Meeting the Challenges.

Third, I've ideatified what I think are the make-or. break situations for EM. I've written those situations :terms of three time periods: near-term, mid-term, an: long-term. I've included these situations under the heading What's Going to Make or Break EM.

In discussing EM, I recognize the contribution and the historical carryover from the Office of Defense Waste and Tramsportation Management (DWTM), the Office of Defease Waste and Byproducts Management (DWBM), and earlier DOE waste organizations.

\section{PROPOSITIONS FOR IDENTIFYING EM'S CHALLENGES}

When considering almout two dozcn programmatic challenges, I found they fit neatly into the framework in Fie 1. Furthermore, I found the "fact of the matter," or proposition, behind the various challenges in each of the framework elements could be summarized into one proposition. Finally, when evaluating and sorting all the propositions and challenges, one superordinate proposition surfaced.

In considering the forces causing the challenges, the superordinate force is the enormous change in EM's world. EM can control part of its world; part of its world it canact. The enormous change external to EM and outside its control effects enormous change within its controh as it responds to the external forces.

Exteral to EM, changes in intensity, kind, and implementation of senutiny and expectation of EM and the effects of DOE's larger mission on EM are forcing 
EM to be different from earlier DOE waste organizations. Forces internal to EM and influenced by changes within DOE cause changes for dealing with external forces. To meet sarytiny changes, EM must change its culture and the munneement process that translates the culture into practice. To respond to more, more-intense, and different expectntions, EM must adjust its resources. In dealing with its share of the DOE mission. EM must develop an effective stratery and ormanization to carry out the strategy.

\section{SUPEAOROINATE FORCE: ENOAMOUS CHANGE}

Extems Forces

serutiny

expoctations

DOE mission
Internal Foreses

culura/management process

resourese

strawey/organization
Fig. 1. The superordinate force of enormous change affects all three pairs of external and internal forces.

The variables within propositions resulting from external forces are generally uncontrollable. Propositions resulting from internal forces are within EM's control, and reflect a corresponding set of external forces. In Fig. 1, I have a one-to-one relationship between three external-internal force pairs.

EM's external forces come from the public the institutions intended to represent the public (e.g. Congress and the media), and other DOE organizations outside of EM. Some challenges clearly refiect one of these constituencies, others reflect several.

The three pairs of propositions in Fie 1 are based on whether EM does or does not have control over the proposition. Knowing the pairs of propocitions belps identify where EM cas marahall its enerpies to manage its response to the challenges, develop prosctive behavior, and forge its direction. The pairs as shown in Fig. 1 are: 1) scrutioy-culture/management-process, 2) expectations-resources, and 3) DOE-miasion-strategy/organization.

Superordinate Proposition: All EM's Internal and exteral challeages derive troen the bat that EM must perform in an arean of chanep unprecedented boch in kind and in mapoltude.

The historically enormous change EM must face will be in legal requirements (e.g, Gramm-Rudman budgeting, eaviroameatal compliance, and conflict of interest): facility changes through modernization; changes in the role of field offices, lead offices, and other organizational relationships; cultural changes; and changes in the type of criticism EM receives.

Change is an often-discussed management problem. No one has clear, always-effective solutions. As I outline the three pairs of propositions and corresponding challenges, the degree, the variety, and the interdependence of changes EM faces is staggering. Neverthelese, EM, DOE, and this country have no other choice than to effectively deal with all the changes.

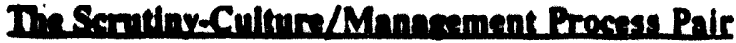

DOE and EM's increased external scrutiny requires an organizational culture and management process that can effectively react to these increased demands and work prosctively to seek input from critics. In these considerations, the word critics (and the reality behind the word) cannot be taken negatively or lightly. Critics implies both an audience (receiver) and feedback (seader). Critics must be considered as a positive influence on EM's change and EM must view the concept of critics with enthusiasm and optimism.

Scrutiny represents the external, or uncontrollable, side of the scrutiny-culture/management process pair of forces in Fig. 1. One of the enormous changes facing EM today is the change in the level of external scrutiny. The inereased serutiny and public awareness leads to the proposition for scrutiny.

Propodition for Serutlay: EM must perform in the bes of enormons chanes in requlatory requirements, more laws and regulations aimed directly at EM sctivities, and lnereased public servitiny because of the liak of mate to public bealth.

EM will have more laws and regulations aimed directly at its activities. EM will see increased formal scrutiny, like auditing or accountability.

DOE's profile has risen among its stakeholders. Intereat in DOE's activities has peaked because of environmeatal awnreseas. This combination of interest and awareneas bas pus the departmeat in the public spotlight, a bot seat EM is not used to occupying and in which very few organizations have been successful.

The Challeapes: EM must either find or build and use good management rools acceptable to the public. E.M 
needs to develop synery among those tools. EM must improve communication stillh to project the best image poesible to critics while communicating effectively with those critics. EM muse equally focus on the quality of internal communications as well. EM must manage innovation. EM must develop personnel training and recruitmeat programs and strategies.

EM's attitude and culture must change so EM can adapt and learn to deal with criticiem. EM needs to manage under personal risk, and deal with the effects of having to abide by "the letter of the law." EM and DOE must set up the procedure and the records to satisfy the scrutiny.

Situatlon: DOE relessed the epidemiologieal data (bealth records of DOE employees containing data on their radiation exposure and medical history) to qualified researchers, something is abeolutely wouldn't do or even address before. They're nol only allowing outside scrutiny, but also enhancing their internal scrutiny with strong quality aseurance and quality control that reports at the highest levels. In January of this year, the Secretary opeaed the department to public scrutiny of its nuclear weapons manufacturing complex for a comprebensive environmental study. The Wall Street Journal reported "By opening a public window into a weapons production process that esseatially had been screened from scrutiny for a generation, Watkins has freed himself to make decisions that departed sharply from the secrecy-first, production-at-any-cost policies of his predecessors" (1).

EM has sought comments from many groups for the development of the five-year plan. DOE has made the five-year plan available to the geseral public and given them opportunities to make comments to be included in the update of the plan.

There have been many media releases of previously guarded information and DOE's high profile hasa't ducked scrutiny--they've been out on the forefront, made mistakes, and said they've made mistakes.

Culture and manapement procear represent the internal, or controllable, side of the scrutinyculture/management process pair of forces in Fig 1. EM's emerging change in culture and its mbangement process corresponds to the increase in external scrutiny and is an enormous change itself. The management process includes the methods managers use to put the culture into practice. Such methods include thine like holding meetings and generating information. The change in culture and the managemeat process leads to the proposition for culture.

Propealtion for Cultures EM is the showease for DOE's new culture, and as such, seoks laput from its stalmbelders which will eracente techuical and manowement approaches aot proviously considered by EM and that EM ran't bare.

EM muse be willing to interface with the public and be available for interviews. DOE and EM can oo longer hide from the spotlight. Openness and bonesty will drive the communication of DOE's aew culture. The five-year plan is an important first step in addressing the concerns of DOE critics. Before it reorganization, wate management activities spread among five or six different DOE organiz: DOE sakebolders dida't know whom to talk to and they heard different and often conflicting reports from these orennizations. Now, these stakebolders can go to one place to get information or provide input to waste managemeat activities. DOE invited these stakeholders, for the first time in its bistory, to belp in the formulation of the five-yes plan. Comments from state and tribal governmeats resulted in the formation of the State and Tribal Government Working Group (STGWG), who represented the stakeholders.

The Conllenoses EM must define and understand its sew culture. The sew culture must be ingrained into the EM orgnization. EM must manage the elements of the new culture and set reasomable expectations with the public for what that culture means to them. EM should recomize and accept new approaches, rationally compare new and old approaches, and select and justify furure approaches.

EM muse thoroughty understand what consensus is and means. They must recopmize when they want consinsus and when they doa'h, and be skilled in aegotiating the right expectations for consensus and meeting throse expectations.

SItwations Scrutiny has increased for DOE. I believe culture change is the firs bis step in properly dealing with scrutiny. The Secretary reorganized the department and I believe be's right. The culture change led by the Secretary has been received well by the critica, eapecially the media. A recent article on the frout pare of the Wall Street Journal (1) reported the Secretary's and the departmeat's actions, specifically the five-year plan and his stanus of Critical DOE Activities" report, in optimistic and positive terms, 
compared to earlier accounts under the old secretive orennization. The five-year plan reflects and communicates the seed for and the character of the aeeded culture change to DOE employees and stakebolders.

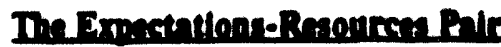

Changes in expectations by the public and its surrogates, as well as DOE employees and contractors, will force EM to rethink and reallocate its resources to address the changes in these expectations. Some expectations will be unrealistic and EM must balance efforts to meet realistic expectations with efforts to change unrealistic expectations.

Expectations represent the externat, or uncontrollable, side of the expectations-resources pair of forces in Fig. 1. New expectations beld by the public for EM call for a combination of little and bis succesces to meet thone expectations. These new expectations lead to the proposition for expectations.

Proposition for Expectations: Larven mpidly

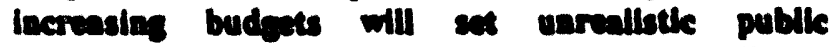
expectatioas; and EM will be oppeted Imandiately and condinually to prodece tandibie evidance of livises up to the promise of the five-gear plan.

Parts of DOE's mission can't be carried out without a strong, succeseful environmental management program. EM's critics need to see minor successes in the shortterm and major succesces in the long-term for its mission to be accomplished in the eyes of the public.

In the Secretary's Poarure Statement sceompanying his FY 1991 budget proponeta be stated thic seed to correct the eavironmental, safety, and bealth problems resulting from too much emphasis on production at the expense of sefety and the enviroamese. He found DOE was not rescting quickty esounth to new challenges coacerning the environment.

The Challewas: EM must speed intellipentty. EM needs to satisfy all its stakebolders. EM must also develop the infraetructure to spead the mosey effectively. EM muet get belp from the National Academy of Sciences (NAS) and other peer technical organizations to do a reality check on DOE's promises. Remember, NAS considered bolk the tive-year plan and the drat of The Applied Recearch, Development, Demonstration, Teating and Evaluation Plan wuch too ambitious.
EM mues learn bow to be effective and efficient and manage quality, productivity, innovation, and quality of work life. EM needs to deal aot only with NIMBY (not in my back yard) but, with NIMTO (not in my term of office) (Frank Feates, personal communication) and oven with NIMPL (not in my professional lifetime). EM muat handle accounatability over the long time periods. Whoever casts the die will not reap the rewards. Congrescional priorities may change mid. stream with many shifts in budget.

Situadion: EM knows of the beadlines and the time frames and requiremeats for doing things differently. But EM bas such a technolosy lag in the area cleasup that it isa't abundantly clear when the : and the deeds are going to merge. EM continues to j.. public commeat and feedback on the budget through an update of the flve-year plan, site plans, etc. A model of the scrutiny-seeking strategies is presented in Fig. 2. (Finure taken from DOE's Environmental Restoration and Wate Management Five-Year Plan (3).)

The allocations of resources represent the internal, or controllable, side of the expectations-resources pair of forces in Fig. 1. Changes in bow EM allocates its resources must address and respond to what the public and other critica expect. The change in resource allocation leads to the proposition for resources.

The world outside DOE will expect the five-year plan to be funded in its entirety. DOE and EM know this will not be the case. EM must compete for funds to implement the plan and Congress will likely grant funding oaly for the action items carrying the most weingte. The five-year plan must face the reality of fiscal responesibility.

The Secretary bas requested in the 1991 budget an increase of more than half a billion dollars for all of DOE over the 1990 budget. This increase is due primarity to the five-year plan. The Secretary has initiated development of a mational strategy to provide a map for DOE policy and funding. This strategy will be developed with public input through bearings, written submincioas, and publicty aveilable reports to reconcile the seed for enery with eavironmental, health, and sefety iasues. The finding of this process will be integrated into DOE's budert beginning in FY 1991, but will be inore clocely alimed in FY 1992 and fully integrated in FY 1993 (4). 


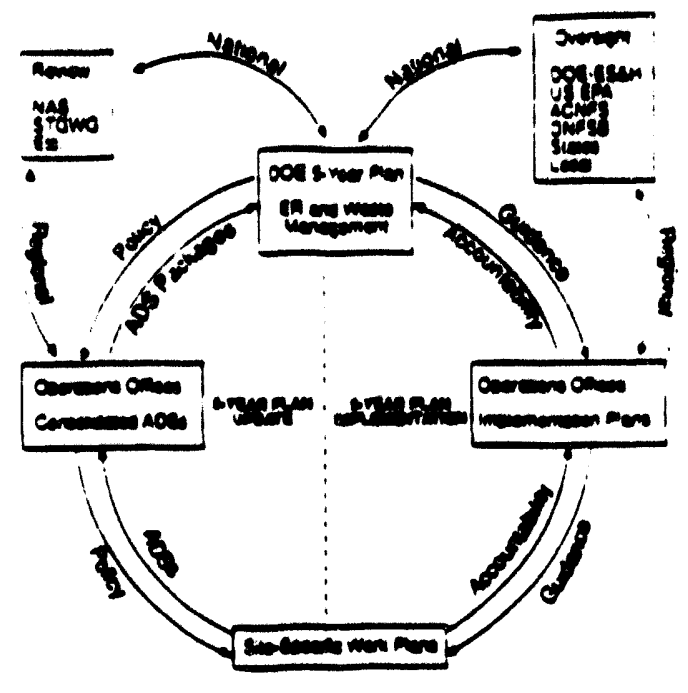

Fig. 2. The annual planning and implementation cycle includes loth internal and external review and accountability. Advisory Committee on Nuclear Facility Safety (ACNPS); Defense Nuclear Facilities Safety Board (DNFSB)

Proposition for Resources: EM will be expected to perform because of the lnereades allocation of resources and must set priorities, standards, characteriantion criteria, and end polata; DM then mames will be decades, aot years; EM will lose lastitutional memory.

The budget for clean-up and compliance is increasing faster than EM's ability to acquire the right resources to live up to the goals of the budget. EM has to have confidence in the data it receives from the field and sends to the field. Long-term and trowlodgenble EM employees will leave and EM will lose inctifutional memory, due to fear (conflict of intereat law), flushins of the system, opportunities world-wide in the wate area, and burt feeling relating to being criticized in derogatory terms.

Today, companies will not bet the farm" to be a management and operations (M\&O) contractor-because of liability problems. DOE is going to have to come to grips with the pressure to reesnmine the PriceAnderson Liability Coverage to make contracting more appealing and leas risty for private enterprice.

The Challeaces: EM lacks trained, qualified hurdworking people to do the work. And DOE lacks the trauning program to rapidly and effectively develop their people. DOE must compete with private waste management firms for qualified people. DOE needs a stratesy to attract, train, and retain good people within the federal pay structure.

DOE must develop training inciuding kindergarten through graduate school plus mid-career training. Where do these people come from? The DOE and their contractors are faced with a potential shortage of qualified human resources. They will be competing with other agencies for the same resources.

EM must develop an effective R\&D program--because that's the only way EM is going to marshall enough resources to meet the expectations.

EM should maintain a balance of 1) keeping enough good people and institutional memory not to make mistakes of repeating past errors or forgetting past promises and people and 2) flushing enough old-c: mind-set people to help the new attitude and cL take hold.

Acquisitioa of information systems will reduce overlap and give beadquarters what they need, when they need it. This will improve turnaround time so the time constant for getting information is shorter than the time constant for reporting it.

EM must develop a basis for priorities and satisfy those people the priorities affect. EM should identify realistic end points.

Situatioa: DOE has lost very valuable people. They may not yet be over that burdle. It must now introduce educational mechanisms. EM has to have confidence in the data it receives from the field and sends to the field.

Quality aecurance is controllable from the inside is the master of its own fate as to whether or not it has - decent QA propram. Similarly, EM controls its deatiny regarding inetitutional memory. Quality control and education work together to retain employees and protect the institutional memory. Activity Data Sheets (ADS's), prepared by organizations involved in all phases of EM's activities, supported the development of the five-year plan. The ADS's were used last year to geserate information as the basis for the five-year plan. This year, the ADS's became budget documents as well a program documents. This is a continual process where the ADS's will be recognized as they come through the system. 


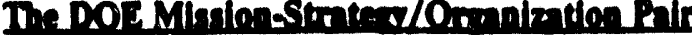

The scope of change in DOE's eavironment bas molded a aew mission for DOE and a resultant change in EM's strategy and organization.

Mission represents the exteraal, or uncontrollable side of the DOE mission-stratecy/organization pair of forces in Fig. 1. DOE and EM need a mission suited to the dual needs of production and protection. The change in mission leads to the proposition for mission.

Propositton for Mission: EM will be smack at the interface of the rock (materials production for natlonal defense) and the hard place (clean-up and miaimization of waste for public health).

DOE has specific production responsibilities associated with Defense Programs, Nuclear Energy, and Energy Research. These programs are connected with DOE's national defense miscion to produce auclear materials for nuclear weapons. To accomplish these production responsibilities DOE operates a large industrial complex which will continue to generate quantities of radioactive, hazardous and mired wastes.

DOE is in a changed social climate. Demands for greater accountability from DOE emerged in the 1970 , and 1980s. These demands mean DOE can so longer adopt a "trust me" attitude when dealing with the public. To meet these demands DOE must change its culture, provide understandable information to the public, and convince the public it is committed to protecting the environment. DOE is in the unenviable position where the demands for producing weapons grade materials has not lessened, and the demands from the public for protection of the environment have increased. In short, DOE is between the "rock" of producing weapons grade materials and the "hard place" of protecting the environment.

The Challeages: EM must underctand the proper balance between the mational defense and the public health. EM should interface with DOE production and the public bealth communities, and promote and maintain that balance for decades.

Situation: DOE raised EM to the Assistant Secretary level; reorganizing and elevating this function three steps in the organization.

In a dramatic change in miscion, DOE is balancing its national security and production mission with an equally or more important mission of public bealth.
This changes EM from a role of supporting the production mission to a role of leading clean-up of waste and restoring the environment. Instead of finding its stratesy and objectives set by nuclear-material. production activities and milestones, EM must set its own strategy and objectives.

Strategy represents the intermal, or controllable, side of the DOE mission-strategy/organization pair of forces in Fig. 1. EM's shift in strategy and organization must reflect the new mission. This new strategy and organization leads to the proposition for strategy.

Propoeiliou for Strates: EM success is essential to DOz's uission.

In the past, EM was driven by the production program and the modernization effort, thereby placing them in a reactive position. Now, EM must change its approach into a proactive pocition. Projects like WIPP are key to the success of other waste projects like Yucca Mountain and to production facilities like Rocky Flats. Instead of waiting for production activities and events to drive EM's activities, EM events will drive production.

Integrating knowledge of other DOE agency activities, such as WIPP and Waste Operations, with EM will help in the development of strategies that will have the greateat net positive effect for all DOE elements. Understanding the relationship between political and technical events will be key to the design of effective strategies to bring success to EM and help accomplish the DOE miscion.

Moat of EM's eavironmental restoration program is in the earlier stages of the work; therefore, they aren't doing many projects. EM is a program involving many problems (ill-defined objectives) and perplexities (illdefined siruations) which one day may become many projects (well-defined situations and objectives). Therefore, EM today deals with less-defined outcomes.

The Headquarters, field office, lead office organization was eatablished at a time of secrecy and fiefdoms. EM must develop sew ways of organizing and implementing EM's stratesy and responsibilities throughout the Headquarters and field site organizations.

Twe Cralleapes: EM should update all documents and statements so they don't contradict the five-year plan. The waste oreanization must be reorganized to support the emergine new culture. EM must learn to be prosctive from a reactive position. EM must promote 
its strategy, culture, five-year plan, and organization throughout its system, including the field.

EM must manage resources, schedule, and quality and the interplay among them. It must develop experienced management able to drive their responsibilities toward processes (repeated well-defined situations and objectives). EM must distinguish between management tools good for problems and perplexities and those good for projects and processes.

Situation: The headquarters-level reorganization under the Director of the Office of Environmental Restoration and Waste Management (EM) has been done. This reorganization has about 70 people but needs 350 people to be fully functional. While the specifies of the reorganization bave been worked out on paper, it will take some time for EM employees to get situated in their new domains of responsibility. The organization structure at headquarters must be conceptually complete before EM can take that structure to the field operations offices to develop mirror-image organizations. Having the operations offices mirror beadquarters will form a more cohesive DOE organization throughout, thereby improving communication within DOE and consequently improving DOE's communications with its critics.

\section{SUGGESTED APPROACHES TO MEDTING THE CHALLENGES}

Potential solutious to the challenges resulting from these, and other, propositions are neither obvious, easy, nor perfunctory. These solutions were derived intuitively and therefore they do sot relate one-to-one with the challenges. Solutions involve such crucial issues as leadership; training communication and developing coslitions; managing culture; atypical project and progrum manapement; and credible, structured management proceses.

\section{Leadorohis}

A good leader and leadership style will drive the success of the other listed solutions. A lender who adopts program champion sterus will pioneer culture change by developing coalitions, communicating the culture effectively throughout the organization, and arranging for training in the sew culture (5). The leader must manage culture change by manaping the elements of culture, managing many widely varying interrelated projects with ill-defined ends, using a credible structured management process.
A good leader should not only communicate the new culture, but reflect the culture to observers both inside and outside the organization. The shift to a more open. honest management style requires the leader to reflect these values and serve as a role model to others in the organization. People need to see these values in practice before they will readily buy-in to the new shared values.

Ideally, the Secretary or the Assistant Secretary should be the leader. But, the road is long and the tenure of these officials is short. So, for good leadership, EM can consider placing a civil servant in the leadership role or make higher officials civil servants.

\section{Trining}

To promote and live up to the new culture, DOE needs a comprehensive training program including technical and management training throughout the organization. Training is an important component of a culture change, especially when part of a personnel development plan. We change culture by teaching culture (6). To change the culture, we need to train all levels starting with management all the way through to the on-site technical implementation levels. Technical representatives don't know what the bard questions are to ask. We need to train technical representatives to know what is required.

\section{Communication and Development of Coalitions}

Managers need to talk less and listen more--inside as well as outside the organization. More important for meeting these challenges than listening to what's happening outside is for the leader to listen to the people and ageacies laslde the organization. Good leaders distinguish themselves through their willingness to listen to their own people. These leaders must also win the truse of their people to improve the quality of their communications. Trust makes people feel that it is not absolutely necesary to figure out what the other person is trying to say, openness and trust therefore influence how and when change occurs (5).

Scanning the eavironment and searching for outside threats and opportunities is certainly important (7). The formation of coalitions works to accelerate the availability of quality excernal information and is a consensus-building strategy. DOE is developing formal relationships with other technical groups across the system like the National Technical Information Service (NTIS) and the National Institute for Standards and 
Technolony (NST, formerly the National Bureau of Standards), the U.S. Environmental Protection Agency, and other agencies. DOE is initiating these interactions and demonstrating leadership. DOE needs to initiate even more of these relationships.

\section{Mnnadne Cullune}

Changing DOE's culture requires the organization to do four things of great difficulty yet of high priority. First, the organization must define and symbolize its new culture. Second, the organization must formulate a strategy for managing the change. Third, this strategy must protect DOE's institutional memory and its crucial experience base. Fourth, DOE must pursue a training and pers onnel development plan with the same vigor and priority as with the five-year plan.

Effective culture change will require the translation of the Secretary's vision of the new culture into a form all DOE employees can understand and communicate. Aligning the DOE organizational structure, plans and policies, and information systems with the new culture can belp DOE get the new culture into place.

The culture change should turn DOE 180 degrees toward open and honest interaction with all its stakebolders. Then, all DOE's managers and seniorlevel employees must understand when and whare consensus is needed and actively pursue a wide range of public interaction.

\section{Atrpical Prolest and Proemm Mnnerament}

Typical project and program management requires the balancing of cost, schedule, and quality (8). For example, if a project manager mua meet a tighter schedule, be or she must know the effect on the specifications and/or the cout. Except in cases of greater efficiency, the specifications must relax and/or the cost must increase.

I believe a fourth criterion plays a superseding role in project and program management. This criterion I simply call critica. As mentioned eartier, the word critics (and the reality behind the word) cannot be taken negatively or lightly. Critics must be considered as a positive influence on EM's change and be greeted with enthusiasm and optimism.

EM has to develop its program management style and system to balance cost, schedule, quality, and critics. EM is so new they don't have a program management plan with checkoffs for reporting the various program elements. EM must identify and recognize its critics and make intelligent resource allocation choices based on the synergistic effects of all four of these criteria.

\section{Crodibles Structurad Mnnnatment Proceas}

In a working management process, the selection of management tools is not random, but planned and consisteat. With such use, the amount of time spent catering to crises is inversely related to the amount of time spent administering a comprebensive structured management process. That is, you spend less time with crises if you spead more time on a good management process. So, to make time for improvement, i.e., culture change, focus on admixistering the management process (9).

Credibility plays an important role in the management process (10). Employees must believe the management process exists to help them do their jobs better. They must trust the management process to direct their work and that of the organization toward a clearly defined and articulated set of gonls.

\section{WHATS GOING TO MAKE OR BREAR EM}

What will make or break EM depends on the actions EM takes and when. I've described, in general, what I believe EM must do in the near-term (one year), the mid-term (three to five years), and in the long-term (beyond five years).

Nieartere thene frame (ose year) -EM must have tangible evidence of progress in decreasing perceived risk. EM must show some winners--that is in the R\&D, in the clean-up arena, and in coming to grips with some of the anaty issues in waste managemeat. EM must show they understand and can live up to the five-year plan, and the first year of that plan. They'll break the faith if they doa't.

Mid-tern inse frame (three to five years)-EM must demonstrate that their investment in R\&D is starting to pay off toward moving the technolosy forward. EM must make sure their education program is starting to bear fruit. Students in kindergarten through graduate school and the university consortia should be included in the educatioanal program. Mid-term, as under the National Defense Education Ach, EM must have people coming out of universities who are interested in working with DOE. By that time DOE's historic image as a cloceted agency must have gone away. If it hasn't, EM is in big trouble. 
Longterm time trime (beyond tive years)-EM must have some major technical successes. For example, they must have cleaned up the ground water at Fernald so the citizens of Obio will have stopped worrying about uranium in their drinking water. EM must have a big hit like this--at the installation level.

So: for the short-term EM needs to show it knows what it's talking about by cleaning up a pit or a pond or a lagoon. In the longer term EM must bring these successes together for a facility. EM needs all the midterm successes to be able to survive in the long term. Through all time frames, the bottom line is that EM must measure their success in terms of how well they satisfy their critics in addition to the old cost, schedule, and quality measures. Today, we measure successful management on a new basis.

\section{ACKNOWLEDGEMENTS}

The ideas presented in this paper represent the cumulative work of hundreds of people over twetve years at Virginia Tech's Management Systems Laboratories. Professional managers from business and industry, and faculty, graduate students, and undergraduates from a wide variety of engineering and other disciplines bave participated in the research and development of these tools and techniques. The author thanks them for their good work. The pronoun " $T$ " is used throughout to separate all of us using management systems (we) from the author (I). However, please recognize " $I$ " is more than one contributor to this work.

The preparation of this paper is funded by U.S. Departmeat of Energy (DOE) Grant No. DE-FG02. 88DP48058. Management Syatems Laboratories thanks the DOE's Office of Environmental Reatoration and Waste Management for providing is a real-world laboratory for the reconrch, developeneach and teating of state-of-the-art manngement tools and the frameworks for understanding how to make them succeseful.

\section{RITLRTNCES}

1. Lippranan, Thomas W. (1990). Reversing Course at the Energy Dept." Wall Street Joumal, January 24, 1990, pp. 1-2.

2. Frank Feates, Department of the Enviroament, Radioactive Waste Division, London, England, persoasl communication, July, 1989, in Woods Hole, Massachusetts.
3. U.S. DOE (1989). Environmental Restoration and Waste Management Five-Year Plan. Springfield, VA: National Technical Information Service.

4. U.S. DOE (1990). "Energy Secretary Watkins Calls for Increased Funding in Environment, Science, and National Security," Press Release (R-90-020), January 29, 1990.

5. Deal, Terrence E. and Kennedy, Allen $A$. (1982). Corporate Cultures, Reading, MA: Addison-Wesley.

6. Schein, Edgar H. (1985). Organizationu. Culture and Leadership, San Francisco: JosseyBass.

7. Thompeon, James D. (1967). Organizations in Action, New York: McGraw-Hill.

8. Kermer, Harold (1989). Project Management: A Systems Approach to Planning Scheduling and Conbolling (Third Edition), New York: Van Nostrand Reinhold.

9. Kurstedt, Harold A., Jr. (1989). "Catering to Crises-How to Escape," IIE Integrated Systems Conference and Society for Insegrased Manufacturing Conference Proceedings (December), pp. 191-198.

10. Kanter, Rosabeth Moss (1983). The Changemasters: Innovation for Productivity in the American Copporation, New York: Simon and Schuster. 
Institutional Factors Affecting DOE Waste Management and

Environmental Restoration Planning

\author{
J. Andrew Walker and Louis I. Middleman \\ Management Systems Laboratories \\ Virginia Polytechnic Institute and State University \\ 1900 Kraft Drive \\ Blacksburg, VA 24060 \\ (703) $231-3501$
}

\begin{abstract}
The magnitude and impact of the U.S. Department of Energy's (DOE) waste management and environmental restoration program requires a drastic change in DOE's culture to include the participation of all levels of government, public forum representatives, and the public. Early in the process of developing a new, comprehensive five-year plan for environmental restoration and waste management, Secretary Watkins invited affected States, Indian Nations, and organizations of elected officials to form the State and Tribal Government Working Group to comment on two formulative drafts of the plan. Management Systems Laboratories of Virginia Tech was asked to help plan and facilitate two review sessions in the spring and summer of 1989, based on a perception of impartiality, experience with similar groups, and active affiliations with State governments. A third session in the fall was devoted to reviewing the draft applied R\&D plan and guiding the scope of a national prioritization methodology. This paper emphasizes three major institutional factors affecting DOE's future: the need for ongoing, pervasive culture change; the need to display this change through truly cooperative planning; and the need to involve the regulatory community in the process of technology development so innovative solutions can be applied with the least possible delay.
\end{abstract}

\section{CULTURE CHANGE}

In its first Five. Year Plan, DOE pledged to evolve from a production-oriented culture to a culture characterized by open communications, clearly understood and demonstrated priorities for environmental stewardship, and accountable management. To begin operationalizing this pledge, DOE invited the participation of affected States, Indian Tribes, and representative organizations of elected officials to review and comment on two formative drafts of the Five-Year Plan. Representatives from Colorado, Idaho, Kentucky, Nevada, New Mexico, Ohio, South Carolina, Tennessee, and Washington joined representatives from the Shoshone-Bannock and Yakima Indian Nations and the National Governors Association, National
Conference of State Legislatures, and the National Association of Attorneys General in Blacksburg, Virginia and later in Chicago. A subsequent session was held in Denver to review and comment on DOE's plans for applied research and the development of a national prioritization system.

Commitments at those meetings reflected both the viewpoints of outside participants and signs of DOE's willingness to demonstrate a change in its culture. Although commendable and encouraging, this beginning leaves much to be done. The future requires increased commitment to an open culture with leadership, patience, and courage in the pace of competing internal and external interests. This is where the challenge lies. We believe the challenges DOE faces can be summarized broadly in two areas. 
Internal Discipline

Though announced last year by formal and informal Secretarial pronouncements, DOE's cultural change must take place against the inertia of traditional mindsets and corresponding funding patterns. DOE's culture, its set of shared values, determines both how DOE (Federal employees and contractors) behaves internally and also how DOE interacts with other government agencies, interest groups, and the public. DOE must exhibit the internal discipline necessary to alter traditional ways of managing. We identify several specific methods that support internal culture changes.

First, DOE's budget process needs to change. Departmental budget requests for environmental compliance and cleanup, including research and development toward such ends, have until lately been defensible only in direct relation to the agency's production mission: nuclear materials and weapons for national security. When something had to give, it was environmental restoration and waste management. DOE must demonstrate that era is past. Current changes in budgeted amounts for environmental restoration and waste management versus production are encouraging signs.

Second, DOE must send a clear message down its chain of command that responsibility and accountability will be strictly monitored, enforced, and rewarded. For example, new and renegotiated Management and Operating (M\&O) contracts are planned to hold contractors liable for compliance violations if the Department funds activities needed to achieve compliance. This will include expanded incentives for contractors to achieve excellence and cost effectiveness in their performance, enhance understanding of performance expectations and performance criteria by both Federal and contractor employees, and tighter controls to ensure corrective action will be forthcoming when contractors do not perform to standards.
Third, DOE must strengthen the independent internal oversight function of the Assistant Secretary for Environmental, Safety, and Health; as well as that of independent external oversight, including the Advisory Committee on Nuclear Facility Safety and the Defense Nuclear Facilities Safety Board. Fourth, the recent decision that DOE, in accordance with the National Environmental Policy Act (NEPA), will prepare two major programmatic environmental impact statements (EIS's) shows DOE's commitment to making sharp changes from traditional management practices.

\section{Extemal Openness}

Although DOE's cultural change must grow from within, a sign of that growth is the increased willingness to listen to the outside. DOE has added six States (California, Florida, Illinois, Missouri. New York, and Texas) and the Umatilla Indian Nation (Washington State) to the State and Tribal Government Working Group (STGWG). DOE must continue to involve this group, as well as environmental special interest groups. The involvement of elected officials and special interest groups is necessary but not sufficient. The participation of the general public is a challenge that DOE recognizes but hasn't been achieved.

Other welcomed cultural openness measures include: a special hotline within DOE headquarters to allow citizens to report specific facility concerns and an appointed Secretarial Panel for the Evaluation of Epidemiologic Research Activities (SPEERA).

While DOE must remain receptive to STGWG and to other interested parties and individuals, it brings up a potentially serious problem. Being open and receptive to outside scrutiny does not mean DOE must agree with or commit to do (unless it is the law or part of a signed agreement) everything suggested. What 
must be achieved is some consensus among groups that have traditionally "earned their living" by disagreeing. In such an arena, players may tocus on the adversarial game itself and not the ultimate goal--cleanup and compliance. This problem arises in the context of cultural change. It's not only DOE's culture, but that of other interested parties, that must change. The danger is a cultural backlash brought on by subtle or overt--or even accidental--pressure on DOE while it's trying to straighten itself out. DOE seems to be genuinely trying to make a 180-degree cultural shift. As DOE demonstrates its willingness to listen to its critics and its ability to meet commitments, trust will begin to increase among all parties and the appropriateness of adversarial postures will decrease. Just as the Department's failures must be exposed, its successes and improvements must be praised. Bob Neil said at a Washington International Energy Group conference last October 31 that DOE should "Publish bad news." He was right. And DOE has been doing the right thing, attempting to become its own severest critic. But fair is fair: if DOE publishes its own bad news, then DOE's critics need to publish its good news. Cultural revolutions, it is clear, come on a tide not of falling but of rising expectations.

\section{COOPERATIVE PLANNING}

The experience of hosting the initial two meetings of the States and Tribes government working group meetings, and observing the third meeting, has given us the opportunity to reflect on lessons learned for involving outside affected parties. These lessons are discussed below.

\section{Session Design}

Planning a successful meeting requires similar thinking to planning the effective design of a document. We suggest consideration of the following formula:

$$
\text { audience }+ \text { purpose }=\text { design }
$$

In our case, we could reasonably anticipate certain audience expectations. For example:

1. STGWG members were expected to be generally knowledgeable about DOE environmental restoration and waste management. Some key issues were evident based on a letter from the governors of ten states sent to the Secretary of Energy prior to the first meeting. The letter called for a comprehensive program to cleanup DOE's defense and research facilities. DOE's invitation to review the Five-Year Plan acknowledged the governor's concern and served to prepare DOE for meeting dialogue and making possible commitments.

2. Participants could be expected to agree on some issues and disagree on others because of competing interests.

3. Participants would be skeptical about DOE's motives and abilities to change.

4. Some disagreements between DOE and the participants over the issues, and their resolution, was inevitable.

The purpose of each session should be clearly established to prevent false expectations. In the case of the STGWG it was clearly to review and comment on the issues and programs defined in the Five-Year Plan. Meeting design must be structured around audience expectations and meeting purpose. For involving diverse, knowledgeable, and skeptical participants the following meeting characteristics are suggested.

- Ensure a minimum of lengthy, technical presentations for participants. This decreases interest and increases suspicion 
and perceptions of an unwillingness to listen and reverting back to the old culture.

Allow sufficient time for an executive session by the participants. This will permit them to collect and discuss group and individual comments. This in turn will minimize differences of opinion between individuals and maximize meaningful discussion.

Don't overplan. A successful meeting plan will bend to the evolving needs of the participants.

Include time for participants to get to know each other. If commitments are met and working relationships are established, trust will be confirmed by actions.

\section{Include Outside Participants Early}

Two points are worth making here. First, include outside participation in the formative stages of developing ideas. Realistically, participation at the beginning is the best time to influence the direction an idea will take. Outside participants know that and will be suspect of de facto conditions. We acknowledge that some issues are sensitive and the risk disclosure to unfriendly observers is possible. But when appropriate, including all participants in formulating ideas will increase trust and ownership of the final product by all stakeholders.

Second, provide review and orientation materials in advance of the meeting date. In very few instances are meetings enhanced by withholding information disclosure until the meeting. Advanced distribution of information allows thoughtful review and comment.

\section{'smmitments}

Meeting participants should be of a level that encourages meaningful dialogue and commitment. For states and tribes representatives, one of the positive attributes of all three STGWG meetings was the ability of DOE persons to make decisions and commitments on behalf of the Department. This does not mean that all requests were met. It does mean that comments were discussed openly and one of three actions were taken: (1) a commitment to change was made, (2) that a commitment to change couldn't be reached without further research and discussion, or (3) an impasse had been reached and no agreement could be reached. In no case should issues be left without resolution through one of these actions.

\section{Elexible Facilitating}

Group meetings can require strong, active facilitating while others are better served by passive facilitating. In either case the ability to recognize and adapt to the requirement is critical. For the states and tribes meetings, a strong facilitator wasn't required because they were practically self-led. However, even a self-led meeting can benefit from a good facilitator. Effective facilitation can (1) better organize and direct group discussion and (2) improve the collection of valid comments.

\section{Meetine Details}

Attention to simple details make the difference between an unsuccessful meeting and a successful one. For example,

- Minimize administrative and logistical chores for the participants. Meeting participants that are informed about arrangements, understand the agenda, and feel cared for are better able to focus on the meeting purpose. 
Provide information about the meeting well in advance. This contributes to the participants feeling the meeting is well organized and worth attending.

- Consideration of protocol can be important. Ensure meeting room details enhance participation by ensuring proper and equal arrangements.

- Consider including a social "ice-breaking" event. This is particularly effective for first time participants.

- Small details like name tags and name tents, participant lists, word processing and administrative services, all contribute to meeting success.

\section{REGULATORY PROCESSES}

RCRA/CERCLA/SARA Impacts on DOE's Technology Development Process

DOE's Environmental Restoration and Waste Management Five-Year Plan (August 1989) included a brief section on Applied Research and Development. Between June and November 1989, DOE produced a substantially expanded draft Research, Development, Demonstration, Testing, and Evaluation (RDDT\&E) Plan to identify the Department's environmental problems and the implied needs for new technologies (and new management structures) to solve these problems. The RDDT\&E Task Force characterized the essential problem as entropy: the dispersion of low-volume, high-value radioactive and hazardous chemical waste streams into high volumes of low-value concentrations. The plume boundaries of these dilute streams are extremely difficult to find; and once found, difficult to access for treatment and often impossible, once accessed to clean to regulatory standards. Leo Duffy, Director of DOE's newly-created Office of Environmental Restoration and Waste Management, has called for and end to DOE's "technology stagnation," an end to the era of "muck, suck, and truck" or "pump and treat."

Entropy was also the management problem: the dispersion of expertise throughout the operations offices and national laboratories; the not-inventedhere syndrome and consequent disinclination to cooperate with other federal agencies, industry, and academia; and the lack of a headquarters focal point for applied RDDT\&E.

As DOE produces the first update of the Five-Year Plan, which will incorporàte a final, edited version of the draft RDDT\&E plan, the new Office of Technology Development (OTD) is acutely aware of the need to align itself and its field elements with the new culture, recognizing that unless DOE can address regulatory concerns in parallel with technical concerns, its proposed solutions may instead become problems.

Technologies for treating, storing, or disposing of RCRA wastes must receive State permits, and it is not uncommon for the permitting process to last eighteen months. Although no permits are required for technologies applied to CERCLA cleanups, such cleanups often yield RCRA wastes. And the waiver of sovereign immunity under SARA makes states active parties in Records of Decision, so that approving the use of new technologies becomes part of approving a remedial action plan.

\section{Benefits of Applying the Quality Circle Concept}

The need for a new, more intimate relationship between OTD and DOE's stakeholders, including institutional stakeholders, is created by the regulatory drivers of environmental restoration and waste operations (including agree-upon 
schedules), discovery of new problems, and technological development outside DOE. DOE has adopted federal facilities agreements, tri-party agreements, etc., as "contracts" for cleanups and compliance. Such agreements define what DOE will accomplish and the schedule for accomplishment. Absent negotiated schedules, meeting these technologies unless OTD establishes confidence that innovative approaches can be delivered without delaying the project. Creating quality circles to conduct cleanups and compliance projects can provide the needed confidence.

Quality circles are teams of experts working together to assure satisfactory project completion. For an environmental restoration project, the quality circle would include, at a minimum, the DOE program manager with responsibility for the site being remediated, the OTD manger responsible for the relevant technology area, the DOE M\&O contractor manager responsible for the site, and representatives of all regulators having jurisdiction at the site. It is the role of the quality circle to help in selecting the technology to be used, to identily where RDDT\&E can help meet project goals, to monitor progress of the RDDT\&E being conducted to support the project, and to propose changes in the scope of the project or of the RDDT\&E as new information of any sort comes to bear.

\section{ACKNOWLEDGEMTNTS}

The ideas presented in this paper represent cumulative work of hundreds of people over twelve years at Virginia Tech's Management Systems Laboratories. Professional managers from business and industry, and faculty, graduate students, and undergraduates from a wide variety of engineering and other disciplines have participated in the research and development of these tools and techniques. The author thanks them for their good work.
The preparation of this paper is funded by U.S. Department of Energy (DOE) Grant No. DEFG0288DP48058. Management Systems Laboratories thanks DOE's former Office of Defense Waste and Transportation Management (now the current Office of Waste Operations) as well as DOE's Office of Environmental Restoration and Waste Management for providing us a real-world laboratory for the research, development, and testing of state-of-the-art management tools and the frameworks for understanding how to make them successful. 


\title{
AUTOMLTION THROUGH THE PIP CONCURRENCE SYSTEM IMPROVES INFORMATION SHARING AMONG DOE MANAGERS
}

\author{
Rebecea M. Imbolz. D. Steves Berube, and Jeffrey L. Peterson \\ Managemeat Systems Laboratories \\ Virgaiaia Polyrechaic Institute and State University \\ 1900 Kraft Drive \\ Blacksburg, Virginia 24060
}

\begin{abstract}
ARSTRACT
The Program Implementation Plan (PIP) Concurrence System is designed to improve information sharing within the U.S. Departmeat of Enersy (DOE) and between DOE and the Field. Effectively sharing information enables DOE managers to make more informed, effective decisions. The PIP Concurreace System improved information sharing among DOE managers by defining the automated process for concurring on a DOE document. thus reducing the time required to concur on the document by $75 \%$. The first step in defining an automated process is to structure the process for concurring on a documeat. Only thoce DOE managers with approved access could review certain parts of a document on a concurrence syrtem. Remember that the concurrence process is a sign off procedure unlike a commentary process in which comments may not be restricted to certain people. The commentary process is the besinning of the concurreace process. The commentary process builds a document; the concurreace process approves it.
\end{abstract}

\section{INTRODUCTION}

DOE managers use information to make decisions setting policy and stratery. They must make these decisions based on intermediate information-o unorganized and informal information that is not always complete. There are two principle reseoses for this. First, it's sometimes difivente for DOE managers, with difierent preferesces, to arree of the information's content. Decinions are then made based on incomplete information since managers have ext resched conesensus on whar's aeeded. Secoad, becaure of the leaphy concurresce chain, decivions muxt often be made before information cas pass through a timecossuming approval process. In both casea, improved management of intermediate information throuch automation can lead to more effective information sharing which leads to more eflective decision makine. This is because automation 1) adds meaningful structure to otherwise, informal information, 2) directs the flow of intermediate information to the appropriate DOE manager for review and approval, and 3) reduces the time involved in concurresce activities.

The PIP Concurrence Syatem automated the proceas required to concur on and produce a DOE planning document-othe Ofice of Defense Waste and Transportatios Manaceemens (DWTM) Progran Implementation Plan (PIP). The PIP had to be coseurred oa by verious DOE managers at the Field and Headquarters (HQ) level.

The 1987 PIP was created reviewed, and concurred os throuph the ues of time-consuming communications tools (mail, phone, facsimile, etc.) withouk a defined concurrence process. Structuring and defining the automated coscurrence process dramacieally reduced the time and overbead required for the coneurrence proceses on the 1988 PIP. However, lescoas ean be learned from the PIP Concurrence Syrem which en be applied to obber documents requiring coscurrence. The PIP 
Concurreace Syatem can be accesced through the Waste Information Network (WIN) System.

\section{THE PROGRAM IMPLEMENTATION PLAN}

To understand bow the PIP Concurreace System was developed. I must first begin with an explanation of the desion and coatent of the PIP document itself.

The PIP document described the curreat DOE approach for managing defease high-level waste (HLW), transuranic (TRU) waste, and low-level waste (LLW) from atomic enerty activities. There were four main sections of the PIP documeat. The firse section was an Executive Summary which gave an overview of the status and management of the three types of defenes radionctive waste-.HLW. TRU waste. and LLW. The next three sections described in ereater detail the status and management of each type of waste. Section nwo conceatrated on HLW. This section was divided into three sections-ethe three sites that contain HLW.oSavananh River Site (SRS), Idabo Natioad Enqineering Laboratory (INEL), and Hanford. Section three concentrated on TRU waste and it too was divided up by the six sites that contain
TRU wase.-SRS, INEL, Hanford, Los Alamos Natioal Laboratory (LANL), Odk Ridge (OR), and Novada Tesu Site (NTS). Likewise, section four diecureed LLW and was divided up by the six sites that costain LLW. In Fig. 1, I show the major active storage/disposal sites for defense radioactive waste (1).

\section{THE PIP CONCURNENCE SYSTEM}

The first step in determining the design of a concurresce syutem is to structure and flow chart the concurresce process itself. Each subsection of the PIP documest was traneformed iato a fle that would evearually reside in the system. I defined a subrection as the larpart continuous section of the PIP document having the same concurreace chain. For example, the SRS paragraph under the HLW section of the document would be conaidered one file. Dividing the documest up in that manner resulted in a total of 72 tiles.

Nexh, I flow charted the concurrence process each file should 80 through. Moux files began at the Field Site (PS) bovel. The files were typically a copy of Lexe Years PIP (Vercion 1 in Figure 2). At that

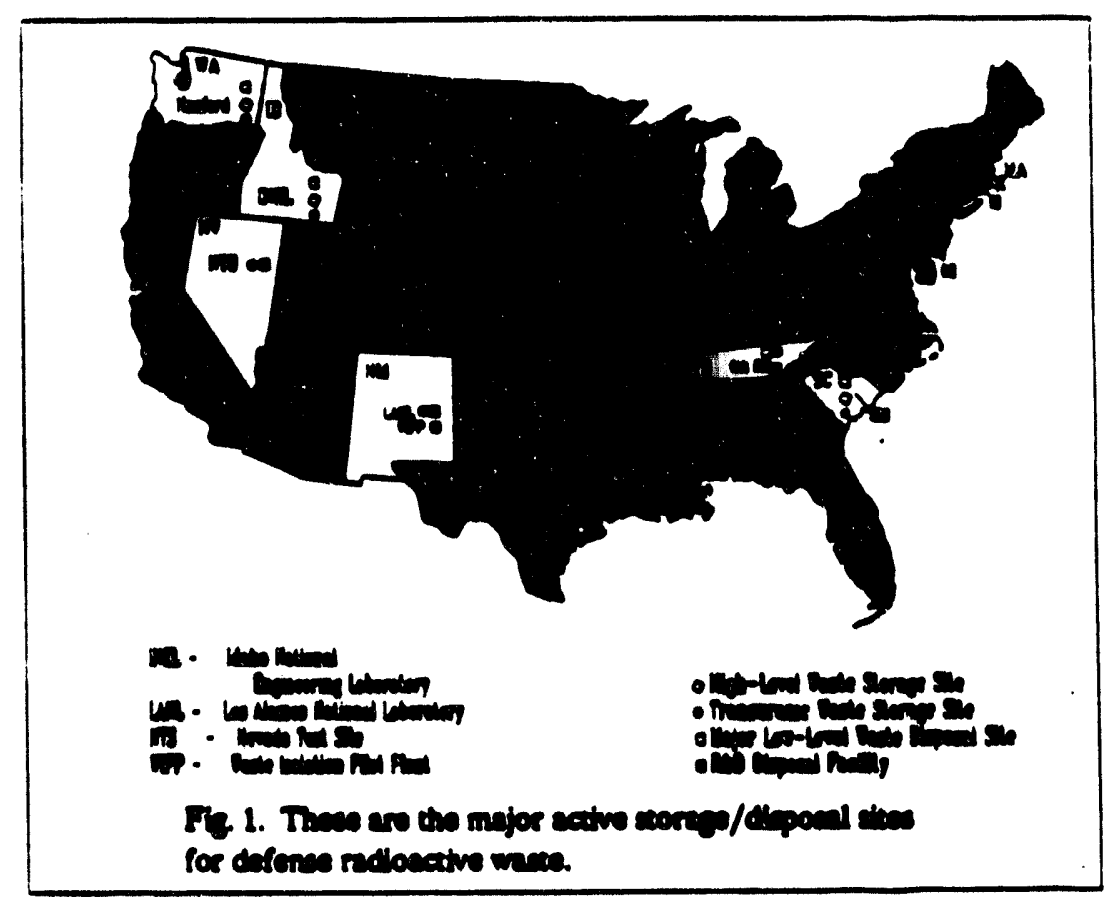


level, they were created or edited (if edited. Version 1 from. Last year's PIP became Version 2) and seat to a FS DOE manager for review and concurrence. If they were concurred on at the FS level they were seat to the aex level of coecurrence for review. This aex level of review depeaded on the waste type. Let's use a SR HLW file. as an example. Since the file discusced the status and managemeat of HLW at the SRS, it would be seat to the HLW Lead Site (LS) for review once it was concurred oa at the FS level. If it was concurred on at the $L S$ level, it was seat on to the appropriate managers at DOE.HO for final review. If at any time during the concurreace procesa, a manager did aot concur on the file, it was thea 'seat back' for further edits, thus creating more Versions) and routed through the concurreace chain again until find approval or coacurrence. Fig. 2 shows the path a file takes when it is 'seat back' for further edits during the review or concurreace process (2).

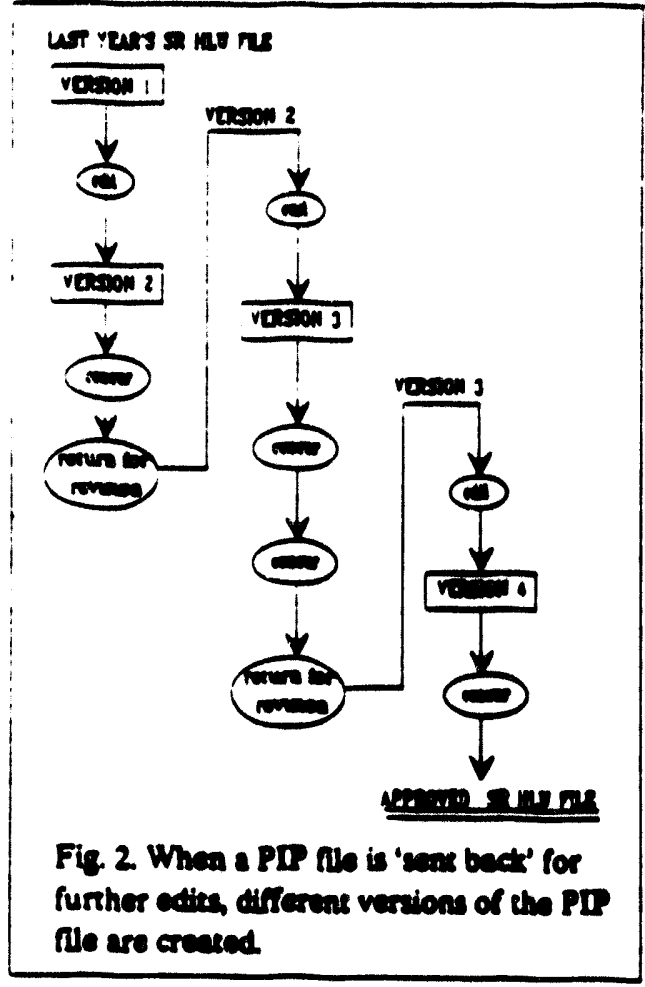

Once the concurresce process wes plotted for a file, the file was acipiped editors and revievers to carty if through the coscurresce chnia (2). Fiz. 3 shows an example of bow a PIP file was axipred users (3).

\begin{tabular}{lll}
\hline & \\
\hline & & \\
\hline
\end{tabular}

To briely reeap, each PIP file starts out as a new version (Version 1), typically a copy of something from last year's PIP. Each PIP file is first edited by someose at the HQ, LS, or FS level. When editing is complete, each editor submits his file(s) (Version 2) to preestablished users for review or coscurreace. The mout direct path a file can take is when all coscurreace people approve the file. Fie 4 shows an example of this direct concurreace path (2).

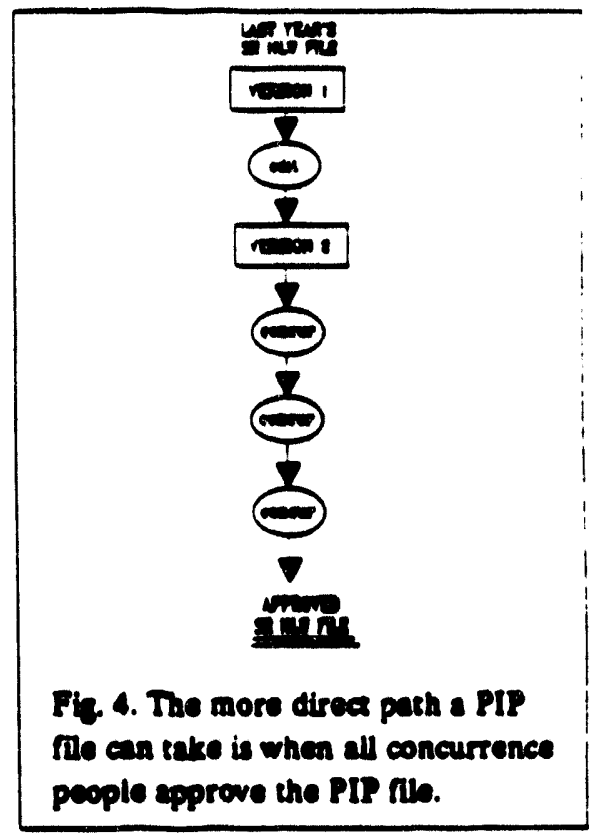

The primary feature of the PIP Coscurreace Syrted wes to track the propreas of fles through the edibie/coneurrence process and provide file acceas/control. A user transerred his file from the 
PIP Concurrence System to the word processor of his choice. Then the user could edit the file uning his own word processing peckage that be was familiar with. Whes finished editing the file, the user transferred it back on to the PIP Concurrence System (2). Fig. 5 shows this process of editing a file (3). Although a file could soo be edited directly from the PIP Concurreace System, a reviewer could read or print the file while in the PIP Concurreace System (2).

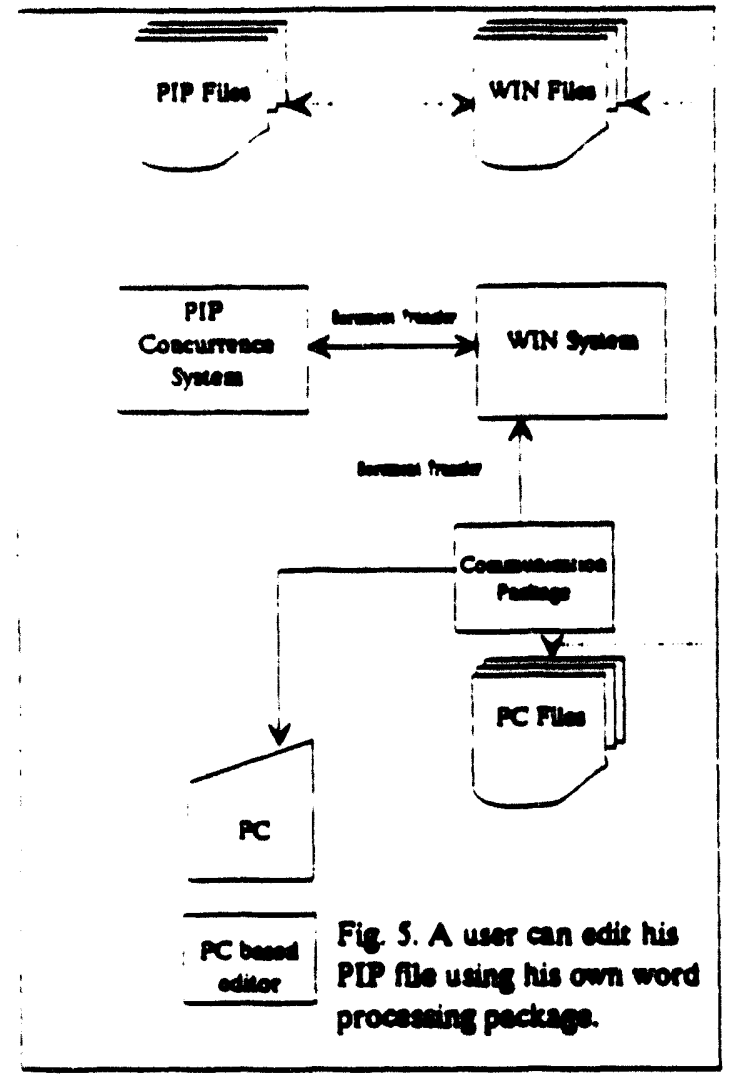

As meatiosed earlier, the PT Concurreace Syrem tracked the propreas of files throuth the editing/concurresce procees. As ars given time, a DOE manaper conld eccess a anme board to determine bow well the coscurresce process was progrescing. Thin festure belped the DOE manacer ideacify bles thet were delayed in the concurreace proceas and who to call to determine the cauce of the delay. Oece a file had completed the eatire concurresce proceses its satus read, - Concurred oa and avilable for copy weaning any user with access to the PIP Concurreace Syrtem could read the approved file or prise it. Thin fearure proved valuable to ueers who seoded the mont current searus of a particular program discuseed in the file. Fiz 6 shoms the PIP Concurreace System Status Board (2).
The PIP Concurreace System DataBase Adminisurator (DBA) function was developed to monitor and facilitate the progress of files through the PIP Concurreace System. The DBA was a desionated user who was given access to the PIP Coscurrence System DBA function. Through a series of screens and commands the DB, in was able to add. delete, and restore files (restore meant to recover files that were accideatally deleted by a user); change the edit/concurrence chain for any file if needed; review the curreat status of all files: and inform ueers of important eveats or upcoming dendlines in refereace to concurreace through a mearage bosed. Fit 7 shows an example of the PIP Concurresce Syutem Mesarge Board (4).

Training a user on the PIP Concurreace System involved educating them on the concurreace process itself so esch user knew his role.

During the first year the PIP Concurresce System was ued to produce the PIP documest, the time required to coseur on the documeat was seduced by 75\%. This is a resule of a aumber of factors, one of them being thex the developmeat of the PIP Concurrence Syrtem clearly defined and implemeated the coscurrence process and maintained concurreacy control. Bernatein and Goodman (1981) define coseurreacy control as the ectivity of coordianting concurreat access to a data base in a multiuser distributed DBMS. Coceurrescy coatrol permits users to access a dacabese in a muliprooramed fachion while preserving the illuaion that esch wer is executing alone on a dodicated system. The main technical difiouly in atrining this gool is to prevent decaberes updates performed by oce user from incertering with dacabase retrievals and updates performed by another (I)." The PIP Concurrence System demonatrated succeschul concurreace coserol. Oaly thow DOE managers who seeded to edit or reviow a fils were gives secess to it, thus eliminating uasolicited comments and review delays. In addition, as a file wes being edited, the syctem prevented the other fire's edicors access to it to eliminnes the ponibility of conflicting versions reaiding on the PLP Coseurreace Syatem, thus escouring date inteprity.

Efrectivaly shariag information is ceatral to DOE's day-to-day operacion. The PIP Concurreace 


\section{AP conctunnea sYste status \\ 1 inger 4 ind}

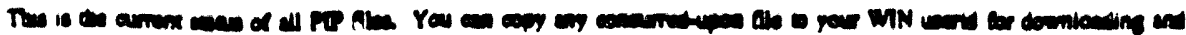

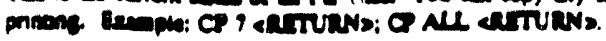

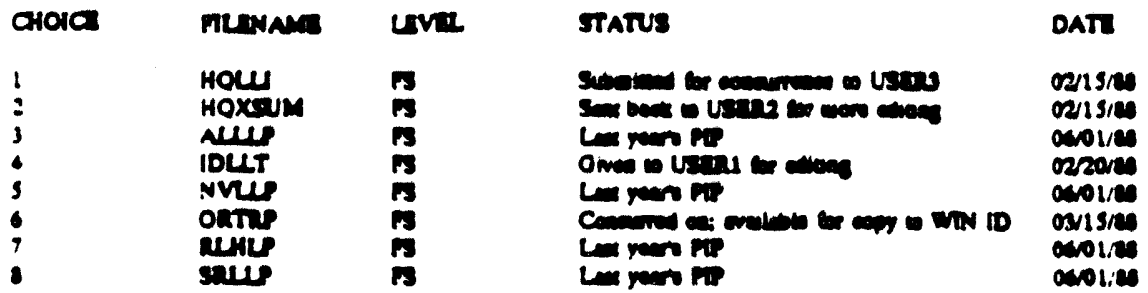

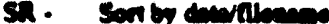

P. Helpwithstus

H. Helowiostatus

Entwe your endia:
CP. Con ab * o me Uent

D - Defley esengunes lin for abs

N Nen parato

- Quis num o mPCO MNN MEN

Fig. 6. The PIP Coscurronce Sysetri Sarve Board displays the curment seans of each PIP Aie.

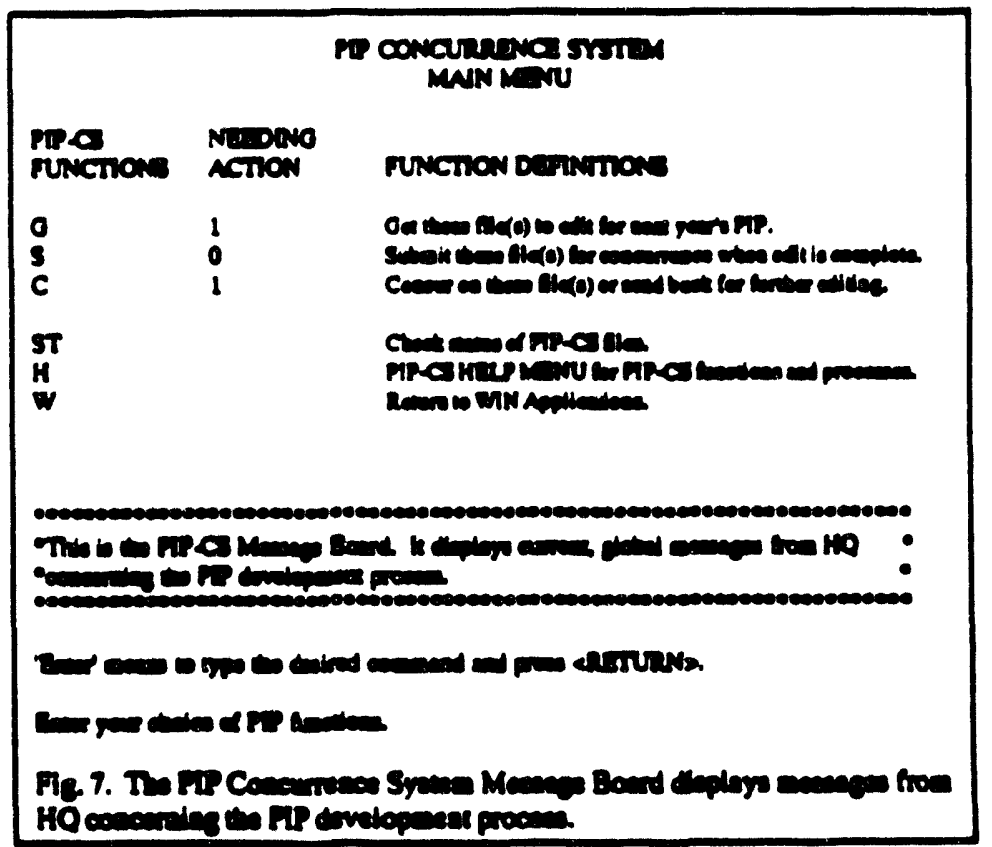

System wet its main objective which was to improve information sharing within DOE. To formulate and execute plane chea to oversee their implementation, requires sharing dace, information, tcole and decivion mechianiams-and shariag them bot oaly between and amoan ofices at one central location, but aleo between thet beation and aumerous of -site feld oflices and contractor offices. Since every function a DOE manager performs involves some kind of sharing it is imperative thet be of she have the cleareat possible bnowledoe abouk every piece of date and the informecion being dealk wich (6).

Thes the PD Concenrrences Syatem was and is viedl to efectively shnring information.

Automation forced me to debine the components of the PIP document and its concurreace process. As 
stated earlier, automatioa improved information sharing among DOE managers because it provided three important features. First, the PIP

Concurreace System added meaningful structure to otherwise, informal information. The intermediate data became part of a concurreace chain so a user always knew who bad last seen the information (a file) and who would see it next. Knowledge of who the ocher reviewers we:e and when they would review a file in the concurrence process belped the user decide what his or ber commeats should be. Second the system directed the flow of intermediate information. Each file had its access restricted by the defined concurrence chain. Thus, only those DOE managers with approved access could provide comment on a file. This restricted access could be viewed as a problem since it may preclude knowledgeable comments by excluding someone in the concurreace chain. However, the benefits from structuring a concurreace chain outweigh the risks since some documents require concurrence (sign off) in addition to a comment period. Lastly, the PIP Concurrence System reduced time involved in concurrence activities because the concurrence process was structured and resided on an electronic system.

The PIP Concurreace System enabled a DOE manager to have access to the most recent data right at his desk. Due to PIP concurrence control mechanisms, this data was not only current, but retained its integrity and reliability. The PIP Concurrence System, as a means to share data and information, was and still is an effective cool and mechanism by which to make more informed decisions to produce useful planning documents. The PIP Concurrence System can be modified to include other DOE documents requiring concurrence.

\section{ACENOWLDDGMENTS}

The ideas preseated in this paper represeas cumulative work of hundreds of people over twetve years at Virpinia Tech's Manacement Systems Laboratories. Profescional manasers trow busineses and indusery, and faculty, eraduate studeats, and undereraduates from a wide variety of engineering and obber disciplines have participated in the research and development of these tools and techniques. The author thanks them for their good work. The pronoun " $[$ " is used throughout to separate all of us using management systems (we) from the author (I). However. please recognize "I" is more than one contributor to this work.

The preparation of this paper is funded by U.S. Departmeat of Energy (DOE) Grant No. DE. FG0288DP48058. Management Systems Laboratories thanks DOE's former Office of Defense Waste and Transportation Management (now the curreat Office of Waste Operations) as well as DOE's Office of Environmental Restoration and Waste Managemeat for providing us a real-world laboratory for the research. development, and testing of state-of-the-art managemeat tools and the frameworks for understanding bow to make them successful.

\section{RERERSNCES}

1. U.S. DOE ASSISTANT SECRETARY FOR DEFENSE PROGRAMS, OFFICE OF DEFENSE WASTE AND TRANSPORTATION MANAGEMENT, Program Implementation Plan, DOE/DP-0042. (June 1987).

2. U.S. DOE OFFICE OF DEFENSE WASTE AND TRANSPORTATION MANAGEMENT, "Program Implemeatation Plan Concurresce Syatem--A User's Manual," (May 1983).

3. MANAGEMENT SYSTEMS LABORATORIES, VIRGINIA POLYTECHNIC INSTITUTE AND STATE UNTVERSITY, What is the PLP Concurteace Syctem? Training Sescion presented to U.S. DOE Otice of Defence Waxte and Traneportation Manapemeath (May 1988). 
4. MANAGENSENT SYSTEMS

LABORATORIES, VIRGINA

POLYTECHNIC INSTITUTE AND STATE

UNIVERSITY, "PIP Concurtence System DBA

Manual." presented to U.S. DOE Office of

Defense Waste and Transportation

Masagement. (February 1988).

5. PA.BERNSTEIN and N. GOODMAN, "Concurrency Control in Distributed Database Systems," Computing Surveys, Vol. 13, No. 2 (June 1981).

6. MANAGEMENT SYSTEMS

LABORATORIES, "Research and

Development of Models and Instrumeats to

Define, Measure, and Improve Shared

Information Processing within Government

Oversight Agencies," proposal submitted to U.S.

DOE's Office of Nuclear Materials, (1986). 


\author{
Regen G. Pulnar \\ C. Patrick Roefllin \\ Anes R. Dase \\ Firald A Eurstadth Jr.
}

\author{
Manapemeat Systems Laboratories \\ Virginin Potyrechnic lactitute and Stace Uaiverity \\ Blacksour Virginin 24060
}

\section{(ABSTRACT)}

Can a group resch conseasus without the rithe information? To support group inceraction, proupware muet provide the correct amoun and tind of information. This pilot study tests the relationahip between iaformation avilability and conseasus. Availability includes ancous of information, a well as the usefulaes of iaformetion. Does the croup here esount information? Does the froup have and une the ride informatioa? Discoveries the iaformetion

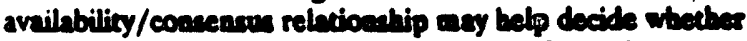
of sol consensus is deaired ueder certini information circumstances.

\section{Introdection}

The relevnece of comenens recenreh is increaning with the

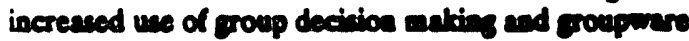

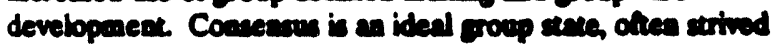
for, but rarely schioved Ideally, concenes enoures complete

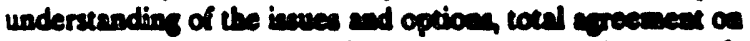
the beat solution, and complete commineen and inpport for thut solution (Gentry, 1922). It is act condtain tor

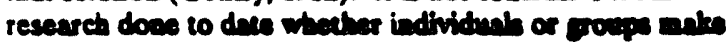
better decinion (Casey and Mebls, 1991). Some of this

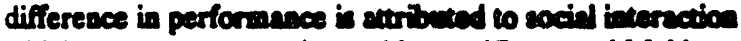

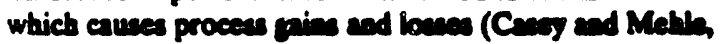

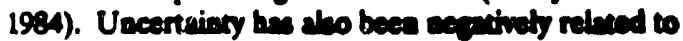

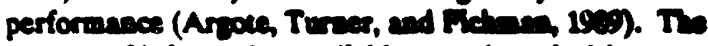
amoun of information aviluble to encle a dachion (uscertainty) contributes to th prosen fins or benes.

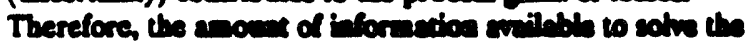

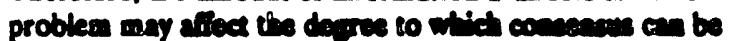

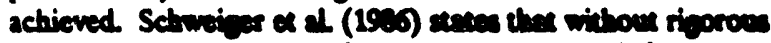
evaluntions and citiques of bo anompoions and inferences

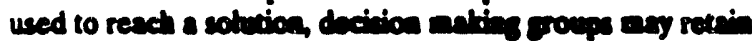

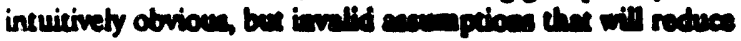

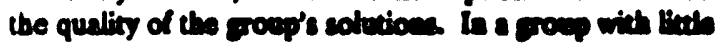

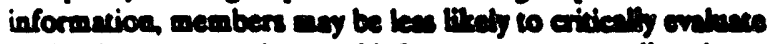

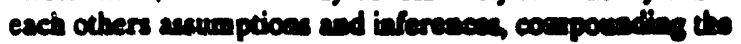

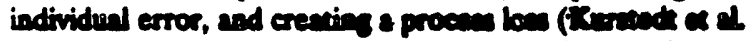

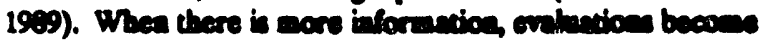
eacies, cresting a proseces prin.

\section{Hypotheses}

This research bots a bow the anoum of informexion provided to a croup afiects four things o) the chiling of the

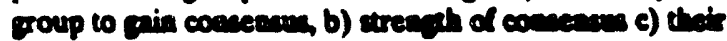
commitreas to the conenem, and d) th quat's of the roep decirion. The pools of this researeh on be ened in fre bypothears:
1. A group cas reach consemsus witbout enough informstion to make the bex decision.

2. Strenpth of cosecesus will sot very sccording to the ancues of information anilable.

3. The qualing of a proup's decinion will be bette the evares of individual decinioes whes the g: bes all the cecceseng iaformation (low uncertaingy).

4. The qual'y of a group's decition will on be better then the everese of individual deciaioses whea the group ton lints information (bigh uncertringy).

5. The more informution a croep hes to make a decivion, the more committed the eroup members will be to the dacinion.

\section{Deanitions}

For thin sudy, the researcher defines conecasus

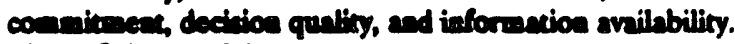
Tne dainitions of these terme were uned to conetruct a quationario.

Comenom is operationally definad a a croup state arrived at

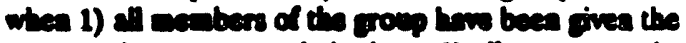

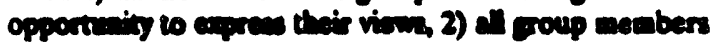

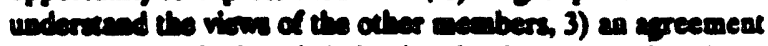
decirion is reacled, and 4) the decinion is mpported to the

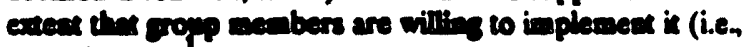
comionets).

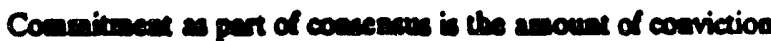

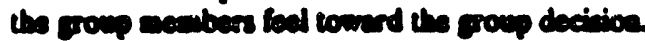

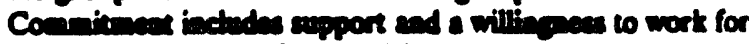

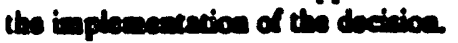

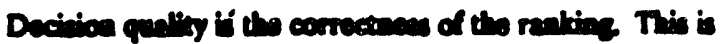

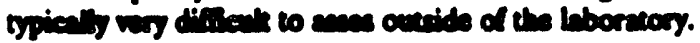

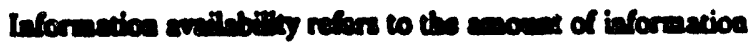

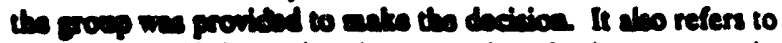

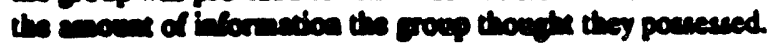

1

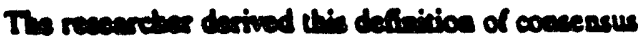
. Grow a drefinion drvaloped by D. Mart Brubaker at

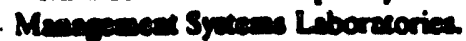


Hetnodoloty

Subjects

Subjects coasisted of 22 undergraduate and 6 gaduste students at Virginia Pobytechaic lastirute and Stace University. There were 12 females and 16 maleh ranging in age from 20 to 33 years. Few of the subjects had any managerial experiesce; work experiesce ranged from nose to engineering positions and instructors.

\section{Task Dealog}

The group and individual tast was to rank 15 items in ordar of importance for survival oe the moon. The "Lous on the Moon" exercise wns used because there is a correct solution and explanstion supplied by NASA (Hall, 1971).

Using the correct answers and NASA's explanation, three information levels were developed. The subjects in the first information level were only pivea a deacription of the situation and a lies of the 15 satveraeble iteme. Subjects in the secoed information level were gives the sinution description, a list of the 15 satvapeable itean and some relevent facts about the moon (preseated a part of the situatioa). Subjects in the third informetion bval ware pives the siruntion description a line of the 15 salvepable inems with pousible survival ueses for esch ited (this we sctually the rationale given in the correct solution), and the same relevan facts abour the moca that reare provided to subjects in the secoed information bevel.

\section{Procodare}

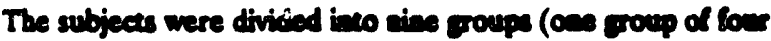

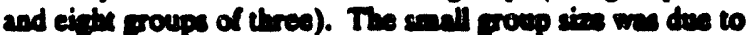

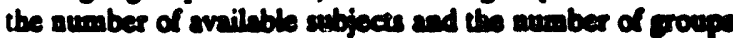
seeded. Exch croup we radoenty enen to en information level Each roup neenber thea received a

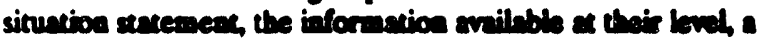
ranking sheet, and a quactionanire deainad to meanore

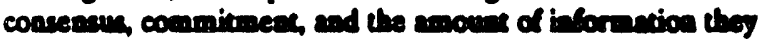
perceived avilable. The perpose of inchating enowe of perceived iaformation on the quectionstive wis to colloct

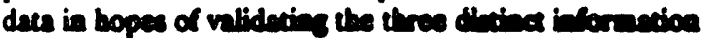

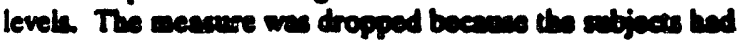

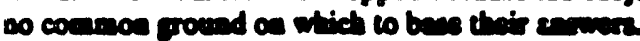

Participants thea read th sinnotion and incormetion provided

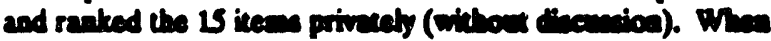
sverpoes finished the individed ren'ing each porp

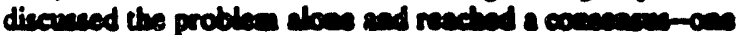

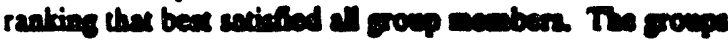

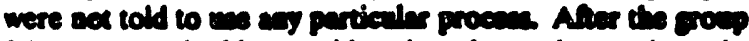

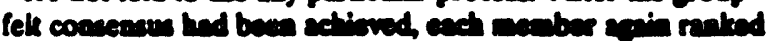

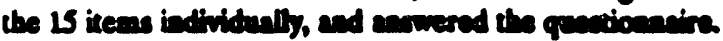

\section{Manom}

The queationanire dexigned for the such connimed of 10

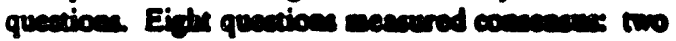

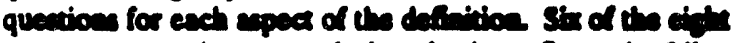

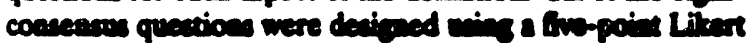

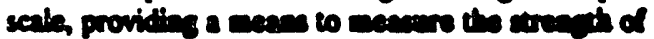

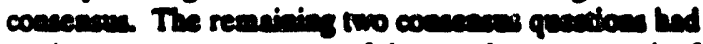
yes/eo responses A ceose of three or bener on enct of the

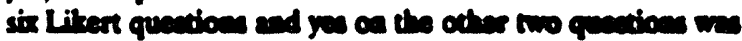
considered surecient evideaces the poren had reachad

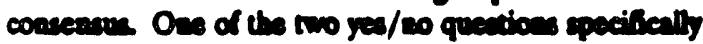
acked subjects if they thoenth theis grow had reached cossesane This quection we compered to the resuls of the olber seves queations.
Commitmeat was measured using two of the eight consensus questions on the questionanire (commitmest was the fourth neceanry coadicion for comensus), and by counting the number of ranking on each individual's secoad ranting that were differeas froe the eroup ranking. The more rankings thes difiered from the group ranking the lower the commitmese. Another meaure of commitmess was obtained by calculatiog the absolute difieresce betiveed the group rankine and each iadividual's second rankine. A large difference between the rankings implies low commitmeat.

Perceived information avilability we measured by tho quection aling the ubjects to rate the uncertainty of the problea. As previouly staced, thase questions were dropped froen the study.

Demographic dat were aleo collected from the queationanire. Dessographics were used to describe the subjecter

Decinion qualiny wes meanred mathematically, indepeadeatly of the quewionnire dece. Ench iadividul and proup ranking we compared to the correct ranking and an absolute

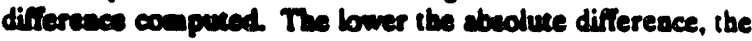

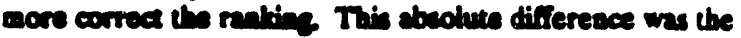
decinion qualing seore. The individual decision qualioy seores were eneread and coepared to the roup decision quality score for esch roup

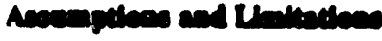

All subjoces were conidered to thre the enene bevil of

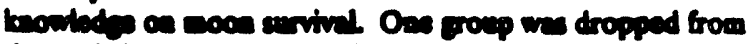

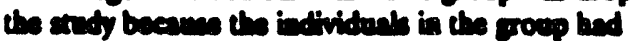

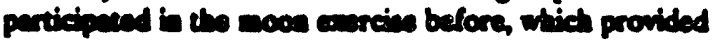

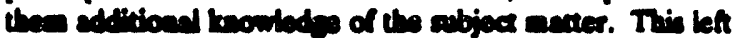
the andy with on proep of lour and eoven croups of three.

The on time coending inposed we the roup hed to complate the tacts during a 75-nimute den period. All groups fininted will in edvences of this thes contring ber sone smbinets seationed tho thomete their roenp would heve schirwad a better eolution if froy had ware time.

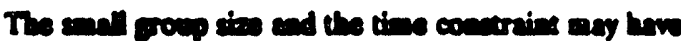

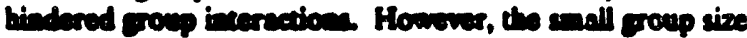
dresened procens loseces end gim

\section{Beralte and Diserecton}

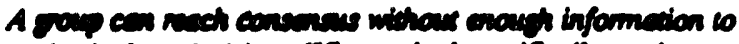

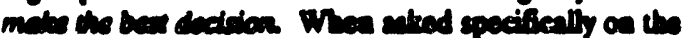

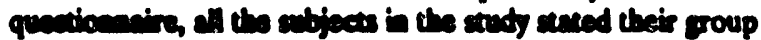
had reacted consenen. Hownwer, when the soven queations

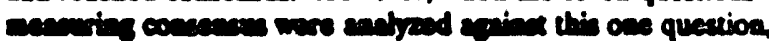

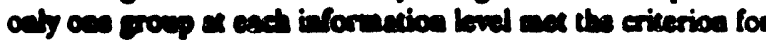

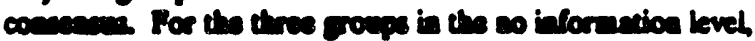

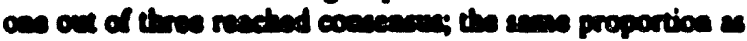

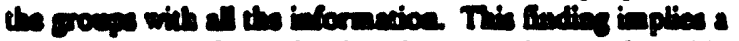

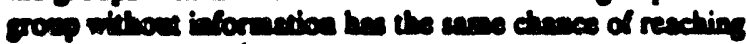

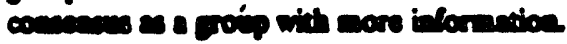

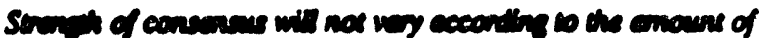

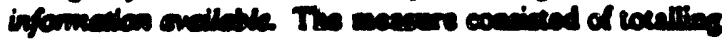

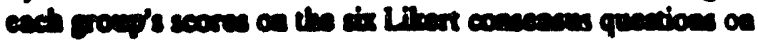

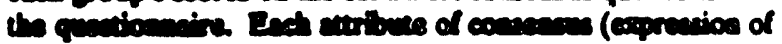

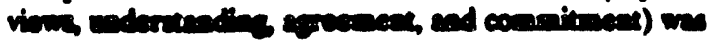

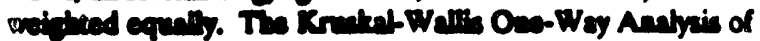

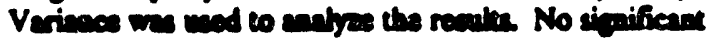

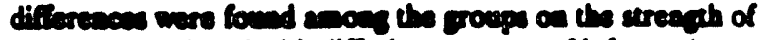

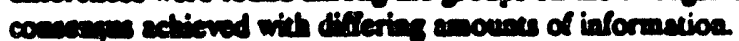

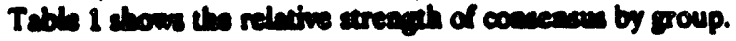




\begin{tabular}{|c|c|c|}
\hline $\begin{array}{l}\text { Information } \\
\text { Level }\end{array}$ & & $\begin{array}{l}\text { Streageh } \\
\text { of } \\
\text { Conseasus }\end{array}$ \\
\hline $\begin{array}{l}\text { Info } \\
\text { Level I }\end{array}$ & $\begin{array}{l}\text { Group } 1 \\
\text { Group } 2 \\
\text { Group } 3\end{array}$ & $\begin{array}{l}22.0 \\
26.3 \\
2.0\end{array}$ \\
\hline $\begin{array}{l}\text { lafo } \\
\text { Level } 2\end{array}$ & $\begin{array}{l}\text { Group } 4 \\
\text { Group } 5\end{array}$ & $\begin{array}{l}23.7 \\
22.7\end{array}$ \\
\hline $\begin{array}{l}\text { Iafo } \\
\text { Level } 3\end{array}$ & $\begin{array}{l}\text { Group } 6 \\
\text { Group } 7 \\
\text { Group } 8\end{array}$ & $\begin{array}{l}23.0 \\
23.3 \\
21.7\end{array}$ \\
\hline
\end{tabular}

Table 1.

Relative Meseures of Streapeh of Consensus

The quality of a roup's decision will be bewor then the oneress of individual decisions when the roup has all the necessersy information (bow uncericing). This bypotbein wes teated by comparing the averase individual decinion qualing score with the group deciaion qualing seore for ench group in the third (bigheut) informecion bevel. The group dection quality seores were simificandly better than the averesp of iadividul decision quality seores ( $p<0.02$ simifieance level) uning the

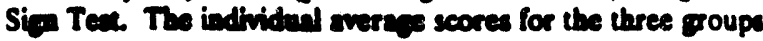
were 32.6, 41.6, and 30 whils the group seores were 20, 30, and 14 reapactively. The secose represents the sbeclute devintion froen the correct anver, so the lower seores are the

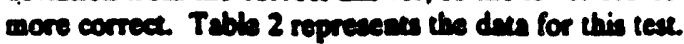

\begin{tabular}{|c|c|c|c|}
\hline $\begin{array}{l}\text { Informaxion } \\
\text { Level }\end{array}$ & & $\begin{array}{l}\text { Averes } \\
\text { Individerl } \\
\text { DO Seons }\end{array}$ & $\begin{array}{l}\text { Group } \\
\text { DO } \\
\text { Secore }\end{array}$ \\
\hline $\begin{array}{l}\text { Info } \\
\text { Loved } 1\end{array}$ & $\begin{array}{l}\text { Grop } 1 \\
\text { Grop } 2 \\
\text { Grop } 3\end{array}$ & $\begin{array}{l}43 \\
43 \\
373\end{array}$ & $\begin{array}{l}380 \\
280 \\
26.0\end{array}$ \\
\hline $\begin{array}{l}\text { Info } \\
\text { Level } 2\end{array}$ & $\begin{array}{l}\text { Group } 4 \\
\text { Groups }\end{array}$ & $\begin{array}{l}393 \\
355\end{array}$ & $\begin{array}{l}30.0 \\
24.0\end{array}$ \\
\hline $\begin{array}{l}\text { Info } \\
\text { Leval } 3\end{array}$ & $\begin{array}{l}\text { Grop } 6 \\
\text { Grom } 7 \\
\text { Grop } 8\end{array}$ & $\begin{array}{l}32.6 \\
41.7 \\
30.0\end{array}$ & $\begin{array}{l}20.0 \\
30.0 \\
14.0\end{array}$ \\
\hline
\end{tabular}

Table 2

Isdivided Verum Gromp Decinion Qunling Secres

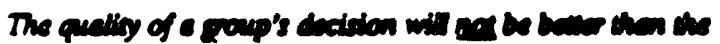

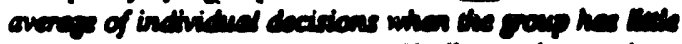

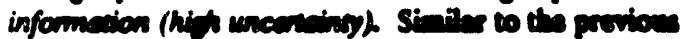

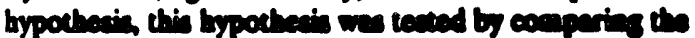

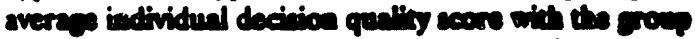

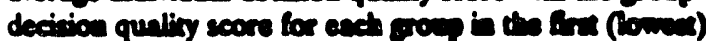

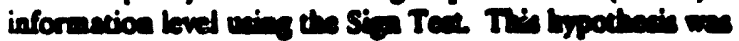

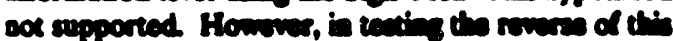

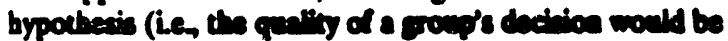

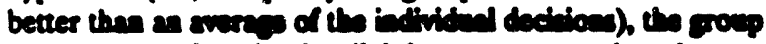

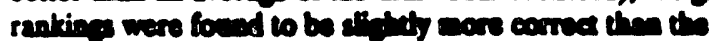

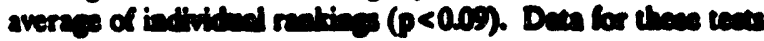
are shom in Trab 2

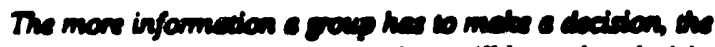

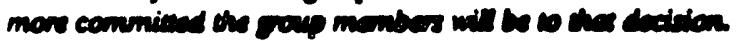

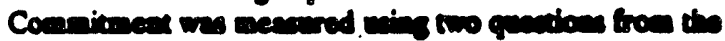

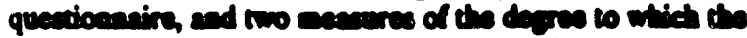

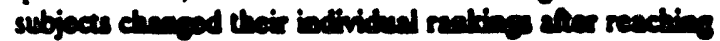

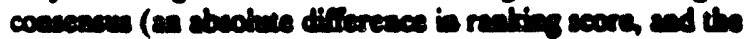

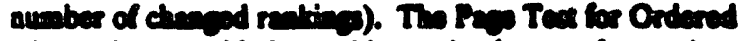

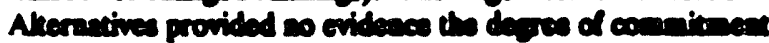

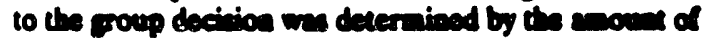

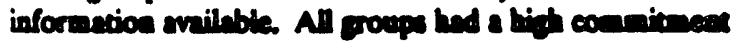
secre.

\section{Coneladen}

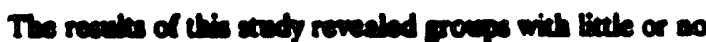

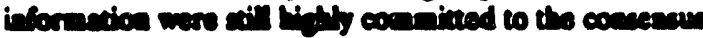

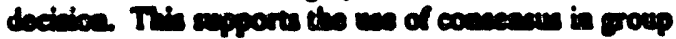

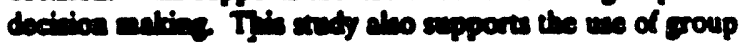

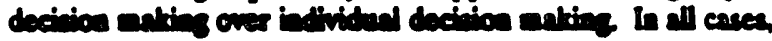

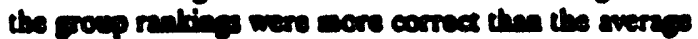
indivitud reations for the romp.

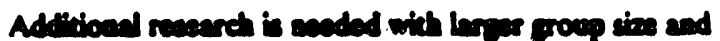

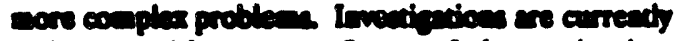

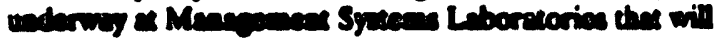

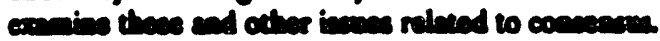

\section{Actoculad inemente}

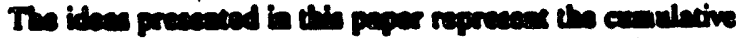

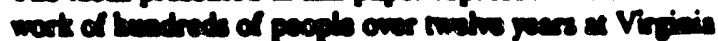

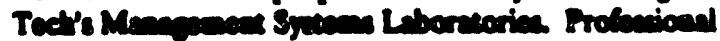

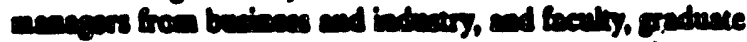

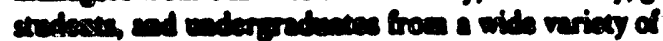

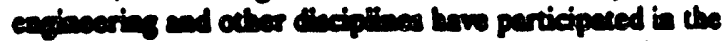

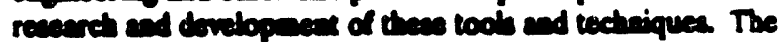

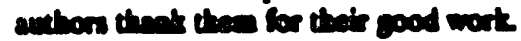


The preparacion of this paper is funded by U.S. Departmeas of Exery (DOE) Grant No. DE-FCo2-6DP 4coses

Manapemeat Syatems Laborktories thanks the DOE's Otives of Environmested Reatoration and Wete Manepenent for providiag us a rest-world laboratory for the rewearch,

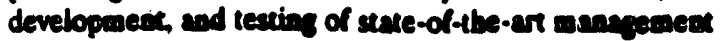
tools and the frameworks for undersandiag bow to mats them succesent.

\section{Refereaces}

Argote, L. Turser, M. En and Fichnen, M. (19:9). To Ceatralize or Not to Ceatralize: The Efects of Uneertainy and Threas on Group Stnecrere and Perforence."

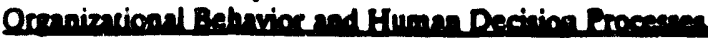
Vol. 43.

Cacey, J. T., and Mehla, T. (19an) 'A Partition of Sanall Group Predecision Performance inso Informatioad and

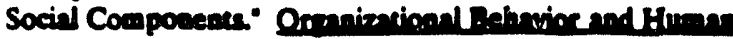
Perfoconnes Vol. 34.
Gesery, M. E. (19:2) "Consensus as A Form of Decision

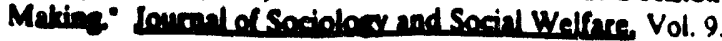

Hill, J. (Nowenter 1971) 'Decisiona, Decisions, Decisions."

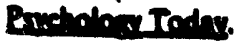

Kurtade, H. A. Jr, Brobnker, D. M., Doen A. R., and

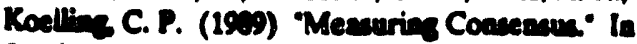

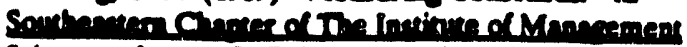
Seinger Jown R. Bater and Robert T. Sunichrau (eds.), ppo36s.

Schmion, D. M. Sandbers, W. R, and Rapan, J. W. (1986) -Gromp Approcedes for improvies Stratepic Decition Making: A Comparain Anstyits of Dialectioal laquiry, Devil's

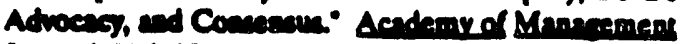
Iongent Vol 29, No. 1. 


\title{
APPLICABIITY OF THE INTEGRATIVE BARGAINING PROCESS FOR NEGOTIATING AND IMPLEMENTING FEDERAL FACIIITY ENVIRONMENTAL COMPLIANCE AGREEMENTS
}

\author{
J. Andrew Walker \\ Management Systems Laboratories \\ and \\ Environmental Design and Planning
} Virginia Polytechnic Institute and State University 1900 Kraft Drive Blacksburg, Virginia 24060 (703) $231-3550$

Submitted for the International Association for Conflict Management Third Annual Conference, Vancouver, Canada Track: "Conflict in the Public Sector"

\section{NeED AND ORTECTIVES}

We live in an increasingly interdependent world where our responsibilities to each other are greater, as are the costs of failure. The concept of interdependency is particularly relevant for government agencies charged with implementing public policy. They must administer public programs in a world of complex, overlapping organizational interfaces (Hanf, 1982; Kingdon, 1984; Nordlinger, 1981; O'Toole and Montjoy; 1979; Pressman and Wildavsky, 1979) and with intense public scrutiny. Individual agencies are incapable of accomplishing program missions independently (Hjern and Porter, 1981; Porter, 1985). Wright (1982) describes our contemporary intergovernmental condition in terms of an overlapping. authority model with three features:

- Substantial areas of government operations involve national, state, and local units simultaneousty;

* areas of autonomy or single-jurisdiction independence and complete discretion are relatively small; and

- the power and influence of a single jurisdiction is very limited. 
Wright's model accurately describes environmental programs in the United States. Nowhere is governmental interdependency, and the need for integrative methods to stimulate cooperation and multiorganizational effectiveness, more apparent than in the administration of Federal and state environmental regulations.

The primary objective of this paper is to determine the potential usefulness of an integrative bargaining process for negotiating and implementing environmental compliance agreements among Federal facilities, the U.S. Environmental Protection Agency, and state environmental regulatory agencies. To operationalize this objective a five-step process is proposed.

First Provide historical background on Federal facility environmental problems and compliance agreements. The focus will be on the U.S. Department of Energy's Environmental Restoration Program as a significant and representative example that seems to call for integrative solutions.

Second Examine the process of determining integrative bargaining model potentiality established by Richard Walton and Robert McKersie (1965).

Third Suggest refinements to Walton and McKersie's process.

Fourth Apply the refined process to the case of negotiating Federal facility compliance agreements.

Fifth Suggest strengths and weaknesses of the integrative approach in negotiating and implementing Federal facility compliance agreements. Tactics suggested by Walton and Mckersie that support integrative approaches will be defined.

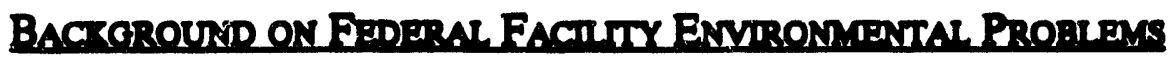

Given evidence of the lack of environmental consciousness displayed by the managers of Federally-owned facilities and their parent agencies over the past 40 years, it isn't surprising the public, Congress, environmental interest groups, and Federal and state regulators have targeted them for increased environmental accountability. It's not been easy to get Federal facilities into compliance with 
laws and regulations designed to protect public health and the environment. Laws and regulations made early to stop pollution and clean up past abuses either didn't apply to or were difficult to enforce on Federally-owned facilities. In most cases loopholes for Federal facilities were no accident. Many Federal facilities were exempted because of their roles in national security and because Constitutional and procedural impediments on Executive Branch agencies enforcing actions upon other Executive Branch agencies.

New Federal laws and administrative procedures, state and local laws, judicial actions, and Congressional and public scrutiny have increased Federal facility accountability and closed old loopholes. Federal facilities are now faced with a host of overlapping and sometimes conflicting Federal, state, and local regulations that include enforcement mechanisms. The new regulations complicate reaching compliance. Conformance with regulations must take place with the cooperation of hostile regulators, and these regulators aren't sympathetic to problems viewed as being largely of the Federal agencies' own making.

To bring its facilities into compliance with Federal, state, and local environmental laws, and to avoid costly litigation, the U.S. Department of Energy (DOE) has adopted a strategy of negotiating Federal Facility Agreements (FFA's). In states where DOE facilities are located, negotiating FFA's involves DOE, EPA, and the host state regulators. FFA's are undoubtedly shaped by the protracted and adversarial process that has been necessary to get to the bargaining table. Of perhaps greater significance is the impact of Federal facility history on agreement implementation. Like all agreements with long implementation periods, the work only begins with negotiations. For cleaning up environmentaly contaminated sites 
many operational decisions must involve the agreement and support of people inside and outside the facilities.

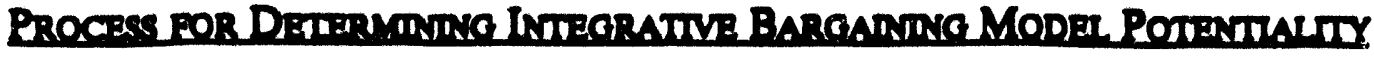

The integrative bargaining model refers to a system of activities for attaining objectives that for the parties involved aren't fundamentally in conflict and can be integrated to some degree. The integrative model is essentially a problem-solving model involving problem identification, search for alternatives and consequences. and preference ordering of solutions and selection. Walton and McKersie establish a three-step process for determining the potential usefulness of the integrative process.

1. Is the subject of bargaining a problem or an issue? A problem is defined as allowing significantly larger joint payofts to all parties although all parties may not share equally in the joint gain. In contrast an issue involves gains to one party and corresponding losses for the others.

2. To what degree do one or more solutions offer all parties a gain in absolute terms over their respective positions in the status quo?

3. To what extent do the facilitating conditions of motivation, information and language, and trust and supportive climate exist?

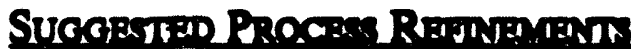

Refinements to the prccess are summarized as:

1. Walton and McKersie don't suggest any priority to facilitating conditions. The final paper will show that motivation must precede information and trust.

2. The relationship between information and trust isn't adequately treated by Walton añd McKersie. An improved process will show information and trust interrelated and growing as they increase through the integrative process. 


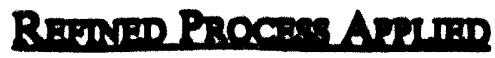

Federal facility managers and regulators have in common the problem of cleaning up environmentally contaminated sites. Integrative solutions offer absolute improvements over the status quo.

Specific motivations vary. Some are different and may conflict, but like problem definition, motivations can be broadly similar. Figure 1 illustrates both divergent and convergent motivations that can be experienced in Federal facility cleanup. Convergent motivations represent overlapping motivations and opportunities for integrative solutions to problems. Motivations and their significance will be elaborated in the final paper.

\begin{tabular}{|c|c|c|}
\hline & $\begin{array}{l}\text { Mon } \\
\text { Divergent }\end{array}$ & Convergent \\
\hline $\begin{array}{l}\text { Federal } \\
\text { Facilities }\end{array}$ & $\begin{array}{l}\text { Production } \\
\text { Mission }\end{array}$ & $\begin{array}{l}\text { Primary: Compliance w/Laws } \\
\text { Secondiry: Cleanup for Continued } \\
\text { Production }\end{array}$ \\
\hline EPA & $\begin{array}{l}\text { Protection } \\
\text { of Federal } \\
\text { Jurisdiction }\end{array}$ & $\begin{array}{l}\text { Primary: Compliance w/Laws } \\
\text { Secondiry: Cleanup to Satisfy } \\
\text { Public Environmental Interest Groups }\end{array}$ \\
\hline $\begin{array}{l}\text { State } \\
\text { Regulators }\end{array}$ & $\begin{array}{l}\text { Protection } \\
\text { of State } \\
\text { Juriadiction }\end{array}$ & $\begin{array}{l}\text { Prtmary: Compliance w/Laws } \\
\text { Secondiny: Cleanup to Satisfy } \\
\text { Public Environmental Interest Groups }\end{array}$ \\
\hline
\end{tabular}

Figure 1. Convergent motivations represent overlapping mottvations and opportunitios for integretive colutions 
The interface between motivation and the information and trust components must be considered. The final paper will discuss these components, their interrelatedness, and their significance.

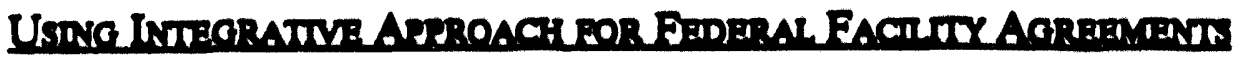

The unhappy environmental history of the Federal facilities will impact both negotiations and the implementation periods of the agreements. Multiple and overlapping regulations, outside scrutiny, huge cleanup cost estimates, long implementation periods; and political realities offer opportunities and problems for using the integrative approach. Walton and McKersie offer helpful strategies and tactics that support integrative processes through attitudinal structuring and intraorganizational bargaining. In the final paper these processes will be evaluated in terms of their strengths for supporting integrative solutions. 


\title{
EXPERIENCE IN DESIGNING AND USING A FLAT STRUCTURE IN A MULTI.PROJECT RLSEARCH ORGANIZATION
}

\author{
Harold A. Kurstedt, Jr., \\ Eugene J. Gardner, and \\ Thomas B. Hindman, Jr. \\ Management Systems Laboratories \\ Virginia Polytechnic Institute and State University \\ Blacksburg Virginia U.S.A.
}

\section{INTRODUCTION}

In early 1986, the organization of the Management Systems Laboratories (MSL) was changed from a standard matrix to a flat organization. The flat organization contributed more negative influences on the organization and its goals than positive ones. One year later, the flat organization was changed to a standard hierarchy and most negative influences were overcome. Before, during, and after the flat organization, MSL saw significant growth in funding and in its resource needs. This paper is an account of an experience with a type of flat organization, why we changed to that organization, what worked and what didn't, why we changed away from that organization, what we learned from the experience, and what we would recommend for research organizations considering flat organizations. The authors include the founder and director of MSL, a senior manager during the experience who informally served as historian, and a manager in the organization that sponsored much of MSL's research during MSL's experience with a flat organization.

\section{MSL'S IDENTTIY}

MSL, an Industrial and Systems Engineering Department research laboratory, conducts research, analysis, and development projects studying management tools. MSL intends for the analysis and development projects to support present or future research projects.

Management tools convert data into information to be used by managers in decision making. Management tools include: organization structure, work-breakdown structure, operations research models, expert systems, paperwork, policies, plans, directives, organizational culture, and the MIS. A manager is anyone who converts information into action. The organization structure is a management tool. MSL is large enough and representative enough to use itself as a research subject to learn about management tools. One reason to convert MSL's organization to a flat structure was to study the effects of a flat structure first-hand.

MSL was chartered in 1981 to demonstrate the proper balance between the business and scholarship of research. Understanding the effect of different organization structures within MSL is part of that demonstration. By 1986, MSL's funding and number of employees had reached $\$ 1.6$ million US and 45 people. Today, MSL annually expends $\$ 7.0$ million US on research and employs 150 people, evenly divided between managers and staff and graduate and undergraduate students. Most of MSL's funding is from the United States Federal government and most of that is from the United States Department of Energy. MSL manages a number of different research grants and contracts from the Department of Energy, other government agencies, industrial organizations of many sizes and functions (banking, manufacturing, utilities, and others), and academia.

\section{WHY MSL CONVERTED TO A FLAT ORGANIZATION}

Before 1986, MSL had evolved from a standard hierarchy to a standard matrix organization for a multi-project environment. By 1986, a Manager of Program Management, a Manager of Research and Development, and a Manager of Operations reported to the Director of MSL. The Manager of Program Management had the research funding in a variety of contractual vehicles managed by a number of program, project, and contract managers, many of whom had formally-identified task managers between them and the people doing the work. The Manager of Research and Development had the research resources (including human talent, laboratories, and equipment) managed by technical managers, several of whom had managers of technical specialties between them 
and the people doing the work. The Manager of Operations provided administrative and logistical support to the organization. The matrix was designed and operated to exercise a healthy conflict between tilose responsible to programmatically serve the needs of the sponsors and those responsible to technically ensure the quality of all products and services. MSL's culture embraces responsiveness, which explains its emphasis on program issues over technical issues.

MSL's Director was the focus of one of the major reasons for leaving the matrix organization. As a university professor, the Director held classroom teaching, student organizations, paper publication and presentation, and university service in high priority. As the founder of MSL, the Director was out of town with present and potential sponsors and was out of the building encouraging the University administration to accept and support MSL's rapid growth. In short, the Director was an absentee director.

Other contributing factors to changing the organization were 1) extensive contract-related travel requirements and personal academic needs of MSL's top managers, 2) lack of experience in MSL's young managers, and 3) inadequate supporting systems to implement the matrix. The available people who could best act on behalf of the Director were absent almost as much as the Director, with the notable exception of the Manager of Operations. Except for the Director, all of MSL's top managers were under forty years old and many came to MSL directly out of their graduate school experience. While researching and developing management tools for others, MSL had insufficient discretionary resources to dedicate to its own supporting systems to provide the level and diversity of information needed for a successful standard multi-project matrix organization.

Five influences motivated a change from a standard matrix to a flat organization. First, the Director was absentee, causing a lack of consistent, MSL-wide direction. Second, potential substitutes were equally absent. The effect of so many top managers being absent was that people didn't know what was expected of them, did what they thought was best, and managers didn't have feedback on progress and results until late, thereby generating last minute changes and crises. Third, there was no clear designee as a formal Deputy Director to act in the Director's absence, because of the candidates' inexperience or personal academic commitments. Fourth, people within the matrix couldn't get status information and resource availability information for contractual reporting requirements. Fifth, tlat organizations were popular, our sponsors were curious, and we could learn from first-hand experience.

\section{MSL'S FLAT ORGANIZATION}

Early in 1986, MSL's Director decided to buy time until some of the managers' personal academic needs were resolved and one or more of the young managers surfaced as a leader. After brief, informal discussions of the tlat organization concept with MSL's top managers, the Director announced the structure and purpose for a reorganization at an annual recognition dinner for all MSL employees. Clearly, the announcement was perceived as a surprise by everyone in the audience. The conceptual nature of the structure and the destruction of past lines of authority threatened almost everyone-especially those in the old authority chain. The new organization was to be so flat, to emphasize its flatness, the Director coined the term "the un-organization" for MSL.

\section{Operations}

MSL's Director introduced the un-organization as a revolutionary concept but was determined to institute an evolutionary process for implementing, evaluating, and modifying the concept. That concept is diagrammed in Figure 1. The new organization was set up to reflect the culture of responsiveness to the sponsor and rotates around continuoushy improving performance. In a multi-project research environment, the structure recognizes five ingredients to support the sponsor and promote marketing opportunities: problem solving, production, innovation, creativity, and performance.

The Director convened a two-day retreat with five of MSL's most experienced people to define the operations of the un-organization. These five included the Manager of Program Management, the Manager of Research and Development, and the Manager of Operations from the old organization. The Director intended to establish the five and himself as a steering group to manage MSL. Each steering group member was to have equal voice. He asked the group for the retreat to effect three changes in MSL: 1) teams should dominate MSL's activities and be 


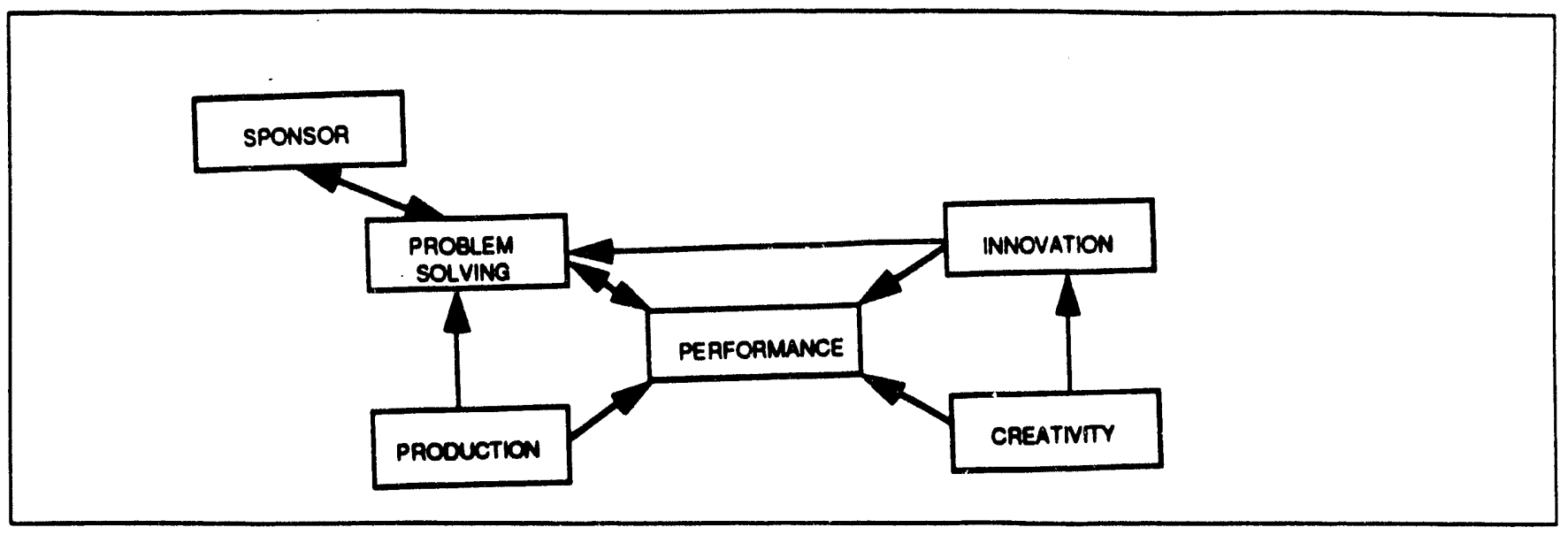

Figure 1. In a multi-project research environment, the structure recognizes five ingredients to support the sponsor and promote marketing opportunities.

assigned to tasks where they are needed or where their interests and capabilities lie to eliminate "turf (territory) protection" situations, 2) the group would develop effective, coordinated direction in MSL's strategic and tactical decision making, and 3) the group would develop more frequent and useful review and feedback of each person's activities and professional development.

The result of the retreat was a plan of action to adopt the un-organization structure in Figure 1 as an interim measure; to evaluate the concept during the year; and, if necessary, to modify the concept at the end of the year. One of the five managers would be the conscience, or proponent, for each of the five ingredients shown in Figure 1 , and, together with the Director, would form a steering group to make the strategic and tactical decisions for MSL. The philosophy for the shared decision making was people would agree the vast majority of times and the Director would seldom have to intervene. Each steering group member would be the conscience for a particular ingredient, taking an active interest in decisions affecting their ingredient. The intent was to ensure each ingredient was considered and balanced against the other ingredients for every task in MSL.

The resulting un-organization would have two levels: the steering group and task teams. Steering group members would also serve on teams. People were assigned to tasks, not to turf. All MSL activities, including administrative, sponsor interaction, social, outreach, and management ones, would be divided into tasks. The steering group would identify tasks and task leaders with input and assistance from involved people. The task leader would be responsible for achieving task objectives, help the steering group select team members, provide information on task progress and receive guidance and suppor from the steering group, and conduct team meetings. The steering group would work with each person to equitably distribute resources across teams and to rank order tasks. MSL people had no designated titles.

The steering group met every Monday, all day and evening. The objective was to concentrate all direction and crisis resolution into one comprehensive weekly meeting, allowing steering group members to travel the rest of the week. Every task leader reported to the steering group each Monday, requiring almost all MSL employees to be in town all day Monday. The steering group had a standard agenda and consistent format guidelines for teams reporting to the group.

Professional evaluation and development was conducted for each person in MSL by the steering group. Group evaluation provided feedback from many different perspectives, rather than just one. The steering group was to conduct quarterly evaluations for all people based on their performance as a team leader and as a team member. The Director evaluated steering group members. The Head of the Industrial and Systems Engineering Department evaluated the Director.

\section{Results}

The steering group meetings were very long; after some months, they were streamlined to fullwork-day meetings. Steering group members 
developed a special language to streamline operations for reports from so many people. The steering group members never championed their ingredients from the concept shown in Figure 1. The ingredients were too qualitative for the members. They had trouble carrying out their responsibilities as conscience for their ingredient because they never formed practical meanings for their ingredient within MSL. The Director had veto power by virtue of his accountability to the University as the only professorial faculty and to the sponsor as the only Principal Investigator (required by the University). Once or twice during the year, the Director exercised his veto to override a decision or resolve a close vote. The veto seemed to have little negative effect, unless it should have been exercised more. Many issues that arose during the week between steering group meetings were held over to the next meeting. Usually, a team realized they should go to any steering group member between meetings for an individual decision if necessary. The steering group members had little trouble making these decisions and accepting decisions by other members.

All steering group members felt responsible for everything in MSL. As a result, 1) they were spread thin in time, talent, and patience; 2) they offended other steering group members because they were perceived as being out of their element; and 3) the sponsor often wasn't sure whom was their primary contact point at MSL. Specific individual conflicts arose between pairs of steering group members, not resolvable by line of authority. The Director brought the pairs together to resolve behavior. He didn't resolve attitudes or feelings.

Because of the steering group, MSL people better understood what was expected of them. MSL people had less, or worse, information because the teams became more independent under the un-organization. The members of the steering group were well informed of everything going on in MSL. Others were not informed outside of their own team activities. In short, the information flowed up the organization well, but not down the organization. Top managers' time was inefficiently used because they had overlapping knowledge of details. The overlapping knowledge wasn't bad, just unnecessary.

Prior to the un-organization, MSL implemented social events such as informal lunches among random groups of MSL people, and a social-type newsletter. The un-organization helped terminate those lateral relations and hinder the informal cultural network because the cheerleader (MSL's Director) was

"committeefied," or reduced to a more balanced emphasis, by a steering group that turned out to comprise mostly introverts.

All MSL people, steering group and team members, had significant trouble with such global lines of authority. They couldn't 1) identify the single person they reported to; 2) delimit who reported to them; 3 ) trace their career path in achievable jumps up through an organization structure; and 4) put a clean, understandable title on their business card.

MSL tried to institute professional development packages for each employee. MSL never put the supporting systems in place to set up reviews, determine the best course of events, or evaluate the progress of and for the people. MSL never set aside the time needed to make sure each person had interaction with the steering group as a body on their expectations and progress. The lack of supporting systems in financial, status, and projection information caused more problems in the un-organization than in the other organization forms.

Very late in 1986, the steering group appointed a team to review the steering group and the unorganization. The evaluation team found:

1) The steering group didn't provide adequate leadership in identifying common goals, describing the corporate vision, and promoting corporate culture;

2) The steering group increased information upflow and the synergy of the upflow;

3) The responsibility and authority the steering group delegated was unclear;

4) The steering group could not provide fulltime management;

5) The evaluation of MSL team members didn't involve team leaders;

6) Support systems in accounting, project management and clerical or sectetarial services were inadequate for the organization structure; and

7) Follow-up on decisions wasn't working well.

MSL abandoned the flat organization in early 1987 partly as a result of the evaluation team and because of four crucial factors. First, one of the steering group members surfaced as a leader and had time to put his personal academic tasks 
behind him. Second, everyone on the steering group identified with each other's weaknesses and member's responsibilities had to be separated. Third, the sponsors preferred clear lines of authority and clearly identifiable accountability. Fourth, the recommendations of the steering group evaluation team could be implemented better through a different organization structure.

\section{LESSONS LEARNED FROM THE FLAT ORGANIZATION EXPERIENCE}

People have trouble seeing their professional progress in terms of more responsibility. Rather, they see progress in terms of an elevated relationship with the top, or a well-defined progression through a multi-tiered organization chart--enough tiers so they can see how far they've come and how far they have yet to go. Young, ambitious, capable people want to know 1) whom they report to, 2) who reports to them, 3) their career path, and 4) their business-card title.

As an organization moves away from a simple hierarchy with well-defined lines of authority toward more-complex diffuse organizations with no, less-defined, or multiple lines of authority, the need for good and more-sophisticated information support systems becomes greater.

When the lines of authority aren't clear, the boundary between the steering group responsibilities and those of other employees is unclear. People are never sure how they are supposed to interact with the steering group. Administration, logistics, sponsor interaction, and other non-direct project activities tend to fall into the cracks.

Issues requiring steering group attention are hard to define in all cases. Those issues, once resolved, don't tend to be communicated well to the rest of the organization.

MSL isn't a one-product or one-project organization. MSL had too many projects with mutually exclusive goals for the un-organization to work. Flat organizations may be best for manufacturing or service (process, not project) organizations that have one or a clearly main-line product or service.
The lessons learned from MSL's specific experience lead to six conclusions for flat organizations in general. Flat organizations 1) need the best information systems possible, maybe better than are now available; 2 ) aren't good for young, fast-growing organizations; 3) aren't good with introverts at the top; 4) aren't good for top managers who haven't already made their mark; 5) need the best professional development and career advancement programs possible, maybe better than are now available; and 6) work better in mature, well-positioned, unchanging organizations.

\section{WHERE MSL IS NOW}

In early 1987, MSL's Director called another retreat and, based on the evaluation team's report, reorganized into a simple hierarchy. The Director appointed a Deputy Director to act in his absence and assigned responsibilities so the Deputy Director would spend more time in town. The Deputy Director decided to separate steering group members as much as possible and made them responsible for autonomous groups. Each group was successful in its own right and the Deputy Director found his job one of resolving perceptions of one-upsmanship among the groups.

In early 1989, MSL's Director, based on the significant growth in size and reputation over the years, was able to hire five experienced managers from government and industry and then assign them to hierarchical positions in the organization. All members of the steering group have management positions in MSL with equal or greater responsibility than in 1986 . However, each one is farther from the Director in the organization than before.

\section{ACKNOWLEDGEMENTS}

The preparation of this paper is funded by U.S. Department of Energy Special Research Grant No. DE FG02-88DP48058. Management

Systems Laboratories thanks the Department of Energy's Office of Nuclear Materials for the research, development, and testing of state-ofthe-art management tools and the frameworks for understanding how to make them successful. 


\title{
Simulation for emergency management: Taking advantage of automation in emergency preparedness
}

\author{
J. Andrew Walker George E. Ruberg John J. O'Dell \\ Management Systems Laboratories \\ V'irginia Polytechnic Institute and State University \\ 1900 Kraft Drive \\ Blacksburg, Virginia 24060
}

Industrial facilities openale in an environment of increased public awareness of hazards, and added regulatory demands. Public awareness and new regulations result from concerns ooer the effuctiveness of safely systems and the consequences of their frilure. Increasingly the public, government regulators, insurers, and stoctideld. ers require coidence that of fallity can be opsrated safely and the accidents can be managed effecetively. Recent federal environmental and occupational sefty regulations reflect the incraning attention. This requirement may lo add ressed by proposing a costeffective approach, which applies simulation kechniques in thre phases lo lake adoontoge of adouncas in emergency prepunel. ness aulomation. The apprench will both I rain and provids soms means of vididating those percons responsible for effectively manning emergencias when thry ocaur.

\section{Need For Improved Emergency Manager Training}

Currently, emergency responders are better prepared than emergency managers 10 aceumplish their critically important functions. By emergency responders we mean those responsible for operational tasks during an emergency, c.g.o fire fighters, emergency medical technicians, and rescue workers. Emergency managers are strategic or tactical decisionmakers in leadership roles, cither in the field or in remote command and control centers (most commonly called Emergency Operations Centers or EOCs), who must manage an emergency, best characterized as an ill-defincd problem with potentially severe consequenees. Emergency managers include plant managers, plant shift supervisurs, and duty officers. In non-industrial settings, they may include senior officials of public safety agencies or other emergency response organizotions.

Training is an integral part of emergency preparedness. Training and standards of proficiency eurrently exist for emergency responders in specific activities. For example, there are approved step-by-step procedures for emergency first responders and rescuc squads in dealing with radiation and hazardous materials incidents (International fire Service Training Association 1987, 1984). In contrast, training and standards of proficiency are leas advanced for emergency managers. Fortunately, understanding the reasons for this difference, and combining those reasons with the increased availability of computers in emergeney management, offer real potential for improvement. This portion of the paper claborates on the need for improvements in emergency management training. The reasons why training for emergency managers is difficult to develop and what currently available methods exist, including their uscfulness and shorteomings, are discussed. 


\section{Difficulties In Developing Eniergency Management} Training

Three difficultics hinder the development of training for emergency managers. First, resources dedicated to preparing for an emergency compete with other needs. It is difficult lo get senior management attention and resources (1) prepare for an event that isn't really cupected to happen, e'.'cially when majur emergencies requiring their significalll purticipation excur infrequently. Any proposed solutiuns for improving emergency management training inust consider the reality of limited time and resources likely to be made available simply for training purposes. Such time and resuurce cunstraints are compounded by high pursonncl turnover ratcs in many areas of both the priviatc and public sectur.

Sccund, management of an emergency often involves an cxtr.ucirdinarily high number of complicated variables. Although sometimes dangerrus, the tasks that emergency respunders must perform are relatively well-defined; having an anticipated scquence, they lend themselves to structure and procedure. Becausc hasic responders have better defined operational respunsibilitics and because they are usually asereriated with a specific tuchnical discipline (fire fighting, emergency medicine, ctc.), these task-oriented jots have gencrally accepted standards of proficiency, with training and testing casicr to measure and certify. By their very nature, such jobs have bencfited from existing computcr-aided training programs and simulators, which provide both instruction and documented evidence of the proficiency of individual responders.

In contrast, there are no widely accepicd, step-by-step prucedures for emergency managers. This type of management docs not reodily lend itself to a high degree of structure or procedure because the anticipated sequence of an emergency cannot be predicted with confidence and precision. The complicated interactions that are a part of any fechnolngical accident are offen not linenr, visible, or understandable. In other words, detailed contingency plans for all possible consequences and all possible paths an cmergency might take may be essentially infinite. Effective emergency management simply cannot follow a rigidly written plan or procedure which can be drilled repeatedly prior in an incident. Rather it means using support systems and toois such as plans, procedures, and computers to suggest a prucess for decisions. The most valuable training would be to develop gencral emergency management skills and 10 practice those skills in an environment that closely resembles the site-specific situation the manager is most likely to encounter in an actual emergency.

Third, emergeney management training is difficult because emergency management is organizationally diverse with no commonly acecpted set of standards and skills for emergency managers. Wic relate this to the following three factors:

1. Emergency management has been slower to develop and organize as a profession than eme gency responder organizations. Professional associations for fire fighters, emergency medical technicians, rescue workers, and other responder disciplines have been organized for a number of years.

2. There is no single, nationally recognized discipline or assuciation for cmergency managers. Many technical disciplines or assoxiations have sumc fraction of its mem. bership involved in emergency management. Examples include the American Institute of Chemical Enginners and the American Nuclear Socicty. While these organizations accurately represent the various individual disciplinus involved with emergency management, they retard development of multidisciplinary standards and skills. This further delays the develupment of training.

3. When an emergency management team is activated at any industrial facility it consists of peuple from various disciplines and backgrounds (tcchnical, administrative, legal, ete.). Even when individuals are well trained in their specific disciplines, their lack of training and experience in large seale emergencies, may cause them to interfere with one another's activitics during such events.

The combination of well organized professional associations, tighter affiliations with specific disciplines, and nure cicarly defined emergency responsibilities has alluwed responders representing individual disciplines to strueture training based on accepted standards. Training for emcrgency managers has not been as fortunate for the reasons identified. Nevertheless, there is a high eost when cmergency managers fail or do not attain adcquate proficiency (U.S. Federai Emergency Management Agency 1983).

\section{Available Methods of Emergency Management Training}

Available methods of training and validation of emergency managers can be summarized in two categoricsclassroom training or participation in emergency drills and excreises. Traditionally, classroom training is basced on seminars and video aids. Although uscful as introductory or general training, these formats by themsclves have the following shortcomings:

- They lack specificity for individuals and the special situations that relate to the sitc-specific variables found in a facility (for cxample, facility hazards, regulations, lucal and corporate management, human and equipment resources, lopography and other environmental conditions, and community relationships).

- They do not provide fully realistic exercises for emergeney managers to practice skills needed for their specific situations.

To be cffective, classrom training must be part of a more comprehensive program.

Extensive experience with drills and excreises shows them to be uscful in addressing the shortcomings of classroom training (Chemical Manufacturers Association 1986, U.S. Federal Emergency Management Agency 1984). Such exereiscs are simulations designed to test and train the clements of the response system and coordination among system components. Various tcchniques include tablctop exereiscs, subsystem drills using role players, and full-scale system exercises. However, just as with classroom instruction, emergency excrcises are not the complete solution. Their drawbacks are principally cost-based and include:

- Assembling the entire response and management tcam, which may include plant and offsite participants, is extremely difficult to coordinate. When it is accomplished, it is very costly. 
- Full-scale excrcises with penple and equipment involve liability risks for accidents and injurics.

- Breaunc of cost and time schertuling difficulties, exercises are infrequent. Participants practiec less often and leam fener lessons.

Many ind ustrial facilities have improved their emergency copatilitics in recent years. Systems have becn cstablished ry' w'riting plans and procedures, implementing responder training programs, working with offsite juriadictions, and devcluping computer support systems (Kutwerg and Tuck 19R4). But, whatever has been acenmplished to date requires further improvement, broader implementation. and continual maintenance and updating. Of perhaps greater future importance will be iraining. particularly em. phasizing management. Additional metherds that can cumplement the currently available and accepted methods uf emergency management training and validation are nerded. Cumputer-based simulaturs can bridge the gap hetween classroom training and live simulations and previde the management training neveded.

\section{Computer-Based Simulators}

At many facilitics computers arc playing an incrossingly hrgier role in prucess safety and cmirgency management (Cnngressiunal Research Survice 1984). These include systems to support decision making in an emergency (Hobcika and Jamci 1985). The essence of this recommendation is not to present a new simulation system that competes with existing computer programs, but to suggest how cencrgency managers can take funther advantage of such systems by also developing them into a training simulator capability.

\section{Basic Automated Emergency Management Systems}

B.isic autumated emergency management systems are wailable for some uetors of industry. They have been installed in many large industrial facilitics and increasingly will he used in smaller faeilities in the immediate future. Alest currently implemented systems are fur decision support, where a databasc feeds subsystems which support sperific functions in managing an emergeney.

A bricf description of subsystems nuw available, or that we belicve will be more broadly availatle in the next few ycars, follow's:

l'lume or Spill Modeling Real-time metcorological and/or other envirunmental data are cumbined with estimated release data to graphically depict the predicted consequences of a relcase in the envirnment (Andrews, Skyllingatad and Clanty. 1988, Heffier and Stunder 1988, Walker 1985).

Hazards Inventory

An up-to-date inventory of hazards resident at a specific faeility which may include their location, quantity, and characteristics (Ward. McCam and Hurton 1988).
Resource Inventory

Identifies personncl and equipment resources available to respond to an emergency. This may include facility resources, other private sector resources, and offsite resources available through mutual aid agrecments (Everson 1986).

Event Logs Records all relevant incident data as they become available. The log becomes a running update of the incident and provides a historical record of all activities and data about the inci. dent (Blackburn 1988).

Board Displaye

Displays current incident infurmation for decision-making by specific categurics; for example, technical infurmation about the relcase, or injurics and medieal infurmation (Emergency Response Facility Safety Parameter Display System).

Notifications Autumated voice notifications of facility management and responders, offsite authoritics, and the public as a part of a public warning system (Peralta, Mayo and Roberts 1988).

Eleetronic Mail

Shares apecific, pre-formatled in. formation with remote locations such as offsite authoritics or curporate offices (Hickman 19(8).

\section{Use and Adaptation of Systems To Simulators}

It is recommended that existing emergeney management decision support systems be adapted and used to function as simulators. Emergency management is a natural area for the application of computer-based simulation. Emergeney managers must make both short and longtcrm mitigation and protective action decisions, often in an atmosphcre of uncertainty, based on many factors. The managers communieate with many different groups, for example: Emergeney Operating Center (EOC) eadre; ficld response teams; senior management: public information center personnel. Information from these sources is disclosed rapidly, but is frequently incomplete (Chartrand 1986, Comfort 1985. Schmidt, Kurstedt, Walker and DeBusk 1985). In addition, a particular incident may not be part of a manager's cxpcrience ur knowledge base. All these lactors complicatc the decision-making environment of the emergency manager or the management team. Emergency managers can achicve improveraents through the simulation for training approach, by increasing their experience and therefore their knowledge base. Most existing automated emergency management systems could be effectively adapted as simulators. Simulators can be expandod, as resources and technology allow, to control scenarios to meet specific objectives, record accomplishod training play "what-if" 
games, and relate consequences to individual or group sctions.

Simulation, in emergency management, involves using the physical environment that an emergency manager faces. The recommended approach is that a facility's automated emergency operations center will sen'c as a training simulaIIr, using historical or artificial incident scenarios. Further it is suggested that a threc-phascd apprnach to implementaliun he followed. Each phase is based on increasing levels of equipment and softuare sophistication and complexity. I'hase I uses a common way to introduce an exereise scenario, by disclosing it through rule players. Participants interact using the existing management system. The principal improvement involves using the existing computer system and on-line checklists to develop a historical tatabase recording the individual actions of the participants. This permits identifying emergency management shills, and validating individuals and the management team on thnse skills.

A l'hase I simulator, for example, could use the commonly autnmated EOC msition of the Mctcorringist (Andrews, Skyllingstad and Clantr. 1988, Sullivan 1988). For instance, this person is usually responsible for asscessing the extent of in airborne dispersion of a hizardous matcrial. In the case "If a chemical incident invelving atmospheric dispersion of a gas, some of the most important items of information an emergency manager may need to know. once the name and quantity of the substance are confirmed, are the wind direction and predieted effects. The wind direction is needed quickly to decide where to evacunte on-site personnel or nearby residents safely. For EOC's which have realtime weather monitoring systems available for this purpose, onec the weather conditions are verified, the available data is typically entered into a dispersion model to calculate the extent of the plume or concentrations, as well as vulnerable zones and cracuation distances. Models such as those cited abuve identify chemical and physical propertics for a farticular substance and provide the emergency manager a basis for determining the immediate and long-term effects 10 populations and the environment.

Thase I systems can also be used for training emergency managers during excreiscs using a realistic secnario for the dain. The emergency manager would get the same informatinn from the same sources whether it is an actual emes. scney or a simulated excreise.

Thase 11 expands the usc of an existing computer system to include automated control and disclosure of scenarios and response to simulator prompts. Phase III further cxpands use of a computer system to include improved interaction with the simulator and give conscquences to decisions and actions of the emergency management team. Figure 1 details specific distinctions of cach phase of the recnmmended appronch.

\footnotetext{
Advantages of the Training Simulation Approach

In general, the odvantages of the recommended approach aduress cach of the difficultics previously identified in developing training for cmergency managers. Technology is not the limiting factor. Additional specific advantages arc:

Stills and Validation - A significant advantage in developing and using simulators at individual facilitics comes from
}

building a bottum-up, experience-based understanding of the skills needed for emergency managers. These simuln. tors will be suitable substitutes for real experience becausc experience with a real emergency is difficult to obtain. It is important to emphasize that validation is not expected to be an expert certification that a manager has acquired a certain amount of knowledge. Success is demonstrated not only by the number of right answers but aleo by the emergency manager's ability to incorporate new information into the management of the response, cuordinate the team decision. making process, and make recummondations for action to various emergency responec groups. No onc can be a certified "expert" because of the variety and cumplexity of emergencies and the number of situational factors involved. But developing and using an emergency management simulator would provide a sound basis for corruborating management skjlls based on the analysis of information and decision-making. Systems will help emergeney manngers do things right but not necessarily specify the right things in do. However, efforts at simulation using this empirically. founded approach will help clarify skills and establish validation criteria by providing supplemental data about manngement performance and effectiveness. Without such systems, this data may be acquired infrequently or unsystematically or not at all during real emergencics or during drills and exercises.

Ineruesed Frequency - Repetition increases knowledge, enhances the ability of emergency managers to function as a toam, and identifies system deficiencies and progress in resolving them. Increasing the frequency of training simulations for managers will help assure these advantages relative to live simulations by reducing the time commit. ments nesded for preparation and reducing management time needed to attend.

Realism - Simulation can be more representative of real. world interactions than classroom training or field excrcises. Participants play an intcractive role with other participants and the support system and by experiencing other characteristics of an emergency, such as stress and time constrained decision-making. As a related advantage. managers from different disciplines and backgrounds learn to function better as an emergeney management team. Realiem is simulated by cvaluating presented dats, making or coordinating decisions, and monitoring progress and obeerving consequences by using the simulator. Use of simulators will be an experience-based approsech in a ficld where real experience of a major emergency is seldom acquired.

System Familierization - To the extent that emergency management is becoming more automated, simulators allow participants 10 practice the use of the computer hard ware and sof ware they would use in a real emergency

Cost-Benefit Ratio - Computer-based simulators are adv(ucated bosed on their attractive cost-benefit ratio. The use of existing aulomated emergency management support systems and the degrec of sophistication can be appropriatcly balaneed with what is neaded and with the cost of available technology. In other words, a Phase I simulator is used if the size of hazard, degree of complexity, and capability of existing automation limit the investinent. Advanecs in available automuted emergency management support systems will mean corresponding advances in 


\begin{tabular}{|c|c|c|c|}
\hline & Approoch to Scenario Design & Mathod of Interaction & Volidotion of Skills \\
\hline$\frac{-}{8}$ & $\begin{array}{l}\text { monuol disclosure of wrillen } \\
\text { ocenorio vaing role ployers orally } \\
\text { releosing inlormotion to } \\
\text { perticiponts }\end{array}$ & $\begin{array}{l}\text { porticiponts use existing } \\
\text { outomoted system } \\
\text { boord disploys ond oral } \\
\text { communicotions }\end{array}$ & $\begin{array}{l}\text { systsm coplures historical } \\
\text { record of ploy } \\
\text { computerized checklist } \\
\text { volidoles thot porticiponts took } \\
\text { specitic octions }\end{array}$ \\
\hline $\begin{array}{l}= \\
: \\
\frac{8}{a}\end{array}$ & $\begin{array}{l}\text { qutomoted method of disclosure } \\
\text { pre-progrommed scenorios } \\
\text { disclosure through outomoted } \\
\text { event logs }\end{array}$ & $\begin{array}{l}\text { porticiponts use existing } \\
\text { oulomoted oystem with simulolor } \\
\text { simulator generotes event log } 10 \\
\text { teed boord disploys } \\
\text { oral communicotions }\end{array}$ & $\begin{array}{l}\text { records porticiponts' actions in } \\
\text { response to simulotor prompls } \\
\text { improved analysis of decisions }\end{array}$ \\
\hline 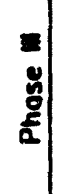 & $\begin{array}{l}\text { outomoted method of disclosure } \\
\text { pre-progrommed scenorios } \\
\text { event logs supplemented by } \\
\text { outomoted messoge injects }\end{array}$ & $\begin{array}{l}\text { porticiponts use exiating } \\
\text { aulomoted systom with simulotor } \\
\text { porticiponts interoct with } \\
\text { simulotor to ploy "whot-if" games } \\
\text { ond receive prompling }\end{array}$ & $\begin{array}{l}\text { expert system rosed } \\
\text { computer generotes reol-lime } \\
\text { consequenses to specitic } \\
\text { octions for porticiponts }\end{array}$ \\
\hline
\end{tabular}

rigure 1. Sprenfic destincuins of each phase of the percomenended epproach.

developing future l'hase II or III simulators. In any event simulators will reduce the cost of emergeney management training by eliminating the need to assemble large response groups 10 train and teat the system. Additionally, they will climinate the liability costs in using pcople and equipment in live simulations.

System /ustification - Emergency management must compete fur rescources with other facility functions. An important advantage of this appronch is that it can further justify cither now systems or systems enhancements beciuse it scrves fliel functions-the primary function is to support the management of an actual einergency and the scecindary function is to serve as a simulator.

Phn Evaluation - The "what ifs" of a simulator, and the ability 10 control these cunditions in an urganized manner, can help to analyec and cvaluate procedures and to develop new strategy and tactics.

Further System Development - Expert system technology in emergency management is just beginning. The field is open for scveral applications (Cass and Chapman 1986, Skylling. stad and Ramsdell 1988). Until these systems are developed and available widely, a basis for them ean come from already installed or planned automated systems which help centralize the important data needed in any effective decision-making process.

\section{Conclusion}

The use of simulators for training is neither new nor unique. Nuclear power plants have simulators specifically buile for training operators. Flight simulators have been used for anany years. The recommended phased simulation training approach differs from the installation of stand alone simulaturs in that modified existing automated systems double as simulators. In addition to the cost. cffectiveness of this approsch we believe it will lend to increased training for the critical management functions that currently are infrequently iraincd. More than the traditional methods of emergency management training computer-based simulation also lends itself to the develop. ment of empirically based skills and criteria for validating emergency managers.

\section{References}

Androma. C.L.; Skyllingaued. E.D.: Clancz, C.S. "Description of Personal

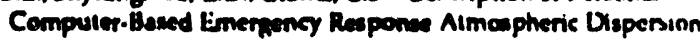

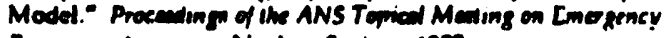
Reprome. Amenean Nucleur Society. 19m.

Mackbum lames A. "Une of the Computerixed Data Link Bunng the func ising liederal liveld linerense." Procendings of the ANS Topical Mearng on Cnargency Ropence. American Nuclear Sudiely. 1 ind.

Chereand. Rober Lex. "Tlusions and Realliy, Using Information Technolngy; in Enerkency Management." In To minel Diemters: Computer

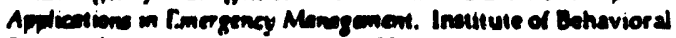
Scienes. Univenity of Colorada, 1 ses.

Oremicel Manulecturen Areodation. "Comanunity Emergency Response Everaves." Wakhingrion D.C. 19se

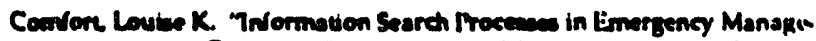
ment: Computer Sinulacion As a Meess of Improving Opganiza. tiond Dudioion-Making Capacty." Precendinge of the Confer. mee en Emegency Planneme. Sodery for Compuler Simulatuon. Le Jolle. Caltifornie. 198.

Congreswenal Researeh Serviee. "Information Tectnolog)" lor Emergency

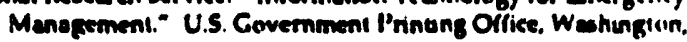
D.C. ises.

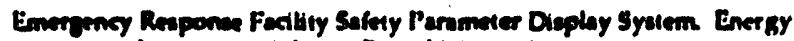
Incopporend, Idaho Falla Idaho, nd.

Everson Prest R. "A Selective Review ol Informotion Responee Technolugy"

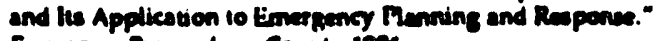

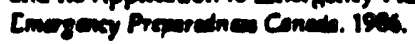

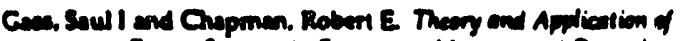

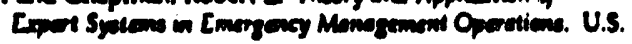
Depparineane of Commerce, ishe.

Ibeffer, Jesome L and Stunder. Barbera J. B. Trogren for Oparational Tra. jectorio (TOD." Precentinge of the ANS Tepicel Meaing on

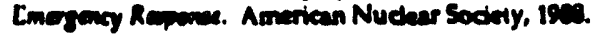


Hickensen Jahn 8. The NRC Emergency Reapones Dats System Fropea"

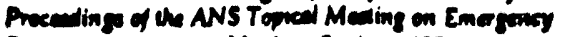
Remone. Amortean Nuclase Sodty, 19.3.

Hobalke, Anwine G. and fanet Bahram. "MASSVAC: A Modd tor Calouldo ing Evecuation Times under Naturd Dievion." Preculinge of the Conformes in Emargency Planning. Sodety fos Cosnoutes Sinulation, Lo Jolla, Caliomia 19a.

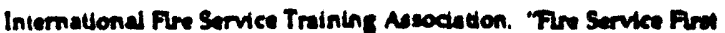
Respondor." First Edtion. Fire Protestion Pullientima, Oulehoon State University, Stllwake, Oblahorna, 1 wat.

International Fire Sarvice Training Associstion. Ilaz Mat Respones Teans Leak and Spall Cuide. Fire Protation Pubticntion, Oulahoon Stale University, Stillwater, OkJahoms, 19:4.

Peralle Raul J.: Mayo. Lowrence E and Roberta, James N. "Applying

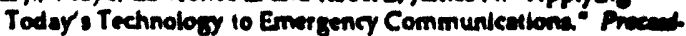
ings of the ANS Topied Moating en Emar gency Rerme. American Nuclear Sodity. 198.

Ruberg Ceorge $E$ and Tuck Kimbarly C. "The Impact of the Suparfund Amendmons and Reouthortention Aet on Emerenoy Prepandnese Response Proppans at DOE Facilities." Preending of the

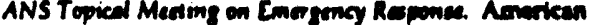
Nucleas Society, 1903.

Schmids, J. Wulian; Kurstedt Ilarold A.Walker.). Andrew: Dricuch Reherd $E$ "Tr-Purpowe Energency Management Model" Procendinge of the Confarence on Emagency Plonwing. Sodety for Computer Simulation, Lo jolla, Caltiornit, 19.3.

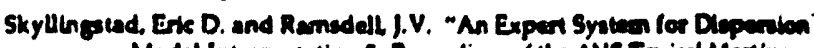
Model Interpretalion." Proceding of the ANS Topied Mentine

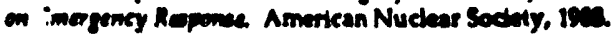

Sullivan Thoone f. "ARAC. Evolution by Acoident." Aresuling of the

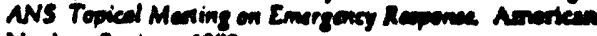
Nuclear Society, $19.1 \%$.

US. Foderal Envergency Manogenent Agency. Guide io Emargung Managoment Esereme. isu4.

US. Federal Emergency Manegenent A pency. "Soction i: Tha Now fes Eneryency Managemant. Incroduction to Enmengy

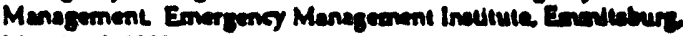
Merland, 1930 .

Walker, Hoy. "Spatial Dus Requitemenis for Fmergency Reppenes" Pre coedinge of the Comference on Emergency Planning. Sodety bes Computer Simulation, Lo Julla Catifomia $19 \%$

Ward. W. M.: MoCam. L. B.; Horton C Y. "Onvelopment of a Compu. terized I lazerd Oavifieation Sritem." Proenting of the ANS

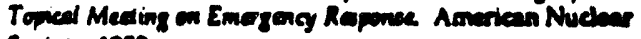
Sodery, 1984.

\section{Acknowledgements}

The authors gratefully acknowledge the support of the U.S. Department of Energy through Special Researeh Grant Number DE-FC05-86DP7033, the Westinghouse Materials Company of Ohio through contract number 277221, and Martin Marictia Energy Systems through contrates 7 M0371 and 19-Y-DY-028C. This support does not constitute an endorsement of the views expressed in this paper.

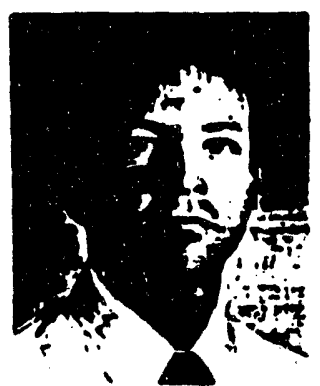

Andrew Walker is the MSL Program Director for Waste Management, which accomplishes rescarch grants and contracts in hazardous materials and waste management and in emergency management. Under his direction, MSL has produced integrated emergency management systems for the Departinent of Encrgy's Oak Ridge Operations Office and Savannah River Sitc, the State and county jurisdictions that surround these locations, and thair oparating and accurity contractors. Andrew also has experience with transportation lesues in emengency management, training, and tabletop and full-ecale emergency exencises. Andrew earned his 8.A. in Political Science from Emory and Henry College and his M.A. in Public Adminiatration from Virginia Tech. He be an active member of the Ameriean Society for Public Administration, especially within the eection on Emergency Management.

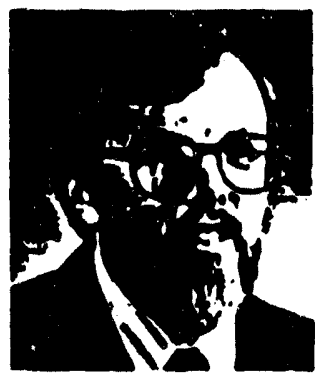

Ccorge Ruberg is a Research Associate and Program Manager at MSL. His research interests include organizational isaucs in emergency managernent, waste management, and transportation. Previously, Ceorge worked for the Cily of New Orleans, where he was Director of the Office of Emergency Managemont and served as a Commissioner of the Orleans Parish Communica.

tions District (911 Bosrd). Ceorge cemed his M.A. in Political Selence from Tulane University and obtained a Bacholor of Sclence from Drexd University.

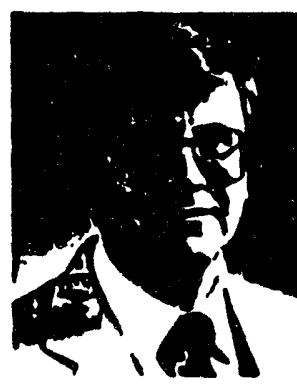

John ODell is a Research Associato at MSL He received a B.S. in Industrial Englncering and Operations Research in 1904 from Virginis Polytechnic Institute and State University. Since 1984, he has worked for Management Systems Laboratorics providing systems support in programming and technical assiscance in the design, development, and implementation of computer applications. He has designed systems for the Department of Energy, Martin Marictla, ALCOA, and Westinghouse. For the last two years he has focusad on automating spectic functions within emergency operations centers induding status boards, checklists, on and offite notification systems, loge, weather, and dispersion modeling. He is currently working on automating the Department of Energy's Oak Ridge Operations Emergency Opurations Center. 


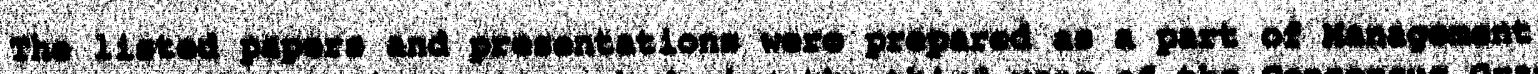

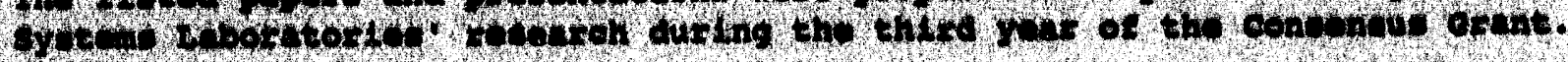

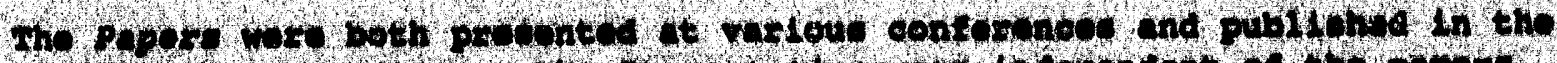

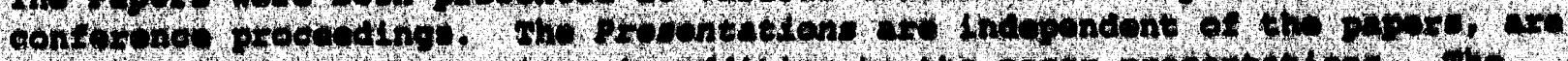

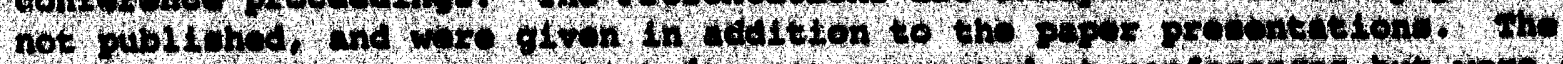

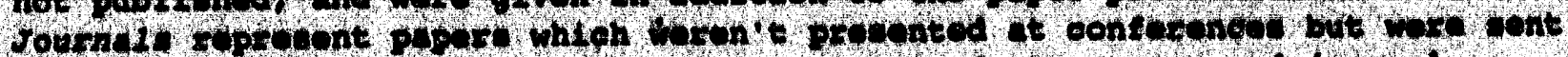

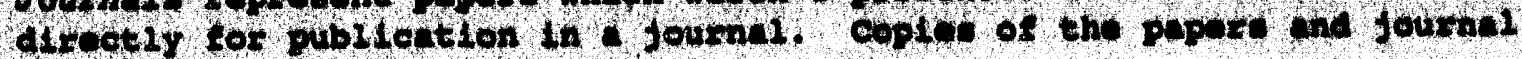
attiblos tollon.

min

nth

papors

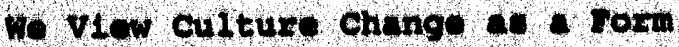

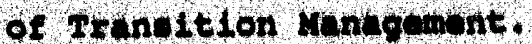

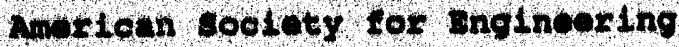
Whapment conternow.

Why Engineers kutet know and Hanige organdzational culture. Procosdinga of the 1990 International Enginoering venagement conference.

To Undoretend conseneus the yust know that the 21emente Are. Southoretern chapter of the Instltute of Hanugement seteneed.

The effect of Experte on the croup consonnus Procese. Southeatern Chapter of the Inotitute of unegement seleness.

Conseneups In Invortigation of the Noed for racliltation. Southeastern Chapter of the Inotitute of Management seleneos.

A solection rool for choosing the Bost particlpative problem-solving toehnigue. Southeastorn chapter of the Inutltute of Uanagement seleneos.

How to Increase Neeting Effectivenose and Ifflelonoy. Southeastern Chapter of the Inetitute of Management solences.
$10 / 90$

$10 / 90$

$10 / 90$
Tundemontel

Contonum Prosuter

Intoralt ton syotene

Tundummtal.
$10 / 90$

$10 / 90$

Fundemantel
Conoonere Procese 
Ditors

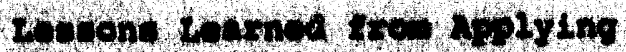

$10 / 30$

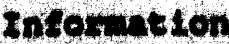

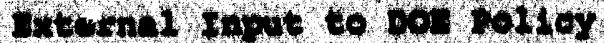

artat

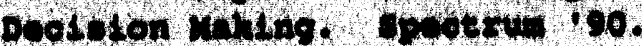

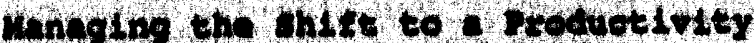

2101

6otinnous

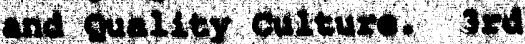
intornt tond and andor on

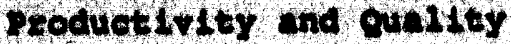
Ronten.

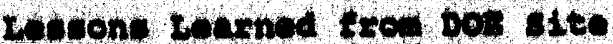

$2 / 91$

Conownetse

cuiture dhange rotivteles and reoente

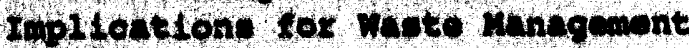

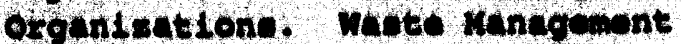

101.

Struotured veeting roehnlques Identify

$2 / 91$

conenneus

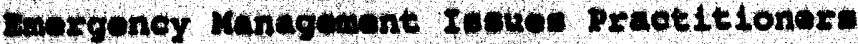

Prodoto

Rovily want to tee Addrowend. Conterenos

on Integrating Dindeter Rewearoh and

Pract 100 .

satiotyling stekcholdore tor

$3 / 91$

Consenneus

suceoseful Project Renagemont.

13th conteronce tor computers and

Induetrlal englinearing.

Case studien on Deslgning uoetings

$9 / 21$

Coneeneus

for Difcetive Inotitut lonal Interactions.

Environmental Remediation 91 .

proeser.

\section{Pronontetione:}

UeIng Expectatione to Improve

$5 / 91$

Fundamental

consentue.

31et TIMB/OnsA Jolnt ratlonal Moeting.

A Consencue Kodel for Doveloping

Fundamentel

strategies In Partieipative Decision

Making.

31et TIMS/ORsA Joint National Moeting.

Problem solving: A subjeot for

$5 / 91$

Consensuse

Educator-Practitloner-Researcher

Procese

Interface.

31et IIMB/OREA Jolnt National Woeting. 


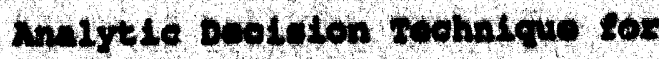

$\mathbf{8} / 91$

Intormation energoney Margere.

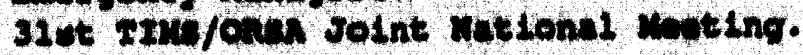

A Unngement Tool to rodeues Coneoneus.

$5 / 91$

rundamentel

310t Ity /onen solnt hetlonal wooting.

How vensogre can vnderstand ind vee engowerwint to ohenge organleatlonal

$\$ / 91$

conennus

culture.

32dt rims/ona Jolnt national voting.

The ves of art in Undergraduate

$5 / 91$

Prooese

ringineoring ranaguent Bdueation.

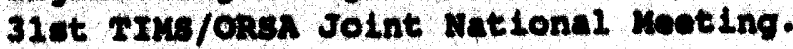

The Role of the Ionder-rollowership

$8 / 91$

coneeneus

Interaction in a syateme Approuch to

procese

culturo Change.

31et Trus/oras Jolnt Mational Mooting.

The Iefects of confllet and Ita

$3 / 91$

rundanontal

Resolution on Dedision quilty and the strength of coneonsus in Coneoneus aroups.

310t TIus/Orsh Joint retional Mooting.

The Effeote of Expert power on the

$5 / 91$

pundamenta1

Group coneoneus Procens.

310t rIus/OasA Joint rational reoting. 


\title{
WE VIEW CULTURE CHANGE AS A FORM OF TRANSITION MANAGEMENT
}

\author{
Harold A. Kurstedt, Jr., Director, Management Systems Laboratories and \\ Hal G. Prillaman Professor of Industrial and Systems Engineering \\ Marilyn S. Cavell, Research Associate \\ Larry A. Mallak, Research Associate \\ E. Morris Howard, Lab Director for Energy Systems \\ Management Systems Laboratories \\ Virginia Polytechnic Institute and State University, Blacksburg, Virginia
}

\begin{abstract}
Transitions create great changes in organizational cultures, and I see culture change as an important tool for managing a transition. The recent change in Presidential administrations represents a very large and far-reaching example for transition management. I analyze a culture change of a smaller scale, yel affected by the Presidential transition and one I have been able to observe. I focus on the U.S. Department of Energy's (DOE) Office of Environmental Restoration and Waste Management (EM).
\end{abstract}

Transitions differ from other types of change. Transitions are planned, evolutionary, and helpful, and are therefore a type of controlled change. I define culture and culture change based on the literature and our experience with government and industry cultures, focusing especially on EM as my example. I then discuss transition management and compare and contrast it with culture and culture change. I can then formulate my picture of culture rhange in conjunction with transition.

\section{Transition is a Form of Change.}

I investigate the differences between managing change and managing transition. Managing change, according to Kanter ${ }^{1}$ demands an "understanding [of] how individuals can exert leverage in an organization to sell their ideas and implement them. Managing transition through a change in culture similarly requires managers to communicate the elements of culture change pervasively throughout the organization. Managers who view culture change as a form of transition management will reap the benefits of a controlled change through a transtormation of the corporate culture.

Transition management differs from day-to-day management in several ways. Managers involved in a transition make different decisions than when they deal with more routine management situations. In routine management, managers use status information, planning information, and verify and evaluate execution against formulation. During a transition, new managers ask "What does my organization do?" and not "What is the status of this milestone?" Managers follow a tutorial to guide them and their staffs through the transition.

Transitions in organizations like EM are especially difficult because of the many entities exerting influence. EM has complex organizational interfaces. The complexity of EM's environment has external and internal aspects. EM encompasses Headquarters in Washington, D.C., and ght operations offices responsible for 15 major DOE . stallations. E.M must respond to the Secretary of Energy's mandate for a new DOE culture. External aspects include Headquarters' interfaces with Congress, other government program offices (e.g., the Environmental Protection Agency, Office of Management and Budget. Department of Defense, and Department of Justice), and other DOE organizations at the Headquarters level. Organizational interface complexity external to EM is increased by its interaction with individual states, Indian tribes, and public interest organizations both directly and through its tield offices.

EM provides a single focal point for DOE compliance and cleanup. These activities were previously spread among DOE Defense Programs, Nuclear Energy, and Energy Research. EM's three programmatic offices-the Office of Waste Operations, the Office of Environmental Restoration, and the Office of Technology Development-have responsibility for distinct program areas concerning environmental restoration and waste remediation.

Changing culture is the difficult part of organizational change. Culture causes organizational inertia and can put a brake on change processes." Culture is "a social energy that moves the corporation intp action"; this action can be either productive or destructive. We want to use our knowledge of culture change to get the most productive action from the culture's social energy and to avoid or mitigate any destructive effects. In most presidential transitions, a government agency must also change its culture.

\section{What is Change?}

Organizations change to achieve a new state with characteristics different from the old state. Organizations can purposefully or accidentally change. In this paper, I am interested in purposefully changing the organization. Purposeful change is intended to achieve an improved state. The improved state would include the characteristics desired by the change master. Kanter ${ }^{4}$ defines the change master as "those people and organizations adept at the art of anticipating the need for, and of leading, productive change." The change master is the decision maker responsible (formally or informally) for the change.

Organizational change can be considered first as a difference in state and second as the process of achieving the different state. The beginning and ending states have characteristics the change master must recognize, define. and determine. The change process has a forcing function, constraints limiting the change, and characteristics of the process. The change master directs and/or implements the change process.

I can characterize the difference in state. Both the old and the new states have real and perceived characteristics. 
Those characteristics include identity, mission, and culture. Identity is like demographic characteristics: who we are. - ission is purpose: why we are. Culture is what we hold ar: what we stand for and how we do things. In this paper, I' $m$ interested in organizations changing their real and perceived states. We want to know 1) what behavior, attitudes, intent, climate, and culure elements to change; 2) how to change those things; and 3) how to measure the progress of culture change.

\section{What is Culture Change?}

Organizational change includes culture change as well as physical, administrative, and other changes. Of these changes, culture change has the greatest effect on organizational change because it deals with qualitative, human issues like values and traditions. In short, without culture change, you get no effective organizational change.

I'll define some terms before proceeding. Culture, simply put, consists of the shared values and beliefs individuals hold dear. Culture gives meaning to events and provides members with rules for behavior. ${ }^{5}$ Elements of culture are particular partitions of culture along some predetermined direction which, when taken together, constitute culture. We accomplish culture change by changing one or more of the culture elements.

When the environment of a strong-culture organization experiences fundamental change, the need for culture change becomes apparent and critical. A life-threatening experience usually precedes culture change. For EM, there will be more work of a different kind and all current stakeholders may or may not be involved. EM will need to ange from a product-driven organization to a processuriven organization. Stakeholders want a voice in EM in the future and must be included in the new culture.

Change and culture change go hand in hand. Changing culture is the difficult part of change. We can readily change the operations, production processes, even how employees are rewarded for their actions, but changing the culture is reputed to require forever and a fortune. This is an exaggeration, but it makes the point: culture change will not happen overnight, and it won't be cheap. Culnure change requires constant management attention and employee involvement. Multiply the cost of all these people participating in the culture change (i.e., everyone in the organization) by the time they will spend in culture change activities and you'll have a rough idea of the financial commitment necessary for successful culture change. Organizations don't have forever or a fortune. Once we start culture change, we have to do it right. We have to manage change and be creative. We must change the momentum caused by the strong culture's organizational inertia and shift to the new culture. As Richard Huber of General Motors put it: "When you ask a bear to dance, you can't quit just because you get tired.'

The changing external forces affect strong-culture organizations most. These organizations value people and process over production. They involve their people in management activities. These organizations must respond to pressures from environmental groups, increasing public scrutiny, diversity in the work force, and participation in

obal markets. They focus on continuous performance .lprovement by making the small things better--little, observable, doable, measurable things. It's not the big things that make the difference--it's the thousand little things we do that please the customer and stakeholder.

\section{Use the Engineering Method for Efrective Culture Transition.}

I believe the engineering method can be simply stated as figuring out 1) where we are (WWA), 2) where we want to be (WWWTB), and 3) how to get there (HTGT). The activities, decisions, and information related to WWA and WWWTB deal with the old and new states in organizational change. In determining the new state, the change master is in a leadership role requiring 1) getting attention through vision, 2) giving the vision meaning through communication, 3) gaining people's trust through positioning to achieve the vision, and 4) gaining people's confidence through healthy understanding of success and failure. In managing the process of changing state (HTGT), the change master must work through the stages of gaining visibility of the organization by monitoring, gaining control by holding the organization at steady state, and improving the organization by changing to a better steady state. The change master does this in the role of what I call administering the management process.

Perhaps the key to successful change is establishing expectations. People are more satisfied if they know what is expected of them. So, in addition to the leadership activity of having a vision for WWWTB, the change master must bring the vision down to earth in communicating that vision in terms of what is expected of each person in the organization. This definition of expected behaviors and attitudes is part of the change process. Once people know what is expected of them, they ll move heaven and earth to live up to the expectations. Obviously, if the change master has defined the right expectations for the desired culture, the change will be successful. To envision the right expectations, the change master must understand his or her organization and how it works.

The most important and most difficult part of change is gaining visibility of WWA and of progress in HTGT. Of course, as we successfully exercise HTGT, WWA will become WWWTB. To get visibility, we must 1) clearly and crisply capture and delimit our responsibilities; 2) identify both the indicators we need to measure to collect data and the standards against which we make the information we need to determine progress; and 3) gather the data, convert them to information, and prepare and present the information in effective ways. Industrial engineers long ago found charting methods to be most effective in capturing and delimiting responsibilities. We can meaşure so many indicators at each step of our change process, ${ }^{8}$ the real problem is in selecting the right measures to evaluate, those few that capture the essence of the important things to change. In culture change today, the only reasonable way to derive standards is through close participation and interaction with all stakeholders: customers, staff, neighbors, and shareholders. I collectively call these entities critics and add them as a fourt 4 criterion to the classical project management triangle of cost, schedule, and quality. Cost, schedule, quality, and critics are interdependent. Not only will managers have to include critics along with cost, schedule, and quality; but cost, schedule, and quality will be more affected by critics than vice versa. Success in the critics criterion can buy latitude in cost, schedule, and quality that these three criteria can't buy without it. 
Today, qualitative issues dominate our top management resources. The manager isn't going to get the qualitative issues to go away. Delegating issues of lower priority requires knowing what the priorities are among issues of all four success criteria. The manager must be able to distinguish the important or difficult issues and intelligently allocate resources and delegate responsibility.

Once we know WWWTB and we gain visibility of WWA and have the ability for visibility of our progress in HTGT, the next stages for the change master are controlling and improving the organization. Visibility is the most important stage because the other stages depend on visibility. Control and improvement are a matter of effective communication between the change master and the people who must make the change and make the new organization successful. The important communication for change is one-on-one between supervisor and subordinate and between any two people in the organization through lateral relations. We can use the cultural network to accomplish lateral relations.

\section{Information for Transition is Training Oriented.}

In managing an organization not undergoing change, decision makers need status and exceptions from standard for the four success criteria: within cost (resources), meeting technical specifications (quality), on time, and satisfying critics. Most information systems are developed intending to provide this type of information. However, transition deals with people (often new people) working with information about new things. For example, in a presidential transition, people in the new administration must come to grips with an unfamiliar organization having an unfamiliar purpose doing unfamiliar things. In short, they must learn about the identity, mission, and culture of the organization. They must do this before they can use the usual management information systems for balancing and optimizing the four success criteria. So, for an organization in transition, the crucial information is tutorial in nature and can't assume prior knowledge and understanding.

Because culture change is so difficult, one fundamental requirement to effect change is a threat to the organization. A second fundamental requirement to effect change is training. Significant portions of the organization's resources must be allocated to teaching the new philosophy, approach, terminology, attitudes, behaviors, outcomes, and outputs. For culture change, people throughout the organization must be trained on the organization's mission, identity, and culture. The information needed for the transition includes definitions and descriptions more so than status and exceptions. In a transition, new managers need to know about what they now manage before they need to know the effects of their management decisions. The systems to supply the tutorialtype information are training systems rather than management information systems. In effect, information systems for management in transition are training systems.

\section{The Case of EM Illustrates an Organization in Transition.}

Most organization talk of culture change; some face up to it. The U.S. Department of Energy's (DOE) Office of Environmental Restoration and Wasie Management (EM) is facing up to culture change--and paying the price. I've heard that more money is made in talking about a new subject than is made in actually doing something about it. That's because it's easier to do things hypothetically than in actuality. Such is the engineer's world. Engineers are used to acting at the point "where the rubber meets the road," things aren't as they seem in theory, and the success factors are at odds with each other.

EM is the showcase for DOE's culture change. They are at the point where the rubber meets the road in a highly sensitized government situation. EM will seek input from stakeholders, which will generate technical and management approaches EM did not previously consider. EM must define and understand its new culture, manage the elements of the new culture, and set reasonable expectations with the public for what that culture means to them.

Critics implies both an audience (receiver) and feedback (sender). EM must view the concept of critics with enthusiasm and optimism. EM will face increased scrutiny and have more laws and regulations aimed directly at its efforts. The combination of stakeholder interest and environmental awareness has put EM in a hot seat that it's not used to occupying. EM must respond to the pressures and actions of external forces including the peace dividend, diversity in the work force, greater environmental awareness, increased public scrutiny, and the removal of the veil of secrecy protecting DOE information.

After March 6, 1989, when Admiral Watkins was appointed Secretary of Energy, he stated he would change the agency by changing its culture. He has mandated that the "trust-me" culture that pervaded DOE and its predecessor agencies for more than 40 years must yield to a "watch-me" culture. He knows the agency must change to survive, and DOE's stakeholders expect the culture to change. I believe Admiral Watkins knows where he wants DOE's culture to be (WWWTB) and he has stated the principles of his vision for DOE's new culture. The Assistant Secretary for EM and his staff also know, understand, and support the new culture. But, the understanding and support isn't being promulgated down through DOE and into the ranks of its contractors. The success of DOE leadership in accomplishing the thorough promulgation of the new culture could spell the survival of DOE and the nuclear industry and significantly influence the lasting impact of DOE's crucial field sites on our environment. DOE hasn't any organizational role models, especially in government, for changing culture. And DOE must bring to reality what it envisions--and it must do so in an evolutionary, planned, and helpful way. EM must lead DOE's transition into Admiral Watkins' new vision by deliberately managing the change.

Based on the principles of organizational change and culture management I outlined earlier, here are the crucial factors to watch in DOE's culture change venture. First, after much study of Admiral Watkins' statements and DOE's needs, I believe I can state and understand the new culture (WWWTB). Admiral Watkins is the champion for the new culture, and a good champion he is; but he doesn't have time to accommodate the full-time job of culture champion. I believe what the Secretary expects of his people is that they design quality into the $D O E$ nianagement and manufacturing process and thereby build quality into the DOE product. The factors of worker and public safety and environmental impact are inherent in focusing on the quality of the process. Also, unless the stakeholders in DOE's process and product are satisfied, there will be no quality. DOE must successfully implement the lessons learned by successful Japanese and West 
German businesses. The lesson is that, in today's world, success comes from focusing on people and process rather than on product and profit (for DOE, budget). But DOE leadership hasn't characterized its vision crisply enough to rapidly pass the understanding of the vision from person to person. DOE hasn't developed the symbology to make the new culture unforgettable for its people. The rank and file in DOE and its contractors haven't heard the vision crisply and can't remember what the vision is. They don't yet know what is expected of them. DOE leadership believes it has spoken, but the rank and file hasn't heard. Watch to see when DOE stakeholders will be able to articulate and implement the vision of the new culture (WWWTB).

Second, DOE management must gain visibility of the process they must design quality into. DOE is right in concentrating its effort here, in that you can't control what you have no visibility of, and can't improve what you can't control. In developing procedures and incident reporting, DOE is trying to get its arms around what has happened and is happening (WWA). Watch to see when DOE management is no longer surprised by its own mistakes.

Third, DOE must have a comprehensive plan to achieve a quality process. EM has made huge strides in its five-year waste management plan for waste cleanup. The organizational change reflecting the culture change will backup cleanup with facility modernization and a new approach to work on the part of its people. The DOE problem is exacerbated by the fact that all DOE's stakeholders (including staff) are impatient. These stakeholders want DOE to do better and they aren't getting much comfort from DOE's past efforts. Watch to see when DOE lays out a comprehensive plan for its future defense complex and sets up systems to monitor and manage the implementation of the plan.

Fourth, DOE must train all its people on the new culture, how to make the new culture happen, and how to work within the new culture. The people who must be trained include DOE and DOE-related contractor people from managers in DOE Headquarters to workers in the field sites. The new culture will affect both how DOE is managed and the technical emphasis in the processes DOE is responsible for. In focusing on process, safety, accountability, and environmental impact must be integrated into technical training. Watch to see if DOE can emphasize training and bring separated training programs into an integrated package reflecting the new culture.

\section{Acknowledgements}

The ideas presented in this paper represent the cumulative work of hundreds of people over twelve years at Virginia Tech's Management Systems Laboratories. Protessional managers from business and industry, and faculty, graduate students, and undergraduates from a wide variety of engineering and other disciplines have participated in the research and development of these tools and techniques. The author thanks them for their good work. The pronoun "I" is used throughout to separate all of us using management systems (we) from the author (I). However, please recognize "I" is more than one contributor to this work.

The preparation of this paper is funded by U.S. Department of Energy (DOE) Grant No. DE-FG02. 88DP48058. Management Systems Laboratories thanks the DOE's Office of Environmental Restoration and Waste Management for providing us a real-world laboratory for the research, development, and testing of state-of-the-art management tools and the frameworks for understanding how to make them successful.

\section{References}

1. Kanter, R. M. 1983. The Change Masters: Innovation and Entrepreneurship in the American Corporation. New York: Simon \& Schuster.

2. Deal, T. E., and A. A. Kennedy. 1982. Corporate Cultures: The Rites and Rituals of Corporate Life. Reading, MA: Addison-Wesley.

3. Kilmann, R. H. 1984. Beyond the Quick Fix: Managing Five Tracks to Organizational Success. San Francisco, CA: Jossey-Bass Publishers.

4. Kanter, R. M. 1983. The Change Masters: Innovation and Entrepreneurship in the American Corporation. New York: Simon \& Schuster.

5. Davis, S. M. 1984. Managing Corporate Culture. Cambridge, MA: Ballinger Publishing Company.

6. Huber, R. L. 1986. "Shaping Values to Form A New Culture," in M. A. Berman (Ed.) Corporate Culture and Change: Highlights of a Conference. New York: The Conference Board, Inc., pp. 44-49.

7. Martin, J., and C. McClure. 1985. Diagramming Techniques for Analysts and Programmers. Englewood Cliffs, NJ: Prentice-Hall.

8. Myers, M. S. 1988. "Let JIT Menà Your Split Culture," Industrial Management, March-April, pp. 11-18.

9. Kurstedt, H. A, Jr. 1990. "Programmatic Issues Affecting the Implementation of the DOE Five-Year Plan," Proceedings of Waste Management '90, Tucson, AZ, pp. 69-76. 


\title{
WHY ENGINEERS MUST KNOW AND \\ MANAGE ORGANIZATIONAL CULTURE
}

\author{
Harold A. Kurstedt, Jr. \\ Larry A. Mallak \\ E. Morris Howard \\ Management Systems Laboratories \\ Department of Industrial and Systems Engineering \\ Pamela S. Kurstedt \\ College of Engineering
}

Virginia Polytechnic Institute and State University Blacksburg, Virginia

\begin{abstract}
The engineering manager's success is being judged more and more on qualitative measures concerning the human elements of their work. These new measures require engineers to become as skilled and at ease with the tools, methods, and techniques for qualitative issues as they are with more traditional quantitative tools, methods, and techniques.
\end{abstract}

To achieve success toward these qualitative measures demands nothing short of a new way of thinking, indeed a new culture embodying new values and traditions. Engineering managers must use culture change mechanisms along with their other management tools so they can better understand and manage culture. They must view concepts such as just-in-time, total quality management, and continuous performance improvement as integral to culture change efforts--these concepts and corresponding programs require an underlying culture to create an environment for change.

Engineers who want to manage and change culture and communicate these changes must become comfortable with hoopla and symbolism to add drama and life to their words and plans. Engineers must understand the elements of culture, become effective communicators, and master the tools, methods, and techniques of culture change.

\section{Introduction}

The success of an engineering manager's work depends more and more on qualitative measures--largely human measures. Traditionally, many engineers chose their profession because they wanted to deal more with structured machines, materials, and processes, logically related through drawings, scientific laws, and mathematical formulae. Today, an engineer's life is indirectly affected by people; who are outside the engineer's immediate contacts who hold a stake in what the engineer is doing. Such stakeholders include the organization's other staff, customers, neighbors, and owners. The engineer's life and success are directly affected by people who interact with him or her each day: the engineer's staff, management, and peers. Together, the people of the corporate staff have a culture, an organizational culture the engineer must be able to diagnose, design, implement, and improve. We can determine the elements of organizational culture and can use tools, methods, and techniques for implementing or changing those ele ments.

The engineering manager must be as skilled at these tools and techniques as he or she is with design or process tools, methods, and techniques. At the core of it, modern concepts like total quality management (TQM), just-in-time (JIT), and zero defects embody the organizational culture principles, elements, tools, and techniques. In short, we $c_{i}$ add a measure of logic and structure to the qualitative criteria we're judged by. Engineers ought to like that.

To change our organization in a fast-paced world, we must change culture; and culture change is the most difficult and important part of changing an organization. I'll describe culture as values and traditions--qualitative ideas representing beliefs and activities each of us holds dear. To change culture, we must replace old values and traditions with new ones. As engineers, we aren't trained or experienced in managing this kind of change. To change closely-held values and traditions, we must use techniques we're not familiar with, like symbols and hoopla. We also must measure things we're not trained to measure, like behaviors and attitudes. But we must develop skills based on our engineering training, like generating a vision.

I'll recognize culture change mechanisms as key management tools in the arsenal managers must use well to effectively conduct business today'. Further, I'll argue that all other management tools must be in harmony with culture change mechanisms for the other tools, or for the culture, to work well. I'll place culture change mechanisms together with all management tools in a control loop analogy to show the role management tools play with other components in a management system.

I'll argue culture management, leadership, continuous performance improvement, and comprehensive planning all focus on values and traditions. So, for example, as the heart of it total quality management and other popular techniques aimed at continuous performance improvement require an understanding of and skill for culture management. Finally, I'll support the importance and use of organizational culture change mechanisms as management tools with anecdotal evidence from government and industry cases.

\section{Oreanizational Culture}

Why must engineers know what organizational culture is and how to manage it? Because today successful organizational change is based on successful culture change.

Organizational culture is capturing a lot of attention recently. The rapid pace of business fueled by advances in telecommunications and information technologies means organizations have less time to react, leaving them more 
vu!nerable to crises. Powertul, visionary leaders are being thrust into new situations with the onslaught of mergers and acquisitions. These new situations call for individuals who know what culture is, how to manage it, and how to measure culture change. Engineers, as agents of change in these organizations, are positioned to be culture change pioneers.

Culture, the once elusive concept reserved for anthropologists, has given way to shelves of new business books on culture, pragmatic journal articles on how to manage and measure culture change, and discussions among engineers and managers on their organizational cultures--what they can do to make their cultures work for them.

Certain elements determine the kind of culture an organization has. Deal and Kennedy [1] identify five elements of organizational culture: 1) business environment, 2) values, 3) heroes, 4) rites and rituals, and $5)$ the cultural network. Of these elements, business environment (better stated as external forces on the organization) has the greatest influence in shaping culture. The external forces tell the organization what must be done to be successful. Organizations reflect aspects of their dominant regional or national cultures in the way they conduct business. Values, the organization's basic concepts and beliefs, define success in concrete terms for employees and establish standards of achievement. Heroes personify the culture's values and act as role models for employees to follow. Rites and rituals, the systematic and programmed routines of day-to-day life, teach people how to behave. The cultural network is a primary means of communicating the values and heroic mythology.

Hoopla and symbolism, in tangible and memorable ways, communicate elements of organizational culture. Symbols should be simple and catchy and reinforce the same messages. Managers should react to and reward seeing the symbols and culture in practice. Ceremonial and recognition rituals such as employee retirement dinners or awards ceremonies exemplify the hoopla. Citibank uses buttons that say "I'm on the QT," where QT refers to the Quality Team. The plant manager at AT\&Ts Richmond Works plant in Richmond, Virginia, symbolizes the passing of responsibility in large group meetings. He calls upon a specific work unit and has a representative of the team stand up. He then passes a football to the representative. This symbolizes the passing of responsibility from the plant manager to the work unit. In the process of passing a football around the auditorium, there is a lot of hoopla, excitement, emotion, and commitment.

\section{Why Cultures Change}

Proper culture change enhances organizational communication. In my experience with service industries, internal communication was at the top of the list of employee-identified weaknesses nearly everywhere I went. Culture change charges management to communicate the new culture formally in several ways: internally--through newsletters, videos, meetings, briefings, and externally-through public relations efforts, advertising, and press releases/media management. These new and enhanced communication techniques to promote the culture change often become part of the new culture. The most powerful and meaningful communication of organizational culture occurs in thi everyday informal words and deeds of managers and employees. We can communicate culture without even thinking about it. That's why to manage culture and culture change, we have to be fastidious about symbols and the cultural messages being sent.

Culture change can be essential to survival, as in the cases of Johnson \& Johnson and Eastman Kodak, because of rapid change in the external forces on the organization.
Kodak's acquisition of Sterling Drug in 1988 symbolized a process of change dating to the early eighties: "Kodak fundamentally changed from a 100 -year-old company with a top-down, functional structure to an organization of business units with a better focus on the customer and financial results. For Kodak, this represented nothing less than a major discontinuity in an established culture" [2]. Culture change may also be the result of a merger or acquisition. USAir has had its share of change and culture change in the wake of acquiring Pacific Southwest Airlines (PSA) and Piedmont Airlines [3]. In June, 1990 Eastern Airlines began a media campaign on its culture of continuous improvement over a 100-day period. Eastern's new culture has been motivated by many fundamental changes in its environment: a major labor strike, slide in market share, and the traveler's shunning and labeling of Eastern as a low-quality airline.

One or both of two forces usually precedes culture change. A life-threatening experience spelling demise for an organization can often motivate culture change. The culture is bedrock and changes because of the pressures of a crisis. Such is the case with Eastern. Strong leadership can also bring about culture change. Lee lacocca led Chrysler to a culture that is more responsive to both the consumer and the global marketplace. Strong leadership is the force changing culture because of a powerful leader's inspiration.

Five situations suggest the need for culture change [4]. A fundamental change in external forces on a strong-culture firm will drive culture change. The divestiture of the Bell telephone companies produced six regional holding companies and several other entities which now had to learn how to compete, when previously they monopolized communications markets. Organizations in a highly competitive industry use culture change as a tool for becoming better competitors. Hospitals, who must now compete for the health care dollar, have waged marketing and public relations campaigns touting the amenities they now offer their "guests."

Becoming more than a mediocre company often begets culture change. Organizations on the verge of becoming Fortune 500 should change their cultures before it's too late. The bureaucratization as the organization grows may threaten the original culture and the underlying values. The culture must be strong if it is to withstand the transition to a large-company environment. Firms experiencing rapid growth may need to change their cultures. The rush of new employees won't know what the organization is all about unless they learn it quickly from the culture. Unless one of these five situations exists, culture change should simply not be undertaken.

In our world of extreme and rapid change, most of us feel global changes in the external forces on our organizations sufficient to force culture change: diversity in the work force, empowerment of workers, informing stakeholders (including stockholders, staff, customers, and neighbors), the need to balance good process (safety and environmental protection) with good product. Further, today we have more specific changes in external forces like mergers, acquisitions, and contract changeovers.

People stick to their old ways. To change what they hold dear, consistency is everything. If you reinforce the new culture 25 ways and keep one remnant of the old culture, people will cling to that one remnant. The champion for the new culture must have external vigilance and notice everything because we can keep reminders of the old culture in small ways. 
The Role of Management Tools Within a Management System

The national and even global shift in values and attitudes toward how we treat individuals in the workplace and how we treat our environment drives today's organizations to adapt their cultures for a better fit with these newly crowned values and attitudes. Culture, simply put, consists of the values and traditions we hold dear. I define culture more precisely, as do Blake and Mouton [5], as patterns of interactions, values, and attitudes, which are derived from traditions, precedents, and past practices and are most visible in the team formations within which most managers work. I'll put culture in an organizational context using the management system model (MSM).

The MSM represents the industrial engineer's structured approach to management (Figure 1). In systems terminology, it's a closed system look at an open system. We study systems as collections of components working together for a given purpose or mission. An organization's purpose is articulated and manifested through its mission statement and helps shape the culture. Therefore, analyzing a management system requires identifying its components and purpose. The purpose of a management system may be the long-term survival of an organization (corporate or board level), the production of a good or service (division or plant level), or meeting a project's deadline (manager level).

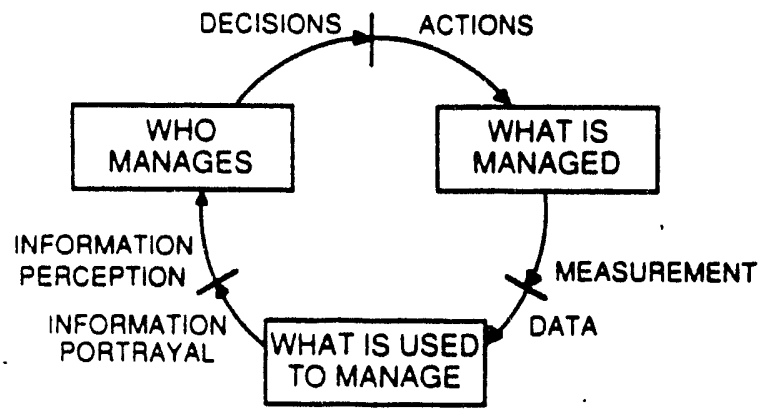

Figure 1. The management system model represents the engineer's systems approach to management.

I represent the MSM as a control loop in Figure 2. The MSM and the control loop have analogous components and relationships. The controller in the control loop is the "who manages" in the MSM, the plant is "what is managed," and the sensors are "what is used to manage;" the interfaces are intact [6]. The comparator shows the manager's bias relative to the bias in incoming information, such that managers in different positions in the organization will respond differently to the same changes [7]. The control loop analog helps study the response of a management system to changes in the system input thereby giving us a qualitative niethod we're familiar with as engineers that we can use to assess culture and culture change.

A valuable MSM feature is separating the ends-the physical things we are responsible for--from the means--the tools we use to manage. The physical things we manage, or "what is managed," include the people, capital, equipment, and materials we are held accountable for. The management tools, or "what is used to manage," include relationships and structures, methods, guides and rules, precedents, and the data-to-information chain [8]. The precedents support the organizational culture, and changing the precedents changes the culture.

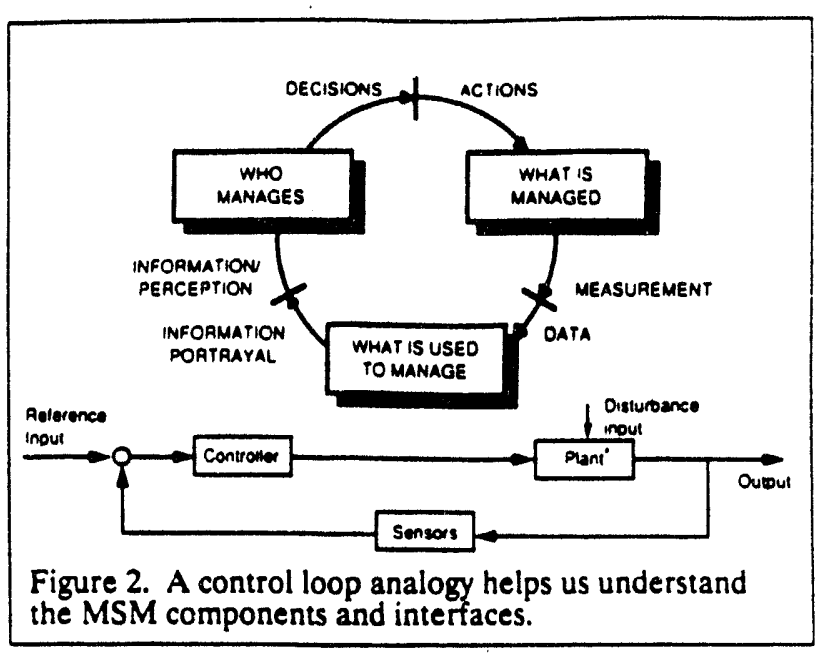

\section{Culture Change Mechanisms as Management Tools}

Culture emphasizes the engineer's responsibility for qualitative aspects of the organization. A strong, wellmanaged culture helps you avert crises and manage them when they do occur. During the Tylenol poisonings of the early eighties, Johnson \& Johnson (J\&J) constantly pointed to their "credo" espousing J\&J's cultural values which guided them successfully through those difficult times. David Collins, a top executive at J\&J during the Tylenol poisonings, put it this way, "Throughout the Tylenol crisis, despite the magnitude of the problems we faced, we were all sure how to proceed because we acted in accord with the values described in the Johnson \& Johnson credo" [9].

Culture change mechanisms, as precedent tools, are part of the five groups of management tools (Figure 3). Culture change mechanisms, more than relationships and structures, methods, guides and rules, or the data-toinformation chain, help us manage people and how they work together in the organization.

Managing culture helps you take advantage of participative management techniques. General Motors' Saturn facility is essentially a $\$ 3$ billion experiment in developing a subculture within a traditional hierarchical assembly line structure. All of Saturn's 3,400 employees must go through a five-day "Saturnization" process designed to teach them how to work in teams and build consensus. They face 100 to 750 more hours of training after the initial Saturnization to cross-train so they have an awareness of how their work fits into the overall scheme $[10,11]$. That's just the beginning; all of Saturn's vendors and suppliers, including second-and third-tier as well as consultants, must be Saturnized [12].

\begin{tabular}{|ll|}
\hline $\begin{array}{l}\text { TOOL GROUP } \\
\text { - Relationships and } \\
\text { structures } \\
\text { - Methods }\end{array}$ & \multicolumn{1}{c|}{$\begin{array}{c}\text { ExAMPLE } \\
\text { organization chant, work } \\
\text { breakdown structure }\end{array}$} \\
- Guides and rules & $-\quad \begin{array}{l}\text { operations research models, } \\
\text { oxpert systems }\end{array}$ \\
- Precedents & $-\quad \begin{array}{l}\text { culture change mechanisms. } \\
\text { social systems }\end{array}$ \\
- Dita-to-information \\
chain \\
Figure 3. Culture change mechanisms are precedent \\
tools, one of the five groups of management tools.
\end{tabular}


In changing the tools and methods for managing culture, the other management tools must change too. That is, all five groups of tools must work together or they will work at odds with one another. And the groups of tools must work for the decision maker or they will work against him or her. So if the new culture highlights participation and empowerment of the workers, then the organization structure (flat organization) and the MIS, or the data-toinformation chain, (relational data bases) must reflect the new culture. The changing culture in the organization must relook at all its support systems, from the mission statement to the hiring and reward systems.

\section{The Relationshio Between Continuous Performance Improvement and Culture}

We recognize the importance of dealing with qualitative issues together with more quantifiable issues like cost, schedule, and technical specifications. We also know qualitative issues influence the quantitative ones and vice versa. We have many attempts at getting our arms around qualitative issues today and bridging from qualitative to quantitative.

The relationships among leadership, culture, and continuous performance improvement illustrate some of the qualitative and quantitative issues managers must understand and differentiate. Schein [13] argues leadership is equal to culture management. I argue leadership is equal to continuous performance improvement and, conceptually, continuous performance improvement is nothing more than doing better at what we do each and every day until we are better than the best. This is a philosophical or qualitative idea engineers try to use. Leadership bridges continuous performance improvement and culture change to a comprehensive planning process that translates global direction into specific activities.

We almost always limit the power of continuous performance improvement when we equate continuous performance improvement with specific tools as statistical quality control (SQC). To be sure, SQC is a valuable tool, but only so when it is integrated into a carefully-focused, synergistic, closed set of tools and techniques for continuous performance improvement. I have described before a closed set of tangible methods and guiding rules for improving performance [14]. To achieve total quality in a fundamental sense is to lead the organization toward the values and traditions underpinning total quality and that means managing culture.

Figure 4 illustrates the tight coupling among the philosophical approaches of comprehensive planning, culture management, leadership, and continuous performance improvement. From Bennis and Nanus [15] I slightly adapt the ingredients of leadership to be 1) gain attention of stakeholders tbrough having a vision, 2) give that vision meaning to the stakeholders through effective communication, 3) acquire the stakeholders' trust by positioning the organization to achieve the vision, and 4) gain the stakeholders' confidence through a healthy understanding of success and failure. The objective is the same: deliberately change the values and traditions of the organization toward a new goal. The tools and methods for one apex of the pyramid in Figure 4 support the other apexes. For real total quality management, or continuous performance improvement, the engineer must change the organizational culture.

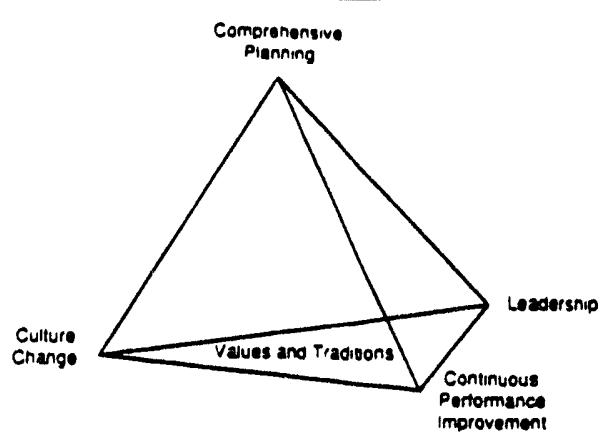

Figure 4. Approaches to culture management are tightly coupled and are rooted in values and traditions.

\section{Conclusions}

Today's engineering managers must be prepared to deal with the only known constant--that there will be change and lots of it. Engineers who know what organizational culture is and how to manage it will be successtul in helping their organizations improve.

Engineering managers must gain competence in the qualitative skills they may have avoided since their years in high school. If success used to mean "meet technical specifications on time and within cost," success now means "do this in a way to satisfy all stakeholders." Engineers must understand the elements of culture, use symbology and hoopla, become effective communicators, and master the inols and methods of culture change.

\section{Acknowledgements}

The ideas presented in this paper represent the cumulative work of hundreds of people over thirteen years at Virginia Tech's Management Systems Laboratories. Professional managers from business and industry, and faculty, graduate students, and undergraduates from a wide variety of engineering and other disciplines have participated in the research and development of these tools and techniques. The author thanks them for their good work. The pronoun "I" is used throughout to separate all of us using management systems (we) from the author (I). However, please recognize "I" is more than one contributor to this work.

The preparation of this paper is funded by U.S. Department of Energy (DOE) Grant No. DE-FG0288DP48058. Management Systems Laboratories thanks the DOE's Office of Environmental Restoration and Waste Management for providing us a real-world laboratory for the research, development, and testing of state-of-the-art management tools and the frameworks for understanding how to make them successful.

\section{References}

[1] Deal, Terrence E. and Allen A. Kennedy (1982). Corporate Cultures: The Rites and Rituals of Corporate Life, Reading, MA: Addison-Wesley.

[2] Samper, J. Phillip (1989). "Changing Corporate Culture: From Shock to Revival," in Organization Designs for the 1990's, Frank Caropreso (Ed.), New York: Conference Board.

[3] Ott, James (1989). "USAir Prepares to Complete Acquisition of Piedmont," Aviation Week and Space Technology, July 17, 1998, pp. 94-95. 
[4] Deal, Terrence E. and Allen A. Kennedy (1982). Corporate Cultures: The Rites and Rituals of Corporate Life, Reading, MA: Addison-Wesley.

[5] Blake, Robert R. and Jane S. Mouton (1968). Corporate Excellence Through Grid Organizational Development. Houston, TX: Gulf Publishing.

[6] Kurstedt, Harold A., Pedro M. Mendes, and Kwang S. Lee (1988). "Engineering Analogs in Management," Proceedings of Ninth Annual Conference of the American Society for Engineering Management, October, pp. 197-202.

[7] Mendes, Pedro, Harold A. Kurstedt, and Patrick Koelling (1988). "Dynamic Aspects of Decision Making," Proceedings of the 1988 IEEE Engineering Management Conference, October, pp. 44-50.

[8] Kurstedt, Harold A. (1986). "An MIS without a Working Management System is Destined to Fail," Proceedings of the Fall 1986 Institute of Industrial Engineers Conference, December, pp. 42-48.

[9] Collins, David E. (1986). "Cultural Heritage and Crisis Management," in Corporate Culture and Change: Highlights of a Conference, Melissa A. Berman (Ed.), New York: Conference Board.

[10] Business Week (April 9, 1990). "Here Comes GM's Saturn," pp. 56-62.

[11] Huber, Richard L. (1986). "Shaping Values to Form a New Culture," in Corporate Culture and Change: Highlights of a Conference, Melissa A. Berman (Ed.), New York: Conference Board.

[12] DePorter, Eldon (March, 1990). Personal communication.

[13] Schein, Edgar H. (1985). Organizational Culture and Leadership, San Francisco: Jossey-Bass.

[14] Kurstedt, Harold A. (1989). "Catering to Crises: How to Escape," Proceedings of the Institute of Industrial Engineers 1989 Irtegrated Systems Conference, November, pp. 191-198.

[15] Bennis, Warren and Burt Nanus (1985). Leaders: The Strategies for Taking Charge. New York: Harper and Row. 


\title{
TO UNDERSTAND CONSENSUS WE MUST KNOW WHAT THE ELEMENTS ARE
}

\author{
Anne R. Doss, Virginia Tech \\ D. Mark Brubaker (student), Virginia Tech \\ Harold A. Kurstedt, Jr., Virginia Tech
}

\begin{abstract}
I've identified the essential elements of successful consensus decisions in the areas of: foreign policy/peace negotiations, emergency relief, Presidential advisory groups (kitchen cabinets), and labor/management negotiations.

Outside laboratory situations, the success of decisions is best determined by history. I researched a number of historically significant decisions and prepared case studies where I could judge success. I looked for whether or not consensus was used and for those elements most important to making the decision successful. I found three elements essential to a successful consensus decision: strong facilitator, relevant information, and shared understanding.
\end{abstract}

\section{INTRODUCTION}

What types of groups are best able to achieve consensus? Why? Can consensus be engineered? How is consensus altered in light of strong leaders or facilitators? Do external forces affect consensus? Is consensus affected by the time spent to generate it? To begin answering some of these questions, I researched historical records of significant events to prepare case studies and see how consensus was achieved in various situations. I tried to find common elements present in the cases where consensus was achieved and also see what was missing when consensus wasn't achieved.

I chose to do case studies from four different areas requiring different types of decisions under different time constraints. From these four areas, I selected the following situations to prepare case studies: the Vietnam Paris Peace Talks, the Cuban missile crisis, the Three Mile Island nuclear accident, the Bhopal disaster, the 1985 Mexican earthquake, the John Kennedy and Andrew Jackson "kitchen cabinets," the GM Saturn agreement with the United Auto Workers, and the 1974 United Steelworkers no-strike agreement. I looked at several perspectives for each case study. First, I identified the participants and wrote a statement of the facts. Next, I identified process issues and the resolution. Finally, I analyzed the consensus decision.

In all cases, some level of consensus was achieved. However, the situations I looked at can be separated into those resulting in successful action following consensus and those resulting in unsuccessful action. This is an important distinction because I'm interested in how consensus can be facilitated to result in successful actions. Across the four areas, the common elements in successful situations and not in unsuccessful ones were: 1) a strong facilitator, 2) possession of all the relevant information needed to make the decisions, and 3) shared understanding among all participants in the consensus process. In the next section, I discuss each of the consensus elements as they relate to the situations chosen from the four areas. 


\section{SUMMARY OF THE CONSENSUS ELEMENTS}

\section{Facilitator}

When successful decisions were made or successful action taken, a strong facilitator commanded respect but considered all points of view and refrained from controlling or biasing discussion. An exception to this was when the public safety was threatened. Then, a consensus of public action took place without a facilitator. For example, in both the Mexican earthquake and the Bhopal disaster, the public immediately responded to help victims despite slow government action.

The lack of a good facilitator was the most frequent problem in cases where a decision wasn't successful. It is apparent that a good facilitator is different from a strong leader. I found strong leaders in four cases, but in only one of these four cases was the decision successful. I look at John Kennedy in the Cuban missile crisis and "kitchen cabinets"; Andrew Jackson in "kitchen cabinets," specifically the bank wars; and Lyndon Johnson in the Vietnam Paris Peace Talks. All three of the these Presidents were strong leaders. The fourth strong leader, I.W. Abel, was president of the United Steelworkers Union in 1974 when the no-strike agreement was signed. Of these four cases, only the Cuban missile crisis can be considered a success.

President Kennedy facilitated his advisors masterfully during the three days the world was on the brink of atomic warfare. He refrained from promoting a course of action but at the same time forced his advisors to give him more options than simply ordering an air strike to take the missiles out. At the end of three days of intense discussions, the best minds in the country came to consensus and recommended a course of action which turned out to be a very successful decision. Not all his advisors liked the blockade, but because everyone had an opportunity to voice their opinion and persuade the group to follow their course of action, they all supported the consensus decision.

In contrast, President Jackson and I.W. Abel were strong leaders who guarded their power fiercely and weren't open to opposing points of view. President Jackson surrounded himself with "yes" men. He completely ignored his cabinet, replacing any advisor who dared to disagree. He tried to crush the National Bank which resulted in the bank wars. While the National Bank powers needed to be curbed, the actions Jackson took left the nation's financial structure on the brink of deflationary collapse.

I.W. Abel also closed off opposing points of view. He negotiated the 1974 no-strike agreement in secret and then used propaganda methods to push it through ratification by the union rank and file. For two reasons, this agreement was the major factor in the subsequent collapse of this country's steel industry. First, the industry couldn't survive the high wages and large pay increases promised in the agreement. Second, by giving management the right to "run the plant," the union made it impossible for blue and white collar workers to work together. This stifled innovation and led to very small increases in productivity.

In Vietnam, President Johnson bargained from a position of weakness. He was leaving office and the North Vietnamese knew they could hold out for the new administration. The Nixon administration also bargained from a weak position because of intense opposition in the United States to the war. Nixon wanted none of the political repercussions of a war Democrats started. They negotiated secretly with North Vietnam leaving South Vietnam no choice but to accept the agreement. This resulted in the communists taking over the country.

Leaders or facilitators were especially needed in emergency crisis situations. In the three emergency cases I looked at, Bhopal, Mexico, and Three Mile Island, no leader emerged from the government to take charge and lead the people 
responsible for handling the situation. In Bhopal and Mexico, the governments hindered rescue efforts more than they helped. The Indian government refused to let doctors use necessary medication or circulate information on how to treat the victims. Because of pride and lack of information, the Mexican government initially refused outside aid and actually had the national guard keep volunteers from rescuing people who were trapped. At Three Mile Island, there were so many Federal and State agencies who converged on Harrisburg, no one knew who was in charge and no one took control.

In Mexico and Bhopal, however, I found many local leaders who led volunteer groups saving hundreds of lives. This led to an easily achievable public consensus based on two crucial ingredients: someone taking charge and a sense of mortality.

\section{Information}

Information is crucial to a successful consensus. I found lack of relevant information to be another major cause of breakdowns in consensus and poor decisions. Jackson and Abel forced consensus decisions affecting large numbers of people. As a result, they overlooked crucial information which might have led to different decisions. Jackson refused to listen to his Secretary of the Treasury, William Duane, and replaced him with someone who wouldn't argue with him. Abel refused to listen to advisors who warned him an oil crisis was possible. The resulting high inflation, combined with the automatic cost of living increases written into the no-strike agreement, led to near destruction of the U.S. steel industry and the union. Abel didn't consider all the information and didn't take advantage of advisors whose expertise might have led to a more survivable agreement.

Often in emergency situations, decisions must be made with incomplete information. Without a time constraint, decision-makers should defer until more information is available. In Mexico, Bhopal, and Three Mile Island, I saw little information available. This led to poor decisions in all three cases. In contrast, during the Cuban missile crisis, President Kennedy didn't allow a decision to be made with incomplete information. The Kennedy advisors, through a process of give-and-take discussion and tedious studying of intelligence information, decided on the blockade which averted a war. If there isn't time to accumulate all the information necessary, knowledgeable and influential leaders must emerge. If time isn't a constraint, the group has to recognize when they don't have enough information and they must refrain from making a decision until they have all the information necessary.

\section{Shared Understanding}

When doing these case $s^{1}$ Idies, I found that those participating in the consensus process came to the group with their own agenda, their own idea of how things should be done, and their own goals and desires. One requirement for achieving consensus is to take a group of individuals' and transform them into a group with the same desires and goals. This requires a shared understanding of all individuals' positions and values.

Repeatedly, I found group discussion broke down because the two sides didn't understand each other. In the Cuban missile crisis, a lack of shared understanding almost led to war. The United States never thought the Soviet Union would attempt to place missiles in Cuba and the Soviet Union never thought the United States would react so strongly and risk a possible war. Better communication and understanding between the two sides would have averted this crisis. As a matter of fact, one outcome from this crisis was a direct line between the Kremlin and the White House to improve communication.

A lack of shared understanding also led to the agreement which devastated the steel industry. Because the union and industry had a history of strikes every time a 
contract came up for renegotiation, the customers would stockpile steel (hedge buying). To overcome this lack of understanding, the union pushed for the no strike agreement which they felt would end hedge buying and increase wages. They failed to realize both the union and industry lacked shared understanding with their customers. Had customers been asked what would end hedge buying, it would've been clear the no-strike agreement wouldn't work. This situation required a consensus decision between union, industry, and customers.

The GM Saturn plant agreement demonstrated how shared understanding and consensus can work successfully. Labor and management both recognized the need for changes in their relationship. They signed an agreement to build a revolutionary new plant which would be highly automated and where union workers would have a larger say in how the plant was managed.

In Vietnam, there was no shared understanding between the United States and the South Vietnamese, our allies in the negotiations with the North Vietnamese. Because of this, the United States negotiated with the North Vietnamese and arranged an agreement pulling the United States out of the war. Unaware we were negotiating the agreement until it was done, the South Vietnamese didn't understand the political pressure President Nixon was under to bring the United States troops home. As a result, South Vietnam ended up agreeing to a treaty which gave the North Vietnamese the power to take over their country.

\section{CONCLUSIONS}

A successful consensus decision is the product of three primary elements: strong facilitator, relevant information, and shared understanding. The facilitator, or leader, must be strong enough and command the necessary respect to direct discussion if needed. He or she must not control or bias the discussion. He or she must also make sure all viewpoints are included in the decision making process.

Shared understanding requires information sharing to improve group cohesiveness. Once you accomplish group cohesiveness, there is less chance the resolution of conflict usually necessary to achieve consensus will split the group into factions.

People need all relevant information to understand the situation and make an informed decision. Consensus will be hindered if needed information is withheld from the group. Also, if information is not made available, the group may reach consensus, but fail to make a high-quality decision.

In the consensus process, I believe the most important person is the facilitator and the main purpose of the consensus process is shared understanding by all the people involved. Also, before all the people can participate in the process, they must have all the relevant information.

Each situation I studied can be considered a problem and through the consensus process, there is a product or a consensus decision. The product or decision was judged to be successful or unsuccessful by subjective evaluation looking back after the fact. Research is needed to determine how to measure the success of a consensus decision.

In the studies of successful situations, all elements were present. In the unsuccessful ones I found one or more elements missing which seemed to cause failure. The results of these case studies allow me to progress to the next stage, testing some of our theories about consensus. I'll use these consensus elements to select key variables to conduct future case studies as well as exploratory and confirmatory research. 
The ideas presented in this paper represent the cumulative work of hundreds of people over thirteen years at Virginia Tech's Management Systems Laboratories. Professional managers from business and industry, and faculty, graduate students, and undergraduates from a wide variety of engineering and other disciplines have participated in the research and development of these tools and techniques. The author thanks them for their good work. The pronoun "I" is used throughout to separate all of us using management systems (we) from the author (I). However, please recognize "I" is more than one contributor to this work.

The preparation of this paper is funded by U.S. Department of Energy (DOE) Grant No. DE-FG02-88DP48058. Management Systems Laboratories thanks the DOE's Office of Environmental Restoration and Waste Management for providing us a real-world laboratory for the research, development, and testing of state-of-theart management tools and the frameworks for understanding how to make them successful.

\section{BIBLIOGRAPHY FOR THE CASE STUDIES}

[1] Abel, E., The Missile Crisis. (New York: J.B. Lippincott Company, 1966).

[2] Abel, I.W., "Exploring Alternatives to the Strike: Basir, Steel's Experimental Negotiating Agreement." Monthly Labor Review 96, no. 9 (September, 1973).

[3] Anderson, Harry, Joseph Contreras, Joseph Harmes, Zofia Smardz, "Against All Odds." Newsweek 106, no. 15(October 7, 1985). pp. 38-40.

[4] Bolton, L.W., "Bargaining Ahead: Major Contracts Expiring in 1974." Monthly Labor Report 96, no. 12 (December, 1973).

[5] Boraiko, Allen, "Earthquake in Mexico." National Geographic 169, no. 5(May, 1986), pp. 654-675.

[6] Bornstein, L., "Developments in Industrial Relations." Monthly Labor Review 96, no. 5 (May, 1973).

[7] Bowonder, B., "The Bhopal accident". Technological Forecasting and Social Change, 32 (1987), pp. 169-182.

[8] Contreras, Joseph, Harry Anderson, Joseph Harmes, Liz Balmaseda, Diane Weathers, "Disaster in Mexico." Newsweek 106, no. 14(September 30, 1985), pp. 16-21.

[9] Dillard, W. C., Sixty Days To Peace: Implementing The Paris Peace Accords (Vietnam 1973): Washington, DC: National Defense University, 1982.

[10] Edid, Maralyn, "A New Labor Era May Dawn at GM's Saturn." Business Week, no. 2904 (July 22, 1985), pp. 65-66.

[11] Edid, Maralyn, "How Power Will Be Balanced on Saturn's Shop Floor." Business Week, no. 2906 (August 5, 1985), pp. 65-66.

[12] Everest, Larry, Behind the Poison Cloud. Chicago, Illinois: Banner Press, 1986.

[13] Goodman, A. E., \& Sullivan, W. H., The Lost Peace: America's Search for a Negotiated Settlement of the Vietnam War. (Stanford, CA: Hoover Institution Press, 1978).

[14] "GM - Auto Workers Saturn contract." Monthly Labor Review 108, no. 10 (1985), pp. 48-50. 
[15] "Griefbusters." The New Republic 193, no. 17(October 21, 1985), pp. 7-8, 10.

[16] Hinh, Maj. Gen. N. D., Vietnamization and the Cease-Fire. (Washington, DC: U.S. Army Center of Military History, 1980).

[17] Hoerr, J.P., "The Steel Experiment." Atlantic Monthly 232, no. 6 (December, 1973).

[18] Hoerr, J.P., And The Wolf Finally Came - The Decline of the American Steel Industry. (Pittsburgh: University of Pittsburgh Press, 1988).

[19] Hoerr, John and Aaron Bernstein, "The NLRB Strikes a Blow for Worker Participation." Business Week, no. 2951 (June 16, 1986), p. 36.

[20] Kochan, Thomas A., Harry C. Katz, and Robert B. McKersie, The Transformation of American Industrial Relations. (New York: Basic Books, 1986).

[21] Latner, Richard B., The Presidency of Andrew Jackson: White House Politics 1829-1837. (Athens, GA: University of Georgia Press, 1979).

[22] MaGill, Frank N., The American Presidents: The Office and the Men. (Pasadena, CA: Salem Press, 1986).

[23] Magnuson, Ed, "A Noise Like Thunder." Time 126, no. 13(September 30, 1985), pp. 35-40, 43.

[24] Maynard, Micheline, "A Labor Deal that Clears the Way for GM's Saturn." U.S. News and World Report 99, no. 6 (1985), p. 22.

[25] "Mexico's Days of Disaster." U.S. News \& World Report 99, no. 14(September 30, 1985), pp. 11-14.

[26] Pope, R. R., Soviet Views on the Cuban Missile Crisis. (Washington, DC: University Press of America, 1982).

[27] Porter, G., A Peace Denied: The United States, Vietnam, and the Paris Agreement. (Bloomington, IN: Indiana University Press, 1975).

[28] Ross, I., "The Remarkable New Pact in Steel." Readers' Digest 103, no. 619 (November, 1973).

[29] Russell, George, "Miracles Amid the Ruins." Time 126, no. 14(October 7, 1985), pp. 36-38.

[30] Schlesinger, A.M., Jr., A Thousand Days: John F. Kennedy in the White House. (Boston, MA: Houghton Mifflin Co., 1965).

[31] Shrivastava, Paul, Bhopal: Anatomy of a Crisis. (Cambridge, Massacusetts: Ballinger Publishing Company, 1987).

[32] Sorge, Marjorie, "Saturn labor pact: New approach." Automotive News, no. 5083 (July 15, 1985), pp. 2,8.

[33] Sorge, Marjorie, "Saturn, UAW seek contract this week." Automotive News, no. 5081 (July 1, 1985), pp. 4. Sciences, 1973). 
[35] . "Trouble After an Earlier Disaster." Time 126, no. 21(November 25, 1985), p. 58. 

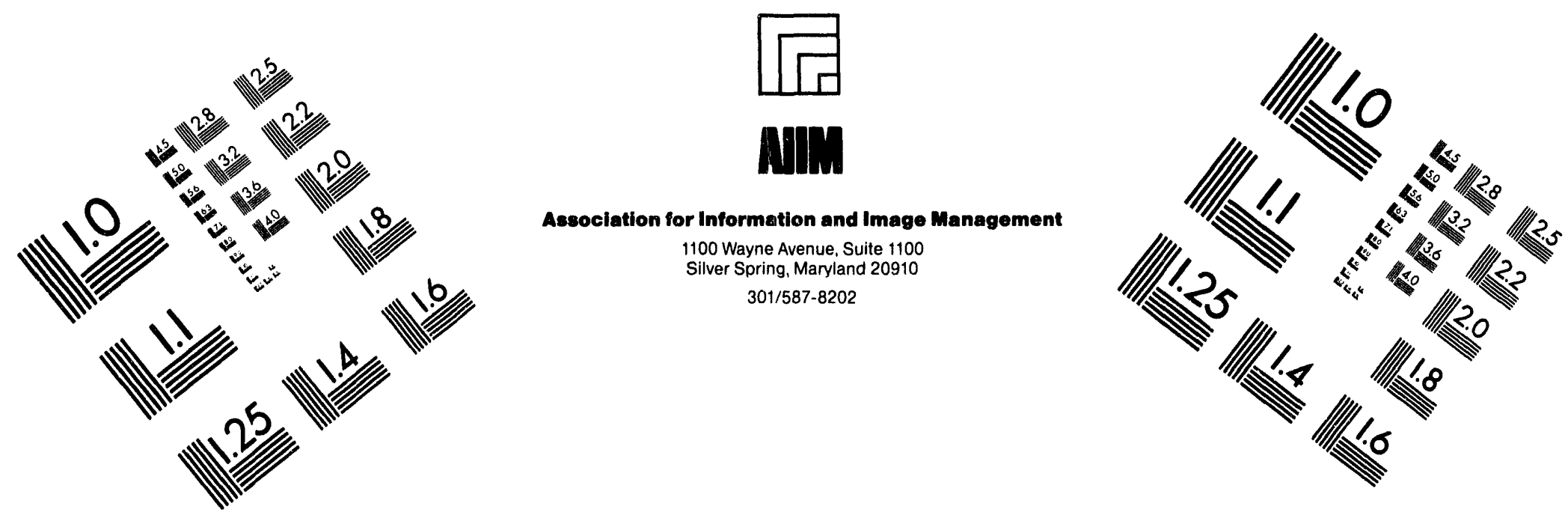

\section{Centimeter}

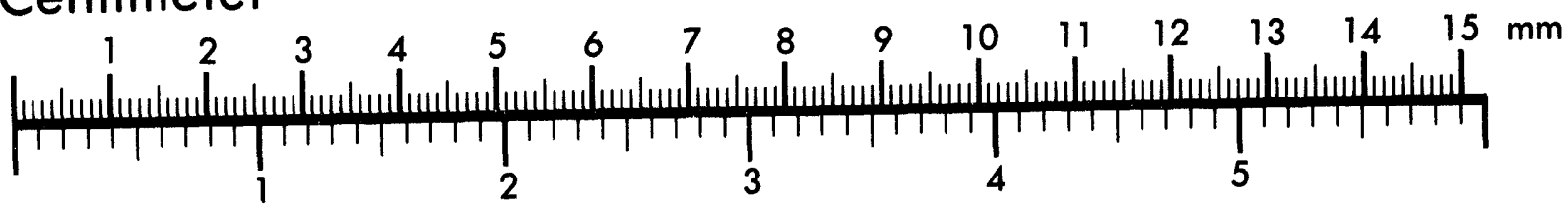
Inches
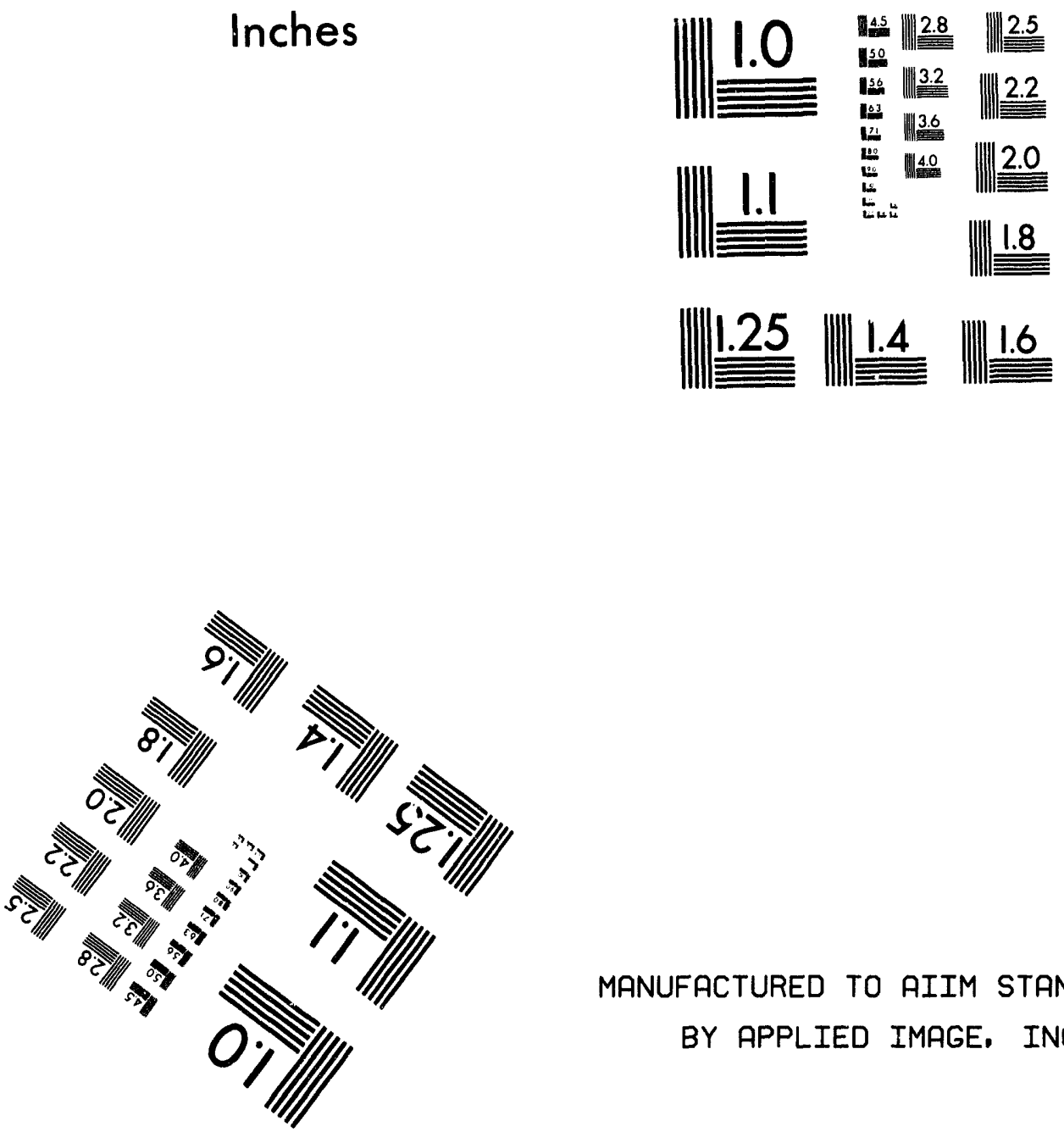

MANUFACTURED TO AIIM STANDARDS

BY APPLIED IMAGE, INC.

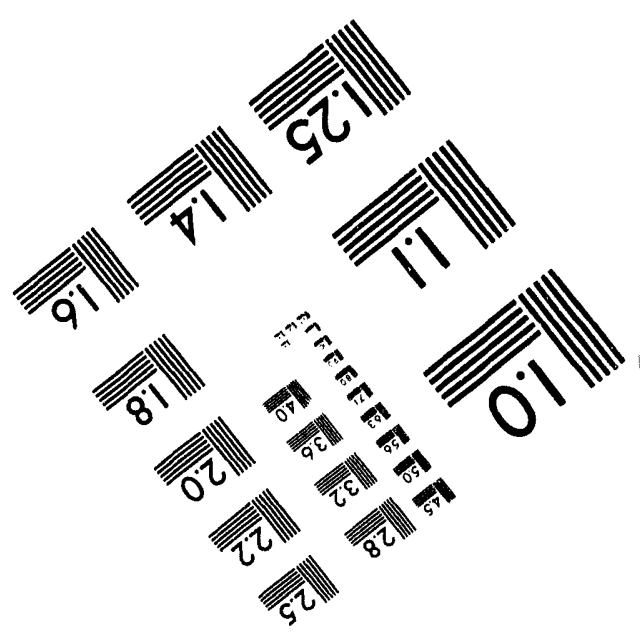



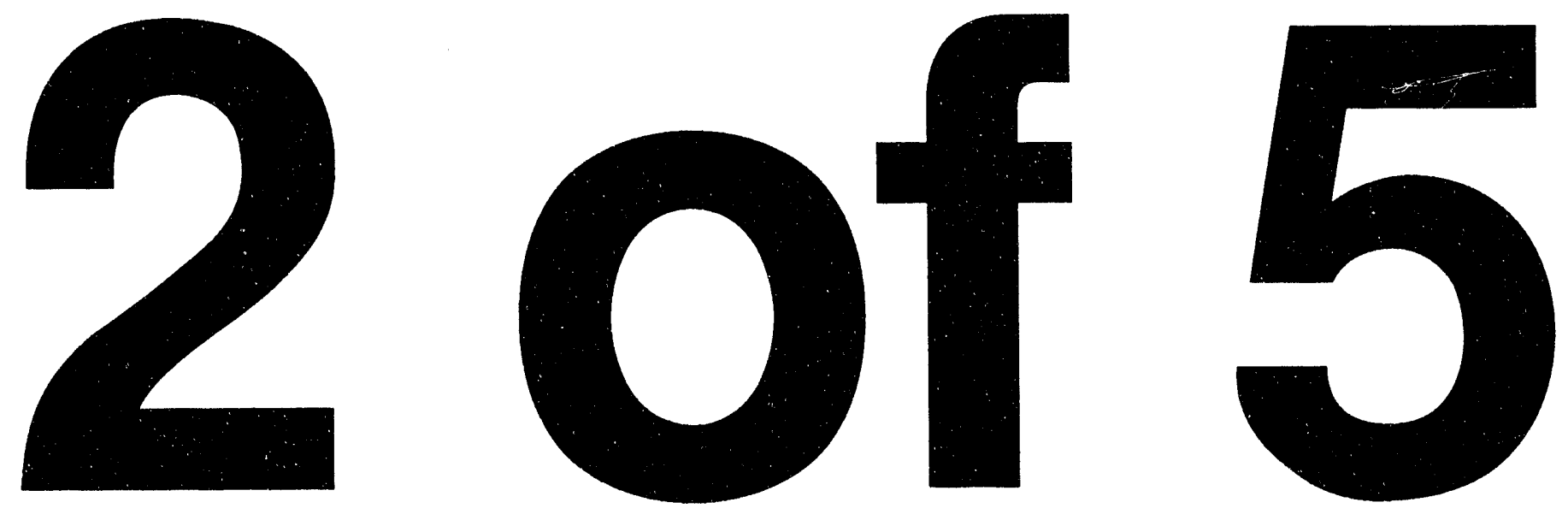


\author{
John W. Polk, Jr. (student), Virginia Tech \\ Harold A. Kurstedt, Jr., Virginia Tech \\ Kathryn A. Welch, Virginia Tech
}

\begin{abstract}
In this paper I present the results of a pilot study examining the role of experts in group decision making. I wanted to evaluate the relationships between the use of expert power in decision-making groups and decision quality, consensus and emergent leadership. Understanding these relationships will help facilitators manage groups better.

Groups with an expert and groups without an expert solved the "Lost on the Moon" [4] ranking exercise. Certain group members were perceived as experts because they had information not available to other group members. However, these experts scored poorly as individuals and therefore, were not true experts on the exercise. In spite of their poor individual scores, the experts gave their groups valuable information, improving the groups' quality scores. The presence of an expert had no effect on group consensus
\end{abstract}

\title{
INTRODUCTION
}

Decision-making groups are usually convened to gain consensus on the highest quality decision possible. Because most decision-making groups include a combination of experts and affected non-experts, expert power will play a large part in group decision making. Experts are asked to participate in group decisions because they have information relevant to the decision topic. If the expert is the only one possessing this information, he or she has a base of power over other group members. Experts can use their power positively by causing the group to consider relevant information, or negatively by forcing the group to accept the expert's solution.

The purpose of this study was to determine the relationships between the use of expert power in decision-making groups and decision quality, consensus and emergent leadership. By understanding these relationships, group leaders, facilitators and members can take steps to ensure experts introduce valuable information into group decision processes without sacrificing consensus.

\section{Expert Power}

One person has power over another if he can perform an act that will result in a change in the other person [1]. I'm interested in how experts change group members' attitudes. French and Raven [3] define expert power as power based on the perception that an individual has some special knowledge or expertise over another person. I use Hinkin and Schriesheim's [5] definition of expert power as the ability to administer information, knowledge, or expertise to another person. This definition implies experts have power because others need their information. Expert power is commonly exercised as rational persuasion, where the expert uses logical arguments with supporting evidence to convince group members of the "correctness" of a position. Successful use of expert power depends on tie expert's 
communication skills and logical ability [9]. If group members aren't convinced by the expert's arguments, they won't be influenced.

\title{
Decisions Requiring Both Consensus and Quality
}

Consensus and quality are two important aspects of a group decision. I define consensus broadly as a state where a common judgment has been arrived at by most of those concerned. Decision acceptance is often used as a synonym for consensus. Maier [7] defines decision acceptance as support of those who are to carry out the decision. Yukl $[9$, p. 112] defines acceptance as the "degree of subordinate commitment to implement a decision effectively." I'm interested in measuring $\% 0$ aspects of consensus/acceptance: the degree individuals accept a decision, and the degree individuals perceive others accept a decision. Yukl [9] defines quality as the objective aspects of a decision affecting group performance. Decision quality is the degree a group's decision matches an accepted standard or "right" answer. I' $m$ interested in decisions requiring both consensus and decision quality because these dimensions are often the most critical aspects of group decision making.

\section{Emergent Leadership}

Emergent leadership is the concept that describes how, in leaderless groups, one individual will be seen as a leader by group members. This usually occurs because someone will appear to have more competence at the task than the rest of the group [9]. The emergent leader gains status and has greater influence over group decisions.

\section{HYPOTHESES}

\section{Relationship Between the Use of Expert Power and Decision Quality}

\author{
H1: Groups with experts will have higher decision quality \\ than groups without experts.
}

Expert power depends on the expert having more knowledge than the group members relevant to the group decision. If the expert uses this power to inject useful information into the discussion, decision quality will increase. For this pilot study, I've assumed the expert won't overuse his or her power.

\section{Relationships Between Expert Power and Consensus}

The relationships considered in hypotheses 2 through 5 are shown in Figure 1. This diagram shows a dual path to reach consensus. Following the upper path, if group members perceive they had the opportunity to express their views, they will support it. "Perception of opportunity to express views" includes the perception that individual views were understood and considered by the group. In Hall's [4] consensus decision-making process, all group members are given a chance to express their views. If members understand the principles behind consensus decision making, and believe these principles were upheld, they will go along with the group's decision even if they don't think it is the best decision. Following the lower path, if members perceive the group decision is high in quality, they will support it. These paths are not mutually exclusive and there is likely a relationship between perception of decision quality and perception of opportunity to express views. The specific hypotheses related to both paths are discussed below. 


\section{FIGURE 1}

\section{Relationships Between Use of Expert Power and Consensus}

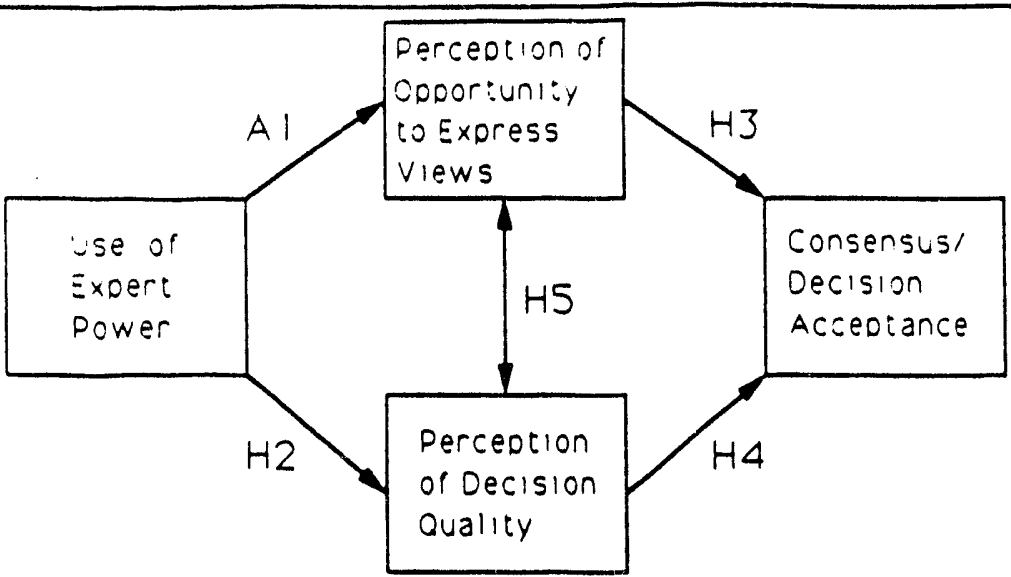

A1: Members of groups with an expert will perceive they had the same opportunity to express their views as groups with no expert.

If experts overuse their expert power, group members may not have the chance to speak their minds. This statement is labeled an assumption $(A)$ rather than a hypothesis $(\mathrm{H})$ because I've assumed experts won't overuse their power. Future studies will investigate the effects of overuse of expert power.

$\mathrm{H} 2 \cdot$ Members of groups with an expert will have higher perceptions of decision quality than members of groups with no expert.

The more experts use their expert power, the more their information is injected into the group decision. If group members perceive the expert's knowledge is accurate, they will perceive that using this knowledge will result in a higher quality decision. Perceptions of accuracy will depend on how salient the expertise is and whether or not the expert's information conflicts with group members' own knowledge. Hovland and Weiss [6] found that subjects' opinions were changed in the direction advocated by a communication to a significantly greater degree when the material was presented by a credible source than when presented by a non-credible source.

H3: The greater the degree group members perceive they had the opportunity to express their views, the stronger the consensus.

Hypothesis 3 says group members will be more likely to accept a decision if they believe their views were considered by the group. "It is generally recognized that the acceptance of decisions by group members is increased through their participation in making the decision" [8]. I believe the more individual group members perceive they had input into the group process, the stronger the consensus. Individuals who have input into a decision tend to identify with it and perceive it to be "their decision" [9].

H4: The higher the group members' perceptions of decision quality, the stronger the consensus. 
Hypothesis 4 says members may accept a decision if they perceive the decision is high in quality. This can occur even if they don't believe their views were considered by the group.

H5: There is a positive relationship between perception of decision quality and perception of opportunity to express views.

Humans are vain creatures; if members see their views in a decision, they will perceive the decision is high in quality. On the other hand, if members perceive a decision is high in quality, they will perceive their views were considered even if they didn't actually express them.

\section{Emergent Leadership}

H6: Experts will be perceived as being leaders.

The amount of influence an expert can exercise over a group is proportionate to the group's evaluation of the person's potential contribution to the group. In this study, "potential contributions" are pieces of information the expert possesses. Because the experts have access to task-relevant information that other group members don't have, they are more likely to emerge as leaders.

\section{METHOD}

\section{Subjects and Group Composition}

The subjects were 20 female employees of Virginia Polytechnic Institute and State University. Subjects ranged in age from 18 to 24. Subjects were assigned so that employees from the same department were in different groups to minimize the effects of referent power (power resulting from one person liking another). All groups consisted of five subjects. There were two experimental groups with one expert each and two control groups. One of the experts was a physics major and the other was an aerospace engineering major. Both had recently taken an astronomy course. Because the experts scored poorly on a pretest, they were given general knowledge about the moon and physics before entering the experiment. The experts had no other knowledge of the nature of the experiment.

\section{Procedure}

The experiment involved two phases. In the first phase, subjects individually completed the "Lost on the Moon" exercise. Subjects ranked 15 items based on importance for survival on the moon. I used this exercise because it has both quality and consensus components. NASA experts have developed the "correct" ranking. Quality can then be measured by closeness to this expert ranking. Groups were instructed that their survival depends on the quality of the decision. In the second phase, five-person groups came to consensus on the rankings using Hall's [4] consensus guidelines. Before performing the group exercise, group members introduced themselves by giving their name, major and any classes they have taken that would help the group solve the problem. This was designed to indicate to other members who had expert knowledge without signaling that person as a leader. 


\section{Measures}

1. Base of expert power. Potential for expert power was measured by the expert's quality score on the individual ranking step. This measurement acted as a manipulation check to ensure experimental groups had one and only one expert and control groups had no expert.

2. Use of expert power was measured using a subset of a 20 -item scale developed by Hinkin and Schriesheim [5] to measure French and Raven's [3] five bases of power. Only the expert and referent items were used because other bases of power were controlled for by design. Questions were modified so they would apply to group members rather than supervisors. Each group member rated all other group members on expert power. Measures of referent power were also given as a manipulation check.

3. Consensus/acceptance was measured with two questions addressing two distinct aspects. One question measured how much group members accepted the decision (individual acceptance). Another measured how much members believed others accept the decision (perception of group consensus).

4. Decision quality was measured by the group's score on the exercise. Scores are computed by summing the absolute differences between a ranking and the ranking given by NASA experts. Because there were only four groups in the pilot study, correlational statistics could not be computed on group measures. To get around this problem, an improvement score for individuals was used as a surrogate measure of decision quality. This score was calculated by subtracting the group score from the individual's initial score.

5. Perception of opportunity to express views was measured with four questions addressing the extent subjects were able to express their views, and have these views understood and reflected in the final ranking.

6. Perception of decision quality was measured with two questions addressing the extent subjects were confident in the ranking and believed it to be the best one.

7. Leadership emergence was measured with a five-item scale from Foti and Lord [2]. This questionnaire was filled out by each member for each other member. Items address leader's contribution to effectiveness, degree of influence, leadership behaviors, and control over activities. Questions were modified slightly to match the format of the power questions.

\section{RESULTS AND DISCUSSION}

\section{Manipulation Checks.}

Both experts failed to score higher on the quality measure than other group members. In fact, one expert had the worst individual score in her group. The other expert scored above average, but one person in her group scored better. This was obviously an unexpected result since the experts were given information about the moon prior to the exercise. However, the exercise also tests some general knowledge, not related to the moon, that the experts lacked. The experts also misinterpreted parts of the scenario and even forgot some of the information they learned previously. In other words, even moon 
experts may not be experts on the "Lost on the Moon" exercise. To check that neither condition had an advantage from higher individual scores, a t-test was run on individual quality scores. This test showed no significant difference $(t=.30, p>.05)$ between expert and no-expert groups on individual quality scores.

\section{The Effect of an Expert on Decision Quality}

Hypothesis 1 received mixed support. Group quality scores are given in Table 1. Combining group scores by condition, the expert groups had a slightly better average group score (lower scores indicate higher quality). Experimenter observations help explain each group's score. In the first expert group, the expert was able to contribute the information she had learned. Even though she was unable to translate this information into the proper ranking on her own, the group was able to use the information and add to it to produce the best ranking. In the second expert group, the expert forgot several key pieces of information, leading the group astray on the rankings of some key items. In both non-expert groups, most or all members contributed to the groups' effectiveness. All groups scored considerably better than the average of their individual scores. This clearly demonstrates the concept of group synergy. Correlation between condition and individual improvement scores was insignificant $(r=.09, p>.05)$. Thus, while hypothesis 1 was supported by descriptive statistics on group scores, it was not supported by correlations on individual improvement scores.

TABLE 1

\begin{tabular}{|llcccc|}
\hline Condition & Group & $\begin{array}{c}\text { Mean } \\
\text { Individual } \\
\text { Score }\end{array}$ & $\begin{array}{c}\text { Range of } \\
\text { Individual } \\
\text { Scores }\end{array}$ & $\begin{array}{c}\text { Expert's } \\
\text { Individual } \\
\text { Score }\end{array}$ & $\begin{array}{c}\text { Group } \\
\text { Score }\end{array}$ \\
\hline Expert & Group 1 & 44.4 & 38 & 40 & 8 \\
& Group 2 & 46.8 & 18 & 60 & 22 \\
\hline \multirow{2}{*}{ No Expert } & Group 3 & 46.4 & 24 & N/A & 22 \\
& Group 4 & 50.8 & 30 & N/A & 18 \\
\hline
\end{tabular}

${ }^{1}$ Lower scores indicate higher quality. Possible range: $0-112$.

\section{The Efrect of an Expert on Consensus}

The correlation between the presence of an expert and perception of opportunity to express views was not significant $(r=-.14, p>.05)$. This supports the validity of the assumption that experts would not overuse their power. Hypothesis 2 was not supported. A correlation between the presence of an expert and perception of decision quality was not significant $(r=-.41, p>.05)$. This is not surprising considering the trained experts were not true experts on the "Lost on the Moon" exercise. Therefore, this hypothesis was not adequately tested. Despite the failure to support hypothesis 2 , the data gave limited support to hypotheses 3,4 , and 5 . With respect to hypothesis 3 , there was a significant correlation between perception of opportunity to express views and individual acceptance $(r=.50, p<.05)$, and between perception of opportunity to express views and perception of group consensus $(r=.45, p<.05)$. Hypothesis 4 was supported by a significant correlation between perception of decision quality and individual acceptance $(r=.58, p<.01)$. However, perception of decision quality was not significantly correlated with perception of group consensus $(r=.38, p>.05)$. Hypothesis 5 was supported by a significant correlation between perception of decision quality and perception of opportunity to express views $(r=.61, p<.01)$. To better understand the 
relationships between these variables, I will conduct studies with greater control and statistical modelling. Individual acceptance and perception of group consensus were not significantly correlated $(r=.29, p>.05)$, indicating that these are separate constructs. Group members may accept a decision without thinking the rest of the group has reached consensus.

\section{Leadership Emergence}

Hypothesis 6 was supported. In both expert groups, the expert had the highest average ratings on both the expert scale and the leadership emergence scale. In other words, even though the experts were not true experts, they were perceived as being experts and leaders. This finding can be explained two ways. First, as subjects introduced themselves, it became clear to the other members that the expert had the best background for solving the problem. Group members had no way of knowing the expert's individual score. All other group members indicated they had practically no knowledge about the moon, heightening the disparity. Second, even though the experts' scores were average to poor, both were able to give their groups useful information. The expert's emergence can not be attributed : :) their being the most-liked individuals because neither expert rateü highest on referent power.

\section{CONCLUSION}

I was unable to test the effects of expert power because the expert manipulation failed to produce genuine experts. However, this study showed that group members with information related to the task can contribute to group effectiveness even if their individual decisions would be poor. Therefore, facilitators must make sure that experts contribute all relevant information without dominating the decision process. Two interesting and unexpected findings occurred. First, perceptions of expertise seemed more important than "true" expertise. Second, individual decision acceptance was not correlated with individual perceptions that others accepted the decision. Both findings indicate a discrepancy between perceptions and reality. These findings will be explored in later studies. This study can only be considered exploratory research. Further research, including controlled studies, will be conducted to more adequately test the hypotheses presented here.

\section{ACKNOWLEDGEMENTS}

The ideas presented in this paper represent the cumulative work of hundreds of people over thirteen years at Virginia Tech's Management Systems Laboratories. Professional managers from business and industry, and faculty, graduate students, and undergraduates from a wide variety of engineering and other disciplines have participated in the research and development of these tools and techniques. The authors thank them for their good work. The pronoun "I" is used throughout to separate all of us using management systems (we) from the authors (I). However, please recognize " $I$ " is more than one contributor.

The preparation of this paper is funded by U.S. Department of Energy Special Research Grant No. DE-FG02-88DP48058. Management Systems Laboratories thanks the Department of Energy's Office of Environmental Restoration and Waste Management for providing us a real-world laboratory for research, development, and testing of 
state-of-the-art management tools and the fiameworks for understanding how to make them successful.

\section{REFERENCES}

[1] Cartwright, D. and Zander, A., Group Dynamics: Research and Theory, 3rd ed. (New York: Harper \& Row, 1968).

[2] Foti, R. and Lord, R., "Prototypes and Scripts: The Effects of Alternative Methods of Processing Information on Rating Accuracy." Organizational Behavior and Human Decision Processes, vol. 39, No. 3 (1987), pp. 318-340.

[3] French, J. R. P. and Raven, B. "The Bases of Social Power." Studies in Social Power, D. Cartwright, ed. (Ann Arbor, Mich.: Institute for Social Research, 1959).

[4] Hall, J. "Decisions, Decisions, Decisions." Psychology Today, vol. 5, No. 6 (1971), pp. 51-54; 86-88.

[5] Hinkin, T. R. and Schriesheim, C. A. "Development and Application of New Scales to Measure the French and Raven (1959) Bases of Social Power." Joumal of Applied Psychology, vol. 74, No. 4 (1989), pp. 561-567.

[6] Hovland, C. I. and Weiss, W. "The Influence of Source Credibility on Communication Effectiveness." Public Opinion Quarterly, vol. 15, No. 4 (1951), pp. 635-650.

[7] Maier, N. R. F., Principles of Human Relations. (New York: Wiley \& $\mathrm{Co}, 1952)$.

[8] Maier, N. R. F. and Hoffman, L. R. "Quality of First and Second Solutions in Group Problem Solving." Joumal of Applied Psychology, vol. 44, No. 4 (1960), pp. 278-273.

[9] Yukl, G. A. Leadership in Organizations, 3rd ed. (Englewood Cliffs, NJ: Prentice Hall, 1989). 


\title{
CONSENSUS: AN INVESTIGATION OF THE NEED FOR FACILITATION
}

\author{
D. Mark Brubaker (student), Virginia Tech \\ Anne R. Doss, Virginia Tech \\ Harold A. Kurstedt, Jr., Virginia Tech
}

\begin{abstract}
In this paper I report observations I made during a year-long experiment with an autonomous work group at Virginia Tech. Management wanted to research if a group of people, with limited experience in group dynamics, could achieve consensus without facilitation. The employees chosen for this experiment were administrative personnel who felt they were treated with little respect and had no power over their working conditions. Thus, the group's morale was low. To motivate the group for this experiment, they were told to come to consensus on ways to improve working conditions. The group met without facilitation for six months and then met with facilitation for three months. I observed the group from the beginning and then became facilitator after the initial six month period. The observations I report in this paper show the need for facilitation to achieve consensus under the conditions of this experiment.
\end{abstract}

\section{BACKGROUND}

Considerable research suggests groups typically make decisions superior to those made by individuals [3] [15]. Vroom [16] found participation in decision making had a positive effect on employee attitudes and job performance. Participation has also been shown to increase motivation [10], decrease role conflict, and increase job satisfaction [6]. These findings have led management to use autonomous work groups to increase job satisfaction and productivity at the same time. I wanted to discover if it is sufficient to bring employees together to reach consensus or if some form of facilitation is necessary.

Intuitively, bringing employees together to discuss work-related problems is a good idea. Let the employees who do the work decide how to do it better. Give employees more control over their work, thereby improving productivity and satisfaction at the same time. This is the idea behind quality circles. Because quality circles were thought to be behind Japan's recent success [13], they became a fad in this country throughout the eighties [17]. However, quality circles haven't been as successful as you might think. In two surveys of firms with quality circles, it was found that about 70 percent of the firms had a savings-to-cost ratio of less than one [17]. These findings suggest bringing people together isn't enough. There must be more.

If you asked several people what consensus means you would get several different answers. Most would probably deal with some form of agreement. Definitions of consensus in the literature range from influence of key group members [7] to perfect unanimity of all group members [4] [11]. A number of authors have included in their definitions the idea that all group members must be able to express their opinions and accept the decision made [16] [14]. Others have defined consensus as some form of agreement [12] [2] [1] [9] [5]. 
To compare the group's ability to reach consensus with and without facilitation, I define consensus to allow me to judge its success or failure. I define consensus as a state where a common judgment has been reached by most of those concerned. Consensus exists when the group makes and supports a decision. In this experiment, for the consensus to be successful, the group had to formulate action procedures and implement their ideas for improving the workplace. A group can easily reach consensus on complaints about their workplace. I didn't consider the consensus successful unless the group generated solutions and supported their implementation.

In this experiment, I wanted to investigate the effect of facilitation on a group's ability to reach consensus, and thereby improving job satisfaction and productivity. Would the establishment of this group increase the morale, productivity, and quality of work life? Furthermore, for this to occur, would the group need facilitation or was it sufficient to let them make their own decisions without direction from facilitation?

\section{METHOD}

\section{Subjects}

The subjects chosen for this experiment were members of the administrative staff at Virginia Tech. Many group members felt they had little power over their working conditions and that they were not appreciated or treated with respect. Management hoped morale would increase if the group was allowed to meet without outside intervention to discuss problems and come up with changes in operations.

\section{Procedure}

The group was originally convened by an outside consultant and given instructions on group dynamics. They were told to achieve consensus on ways to improve their work environment and then consult with their supervisor to implement their ideas. Management supported the experiment.

To build a team concept, the group's first task was to write a contract which each member signed voluntarily. The contract detailed how the meetings were to be run and specified everything discussed would be kept confidential.

Because I believe conflict has 10 be surfaced and resolved to achieve consensus, the group was instructed to make sure different roles were present during all discussions. Assigning roles to make sure every side of an issue is discussed is an effective way to regulate conflict [15]. Furthermore, regulated responses to conflict allow a group to attain more accurate decisions and allow them to be more satisfied with the interaction occurring in the group [8].

Different roles were discussed. The group was asked to be aware of the following roles: facilitator ("honest broker," mediator, impartial third party); issue presenter (someone bringing a concern); prosecutor (challenger, explorer of facts); defender (advancer of the issue); information giver (others in the group having pertinent knowledge about the issue); and question caller (someone recognizing the need to wrap up discussion and move on to deciding on a course of action). 
The group allowed me to sit in on their meetings to observe for research purposes. They met without facilitation for six months after which time I became facilitator and we continued to meet for three more months.

\section{RESULTS}

The results of the group consensus process without facilitation were unsuccessful. The group couldn't achieve consensus on anything but minor issues. The group failed to follow the guidelines set up in the beginning and eventually became disillusioned with the process and wanted to disband.

The first couple of months went well. There were some personality conflicts but they were dealt with and the group started to become cohesive. Initial success on some issues was achieved. The group planned a surprise party for their boss, a Christmas party, and implemented other ideas to make their workplace more enjoyable. However, after enjoying this initial success the group never followed up with action on more important issues. They considered issues like the way they were treated, problems with late paperwork, linited access to upper management and a perceived breach of confidentiality in their group. They failed to follow the guidelines originally set and only looked at these issues from one viewpoint and didn't take action on solutions. Eventually the group wanted more guidance on what to do.

The group experienced team building and the power accompanying it but became disenchanted when there were no results to prove they weren't wasting their time. Consensus without resolution and final implementation of action is unsatisfactory.

The issue finally destroying the group's morale and effectiveness was a perceived breach of confidentiality. This completely polarized the group and destroyed group cohesiveness. At this point I became facilitator and we started over.

The formal facilitation phase was going well when the experiment was ended prematurely. No results are reported or conclusions made from the formal facilitation stage due to its early end.

\section{CONCLUSIONS}

I have been involved with many different groups over the past few years. Some have worked well with minimal facilitation and others have worked well with no facilitation. These groups have been made up of people with experience in government and group dynamics. I wanted to see if a group of employees without this experience could reach consensus without outside facilitation. From this experiment, I concluded they couldn't. because of three factors. First, the group felt they had no power within the organization to change anything. Second, they feared failure. The group believed that if they tried new ideas which were unsuccessful, they could lose their jobs. Third, the perceived breach of the confidentiality agreement played on the fears associated with the first two factors to completely destroy group unity.

For the manager facing a need to involve employees in decision making I believe the following actions will increase the chance of success. First, make sure the employees receive formal training in group dynamics and team building. Second, make sure there is 
independent facilitation until the group is confident with their abilities. Then gradually reduce the role of the facilitator and let them take over their own meetings. Third, initially assign issues or problems to solve. This will give the group confidence that they will be listened to and increase the chance of their solutions being implemented. After a number of successes, broaden the group's purpose to a more general statement like finding ways to improve the work place.

I think groups such as this are a benefit to the work place. However, forming an autonomous work group is not a sure cure for all the manger's ills. The group's creation and subsequent meetings must be carefully facilitated for maximum benefit. More research needs to be done on facilitation of consensus. We need to know in what situations and with what people different facilitation techniques will be most effective.

\section{ACKNOWLEDGEMENTS}

The ideas presented in this paper represent the cumulative work of hundreds of people over thirteen years at Virginia Tech's Management Systems Laboratories. Professional managers from business and industry, and faculty, graduate students, and undergraduates from a wide variety of engineering and other disciplines have participated in the research and development of these tool and techniques. The authors thank them for their good work. The pronoun "I" is used throughout to separate all of us using management systems (we) from the authors (I). However, please recognize "I" is more than one contributor.

The preparation of this paper is funded by U. S. Department of Energy Special Research Grant No. DE-FG02-88DP48058. Management Systems Laboratories thanks the Department of Energy's Office of.Environmental Restoration and Waste Management for providing us a real-world laboratory for the research, development, and testing of state-of-the-art management tools and the frameworks for understanding how to make them successful.

\section{REFERENCES}

[1] Beck, M. P. \& Benjamin, W. L., "Some Heuristics for the Consensus Ranking Problem." Computing and Operations Research, vol. 10, No. 1 (1983), pp. 1-7.

[2] DeStephen, R. S., "High and Low Consensus Groups - A Content and Relational Interaction Analysis." Small Group Behavior, (May, 1983), pp. 143-162.

[3] Hall, J., "Decisions, Decisions, Decisions." Psychology Today, (November 1971).

[4] Hirokawa, R. Y., Emergent Issues in Human Decision Making. (Carbondale IL: Southern Illinois Press, 1985).

[5] Holder, J. J. "Decision Making by Consensus." Business Horizons, (April, 1972), pp. 47-54.

[6] Jackson, S. E., "Participation in Decision Making as a Strategy for Reducing Job Related Strain." Joumal of Applied Psychology, vol. 68, No. 1 (1983). 
[7] Kacprzyk, J. \& Fedrizzi, M., "A 'soit' Measure of Consensus in the Setting of Partial (fuzzy) Preferences." European Journal of Operational Research, vol. 34, (1988), pp. 316-325.

[8] Pood, E. A., "Functions of Communication: An Experimental Study in Group Conflict Situations." Small Group Behavior, vol. 11 No. 1 (February, 1980), pp. 76-87.

[9] Price, J. L., Handbook of Organizational Measurement (Lexington, MA: D. C. Heath \& Co., 1984), pp. 79-83.

[10] Rabinowitz, W., Valentine, C. G., Falkenbach, K., Weener, P., \& Travers, J. R., "Worker Motivation: Unsolved Problem or Untapped Resource? California Management Review, (January 1983).

[11] Rawlins, W. K., Emergent Issues in Human Decision Making. Phillips, G. M. \& Wood, J. T., (Eds.). (Carbondale, IL: Southern Illinois University Press), pp. 19-37.

[12] Scheff, T.J., "Toward a Sociological Model of Consensus." American Sociological Review, (1984), pp. 32-46.

[13] Szilagi, A. D., Management and Performance. (Glenview, IL: Scott, Foresman \& Co., 1984).

[14] Tjsovold, D. \& Field, R. H. G., "Effect of Concurrence, Controversy, and Consensus on Group Decision Making." The Joumal of Social Psychology, vol. 125, No. 3 (1985), pp. 355-363.

[15] Tjsovold, D. \& Field, R. H. G., "Effects of Social Context on Consensus and Majority Vote Decision Making." Academy of Management Joumal, vol. 26, No. 3 (1983).

[16] Vroom, V. H., "Some Personality Determinants of the Effects of Participation." Joumal of Abnormal and Social Psychology, vol. 59 (1959), pp. 322-327.

[17] Wood, R., Hull, F. \& Azumi, K., "Evaluating quality circles: The American application." Califormia Management Review, (Fall, 1983), pp. 37-53. 


\title{
A SELECTION TOOL FOR CHOOSING THE BEST PARTICIPATIVE PROBLEM-SOLVING TECHNIQUE
}

\author{
John F. Keeling III (student), Virginia Tech \\ Harold A. Kurstedt, Jr., Virginia Tech \\ James E. Hughes, Virginia Tech
}

\begin{abstract}
The primary purpose of this paper is to provide managers and group facilitators with a tool for selecting the best technique(s) to help a group solve a problem. The selection tool was created by matching available techniques to problem component examples using a contour map. The secondary purpose is to: (1) describe (by example) three components of a problem that requires a group to solve, and (2) describe available techniques for group problem solving.
\end{abstract}

\section{INTRODUCTION}

Managers and group facilitators (hereafter referred to as "managers") face complex problems involving groups of people with diverse internal and external interests. According to Shaw [12] and Hall [4], participative problem solving provides higher quality solutions to nonroutine problems.

We can use many familiar group decision-making techniques to help us with participative problem solving, such as Brainstorming, Nominal Group Technique (NGT), and Devil's Advocacy. Each technique has its strengths and weaknesses. For example, NGT is successful for idea generation, but unsuccessful for consensus gaining [2] [9].

One problem faced by managers is selecting the best technique for a given problem being solved by a group of people. The manager can use many techniques, each with different applications, strengths, and weaknesses. I present a selection tool to give the manager the information he ${ }^{1}$ needs to select the best technique. The selection tool uses a contour map [13] for matching available techniques to example problem components. Together, problem compcinents structure the group decision process. Each component must be addressed to solve the problem properly. In this paper, I select three problem components to address: purpose, people, and participation.

I represent the problem components with examples. For instance, the "people" component is either heterogeneous or homogeneous. I match the techniques to the component examples based on three criteria: (1) what the technique was designed for, (2) the technique's strengths demonstrated by empirical testing and comparison to other techniques, and (3) my own judgment based on my understanding of 1 and 2.

To use the selection tool, a manager must first have a problem requiring a group solution. The manager gathers information to understand the components of the problem. Then the manager (group facilitator) uses the tool to select the best technique to help the group solve the problem.

${ }^{1}$ Or she - the traditional masculine pronoun is used for lightness of style. 
I begin by explaining the selection tool's three components and describe the examples for each component. Next, llook at the selection tool's techniques and explain the matching of the techniques to the examples for each problem component. I conclude with two hypothetical applications to illustrate the use of the selection tool.

\section{COMPONENTS OF THE SELECTION TOOL}

Figure 1 illustrates the selection tool. The components of the tool are the purpose, people, and participation. Since this isn't meant to be an exhaustive typology, some components may be missing. Purpose is why the group is acting or what the group wants

FIGURE 1

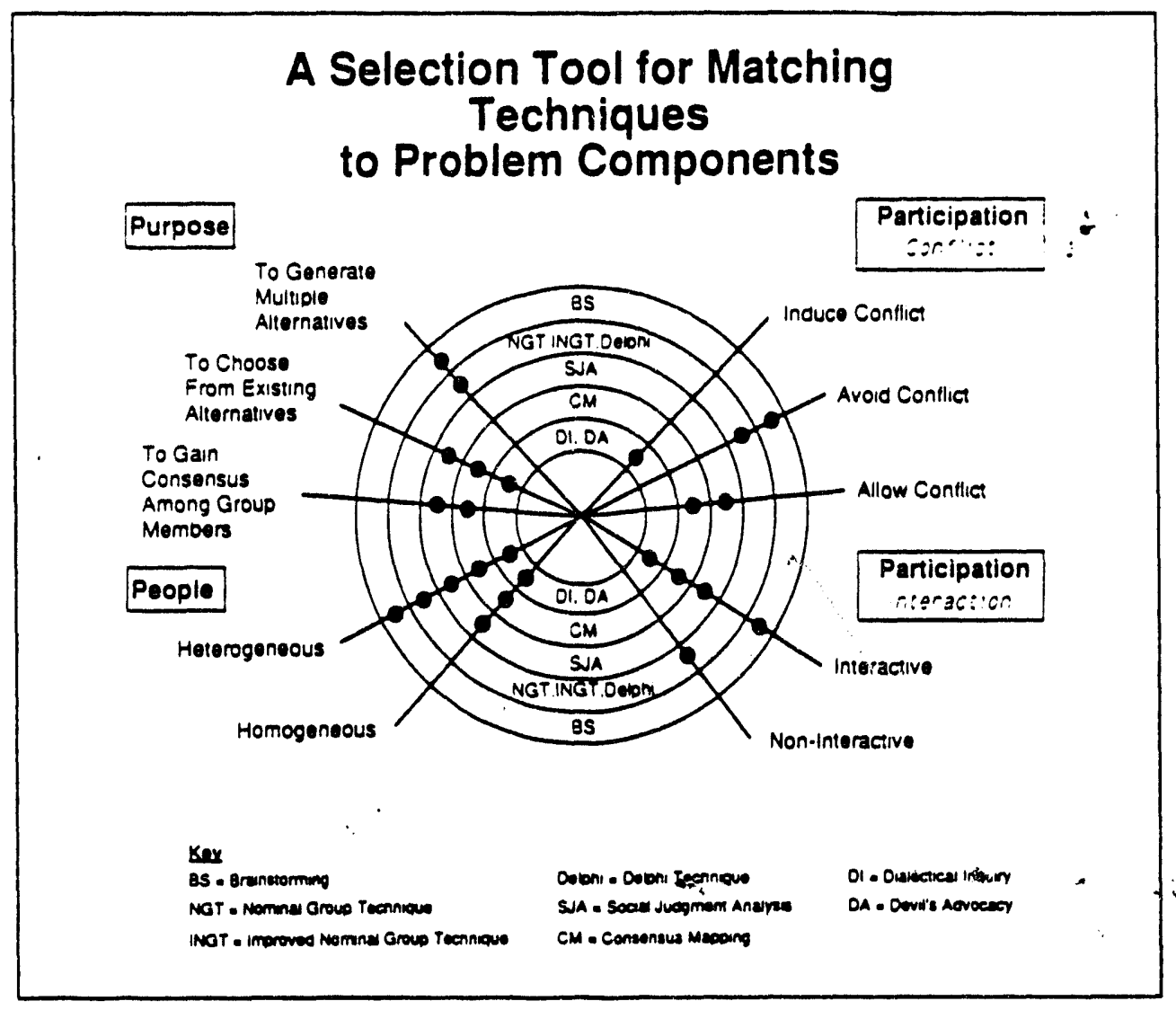

to achieve (i.e., the mission). As shown in Figure 1, purpose is divided into three examples: to generate multiple alternatives, to choose from existing alternatives, and to gain consensus among group members. The purpose could be one or a combination of these examples. The group's purpose could be to generate as many alternatives as possible. Alternatives are ideas that are all or part of a solution. If the group already has alternatives, they may want to analyze their individual and group judgments to obtain a higher quality recommendation. The purpose may also include gaining consensus among group members. Consensus relies on "a thorough, open, and constructive discussion and examination of the recommendations and underlying assumptions developed individually by group members ... each group member should have the opportunity to present his recommendations, the underlying assumptions, and relevant facts and data in as clear and logical a manner as possible" [11].

People are participating to achieve the purpose. Two examples of people are heterogeneous and homogeneous. A heterogeneous group 
is characterized by "widely varying personalities and substantially different perspectives on a problem [2; p. 26]." In a homogeneous group, people have similar personalities and perspectives.

The participation is how the participants go about achieving the purpose. I divide participation into two subcomponents: conflict and interaction. Each subcomponent has additional examples. Conflict occurs when "two systems (persons, groups, organizations) interact directly in such a way that the actions of one tend to prevent or compel some outcome against the resistance of the other" [8; p. 613]. When occurring at proper levels, conflict can increase decision quality [1] [15]. Conflict can be induced, avoided, or allowed. When conflict is induced, participants are forced into a conflictual situation. The manager can avoid conflict by restricting interaction among participants. If conflict is not specifically avoided or induced, then it is allowed to occur at any time during the participation. I define interaction to be verbal and non-verbal communication between the people in the group. The manager either allows interaction (to various degrees) or avoids it. I select two extremes of interaction examples: interactive and non-interactive. In an interactive participation, group members discuss the problem face-to-face an: are allowed to communicate freely. In non-interactive participativin, group members are restricted from open discussion.

\section{MATCHING TECHNIQUE TO COMPONENT EXAMPLES}

I looked at three components (purpose, people, and participation) of a participative problem and selected examples for each. The selection tool matches available techniques to the component examples (See Figure 1). Techniques are represented by rings on the diagram. Component examples are connected to the diagram with a line. A match of technique to component example is designated by a dot on the line corresponding to the example and within the technique ring. I use italics for all references corresponding directly to the tool. As mentioned, matching is based on three decision criteria (in priority order): (1) what the technique was designed for, (2) the technique's strengths demonstrated by empirical testing and comparison to other techniques, and (3) my own judgment based on my understanding of 1 and 2. I briefly describe each technique (and any closely-related technique) and explain the matching of the technique(s) to the respective componient example.

Brainstorming is an interactive technique for generating multiple alternatives. Participants voice ideas out loud. These ideas tend to spark ideas from other members, resulting in a large pool of ideas. Because this technique is only used for generating ideas, it avoids conflict. Heterogeneous groups will be more effective than homogeneous groups using this technique because they generate both a higher quantity of ideas and higher quality ideas [2] [6] [7].

The Nominal Group Technique (NGT) involves nominal alternative idea generation (i.e., participants work side by side, but don't interact), round-robin recording of alternatives, interactive group discussion of alternatives for the purpose of clarification and evaluation, and individual voting on priorities through a ranking or rating procedure. The group decision is the "pooled" outcome of individual votes [14]. Two techniques closely related to NGT are Improved NGT (INGT) and Delphi. INGT differs from NGT in that participants generate the first set of alternatives at home and anonymity is maintained throughout the process. The Delphi 
technique differs from both of these techniques in that participants work independently at home during the entire process. These three techniques are non-interactive $e^{2}$ and are designed to avoid conflict. Empirical studies show they are effective techniques for generating multiple alternatives [2] [3] [9]. Heterogeneous groups will produce better results using these techniques, because, as mentioned, they generate both a higher quantity of alternatives and higher quality alternatives.

Social Judgment Analysis (SJA) is an interactive technique used to analyze and improve group judgment in making a decision. Therefore, I believe this technique is useful for choosing from existing alternatives. SJA allows conflict and uses it to promote understanding. Group members understand the factors influencing their judgment and therefore are better able to understand the underlying differences between individual judgments. This technique also produces a stronger consensus among group members compared to NGT [10]. I recommend SJA for both homogeneous and heterogeneous groups.

Consensus Mapping (CM) is an interactive technique used to categorize and integrate ideas to form a final solution (i.e., the consensus map). The process begins after ideas are generated, clarified, and evaluated (e.g., with a rating or prioritization). Subgroups work individually to combine related ideas into classification schemes. Classification schemes from sub-groups are consolidated by a committee (separate from the groups) to form a first cut at the solution (i.e., the consensus map). The first cut is improved by the sub-groups. The improvements are consolidated to form the consensus map. CM is useful for complex and interrelated problems involving categorization, analysis, and integration of ideas. Because of this, I believe this technique is useful for choosing from existing alternatives. CM, like SJA, allows conflict, but does not specifically use it to promote understanding. CM "enables group participants with differing perspectives to arrive at a shared image of a problem and a mutually agreeable solution" $[5 ;$ p. 598]. Therefore, the technique is useful for gaining consensus among group members. I recommend CM for both homogeneous and heterogeneous groups.

Dialectical Inquiry and Devil's Advocacy are interactive techniques that induce conflict. Dialectical Inquiry starts with two recommendations and uses a debate to arrive at a final decision. Devil's Advocacy uses one recommendation and exercises criticism and modification to arrive at a final decision. These techniques increase decision quality [11] by revealing underlying assumptions, information, thoughts, and/or feelings group members may be withholding. I believe this is necessary for effective analysis of one or more potential solutions. Therefore, I recommend them for choosing from existing alternatives. I recommend these techniques for both homogeneous and heterogeneous groups.

\section{APPLICATIONS OF THE SELECTION TOOL}

I introduce two hypothetical applications of this selection tool to demonstrate how it may be used on typical real world problems involving participation. You use the tool by matching your particular problem's component examples to the techniques to determine which technique is most appropriate. As mentioned, a match is designated by a dot on the line corresponding to the example (See Figure 1). The

2 While NGT has an interactive phase, it is mainly a non-interactive technique. 
technique(s) having the most matches to your problem's component examples would be the best technique(s) to use.

Participative Problem \# 1 - How to Improve Management Relations

Participative problem \# 1 involves a group with diverse personalities and opinions whose positions range from first-line production supervisor to CEO. The purpose of the meeting is to get some recommendations about how to improve relations between upper and lower-level management. The convenor wants to allow equal participation, while focusing on a few good recommendations.

Based on the problem requirements, we can assume the group is heterogeneous and the purpose is to generate multiple alternatives. A meeting implies interactive participation. To ensure equal participation in an interactive setting involving a wide range of job status levels, the technique should avoid conflict. Using our selection tool (Figure 1) and our problem specifications, we can eliminate all but four techniques, namely NGT, INGT, Delphi, and Brainstorming. Delphi can be eliminated because the participants are meeting faceto-face. The requirement of focusing on a few good recommendations eliminates Brainstorming because it is simply an idea-generation technique. So we are left with NGT and INGT as our two possible choices. Unless the convenor prefers INGT, I recommend NGT for this group problem because of its simplicity and proven effectiveness.

\section{Participative Problem \#2 - How To Safely Dispose Nuclear Waste}

Participative problem \#2 involves a group of people who have worked together for some time and who have fairly similar views. The purpose of the group is to develop the best plan for the safe and effective disposal of waste from several nuclear reactors in the area. The meeting should be open and participants should be encouraged to surface all thoughts and feelings to ensure successful implementation of the plan. All group members must be able to accept the final plan.

This problem implies the group is homogeneous since they have similar views. There isn't a specification for the process, but since we are working with a homogeneous group we should induce or at least allow conflict to improve decision quality. The requirements of having an open meeting and the need for successful implementation imply we must gain consensus among the group. Therefore, using our selection tool, the two possible techniques are CM and SJA. Because a complex plan often involves the categorization, analysis, and integration of ideas, I recommend CM for this problem.

\section{CONCLUSION}

Managers often find themselves involved with problems requiring participation. The manager can use a technique to help the group become more effective. There is no one test technique for all situations. Different techniques are useful for different applications. The difficulty is choosing the best technique for your particular problem. I have presented a selection tool to assist in this task. The tool matches problem component examples to available techniques. If the manager understands the components (and examples) of his group problem components, he or she can use this tool to choose the best technique. However, no tool eliminates the need for good judgment. Mangers should weigh the recommendations of the tool against their own experience. 
The simplified version of this tool can't distinguish among all possible techniques. For instance, SJA and CM are matched to the same criteria even though they are very different techniques. I recommend further research to enhance the number of components and examples (to provide for better distinction) as well as techniques to be matched. I also encourage managers to develop their own selection tool using techniques they are familiar with.

\section{ACKNOWLEDGEMENTS}

The ideas presented in this paper represent the cumulative work of hundreds of people over thirteen years at Virginia Tech's Management Systems Laboratories. Professional managers from business and industry, and faculty, graduate students, and undergraduates from a wide variety of engineering and other disciplines have participated in the research and development of these tools and techniques. The author thanks them for their good work. The pronoun "I" is used throughout to separate all of us using management systems (we) from the author (I). However, please recognize "I" is more than one contributor to this work.

The preparation of this paper is funded by U.S. Department of Energy (DOE) Grant No. DE-FG02-88DP48058. Management Systems Laboratories thanks the DOE's Office of Environmental Restoration and Waste Management for providing us a real-world laboratory for the research, development, and testing of state-of-the-art management tools and the frameworks for understanding how to make them successful.

\section{REFERENCES}

[1] Brown, L. David, Managing Conflict at Organizational Interfaces (Reading, Massachusetts: Addison-Wesley Publishing Co., 1983).

[2] Delbecq, Andre L., Van de Ven, Andrew H., Gustafson, David H., Group Techniques for Program Planning: a guide to nominal and delphi processes (Glenview, Illinois: Scott, Foresman \& Co., 1975).

[3] Fox, William M., Effective Problem Solving - How to Broaden Participation, Improve Decision Making, and Increase Commitment to Action (San Francisco, California: Jossey-Bass Inc., 1987).

[4] Hall, Jay. "Decisions, Decisions, Decisions." Psychology Today, vol. 5, No. 6 (1971), pp. 51-88.

[5] Hart, Stuart, Boroush, Mark, Enk, Gordon, Hornick, William, "Managing Complexity Through Consensus Mapping:

Technology for the Structure of Group Decisions." Academy of Management Review, vol. 10, No. 3 (1985). pp. 587-600.

[6] Hoffman, L. Richard., "Homogeneity of Member Personality and its Effect on Group Problem-Solving." Joumal of Abnormal and Social Psychology. vol. 58, No. 1 (1959), pp. 27-32.

[7] Hoffman, L. Richard, Maier, Norman R.F., "Quality and Acceptance of Problem Solutions by Members of 
Homogeneous and Heterogeneous Groups." Joumal of Abnormal and Social Psychology. vol. 62, No. 2 (1961), pp. 401407.

[8] Katz, Daniel, Kahn, Robert L., Social Psychology of Organizations (New York, NY: John Wiley and Sons, Inc., 1978)

[9] Mahler, Julianne G., "Structured Decision Making in Public Organizations". Public Administration Review, vol. 47, No. 4 (1987), pp. 336-342.

[10] Rohrbaugh, John, "Improving the Quality of Group Judgment: Social Judgment Analysis and the Nominal Group Technique." Organizational Behavior and Human Performance, vol. 28, No. 2 (1981), pp. 272-288.

[11] Schweiger, David M., Sanberg, William R., Ragen, James W., "Group Approaches For Improving Strategic Decision Making: A Comparative Analysis Of Dialectical Inquiry, Devil's Advocacy, And Consensus." Academy of Management Joumal, vol. 29, No. 1 (1986), pp. 51-71.

[12] Shaw, Marvin E. Group Dynamics: The Psychology of Small Group Behavior. (New York, NY: McGraw-Hill, 1981).

[13] Shin, Seung, Riel, Phillipe F., Sink, D. Scott, "Productivity Measurement: A Situational Approach" Proceedings, IIE Spring Conference, Orlando, Florida, May 1988.

[14] Van de Ven, Andrew H., Delbecq, Andre L., "The Effectiveness of Nominal, Delphi, and Interacting Group Decision Processes." Academy of Management Journal, vol. 17, No. 4 (1974), pp. 605-621.

[15] Wall Vincent.D., Galanes G.J., and Love, S.B. "Small, TaskOriented Groups: Conflict, Conflict Management, Satisfaction, and Decision Quality." Small Group Behavior, vol. 18, No. 1 (1987), pp. 31-55. 


\section{HOW TO INCREASE MEETING EFFFCTIVENESS AND EFFICIENCY}

Martin Grunau (student), Virginia Tech

Harold A. Kurstedt, Jr., Virginia Tech

\section{ABSTRACT}

In this paper. I present a model adapted from control theory to gain insight into the dynamics of meetings. I relate the system controller and feedback sensor in the model to the role of the facilitator in meetings. By drawing parallels between the control theory model and the adapted model for meetings, I came up with an operational definition of a group facilitator and derived the following hypothesis: Providing facilitators with information about group members prior to the meeting will improve their effectiveness and efficiency in facilitating the meeting. Such information, for example, could include the group members' personality types measured with the MyersBriges Type Indicator $[10)$ or their conflict modes measured through the Thomas-Kilmann Conflict Mode Instrument (12)

\section{INTRODUCTION}

We use group facilitators hoping they will improve the effectiveness and the efficiency of meetings. What role does the facilitator play in meetings and how does it affect meeting effectiveness and efficiency? I answer this question by introducing a conceptual model adapted from control theory. The model is a representation of general systems and their feedback loops.

Although this model was originally developed for engineering applications, I find it applicable to group dynamics as well. The model helps us better understand the roles played by group facilitators and how inputs and outputs of meetings relate to them. I use the model to help shape a definition of group facilitators in view of increasing the meeting's efficiency and effectiveness. Both the conceptual model of meetings and the facilitator definition lead me to the mpothesis that meeting efficiency and effectiveness are increased by giving the group facilitator information about the rroup members prior to the meeting.

\section{MEETtNGS}

What are meetings? Meeting are events with three general purposes: (a) 10 share information (7) (b) to make decisions and (c) to build teams (3). These three purposes are not mutually exclusive. For example, making decisions requires sharing information to increace the pool of available information and also involves the actual decision-making process. This is eccomplished through the interplay of group process and rroup participants. As the meetine purpose moves From simple information sharing to deciaion making, system complexity increases and so does the need for a group lacilitator. Hence, we need to define the components of meetings and the role of facilitators more systematically. This will help us gain better visibility of how to improve facilitator and meeting efficiency and effectiveness.

\section{CONTROL THEORY}

I use control theory to help us with the analycis and design of systems. What are systems? Blanchard and Fabrycky 2 define a system as an "aseemblage or combination of elements or parts forming a complex or unitary whole." Kurstede [9] says systems "convert inputs into outputs resulting in throughput to meet some objective and incorporate measures of performance to determine how well the objectives are beins met." More specifically, control theory deals with dynamic responses of systems to given inputs. These dynamic responses result through the interaction of three system components (Figure 1). They are called plant, controller and sensor. The plant is the system's component to be controlled. The sensor acts as the feedback component in the control loop measuring the behavior of the plant and sending the information back to the controller. The controller modifies the plant behavior according 10 input from the sensor and external input. Given a plant, the system designer can choose sensor and controller characteristics to manipulate the system performance to reach pre-established output specifications. The component characteristics include accuracy and system stability which I explain lacer.

\section{SIMILARITIES BETWEEN MEETINGS AND CONTROL THEORY}

Meetings and control theory share some similarities. Both make use of the system concept: Inputs are transformed by some procese into outpuls. In meetines this process consists of interactions berween facilipators and the group members. In control theory, the process is the interplay of the three system components, plant, sensor, and controller. Control theory systems and meetings exist because they are to accomplish a particular purpose. Their quality or success is measured by the degree to which the output of the systems matches their purpose. These similarities led me to the conclusion that it should be possible to superimpose the concepts of control theory onto meetings.

\section{MEETINGS FROM A CONTROL THEORY PERSPECTIVE}

Figure 2 is a modification of the feedback loop from control theory, to represent a meeting as a system.

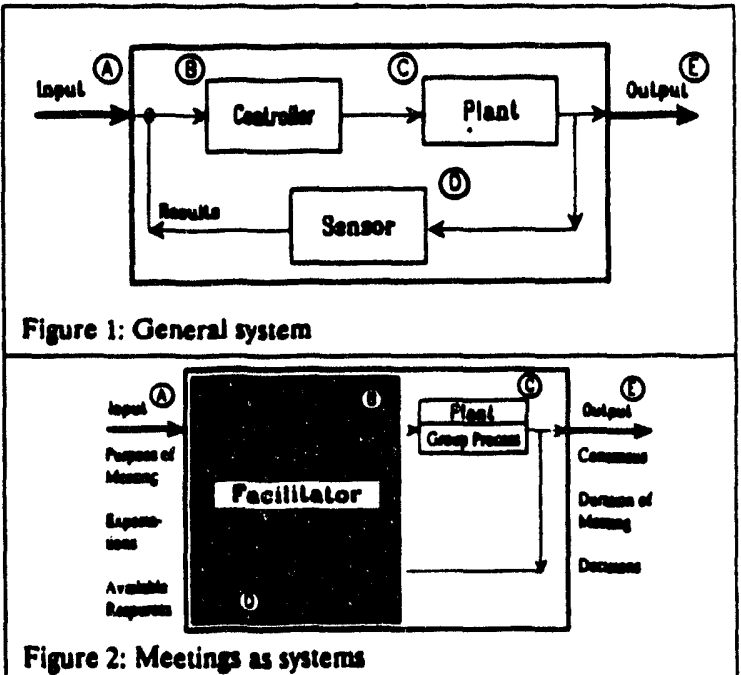

Figure 2: Meetings as systems

To avoid coafmion, wote tha I ande a distinction between process and group procens. The procens is the incerplay of the three syuem

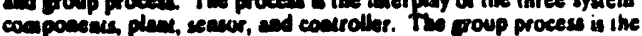
increction asom, ive group menbers within the plas composent ouly. 
Approsching group meetings more systematically, I use the three purposes of meetings as inputs to the system (A). Other inputs to meetings include facilitators. group member personalities, expectations of group members, available resources, and the group composition. The outputs (E) of meetines include decisions, increased knowled ge of group members, group members' satisfaction and a change in group cohesion. These outputs match the three purposes of meeti iges.

Clarifying Kursted's concept of throughput will be beneficial to understand how to control or minimize the discrepancy between anticipated and actual output (accuracy).

Throughput includes the controller, the plant, and the sensor as well as their dynamic interrelationships. Managing the throughput well allows us to drive the system to the desired output and therefore increase the accuracy. Fig. 2 shows the three components of the system: plant (C), controller (B), and sensor (D).

The system's plant is the part to be controlled. In a meeting it is equivalent to the group process. Elements of the group process to consider are discussion monopolization by few individuals. group morale and atmosphere possibly undermined by conflict, group members' opportunity to express their opinions, or the degree to which are they bored by the meeting.

In general systems, the sensor acts as the feedback component in the control loop. It measures the behavior of the plant. The controller then deals with two input signals, one external input signal and the output of the sensor. Both signals are read and acted upon by the controller. The controller's action has direct impact on the input of the plant. I believe the sensing and controlling functions are facilitator responsibilities. Facilitators collect, measure, or sense the output of the group process (e.g. members' yawning loss of eye contact, or dysfunctional side conversations). Facilitators then compare the data to their own knowledge, judge it to determine what is wrong with the group process and how it should be modified to improve it. Finally, facilitators actively change the control of the group process according to the sensor output while at the same time considering the external input $(A)$ as weil.

Fox [4] expands on this idea. He says group members have knowledge about the task of the meeting. while facilitators have knowledge about the group process. Facilitators are responsible for creating and maintaining a proper climate, and aiding participation by presenting choices to the group rather than making the decision for the group. Also the facilitator sets guidelines established before the meeting. Essentially the facilitator is a referee. In this way the facilitator makes sure group members have an opportunity to express their views, are encouraged to participate, and are protected from unhealthy criticism. I like these guidelines, since they focus the facilitator's attention on the group process (C), rather than specifically task-related issues. Kaplan (8) and Prince [11] agree with Fox's emphasis on the group process. They say facilitation supports a process 10 achieve a foal through facilitators' nonevaluative feedback. Based on Fox and Kaplan, I define a successful facilitator as a thind pany able to assist and/or adapt a group process efficiently and efjectively 10 the extens that the group will strive to execuse the puppose of the meeting.

The goal of the facilitator is to effectively and efficiently move the group process closer to achieving the purpose of the meeting. Facilitators make meetin effective when they can make the meetins outcome match the purpose of the meeting or the convenor's expectations. Facilitators make meetings efficient when a maximum amount of work is accomplished in the shortest amount of time.

\section{MEETING EFFECTIVENESS AND EFFICIENCY}

Using the analosy of control loops and the definition of successful facilitators, I propose three criteria from control theory which should be taken into consideration when designing an efficient and effective meeting:

1. Should the system be controlled (open- vs. closed-Inop system)?:

2. Is the system stable [5]?; and

3. Does the system have acceptable accuracy [5]?

I believe the first question is most important because it is affected by the availability of resources such as money and time. Controlled systems are more expensive than uncontrolled systems since they require a sensor, i.e., a facilitator. So the convenor must first determine whether the system is controlled or uncontrolled. If the convenor decides on a controlled syatem requiring a facilitator, the question becomes how to choose the facilitator to best drive the group process towards the desired outcome. The concepts of stahility and system accuracy become key issues.

Stability is easily understood. A system is either stable or it isn't. A system is said to be stable if it eventually reaches a steady state or steady result. Unstable systems are expensive since they eventually self-destruct. I belleve the meeting has to be stable to the degree that the group dynamics will not self. destruct. Otherwise group members will leave the meeting in frustration and anger because group process permitted aggressive dysfunctional verbal behavior.

System accuracy is specified in terms of acceptable steady-state errors. A steady-state error is the difference between desired output and actual output. The meeting should reach an accurate steady result (output). The meeting accomplishes its purpose when the right solution is found to the right problem; the meeting fails if it solves a problem it did not intend to solve. Continued unsteady results may otherwise translate into groups unable to continue operating functionally as a sroup, or a group solution not in compliance with the purpose of the meeting-in short, undesirable system behavior.

My analysis using control theory to model meetings as systems together with my definition of the facilitator role in meetings leads me to pose a hypothesis for improving meeting effectiveness and efficiency.

Hypothesis:

Group facilitosors, meeting effectiveness (desired accuracy and stabiliny) and eficiency (desired accuracy/rime needed. stability/time needed) will be improved by siving facilitators information about group members prior to the meeting.

Providing facilitators with information or knowledge about group members is equivalent to tuning the sensor in the system - making the sensor more aware of the correct things 10 measure during the meeting or more in tune with potential dificulties. Obviously the sensor will not measure just one thing but several group process characteristics in serial and parallel. Such information could be the group members' personality traits and implications for their information perception and evaluation preferences measured through the Myers-Briess Type Indicator (MBTT) [10], or their favored method of dealing with conflict measured through the Thomas. Kilmann Conflict Mode Instrument (12).

Increased meeting efficiency and effectiveness has implications on the group process itself. An increased amount of information or knowledge about group members will allow facilitators to create a flexible group process meaning they can change the direction the group process is heading quicker according to the needs of the group. 


\section{IMPLICATIONS FOR MANAGERS}

Manazers can model their meetings as a system so they can apply system analysis techniques. Viewing their meetings as systems defined by control theory adds a structured perspective for the identification, definition, measurement, and feedback of the system's components--the facilitator, group members, and the group process, and its inputs and outputs. Managers can determine if they have all the necessary system components and characterize those systems using structured techniques.

Managers should learn what the role of the facilitator should be, and my definition of the facilitator should help. Managers should evaluate the roles their facilitators play in their meetings. Often, managers themselves assume the role of facilitaior. Group members perceive managers acting as facilitators as biased. and susceptible to exerting their positional power to influence or intimidate group members.

Managers can experiment with giving the group facilitator more information atout group members before the meeting This additicnal knowledge might help the facilitator avoid Janis' phenomena of groupthink [6]. In a groupthink situation, the capability of groups to make good decisions is diminished hecause of lack of conflict during the group process. For example, if all group members are classified with the same MBTI personality type and all score high on conflict avoidance on the Thomas-Kilmant Conflict Mode Instrument, chances for groupthink are high. This information about the group members will help the facilitator intervene in a groupthink situation at an early stage once the first symptoms become visible.

Earlier. I mentioned Fox's [4] proposed facilitator responsibilities: to give all group members an opportunity to express their views, encourage and motivate them to participate, and protect them from other group members' hostile attacks such as personal accusations. These three responsibilities require a facilitator to be knowledgeable of the group members' attitudes concerning conflict as well. Are they very angressive, or do they tend to avoid conflict? The facilitator will be tuned in to possible difficulties arising during the meeting with particular group members. When the facilitator can match dysfunctional group behavior with the knowledge he has about the individual, the facilitator can take "controlling" action to correct and redirect the group process.

\section{SUMMARY}

How can we increase meeting effectiveness and efficiency? I looked at wwo key points before arriving at my hypothesis for increasing meeting efficiency and effectiveness: (a) Meetings are systems which can be modeled using control theory. They have inputs, outputs, and the three system componentscontroller, plant and sensor-of which the sensor and the controller constitute the facilitator's role. (b) I verbalized a facilitator definition based on the conceptual model and on a literature review. I define a successful facilitator as a third party able to assist and/or adapt a group process efficiently and effectively to the extent that the group will strive to execute the purpose of the meeting. So the facilitator is primarily concerned with the group dynamics, not with the task itself. These two key points led to the hypothesis that group facilitators and meeting effectiveness (desired accuracy and stability) and efficiensy (desired accuracy/time needed, stability/time needed) will be improved by giving facilitators information about group members prior to the meeting.

Future research can test the correctness of this hypothesis qualitatively as well as quantitatively. As the model is further developed, researchers may use mathematical relations to calculate the necessary amount of information facilitators should have about their group members to increase meeting effectiveness and efficiency.

\section{ACKNOWLEDGEMENTS}

The ideas presented in this paper represent the cumulative work of hundreds of people over thirteen years at Virginia Tech's Management Systems Laboratories. Professional managers from business and industry, and faculty, graduate students, and undergraduates from a wide variery of engineering and other disciplines have participared in the research and development of these tool and techniques. The authors thank them lor their good work. The pronoun " $i$ " is used throughout to separate all of us using management systems (we) from the authors (1). However, please recognize "F is more than one contributor.

The preparation of this paper is funded by U.S. Department of Energy Special Research Grant No. DE-FG02-88DP48058. Management Systems Laboratories thanks the Department of Energy's Office of Environmental Restoration and Waste Management for providing us a real-world laboratory for the research, development, and testing of state-of-the-art management tools and the frameworks for understanding how to make them successful.

\section{REFERENCES}

[1] Bailey, R.W., Supponing Human Performance: Human Performance Engineering (Englewood Cliffs, New Jersey: Prentice-Hall, Inc., 1989).

[2] Blanchard, B.S., and Fabrycky, W.J., Systems Engineering and Anatysis (Englewood Cliffs, New Jersey: Prentice-Hall, Inc., 1981).

[3] Buller, P.F. The Team Building Task Performance Relation: Some Conceptual and Methodological Refinements," Groups and Organizations Studies, vol.11. No.3, (September, 1986).

[4] Fox, W.M., Effective Group Problem Solving: How so broaden panicipation improve decision malaing and increase commilment to action (San Francisco: Jossey-Bass Inc., 1987).

(5) Hale, F J., Introduction 10 Control System Analysis and Desion (Englewood Cliffs, New Jersey: Prentice-Hall. Inc., 1973).

[6] Janis, I.L., Victims of Groupthink (Boston, Massachusetts: Houghton Mifflin, (972).

[7] Jay, A. "How to Run A Meeting," Harvard Business Review (March-April, 1976).

(8) Kaplan, R.E. "Some Hidden Elements Of Control In Group Facilitation - Appreciating the Bounded and Binding Aspects of Openness," Small Group Behavior. vol.16, No.4, (November, 1985).

[9] Kurstedh, H., Working Draft 1: The Industrial Engineer's Systematic Approach To Mangement (unpublished lecture notes), (Virginia Tech, Fall 1989).

[10] Myers, 1. Inuroduction to Type: 1 description of the theory and applications of the Myers-Briges Type Indicator, (Palo Alto, CA: Consulting Psychologists Press, 1987).

[11]Prince, M.G., "How to Be a Better Meeting Chairman", Harvand Business Review, (January-February, 1969).

[12] Thomas, W.K., and Kilmann, R.H. Thomas-Kilmann Conflict Mode Instrument, (Tuxedo, New York: XICOM, Inc., 1974). 


\title{
LESSONS LEAR.NED FROM APPLYING EXTERNAL INPLT TO DOE POLCY DECISIOY MAKNG
}

\author{
Rebecea M. Imbolz. Thomas B. Hindman, Jr., and D. Mark Brubaker
}

\author{
Management Systems Laboratories \\ Virginia Polyrectaic Instinute and Sute U'aiversity \\ 1900 Kratt Drath \\ Blacksburg Virginia 24060 \\ (703) 231.3501
}

\section{NTRODLCTION}

Our nation has entered an era in which the public is demanding clean up and restoration of its eavironmeah, understandable information. and participation in decision matiog. The U.S. Deparment of Energy's (DOE's) culure, which Gew out of the Atomic Esery Commiction AEC) culnure of classification. compartmearalization and strict-aeed-10-know dissemination of information, whe in direct conflict witb this demand for public irvolvereace The DOE recomized this and commitred to changing their culture inco one of openness and public involvereat in decision matang and policy direction.

As a result, DOE created a numbar of exteral review groups, one of bem being the Stace and Tribal Government Worting Group (STGWG). The STGWG wes cresed to revicw the first Eovironmental Restornton and Wate Managemeat Five. Year Plan The Five-Yeas Plan establishes an aread for complinecs and cleanup of DOE insenllations aprinst which progress can be menrured

The STGWG is now an oa-poing roup which continues to reviow drese of turns Fiv. Year Plans and othor ineses the DOE brin: before them or ieneses which the Stan and Tribed participents themelves bitas up for disomion.

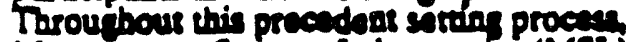

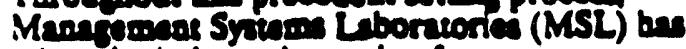
cakes the independans rols of observer/Gciliences. In this peper. I will dicanes the obsendes excountered bow they were solved and what MSL bas learecd about applyin exterad inpue to DOE poligy decision onting.

\section{CREATION OF STGWG}

In March of 1989. Admiral James D. Wathing the Secretary of Eaergy, coubrutied :0 the developmeat of a comprebensive Five-Year Plan for eavironmeatal restoracion and waste mnnagemoat activities at DOE auclear-related producton, restin and research and developoneat (RAD) insellations and for improved complinses mith applicable regulacions: Federl state and municipal. This resuled in the Ervironmental Restoration and Waste Mann soued Five. Yesp Plan. The DOE crested the STGWG and asked them to perticipate in the developmeat of the Five. Yess Ples to emphesize and suppors the DOE's new culture of opanones and to eive the DOE's agends for compliance and cleanup nauonal visibiling.

The DOE asked MSL to bost and he!p facilienes the first Stase and Tribal review of the Five-Yen Plan to mite dear the unbiased and indapondons asure of this review. The DOE. in conjunction with MSL decided that the objectsve for his group would not accesenty be comeneris Th DOE did oot wene the

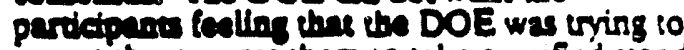
conos them or cer boa wo vits a unified stand. The DOE wand impus from every reprenatency wbothar all vicus were in apreamas or woe If the ead representalives ofree mos in cloned seasions and crome 10 the DOE with consenares gritigues abous the FiveYese Plin which tho DOE then addreseed. 
After these meeting DOE. follown 3

STGWG participanes were chosea based on a letrer Adniral Watkins received in April 1989. This letter, so.signed by ten governors of states with DOE facilities within or adjacent to their borders, outlined the governors' conceras regarding federal commiement to the cleanup of all the DOE installations. It outlined some possible courses of action and called for a comprehersive national plan for eavironmeatal iestoratuon - Lising this lenter as an indicanon of the most seriously affected states, DOE irvited the following states and proups to participate in STGWG: Colorado. Idabo, Kearucky, Nevade Vew Mexico, Ohio, South Caroline Tennesere, Washingror Yakima Lodian Nation, Sboshone. Bannock Tribes Nacional Govereors'

Association. Vational Confereace of Stace Legislanures, and the National Aleociation of Altorneys Geaeral. The Governor of Oregon aiso signed the April 1989 lenter to Admira Watkins; however, Oregon indicated informally that at that inse chey could be sepreseated by the State of Washington and thus the DOE did not invite them to this initis meeting Except for Nevada, all ioviced seos representatives io this initial meeting. However. Nevada bas atrended subsequen STGWG meeting

\section{STGWG EFFECTS ON DOE POUCY}

The ioitial meeting was beld on the Virginis Tecb campus (to explanias an unbiased atmosphere) in Blackboure. Vir inis and bosted by MSL During this meetine Secrenary Watbins was in clow rouch with DOE personel so be could provide real.time reapones to STGWG commeats. As a reaule no myjor changes in DOE policy were writran ineo the Five-Year Plan the tirst was a dimptrame for the cleanup of DOE instuliations Semorny Watbins a greed to a 30year god for the clanum of all DOE installation The DOE aleo apend to incorporate ine the Five-Yent Fina a cless ackoowledgement of States autbority to set regulatory requiremoats in the deamip proeses

At subsequast wanting in Chicaso and Deaver, the DOE arped to arpand this

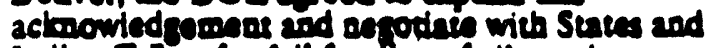
Lodian Tribu for full hunding of all repulatery activities directy relued to the DOE installadoen. TH DOE also apeed to seop pushing for antiond uniform repulatory standards TDe DOE appanded on the 30 yes clesnup goel by apeein to support lecishation through Confren earbbishing andional comminedet to a 30-yess timptrana.
STGWG's recommeadaroo of inving Siaces and Indian Nacions with DOE installations insicie their borders. ioviced the followng 10 paric:pa:e in turure STGWO meetiogs: Caluomia Floric1 Ullinois, Miseouri. New York Texas. and Confederated Tribes of the Umatula Indian Reservacion. All invited exeepe New Yopk have sent repressectatives.

In Jaouary 1990, following the August 1989 publicatios of the enst Five-Year Plas the DOE began work on the 1990 Five.Year Plan :Or Fiscal Years 1992 - 1996. Agaia STGWG was asked to meet and review the Erst predecisiona! drats of this pien This March 1990 meetiag was held in Kanas City. The major outcome was a call by STGWG for DOE commitment to legislation establishing an account which would provide loagterm stable funding to ensure clesaup. This zecounh boown as a Near. Term Respones Fund would be a separate set of tinds available for unnoticipared cleanup coses 10 mee: regulatory requiremeats. The STCWG abo streand the ooed for instintionaliziog the 30year cleanup goal.

Ln subesquent meedings beld in May of 1990 in Artingon. Virinin and later in June of 1990 in Se. Louis the STOWO met to review DOE's respoesen to their requeas made in previous meating as well as budgot aumbers is the 1990 Five-Yas Ptan As this ime, the State of Oregoa indicaled thre thoy did want 10 be irvolved with STOWO. Tour they were ionied to atrend bocts of these meetings but were odly able to arned the St Lovis meetine. One of the major discusaton points throughour the Se Lous meeting was the starus of insurutionalising tbe 30- Year Clesoup Goel, which DOE had agreed to support and the sentus of DOE's commitmeat to serting up a Near.Term Response Fund. DOE sud that due to minpower and time conserainas shey had beas unable to solicit suppon for this logislation through Conprese. Tous STCWO docided to form a subproup of STGWG paridipanes to moet later in the Summer of $19 \% 0$ to drat and suppors this lecislation DOE would aleo be urvised to parieipate and MSL would coordinate and faciliente this mootine. 
LESSONS LEARNED FROM INVOLVING EXTTRNAL PARTIES IN DOE POUICY DECISION MAKNG

Meeting Design

MSL has either bosted facilitated or observed all of the STGWG meecings. This expernence has guven me an oppormaity to think abour lessous learned from designing chese meetings for involving oulside affecred parties.

Planning a successtul meeting is similar to planning the desigo of a documeat. I suggest considering the following formula:

$$
\text { audiesce + purpose }=\text { desigor }
$$

With our audience, I could anticipate the following characteristics?

1. STGWG members would be fairty knowtedgeable about DOE eovisoomental restoration and waste managemeor activities and isnues.

2 STGWG participanes would aree on some iscues and disagree on otbers because of compeciog interesta.

3. Some disagreemenes berwees DOE and the Suate and Tribal participans over issues and their resolution were expecred.

4. Suate and Tribel participans would be skeptical about DOE's abilities and motives to change.

Before a meeting is purpoes should be clearty established to prover files expectations. in the case of STCWO, the purpees ins to review and coononat on the ingiea and programs defieed in the Five-Year Pras ${ }^{3}$

Meating desing must be structured around weticoen epperations and meeting purpoes For involving diverse bowledgesble. and stepded perricipans the following meeting charasteristos are sirgened ${ }^{3}$

- If poscible provide poviow and oneatsion materinis before the meeting dace. Advanced distribution of information allows for thoughoful review and commenc.
- Ensure a minimum of lengthy, iecricica: presentations for participanes. Leng:... technical presencations dectease naterest and increase suspicion and percestion il an unwillingoess to listea and rensm:0 the old culrure.

- Allow time for an executive session ior State and Tribal paricipants. This permits them to collect and discuss group and individual comments and thus minimize differences benweed indindusis and marimize discussion.

- Do aot overplan A successful meet:r.z plas will beod to the evoiving needs of ibe participants.

- Inciude time for participars to get :0 know one anotber. If commimenis are wer and working relationsbips establisted, truse will be confirmed by action

Meeting Faciliution

It's imporant to recogmize whether or no a meeting requires a stroug racilieator. For the STGWG meeting, a strong facilizator wasa't required becuse bey were bacically self-led. However, even a self-led meeting cas bedefit from an efiective facilitator. Effective facilitation en (1) betrer orgnaize and direct group discusion, apd (2) improve the collection of valid commenes. 3

DOE-Leseona Learned

The DOE also leareed some lessons about bow to effectivety work with exteraal parties to gain input inco DOE policy making

- Disaribute matarials the participanes will be discusaing before the meeting. It $\mathrm{DO}$ onky allows tim for boughoful review. but seduces suspicion that they ase being cllled to a meeting where the DOE is not prepared to releare mat sial for dicausion.

- Seod out the menting plan before the meeting Participants teel more in control of the monting if thoy bave as idea of whas is expected. 
- DOE paricipanis must have authority 10 make commitrnents on-the-spot or have quick access to a higher authonty that can make these commitments. Otheruise. State and Tribal paricipants feel little or no progress is made during the meeting.

- The DOE should make feasible commitments. If the DOE cannot 'make good' on their commitrnents, suspicion and mistrust will take place benween the

- During the meeting it is important for the DOE to discuss the stans of coonmitments made be previous meeting. Bringing participanes up to-date demonstrates the DOE's 'rood faith' in following through with commitments. DOE and the State and Tribal participants.

\section{CONCLLSION}

The relationstip berween the DOE and the State and Tribal members bas been beneficial to both. The DOE has been given an oppormanity to listen to State and Tribal conceras and respond to them. The State and Tribel members in num have voiced their support to the DOE as long as the DOE continues to actively pursue the 30-Year Cleasup Goal.

\section{ACKNOWLEDGEMENTS}

The ideas presented in this paper represent cumulative work of bundreds of people over twelve years at Virginia Tech's Managemeat Systems Laboracories. Professional managers from business and industry, and faculy, pracuate studeats and undergraduates from a wide variety of disciplines bave participated in research and development of these tools and technigues. The author thants them for their good wort? The procoun " $\Gamma$ is used throughout to separate all of us using managemeot systems (wo) trom the author (D). However, please recomins $T \mathrm{~T}$ is more than one contributor to this wort.

The preparation of this papar is tunded by U.S. Deparmens of Exery (DDE) Groms Na. DEFG0285DP48058 MSL thants DOE's Otwen of Environmental Rearontion and Wasto Manrgement for providing us a real-world labortory for the research, development and testing of state-of-che-ert manajement wools and the trameworts for understanding bow to matse them successful

\section{REFERENCES}

1. U.S. Deparment of Energy, "Enviroamental Restoraulon and Waste Management Five-Year Plan.' Fisca! Years 1991.1995, August 1989.

2. Govemor Booch Gardner, State of Washingron: Govemor Cecil D. Andrus. State of Idaho; Govemor Gartey E. Carruthers, State of New Mexico: Governor Ned Ray McWherter. State of Tennessee; Govermor Cartoll A. Campbell. Jr.. State of South Carolina: Governor Richard F. Celeste. State of Ohio; Goverpor Neil Goldshmidh Siate of Oregon: Governor Roy Romer. State of Colorado: Governor Bob Miller, State of Nevada; and Goveraor Wallace Willineor, Stave of Keanucky. Defense Wasee Cleanup: A Proposa for A Nationd Solution" pp. 1.2 April 1989.

3. J. A. Walker and L I. Middleman. Tnstinutions Factors Affecting DOE Waste Mrangemeas and Eovironmental Restoration Planning." Waste Managemear 90 Proceedinge, in press. 
MANAGING THE SHITT TO A

PRODUCTIVITY AND QUALITY CULTURE

Larry A. Mallak

Harold A. Kurstedt, Jr.

Kathrya A. Welch

Management Systems Laboratories

Department of Industrial and Systems Engineering

E. Scont Geller

Departmeat of Psychology

Pamela S. Kurstedt

College of Engineering

Virginia Polytechnic Institute

and State University

Blacksburg, Virginia 


\begin{abstract}
Managers can exploit the similarities of culture management and efforts to improve productivity and quality because they both rely on values and traditions. Both are continuous, value-driven processes requiring daily monitoring and evaluation. And both demand strong effective leadership and commitment from all employees.

We must shift from organizations that strive for productivity and quality in their final products and services to organizations that value productivity and quality as part of their culture and build these into their processes.

Organizations need to adopt the values and traditions surrounding good productivity and quality so productivity and quality become the norm--the core supporting daily decisions. A strong, supportive culture guides decision making at lower levels in the organization by clearly communicating the expectations. Work can then proceed with fewer interruptions, with workers becoming empowered, and with managers having more time to manage.
\end{abstract}

We offer several approsches and techniques for achieving a productivity and quality culture. They are: 1) continuously improve productivity and quality, 2) build productivity and quality into your culture, 3) collect culrure change evidence, 4) link your efforts to a comprehensive plan, and 5) build strong, effective leaderahip. Coasistent application of these approaches and techniques will belp managers forge new cultures that value productivity and quality and work for continuous improvement.

(KEY WORDS: corporate culture; culture change; continuous performance improvement) 


\section{THE SIMILARITY OF CULTURE MANAGEMENT AND EFFORTS TO IMPROVE PRODUCTIVTTY AND OUALITY}

We believe the shift to productivity and quality thinking is a culture management issue. Culture management, along with continuous performance improvement and leadership, rotates around the qualitative issues of values and traditions. Schein (1985, p. 317) draws parallels between culture change and leadership, saying "the unique and essential function of leadership is the manipulation of culture."

When faced with the god of improving performance or changing the organizational culture, today's managers tend to focus on one of two dominant approaches. They primarily prefer either top-down or bottom-up approaches. Put another way, some prefer the use of continuous performance improvement emphasizing the day-to-day interactions between supervisor and subordinate (a bottom-up approach). Other managers prefer vision definition and communication coupled with a comprehensive planning process (a top-down approach). A comprehensive plan covers all time frames: long-term planning (5-100 years), strategic planning (3-5 years), tactical planning (1-3 years), and action planning (0-1 year).

To achieve the desired culture and provide an intelligent mix of top-down and bottom-up approaches, managers must build continuous improvemeat into their organizations and integrate this process with the leader's vision. Organizational commitment to continuous performance improvement involves bottom-up mechanisms for both individual and group empowerment. Empowerment refers to the process of individuals or groups taking responsibility for their own actions and the success of their own achievements. Empowerment occurs when an individual or group receives power, control, authority, or self-efficacy and is thus positively reinforced and intrinsically-motivated for continuous improvement (Byham, 1989). Between the supervisor and subordinate, continuous performance improvement aims at immediate specific gnins on a step-by-step basis. In this way subordinates get early eratification for improvement because there is an increased probability of preseating them with a simple, understandable system.

Continuous performance improvement and culture change complement each other in chnnging values and traditions: 1) both are value-driven processes, rebring on internalization of values for success; 2) both are continuous proceaces requiring daily monitoring and evaluation; 3) while the productivity and qualiny miecion is communicated through a top-down approach, daily succeaces resulting from continuous performance improvement support the mincioa thrount a bottom-up approsch; 4) both culture management and continuous performance improvement require strong, effective leadership and commitment from all employees; and I) both can effectively guide leadership in manaping the shift to a productivity and quality culture. 


\section{APPROACHES AND TECHNIQUES FOR ACHIEVING A PRODUCTIVITY AND QUALITY CULTURE}

\section{Continuously Improve Productivity and Ouality}

The concepts of just-in-time (JIT), total quality management (TQM), and others are more than just programs desigened for specific aspects of operations and process control. These are ways of thinking about and approaching continuous performance improvement. These concepts have been adapted, in part, from the Japanese style of management. The difference is the Japanese cultural values mandate quality and productivity be built into the process, aegating the aeed for a particular program.

Managers forge a new culture by managing the methods of continuous performance improvement. The methods include: 1) setting expectations, 2) charting, 3) defining indicators, 4) collecting data, 5) converting data to information, 6) organizing and presenting information, 7 reviewing progress, 8) developing personal effectiveness, and 9) appraising people's performance (Kurstedt, 1989). To manage these methods managers need to define performance in measurable terms, obtain baseline data, measure day-to-day, and compare today with yesterday to realize continuous improvement.

Managers can use empowerment to change values and traditions by encouraging participation and commitment in the organization. All members of the organization must be invited to shape the new productivity and quality culture through involvement in continuous definition, intervention, and evaluation phases. The communication network should accommodate topdown as well as bottom-up linkages to supply feedback necessary for keeping actions generated by the change mechanisms aligned with the mission.

\section{Build Productivity and Ounlicy Into Your Culnure}

Culture, as a set of shared values, is requisite for productivity and quality improvement. Leaders desiring high-performing systems must understand and clearly communicate their organization's purpose and objectives and direct the organization toward its value system. "Productivity, particularly in white-collar work, can increase only after a major commitment is made to long-term quality improvement objectives that are shared throughout the organization. Total quality performance requires total organizational dedication and involvement, espocially from lop managemente [italies added] (Schein, 19:8, p. viii).

Leaders muat briild productivity and quality into their cultures. Reward syatems must support this by motivating employee behavior toward common coak. Employees must think productivity and quality throughout all aspects of their jobs. Ouality must be viewed as a processen not as an end product, and muat be measured constantly, not juat before (or even after) the product or service is delivered to the customer. 


\section{Collect Cullure Chnore Evidence}

Culture management processes will produce successive beneficial modifications in the organization's culture. We can assess bow well we've built productivity and quality into culture and measure the effects of these successive modifications by looking for productivity and quality culture evidence. I describe culture change evidence based on Sathe's (1985) work. In general, culture change is evidenced by observing: 1) old-culture dissonant behavior, 2) self-initiated behavior, and 3) intriasically-motivated employees.

Old-culture dissonant behavior refers to behavior that is contradictory, or dissonant, with the old culture. As the transformation to the aew culture begins, mesh of old and new culture values and traditions exists. Managers can compare them to what's expected and desired under the new culture. Managers can identify and log behaviors that contradict the old culture, supplying measurable evidence of effective culture change.

Employees with self-initiated behavior know how to act when faced with uncertain situations, even without the wisdom and counsel of experienced management. Throughout the Tylenol poisonings of the early eighties, Johnson \& Johnson employees knew what to do even though they could never have anticipated such a crisis. The culture at Johnson \& Johnson had taught its people the value of self-initiated behavior, which they put into practice during the Tylenol crisis.

Intrinsically-motivated people behave consistently with an organization's culture because they have internalized the culture's values and traditions. When people act in accordance with the organization's culture, but have not intermalized the attendant values and traditions, they tend to revert to previous values and traditions in the absence of visionary management and reinforcement of the new culture attributes.

Link Your Efforts to Comprehensive Plnn

Managers must learn bow to link their productivity and quality improvement efforts to a comprehensive plan to get both short- and long-term results. This calls for leadership that can intelligently mix top-down and bottom-up approsches to manage the shift to the new culture.

Planning must span all levels of the organization-from strategic to operational. Continuous performance improvement gives us the day-in, dayour setting of expectations. To ensure the desired culture change occurs, planning must integrate the sctivities of all facets of the organization. Compreheneive plans instirutionalize and document the culture change and provide ways to mearure the effects of successive modifications to the culture.

Managing profound organizational change means adopting sew values with management and employees collaborating to forge the sew culture. To be succeseful, managers attempting culture change need a compreheasive plan to: 1) organize, communicate, and provide a basis for mensuring culture change and 2) translate long-term phans into shorter-term action plans. 


\section{Build Strone Effective Leadership}

Leadership emphasizes the human aspects of culture management. Individually, culture management, comprehensive planning, and continuous performance improvement cannot produce the desired culture change. Blake and Mouton (1968, pp. 273-274) call culture a massive fabric adding that "any attempt to change one thread is wasteful and inefiective." Leadership catalyzes the sypergy among culture management, comprehensive planning and total quality management.

During times of agonizing doubts and uncertainties, nothing serves an organization better than leadership that 1) knows what it wants, 2) communicates those intentions, 3) positions itself favorably with external forces, and 4) empowers its work force. Leadership must create an appropriate and compelling vision of the desired culture; direct activities toward the organization's values and mission; give the mission meaning through communication; and position the organization so it can design and control relationships with major constituencies in a complex, ambiguous, and uncertain eavironment (Bennis and Nanus, 1985). The leader must ensure employees property perceive the vision and operationalize it with regard to their daily work activities so their bottom-up approaches can work. Leaders who can sustain appropriate behaviors and attitudes will achieve beneficial culture change in their organizations.

\section{CONCLUSIONS}

Most firms claim they value productivity and quality, and indeed they probably do. But unless there is both high intensity--meaning great amounts of approval/disapproval attached to expectations regarding productivity and quality--and consistent sharing of these values (i.e., consensus) within the organization, a strong productivity and quality culture will not exist (O'Reilly, 1989).

For managers to lead the shift to a quality and productivity culture, they must learn leadership stillh. Good leadership creates a vision of the desired culfure and worts to suatain positive change while encouraging continuous performance improvement. Through this intelligent mix of top-down and bottom-up approacher, these leaders can view culture management and efforts to improve productivity and quality as similar and congruent activities. Research belps us underatand, in a structured fashion, the relationships among culture concepts and theories and productivity/quality improvement insues. Our experience leads us to sugrest several approaches and techniques for manaping the shif to a productivity and quality cultures discussed earlier:

1. Continuously improve productivity and quality.

2. Build productivity and quality into your culture.

3. Collect culture change evideace.

4. Link your efforts to a comprebensive plas.

5. Build strons, effective leadership.

By consietently applying these approsches and techniques, managers can 
forge cultures in high-performing organizations that value productivity and quality ard work for continuous improvement.

\section{ACKNOWLEDGEMENTS}

The ideas presented in this paper represent the cumulative work of hundreds of people over twelve years at Virginia Tech's Management Systems Laboratories. Professional managers from business and industry, and faculty, graduate students, and undergraduates from a wide variety of engineering and other disciplines have participated in the research and development of these tools and techniques. The author thanks them for their good work. The pronoun " $I$ " is used throughout to separate all of us using management systems (we) from the author (I). However, please recognize " $I$ " is more than one contributor to this work.

The preparation of this paper is funded by U.S. Department of Energy (DOE) Grant No. DE-FG02-88DP48058. Management Systems Laboratories thanks the DOE's Office of Environmental Restoration and Waste Management for providing us a real-world laboratory for the research, development, and testing of state-of-the-art management tools and the frameworks for understanding how to make them successful.

\section{REFERENCES}

Beanis, Warren and Burt Nanus. (1985). Leaders: The Strategies for Taking Charge. New York: Harper and Row.

Bytam, William C. (1989). Zapp: The Lighoning of Empowerment. Pittsburgh: Development Dimensions International Press.

Blake, Robert R. and Jane S. Mouton. (1968). Corporate Excellence Through Grid Organizational Development. Houston: Gulf Publishing.

Kurstedt, Harold A. (1999). Catering to Crises--How to Escape, Proceedings of the Instinute of Industrial Engineers 1989 Integreted Systems Conference, November, pp. 191-198.

O'Reilly, Charles (1999). Corporations, Culture, and Commitment: Motivation and Social Control in Organizations. Califomic Management Review, 31:4, 9-25.

Sathe, Vijay (1935). How to Decipher and Change Corporate Culture. In R.H. Kilmane, MJ. Sartoa, R. Serpe, and Aseciates (Eds.), Gaining Contool of the Coporete Culture, San Francisco: Joceey-Base, pp. 230-261.

Schein, Edear H. (1935). Onganizational Culture and Leadership. San Francisco: Jocecy-Base, p. 317.

Schein, Lawrence. (1988). In L. Schein \& M. A. Berman (Eds.), Total Qualiny Performance: Hightights of a Confenence. New York: Conference Board. 


\section{AUTHOR BIOGRAPHIES}

LARRY A. MALLN is a Research Associate with MSL. He assists in the labs' research activities and bolds responsibilities for academic papers, presentations, and propoeal writing. He received his B.S. from the University of Illinois and his M.S. from Virginia Tech, both in industrial engineering. He is currently working on his Ph.D. in management systems engineering at Virginia Tech. He has a broad base of experience in both academia and industry, having held positions with the University of Wisconsin--Madison, the Otio State University, and SunHealth Enterprises Inc. He is a Mass Media Fellow of the American Aseociation for the Advancement of Science.

HAROLD A. KURSTEDT, Jr. is the Director and founder of MSL. He conducts research on the use of management tools, participative management procesces and techniques, propram and project management, and organizational culture. He has been with Virginia Tech since 1974 and is the Ha G. Prillaman Professor of Industrial and Systems Engineering. He received his B.S. from the Virginia Military lnstitute in civil engineering and his Ph.D. from the University of Illinois in auclear engineering. He bas served on the mechanical, auclear, and industrial enoineering faculties at the Virginia Military Institute, the Ohio State University, and Virginia Tech. He has been chairman of ar slear engineering at Ohio State and Virginia Tech. He has had extensive induatrial experience at Babeock and Wilcox Co., the Ballistics Research Laboratories, Industrial Nucleonics Corporation (now AccuRay), Managemeat Horizons Data Systems, and Systems Research and Applications Corporation.

KATHRYN A. WELCH is a Research Aseociate with MSL. Her responsibilities include writing proposale, ideatifying and implementing research designs for erants, identifying strategies for data collection and analysis, defining appropriate statistical procedures, designing instruments such as surveys, and anslyzing results. She bolds Ph.D. and M.S. degrees in industrial-organizational paycholoy from Virginia Tech. She also bolds a B.S. in poycholo y from Chrkeon University and a B.A. in English from Marymount College. Her tesching, research, and consulting background includes the areas of leadership, motivation, industrial training, and largescale systems.

E. SCOTT GELLER is Profeesor of Psycholowy in the Applied-Experimental Psychology Propram and in Director of the Center for Applied Behavior Syatems at Virjinin Tech. He conducts applied behavior andysis research focusiog on lifter control, eserey and water conservation, and safety belt promotiona. He bolds Ph.D. and MA. degrees in experimental poycholocy from Southern Itinois University, and a BA. in poyeholory from the College of Wooter. He is the current editor of the Joumal of Applied Behovior Arabois.

PAMELA S. KURSTEDT is Arcistant Dean of Virginin Tech's College of Encineerine. She bns academic and administrative responsibilities relating to the undergradunte student body. She bolds a B.S. in mechanical engineering and an M.S. in industrial engineering from Virginia Tech. Her research interests are service sector mansement and productivity. She consults with Citibank on their manserment procese. She is pursuing ber Ph.D. in induatrial engineering at Virginin Tech. 


\title{
LESSONS LEARNED FROM DOE SITE CULTURE CRANGE ACTIVITIES: IMPLICATIONS FOR WASTE MANAGEMENT ORGANIZATIONS
}

\author{
Harold A Kurstedt, Jr. \\ E. Morris Howard \\ Anne R. Doss \\ Larry A. Mallak \\ Management Systems Laboratories \\ Virginia Polytechnic Institute and State University \\ 1900 Kraft Drive, Blacksburg, VA 24060
}

\begin{abstract}
Management Systems Laboratories (MSL) has worked with the U.S. Department of Energy (DOE) and several of its contractors as they understand and assess the DOE culture change and change the contractor culture to serve DOE's needs. Primarily, these contractors have been those whose responsibilities include starting up and operating weapons materials facilities. The number and scope of these activities have escalated and expanded to contractors at DOE sites such as Westinghouse at the Savannah River Site (SRS) in Aiken, South Carolina, EG\&G at the Rocky Flats Plant (RFP) in Golden, Colorado, and Westinghouse at the Feed Materials Processing Center (FMPC) in Fernald, Ohio.

The point of this paper is not to compare or contrast the relative merit of one site over another. It is to show the lessons, good and bad, and use and communicate those lessons, especially those lessons transferable to other sites in similar situations. I've looked at what's transferable from all of our experiences. I want to take both the good and the bad lessons learned from any of the sites and make these lessons available for use in future work. No one site was perfect and no one site was terrible. So, for this reason, it's neither appropriate to identify sites by name nor to use generic labels like Site A and Site B, which would show how many situations originated in a particular site. I have taken care to protect the identities of the individual sites involved.

Instruments designed to assess culture have helped to begin identifying behaviors counter to the desired culture. Meetings, workshops, motivational speeches, and other means help communicate and illustrate culture change activities. Hoopla (e.g., dinners, awards ceremonies) and symbolism play important roles in getting employees aware and motivated to become involved in the culture change while generating enthusiasm and displaying symbols of the new culture.

Experience and leseons learned in these DOE and DOE contractor organizations should apply in other similar organizations, including those focusing on waste management at DOE sites. Waste management organizations face many issues, such as public scrutiny, heightened expectations, changing mission, resource shifts, and strategies to accomplish their mission (1). Combined with the Secretary of Energy's call for a new culture, based on DOE's being "a victim of long-term cultural misdirection" (2), the rapid fundamental change in environment beckons the need for culture management in all DOE organizations.
\end{abstract}

MSL has responded to managers and senior managers throughout many DOE organizations, including headquarters in Washington and field sites throughout the 
country. From these experiences, I can relate many lessons learned about culture change in these organizations.

Experience in DOE sites has demonstrated some success of several culture change activities. Organizations dealing with hazardous materials such as waste management organizations can add to their efforts in culture change by considering the lessons learned. Based on these experiences, waste management organizations and their associated contractors can put the lessons learned to work for their organizations to design and implement their culture change programs.

\section{INVOLVEMENT WTH DOE CULTURE CBANGE}

MSL's involvement with the DOE and culture change over the past year and a half has covered many of the preliminary steps an organization should take when contemplating culture change. A series of papers captures some of what MSL has learned to date about organizational culture change in practice $(3,4,5)$. I began by briefing top managers on culture--what it is and what its elements are. I progressed to more detailed briefings emphasizing empirical issues such as observation, identification, and measurement of key cultural elements. I assisted in the managers' need for hoopla and symbolism (e.g., buttons, plaques, posters, and audiovisual materials) in generating enthusiasm for the new culture. At one site, I designed a survey to measure attitudes regarding the new culture.

MSL's recent intensified involvement with DOE and culture change began in late 1989 with the start of a series of organizational culture presentations. The first wave of these presentations covered the basics: defining organizational culture, examining why managers need to understand culture, and explaining various concepts associated with organizational culture. The second wave of these presentations, starting in March, 1990, went into more depth. In the second wave, I presented definitions and concepts important to an understanding of organizational culture, and began developing an approach to culture change and the need for managers to not only understand culture, but to be able to change culture as well. I gave them examples of other organizations' culture change and then I shared with them what their culture was changing to and what was causing that culture to change. I worked with the sites to decide: 1) whether or not the culture will change, 2) what the new culture was going to be, and 3) what sorts of things needed to happen to make that culture change. Those ideas were consistently communicated to very large groups of people, 160 top managers, who in turn communicated these ideas to their suborganizations. I developed a "culture change map," a detailed flow chart outlining an initial view of the elements involved in identifying what to change in the culture, how to change those elements, and how to measure culture change. This culture change map was prepared in response to one DOE manager's comment concerning the lack of operationalization of organizational culture change. I consider the map to be rudimentary, a first cut at a seemingly boundless domain but one that needs structure so managers can take action to manage culture to their benefit.

My presentations were concentrated at three DOE field sites. At one site, I made presentations starting with top executives, working my way into several groups of 100-150 managers at a time. This broad coverage communicated the same message to large groups of people within a short three-month time frame. These senior managers then carried the message to their people to further communicate the presentation content to the thousands of professionals and hourly workers at the site. At another site, I made 
presentations to executives and a few select managers. At the other site, I made presentations to their advisory group.

In addition to presentations, I designed a survey instrument to capture general and specific opinions and attitudes regarding the change in O\&M contractors. I administered the survey, and collected and analyzed the results. This application supplied valuable data for site managers to evaluate the impact new management had on the site based on a variety of measures. I found people were in tune with culture change. I found people felt the direction of the change in culture was appropriate. But, I also found they didn't believe management backed it.

I've dealt with some of the symbolism. Hoopla played an important part in communicating the culture. I designed buttons espousing the new values and the new organization and distributed them to site managers. I've also put together statements of shared values for managers to display on their desks. In addition, one of the site executives went further in promulgating the new culture. He had posters put up espousing elements of the new culture. He had other symbolic articles made with the plant's slogan about its new culture affixed.

Organizational culture consists of values and traditions--the things we hold dear. These aren't changed as easily as other aspects of our organizations (e.g., introducing new manufacturing processes, new technologies). Changing organizational culture is an emotional issue, and we must act on it emotionally. Just as membership in a family, church, or university instills particular values and traditions in us, so does our membership in an organization. Members who have held the culture close to their hearts for many years will resist efforts to change. Culture change efforts, to be successful, must take the human concerns of their members into account.

At DOE sites, the culture change has not been completed. I can't say what will work and what won't work with any degree of finality. I can identify things that seem to be working and that are making progress and people are happy about and I can identify things that seem not to be working and that have been discarded for that reason.

One site, in particular the production staff, was aversive to the use of procedures, preferring to depend on the historical means of performing their jobs. They know now they have to use procedures. They don't like everybody saying "You gotta do this safely no matter what happens." I'm seeing acceptance of the use of procedures and increased awareness of the need for safety in operations; however, they are not convinced that senior management really believes in the new procedures and safety in operations over production. At this site, the lack of confidence in management commitment to procedures and safety lead to a great reluctance of the staff to report or identify nonconformities. The behavior is changing; the attitude is not. What I'm finding at this site is behaviors such as adherence, writing of procedures, and practicing safety are changing. The attitudes, as ascessed through attitude surveys, are not keeping up with the behaviors. The attitudes may not have changed at all. Changes in behavior are believed to lead, over time, to changes in attitudes (6). Individuals engaging in behaviors that are incongruent with their attitudes are to be expected-they're experiencing a condition termed by psychologists as cognitive dissonance. 


\section{GENERAL CEARACTERISTICS AND INFLUENCES}

I've seen many general characteristics and influences of culture change that hold implications for all organizations, in particular those organizations involved in potentially hazardous activities such as waste management. These general characteristics and influences help us understand more about organizational culture change in many different types of organizations and set the stage for the discussion of the specific lessons Il learned while working with several DOE sites and DOE headquarters.

In many cases, it wasn't the organization that changed. Rather, the world changed and the organization stayed the same. So the need for a change in culture occurred because of the need for the organization to interact better with its environment. In the example of DOE, the increased levels of scrutiny and requests for information led to the development of the Five-Year Plan for environmental remediation and waste management. Similarly, changes in the world are affecting other organizations as well. Organizations today face a more well-informed and information-demanding consumer. The implications of this shift in consumer behavior have caused food packaging to contain information on cholesterol, sodium, and fat content, chemical firms to juriciously monitor the quality of effluents, and politicians to be more accountable to their constituents.

Consequently, in many organizations today, there is an awareness but not the recognition that the culture must change. Managers and executives must listen carefully to what their people are saying. Listening to members of their organizations will give cues to what will work and what won't and how managers and executives can begin culture change efforts in their organizations. People and their organizations know there is a move afoot to a certain culture, but they haven't begun taking the necessary steps toward that culture.

In the larger world, changes in personnel are becoming more commonplace in the current wave of merger and acquisition activity. Also, with the popularity of contract management for large facilities, as is done for the DOE at its field sites, the periodic changeover in contractors brings with it new personnel, usually at the upper levels of management.

The rank and file view contractor changeovers and the subsequent "new" ideas about change with skepticism and are reluctant to change. These workers have been at the site all along and they consider everything to be fine. Their implied comment is, "Why change now? This must be some management notion we can ignore. All things pass and so will this." Culture change efforts should counter this notion, showing how the culture will change regardless of who the contractor is. This is discussed in more detail under "Lessons Learned."

These contractor changeovers and other causes of personnel changes produce a mix of different people representing different cultures. The tendency in these cases is to tear down the old culture and build a new culture. What should ideally be done, as I'll discuss under "Lessons Learned," is to forge a new culture from the different cultures people bring to the organization. This reduces the feelings of wholesale dissolution of previous cultures and molds the organization in a specific, desired direction.

Many people in organizations are experiencing the pressure from outside forces. These outside forces are driving them toward a defensive stance. The successful will control . their own destiny. These organizations are faced with choice of molding to fit or forcefitting. These organizations got involved with culture change simultaneously with the 
increased pressure from outs,de. They dealt with the forcing functions to change culture in the midst of other pressing issues. Their culture change efforts improved their ability to stabilize the pressured situation. Pressure was largely a matter of perception, but without a systematic and comprehensive approach many organizations lost the ability to mold their own cultures.

\section{LESSONS LEARNED}

I observed and noted example successes and failures when undertaking culture change. Some culture change activities worked successfully at one site, yet were ineffective at other sites. Most of my lessons relate to actual experiences at DOE sites. Knowing what works and why it works gives other organizations attempting culture change a body of experience to draw on.

\section{Get yourself a champion.}

Perhaps the most dramatic lesson I learned in my culture change efforts with DOE sites was the need for a champion. Champions lead the culture change and see the process through to the end. Champions are known throughout the organization, have authority, and are respected. Success in the organization marks the champion, and he or she displays belief in the new culture through emotional leadership--cheerleading. Above all, champions enjoy what they do. My experience points clearly to this lesson: organizations can't change culture without a champion.

The champion must be positioned at a high level in the organization, have authority, and be respected by the employees. In one case, the champion was a senior line executive. I emphasize line and not staff. It wasn't an advisory group, it wasn't a staff position, it was a key line manager. In another case, I had an interested senior manager without support from his counterparts and his senior management.

The organization at one site supported the concept of culture and met my definition of champion. Another site had a lone wolf who wasn't able to muster support for culture change activities. None of the other managers participated. The manager at this second site had very limited participation from a very limited number of people. What this site had was an honest attempt by one man to do what the total organization should do. He just couldn't build the organization.

The champion's characteristic of being a "cheerleader" is important but not totally essential to his or her success. Champions who are strong managers anchor their success on their strength of belief in the culture change and in the new culture. The deeper the abiding belief and the more relentless the work ethic, the more successful the champion. Managers under these champions "take," that is they pick up on the enthusiasm and the need for change and broaden the involvement and scope of the effort throughout the organization. The difference between a champion who "takes" and one who doesn't is like the difference between an orchestra and a one-man band. These managers should build a supportive management style--founded on openness and trust. These managers, and hopefully champions, should be insiders with a stake in the change being initiated.

Culture change is working where I can identify a champion; it's not working where there's more of a token champion. By token champion, I mean someone lacking management support or authority to speak for and act on behalf of the organization. The site with the token champion tried to take the cream off the top in their culture 
change efforts. They tried to do the easy things and they never did the long-term things. Because of a lack of management dedication to the culture change, what they wanted to do was make a show of culture change. They did the short-term things that gave instant gratification and instant visibility and they didn't do the long-term things. The lesson here is undertake both short- and long-term efforts. Lay the foundation for real culture change, get instant gratification so people feel good about the culture changing while they're waiting for the more long-term things to take hold.

\section{Beware of tokenism.}

At one site, the only evidence of culture change was one sign put up along the highway leading to the plant. The implied message was, "Let's do something." But this was tokenism, merely checking the squares. At another site, every office, all the signs, buttons, and tags said something about culture change. The implied message was, "These guys are serious, they're not going to abandon the culture change effort." In the beginning some people thought these efforts humorous. In the end these people took the change seriously.

At one site, all the senior managers were genuinely involved in the culture change and were interested in the concepts necessary for culture change. At another site, there was obvious tokenism. All but one of the senior managers sent their administrative assistants or chiefs of staff to culture briefings and meetings. They didn't even send their second line manager.

\section{Manage symbolism.}

As I hinted in the tokenism lesson, symbolism is very important for communicating and getting buy-in to culture change. Having the culture message promulgated everywhere sends clear signals that management is behind this change, and it has value for message content in espousing the organization's values in clear, simple terms.

One site had vision and mission statements that were posted on just about every wall. They had buttons, notepads, newsletters, suggestion boxes, and various types of symbolism. At another site, they had one sign on the roadway.

I went to one of the sites and asked to see their mission statement. They pulled out a four-page document that apparently did not reach many of the staff and where it did, it was received as just another management "procedure." In contrast, a different site's mission statement was brief and therefore could be framed with large print and put everywhere. They promoted it so much, people questioned how much they promoted it. No one at the first site ever questioned how much their mission statement was promoted, because they never knew it was promoted, and they were right.

One site integrated quality into its culture and even held a banquet to hand out rewards to employees who have conformed to the quality expectations of the new culture. Another site had nothing of the sort.

\section{Align actions to the new culture.}

I learned that all the rituals, the values, and the heroes-everything--should be aimed in the same direction. One site's actions, for the most part, were aimed in the same direction. At another site, management held one set of values yet had rituals espousing a different set of values. Not only do the people become confused, the whole process of culture change becomes confused and ineffective as well when it lacks alignment. So, all 
management actions must be aligned with the new culture. A manager can take twentyfive actions aiming at the new culture and one action aiming at the old culture, and the single misaligned action will do more damage than the twenty-five actions do good because of people's inertia in changing anything, especially values and traditions.

At one site, the people sincerely wanted to change, but management did not support or empower them. They didn't have the power to change from the bottom-up like Procter \& Gamble. At P\&G, the people at the bottom wanted to change the culture, and they were formally empowered. In this case, the people wanted to change, management said go ahead but didn't empower them and, in some instances, chastised them when they identified legitimate concerns. Management deeds didn't align with the words.

Management mouthed the new culture, but their deeds were against the new culture. The words caused behavior change, but the words alone could not cause attitude change.

I came to three major findings at this site: 1) behaviors are changing--I observed and measured the change, 2) attitudes are not changing to keep pace and I observed and measured this lack of change, and 3) desired behaviors are not being reinforced on a regular basis, and this portends danger in undoing the behavior change. Behavior change is fragile--that is, it may not last long. I learned behavior can change quickly, but attitude change takes time.

This site changed behaviors by writing procedures, training people on the importance of using the procedures (this was one of this site's successes), holding safety meetings and talking up safety. Employees were disciplined for noncompliance with safety procedures.

Forge the new culture.

One site was busy trying to undo the old culture and busy pointing fingers. Another site busily forged a new culture. The first site did not have a supportive culture. A comment frequently heard was "I've done it this way for 10 years and I'm going to continue to do it this way." The second site had a well-defined culture that was harder to change. The first site could have had an easy time of culture change if they wanted to--they had a more informal culture, while the other site had a more formal culture.

Both had a large number of subgroups (around five) that brought different cultures to the organization. These five or so groups played new, additional, and different roles. Each group brought its own culture on top of the existing culture. The first site talked about the old culture, pointing out its weaknesses and pointing fingers to blame. The other site came up with a brand new culture different from the five and different from the old causing the five to pull together toward the new culture. The second site didn't consume its time with blaming the old culture.

DOE overall is trying to shift from a culture emphasizing production at any cost to one more centered around safety: Most DOE facilities "were constructed when still-evolving applicable nuclear safety and environmental standards were significantly different from what they are today and when DOE (and Congress) emphasized production over environmental or safety observance" (7).

My experience strongly suggests the forging of a brand new culture containing pieces from all the diverse groups. This forging produces a mixed and not a compromised culture. The resulting culture is new and does not completely replicate the old culture or any of the diverse groups, though several elements of the old culture may be modified slightly to reflect the new culture. Success requires forging a unified culture 
from several cultures. This, I believe, is the most valuable lesson I've learned to date in culture change involving new groups of people from several cultures.

Satisfy stakeholders along the way.

DOE and its field sites are currently experiencing an increased wave of scrutiny. Their well-known professional ability and integrity must buttress this new scrutiny. The abilities and integrity of technical staff at DOE field sites are not being questioned; the stakeholders are exercising their rights--in all technical fields. Nuclear technical people have historically been a closed society, but continued confidence and credibility absolutely depend on integrity and openness.

In a new culture, candor and openness come first. The new culture demands faith from its stakeholders. One site experienced success when the stakeholders believed and said managers and technical people at that site knew what they were doing. However, and this is a key point: this site didn't communicate the culture change to nor did they get any participation by the stakeholders. Had they gotten the participation from and informed all the stakeholders, they feasibly would have reduced the number and scope of problems they faced.

A second site with fewer stakeholders had an easier job. This site wasn't perceived as being the problem at the same magnitude as first site was. Managers and technical people at this site were probably competent and acted in accordance with government and industry standards and expectations. From now on, though, competency and compliance will have to be proven, for all sites are under the magnifying glass.

\section{Document everything.}

One site documented everything they did. This strengthened their position with DOE and stakeholders in general. Their management was able to show the direct implications of taking specific actions. Their management style was tight, organized, and accountable.

Another site was never able to come to grips with the issues underlying problems which reduced their effectiveness in communicating with the stakeholders. This inability to effectively and accurately communicate with the stakeholders adversely affected perceptions of management competency and reliability.

Managers at these sites should exercise discipline and thoroughness in management of operations. They must demand explanation and rationale to justify strategy, decisions, and actions. They should expect clear documentation that standards were met. And they should judge their site's performance against commercial values, practices, and standards. Meticulous documentation should improve the ability to deal coolly with the facts by making those facts more readily available.

\section{Define the new culture.}

I can estimate the direction of DOE's new culture. DOE will centralize from the field into headquarters and create a very prescriptive workplace. DOE will increasingly try to hold contractors financially responsible for their work and work-related infractions. The new culture will demand risk-free operation and must be ready to concede to environmental issues raised by stakeholders. New loyalists will replace resigning or retiring employees. 
The old DOE culture is a dichotomy of technical people and managers. In the old culture, roles were ill-defined and qualifications were undocumented and unmonitored. Managers managed and left technical details to technical people. Ability was judged by degrees and experience rather than by performance. The new DOE culture will stress technical inquisitiveness, that's not a function of production. In the new culture, people will learn how to ask the right questions and won't take the first answer for granted.

In the days of the Atomic Energy Commission, the world believed and had faith in technical giants. The new scrutiny strikes at this belief, which is the heart of current happenings in waste management and in everything else. The professional ability and integrity of $D O E$ is being questioned from both inside and outside.

\section{Increase the half-life of change.}

Without the long-term to support the short-term, there's a very short half-life for culture change or any other type of organizational change. Feelings and excitement about the short-term changes erode without the longer-term foundation. The lesson I learned is: taking long-term actions simultaneously with short-term actions gives those short-term actions a longer half-life.

At one site, management gave culture change lip service. Management's deeds didn't match what they said. Meetings related to the new culture were sparsely attended. Another site used many forms of symbolism: mission statements, management input campaigns, meetings, motivational speakers, and much more.

\section{Importance of culture is a relative issue.}

There is an awareness of the importance of culture change, but a shortage of accompanying actions. In other words, when the subject of culture is raised, people's ears perk up, but some of them say, "Yes and we've got to change it" or some of them say "Yes, I've heard about that and I am not going to change it." They are all aware that something is happening to their culture.

The importance of culture and culture change is perceived relative to other changes facing the organization. Organizations should think through organizational culture issues before these issues become hot topics of public interest and debate. Organizations must become proactive with regard to culture. One site has been through the roughest part of its culture change and is now able to put culture change in perspective with its balance of organizational issues. There was a time when they were "in the hot seat and everybody was staring right down their throat," to quote an unidentified source. Before that turn came at this site, before they were seen as moving off the hot seat, they were involved with culture change.

Successful organizations focused on culture change before the pressure relaxed. And, given my limited sample, that could imply that it was the culture change efforts that affected the pressure turn-around, rather than vice versa. In other words, it was not a situation where, "Gee, the pressure is off, now we have time to change the culture." It was, "The pressure is on, but we are going to pay attention to culture," and that paying attention to culture helped relieve the pressure. Pressure, like culture, is largely a matter of perception. 


\section{Make lemonade out of lemons.}

It used to be "Just say no" was the catch phrase. That's okay for drugs, but bad for culture. We need to follow the Nike call to "Just do it." As I'll talk about later, the culture change is inevitable: either we can take steps now to change it, or get all embroiled (and perhaps roasted) by those who are critical of our late response. Successful organizations take a positive attitude and look for ways to make lemonade out of lemons--that is, take a bad situation, find the positive angle, and turn the bad situation into a good one.

One site took a lead on culture change. Their management was assertive with the goal of dominating their environment and not being controlled by outside forces. Another site fell prey to a defeatist attitude, believing culture is uncontrollable and that "it's going to get us."

\section{Culture will change when threatened.}

Culture change can be a threatening prospect, but very often organizations won't feel the need to address culture change until they're faced with a life-threatening condition. It is in life-threatening conditions that the relative importance of organizational culture change suddenly increases dramatically. The threat of the organization's demise (or individual's demise) makes many ideas palatable that weren't so before the threat. These organizations are having to deal with the forcing function from their environment to change the culture in the midst of other very significant issues in their lives.

At one site, the leader told management and workers that the culture is going to change regardless of who remains in the organization or who holds the O\&M contract for the site. He urged them to face up to the inevitable change. If I could have had the management say what they felt about culture change, it might resemble this: "Hey, this is bigger than all of us. This is going to happen. If we go away, this is still going to happen. If we help it, it's going to happen faster. If we thy to beat it down, it's still going to happen. It may not happen quite as fast but it's going to happen." Another site held the notion that they would "bring in the culture," that they knew the answers, "we've done this before."

\section{Differentiate between urgent and important.}

The manager's unrelenting focus from one urgent activity to another means the important activities have not been addressed early enough. I must differentiate between urgent-activities which have reached crisis level and now demand our immediate attention-and important-activities having to do with results and contributing to the organization's mission, values, or high priority goals (8). Important activities, once neglected, progress into urgent activities, thereby disrupting the manager's coverage of important activities. The technical/production/operational/environmental issues were considered at least more urgent, if not more important than the social/culturalhuman issues.

\section{Bypass the conventional wisdom.}

These sites undergoing culture change could not abide by the conventional wisdom that culture change takes forever and costs a fortune. They don't have forever or a fortune. They had to be creative enough to change their cultures with a reasonable budget and within a foreseeable timeframe. We have to be assertive and manage culture change, and not just let it happen or overwhelm us. 


\section{Emphasize the practical.}

Many experts on organizational culture emphasize the anthropological issues such as the rites and rituals of corporate life, cultural artifacts, symbols, and precedents. But managers have a bias for action, and put emphasis on the practical. Correspondingly, I learned to emphasize the practical by including anthropological issues only as they relate to changing the workplace culture.

I could have addressed what's wrong with the old culture and what's wonderful about the new culture, but I essentially had to work through three steps: 1) I had to identify what elements of the old culture must change, 2) I had to address how to change those elements, and 3) I had to begin devising ways to measure to see if and how those things changed. By going through this three-step process, I learned I could better meet the practical expectations managers had regarding culture change.

\section{Emphasize the positive.}

There's always a positive way and always a negative way to say something or to approach a situation. I observed the effects of framing statements about the old or new culture or process of change in both positive and negative terms. One of the sites took a primarily positive approach to culture change, while another site took a mostly negative attitude toward culture change. Statements framed in negative terms typically worked against managers, while those framed positively worked for the managers.

Embed the old culture in the new.

Another piece of conventional wisdom claims that it is harder to change a strong culture than a weak culture. In a strong culture, the people understand ritual, they understand symbolism, they understand the elements of the culture, so the culture may change, but the rituals and symbolism are still present. In the site generally considered to have a strong culture, complete with a variety of well-respected rituals, the change in culture didn't require the building of rituals from ground zero. Instead of taking the rituals and demolishing them, management at this site took the rituals and adjusted them slightly. These were the same rituals, different culture. But these rituals had slightly different values. This site was successful when they found a way to embed the values of the old culture inside the values of the new culture.

\section{CONCLUSION}

I've concentrated the lessons learned by discussing my experience with two DOE sites, yet these lessons represent the accumulation of years of working with DOE headquarters and many of its field sites over the past ten years. The following is clear: the DOE culture is changing and will continue to change until it is aligned both with its stakeholders and with the vision set forth by the Secretary. Resistance to this change will only be met by the inevitable: change or be changed.

Managers who are too busy to manage culture and culture change should simply not get involved in the first place. As Figure 1. shows, once we start the culture change process, we can't stop just because it becomes unpleasant. Many of these managers practiced benign neglect and stonewalling wherever necessary. Their common line was, "As soon as we do $X$, we'll start our culture change activities." Well, $X$ never happened either. 


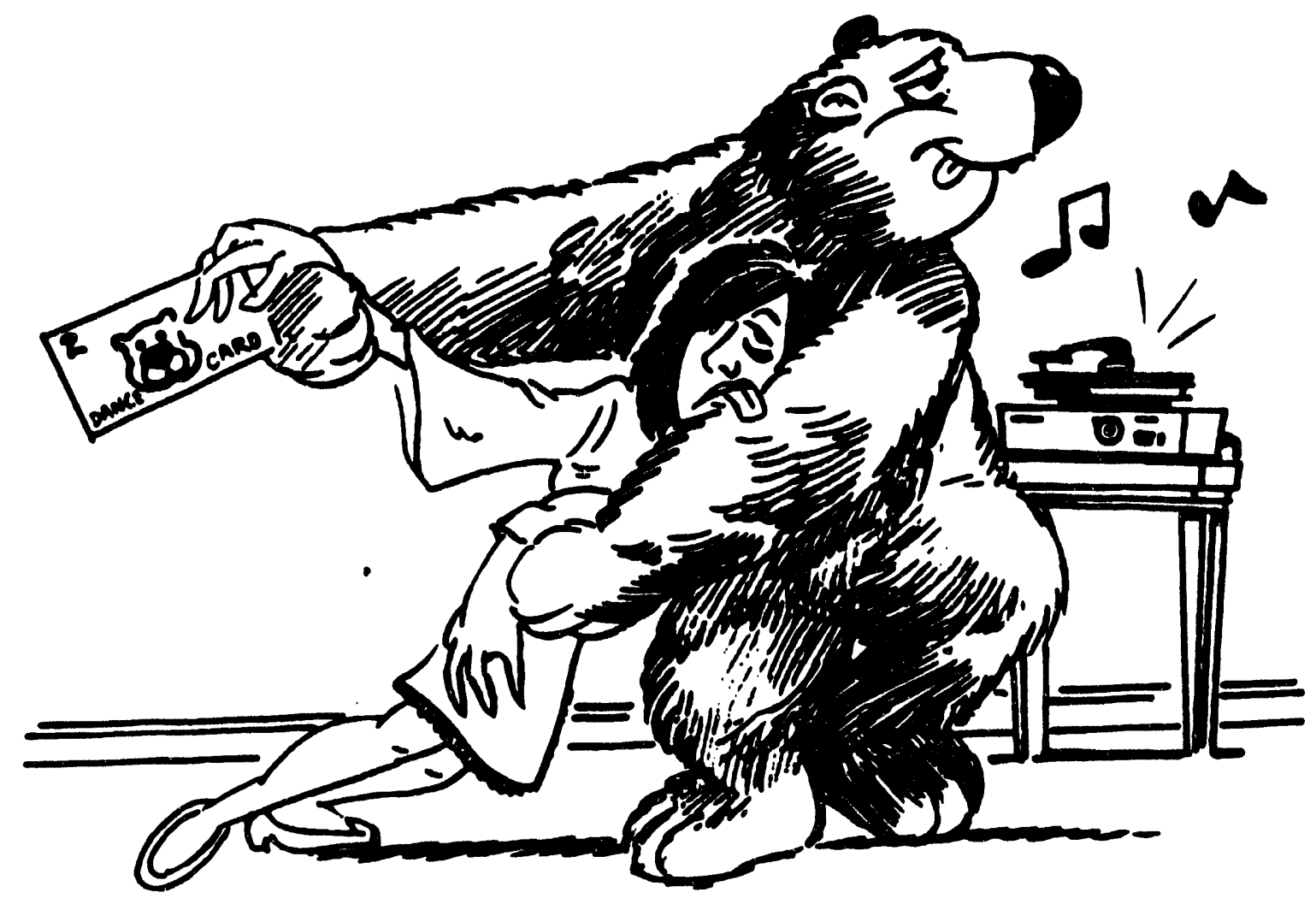

Figure 1. If you ask a bear to dance, you can't quit just because you get tired.

The lessons I've learned bear implications for waste management organizations both within and outside DOE, and can even be generalized to many other types of organizations. My list of lessons is only the beginning, but I believe it to be a valuable start to culture change. By learning from these lessons, we'll be better prepared to tackle the myths and perceptions that work in our organizations against the processes of culture change and enable the building of cultures and organizations aligned simultaneously with the values of those both inside and outside the organization.

In summary, here are the lessons:

Get yourself a champion.

Beware of tokenism.

Manage symbolism.

Align actions to the new culture.

Forge the new culture.

Satisfy stakeholders along the way.

Document everything.

Define the new culture.

Increase the half-life of change.

Importance of culture is a relative issue.

Make lemonade out of lemons. 
Culture will change when threatened.

Differentiate between urgent and important.

Bypass the conventional wisdom.

Emphasize the practical.

Emphasize the positive.

Embed the old culture in the new.

\section{ACKNOWLEDGEMENTS}

The ideas presented in this paper represent the cumulative work of hundreds of people over thirteen years at Virginia Tech's Management Systems Laboratories. Professional managers from business and industry, and faculty, graduate students, and undergraduates from a wide variety of engineering and other disciplines have participated in the research and development of these tools and techniques. The author thanks them for their good work. The pronoun "I" is used throughout to separate all of us using management systems (we) from the author (I). However, please recognize "I" is more than one contributor to this work.

The preparation of this paper is funded by U.S. Department of Energy (DOE) Grant No. DE-FG02-88DP48058. Management Systems Laboratories thanks the DOE's Office of Environmental Restoration and Waste Management for providing us a real-world laboratory for the research, development, and testing of state-of-the-art management tools and the frameworks for understanding how to make them successful.

\section{RETERENCES}

1. Kurstedt, Harold A. (1990). "Programmatic Issues Affecting the Implementation of the DOE Five-Year Plan," Proceedings of Waste Management 90 Symposium, 69-76.

2. Brown, II, Omer F. (1990). "Energy Department Contractors and the Environment: A More "Special Relationship," Federal Bar News \& Joumal 37, February, 86-89.

3. Kurstedt, Harold A, Cavell, Marilyn S., Mallak, Larry A, \& Howard, E. Morris. (1990). "We View Culture Change as a Form of Transition Management," Proceedings of the 11th Conference of the American Society for Engineering Management 52-56.

4. Kurstedt, Harold A, Mallak, Larry A, Howard, E. Morris, \& Kurstedt, Pamela S. (1990). "Why Engineers Must Know and Manage Organizational Culture," Proceedings of the IEEE Intermational Engineering Management Conference (Santa Clara, CA), 159-163.

5. Mallak, Larry A, Kurstedt, Harold A, Welch, Kathryn A, Geller, E Scott, \& Kurstedt, Pamela S. (in press). "Managing the Shift to a Productivity and Quality Culture," Proceedings of the Thind Intemational Conference on Productivity and Quality Management (Miami).

6. Geller, E. Scott (1987). "Applied Behavior Analysis and Environmental Psychology: From Strange Bedfellows to Productive Marriage," in D. Stokols \& I. Atman (Eds.), Handbook of Environmental PJychology, Vol 1, pp. 361-388. New York: John Wiley \& Sons. 
7. Brown, II, Omer F. (1990). "Energy Department Contractors and the Environment: A More "Special Relationship," Federal Bar News \& Journah, 37, February, 86-89.

8. Covey, Stephen R. (1989). The Seven Habits of Highly Effective People, New York: Simon \& Schuster. 


\title{
Structured Meeting Techniques Identify Emergency Management Issues Practitioners Really Want to See Addressed
}

\author{
by \\ George E. Ruberg \\ John F. Keeling III \\ Management Systems Laboratories \\ Virginia Polytechnic Institute and State University
}

\section{Introduction}

Emergency Management (EM) researchers need a proactive and systematic approach for obtaining research topics practitioners really want to see addressed. Because of the inter-disciplinary nature of EM, traditional research hasn't provided practitioners with the information and management tools they can readily use. Furthermore, because historically EM practitioners haven't been academically oriented, they haven't been inclined to make use of the research provided. We feel this is changing as evidenced by such activities as an increased emphasis on professional standards and training: One example is the National Coordinating Council on Emergency Management's project to develop professional standards for emergency managers. Our method adds another link to the connection between research and practice to better meet the needs of emergency managers.

The primary purpose of this paper is to introduce three structured meeting techniques that can be used to determine EM issues practitioners really want to see addressed. The secondary purpose is to list the benefits of using these techniques and provide recommendations for research based on three applications of the techniques.

\section{Approach}

Three group techniques were used for generating initiatives for EM research: a modified nominal group technique (NGT), role playing combined with a modified NGT, and a table top exercise combined with role playing. In the sub-section for each technique I:

1) Describe the standard or traditional methodology and present empirical background information for the technique used (Background). 
2) Present a brief overview of the meeting where the technique was applied and explain why the technique was appropriate for the application. I briefly describe the circumstances under which the technique was used, noting any modifications made to the original technique and explaining why these changes were more appropriate for the EM application (EM Application).

3) Give a brief list and description of selected outputs from the technique we feel are applicable to research (Outputs).

\section{AModification of NGT}

\section{Background}

Nominal Group Technique (NGT) is one of the most widely used group techniques. NGT has four primary steps [1]. First, each group member silently generates ideas in writing. Second, group members voice their ideas in a round-robin fashion. The facilitator of the group records each idea in a terse phrase, usually on a flip chart. Third, a discussion is held for the clarification and evaluation of each recorded idea. Finally, group members individually rank and vote on priority ideas. The group decision is mathematically derived through rank-ordering or rating of the individual votes.

NGT is an effective technique for generating multiple alternatives [1] [3] [4]. Other reasons why NGT has been effective include its high focus on the task, its equality of group membership, and its structured steps usually leading to a high sense of closure [7].

\section{EM Application}

EM practitioners representing many different types of national organizations (industry trade groups, public interest groups, and Federal, State, and local governments) and many different fields related to emergency preparedness (fire fighting, law enforcement, medical services, etc.) attended a workshop in September 1990 to develop a list of initiatives for the newly formed National Task Force on Hazardous Materials Emergency Preparedness. The purpose of the National Task Force is to:

1. Enhance the Nation's capabilities to protect life, health, property, and the environment during hazardous materials emergencies, and

2. Improve management of the response to hazardous materials emergencies. 
Participants at the workshop were divided evenly into four groups: Training and Education, Emergency Planning, Prevention and Enforcement, and Response and Recovery. Each group worked independently using a modified version of NGT. NGT was chosen because it has proven itself as an excellent technique for generating ideas, and the purpose of the meeting was to generate initiatives.

The modified NGT used in the workshop differed from standard NGT in three ways. First, the clarification step was expanded to include a broader range of discussion. This allowed participants to better understand each initiative and why it was offered. Second, the workshop technique didn't use NGTs step of formal (mathematical) ranking and voting on priority ideas. The workshop technique used a quick, more subjective ranking instead. The designers of the workshop felt it was more important to spend the time on detailed development of the priority.ideas than on formal voting and ranking. Finally, a step was added to standard NGT to develop programmatic ideas for the top-rated initiatives. This allowed group members to develop a rough outline of what the initiative needed to accomplish and who needed to take part in its implementation.

\section{Outputs}

The four groups attending the workshop came up with a list of over eighty EMrelated initiatives, some of which were developed in greater detail (e.g., by further describing proposed outcomes and significant milestones). The following initiatives are examples of those applicable to research.

\section{Training \& Education:}

o Compare, analyze, and evaluate existing certification programs and develop model programs including a core curriculum and training standards and competencies.

o Develop improved delivery systems and quality assurance mechanisms for training programs.

o Evaluate the instructional methodology in existing emergency preparedness courses.

o Evaluate public awareness methods.

\section{Emergency Planning}

o Improve hazard analysis methodologies (e.g., for hazardous materials transportation).

o Improve decision support models and tools for public officials.

o Improve needs assessment methodologies.

\section{Prevention and Enforcement}

o Develop engineering solutions to address the causes of accidents. 
o Identify economic incentives to industry for prevention actions.

Response \& Recovery:

o Establish standards for a uniform Incident Command System.

\author{
Role Plavine with Another Modification of NGT
}

\title{
Background
}

The technique of role playing involves people interacting in imaginary situations. Role playing forces a person to put himself or herself in another person's place, to feel and behave as the other would. A successful role play increases one's understanding of others involved in the situation. Using role playing for communication can help improve attitudes and desired behavior [5]. For example, role playing can help doctors communicate better with patients, salespeople with customers, or scientists with government administrators who lack technical expertise. See the previous section for background on NGT.

\section{EM Application}

U.S. Department of Energy (DOE) representatives attended a workshop with over twenty local government officials from around the nation in April 1989. The DOE representatives and the local government officials were primarily senior and midlevel managers with an interest in hazardous materials transportation and emergency management. The purpose of the workshop was to address issues of mutual concern and to identify areas of productive action for DOE.

The workshop participants used role playing with a modified version of NGT to develop their programs. The programs targeted hazardous and nuclear materials transportation and emergency preparedness related to incidents involving the shipments of those materials. The workshop used role playing to promote a constructive atmosphere for identifying the action items. The designers of the workshop felt that by asking local officials to play the roles of DOE employees, the workshop would focus discussion on improvements rather than deteriorating into a gripe session. NGT was combined with role playing because idea generation was a major goal of the workshop.

Participants at the workshop were told to play the role of a DOE staff member. Their DOE division was responsible for the transportation of hazardous (including radioactive) materials and emergency preparedness related to incidents involving those materials. Participants were divided into three teams. Each team was given a mock report of planning assumptions and issues local government identified as 
concerns for them. Teams were asked to develop a program plan to address as many local government concerns as possible.

The workshop technique differed from standard NGT in four ways. First, each team was assigned both a leader and a facilitator. A local government team leader was chosen for each group because the designers felt that would help reduce possible feelings of manipulation of the group's outputs. The leaders of each group were the moderators and also reported the group's results to the other groups. The facilitator played a different role than a standard NGT facilitator, recording ideas and making sure the groups followed the steps of the technique. The second difference involved an informal ranking of initiatives, instead of using NGT's standard mathematical approach, because the workshop designers felt it would be more useful to work on developing content given the time available. The third difference was an additional step of asking the teams to develop more detailed programmatic descriptions of the top-ranked initiatives. The fourth difference involved merging initiatives by team leaders and facilitators after the round-robin listing. The merged initiatives were reviewed with the other team members to make sure they agreed with them.

Several steps used in the workshop technique, in addition to the standard NGT steps, were used to build a composite program plan for the hypothetical DOE office. After teams ranked their top five initiatives, they used scoping forms to guide them through the process of developing an initial implementation plan for each initiative. Team leaders presented their team's work to the other workshop teams. Finally, a composite plan was developed by all the workshop participants using the various initiatives developed by the three teams.

\section{Outputs}

The three teams initially came up with over fifty action items. They selected the following nine as the most important and developed detailed program suggestions involving statements of work, milestones, resources, and costs. Many of these top items are applicable to research.

1. Develop a central database of radioactive materials transportation information with electronic access by local governments.

2. Expand the existing public information program.

3. Facilitate the development of national standards of operation and training for emergency responders. 
4. Establish a Nuclear Transportation Advisory Committee made up of interested Federal agencies, States, and local governments.

5. Develop a cooperative Federal training program for local first responders and managers.

6. Support general public education on transportation and repository programs to rebuild credibility with targeted audiences.

7. Develop an integrated training program to certify first responders for radioactive materials transport accidents.

8. Provide policy guidelines and procedures for local governments to incorporate into their overall emergency plans.

9. Require States to notify local jurisdictions about some radioactive material shipments through Federal legislation or regulations.

\section{Tabletop Exercises}

\section{Background}

Tabletop exercises are a way to practice, develop, improve and test skills in EM operational procedures or systems [2] [6]. The participants assembled together are provided with a "what if" incident or scenario. The scenario is usually broken down into several phases. Each phase includes information inputs, which may be warning messages, hazard impact reports, damage assessments, assistance requests, or other intelligence about the effects of a hazard and the need for protection of life and property. Participants make decisions about the inputs at each phase to determine appropriate actions for minimizing the effects of the hazard. Participants must consider the decisions and actions they have made at previous phases. Outputs are decisions or actions participants describe verbally to the whole group. These may include resource allocations, action directives, and other messages.

Participants are selected so they will be consistent with the exercise's goals and objectives. A moderator and recorder are assigned. Evaluators and observers are optional. The moderator controls the play of the scenario. A recorder, evaluators and observers are optional. A recorder notes problems requiring attention and documents agreements or suggestions. An evaluator uses appropriate tools, such as time lines and checklists, to evaluate the exercise and participant responses. Observers simply watch the play of participants but may participate in the critique. 
Finally, the design should consider all the details of implementing the scenario exercise (e.g., time, place, and duration).

Tabletop exercises complement traditional development and maintenance programs which rely on individual review or major field exercises. Drawbacks of individual review include:

o No individual or group perception of the system as a whole (i.e., no observation of the entire response),

o Incomplete data collection, and

o No mental stimulation.

Tabletop exercises offer a group perception of the whole system and allow participant observation of the entire response. They generate more complete and better quality data than individual review because the groups discover gaps, overlaps, and inconsistencies. They pinpoint needs for upgrades in training, equipment, and the system itself.

The major drawbacks of field exercises include their cost. Some can run over hundreds of thousands of dollars. Furthermore, field exercises are highly visible. Testing a poorly developed system can result in low morale or bad publicity. Tabletop exercises are relatively inexpensive. Direct participation by all system elements is not required. This saves the expense of facilities, equipment and people. It also eliminates any safety and liability concerns. Tabletop exercises can be done with less media attention while gaining a preliminary confidence in the system.

\section{EM Application}

Federal and local government representatives from around the country attended a workshop in May 1989 to design and develop tabletop exercises they could take back home and implement. The participants worked in teams to develop exercise scenarios and used role playing to gain insights into the EM systems in their communities and how a tabletop exercise program would fit in.

Facilitators aided workshop participants in following a structured procedure for designing and developing their tabletop scenarios. Participants were specifically asked to draw on their existing knowledge of their own communities. Design involved defining the goals and objectives of the exercise and selecting exercise players based on these goals. For example, a goal developed by an exercise design team at the workshop was to "improve City response, and assistance from State and Federal agencies." Similarly, an objective was to ensure that the "roles and 
responsibilities [of the City's Emergency Operating Center personnel] effectively interface and support those of the Command Post."

Development of the exercise also involved selecting leadership and participants, choosing a credible scenario, phasing the scenario, and converting each phase into details necessary to stimulate play. To choose a credible scenario, participants needed to ensure it was factual and it supported their goals and objectives. This involved analyzing hazard technical data, facility status, jurisdictional involvement, possible weather conditions, and historical information. Phasing the scenario involved breaking it down into phases based on critical features. Converting each phase into details involved determining all messages the participants would receive, determining moderator questions, and determining expected participant responses.

After developing the scenarios, the teams broke up into role-playing groups to act out a sample of their materials to the other teams. Before the teams ran through their scenarios, team moderators presented reports to the rest of the participants, describing their scenario implementation exercise.

\section{Outputs}

Based on the scenario design portion of the workshop, the teams identified the following major issues and concerns. Again, several of these issues are applicable to research.

o Interface between the Emergency Operations Center and the Field Command Post, especially coordination among different levels of government

o Effectiveness of emergency public information systems

o Mobilization during off-hours

o Coordination of information flow

\section{Conclusions}

I have described three structured meeting techniques that can be used to determine EM issues practitioners really want to see addressed. These techniques were used in workshops with emergency management practitioners. The workshop techniques are based on proven group techniques. The modifications made them more applicable to the purposes of the workshops.

We believe the meeting techniques as used in our workshops successfully meet the objective of identifying research ideas because (1) we designed structured 
approaches which clearly spelled out the steps the participants needed to take, (2) we used techniques which gave people time to generate and list ideas before they were discussed and elaborated on, and (3) we used techniques which encouraged participation and team-building and even made it fun. These techniques have broad application for both researchers and practitioners because they

o systematically and proactively identify the needs of a diverse EM community

o focus on idea generation using adaptations of proven group techniques

o put researchers in direct contact with practitioners

o lay the foundation for program evaluation and improvement

o establish a collegial atmosphere which emphasizes a team effort

o generated many initiatives applicable to research

The workshop techniques were used to produce numerous initiatives the EM practitioners wanted to see addressed. Many of these initiatives, including the most important ones, are applicable to research. For example, an initiative from modified NGT suggests research in comparing, analyzing, and evaluating existing certification programs. This would help practitioners develop a model training program for emergency managers. An initiative from the tabletop exercises suggests developing a method for testing the effectiveness of emergency public information systems. These techniques do not replace other research but are a good first step in linking research and practice to better meet the needs of emergency managers.

\section{References}

[1] Delbecq, Andre L, Van de Ven, Andrew H., Gustafson, David H., Group Techniques for Program Planning: a guide to nominal and delphi processes (Glenview, Illinois: Scott, Foresman \& Co., 1975).

[2] Federal Emergency Management Agency. Exercise Design Course: Guide to Emergency Management Exercises (June 1984).

[3] Fox, William M., Effective Problem Solving - How to Broaden Participation, Improve Decision Making, and Increase Commitment to Action (San Francisco, California: Jossey-Bass Inc., 1987). 
[4] Mahler, Julianne G., "Structured Decision Making in Public Organizations". Public Administration Review, Vol. 47, No. 4 (1987), pp. 336-342.

[5] Shaw, Malcom. E., Corsini, Raymond, J., Blake, Robert, R., Mouton, Jane, S. "Role playing: A practical manual for group facilitators." (San Diego, California: University Associates, Inc, 1980).

[6] U.S. Environmental Protection Agency. Guide to Exercises in Chemical Emergency Preparedness Programs. (May 1988).

[7] Van de Ven, Andrew H., Delbecq, Andre L., "The Effectiveness of Nominal, Delphi, and Interacting Group Decision Processes." Academy of Management Journal, Vol. 17, No. 4 (1974), pp. 605-621.

\section{Acknowledgements}

The ideas presented in this paper represent the cumulative work of hundreds of people over twelve years at Virginia Tech's Management Systems Laboratories. Professional managers from business and industry along with faculty, graduate students, and undergraduates from a wide variety of engineering and other disciplines have participated in the research and development of these tools and techniques. The authors thank them for their good work.

The preparation of this paper is funded by U.S. Department of Energy (DOE) Grant No. DEFG02-88DP48058 and by Urban Energy \& Transportation Corporation Cooperative Agreement No. DE-FCO2-87CH10343 and No. DE-SC0690RL1180. The authors thank DOE's Transportation Management Division for providing us a real-world laboratory for the research, development, and testing of state-of-the-art management tools and the frameworks for understanding how to make them successful. 


\title{
SATISFYING STAKEHOLDERS FOR SUCCESSFUL PROJECT MANAGEMENT
}

\author{
Larry A. Mallak \\ Management Systems Laboratories, Virginia Tech \\ Gerold R. Patzak \\ Technical University of Vienna, Austria \\ Harold A. Kurstedt, Jr. \\ Management Systems Laboratories, Virginia Tech
}

\section{INTRODUCTION}

Successful project management has been defined as balancing the triangle of the cost, schedule, and quality criteria (Kerzner, 1989). For example, if the project manager must meet a tighter schedule, he or she must know the effect on specifications and/or cost. Except in cases of greater efficiency, the specifications must relax and/or the cost must increase. This classical triangle applies to all levels of management, including program management.

Today, successful project management cannot be secured by meeting just these three traditional criteria. We must meet the cost, schedule, and quality criteria and, in doing so, we must satisfy the stakeholders of the project. Why are stakeholders now an issue? Haven't project managers always had to contend with stakeholders? I'll address why stakeholders have risen in visibility, power, and influence in project management.

To satisfy our stakeholders, we must know who they are. I'll identify categories of stakeholders, identify their main interests, assess the power of each category, locate the channels this power is exercised through, design ways to address stakeholder expectations, and discuss how stakeholder satisfaction strategy can be implemented in project management.

I argue that satisfying stakeholders is a compromise. Stakeholder satisfaction relies on information about the three success criteria plus assurances on data and information accuracy and reliability (and also assurances past problems have been fixed and reoccurrence minimized). Project managers will dramatically increase their potential to manage projects successfully if they understand who their stakeholders are, each stakeholder's agenda, stakeholder power and how it's used, how to satisfy stakeholders, and ways to actively and sincerely seek and address stakeholder concerns.

\section{THE RISE OF STAKEHOLDERS IN PROJECT MANAGEMENT}

Why have stakeholders risen in visibility, power, and influence in project management? We've always had stakeholders--they just weren't recognized and accounted for. But today's stakeholders are more sophisticated, informed, and vocal. They have greater and more timely access to information and are more apt to seek information. We have to go beyond pleasing the customer--we must please many different categories of stakeholders. Today's stakeholders are more powerful and demand more attention than their predecessors. They have more avenues (media, special interest groups, responsive legislatures, etc.) to voice to their views. 
Stakeholders have a better understanding of the underlying processes at work in government and industry. They know more about how government and corporate actions affect us, our neighborhoods, our interests, and our environment. They are concerned about institutional, technical, and cultural actions. The stakeholders are more knowledgeable, too, and often demand more than a lay person's explanation. They ask tough questions and expect quick, decisive, accurate answers.

Stakeholders are more powerful today than ever before. The combination of knowledge and education gives stakeholders expert power, adding to the position power exercised through influence in the political process. Our modern democratic government systems have matured to the point where citizens have formal means and authority to exert greater influence on organizations and their projects. Communications are instantaneous via fax, electronic mail, and other means. Citizens are more inclined to call a newspaper or television station to air their dissatisfaction with government or industry activities. And the media, reflecting various segments of public interest and opinion, are quick to act because these stories appeal to their readership and present potential to expand their audiences. Media have become more of an influence in covering projects such as the Prince William Sound cleanup, the Chernobyl accident, the "Chunnel" connecting England with the rest of the European continent, and the investigation of airline crashes.

Few events and activities go unnoticed and unreported, at least in some circles. Smaller, regional news may not make page one of the New York Times or USA Today or be on CNN Headline News, but may be reported by your local paper or television station, in alternative publications, or on handbills tacked up in your grocery store. The grass roots have grown.

We've always had stakeholders. Research and analysis of stakeholders isn't new. Ackoff (1981) discussed a stakeholder's view of the organization, while Mason and Mitroff (1981) used stakeholder analysis to sort out faulty assumptions in strategic planning. Katz and Kahn (1978) divided the organization's environment into five sections, where classes of stakeholders interact with the organization. Cleland and King (1983) spoke of the need to consider the behavioral dimensions of project management through satisfaction of economic, social, and psychological concerns. We've simply started putting more emphasis on stakeholders and begun to deal with them in a methodical way because they've become more vocal, they know more, and they have more power to influence our projects. We now worry more about several stakeholders, including the project team, instead of focusing almost exclusively on one stakeholder: the customer. We've increased our awareness of other stakeholders.

\section{CATEGORIES OF STAKEHOLDERS}

To manage and design strategies for satisfying the stakeholders, we must know the categories of stakeholders and their special interests, agendas, power, and influence regarding the project. We can't fully meet all the stakeholders' expectations, so an analysis of different stakeholder categories helps us rank and focus our activities. My discussion of stakeholder categories begins inside the organization and moves outward to non-human stakeholders, as shown in Figure 1. I've identified and will discuss the following categories of stakeholders: 1) workers involved with the project, 2) corporate division/governmental agency, 3) parent corporation/government, 4) customers, 5) capital suppliers, 6) subcontractors (consultants, contributors to the project), 7) users of the project, 8) authorities and regulatory agencies, 9 ) the public (as represented by the media, special interest 
groups, lobbyists), and 10) non-human (e.g., scientific environment, natural environment). Especially for government projects, the customers, capital suppliers, subcontractors, users, and the public may be the same people; however, for discussing project management in general, they are different categories of stakeholders. Figure 1 shows the potential overlap by grouping these categories together.

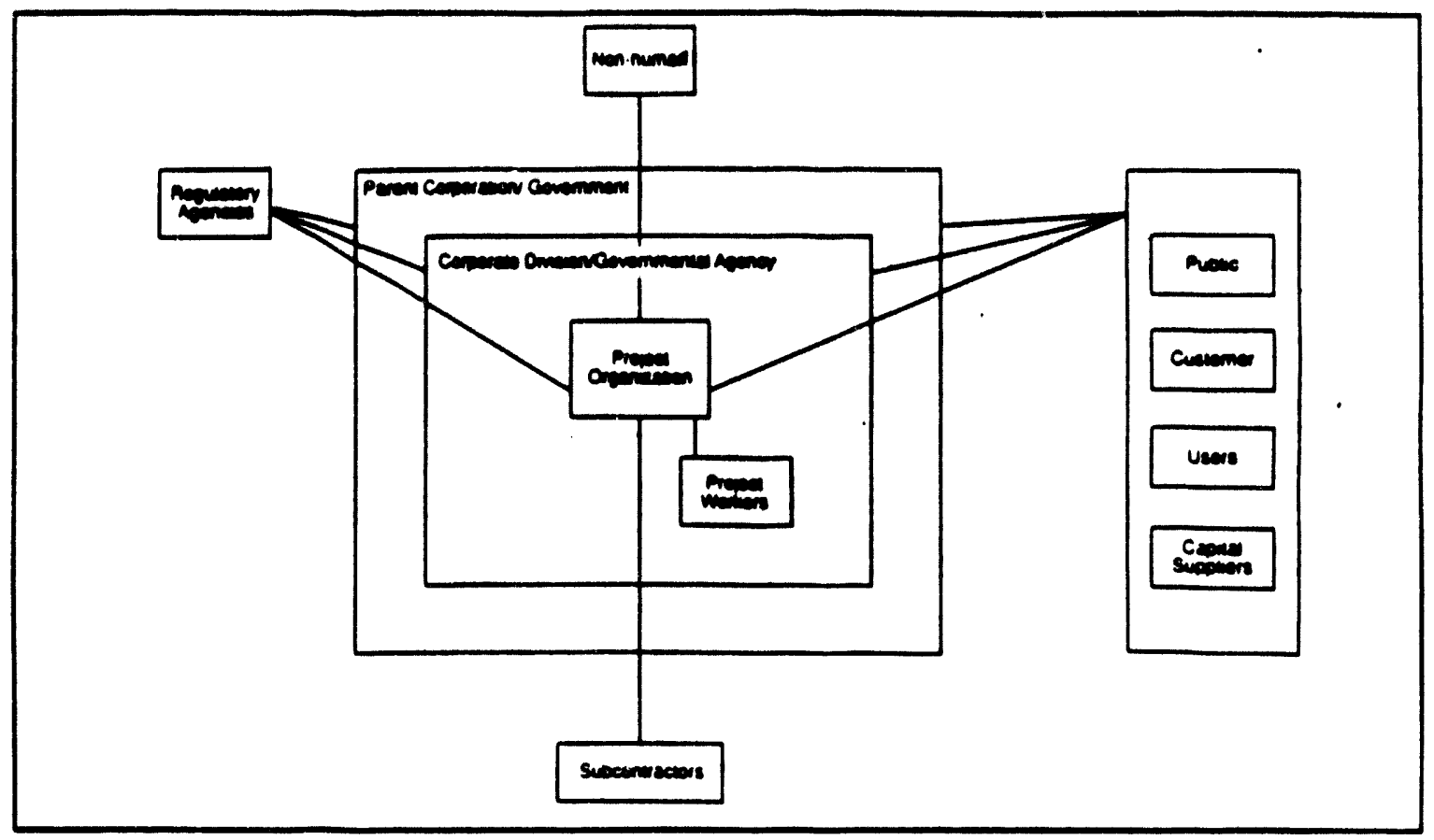

Figure 1. Many stakeholders influence the project organization through a combination of direct and indirect means.

Workers involved with the project

Description: This stakeholder category includes the project team and other organizational members both up and down the organization who become directly involved with the project, including the workers responsible for implementing project steps (e.g., pouring concrete forms for a building, wiring electrical networks, charting the project).

Main interegta/agenda: The project responsibility rests locally with this group of stakeholders, so their agenda is straightforward: get the project done on time, within cost, in conformance to quality specifications, and now, to the satisfaction of stakeholders. The workers' interests include: the different types of reward systems, work satisfaction, professional development, career steps, and social climate (Patzak, 1985). Because of their proximity to and responsibility for the work, this group has special interest in the project's technical considerations.

Power assessment: The project team often holds decision-making authority for most project-related decisions, especially once funding has been agreed on and dispersed. Power rests with the project team corresponding to the positions the team members hold in the organization. The project team must sometimes yield to higher management decisions regarding changes in funding and deadlines, often expecting quality to be unaffected. 
Power channels: The project team exercises its power through its project management decisions and the level of influence it exerts on higher management levels to make favorable project decisions.

How to satisfy: Because the responsibility for satisfying stakeholders rests with the project team, the project team should address satisfying non-project team member expectations. Give workers both a clear understanding of how project success is measured through stakeholder satisfaction and a clear set of instructions for the work they're expected to do. They should be trained on project activities. Apply more-traditional management principles to make the workplace desirable and to motivate performance through a goal-directed reward system. When working with non-project members, consider the workload the project adds to their efforts so their responsibilities contribute to the project instead of the project disrupting their work.

Staff the project well, getting the right people in the right positions having the right responsibility, authority, and accountability. Help set realistic, achievable deadlines and define acceptable and affordable quality levels by understanding the workers' work. Inform project workers of how their efforts affect the project.

\section{Corporate division/Governmental agency}

Description: This stakeholder group comprises the balance of the organization not directly involved with the project. The balance of the organization includes functional areas, such as purchasing (procurement), finance, and top management. This category doesn't extend to any parent organization. I treat the parent organization as a separate category.

Main interests/agenda: The corporate division or governmental agency is concerned with financial and perception aspects of the project. Functional departments such as purchasing and finance, want to know: 1) how well the cost criterion is being met, 2) whether there are cost overruns or gaps in funding that hold up the project, and 3) if resource surpluses could be better applied elsewhere in the organization. Functional departments want to avoid disturbances caused by sharing limited resources with the project. Top management looks at how the project: 1) tits into the organizational picture, 2) impacts the whole organization, 3 ) is being perceived by stakeholders, and 4 ) is performing financially.

Power assessment: Functional areas can work for or against the project through control over the purchasing and delivery of materials, project funding, performance reporting, image management (advertising and public relations), certifications, permits, and the many paper traps that can slow down or stop a project. Top management makes decisions about: 1) how and when the project will be tunded, 2) the project's priority for funding and completion, and 3) project team staffing. Top management, at times, can terminate the project.

Power channel:: Functional areas exercise their power by carrying out their responsibilities as directed by top management. They often are at the operational end of management decisions regarding timing and allocation of funds, and can exert considerable power through the paperwork associated with their responsibilities. Top management, by definition, makes strategic decisions for the project and wields considerable power through the organizational hierarchy.

How to satisfy: Satisfy functional areas by checking their boxes--complete paperwork on time. Keep functional areas posted on project performance and deviations from plan. Minimize surprises, such as project hold-ups due to late material deliveries or not completing proper paperwork. Satisfy top management by regularly updating them on project status and justifying changes from plan. Also, be responsive to top management's requests for information so they can make informed 
decisions about the project. Make the project team leader or a designee available to top management to provide immediate answers or serve as point of contact for further inquiry.

Learn the paperwork and procedures required by functional areas and include them in the project plan. One person on the project team (depending on the size of the project) should act as liaison to the functional areas so both the team and the functional areas have a central point for exchanging information. Also, for top management, make a contact person available for questions and information requests. This contact person should be the project manager. Before and during the project, ask for and anticipate the information top management needs and ensure its availability.

\section{Parent corporation/goverament}

Description: The parent corporation or government (i.e., local, state, federal, international), can be a separate authority level or a legal entity containing the project's corporate division or governmental agency. The top executive of the corporate division or governmental agency is the linking pin with the parent. The . parent corporation is usually either a holding company created primarily for the purpose of merger, acquisition, and diversification activities or a larger firm that has acquired a new business or given one of its divisions a separate corporate existence for strategic reasons. The local, state, federal, or international government is the jurisdiction under whose budget the project is funded.

Main interests/arenda: The parent's interest with respect to a particular project may be very little or nonexistent. The parent concerns itself with the overall financial picture of the corporate division or governmental agency and stays out of day-to-day management. Depending on the nature of the relationship between the parent and project organization, the parent and division or agency could be at crosspurposes. The parent may have responsibility for the division or agency for reasons other than an interest in continuing its operations. The parent government may be interested in closing the agency thereby terminating the project and avoiding further budget outlays. The parent corporation may close down the division to avoid further losses or sell the division to generate cash for operations. The scope and visibility of the project may affect the involvement of the parent regarding perception and politics.

Power assessment: The parent's power is typically limited, except through conformance to organization-wide policy or by virtue of funding the entire corporate division or governmental agency. The potential exists, depending on the particulars of the legal organizational structure, for micromanagement should top management of the division or agency not perform as expected.

Power channels: The parent can exercise its power through the division's or agency's top management. The parent typically can't directly affect a project.

How to satisfy: Because of the indirect relationship between the project and the parent, providing periodic updates on the project is typically not advised, and can signify the desire for micromanagement by the parent. (This may be desirable at times, as in the case of ineffective division/agency top management.) Because the top executive of the division or agency is the linking pin to the parent, provide him or her with information necessary for doing his or her job. Tight tinancial management will typically keep parent managers at ease.

Find out through top management or other informants what the parent wants to know about your project, if anything. Then deliver that information to the proper people through the proper channels. 


\section{Customers}

Deacription: For the purposes of this analysis, customers are those people and/or organizations outside the project organization who provide the agreements for scoping the project. The customers are not always the users of the project. For example, the customer for a bridge might be a city or municipality. Internal customers, such as the balance of the division or agency or the parent, have already been discussed for their involvement in the project. Their participation as a customer will be similar to those outside the project organization, except for their power and power channels.

Main interests/agenda: The customer wants the project completed as planned, quickly, inexpensively, and at the best quality possible. These are competing interests, given the interdependencies of cost, schedule, and quality. The customer wants project updates on demand and expects dutiful justification for project plan deviations. The customer will often try to expand or change the project once underway and not expect to pay more.

The customer also wants information plus assurances that the project is going well. The customer wants to be proud of the project for intrinsic reasons and for the public relations value of the project for the community. Nevertheless, pride is important to the customer, and therefore, should be important to the project manager.

Power ascessment: The customer's power is acquired through the agreement to perform the work and through relationships (whether professional, financial, or personal) with other parts of the organization. Typically, the customer has the right (or could be financially forced) to stop the project once underway. The project's success ultimately rests with the customer. The customer may wrestle more power over the project team through hints of future work based on their experience with the current project.

Power channels: The customer exercises power: 1) by being the agent of the agreement and therefore the reason for the project, 2) through current relationships with the organization, 3 ) through hints of future work, 4) by communications with non-project team members (especially top management), 5) through promises they make to their stakeholders ("We promised our clients we'd have this tinished by the end of the year."), which pull "second generation" stakeholders into the picture and put pressure on the customer.

How to satisfy: Meet and try to exceed the terms of the contract or agreement. Prepare project plans tor audiences both inside and outside the organization. The inside plan gives challenging but realistic targets for the project team. The outside plan has built-in financial and schedule buffers to enhance the probability of staying on budget with the agreed timetable. Anticipate project addons the customer will request later and put into the original project plan. Clearly define what work will be done and when. Identify what isn't within the scope of the project, and determine cost and time estimates for later justification.

Find out both what the customers want and need from the project. Anticipate project changes. Provide accurate and timely information on project progress.

The project manager and team should work to create a calm mood or atmosphere surrounding the project to give the customer a sense of pride and peace of mind throughout the project. Invite the customer to critical decision meetings. Participation helps satisfy the customer's interest and projects confidence to the customer's organization and even to the community. 


\section{Capital suppliers}

Description: This group of stakeholders supplies the capital for the project. In government, the principal capital supplier is the taxpayer. Capital suppliers in government are most often different from the customer. That is, state taxpayers provide the capital to pay for a bridge serving a small portion of the constituency, such as a bridge on a small country road. In some cases, the customer may be the sole supplier, but often banks, thrifts, and other investors put up the funds.

Main interests/agenda: In the private sector, suppliers of capital are primarily interested in the financial return on their investment, though others may be interested in equity ownership of the project once completed. Retail ventures such as shopping centers often attract capital in exchange for equity. Capital suppliers assess projects based on two criteria: 1) the interest rate and 2) the probability that they'll get their investment back. What is the risk associated with their investment? Will the project be successful? Competitors may supply funds, often disguised as a third party investment to buy out the firm if successtul or to exert other influences.

Capital suppliers want more information so they can assess the risk of their investment and make decisions on funding disbursements. They want to improve their chances of getting a better return. European bankers are so concerned with the importance of project management that they: 1) require the use of project management methods so they can assess risk (both technical and financial) and 2) have project managers on their staffs who oversee and judge the project team's use of project management methods.

In government, taxpayers expect their interests to be represented in decisions made by their legislatures. The roles and expectations of a taxpayer differ from the private sector capital supplier who expects a commensurate return on his or her investment. The taxpayer realizes one action of a democratic form of government is to redistribute a portion of its tax collections to social programs. However, the taxpayer holds the government accountable (through legislative representatives) for actions deemed necessary to protect and develop public interests such as transportation, safety and security, law enforcement, emergency assistance, and others actions having more direct benefit to the taxpayer.

Power assessment: These stakeholders hold power usually in direct relation to their funding level, though some prestigious investors may harness more power with respect to the project by their "pack-leading" phenomenon. Investors often enter into or exit from funding projects based on the presence of these prestigious investors, so if the prestigious investor pulls out, others may follow because of the respect they have for that investor.

Power channels: Power is typically exercised through the availability of or legal promise to supply funds to the project. Professional and personal relationships of the capital suppliers with project team or other organizational members may offer an additional power channel.

How to satisfy: Demonstrate and produce expected (or better) returns on investment. Keep detailed financial records and allow independent auditing. Provide financial information as required or requested.

Carefully design the funding package to produce desired levels of funding at the lowest risk, and comprising reliable investors who'll stick with their promises and stay out of day-to-day management.

\section{Subcontractors}

Description: This stakeholder category consists of consultants and other contributors to the project. Subcontractors are responsible for portions of the project, and have typically been contracted by the project team. 
Main interests/arenda: Because the subcontractors don't have the full picture or final responsibility for the project, they'll tend to suboptimize by doing a good job on their part of the project. But, the linkage with the rest of the project is the project team's problem. The lack of a big picture means subcontractors don't realize or have to endure the ripple effect of missed deadlines or work that must be redone.

However, subcontractors typically want to continue working with the organization on subsequent projects. The subcontractor seizes the opportunity to gain knowledge and expertise in its field, and strives to build its reputation in the field.

Power assessment: Subcontractors exert power only to the extent the project and project team rely on their part of the project. They're involved in decision making usually as advisors, sometimes as technical experts; and their decision making is limited to their part of the project as defined or agreed upon at contract time.

Power channels: Subcontractors exercise their power through delaying the ir part of the project, increasing the price in mid-stream, or through technical considerations forcing decisions on cost, schedule, or quality.

How to satisfy: Satisfaction of these stakeholders should be a lower priority, assuming they have what they need to get the job done-specifications for the job, . deadlines, necessary information, and a project team contact person. Subcontractors should worry more about satisfying the project team, and not vice versa.

Set clear expectations for the subcontractors and follow up on them regularly to avoid surprises. Give them what they need to get the job done right and on time. Brief them on how what they're doing fits into the project and what the impact of late or substandard work is on the project and on their future as a subcontractor with you.

\section{Users of the project}

Description: Often, the users of the project are the same as the customer--as in the case of a manufacturer contracting with a design/build firm to add 100,000 square feet of warehouse space to an exasting facility. We'll consider users of the project when those users are different from the customer. An example stakeholder in this category is a commuter who uses a bridge contracted by the responsible governing body. The commuter, as a taxpayer, supplied some of the funds needed to build the bridge, but the tax monies used to finance the bridge were collected from many other taxpayers who will never use the bridge.

Main interesta/arenda: Project users want the project to meet their objectives. Their agendas range from apathy to radical activism. The users are a collective group for whom the project is designed.

Power assessment: Users' power is usually limited to how well they can influence the project through the customer. In aggregate, the users can exert considerable pressure on the project through public relations, financial, or legal means. They can occasionally affect the project or the project team directly. Power channels: Users' power must usually be exercised through an agent, such as the customer or through a voting mechanism or letters to government officials, and not directly with the project team or its organization. In municipal projects, the users' desires are taken as consultative or advisory. In other projects, especially those designed for economic gain, the users' desires are more binding and greater care is taken to attend to them as a group or as a collection of subgroups. Individual users can rarely exert power over the project team unless they represent the group or a significant subgroup of users.

How to satisfy: Get their input and ideas on the project. Respond to their concerns through the customer, because they are the customer's chief stakeholder. 
Be sure the customer understands this group of stakeholders because, in aggregate, they represent a powerful force.

Find out how the project will affect the users and design ways to minimize negative and maximize positive effects. Design for the masses; try to satisfy the majority of the users.

\section{Authorities and regulatory agencies}

Description: These stakeholders consist of all agencies having regulatory or other authority regarding the project. Example agencies could be OSHA, EPA, municipalities responsible for enforcing building codes, and state governments.

Main interests/agenda: These stakeholders are interested in the project only to the point of checking their particular box (e.g., making sure the project meets the ir regulations, ensuring proper paperwork has been completed and filed). They typically have an administrative interest in the project; their technical interest extends only to their particular jurisdiction (e.g., building codes, waste disposal procedures, tax collection). These agencies seek to minimize their problems with the project.

Power assessment: These stakeholders hold specific powers defined by law, usually. The nature of these stakeholders' power can vary based on how their agency affects the project.

Power channels: Authorities and regulatory agencies exercise their power through holding up the project or forcing redesign of the project because their needs weren't met. In more extreme cases, they can exercise legal power through threats of sanctions or court orders.

How to satisfy: Identify all agencies relevant to the project and find out what regulatory or other authority they have for the project. Then design the project plan recognizing the requirements of these agencies.

\section{The public}

Description: Project managers concern themselves with representatives of the public, rather than random members of the public. Representatives of the public often include the media, special interest groups, lobbyists, and neighborhood groups. In government, the customer, user, capital supplier, and public all overlap and are often the same. For this reason, I consolidate these four stakeholders in Figure 1. Main interests/apenda: The public's interests are wide-ranging. Like the users of the project, the public's agenda can range from apathy to radical activism. Many of the public's agendas are driven by concerns for personal financial situations, transportation infrastructures, neighborhoods, the environment, or attitudes and beliets regarding the project. Members of the public often formally organize to voice their concerns.

Poswer aresesmens: Again, like the users of the project, the public's power derives from their ability to influence an agent such as the customer or an authority/regulatory agency, rather than trying to influence the project team directly. Power channels: The public's power must usually be exercised through an agent, such as the customer or through a voting or petition mechanism, and not directly with the project team or its organization. Individual users can rarely exert power over the project team unless they represent the group or a significant subgroup of the affected public. 
How to satisfy: Identify the expected special interest groups and their concerns relative to the project. Address these concerns in light of the overall project objectives or state why addressing them is not appropriate.

Speak with these groups to find out their true intent. Often, the public has received a biased view from the media or a special interest group. The project manager should try to give the public a balanced view of the project, and offer explanation and interpretation of critical or controversial project-related events.

\section{Non-human}

Description: The non-human stakeholders such as the 1989 Time Magazine Planet of the Year (Earth) and the scientific/technical environment deserve to be a stakeholder category because projects are being evaluated more and more for their environmental impact and for their effects on scientific and technical developments. Non-human stakeholders are usually represented by one of the other categories, such as the public or users.

Main interests/agenda: Their interests, as voiced through their agents, are chiefly protection from adverse impact.

Power assessment: Non-human stakeholders have power in that they motivate other stakeholders, like the public.

Power channels: The natural environment can exercise its "power" through the deterioration of living conditions to the point where air, water, and other lifesustaining substances are contaminated to the extent that quality of life or the capability to survive is diminished. Scientific and technical environments can retaliate through scientific activities or technologies that overpower human capabilities. An uncontrolled chemical experiment provides an example. The proliferation of a uncontrolled chemical reaction would overwhelm us and would create adverse impact on the natural environment, which would in turn deteriorate the quality or survivability of life on the planet. Human stakeholders would react to the effects on the non-human stakeholders and exercise their power.

How to satisfy: We need to know and understand the potential impact of the project for both the natural and the scientific/technical environments and design ways to eliminate or minimize any adverse impact or uncontrollability. Listening to and consulting agents of other stakeholder categories can give us more (albeit biased) information about these non-human stakeholders. We can then incorporate this information in our project management activities.

\section{CONCLUSION}

The process of satisfying stakeholders is always a compromise. We can't completely satisfy the customer because the customer would want it all tomorrow for free. Because the customer pays, we're always in a compromise situation-fees are negotiated, quality is decided based on technical constraints, or schedule is dictated by labor and material availability and technical characteristics of the project. We can be in a better position to meet the stakeholder's expectations if the stakeholder buys into the expectations given the constraints.

To satisfy our stakeholders, we supply them with accurate, timely information about our project's cost, schedule, and quality. Buî satisfaction goes beyond merely providing information-satisfaction depends on the mode of information portrayal and delivery. (A memo sent in the mail is different from a personal visit by one of the project managers.) Satisfaction is information plus assurance, assurance that the 
project is working toward meeting or exceeding the stakeholder's expectations. Stakeholders must feel the project is being managed with their interests in mind.

As project managers, we must manage the perception as well as the project. We must consider how our actions are viewed by the various stakeholders. Sometimes actions we take with good intentions and expected good consequences may have alternative interpretations. We need to understand the interpretations and provide information and assurances to clearly communicate so we understand what stakeholders are thinking.

Project managers can use several tools to manage stakeholders: 1) systems to provide information and assurances; 2) conflict management processes; 3) consensus and persuasion processes; 4) interpersonal communication; and 5) stakeholder analyses. These are not typical project management tools, but they are management tools you must apply to projects. This paper has touched on some of the applications of these tools to satisfying stakeholders, but its focus has been on the use of a structured analysis of stakeholder categories to assist the development of stakeholder satisfaction strategies.

So, how do we know how well we've satisfied our stakeholders? We can simply ask them. We can meet with them regularly or periodically. We can call them on a regular basis to provide updates and answer their questions and concerns. For larger groups, we may wish to conduct a survey. Press conferences help in satisfying the more organizationally-distant stakeholders such as the public, users, neighborhood groups, and environmental groups. Johnson \& Johnson made successful use of press conferences in managing the Tylenol incident (Collins, 1986). Project managers must learn to be proactive toward their stakeholders, supplying them with information before they ask for it or demand it. Project managers who understand who their stakeholders are, the individual stakeholder agendas, stakeholder power and how it's exercised, how to satisfy their stakeholders, and actively and sincerely seek and address stakeholder concerns will dramatically increase their potential to manage projects successfully. 


\section{ACKNOWLEDGEMENTS}

The ideas presented in this paper represent the cumulative work of hundreds of people over fourteen years at Virginia Tech's Management Systems Laboratories. Professional managers from business and industry, and faculty, graduate students, and undergraduates from a wide variety of engineering and other disciplines have participated in the research and development of these tools and techniques. The author thanks them for their good work. The pronoun " $I$ " is used throughout to separate all of us using management systems (we) from the author (I). However, please recognize "I" is more than one contributor to this work.

The preparation of this paper is funded by U.S. Department of Energy (DOE) Grant No. DE-FG02-88DP48058. Management Systems Laboratories thanks the DOE's Office of Environmental Restoration and Waste Management for providing us a real-world laboratory for the research, development, and testing of state-of-the-art management tools and the frameworks for understanding how to make them successful.

\section{REFERENCES}

Ackoff, Russell L (1981). Creating the Corporate Future: Plan or Be Planned For. New York: John Wiley \& Sons.

Cleland, David I. and King, William R. (Eds.). (1983). Project Management Handbook. New York: Van Nostrand Reinhold.

Collins, David E. (1986). "Cultural Heritage and Crisis Management," in M.A. Berman (Ed.) Corporate Culture and Change: Highlights of a Conference. New York: The Conference Board, Inc., pp. 13-19.

Katz, Daniel and Kahn, Robert L. (1978). The Social Psychology of Organizations. New York: John Wiley \& Sons.

Kerzner, Harold. (1989). Project Management: A Systems Approach to Planning, Scheduling and Controlling. New York: Van Nostrand Reinhold.

Mason, Richard O. and Mitroff, Ian I. (1981). Challenging Strategic Planning Assumptions. New York: John Wiley \& Sons.

Nutt, Paul C. (1984). Planning Methods for Health and Related Organizations. New York: John Wiley \& Sons.

Patzak, Gerold R. (1985). Systemtechnik. Beciin/Heidelberg: Springer-Verlag. 
Case Studies on Designing Meetings for Effective Institutional Interactions

\author{
Rebecca M. Imholz \\ George E. Ruberg \\ D. Mark Brubaker \\ Management Systems Laboratories \\ Virginia Tech \\ Blacksburg, VA 24060
}

\begin{abstract}
The U.S. Department of Energy (DOE) has been actively pursuing opportunities for external input into its programs and plans. This pursuit for external input is a result of DOE's commitment to a culture of openness and public involvement in decision making and policy direction. This input has included interactions between DOE and other institutions such as state and local governments and Indian Nations. One way these interactions occur is in the form of meetings to discuss issues or concerns the various participants have regarding DOE policies. Our involvement with this type of interaction has been as the meeting designer, coordinator, and facilitator for two external review groups - the State and Tribal Govermment Working Group (STGWG) and a group comprised of DOE transportation officials and local government officials from cities and counties around the country. We'll refer to this second group as the Local Government Network. We coordinate the design and facilitation of the Local Govermment Network with the Urban Energy and Transportation Corporation.
\end{abstract}

When designing the structure of a meeting I rely on the formula:

$$
\text { Audience }+ \text { Purpose }=\text { Design }
$$

In order to design an effective meeting, it's important to know who your audience is and the purpose of the meeting objectives. Our paper will discuss what we've learned about effective meeting design from our involvement with the two external review groups mentioned above. We'll also discuss the results of meeting evaluations participants filled out following a meeting to help us determine if our meeting designs met their purposes.

\title{
FORMATION OF STGWG
}

In March of 1989, Admiral James D. Watkins, Secretary of Energy, committed to the development of a comprehensive Five-Year Plan for environmental restoration and waste management activities at DOE nuclear-related production, testing and research and development (R\&D) installations, and for improved compliance with applicable regulations: Federal, state, and municipal. This resulted in the Environmental Restoration and Waste Management Five-Year Plan. The DOE created STGWG to participate in the development and review of the Five-Year Plan as a means to improve the plan by considering state and tribal concerns in the development stage, to emphasize 
and support DOE's culture of openness, and to give DOE's cleanup ind compliance agenda national visibility.

\section{STGWG Participants}

STGWG participants were chosen based on a letter to Admiral Watkins received in April 1989. This letter, co-signed by ten govemors of states with DOE facilities within or adjacent to their borders, outlined the govemors' concems regarding federal commitment to the cleanup of DOE installations. The letter outlined some possible courses of action and called for a comprehensive national plan for environmental restoration (1). Using this letter as indicative of the most seriously affected states, DOE invited the following states and groups to participate in STGWG: Colorado, Idaho, Kentucky, Nevada, New Mexico, Ohio, South Carolina, Tennessee, Oregon, W ashington, Yakima Indian Nation, Shoshone-Bannock Tribes, National Governors' Association, National Conference of State Legislatures, and the National Association of Attomeys General. Since STGWG's first meeting in June 1989, the group has expanded to also include California, Florida, Illinois, Missouri, New York, Texas, and the Confederated Tribes of the Umatilla Indian Reservation.

\section{STGWG Today}

STGWG's purpose is to give affected states and Indian Nations a voice in the annual review and development process of the Five-Year Plan. Today, STGWG meets approximately four times a year with DOE to plan, formulate, and review the Five-Year Plan and discuss related issues.

\section{DOE'S EFFORT TO NETWORK WTTH LOCAL GOVERNMENTS}

DOE's Transportation Management Program (TMP) is responsible for supporting DOE programs in the movement of all materials, including the development of packaging and systems for transporting radioactive materials. TMP also coordinates DOE's program for regulatory and policy development in this area. In 1989, TMP began an effort to establish a network of local government contacts with interests in transportation issues including emergency management, enforcement, and training.

Since 1989, DOE has hosted several interactive meetings with local government officials from cities and counties around the country. The DOE representatives are from TMP and the local officials are senior and mid-level managers involved in transportation and emergency management.

The purposes of the meetings are to:

* share information that local government officials can apply to their own communities; 
* exchange experiences and ideas applicable to other emergency management programs; and

* identify areas of productive action for DOE and local governments to address issues of mutual concem (2).

USING AUDIENCE + PURPOSE TO DESIGN A MEETING

\section{Developing the STGWG Meeting Design}

MSL has hosted, facilitated, or observed each of the ten STGWG meetings to date. This experience gives us an opportunity to relate lessons learned from designing these meetings for this particular group.

Planning a successful meeting is similar to planning the design of a document. Let's return once again to our formula:

$$
\text { audience }+ \text { purpose }=\text { design } .
$$

The STGWG participants demonstrate the following characteristics:

1. STGWG members are knowledgeable about DOE's environmental restoration and waste management activities;

2. STGWG members agree on some issues and disagree on others because of differing interests;

3. There are some disagreements between DOE and STGWG over issues and their resolutions;

4. STGWG members are, at times, skeptical about DOE's abilities and motives to change; and

5. STGWG members remain the same from meeting to meeting.

The STGWG meeting process has been structured to meet these audience expectations and the meeting's purpose which is, as mentioned earlier, to review and comment on the issues and programs defined in the Five-Year Plan.

For involving diverse, knowledgeable, and skeptical participants, we suggest the following meeting characteristics which represent the STGWG meeting structure: (3)

Involve a subgroup of STGWG - a planning committee - to help you set the location, agenda, and meeting process with DOE. This supports DOE's commitment to openly receive input into their planning process and improves the chances for a meaningful dialogue at the meeting if both groups, DOE and STGWG, feel they have contributed to the meeting agenda. 
Provide review and orientation materials before the meeting. Advanced distribution of information results in thoughtful review and comment.

Ensure a minimum of lengthy, technical presentations for participants. Lengthy, technical presentations decrease interest and increase suspicion and perception of an unwillingness to listen and return to the old culture.

Allow time for an executive session of STGWG members. This permits them to freely collect and discuss group and individual comments and thus minimize differences between individuals and maximize discussion when discussing issues with DOE.

Don't overplan. A successful meeting plan will bend to the evolving needs of the participants.

Include time for participants to get to know one another. If commitments are met and working relationships established, trust will be confirmed by action.

Discuss, during the meeting, the status of DOE's and STGWG's commitments made the previous meeting. Bringing participants up-to-date demonstrates both DOE's and STGWG's 'good faith' in following through with commitments.

Make feasible commitments on-the-spot or have quick access to a higher authority to that he or she can make these commitments. This is especially true for DOE participants; if DOE is unable to make feasible commitments, STGWG will feel little or no progress is being made.

Inspect, don't expect meeting logistics. Meeting details can make or break it. Taking care of details for participants ensures their time is spent concentrating on the meeting at hand.

The meeting process for STGWG has evolved over the years to include three main areas:

EXECUTIVE SESSION: During this session, only STGWG members meet. This is a self-led session. They use this time to gather and discuss comments as a group before giving them to DOE.

ROUND TABLE DISCUSSION: STGWG members and DOE participants meet together to discuss issues and comments, many of them derived from the EXECUTIVE SESSION. This session is led by a facilitator.

PRESENTATIONS: Both STGWG members and DOE participants present information on topic areas suggested by the STGWG Planning Committee prior to the meeting. This time is also used by the facilitator to update meeting participants on the status of action items since the last meeting.

Developing the Local Government Network Meeting Design

Let's return again to our formula: Audience + Purpose $=$ Design. The audience or participants of the Local Government Network demonstrate the following characteristics: 
1. Participants are local government officials (mid- to senior-level managers) with interests in transportation and emergency management

2. Workshop participants are chosen nationally based upon the topic area for discussion. Therefore, the participants are not the same from workshop to workshop.

The purpose of the Local Government Network Meetings is for DOE and local government officials to share information and identify areas of action for both parties to address issues of mutual concem. Since the workshop audience changes frequently and the purpose involves issue identification and resolution, we've used a Program Planning Exercise during the workshop process.

The Program Planning Exercise uses meeting techniques designed to develop ideas for a DOE program plan to address local government concerns about transportation issues such as emergency preparedness for nuclear materials transportation incidents. We ask participants to play the role of a staff member for a DOE administrator, to review a report identifying local government concems (the "report" is based on the actual product of an earlier workshop), and to develop a program plan to address those concems (2).

The meeting process is designed to accomplish the objective and maximize limited time. The process is interactive, collectively building a consensus plan based on both individual participation and team thinking. The exercise places participants in the role of a Federal manager faced with a difficult job--trying to satisfy transportation concems while staying within organizational constraints. The result of the participative exercise is to (1) generate a program of initiatives to address local concerns and suggestions for their implementation, and (2) foster a better mutual appreciation of the problems facing both DOE and the local governments in developing such a program (2).

The exercise has three main parts. First, we divide participants into teams for the purpose of designing programs to meet the meeting objective. Second, we reconvene the participants to have team leaders report back to the full group on the initiatives and implementing steps developed by each team. Third, the participants discuss various aspects of each plan and achieve consensus on a composite program plan that might serve as a model for continued cooperation between DOE and local governments (2).

\section{MEETING FACILITATION}

Some group meetings require strong, active facilitating while others are better served by passive facilitating. In either case, it's important to recognize the type of meeting facilitator needed and adapt to the requirement. For STGWG, strong meeting facilitation has not been needed because the meetings are mostly self-led. This is because the STGWG participants remain the same and thus are used to interacting with each other as a group. In addition, the purpose of the meeting is for review and discussion of the FiveYear Plan; group consensus on issues and a set deliverable or program plan from the meeting is not required. However, even a self-led meeting can benefit from an effective facilitator because effective facilitation can: (1) better organize and direct group 
discussion, (2) improve the collection of valid comments, and (3) make sure group members have an equal chance to participate (3).

The Local Government Network requires stronger or more aggressive meeting facilitation with a more structured meeting process. This is because the participants do not remain the same; the individuals vary from meeting to meeting. So they are not as capable of being a self-led group. Also, the purpose of the meeting is to list issues or ideas and develop plans for resolving them. The facilitator uses a structured modified Nominal Group Technique which has been proven effective for idea generation (4). The facilitator leads breakout sessions with role playing for participants to develop plans and recommendations.

\section{EVALUATING EFFECTIVENESS OF MEETING DESIGNS}

Results of STGWG Meeting Evaluations

Following the conclusion of the last two STGWG meetings, we gave participants, both STGWG members and DOE participants, meeting process evaluation forms. We designed these forms to measure participants' perceptions of their opportunity to express views and the degree their meeting expectations were met. The form also asked for unstructured comments about the meeting logistics and process.

The following scale was used for all questions:
(1) strongly disagree
(2) disagree
(3) moderately disagree
(4) neither agree nor disagree
(5) moderately agree
(6) agree
(7) strongly agree

All means were between 5 (moderately agree) and 6 (agree). The average response rate for both groups was $64 \%$.

We'll be discussing the results to two sets of questions. The first referred to the Round Table Discussions where both STGWG and DOE meet together. Both STGWG and DOE agreed moderately that their individual views were heard, understood, and given full consideration by all participants. Both STGWG and DOE also moderately agreed they were given enough time to express their views. Regarding whether or not meeting expectations were met (which were established at the beginning of the meeting), STGWG participants moderately agreed they were met and DOE participants agreed they were met.

The second set of questions referred to the Executive Sessions (which are for STGWG members only). STGWG participants moderately agreed on the following: their individual views were heard, understood, and given consideration by other members; they had enough time to express their views; and their meeting expectations were met. 
There was also a set of unstructured comments regarding logistics and the meeting process. Many of the participants' comments were addressed earlier in the paper under the discussion of suggested meeting characteristics for this type of extemal review group. However, we will summarize some of the comments made on the evaluation form. The majority of the participants felt the meeting logistics were handled very well and that the process allowed for an open atmosphere which is non-threatening and provides people with an equal opportunity to speak. However, a minority of participants did note the tradeoff between giving everyone a chance to speak and managing the process efficiently. The facilitators have tended to lean towards giving everyone a chance to speak at the expense of running a more efficient process. Encouraging participants to be more concise should help improve the efficiency without limiting the opportunity to express views.

\section{Results of Local Govemment Network Evaluations}

Here is a summary of comments made by participants following the Program Planning Exercise at a past Local Govemment Network meeting. All of the participants who filled out an evaluation form believed the Program Planning Exercise was useful in providing them with an opportunity to develop specific program ideas for DOE which directly addresses their needs. Some of their recommendations included adding more time during the exercise for thorough discussion (increase the time from a half day to a whole day.) (Since that comment was made some workshops have included a lengthened time for the planning exercise if the topic warrants it.) Participants said workshops should be on a regional basis instead of a national one. Currently, participants are chosen nationally depending upon the workshop topic. The location of the workshop is not necessarily tied to its purpose (5).

\section{CONCLUSION}

As we've discussed, the key or formula needed to design an effective group meeting is: Audience + Purpose $=$ Design. I've discussed how I've applied this formula to two meeting groups, STGWG and the Local Government Network.

We've learned that with STGWG a flexible and open forum is needed. The STGWG audience is members who are knowledgeable about DOE's environmental programs, yet skeptical about DOE's ability to change to a culture of openness and emphasis on cleanup. The STGWG audience remains the same through the various meetings and there is little changeover of members. The purpose is to have open an dialogue with DOE about DOE's Five-Year Plan and related issues. Group consensus on issues and a resulting meeting deliverable is not required. Therefore, we use a flexible, unstructured meeting process which bends to the evolving needs of the participants. We've developed a STGWG Planning Committee to help design each meeting agenda. Since the meetings are self-led, the facilitator is passive, yet still very effective in ensuring that all participants comments are heard, group discussion is directed and organized, and action items followed.

We've learned that with the Local Government Network, a more structured meeting process is required with a more aggressive, yet still effective facilitator. The audience for this group changes constantly depending upon the topics being discussed. The purpose of the meetings is to share information about transportation related issues and develop 
some areas of productive action to address these issues. Therefore, we believe the Program Planning Exercise is a successful component of the meeting because: ( 1 ) it is a structured method which clearly spells out the steps participants need to take to reach an objective, (2) it uses a modified nominal group technique which gives people time to generate issues and list ideas before they are discussed and elaborated on; and (3) it incorporates consensus-building teaming and role playing techniques, which encourages participation, creativity, and even a little fun!

\section{ACKNOWLEDGEMENTS}

The ideas presented in this paper represent cumulative work of hundreds of people over twelve years at Virginia Tech's Management Systems Laboratories. Professional managers from business and industry, and faculty, graduate students, and undergraduates from a wide variety of disciplines have participated in research and development of these tools and techniques. The author thanks them for their good work.

The preparation of this paper is funded by U.S. Department of Energy (DOE) Grant No. DEFG0288DP48058. MSL thanks DOE's Office of Environmental Restoration and Waste Management for providing us a real-world laboratory for the research, development and testing of state-of-the-art management tools and the frameworks for understanding how to make them successful.

\section{References}

[1] Govemor Booth Gardner, State of Washington; Governor Cecil D. Andrus, State of Idaho; Governor Garrey E. Carruthers, State of New Mexico; Govemor Ned Ray McWherther, State of Tennessee; Governor Carroll A. Campbell, Jr., State of South Carolina; Govemor Richard F. Celeste, State of Ohio; Govemor Neil Goldshmidt, State Oregon; Governor Roy Romer, State of Colorado; Govemor Bob Miller, State of Nevada; and Governor Wallace Wilkinson, State of Kentucky, " Defense Waste Cleanup: A Proposal for A National Solution," pp. 1-2, April 1989.

[2] J. Andrew Walker, George E. Ruberg, and Susan H. Denny, "Radioactive and Hazardous Materials Transportation: What Local Officials Are Telling Us," Waste Management '90 Proceedings, Volume I, pp. 211-213.

[3] Rebecca M. Imholz, Thomas B. Hindman, Jr., and D. Mark Brubaker, "Lessons Leamed from Applying External Input to DOE Policy Decision Making," Spectrum '90, pp. 12-15.

[4] Andre L. Delbecq, Andrew H. Van de Ven, and David H. Gustafson, "Group Techniques for Program Planning: A Guide to Nominal and Delphi Processes," Scott, Foresman \& Co., 1975.

[5] Management Systems Laboratories, Energy Task Force Management Corporation, "Final Report: Nuclear Materials Transportation and Emergency Management," 1989 Conference and Workshops, pp. IV 29. 30. 


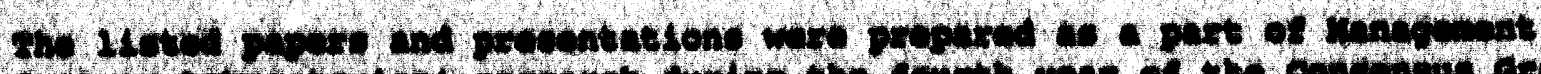

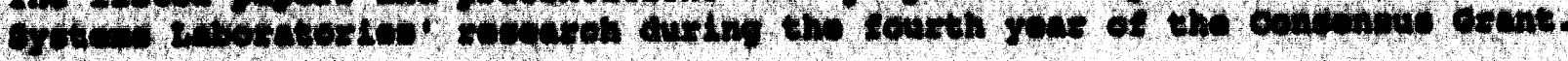

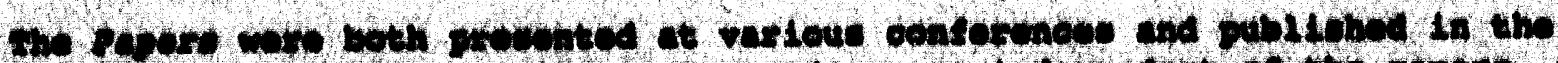

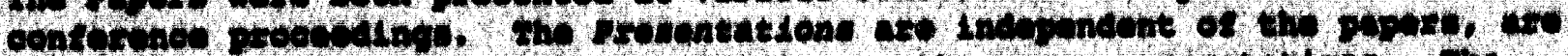

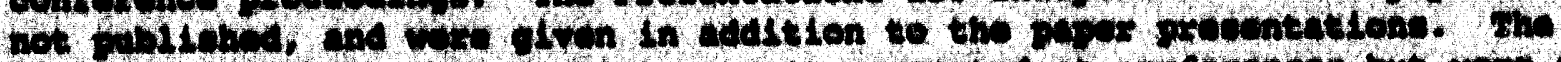

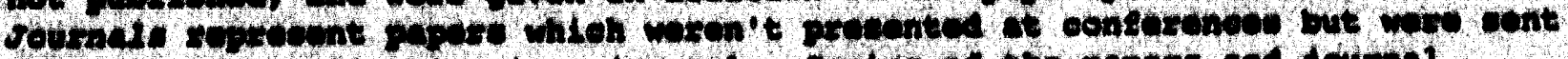

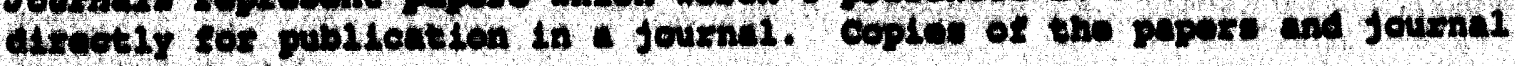
ortiolos tollow.

othen

roperas

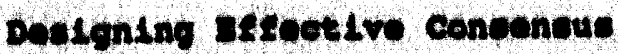

Planking Eyotene.

Southoutein cheres of the Inotitute

os Vhnegume selences.

culdellnoe tor conoultative and

part lelpetive eroupe.

douthosotorn ohspter of the Inotitute

of unnguent selenees.

The It teete of Inoontives on contilot

Manughinat, Poreolved strength of

coneonsue, and Deolelon guellty.

Southeactern Chapter of the Inotitute

of vanagenent selenees.

Eopand guality Manggement Into tho

cuntemer' Invironeunt to sotablieh

Ffteot 1 ve rearures and standarde.

1ht International syaposiun on

productivity and quilty Improvemont,

- toous on eovernment.

The Role of Lesderentp in

Implewonting rotal guallty Kanagument.

1et Intornutional symposium on

produetivity and oulity raprovement,

- Eocus on Covernmint.

The Environmental Trilogy Project:

Balanolng rochnical, Inotitutional,

and Cultural porapectives to

Environimontal Managenent.

Maste Munagement 92 .

Are dA's Communieation Toole Effective?

Ivaluntion Renesech of Two AN Publications. Waste Management 192.
$10 / 91$

runderentel

nith

Pantin,

$10 / 91$

Fundrandel

$10 / 91$

rundaronted

$2 / 92$

Coneonous

proeeses

$2 / 92$

conerneus

procees

$3 / 92$

Consensus

Procena

$3 / 92 \quad \begin{aligned} & \text { Information } \\ & \text { syoteme }\end{aligned}$ 


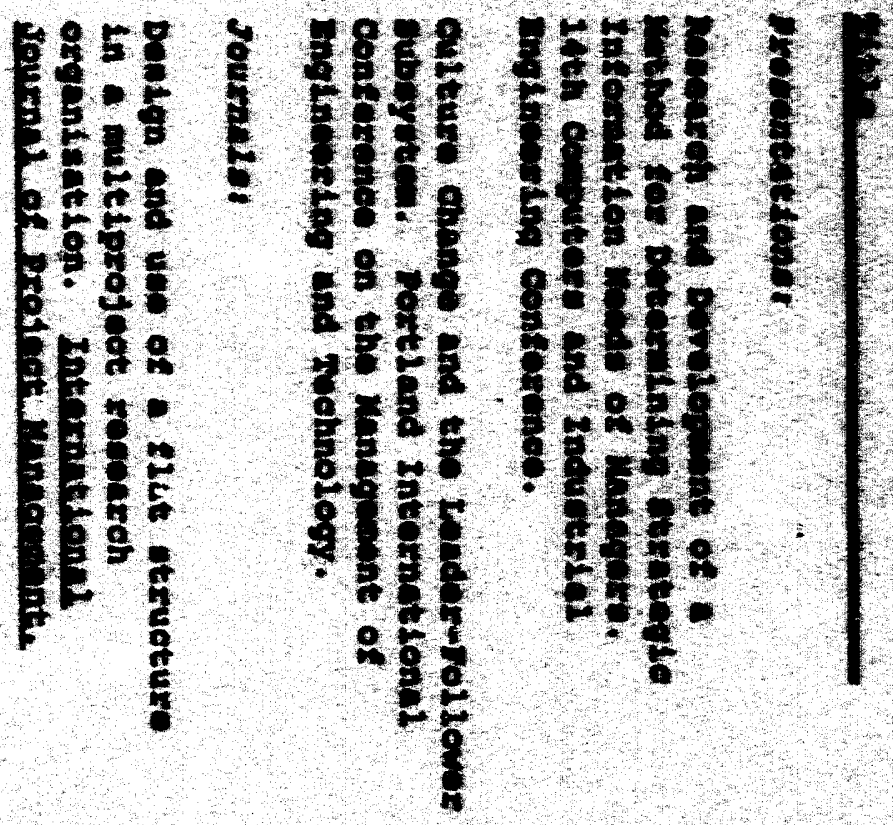

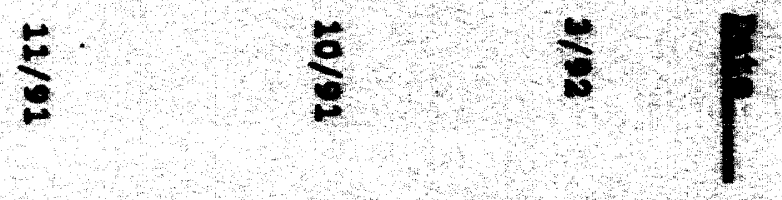

8

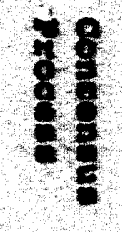

$8 \frac{8}{8}$

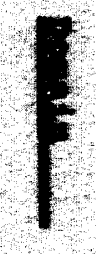




\title{
DESIGNING EFFECTIVE CONSENSUS PLANNING SYSTEMS
}

\author{
Jana L. Moore (student), Virginia Tech \\ Harojd A. Kurstedt, Jr., Virginia Tech
}

\begin{abstract}
I classify planning approaches into autonomous, consultative, committee, and consensus planning. Then, I offer guidelines for designing successful consensus planning systems. During consensus planning, a team of stakeholders has the opportunity and the authority to develop a plan by reaching a common judgment. Planning members have an opportunity to share information and express their views to develop a plan they can accept. Consensus planning is helpful when the organization is heterogeneous, when the situation is complex and controversial, or when stakeholder support is needed for implementation. However, consensus planning requires time and effort to prevent unproductive discussion, undue influence of experts, or groupthink. The unique characteristics of consensus planning require a structured process, interactive participation, and simple but powerful information and analysis tools.
\end{abstract}

\section{INTRODUCTION}

This paper is for managers who are considering using a consensus process during organizational planning. I'll present a classification of planning approaches based on the literature and my own experience. I'll characterize, and differentiate four types of planning: autonomous, consultative, committee, and consensus. I'll then concentrate on consensus planning. I offer six guidelines for successfully implementing consensus planning. First, determine whether team planning is appropriate. Second, determine whether you would benefit from consensus planning. Third, consider whether you have the time and ability to successfully implement consensus planning. Fourth, if you decide to use consensus planning, choose a process to guide plan development and implementation. Fifth, choose an interactive group participation method. Sixth, choose simple but powerful information and analysis tools. I'll discuss each of these guidelines and make recommendations. Finally, I'll discuss general conclusions.

\section{CHARACTERIZING P.LANNING APPROACHES}

You can delegate planning activities to trained planners, managers, other employees, or consultants [8] [12]. From this point on, I'll refer to all of these people generically as planners. Plans are powerful: they guide your management decisions and actions by forming explicit strategies for the future [8]. Thus, many people expect participation and involvement in organizational planning [12].

I classify planning approaches based on the type of planning member participation. I differentiate individual from team planning, which is planning conducted by two or more people [12]. Team planning can include stakeholders (for example, managers, other employees, customers, or governmental-agency representatives) in the planning process and allow these stakeholders to assist in plan development and implementation. I further divide team planning into three types: consultative, committee, and consensus [2] [4] [11]. The classification is as follows:

\section{Individual Planning}

Autonomous: One planner has access to all required information and formulates a plan. Autonomous planning is conducted by one planner who may decide to use other people as information sources but who has authority to write the plan. 


\section{Team Planning}

Consultative: During consultative planning, multiple planners provide input. Thus, you can include a wide base of expertise. Planning members participate by generating information, critiquing alternatives, or reviewing plan drafts. However, final planning authority is not shared with the entire group. One or more planners collect, synthesize, formulate, and disseminate a plan based on input from the rest of the group.

Committee: Committee planning divides the responsibility, effort, and authority, usually by area of interest or expertise. Each subgroup or individual planner constructs part of the plan.

Consensus: During consensus planning, planners have both opportunity and authority to develop a plan by reaching a common judgment. Consensus planning allows for group interaction. Planners share information, express their views, and are able to accept the final plan even if they don't completely agree with every part of the plan. Although we can measure the level of consensus among stakeholders (levels of agreement, acceptance, willingness to implement, and commitment) for any plan, only a portion of plans are constructed using a consensus process.

In the remainder of this paper, I'll concentrate on consensus planning.

\section{GUIDELINES FOR USING CONSENSUS PLANNING}

Now I'll discuss six guidelines for successfully using consensus planning.
1) Determine Whether to use Team Planning

Because consensus planning is a type of team planning, I'll first discuss when to use team planning. I recommend using multiple planners if the organization is heterogeneous or if the situation is complex and no one has all the required information, expertise, or authority [2]. If the organization is characterized by heterogeneity (conflicting goals, agendas, information, perspectives, or judgments), team planning will increase the likelihood everyone's opinion is considered and assimilated before the plan is formulated [1] [2] [5] [6] [7]. A team of planners can synthesize goals, information, and expertise from multiple sources. If the plan must exhibit originality, creativity, or multiple options, information sharing will facilitate idea generation [2]. Also, multiple planners will be able to crosscheck the validity of assumptions and identify potential sources of error [2].

2) Determine Whether to use
Consensus Planning

After you decide team planning would be advantageous, then determine whether a consensus process would be appropriate. Consensus means everyone's point of view is heard and seriously considered [2]. A consensus process is especially beneficial when the plan is characterized by conflict and controversy, or when the support of several people is required for successful plan implementation. Consensus planning can increase group cohesion by developing a common goal, sharing information, and resolving conflict among planners. You can also use consensus planning to make trade-offs, balance needs, divide scarce resources, or coordinate agencies or departments. You can benefit plan implementation if you include a 'vide base of stakeholders and they can develop a plan with a high degree of consensus--agreement with the plan, acceptance of the plan, willingness to help implement the plan, and commitment to the plan [1].

\section{3) Determine Whether Consensus Planning Justifies the Cost}

I've briefly mentioned some of the :dvantages of consensus planning: pportunity to develop a common goal, shared understanding, increased agreement, acceptance, willingness to 
implement, commitment, etc. However, consensus planning can be expensive in time and effort. Carefully weigh the costs and benefits. Because consensus planning requires a commitment of time from multiple planners, logistics of coordination and scheduling may be significant. Consensus planning also requires time for information sharing, discussion, and conflict resolution.

In addition, consensus planning requires effort to avoid unproductive discussion, undue influence of experts, and groupthink. To minimize unproductive discussion, consensus processes require facilitation [3]. Facilitation ensures the discussion isn't dominated by a few individuals, prevents unnecessary repetition or tangential discussion, keeps the group focused on the task, and maintains a systematic, logical planning process. Undue influence of an expert may occur if a planner claims to have expertise in an area the others are deficient in. You need to prevent an expert from dominating a group or a false expert from misleading the group [11]. Have planners seek input from multiple experts or, as a minimum, carefully challenge the assumptions of experts. Finally, if the plan is formed with little discussion, a condition known as groupthink may result [6]. Planners may prematurely develop their plan without adequately generating and critiquing alternatives, testing assumptions, or identifying weaknesses [6]. Inducing and resolving conflict can minimize groupthink.

\section{4) Choose a Structured Planning}

Unless you select and use an explicit process, planners may become confused and inefficient if they have different ideas about how to develop and implement the plan [12]. Planning processes can be divided into two classes: those intended to give global direction and those intended to provide step-by-step guidance.
The global processes usually provide an outline. Examples include policy analysis, goal setting, and the Creative Planning Process [2] [4]. Global processes provide general structure and recommendations, not specific actions. For example: accurately define the problem, explore various alternatives and evaluate them, make the decision, and implement the solution [2]. The global processes may be better suited to experienced planners or groups who seek autonomy and control.

Step-by-step processes are more structured, more guided, and less flexible. Examples include the Performance Improvement Pianning Process [4] [1" and the Analytic Hierarchy Process [1

Step-by-step processes may dictate participation methods, and information or analysis tools within the process itself [4] [12]; however, participation methods and information and analysis tools should be chosen independently. Step-by-step processes may require training for novices or leadership if the issues are complex.

\section{5) Choose an Interactive Participation Method for Meetings}

Ideally, you would choose a participation method to: facilitate group discussions [3], encourage interaction and mutual understanding [3], induce and help resolve conflict without resorting to bargaining or voting [5] [6], and balance status differences [11]. Since we don't have a participation method to meet all these demands, you can either make tradeoffs or use more than one technique. For example, brainstorming encourages group interaction [7], devil's advocacy induces conflict [7], and the Nominal Group Technique and Delphi balance unequal status differences [7].

\section{6) Choose Simple but Powerful Information and Analysis Tools}

I recommend using the simplest information or analysis tool available to portray the necessary information to the group. For example, you can use graphs, diagrams, tables, matrices, or checklists 
[4]. Since planners won't have the same knowledge, skills, or abilities, developing a shared understanding may be difficult. More complex tools, such as flow charts, process diagrams, or computerized models, may be confusing to some planners in the group.

Information tools assist planners by collecting, summarizing, and displaying data and information. Analysis tools use relationships, algorithms, and models to suggest alternatives or solutions [13]. With a small group, you may be able to custom-tailor the tools to fit backgrounds, abilities, and preferences of group members. However, if the tools aren't custom-tailored, they must be understandable to everyone in the group.

\section{CONCLUSION}

Consensus planning can result in plans with increased acceptance, agreement, willingness to implement, and commitment if planning systems are properly designed and managed. Before you decide to use consensus planning, realize the planning process may be expensive in time and effort. However, stakeholder participation, familiarity, and buy-in with the plan will save management time and effort later. In addition, consensus planning assumes groups have the authority and the opportunity to develop plans for implementation. Thus, consensus planning may result in plans contrary to your organizational vision or management style. If you carefully choose planning processes, participation methods, and information and analysis tools, the group should work together to develop high-quality plans.

\section{ACKNOWLEDGEMENTS}

The ideas presented in this paper represent the cumulative work of hundreds of people over fourteen years at Virginia Tech's Management Systems Laboratories. Professional managers from business and industry, and faculty, graduate students, and undergraduates from a wide variety of engineering and other disciplines have participated in the research and development of these tools and techniques. The authors thank them for their good work. The pronoun "I" is used throughout to separate all of us using management systems (we) from the authors (I). However, please recognize "I" is more than one contributor.

The preparation of this paper is funded by U.S. Department of Energy Special Research Grant No. bE-FG0288DP48058. Management Systems Laboratories thank the Department of Energy's Office of Environmental Restoration and Waste Management for providing us a real-world laboratory for research, development, and testing of state-of-the-art management tools and the frameworks for understanding how to make them successful.

\section{REFERENCES}

[1] Doss, A.R., Brubaker, D.M., and Kurstedt, Jr., H.A, "To Understand Consensus We Must Know What the Elements Are," Proceedings of the 26th Annual Meeting, Southeastern Chapter of the Institute of Management Sciences, October, 1990, pp. 119. 121.

[2] Bradford, D.L Group Dynamics, Modules in Management Series, (Chicago, Illinois: Science Research Associates, 1984).

[3] Brubaker, D.M., Doss, A.R., and Kurstedt, Jr., H.A, "Consensus: An Investigation of the Need for Facilitation," Proceedings of the 26th Annual Meeting, Southeastern Chapter of the Institute of Management Sciences, October 1990, pp. 103-104.

[4] Coleman, G.D., "A Critique of the VPC's Planning Methodology," (Unpublished Masters Thesis, Blacksburg, Virginia, Virginia Polytechnic Institute and State University, December, 1988). 
[5] Hall, J. "Decisions, Decisions, Decisions," Psychology Today, vol. 5, No. 6, (1971) pp. 51-54; 86-88.

[6] Janis, I.L (editor), Stress Attitudes, and Decisions: Selected Papers (New York: Praeger Publishers, 1982).

[7] Keeling, J.F., Kurstedt, Jr., H.A, and Hughes, J.E., "A Selection Tool for Choosing the Best Participative Problem-Solving Technique," Proceedings of the 26th Annual Meeting, Southeastern Chapter of the Institute of Management Sciences, October 1990, pp. 122-124.

[8] Mintzberg, H. "Strategy Making in Three Modes" in R.C. Shirley, M.H. Peters, and A.I. El-Ansary (editors), Strategy and Policy Formation: a Multifunctional Orientation, (John Wiley and Sons, 1976).

[9] Mitroff, I.I., Shivastava, D., and Udwadia, F.E., "Effective Crisis Management," The Academy of Manarement Executive, vol. 1, No. 3, (1987) pp. 283-292.

[10] Polk, J.W., Kurstedt, Jr., H.A, and Welch, K.A., "The Effects of Experts on the Group Consensus Process," Proceedings of the 26th Annual Meeting, Southeastern Chapter of the Institute of Management Sciences, October 1990, pp. 115-118.

[11] Saaty, T.L., Decision Making for Leaders: the Analvtic Hierarchy Process for Decisions in a Complex World, 2nd ed. (Pittsburgh, Pennsylvania: RWS Publications, 1990).

[12] Sink, D.S., and Tuttle, T.C., Planning and Measurement in Your Organization of the Future. (Norcross, Georgia: Industrial Engineering and Management Press, 1989).

[13] Turner, W.C., Mize, J.H., and Case, K.E., Introduction to Industrial and Systems Engineering. (Englewood Cliffs, New Jersey: Prentice-Hall, 1987). 


\title{
GUIDELINES FOR CONSULTATIVE AND PARTICIPATIVE GROUPS
}

\author{
Christine M. Walsh (student), Virginia Tech \\ Harold A. Kurstedt, Jr., Virginia Tech
}

\begin{abstract}
My contribution is a set of guidelines for managers to increase participation in task groups. Based on situational leadership theories, I explain the situations when group decision-making is appropriate and provide specific guidelines to help managers increase participation in task groups. Managers should seek subordinates' participation in the decision process when the decision is nonroutine, information is nonstandardized, time is not critical, and subordinates are independent and motivated. By increasing participation, managers can increase subordinates' consensus and commitment to the decision without decreasing decision quality.
\end{abstract}

\section{INTRODUCTION}

This paper will offer managers guidelines to increase the effectiveness of consultative and participative group meetings. From research on situational leadership and participation techniques, I identified situational variables which can direct a manager to the appropriate decision-making style. From these situation variables, I developed guidelines for consultative and participative meetings.

\section{GROUP DECISION MAKING}

To be successful, managers must be adaptive and use the correct decisionmaking style given the situation, ranging from autocratic to participative [1, p. 14]. I limited the scope of this paper to consultative and participative decisionmaking styles. However, groups are not always a feasible way to solve problems. Managers should use groups to solve a problem if the following situational variables exist:
1) The problem is ambiguous $[13, p$. 113][12, p. 110];

2) The problem requires the generation of multiple alternatives [1, p. 12];

3) The problem isn't covered by any current policies [12];

4) The benefits are worth the group's time [12, p. 96];

5) The decision doesn't require any

severe time pressure [12, p. 110];

6) The manager wants to offer subordinates professional development and practice at divergent thinking [12, p. 111];

7) The manager does not have the necessary expertise or information [1][3, p. 303] [13, p. 109];

8) Subordinates have valuable information or expertise [12, p. 96]; and

9) Subordinates are affected by the decision [12].

Once the manager has determined the situation warrants a group, then he or she must determine whether to use a consultative or a participative group. Vroom and Yetton define these decisionmaking styles as:

Consultative - The manager shares the problem with subordinates as a group, obtaining their collective ideas and suggestions. Then he or she makes the decision, which may or may not reflect the subordinates' influence.

Participative - The manager shares the problem with subordinates as a group. Together subordinates generate and evaluate alternatives and attempt to reach agreement (consensus) on a solution. The manager's role is much like that of a chairman. He or she does 
not try to influence the group to adopt his or her own solution and is willing to accept and implement any solution having the support of the group [13, p. 114].

\section{CONSULTATIVE GROUPS}

A consultative decision-making style has several advantages over individual decision making. It increases decision quality, enlarges subordinates' jobs, and allows the manager to remain in control. A manager should use a consultative group to solve a problem when:

1) The decision quality is important, yet the benefits are not worth the extra time for a participative group [1, p. 14];

2) Subordinates do not have the same goals as the organization [1][13, p. 116];

3) The manager is not willing to accept the risk of sharing with the group the responsibility for making the decision [12, p. 97]; and

4) The manager needs to remain in control [12].

Once the manager has decided to solve a problem using a consultative group, he or she must begin to plan the meeting. Consultative meetings allow subordinates to offer suggestions and to give opinions on other members' ideas. Consultative meetings need to be well-structured to maximize idea generation and to reduce conflict. The main purpose of consultative decision making is to increase decision quality by using a variety of viewpoints and information sources.

Prior to the meeting, the manager needs to decide on the problem he or she wants to solve and gather the necessary information. The manager should distribute the meeting's agenda and the information to group members several days before the meeting. When designing the meeting, the manager needs to consider what he or she wants to accomplish and the purpose of a consultative meeting. Since consultative meetings do not require the group members to come to consensus, the manager can invite more stakeholders than to a participative meeting. A group of 6-12 members will have a variety of information sources and still allow free communication and participation $[10, \mathrm{p}$. 172]. Larger groups are better for consultative groups because innovation benefits from exchanges of ideas that stem from many different viewpoints [9]. To increase the variation of viewpoints and the quantity and quality of ideas, the manager should have a heterogeneous group [4][5, p. 146][7, p. 123]. The manager should consider a facilitator, experts, and stakeholders as possible group members. Group members need to have both expertise and motivation [9, p. 354]. If the manager does not have experience with structured-group approaches, he or she should have a facilitator run the meeting.

At the beginning of the meeting, the manager or facilitator (whoever is running the meeting) must set the group's expectations [8][9, p. 371]. The facilitator needs to explain to group members that their ideas are crucial for the solution of the problem; however, the manager will make the final decision alone. This will help decrease the subordinates' discontent if the manager does not follow the group's alternative.

The meat of a consultative meeting is the idea-generation phase. To produce the most ideas, the facilitator needs to separate idea generation from idea evaluation [10][5, p. 145]. The group should be allowed between 20 and 30 minutes for this phase [11]. During the idea generation phase, group members need to practice divergent thinking. I would recommend a combination of brainwriting and brainstorming. These techniques allow maximum idea generation and avoid conflict [7]. Brainwriting gives group members a chance to consider the problem silently (no interaction) and write down any solutions they can think of. In brainstorming, the facilitator challenges 
the group into a rapid-fire generation of ideas, inviting new and modified ideas. Rules that apply to brainstorming include: 1) no criticism of ideas is allowed; 2) wild ideas are encouraged; 3 ) idea quantity is encouraged; and 4) combining and improving ideas are desirable $[10$, p. 171]. The quality of the solution produced is improved if these rules are followed.

The facilitator should explain the brainwriting and brainstorming process to the group. Then, he or she should allow members five to ten minutes to brainwrite $[10$, p. 180]. Brainwriting eliminates conformity pressure, therefore decreasing participation differences caused by status differentials [7][10]. The next step is idea recording. The facilitator should sequentially ask members to read one idra in turn (still no discussion permitted on ideas). Finally, the facilitator should explain the brainstorming rules and challenge members to give ideas. One advantage of brainstorming is that it allows members to build from other members' ideas. Other members may correct errors or add suggestions to previous ideas resulting in higher quality alternatives $[10$, p. 172]. When a few group members tend to dominate the process or some group members seem inhibited, the facilitator can use a procedure called sequencing. Sequencing offers some structure by giving each group member a chance to suggest an idea in turn [10][5, p. 145].

After the group has generated ideas, the group needs to identify the criteria necessary for an acceptable solution. The group should evaluate each alternative against the criteria $[12$, p. 100$][11$, p. 212]. Finally, the manager needs to thank the group for its ideas and conclude the meeting.

After the manager has considered the groups ideas, he or she needs to make the decision alone and develop an action-item list for implementation. The manager should inform group members of his or her final decision and provide reasons for any differences from the group's alternatives. To establish accountability for implementation, the manager should distribute the action-item list. The manager needs to regularly check on the progress of action items.

\section{PARTICIPATIVE GROUPS}

In today's rapidly changing organizations, managers are increasingly using participative approaches. Participative decision making has many advantages, including the following effects upon group members: increased productivity, reduced resistance to change, higher task motivation, higher job satisfaction, better teamwork, stronger commitment to decision, and an increase in self-esteem $[12$, p. 114]. Managers need to be careful, however, because participative decision making takes large quantities of time, reduces managerial control, and can lead to a reduction of the group's evaluative powers if the group becomes too cohesive $[12$, p. 115]. Managers should use participative decision making when:

1) Subordinates are necessary for implementation and their support and commitment are imperative [12,

p. 111];

2) Creative or divergent thinking is imperative [12, p. 110];

3) An integrative, high-quality decision is necessary [12];

4) Subordinates have adequate maturity levels [13, p. 106] and share organizational goals [12, p. 111]; and

5) The manager wants to generate trust with the subordinates [12].

The manager should act as a leaderfigure; however, he or she shouldn't dominate the discussions. The leader's primary role is to participate as a group member; however, he or she should also facilitate the group process. The leader should ensure that everyone participates, conflict is resolved, and one member doesn't dominate the discussion.

As in the consultative case, the manager still needs to determine the problem to be solved, obtain the necessary information, 
develop an agenda, and distribute the agenda and informatior. prior to the meeting. A participative meeting has different purposes than a consultative meeting with an increased interest in the satisfaction of its members and, therefore, should have a different structure. The group composition should still be heterogeneous; however, the members need to be more mature $[13$, p. 106] and independent [3, p. 303]. Since the group will need to come to an agreement, the group should only have tive to seven members [2, p. 45]. This number will optimize both the group's communication and their problem-solving ability [2]. The group should be arranged around a circular table because it is more conducive to open communication and informality and reduces status differentials $[13][9, p$. 245].

At the onset of the meeting, the manager needs to set the group's expectations explaining the group will determine the final decision and will be responsible for developing a high-quality solution $[8][9$, $p$. 371]. The group should discuss the organizational goals and the problem to be resolved. It needs to spend time to define and diagnose the problem and establish a criteria for an acceptable solution. The leader should encourage constructive conflict and an open atmosphere. The idea-generation phase should be less-structured than the consultative case. Since the group members are more mature, independent, and autonomous; the group does not require as much facilitation. The technique I recommend is brainstorming. Since one of the primary reasons for participative decision making is to increase subordinates' satisfaction; an interactive, open discussion is preferred. Brainstorming produces high-quality solutions since members can improve and change ideas.

During the idea-evaluation phase, the leader should assign or assume the role of devil's advocate. This person surfaces assumptions in solutions and challenges them. This will induce conflict, which should be resolved with a combination of collaboration and compromise [2, p. 15]. Hall's Consensus Guidelines can be used to effectively manage conflict. Once all the possible altematives have been generated, the group needs to compare alternatives to sriteria and decide on a solution. The group then needs to determine a plan for implementation and develop an action-item list to establish accountability. After the meeting, the manager needs to regularly check on the action-item list.

\section{CONCLUSIONS}

This paper presented guidelines to 0 used in consultative and participative meetings. I've summarized my guidelines in the following table.

\section{TABLE 1: GUIDELINES FOR GROUP MEETINGS}

However, there is no one best technique for every situation. Managers should understand when to use groups and how to use them effectively. This paper gives general guidelines; however, techniques should to be tailored to the situation.

\section{ACKNOWLEDGEMENTS}

The ideas presented in this paper represent the cumulative work of hundreds of people over fourteen years at Virginia Tech's Management Systems Laboratories. Professional managers 
from business and industry, and faculty, graduate students, and undergraduates from a wide variety of engineering and other disciplines have participated in the research and development of these tools and techniques. The authors thank them for their good work. The pronoun "I" is used throughout to separate all of us using management systems (we) from the authors (I). However, please recognize "I" is more than one contributor.

The preparation of this paper is funded by U.S. Department of Energy Special Research Grant No. DE-FG0288DP48058. Management Systems Laboratories thanks the Department of Energy's Office of Environmental Restoration and Waste Management for providing us a real-world laboratory for research, development, and testing of state-of-the-art management tools and the frameworks for understanding how to make them successful.

\section{REFERENCES}

[1] Bradford, D.L Group Dynamics (Chicago, Illinois: Science Research Associates, 1984).

[2] Brightman, H.J. Group Problem Solving: An Improved Managerial Approach (Georgia: Business Publishing Division, College of Business Administration, Gerogia State University, 1988).

[3] Cohen, Allen R. et al. Effective Behavior in Orcanizations (Homewood, Illinois: Richard D. Irwin, Inc., 1984).

[4] Delbecq, Andre L, Van de Ven, Andrew H., Gustafson, David H. Group Techniques for Program Planning: A Guide to Nominal and Delphi Processes (Glenview, Illinois: Scott, Foresman \& Co., 1985).

[5] Guzzo, R.A. Improvine Group Decision Makins in Organizations (New York: Academic Press,
1982).

[6] Hall, J. "Decisions, Decisions, Decisions" Psychology Today Vol 5, No. 6 (1971) p. 51 - 58.

[7] Keeling, J.F., Kurstedt, Jr., H.A, Hughes, J.E. "A Selection Tool for Choosing the Best Particpative Problem-Solving Technique," Proceedings of the 26th Annual Meeting, Southeastern Chapter of the Institute of Management Sciences, October 1990, pp. 122 124.

[8] Keeling, J.F., Kurstedt, Jr., H.A. Hughes, J.E. "The Effect of Meeting Group Members' Initial Expectations on Strength of Consensus," Proceedings of the 27th Annual Meeting, Southeastern Chapter of the Institute of Management Sciences, October 1991, in press.

[9] Nutt, P.C. Making Tough Decisions: Tactics for Improving Managerial Decision Making ( San Francisco, CA: Jossey-Bass

Publishers, 1989).

[10] Van Gundy, A.B. Managing Group Creativity (New York: American Management Association, 1984).

Yan Gundy, A.B. Techniques of Structured Problem Solving (New York: Van Nostrand Reinhold, 1988).

[12] Wynn, R., Guditus, C.W. Team Management: Leadership by Consensus (Columbus, Ohio: Charles E. Merill Publishing Co., 1984).

[13] Yukd, G.A. Leadership in Organizations (Englewood Cliffs, New Jersey: Prentice Hall, 1989). 
THE EFFECTS OF INCENTIVES ON CONFLICT MANAGEMIENT, PERCEIVED STRENGTH OF CONSENSUS, AND DECISION QUALITY

Martin H. Grunau (student), Virginia Tech Harold A. Kurstedt, Jr., Virginia Tech

\section{ABSTRACT}

I describe a model relating individual vs. group incentives and collaborative vs. competitive conflict management to perceived decision quality and group consensus. Based on a pilot study and the literature, I attempt to understand the consequences of incentive structures on conflict management, consensus, and perceived decision quality. Understanding these relationships helps managers, facilitators, and group members guide meetings better.

In the pilot study one set of groups was exposed to an individual incentive structure while the other was exposed to a group incentive structure. In the experiment, all groups watched one-third of the movie "12 Angry Men" and ranked the order in which the 12 jurors changed their verdict from guilty to not guilty. However, the individual incentive structure did not produce the anticipated competitive behaviors during the group process. Despite this limitation, I was able to support one of the hypotheses: consensus is highly correlated to perceived decision quality $(r=.93, p=.0001)$. The correlations between collaborative conflict management and consensus and perceived decision quality were both marginally significant $(r=.55$, $p=.07$ ).

\section{NTRODUCTION}

Decision-making groups trying to achieve consensus are usually made up of people with a variety of predispositions. These predispositions cause social pressures which naturally lead to conflict [1][2]. If conflict is not managed properly, it will be detrimental to the group and the meeting's products. However, providing a conflict environment that stimulates creative thinking and open information sharing can enable groups to reach consensus and a high quality decision.
The purpose of this pilot study was to examine the effects of two conflict management styles, collaboration and competition, induced through group and individual incentive structures respectively, on the perceived quality of the group decision and the strength of consensus. Convenors, facilitators, managers, and group members need to understand and learn the possible consequences of incentive structures and conflict management strategies so they can intervene in the group process of meetings before damage occurs.

\section{A MODEL FOR MEETINGS}

Figure 1 shows a very broad conceptual model of conflict in meetings. My concept is that people bring the ingredients for conflict to meetings. The ingredients include group members' different backgrounds, personalities, goals, opinions, and expectations.

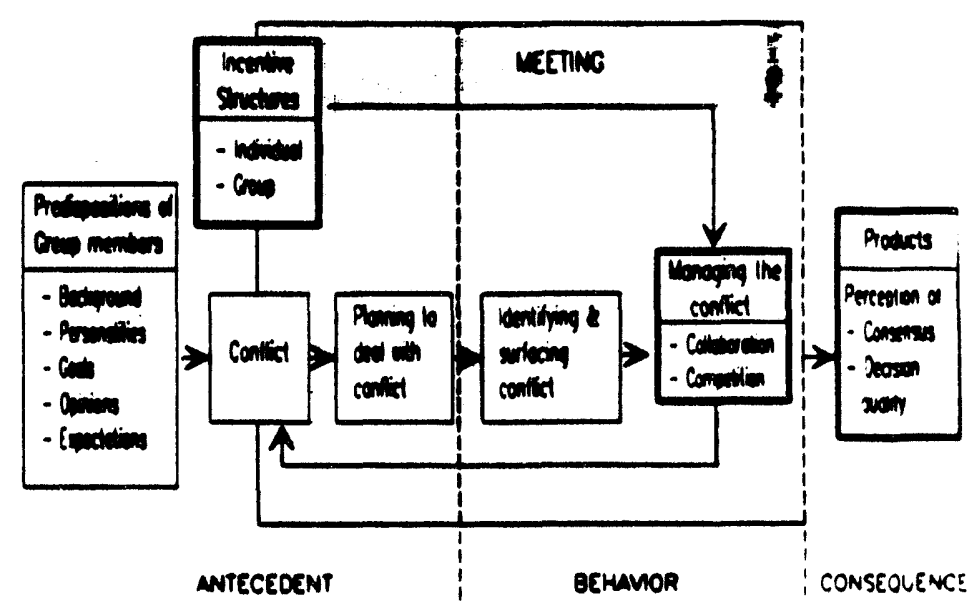

Fizure 1: Broad Conceptual Model of Con liat in Meetingas

This conflict might be based on who the group members are attending the meeting, what the problem is that will be dealt with during the meeting, how the people in the meeting expect to interact with one another, and why the group members come to the meeting.

Conflict may not be perceived by all members of the group. Unless conflict is brought out in the open and managed, it will more than likely go undetected. Using the model to get the best products of a meeting, group members must make the choice to deal with existing conflicts. Then we can identify, surface, and finally manage the conflicts by displaying functional behavior. 
The type of behavior displayed by group members in meetings can be influenced through pre-imposed meeting incentive structures. Depending on the type of incentive (group or individual) the type of conflict management strategy used during the meeting can be influenced before or during the group process.

Essentially, there are three ways to manage conflict. Group members can escalate conflict, reduce conflict, or they can do nothing about the conflict. Raising the conflict may mean exploring the underlying reasons in more detail or making sure all possible disagreements are aired and dealt with. A simple way to reduce conflict temporarily could be to intentionally avoid or to smooth over the conflict.

The justification for the feedback loop between the conflict management box and conflict itself lies in their reciprocating actions. Frequently it is the characteristics of a conflict resolution attempt that spurs a new conflict Therefore, the consequence of managing one conflict can produce a new conflict. All attempts to deal with conflict have some kind of effect on the group process. I call the effects "products."

The products box in Figure 1 contains variables that reflect the state of the group and its interactions at the end of the meeting. These variables, for example, include the degree to which a group has come to consensus on a decision or how strongly the group believes they have reached a high quality answer.

\section{Distinction Between Connict and Connict} Management

In the literature, conflict has been viewed primarily from two different angles. Some researchers believe conflict is internal such as someone's belief or perception of disagreement [2][3], while others stress conflict to be extermal reflected in people's contradictory actions and behavior [4][16]. I support the notion that conflict is internal. I define conflict as a state and label it uncommunicated disagreement while I define conflict management as the process following the state of conflict. The process is the communication of the disagreement. Therefore, conflict management relates to a behavior aimed at resolving the conflict.

\section{Potentlal Conflict Consequences}

The potential negative consequences of mismanaged conflict have been described in the literature. Mismanaged conflict can reduce tolerance among group members [S], commitment or support of the group and the final decision [6][7], trust among group members [2][8], amount of information shared among the 8 members [2][9], and the quality of the grour decision [10].

The potential positive outcomes from wellmanaged conflict are increased information sharing and a greater number of creative alternatives generated within the group [11], increased satisfaction with group process and the resulting decision [12], increased interpersonal attraction [13], and a better decision [2][14].

\section{Collaborative and Competitive Connict \\ Management}

There is no one best conflict resolution strategy that group facilitators can use in all conflict situations. Here, I focus on two very different conflict management styles: collaboration and competition. Collaborative efforts are defined as being assertive and cooperative [15]. They are most applicable for decision-making groups, where management has allocated sufficient resources such as time. Collaborative conflict resolution attempts permit the sharing of information in a nonthreatening environment, producing a shared understanding of this information and a high degree of group satisfaction. Collaboration results in a win/win situation. One party can win only if the other wins also. Competitive efforts are defined as being assertive yet uncooperative [15]. They are applicable in situations requiring quick and decisive actions, and require fewer resources. However, competitive conflict resolution attempts can create threatening environments reducing the amount of information group members are willing to share and decreasing shared understanding as well as satisfaction with the group decision. Competition results in a win/lose situation. One party can win only if the other loses.

\section{Incentive Structures}

Thomas [1], Deutsch [2], and Coser [14] list incentive structures as one way to manipulate behavior when managing a conflict. Coser 
hypothesized incentives geared toward the individual produced social pressures to compete againat other individuals causing interpersonal competition. On the other hand, incentives geared toward groups give groups a common goal. This causes intergroup competition, which in tum causes intragroup collaboration. Sherif [16] supported this hypothesis with the "robbers' cave" studies. After strongly competitive behaviors occurred among individuals in groups, superordinate group goals were introduced. These goals gave all individuals a common goal and focused their attention away from the internal conflict. Competitive behaviors within the group shifted towards competitive behaviors between the group and the outside. The introduction of a superordinate goal can be compared to the introduction of a new incéntive, producing different social pressures and causing a shift in the conflict management strategy.

Percelved Consensus and Decision Quality Consensus and decision quality are two variables we can measure to determine the success of group decisions. I define consensus to be a state where a common judgment has been reached by most of those concerned. Consensus exists when a group makes and supports a decision. I believe the following five subcomponents can be used as measures of consensus. The subcomponents are group members' agreement with the decision [17], acceptance of the decision [18], support or commitment to the decision [19][20], an understanding of the information shared to come up with the decision [17], and satisfaction with the group process and the decision [11][20]. Therefore, I can expreas consensus as a function:

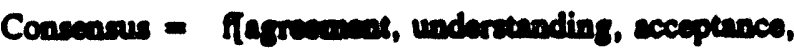

$$
\begin{aligned}
& \text { setiofuction, commitument] }
\end{aligned}
$$

In this research, I view consensus not as a binary variable, but as having different levels of strength.

Often the quality of a particular decision can't be determined right after the decision is made; especially if a problem doesn't have one correct answer. Frequently, the decision needs to be implemented and its quality judged, based on the consequence its implementation had. Therefore, since it is difficult to measure the actual quality of a decision directly, I believe it is more appropriate to consider the process elements required to achieve a particular level of decision quality. Janis believes the primary requirement for high-quality decisions is the sharing of relevant information [10]. Coser [14] argues decision quality is primarily influenced by the generation of a multitude of alternatives from which the group can choose from. I believe there is no one variable solely responsible for a group process ensuring high decision quality. Therefore, I measure decision quality through the subject's perception of two components which are believed to be important for high decision quality. I express perceived decision quality as a function of two variables:

$$
\begin{aligned}
& \text { Perceived decision quality - } \text { fperceived opportunity to } \\
& \text { expreas views, percoived } \\
& \text { gecerncion of alteratives }
\end{aligned}
$$

\section{HYPOTHESES AND ASSUMOPTIONS}

Based on my model for meetings, I formulater expected relationships in a set of five hypotheses. I formally state two assumptions that provide the foundation for investigating these hypotheses.

A1: An individual incentive structure causes competitive conflict management during the group process. (Manipulation check.)

A2: A group incentive structure causes collaborative conflict management during the group process. (Manipulation check.)

H1: Collaborative conflict management is positively correlated with consensus.

H2: Competitive conflict management is negatively correlated with consensus.

H3: Collaborative conflict management is positively correlated with the perception of high decision quality.

H4: Competitive conflict management is negatively correlated with perceived high decision quality.

H5: The perception of decision quality is positively correlated with the perception of strength of consensus. 
The two assumptions are a manipulation check to $s e 0$ if the two different incentive structures were able to produce the desired collaborative and competitive behavioss in the group process. Hypotheses 1 through 4 show the relation between the perceived behaviors of the group process and the group products of consensus and perceived decision quality. The last hypothesis is the result of the continuous debate over what consensus actually is. It just may be possible that the perception of having a good solution may be a part of consensus and should be measured as a part of the consensus scale. All data was collected via questionnaires.

\section{METHOD}

\section{Subjects and Group Composition}

The subjects were 7 male and 5 female engineering students of Virginia Tech. Subjects were either 21 or 22 years old and were randomly assigned to four groups. All groups consisted of three group members. Two groups were exposed to the group incentive structure, while the other two groups were exposed to the individual incentive structure.

\section{Procedure}

The experiment consisted of two phases. During the first phase, all subjects were told they were taking part in a decision making exercise. The proctor told them they would have to predict the order in which 12 jurors in a film would change their vote from guilty to not guilty. The subjects then watched the first 35 minutes of the movie " 12 Angry Men" with Henry Fonda. After viewing 35 minutes of the movie, the subjects individually ranked the order in which the jurors changed their vote.

In phase two, the subjects were divided into their respective groups and were told they were consensus groups and had to make the same decision again as a group. Just before the group interaction began, the groups were exposed to the two different incentive structures. The individual incentive structure was: "The individual in this group whose individual ranking is closest to the group ranking is rewarded with S10." The group incentive was: "You are in a contest with other groups doing the same experiment. The group which has the most correct answer according to the ranking in the movie is rewarded $\$ 10$ per group member." After the group had come up with the decision, all group members were asked to fill-out a 32-item questionnaire consisting of 28 Likerttype items and 4 open-ended questions.

\section{RESULTS AND DISCUSSION}

Due to the very small sample size $(n=12)$ in two groups per condition, the findings of this pilot study are not generalizable. However, results indicate that the type of questions I used to measure the various constructs have high internal reliability.. The Cronbach's alpha values for the different scales were:

$\begin{array}{ll}\text { Collaboration: } & \text { alpha }=0.66 \\ \text { Competition: } & \text { alpha }=0.79 \\ \text { Consensus: } & \text { alpha }=0.84 \\ \text { Perceived Decision Quality: } & \text { alpha }=0.88\end{array}$

I used a t-lest to test if assumpoions 1 and 2 were supported. The effects of the incentive structures to either produce competition collaboration were not supported $(t=.79, p>.05 ; t=.76, p>$ $.05)$. This means the manipulation did not work. It turned out both groups collaborated strongly. There are several possibilities why this occured. In the experimental procedure all groups were told they were consensus groups. This statement and the individual incentive structure may have had a confusing effect on the subjects. They might have focused on being a consensus group rather than on the individual incentive structure. Another reason may have to do with the sample of students I had in the study. All students were senior engineering students. Seniors may have known each other for four years reducing the possibility they were willing to compete against one another. Further, engineering students are taught to be problem solvers. One characteristic of a problem solver in my opinion is a collaborative orientation to gather as much valuable information as possible. Another reason for the failure of the manipulation may have been methodological. It is possible that the incentive of $\$ 10$ was too low for them to risk being competitive, or money was simply not an incentive for people to compete. 

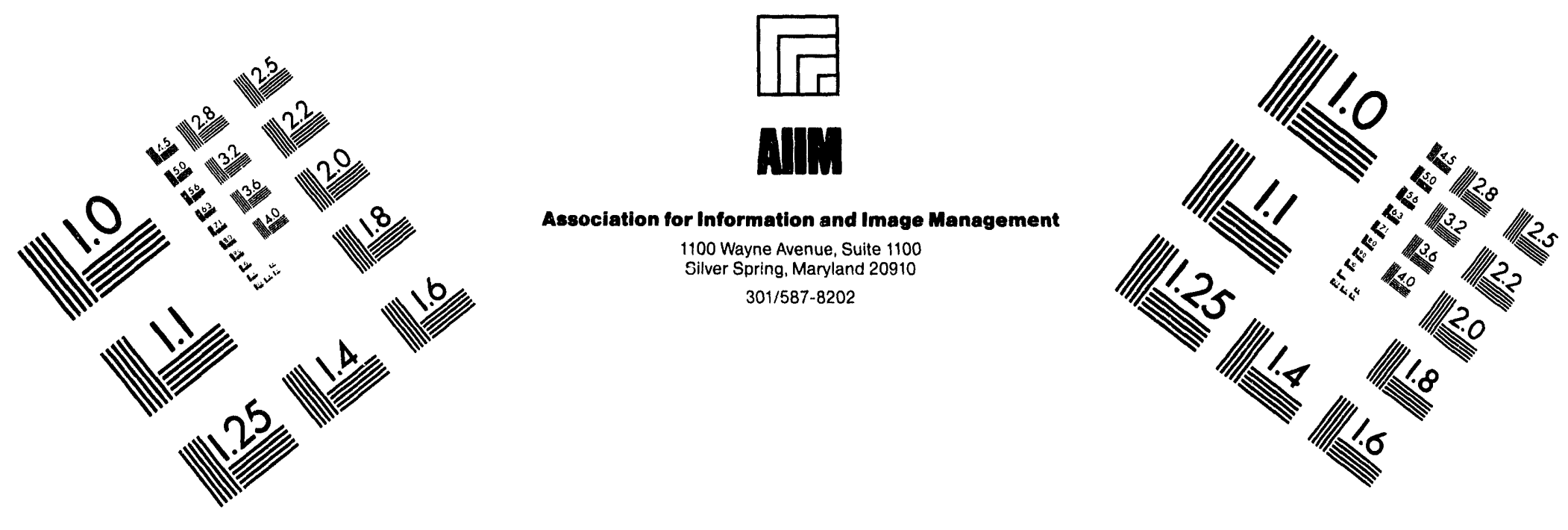

\section{Centimeter}

$\begin{array}{llllllllllllllll}1 & 2 & 3 & 4 & 5 & 6 & 7 & 8 & 9 & 10 & 11 & 12 & 13 & 14 & 15 & \mathrm{~mm}\end{array}$

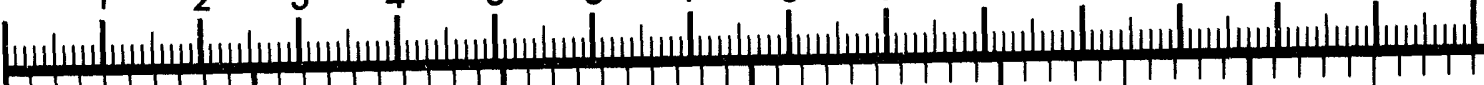

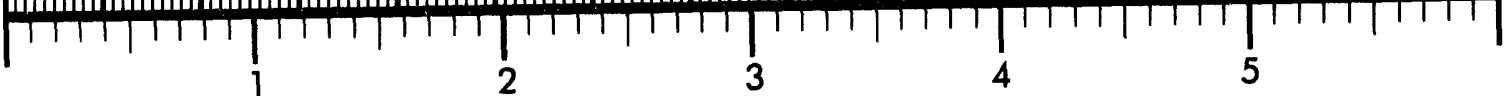
Inches
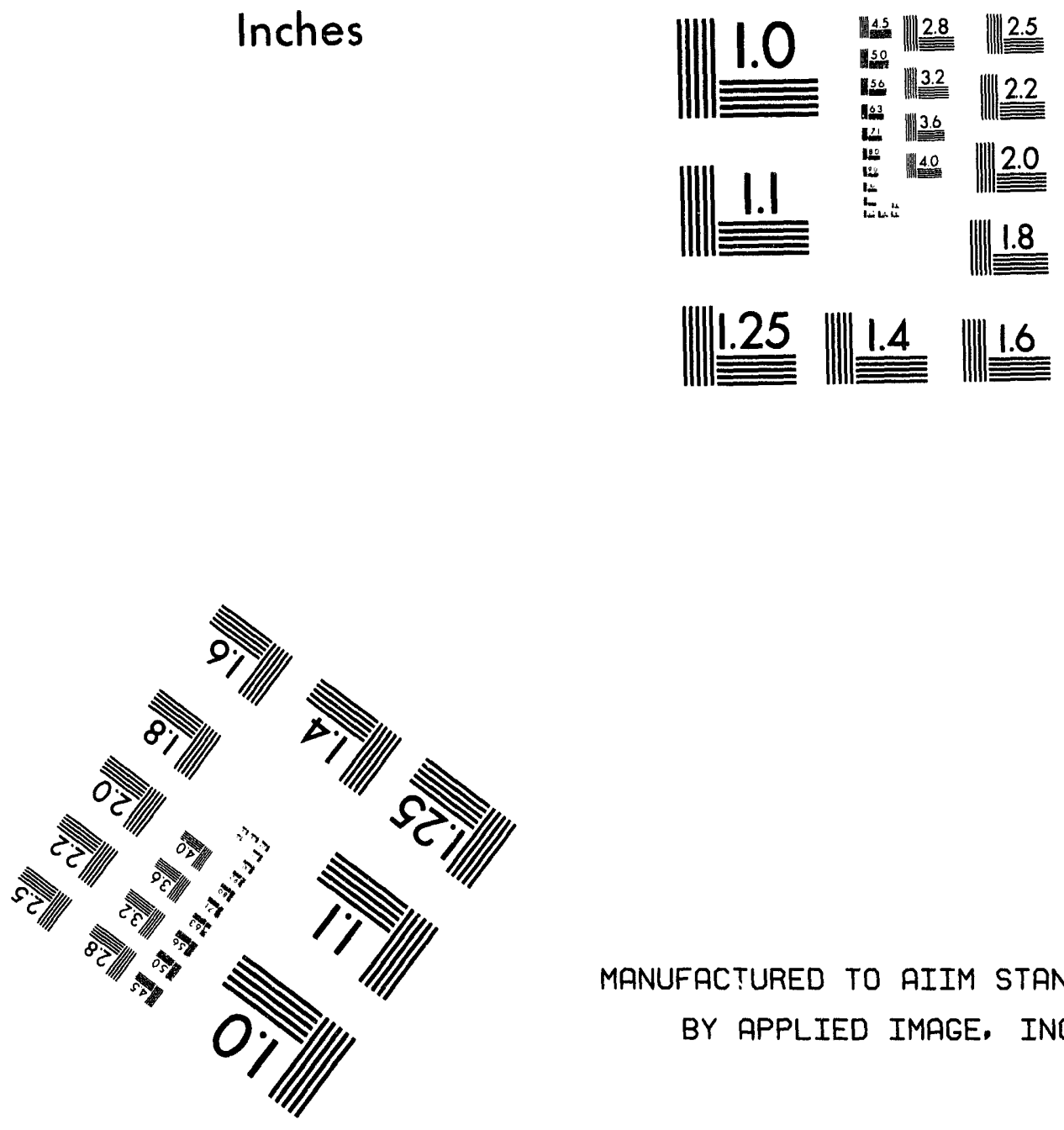

MANUFACTURED TO AIIM STANDARDS BY APPLIED IMAGE, INC.

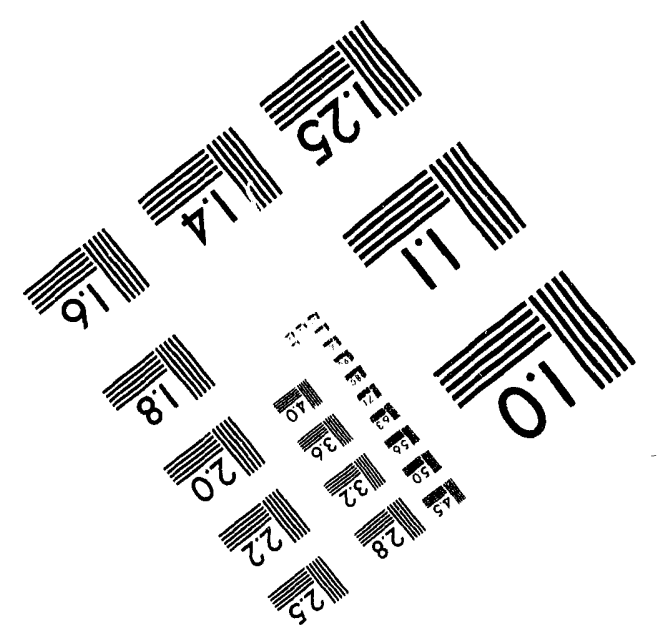



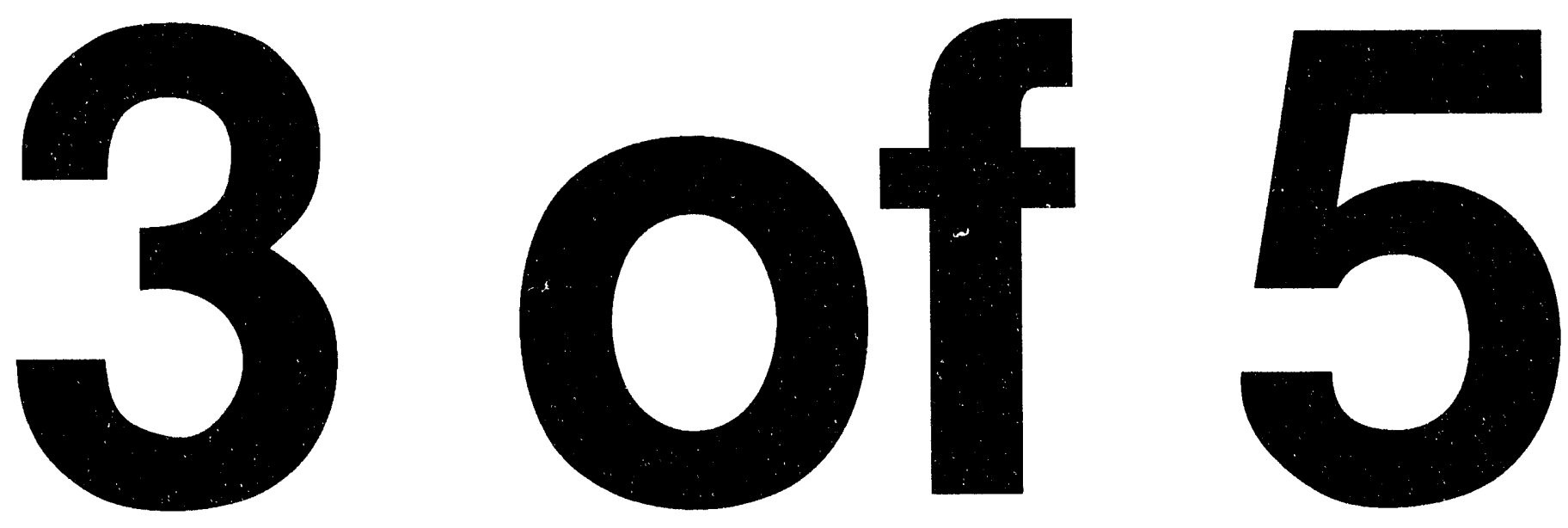
Hypotheses 2 and 4 related to the effects of competition and were not supported since none of the groups competed. However, hypotheses 1 and 3 were marginally supported $(r=.55, p=.07 ; r$ $=.55, p=.07)$. An increase in sample size will increase the power in the statistical test and may result in statistical significance for these two hypotheses.

Hypothesis 5 stated there was a positive correlation between perceived decision quality and consensus. This correlation was found to be significant $(r=$ $.93, p=.0001)$. This result indicates that it may be necessary to look at the phenomenon of consensus again and rethink if it may actually include the elements used to measure decision quality. These elements were "perception of opportunity to express views" and the "perception of generating sufficient alternatives."

\section{SUMMARY}

I have presented a model relating individual and group incentive structure to conflict behaviors, consensus, and perceived decision quality. I believe the methodology attempting to test this model in a laboratory experiment worked well with the exception of the incentive structure itself. Modifying the experimental procedure by not pressuring the subjects into consensus roles and changing the sample to include younger subjects who don't know each other might also help me in a large scale study to test my hypotheses better.

However, this study has indicated that rollaborative conflict management may influence the strength of consensus and the perception of the decision quality. Furthermore, the question must be raised to what extent the construct of consensus and perceived decision quality are really different. The results might suggest to measure perceived decision quality as part of the construct of consensus.

\section{ACKNOWLEDGMENTS}

The ideas presented in this paper represent the cumulative work of hundreds of people over fourteen years at Virginia Tech's Management Systems Laboratories. Professional managers from business and industry, and faculty, graduate students, and undergraduates from a wide variety of engineering and other disciplines have participated in the research and development of these tools and techniques. The authors thank them for their good work.

The preparation of this paper is funded by the U.S. Department of Energy (DOE) Grant No. DEFG02-88DP48058. Management Systems Laboratories thanks DOE's Office of Environmental Restoration and Waste Management for providing us a real-world laboratory for the research, development, and testing of state-of-theart management tools and the frameworks for understanding how to make them successful.

\section{REFERENCES}

[1] Thomas, K. (1976). Conflict and Conflict Management. In M. Dunnette (Ed.), Handbook of Industrial and Organizational Psychology, 889-935. New York: Rand McNally \& Co.

[2] Deutsch, M. (1973). The Resolution of Conflict. New Haven: Yale University Press.

[3] Schmidt, S.M., Kochan, T.A. (1972). Conflict: Towards Conceptual Clarity. Administrative Science Quarterty, Vol.17, 359-370.

[4] Katz, D., Kahn, R.L. (1972). The Social Psychology of Organizations. 2ed. John Wiley \& Sons, INC, New York.

[5] Simmle, G. (1955). Conflict and the Web of Group-Affiliarion. New York: Free Press.

[6] Pettigrew, A. (1973). The Politics of Organizational Decision Making. London: Travistock.

[7] Pfeffer, J. (1981). Power in Organizations. Boston: Pittman.

[8] Tjosvold, D., Andrews, I.R., Jones, H. (1983). Cooperative and Competitive Relationships between Leaders and Subordinates. Human Relarions, Vol.36, 1111-1124.

[9] Rabbie, J.M., Wilkens, G. (1971). Intergroup Competition and its Effects on Intra- and Intergroup Relations. European 
Journal of Social Psychology, Vol.1, 215234.

[10] Janis, I. (1972). Victims of Groupthink. Boston: Hougthon-Mifflin.

[11] Hoffman, L.R., Harburg, E., \& Maier, N.R.F. (1962). Differences and Disagreements as Factors in Creative Group Problem Solving. Journal of Abnormal and Social Psychology, Vol.2, 235-244.

[12] Wall, V.D., Nolan, L.L. (1986). Perceptions of Inequity, Satisfaction, and Conflict in Task-oriented Groups. Human Relation, 1033-1052.

[13] Johnson, D.W., Johnson, R.T. (1974). Instruction Goal Structure: Cooperation, Competitive, or Individualistic. Review of Educational Research, Vol.44, 213-240.

[14] Coser, L.A. (1956). The Functions of Social Conflict. New York: The Free Press.

[15] Thomas, W.K., Kilmann, R.H. (1974). Thomas-Kilmann Conflict Mode Instrument. Tuxedo, New York: XICOM, INC.

[16] Sherif, M., Harvey, O.J., White, B.J., Hood, W.R., Sherif, C.W. (1961). Intergroup Conflict and Cooperation: The Robber's Cave Experiment. Norman, OkJahoma: University of Oklahoma Press.

[17] Scheff, T.J. (1984). Toward a Sociological Model of Consensus. American Sociological Review, 32-46.

[18] Tjosvold, D., Field, R.H.G. (1983). Effects of Social Context on Consensus and Majority Vote Decision Making. Journal of Social Psychology, 500-506.

[19] Johnson, D.W. (1975). Joining Together. Englewood Cliffs, NJ: Prentice Hall.

[20] DeStephen, R.S.(1983). High and Low Consensus Groups: A Content and Relational Interaction Analysis. Small Group Behavior, 143-162. 


\title{
EXPAND QUALITY MANAGEMENT INTO THE CUSTOMER'S ENVIRONMENT TO ESTABLISH EFFECTIVE MEASURES AND STANDARDS
}

\author{
Harold A. Kurstedt, Jr. \\ Larry A. Mallak \\ Linda C. Pacifici \\ Management Systems Laboratories \\ Department of Industrial and Systems Engineering \\ Virginia Polytechnic Institute and State University
}

\begin{abstract}
I show how a structured management procesy can help establish measurements and standards for customer satisfaction. The key to establishing better

measurements of customer satisfaction is a structured management process proven to gain quality within an organization and expanded to involve the customer. My focus is on performing the functions and procedures to implement continuous improvement leading to TQM. I emphasize setting expectations, charting workflow, and defining indicators and standards to formulate quality. standards for customer satisfaction. I base my discussion on experience in a research organization-a state government entity--serving primarily federal government organizations. The management process I use seeks to improve customer satisfaction by reducing the opportunity to make errors and catching the small problems before they become big, unmanageable problems with destructive effects.
\end{abstract}

\section{INTRODUCTION}

In government organizations, we've traditionally measured customer satisfaction by counting complaints, administering questionnaires, and communicating through informal networks such as phone calls or personal visits. But these are merely perceptual measures designed to capture attitudes and dispositions toward aspects of product or service delivery and utility. We need more detined, concrete, meaningful, rich measures, and we can get these by expanding the quality management function beyond the organization and into the customer's environment. These higher quality measures give us better information on our processes so we can carefully tailor our products and services to best meet the customer's expectations.

The key to establishing better measurements of customer satisfaction is a structured management process proven to gain quality within an organization and expanded to involve the customer. We use a system of nine functions to help us implement the management process. I'll focus on setting expectations, charting workflow, and defining indicators and standards to formulate quality standards for customer satisfaction. Six other functions complete the management process. These functions maximize and ensure the effectiveness of the formulating functions.

Many govemment organizations, especially those I work with, don't necessarily n. ?nage physical things but, rather, information about those physical things. Government, especially in the United States, is becoming more concerned with its stakeholders--its customers. These stakeholders are demanding better quality from their government in terms of constituent representation, equitable decision making based on high quality and low-bias information sources, accountability for government and government contractor actions, and assurances of health and safety regarding those actions. Building the customer - the public and other stikeholders-into the governmental workflow builds in measures the government organization can use to gain better information about their processes and to make decisions about current or needed activities to satisfy those stakeholders.

Within an organization, both supervisor and subordinate wort together to practice the nine functions cooperatively to meet the organization's goals for its stakeholders. The supervisor-subordinate relationship drives the internal management process. Expanding the management process to the customer's environment changes the driving function. Both the provider and the customer are working toward the same end of providing a quality product using a quality process. There isn't a supervisor-subordinate relationship. However, there needs to be another type of intense coonerative relationship between people who are peers throughout the provider and customer organizations. The tonls used for setring expectations between provider and customer and charting workflows to define indicators and standards become the prime link to expanding the functions to the customer's environment. In this paper, I'll show how we can apply a structured management process to establish measurements and standards for customer satisfaction.

\section{OURAPPROACHTO TOTAL OUALITY MANAGEMENT}

The concept of rotal quality management (TQM), is more than just a program designed for specific aspects of operations and process control. This is a way of thinking about and approaching continuous performance improvement [1]. We can approach total quality management (TQM) from many different perspectives. We can understand TQM on the conceptual/theoretical level-a top-down approach. TQM can be articulated from definitions and descriptions. TQM can be practiced through functions and procedures implemented with leamed skills and techniques-a bottom-up approach. We believe a dualpath (both top-down and bottom-up) approach fills the long and arduous gap between a novice TQM 
organization and an organization with profound knowledge about TQM. On the conceptual level, we find our system worke through shared values of coordination and cooperation. Writing scoping agreements, charting workflows, logging data, developing indicators and standards, and organizing and portraying information are functions and procedures implemented through learned skills and techniques. My focus is on performing the functions and procedures to implement continuous improvement leading to TQM.

Often, the process of satisfying a customer is an inefficient back-and-forth process because of the need to clarify information, correct errors, obtain missing information, get approval, and communicate up and down the organization [2]. The process of satisfying a customer frequently becomes a reactive process. The management process seeks to improve customer satisfaction by reducing the opportunity to make errors and catching the small problems before they become big, unmanageable problems with destructive effects. The management process is predicated on continuous improvement, which White has called "the most powerful concept for guiding operating management" 3]. We want a proactive process to eliminate errors before they happen.

Our organization, Management Systems Laboratories (MSL), as part of a state university, is a state government entity subject to the laws, regulations, guidelines, governance, and cultural practices of a state government. MSL primarily works with federal government cabinet-level agencies, their field sites and contractors. So we are a government organization serving another government organization whose customers range from still other government organizations to the public and their elected and appointed officials. The organizations we serve have both internal and external customers. This paper reflects this experience.

\section{EXPANDING THE FUNCTIONS TO THE CUSTOMER}

Nine functions implement continuous improvement. The nine functions together represent a closed set addressing the formulation, execution, and verification of continuous improvement cooperative management. Expanding the management functions into the customer's environment requires systematically considering the effect on the customer and how the customer in turn affects the formulation functions. I'II describe the systematic interaction between the formulation functions and the customer.

\section{Eormulation Functions}

The formulation functions and the customer are affected by a series of interactions. The formulation functions affect the customer in several ways. First, the customer no longer holds sole responsibility for the supplied product or service and is no longer the only one doing something about managing its quality throughout the process. Expanding measurement into the customer's organization means both the provider and the customer are working toward the same end of providing a quality product using a quality process. Second, the customer has an additional reference point for the quality of its product based on the specifications of the provider, especially those specifications relating to what the customer does with the product. Third, the customer benefits from the provider's knowiedge regarding the supplied product. The provider should be able to recommend measures that more effectively capture critical aspects of product or service performance.

The customer, in turn, affects the formulation function. First, the customer gives input to the developmens or selection of internal measures based on its goals and objectives and on its expectations regarding performance. This requires communication of those goals, objectives, or expectations to the provider, so the provider can incorporate them into the expansion of the measurement and management processes. Second, the customer affects the internal measures by the particular interpretation of those measurements which may not be the same interpretation as that of the provider. Here, customer and provider should discuss their differing interpretations, noting the points of departure, and try to develop a single interpretation they can both agree upon. Third, the customer can also affect the internal measures by the implementation of those measures. Specifically, the provider may suggest daily production be logged and note critical incidents which disnupt production. The customer may feel production needs to be logged weekly or for each shift and may not take the time to note the critical incidents. Both the implementation and the interpretation of the measures should be agreed upon by the customer and the provider. This mutual agreement facilitates effective measurement so the data and information have meaning for both parties individually, thereby, allowing meaninghul comparisons.

Formulation functions help the manager isolate crises and plan what to look for to identify crises and their causes. Misst crises are preventable, yet we allow them to happen. We choose to ignore a signal that tells us we've done something (or haven't done something).the type of signal that can come back to haunt us. The crises happen because we don't take action to keep them from happening. Other crises are never preventable. They will happen no matter what we do. To help in proactively managing to avoid crises, I'll discuss the three functions making up formulation activities: setting expectations, charting, and defining indicators and standards.

Settine expectations: Productive workers need to know what is expected of them. Setting expectations transsates long-term plans and goals into daily, weekly, and monthly expectations. We must interpret the long. term plans and goals to generare the qualitative, quantitative, and milestone expectations each person can do to contribute to the larger organization's plans or goals. Different tools can document expectations for both supervisor and subordinate so the expectations are nailed down. Objectives for production, scoping agreements for projects, and priority lists and checklists for varied tasks are three tools to set expectations for daily or weekly timeframes within one's own organization.

Setting expectations in the customer's environment is critical for evaluating the quality of product and service delivery. The process of arriving at these expectations in the customer's domain requires 
processes emphasizing mutual cooperation. A scoping agreement (Figure 1) gets down on paper a joint statement of expectations for both parties. The scoping agreement is a valuable tool for projects within an organization, but increases in effectiveness as parties to the agreement become more diverse either in background or in organization.

1. Identificaton of Generd Informanon

1) Idenufy partes of agreement including doer and receiver.

2) Idenafy the tast wo be done or the reatit of the apreanent.

3) Idenafy whas injeared the apreecent (e.8., concret management plas. updaw moseng).

4) State what tanpble outcome is needed and/or expected by receiver.

5) Idenafy une tunding vehicte or pach.

11. Descripoion of Tast and Wha is of Follow the Task

1) Describe whas the ones or resule looks liks (be physica).

2) Sute whas follows from complecing the usts-whas the cast leads 10.

II. Description of Does and Recaiver Responsibilities

1) Desersbe what is pronded by does.

2) Describe what is needed troos receiver.

IV. Description of Bectround and Supporing Informusion

Figure 1. A scoping agreement reduces the equivocality of an expectation-setting process between customer and provider.

A scoping agreement can help improve product and service delivery given the diverse interests and goals existing between the provider's organization and the customer's organization. Items in the agreement state the responsibilities of both parties (doer and receiver of actions) and the expected tangible outcome. Stating what the task leads to helps in capturing a system focus so we don't suboptimize by making this task incompatible with subsequent tasks. As a provider of a service, we must be concerned not onty with the customer's initial receipt of the service, but with what transformation processes they apply to it. Expanding total quality management into the customer's domain is an expansion of our organization's boundaries beyond the typical "send to customer" milestone and, thereby, broadens our system focus.

Charting: The second function, chartine, identifies every entity in an organizational unit and shows the relationships among the subentities. Charting can identify indicators to be measured, delimit responsibilities, and identify interfaces with other units to enable the manager to determine ways to streamline or change the workflow. Worlflow charts, Gantt charts, organizational charts, and charts of financial resources are used for different types of work. The variations of charts used as tools for laying out the unit's work. and resources is almost limitless. Clearly, charting gives us the visibility to determine alternatives and see cause and effect in measuring performance and improving the work and use of resources.

Charting the provider organization's workflow doesn't end when the product or service is delivered to the customer organization. The success of a product or service is ultimately determined by the end user and is partially determined by midstream customers who perform a transformation process. What does the customer organization do with our product or service? We must expand our quality management efforts to include charting what the customer organization does with our product. We must expand our efforts far enough to meet the customer's expectations and meet their standards. Deming writes of the need to break down barriers between staff areas as one of his principles for the transformation of American management. He gives an example to illustrate the need for teamwork and cooperation: "Everyone was doing a superb job, and had been doing so for years. Nobody had any problems. Yet somehow or other the company was going down the tube. Why? Each staff area was suboptimixing its own work, but not working as a team for the company" [4]. We go a step further and extend our charting initiatives beyond our internal staff areas to include the customer organization.

I see two dimensions to charting processes in the customer's domain. The first one is common to all charting: to what level of detail do we wish to carry the charting? We could chart ad infinitum if we were to capture every task and every contingency. We must decide the level of detail for charting to be effective and supply meaningful indicators, standards, and management information. The second dimension is how far to carry the charting into the customer's domain. We should establish this length so we can help them tailor their products and services to best meet the needs of the customer given the impact and benefit of our involvement. Of course, we must have charted the product in our own organizations first for the charting process in our customer's organization to have meaningful and valid reference points. In addition, some indicators may not be in the customer's organization.

We meet some obvious barriers when trying to chart product workflow in our customer's environment. These barriers aren't unique to charting, yet they pertain to implementing most functions of the management process. The necessity of customer-provider cooperation in charting and charting's position in the management process surface these barriers early on. First, we don't have the authority to observe and collect data for charting as we do in our own organizations. Second, we must convince the customer of the value of allowing us (or them) to chart the product workflow as part of our total quality management initiative. Third, if we rely on the customer to supply us data, we must sift through their biases and institute mechanisms to ensure we have valid data.

Definineindicators and standardy: Charting worlfiow in our own organization and into the customer's organization supplies the preliminary analysis for defining indicators and standards. By identifying the critical points in the charted workflow, we can target specific indicators to measure in our efforts to improve product delivery and performance. quality. Flow charts help the manager understand the work and choose the right indicators. We set indicators by scrutinizing the charts and deciding the important things to measure. Comparing indicators against standards provides 
information on the performance of the unit. To gain control, the manager must establish a reference point for every indicator. Through the reference point, the indicators have the potential to become information about performance. When a reference point is well understood, attainable, and considered significant, it becomes a standard. By comparing indicators to standards, the manager can find indications of successes and failures and impending problems. Milestones are indicators for projects. Objectives are indicators for people. Clearly, we can't evaluate or manage what we don $t$ measure; and measuring the right things is critical to continuous improvement.

Having chared our workflows and developed indicators and standards for points within our organizations, we must now identify indicators relevant to the customer's use of the product. The indicators we choose should be tightly coupled to what we see as critical success factors. Charting helps us gain visibility of both the provider's and the customer's processes. Most managers will neither have the time nor the background for choosing indicators valid with respect to desired goals, but will choose indicators they feel are useful for success. Knowing these indicators will work their way into reviews and appraisals raises the concern about their validity in litigation or other contested cases. Establishing indicators using methods of job analysis [5] will help in choosing those indicators having high criterion-related validity. Not only will proper selection of indicators keep us safe legally, we should also have confidence in knowing our indicators are valid and good predictors of the criteria we have linked them to.

A more subjective yet valuable and rich means of gathering information on indicators and standards is through focus groups. A locus group is used frequently for marketing purposes to support new product development and testing with representatives of the actual user. Focus groups employ a neutral facilitator to solicit feedback regarding the product or service. More generally, focus groups focus on a limited set of issues or topics under the direction of a facilitator who promotes interaction and keeps the discussion on track [6]. Observations made by the facilitator and other observers supply subjective data to consider along with a scientifically designed mail or telephone survey or interviews. Focus groups serve as an additional tool for gathering information on indicators and standards.

In conducting these measurements, either on the customer or provider side, we need valid reference points or standards One way to derive these reference points or standards is through a benchmarking proceses. Benchmarking is "the search for industry best practices which will lead to exceptional performance through the implementation of these best practices" [7]. The key to benchmarkine, and to the management process, is the focus on implementation-getting something done. As an example, different industries and jobs within those industries have different demands on their time and therefore experience varying levels of urgent activity. Looking at the percentage of time government managers in a deputy assistant secretary level spend on urgent or crisis activity and comparing this across other similar government organizations and against comparable positions in private industry gives us an idea of how we compare. An example of a benchmark is the desired percentage of a workday spent catering to crises coupled with a plan to achieve that desired level.

For our purpose of setting standards, we want to involve representatives of the customer's firm who have sufficient knowledge and responsibility to decide on effective measures that can be readily implemented. Standard-setting requires research and technical expertise to choose standards that can stand up to legal and other tests. Again, as in defining indicators, we want standards that give us valid information about our indicators and ultimately about the criteria those indicators are based on.

Standards, whether in-house, from industry associations, or from the government, supply us with a range of choices. Competent reviewers from the provider organization and from the customer should decide which standards to choose and whether to modify them based on their experience, training, and expert judgment. Indicators and standards should be systematically selected to ensure they are properly linked to the criteria they intend to measure and that a valid process is followed. Indicators and standards, properly selected and implemented, represent a powerful means of predicting success.

\section{Execution Functions}

Collecting and logaing data, converting data to information, and organizing and presenting information are three functions used to execute activities in continuous performance improvement.

Collectine and logring data: We gain visibility of our workflow through measuring and logging items we've identified in the defining indicators and standards function. When we gain visibility of exactly what's going on in detail, we can control the worloflow and better focus on what needs to be done. Collecting data and choosing the right data to collect are important. The measures we define and $\log$ will be reported in the management information system (MIS). Logs are designed for production activities and projects (workstation activities). In real life, people need constant encouragement to log, but if done properly, logs will bring great rewards. Properly done logs contain only dats the keeper and the user of the logs know are valuable for improvement. Well-designed logs are easy to keep and ure.

Once we've defined indicators and standards for the product workfow, including those indicators and standards inside the customer's organization, we need to develop ways to capture or log the significant data in the customer's domain. We can take two different approseches to logaing: we can either work with the customer to heip them develop loos or we can let the customer do their own loge. Working with the customer is preferable in developing loog because it gives us an opportunity to dovetail our measurements with theirs, thereby, facilitating comparisons against the same reference point. To develop logs with our customer, we can either assist them in deciding how and what to log or we can share the logs we've developed for our organizations and let them apply these logs to their organizations. Alternatively, we could have them develop their own loga. This has the potential of improving ownership, but leaves compatibility and comparability of measures to chance. 
What we'd most likely see in practice is a set of informal communication linkages in various levels of the organizations. Our organization would drive the process through telephone calls to corresponding people in the customer's organization. We'd call and tind out, for example, if they received the material, and mark our logs. The data for these logs wouldn't be captured realtime, but we would schedule the frequency of phone calls to match the need for the data collection. For example, material delivery in one part of the production process may need to be tracked daily; elsewhere it may only need to be tracked weekly.

Convertine data to information (MTS): Entries in logs are data. Since we can't make decisions on data, we convert data to information through the management information system, the MIS. The MIS comprises aggregations and exceptions. In the MIS we aggregate data and compare indicators or aggregated data to reference points or standards to make information. The aggregations and exceptions from the logs must be carried to appropriate management levels where decisions can be made. We highlight exceptions in the data and those found when comparing indicators to standards. Aggregating data and highlighting exceptions help supervisors get the information they need quickly. Where appropriate, we can also use statistical analysis to aggregate the data. If we don't have enough data for a statistical analysis, we can rely on frequency distributions and other methods of data-reduction techniques. Production, projects, people, and financials provide data for the MIS.

As mentioned earlier, the conversion of data to information takes data captured on the logs, compares it to a standard, and this comparison results in information. We want to expand this conversion process-the MIS-to the customer's organization. An analysis of information flows to and from the customer will help in the design of the MIS. The MIS should be linked at the bottom and at the top of the organization. At the bottom of the organization, I'm referring to the operational data specific to products and services that must be shared to allow data-to-information conversion. At the top, l'm referring to aggregate reports on wide. ranging aspects of the business, often portrayed in financial terms.

Organizine and presenting information: The management process we espouse depends on daily low, MIS, and reviews for production. For projects, logen, MIS, and reviews are maintained weekly. Also, the data from daily reports should be agregented to show trends for the week and presented with project information as weekly reports. The MIS for projects can be based on a master schedule. Monthly review books allow the manager to see aggregated reviews of the weekty reports, project progress, financial information, and people information. The daily, weekly and monthly MIS review format needs to stay consistent so the managers can concentrate on substance.

Once we have the information from the MaS, we need to communicate it to interested parties. We do this through organizing and presenting the information using a variety of information portrayal techniques designed to communicate effectively given the nature of the information and the characteristics of the audience, particularly the cognitive style of the primary audience. The frequency of reporting information from the MIS should match the needs of the operations being reported and the demands and priorities of those reviewing the reports. The frequency of reporting should parallel the review cycle to be most effective.

\section{Verification Functions}

Three functions verify the work in continuous performance improvement. Reviewing status and progress, exercising personal effectiveness, and appraising performance complete the nine functions. A discussion focusing on the formulation functions is incomplete without closing the loop (Figure 2) by showing how to review and appraise the information produced through the previous six functions. All the functions work together synergistically and maximize the effectiveness of formulation functions. I've focused on the formulation functions of setting expectations, charting, and defining standards and indicators. The execution functions, discussed earlier, result in information to feed the review and apprs isal functions.

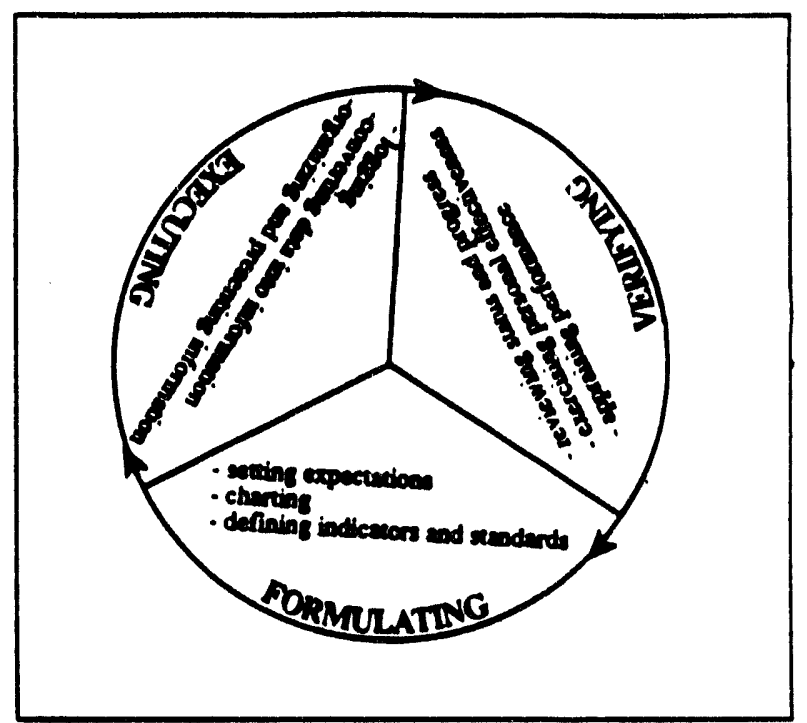

Figure 2. Reviews and appraisals complete the cycle begun by the formulation functions.

Bevieming aratur and progrear: Daily, weekly, and monthly reviows are tace-to-face meetings between supervisor and subordinute. The guarantee of regular and frequent times to meet with the supervisor can relieve staff rension and frustration. A daily review focues on daily expectations, status, and progress and should take no more than three minutes. Brief reviews (or any lind of meeting) depend on 1) no interruptions, 2) recomiring and rescheduling topical discussions, and 3) prepared information from the logs or MIS. When each person has a log, it takes only a few minutes to review. The weekly review sums the daily reports for a week through a mana rement summary. Why review weekty as well as daily? The weekly reviews help us more readily identify trenda. In monthly reviews, we summarize information for the unit to integrate the data for production, projects, people, and financiala. We should compare the monithy review of actuals to the operating plan projections. The monthly review also 
looks forward to next month and helps us in forecasting for the rest of the year.

Review the reports with the customer. As stated earlier, these reviews should be crisp, structured, and frequent. The schedule for review should match the reporting frequency for most effective use of a total quality management system. When the reporting and reviewing frequency schedules are different, we're either reviewing more than one report at a time and trying to combine the report mentally or we don't have anything new to review at every other meeting.

Reviews should be conducted at all levels of the organization. Daily reviews on operations should be conducted between first line supervisor positions at each organization. Whether or not reviews should be conducted daily at middle and top management levels is debatable. What is not debatable is that these reviews should occur regularly-at least once if not twice per week--at all levels in the organization.

By expanding the quality management function into the customer's environment, we lose the clear ability to carry on the supervisor-subordinate analogy. The customer, acting as a supervisor, probably won't initiate reviews. So our organization, as the subordinate, will have to initiate the reviews.

These reviews are mini-appraisals for quick feedback correction. Reviews should note status and exceptions. The subordinate role is typically interested in "where we are" with the supervisor role being interested in "where we want to be." The tone of these meetings is on feedback and help and joint ideas on "how to get there."

The reviews should be conducted at the same time and same place each day. There should be safeguards against interruptions, and the reviews should identify topical meetings rather than become topical meetings Most often, the two people reviewing status aren't the best people to discuss a topical matter. The relevant expertise should be assembled to analyze and resolve the topical matter.

\section{Exercisine personal effectivener: We define} personal effectiveness to encompass time manarement and communication. Exercising personal effectiveness in the customer's domain means helping them manage their time better, working with them in a time-eficient manner, and improving communication procesces. The ability to discern between the urgent and the important is crucial to time manarement. Too many times urgent activities pull us away from our important activities. We need a management process to reduce these urgent activities that rob our time and diarupt our schedules. Instead of reacting to the urgency of crises, which can be satisfying and even rewarded by mamy organizations, providers and customers must develop the ability to be proactive. We may need to examine our customer's reward systems and organizational culture to see what the consequences of crises and reactive management are. At first, we'll endure the necessity of reacting to crises before we stabilize them and implement the management procese.

Communication enhances personal effectiveness when we have the opportunity to communicate the improvements both inside and outside our organizations.
We should take advantage of effective communication to reduce uncertainty and equivocality to shift our reacting to crises and problems to more proactive activities.

Appraising: Appraisals complete the nine functions. Within an organization, appraisals evaluate how well supervisors and subordinates set their expectations and how well they meet these expectations. When expectations aren't met, we have four alternatives: 1) adjust expectations, 2) train the doer to better meet expectations, 3) improve the process and system supporting the doer, and 4) improve the match between the doer and the expectation. Through regular open and frequent reviews, the supervisor and subordinate can objectively evaluate the four altematives on a daily basis, select a course of action, and follow-up and follow. through to meet realistic expectations. Clearly, appraisals aren't an annual rite. Appraisals must be continuous for improvement to be continuous.

We conduct mini-appraisals in our regular reviews. Appraisals follow up the reviews on a monthly or quarterly basis. Along with the customer, we identify trends and accumulations from the logs. To.best support our management process and quality management function, appraisals should be supportive, open and honest, non-threatening factual, uninterrupted, and organized. We should know clearly what is to be covered in the appraisal.

In our work with a federal govemment agency, we conduct informal appraisals at several levels. At the top level of most organizations, the chief executive is often too busy to regularly review status with the customer because of myriad demands on time. In the MSL director's stead, the appropriate laboratory director calls a high level official of the government organization to get a reading on how we're doing and what we can do better. These interactions must be data-driven and not rely just on feelings of success or progress. Reviews must have a measure of consistency and provide a means of seeing trends.

Two issues come into play in MSL's appraisals. First, the president-to-president connection is often umworkable because of time demands and priorities; the process will fall down if left to the presidents of the organizations. Second, we don't have logs and an MIS in place yet to support the process. Our process is still valuable for gothering subjective data and affective responses, but its value increases if we have $\log$. Our chief task here is to comince the higher level people to begin logring their interactions with the customer.

\section{CONCLUSTON}

Implementing the management process in a govemment orgnnization first requires attention to formulation functions. Frequent reviews and honest appraisals support quality efforts in both the provider and customer orgnizations. In conclusion, I present two general statements about manngement process implementation.

Doa't start unlans you intend to do it and stay with it. Figure 3 likens this conclusion to dancing with a bear. Done correcthy, the management process will surface negatives. Bringing negatuves to the surface shouldn't 
Overwhelm us-it should inspire us to start fixing them. We should leave the negatives submerged unless we intend to fix them. I call this the dilemma of management process implementation-we can't implement the process unless we plan to follow through or we'll have a worse situation on our hands than when we started. The process will work against us if we surface the negatives and don't fix them. We can't assume ignorance. We must carry the process beyond the negative stage. The customer is more likely to stay with us if we begin fixing the negatives because 1) our service delivery quality is known and we're improving our service delivery, intending to get better and 2) once we start fixing their problems the unknown becomes how much better we're going to become. With another provider, what they have done, what they can do, and what they intend to do are all unknown.

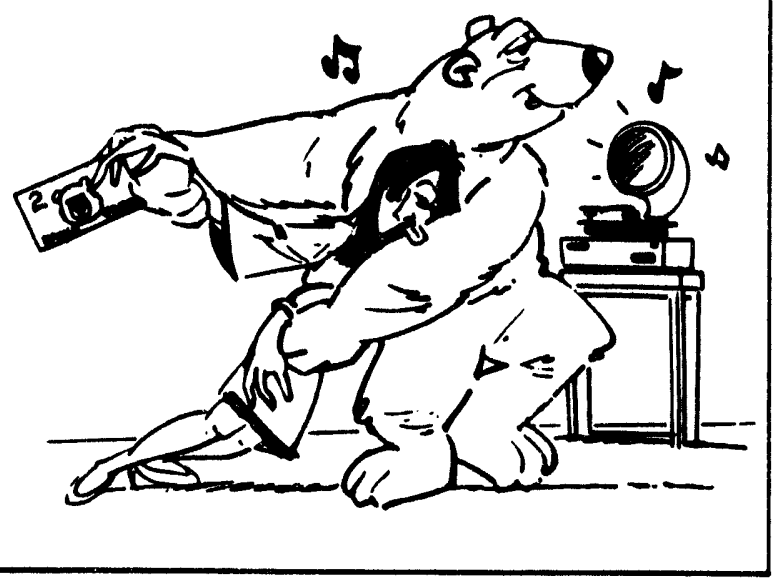

Figure 3. Once you start dancing with a bear, you can't stop just because you get tired.

Surfacing negatives can help us provide better service to the customer. Customers can tell us why they left us (giving us feedback on our performance so we can fix it). Otherwise, they leave us not telling and not knowing why they made that decision. So, customers who know the negatives 1) can tell why they left, or 2) won't leave when we're fixing their problema. A new provider would have too much start-up time and learning time that such a decision wouldn't be cost. effective, unless there is no evidence of continuous improvement.

Be procectlve. This is what building quality into the customer's environment is all about. We need to become better and better, faster and faster. We need to drive the management process beyond the reactive phase and into problem solving. We need to inprove before the fact. By the time the customer has complained to us, the problem has gone too fas. We need indicators and standards in place in the customer's organization so we can have earty, frequent, and accurate readings regarding our products and services. The more we can find out while the product or service goes through the customer's organization, the more able we'll be to continuousty improve our processes to best suit the customer. And the customer's job becomes easier because they can shift their attention away from our product or service and onto theirs. That is, after all, why they're in business. The management process helps our customer stay in business and stay on top of the business so we can too.

\section{ACKNOWLEDGEMENTS}

The ideas presented in this paper represent the cumulative work of hundreds of people over fourteen years at Virginia Tech's Management Systems Laboratories. Professional managers from business and industry, and faculty, graduate students, and undergraduates from a wide variety of engineering and other disciplines have participared in the research and development of these tools and techniques. The author thanks them for their good work The, pronoun "I" is used throughout to separate all of us using management systcms (we) from the author (I). However, please recognize "I" is more than one contributor to this work.

The preparation of this paper is funded by U.S. Department of Energy (DOE) Grant No. DE-FG0288DP48058. Management Systems Labioratories thanks the DOE's Office of Environmental Restoration and Waste Management for providing us a real-world laboratory for the research, development, arid testing of state-of-the-art management tools and the frameworks fo: understanding how to make them successtul.

\section{BEEERENCES}

[1] Mallat, L A, Kurstedt, Jr., H. A, Welch, K. A, Geller, E. S. and Kurstede, P. S. "Managing the Shift to a Productivity and Quality Culture," in D. J. Sumanth, J. A Edoanmwan, D. S. Sink, and W. B. Werther, Jr. (Eda) Productiviny and Qualiny Managemens Fronticers-III, Norcross, GA: Institute of Industrial Engineers, 1991, pp. 456462.

[2] Dorman, G. C. (1988). Go with the Flow-. Measuring Information Worker Quality. In Total Quality Pefformance: Highlights of a Conference, L Schein \& M A Beman (Eds.). New York: Conference Board.

[3] White, B. J. (1988). Accelerating Quality Improvement. In Total Qualiny Performance: Highlighs of a Conference, $L$ Schein \& M. A Berman (Eda). New York: Conference Board.

[4] Deming W. E (1986), Out of the Crisis. Cambridfe, MA: MIt Center for Advanced Engineering Study.

[5] Sparks, C. P. (1988). Job Analysis. In Readings in Personnel and Human Resource Managements (3nd Ed, RS. Schuler, S.A. Youngbload, \& V.L Huber (Eda), St. Paul, MN: West Publishing.

[6] Stewart, D. W. Shamdasani, P. N. (1990). Focus Groups: Theory and Prectice. Newbury Park, CA: Sage Publications.

[7] SunHealth Enterprises Inc. (1991). Benchmarking the SunHealth Way. (internal document). 


\section{AUTHHOR BIOGRAPHIES}

HAROLD A KURSTEDT, Jr. is the Director and founder of Manapement Systems Laboratories, a research arm of Virginia Tech's Department of Industrial and Systems Engineering. He conducts research on the use of management tools, participative management processes and techniques, propram and project management and organizational culture. He has been with Virginia Tech since 1974 and is the Hal G. Prillaman Professor of Industrial and Systems

Engineering. He received his B.S. from the Virginia Military Institute in civil engineering and his $\mathrm{Ph}$. D. from the University of Illinois in nuclear engineering. He has served on the mechanical, nuclear, and industrial engineering faculties at the Virginia Military Institute, the Ohio State University, and Virginia Tech. He has been chairman of nuclear engineering at Ohio State and Virginia Tech. He has had extensive industrial experience at Babcock and Wilcox Co., the Ballistics Research Laboratories, Industrial Nucleonica Corporation (now AccuRay), Management Horizons Data Systems, and Systems Research and Applications Corporation.

LINDA C. PACIFICI is a Research Associate at Management Systems Laboratories. Her responsibilities include coordinating and preparing academic papers and presentations, assisting with research needs and managing the director's undergraduate and graduate clasees at Virginia Tech. She received her B.S. in sociology from Virginia Tech and an M.S. in curriculum and instruction from Radford University. She has taught in private and in public schools in Montgomery County, Virginia and has presented papers on teacher empowerment.

LARRY A MALLAK is a Research Associare with Management Systems Laboratories at Virginia Tech He assists in the labs' research activities and holds responsibilities for academic papers, presentations, and proposal writine. He received his B.S. from the

University of Illinois and his M.S. from Virginia Tech, both in industrial engineering. He is currently working on his $\mathrm{Ph} . \mathrm{D}$. in management systems enqineering at Virginia Tech. He's worked in both academia and industry, having held positions with SunHealth

Enterprises, the University of Wisconsin-Madison, and Ohio State. He is a member of the Institute of Industrial Engineers, the Academy of Management, the American Society for Engineering Management, and a Maw Media Fellow of the American Aseociation for the Advancement of Science. 


\title{
THE, ROLE OF LEADERSHIP IN IMPLEMENTING TOTAL QUALITY MANAGEMENT
}

\author{
Harold A Kurstedt, Jr. \\ Kathryn A Welch \\ Larry A. Mallak \\ Management Systems Laboratories \\ Department of Industrial and Systems Engineering \\ Virginia Polytechnic Institute and State University
}

\begin{abstract}
Recent trends in quality-driven culture change have provided much discussion about how to achieve quality improvement but insufficient attention to the roles of participants in the change. Three such roles (leader, champion, and cheerleader) are the focus of the present paper. ['ll argue that the presence of individuals playing all roles heightens the potential for quality improvement through organizational culture-change efforts.
\end{abstract}

\section{INTRODUCTION}

Quality improvement and quality culture are terms increasingly heard in all types of oryanizations, especially in government organizations. Limited financial resources and increased demands by stakeholders require government organizations to become more effective and efficient than ever before. Recent trends in quality-driven culture change-whether Total Quality Management (TQM) or "Big Q"-have focused on recommendations for roles organizational members can play. However, the complexity and diversity of culture-change roles have not been fully explored. The quest for quality in industry and government today requires leaders--leaders who are champions and leaders who are cheerleaders. Leader, champion, and cheerleader are three roles involved in the promulgation of culture change.

Organizational culture is a state characterized by values, norms, and traditions $[2,14]$. "Culture is learned through a variety of explicit and implicit mechanisms" $[14$, p. 221). Role theory $[5,7,11,13]$ explains the social psychological context for such change. Specifically, role theory suggests that human social interaction is a function of roles people assume or "play." However, despite the sugrestion of expectations accompanying each role, thie theory permits individual variation in the way the pars are "played."

A number of relevant questions arises. What roles do leaders play on the culture-change stage? What roles do champions play? Is the champion ahways the lender? What leadership skills do champions need? How does a champion work in a top-down versus a bottom-up approach to culture change? How are cheerleaders different from champions? In this paper, I'll examine these and other issues regarding the implementation of beneficial culture change in government organizations. I'll define the roles of leader, champion, and cheerleader. I'll examine how different roles influence the change to a productivity and quality culture against the backdrop of several organizational examples. In one case, beneficial culture change occurted probably because it had a champion. In another, beneficial culture change didn't occur because there was no champion. Several other relevant examples will be highlighted. First, I'll brielly define some relevant culture-change roles.

\section{DEFINING THEROLES OF LEADER, CHAMPION ANDCHEERLEADER}

Leadership is a "system of relationships and constraints as well as opportunities" [6, p. 179]. This definition underscores the current view of leadership as an interactive or bi-directional process. Hollander and Offerman [6] identify leader-follower subsystem constraints as task requirements and expectations and commitments of followers. In this interactive view, leaders provide things followers want (e.g., information, rewards, pay, resources, and leadership).

Followership involves the empowering of the leader by followers. Followers provide things leaders need (e. p, quality work, respect, cooperation, and followership).

A leader may be distinguished from a supervisor in the following way. A supervisor is a job title specifying certain directing behaviors to be performed by the incumbent [10]. On the other hand, leadership suggests how (in a less tangible way) the job must be done [10]. We define a manager as anyone who uses information to make decisions afiecting what he or she manages. I believe a leader is a role decision makers may play. A leader brings vision and judgment to the workplace [1].

To facilitate quality-driven cuiture change, it's not enough for leaders to tell followers to "do it right the first time" [3]. Organizations need leaders who adopt program champion status $[8,12]$. Champions are catalysts for change. By contrast, the cheerleader takes the catalyst activities to a greater level of intensity than does the champion. Cheerleaders act out and promote behaviors congruent with the new culture.

Cheerleaders may display more outward enthusiasm than champions. However, cheerleaders may not have internalized the beliefs, values, and traditions of the quality culture to the same extent that champions do. I'll argue these roles may coexist within the same individual. 


\section{LEADING OUALITY.DRIVEN CULTURE CHANGE}

To produce quality culture, management and supervision are not enough. Leadership is essential. In shor, TQM requires the careful orchestration supplied by leadership to create a quality culture. As indicated earlier, this orchestration can only be accomplished by a leadership that knows where it's going, communicates those intentions, positions itself favorably with both internal and external forces, and empowers its own work force [1]. By communicating a vision of the desired change and encouraging participation and commitment at all levels in the organization, leaders can use empowerment to change values and traditions.

All members of the organization must be invited to shape the new productivity and quality culture. Despite the interactive nature of relationships in a dynamic system, we often forget that recommendations for culture change need to parallel the dynamism of the target organization. Thus, it makes little sense to make only top-down (from the ieade: down)

recommendations for culture change. Although it's prudent for leaders to gain attention through vision, realize meaning through communication, earn trust through guidance, and build confidence through understanding of success and failure [1], they must concern themselves with the crucial role of followers.

We often forget the leadership process is not just a matter of supervisors influencing subordinates. In a variety of ways, subordinates influence their supervisors. Subordinates influence supervisors in much the same way supervisors influence subordinates--through physical evidence and through example. If the subordinate is productive, is on-time, and produces high quality work, the supervisor will be influenced. Supervisors don't want admonitions or platitudes. They want evidence of performance. So, subordinates should practice guality, thus influencing supervisors to see quality. Quality-driven culture change requires a bottom-up detail-oriented approach.

However, to change to a quality culture, what's needed is neither a bottom-up approach alone nor a top-down approach alone, but rather a bi-directional or dual-path approach (top-down and bottom-up). By topdown and bottom-up, I'm talking in terms of both function and organization. Most people recognize the top-down functions such as projecting a vision, defining the organization's reality for followers, and planning. In functional terms, bottom-up leadership occurs at the smallest partition of a functional domain. Designers of quality improvement can build a step-by-step context ior improvement. Most also recognize the top-down organizational areas such as the chpin-of-command, and legitimate power. There is also a bottom-up approach in an organizational sense. Here, efforts focus on the lowest level of the organization, that is, efforts occur from individual follower to the highest leader. Qualitydriven culture change must also be top-down and bottom-up. Top-down and bottom-up approaches work together [9]. As an organization's mission, vision, and principles are conveyed to organizational members topdown, day-to-day quality improvement occurs bottomup.

Through empowerment, leadership integrates top- down and bottom-up approaches to TQM. Although crucial, the leader may not be enough. Empowerment may depend greatly upon a champion. Members will be more inclined to participate if a champion effectively engages them in culture change. The champion possesses the ability to stimulate, excite, and inspire those around him or her. The champion needs to possess sufficient skill to drive culture change.

\section{CHAMPIONING OUALITY.DRIVEN CULTURE CHANGE}

Champions crusade for culture change. To do so effectively, champions must believe in the new culture; possess the visibility, position power [4], and referent power [4] to affect culture change; be committed to affecting culture change; and communicate that commitment to followers. Position power arises from the legitimate power one possesses by virtue of one's place in the organizational hierarchy. Referent power arises from the respect one receives from organizational members. From the list of characteristics identified above, it's apparent that any organization may possess a number of individuals meeting these requirements. Champions aren't always formal organizational leaders, but champions could be such leaders. While not necessarily the leader, a champion may nevertheless possess leadership skills: vision, communication, and positioning One of the biggest impediments to qualitydriven culture change is the belief of organization members that this (culture change) "too shall pass " The champion must counter these views with advocacy. Thus, champions serve as activators of the motivational components of the change-management process. Champions activate the dual-path mechanisms involved in system-wide culture change. Leaders communicate a vision of where they want to lead followers. Champions make the vision happen by eliciting buy-in. They exemplify the followers' potential selves to followers. I emphasize the presence of a champion implies more than a one-directional effect. Thus, the champion is the catalyst for change. In addition, the leader who's also a champion can strengthen the intensity with which the leader-follower interaction combines to affect organizational culture change.

\section{CHIEERIEADING OUALITY-DRIVEN CUILTURE CHANGE}

As indicated earlier, culture-change cheerleaders act out and promote behaviors congrient with the new culture. They market culture change within the organization. Cheerleaders are the internal advertising agents for culture change. (They may be external marketers as well.) They capture and captivate their internal market. the organizational cheerieader can bring heightened enthusiasm to bear on the lengthy culture-change process. Cheerleaders instill a contagion about their enthusiasm 20 those around them want to become involved. They inspire the crowds of "fans." Cheerleaders engage in more "hoopla and razle dazzle" (12) than do champions. It's a matter of both content and degree. The cheerleader is illustrated in the. accompanying figure.

These culture change facilitators communicate a vision through symbolism For example, AT\&T uses a football ceremony to symbolize its culture. When Martin Luther King led the civil rights movement, he 
maximized a combined champion-cheerleader role by engaging, inspiring, and igniting followership. Champion-cheerleaders perform their role both topdown and bottom-up. Followers who can be "sparked" likewise kindle enthusiasm in their leader. Attentive students can "fire up" a teacher. Conversely, leaders and teachers confronting an apathetic work group or class may feel the energy drain from them. Yet teachers are often expected to bear sole responsibility for their students' attentiveness. So, a good cheerleader alone can't shift the organization to a quality culture. The cheerleader needs a group of attentive, enthusiastic, and motivated followers to successfully accomplish the shift.

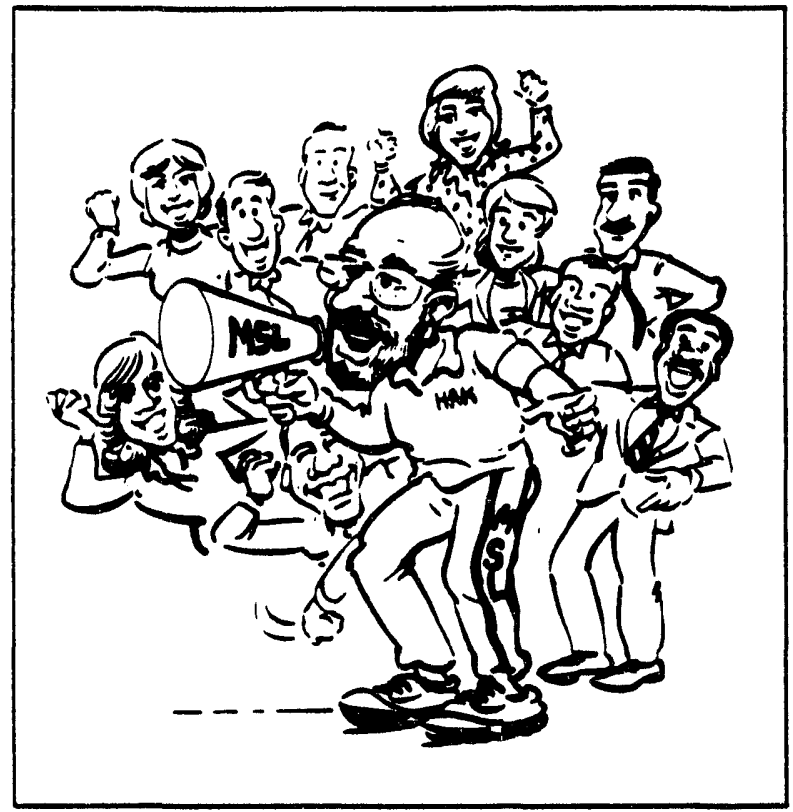

Figure 1. The culture-change cheerleader acts out and promotes behaviors congruent with the new culture.

Sometimes a cheerleader may be an external facilitator. When the senior author consults in organizations, he sometimes provides a cheerleader's voice to the beliefs of the organizational leader. He acts as a missionary for culture change.

\section{COMBINING THEROLES OF LEADER. CHAMPION AND CHEERLEADER}

I've identified different culture-change facilitation roles. Although these roles are independent, a given individual may play more than one role. Indeed, culture change is best served by the presence of all of these roles-ideally played by the organization's leader. However, in practice, individuals may play all of the roles or some combination of them. Not all individual are skilled enough or well-suited to play all the parts. For example, realistically, not all leaders can effectively play both champion and cheerleader. Ideally, complementing such a leader would be additional organizational champion-cheerleaders, a supporting cast of others in the organization who'll play the supporting roles.

For example, in one organization where we've vorked, the leader was not the cheerleader. He wanted to move his organization to a new quality culture. To conmunicate his commitment, he told managers they cou'd leave if they didn't buy-in. The cheerleader role was played by another manager who elicited follower enthusiasm for culture change. Because of combined effects of the presence of the cheerleader and champion, movement to a quality culture was possible.

\section{EXAMPLES OF CHAMPION-PRESENT AND CHAMPOON-ABSENT CUILTURE CHANGE}

Imagine you're in an organization and the champion of the culture change stimulates enthusiasm and communicates the new culture vision. Imagine, on the other hand, you're in the midst of culture change and you have no such champion. The need to change has been mandated, but no one-either at the top of the organization or elsewhere-champions the change. This "tale of two cities," exists in both corporate and governmental America.

For example, in one government organization, the re was a champion. While under his direction, TQM had begun to take hold. However, quality concerns were not fully ingrained. When the champion left the organization, people believing in TQM were without an advocate or catalyst. None of the "survivors" stepped in to assume the role of champion-catalyst and there was a reversal in the quality culture emphasis. Of course, the co-occurrence of events (presence of a champion and improved quality culture) does not prove that one causes the other. However, events following the departure of the champion (approximating a reversal-baseline design) provide compelling sugrestive evidence. In reversal-baseline desigons, a treatment is applied. Then, observational data is gathered. Treatment is withdrawn and more data is collected. If application of the treatment leads to the expected result and removal of the treatment leads to a reversal of the result, causality is inferred. Additionally, in another government organization, quality-based culture change was attempted in the absence of a champion. Here too culture change efforts failed. So, we have additional evidence for the importance of a champion.

At Management Systems Laboratories (MSL) our Management Process uses a TQM perspective. The advantage at MSL is we have a director who's both champion and trainer. There is consistency in both symbol and mesage. What's more, a single champion serves in both top-down and bottom-up capacities. MSL's director is also the organizational cheerleader. There may be auciliary roles enacted also when program directors or lab supervisors cheerlead and coach too. Throughout the organization, individuals "religiously" foster responsiveness and meticulosity, the laboratorywide credos. These are taught from the time employees join the organization and reinforced throughout day-today functioning. In our graphics laboratory, the supervisor knows the management process because she not only has been trained by the director/champion, but also she has managed the production of our manarement-process publications. She can be heard reminding her subordinates of maxims (e. g., "Focus on what you can do. Pay attention to Detail...."). Similarly, others throughout MSL play auxiliary culture-change roles. 


\section{CONCLUSIONS}

Quality improvement in organizations is no accident. Such improvement requires the vision. planning, and managing of individuals serving a variety of change-facilitation roles. This paper has emphasized three of these roles: culture-change leader, champion, and cheerleader. From my review of the literature, my research, and my experience in organizations, I believe that these roles are critical to an organization's shift to a quality emphasis. In my work with managers from large banking organizations to top executives in cabinet-level government organizations, l've found these managers acted as champions and as cheerleaders. In each case, the role the individual manager played can be linked to the success or failure of total quality management implementation.

I've examined the culture facilitation roles, emphasizing the bi-directional aspects of them. In particular, an interactive view of leadership underlies this adaptation of role theory. Just as leaders (or champions or cheerleaders) affect followers, so also do followers affect their leaders. I've emphasized that because all members of the organization must be invited to shape the new culture, this interactive approach with its dual-path features is particularly useful.

To be a champion of culture change, one must be in a position to make a difference and be committed to making a difference in quality-driven culture change. Champions must be advocates, catalysts, and activators of the dual-path mechanisms which lead to quality improvement. Cheerleaders take the enthusiasm for and dedication to culture change to a higher level than champions do. This role involves bringing a zest for the change to the internal stakeholders.

Leaders, champions, and cheerleaders are roles that may be played by the same individual or by different individuals. Although I've argued that, ideally, the same individual plays all three roles, it's possible that various individuals play leadership, champion, or cheerleader roles. Indeed, in the shift to TQM, it's possible that each member of the organization can be a champion.

\section{ACKNOWLEDGEMENTS}

The ideas presented in this paper represent the cumulative work of hundreds of people over fourteen years at Virginia Tech's Management Systems Laboratories. Professional managers from business and industry, and faculty, graduate students, and undergraduates from a wide variety of engineering and other disciplines have participated in the research and development of these tools and rechniques. The author thanks them for their good work The pronoun "I" is used throughout to separate all of us using management systems (we) from the author (I). However, please recognize that "I" is more than one contributor to this work

The preparation of this paper is funded by U.S. Department of Energy (DOE) Grant No. DO.FG02. 88DP48058. Management Systems Laboratories thanks DOE's Office of Environmental and Waste

Management for providing us with a real-world laboratory for the research, development, and resting of state-of-the-art management tools and the frameworks for understanding how to make them successful.

\section{BEFERENCES}

[1] Bennis, W. \& Nanus, B. (1985). Leaders: Strategies for taking charge. NY: Harper \& Row.

[2] Deal, T. E. \& Kennedy, A. A. (1982). Corporate Cultures. Reading, MA: Addison-Wesley.

[3] Deming, W. E. (1986). Out of the crisis. Cambridge, MA: MiT Center for Advanced Engineering Study.

[4] French, J. R. P. \& Raven, B. H. (1959). The bases of social power. In D. Cartwright, (Ed.), Studies in social power. Ann Arbor: University of Michigan, Institute for Social Research.

[5] Goffwan, E (1959). The presentation of self in everyday life. NY: boubleday.

[6] Hollander, E. P. \& Offermann, L. R. (1990). Power and leadership in organizations. American Psychologist 45, 179189.

[7] Katz D. \& Kahn, R. L (1966). "re social psycholozy of organizations. NY: Wiley.

[8] Kurstedt, H. A. Jr., E. M. Howard, A. R. Doss, \& L A. Mallak (1991a). Lessons leamed from DOE site culture change activities: Implications for waste management organizations. Proceedings of Waste Management 91 Symposium, February, 1991, in press.

[9] Kurstedt, H. A., Jr. (1991b). How to change to a quality culrure. Part of the teleconference series, Virginia Tech Perspectives: Quality Leadership and Management. Blaclasburg, VA

[10] Landy, F. J. (1989). Pyychology of work behavior. Pacific Grove, CA: Brooks/Cole.

[11] Newcomb, T. M., Tumer, R. H., \& Converse, P. E. (1963). Social poychology: The study of human inseracion. NY: Holt, Rinehart, \& Winston.

[12] Peters, T. J. \& Waterman, R. H. (1982). In search of accellence. NY: Warner Books.

[13] Sargent, S. S. \& Williameon, R. C (1958). Social psycholog (2nd ed). NY: Ronald Press.

[14] Schein, E H. (1985). Organizarional culture and leadership. San Francisco: Jossey-Bass.

\section{AUTTHOR BIOGRAPHIES}

HAROLD A KURSTEDT, Jr. is the Director and founder of Management Systems Laboratories, a research arm of Virginia Tech's Department of Industrial and Systems Encineering He conducts research on the use of management tooks, participative management processes and techniques, program and project mana gement and ormaizational culture. He has been with Virginin Tech since 1974 and is the Hal G. Prillaman Profeseor of Industrial and Systems Engineering He received his B.S. from the Virginia Military Institute in civil encineering and his Ph.D. from 
the University of Illinois in nuclear engineering. He has served on the mechanical, nuclear, and industrial engineering faculties at the Virginia Military Institute, the Ohio State University, and Virginia Tech. He has been chairman of nuclear engineering at Ohio State and Virginia Tech. He has had extensive industrial experience at Babcock and Wilcox Co., the Ballistics Research Laboratories, Industrial Nucleonics Corporation (now AccuRay), Management Horizons Data Systems, and Systems Research and Applications Corporation.

KATHRYN A WELCH is a Research Aseociate and Program Director for Research Methods at MSL. Her responsibilities include managing proposal writer teams, identifying and implementing research designs for grants, identifying strategies for data collection and analysis, defining appropriate statistical procedures, designing instruments such as surveys, and analyzing results. She holds $\mathrm{Ph}$.D. and M.S. degrees in industrialC'ganizational psychology from Virginia Tech. She also holds a B.S. in psychology from Clarkson University and a B.A. in English from Marymount College. Her teaching, research, and consulting background includes the areas of leadership, motivation, industrial training, and large-scale systems.

LARRY MALLAK is a Research Associate with Management Systems Laboratories at Virginia Tech. He assists in the labs' research activities and holds responsibilities for academic papers, presentations, and proposal writing. He received his B.S. from the University of Illinois and his M.S. from Virginia Tech, both in industrial engineering. He is currently working on his $\mathrm{Ph} . \mathrm{D}$. in management systems engineering at Virginia Tech. He's worked in both academia and industry, having held positions with Sunfiealth Enterprises, the University of Wisconsin-Madison, and Ohio State. He is a member of the Institute of Industrial Engineers, the Academy of Management, the American Society for Engineering Management, and a Mass Media Fellow of the American Association for the Advancement of Science. 


\title{
The Environmental Trilogy Project: Balancing Technical, Institutional, and Cultural Perspectives to Environmental Management
}

\author{
Pamela S. Kurstedt \\ College of Engineering \\ Virginia Polytechnic Institute and State University
Russell Jim
Environmental Restoration and Waste Management Program
Yakima Indian Nation \\ Bonnie C. W. Wadsworth \\ Museum Director \\ The Shoshone-Bannock Tribes \\ William H. Burke \\ Confederated Tribes of the Umatilla Indian Reservation \\ Harold A. Kurstedt, Jr. \\ Management Systems Laboratories \\ Virginia Polytechnic Institute and State University \\ 1900 Kraft Drive, Blacksburg, VA 24060
}

\begin{abstract}
SUMMARY
"The significant problems we face cannot be solved at the same level of thinking we were at when we created them." Albert Einstein
\end{abstract}

I've identified an initial set of three perspectives important to building an integrated, comprehensive approach to managing the environment--technical, institutional, and cultural. I've constructed an holistic model (called the Environmental Trilogy) for environmental management, encompassing at least these three perspectives and their interrelationships. In this paper, I outline the model and report the results of a working session facilitated at Virginia Tech in Blacksburg, Virginia, in October 1991, involving three representatives from each of the technical, institutional, and cultural perspectives. The institutional members of this group were people who understand institutional effects, rather than those who represent institutions. The working group discussed and analyzed the technical-institutional, technical-cultural, and institutional-cultural interrelationships of the environmental trilogy. The goals of the working group were to put structure on the environmental trilogy model, to facilitate the definition of variables, and explore relationships between and among variables. The working group members are to continue studying issues and components, perspectives, connections, and cause-and-effect in the models and report back to interested parties. The outcome is projected to be a more holistic, integrated view of the environment.

\section{MOTIVATION}

Discussions with Department of Energy, Native American, and states members in the State and Tribal Government Working Group (STGWG) revealed communication issues in dealing with environmental management. Stakeholders with differing perspectives, problems, and solutions inhibit the work of environmental managers who work from many disciplines, from many separate 
public and private agencies, businesses, and organizations. Translators are needed to define and relate the perspectives for those involved in environmental management. One of my objectives in developing this model is to provide a framework for teaching and supporting these translators. Through an understanding of these perspectives, translators then can help refine our understanding of the environmental trilogy to support environmental management.

I believe a holistic approach using the environmental trilogy is unique in its significant impact on real-world environmental problems (e.g., preparing an EIS). The integrated outputs coming from the environmental trilogy project will allow for a waste cleanup plan, EIS, or other environmental management tool to contain the spirit or essence of the environment, i.e., to have "soul." The Native Americans' environmental culture practices alert us to the importance of having a plan relating to the spiritual side of nature and strengthens the cultural component of the environmental trilogy, balancing the three perspectives. We can't put "soul" in an environmental management plan without an understanding of the environmental trilogy. We can't help society understand the environmental trilogy until we have translators who understand the three perspectives and the connections among them.

The Environmental Trilogy (1) model is shown in Figure 1. The technical perspective relies on scientific principles, laws of nature, and methods for implementing knowledge of those principles and laws into programs of both preventive and remedial nature. The institutional perspective anchors on regulations, laws of society, and policies. We usually approach and explain culture in human terms: values, norms, traditions, beliefs, behaviors, and attitudes. By broadening our perspective, we can study environmental culture where humans are just one component. Thus the cultural perspective recognizes the values, traditions, and norms of the environment as opposed to the values, traditions, and norms of the societies interacting with the environment. I don't believe anyone has fully drawn the connections among these perspectives--institutional, technical and cultural--and we therefore aren't recognizing the full cause-and-effect implications of the interrelationships among them.

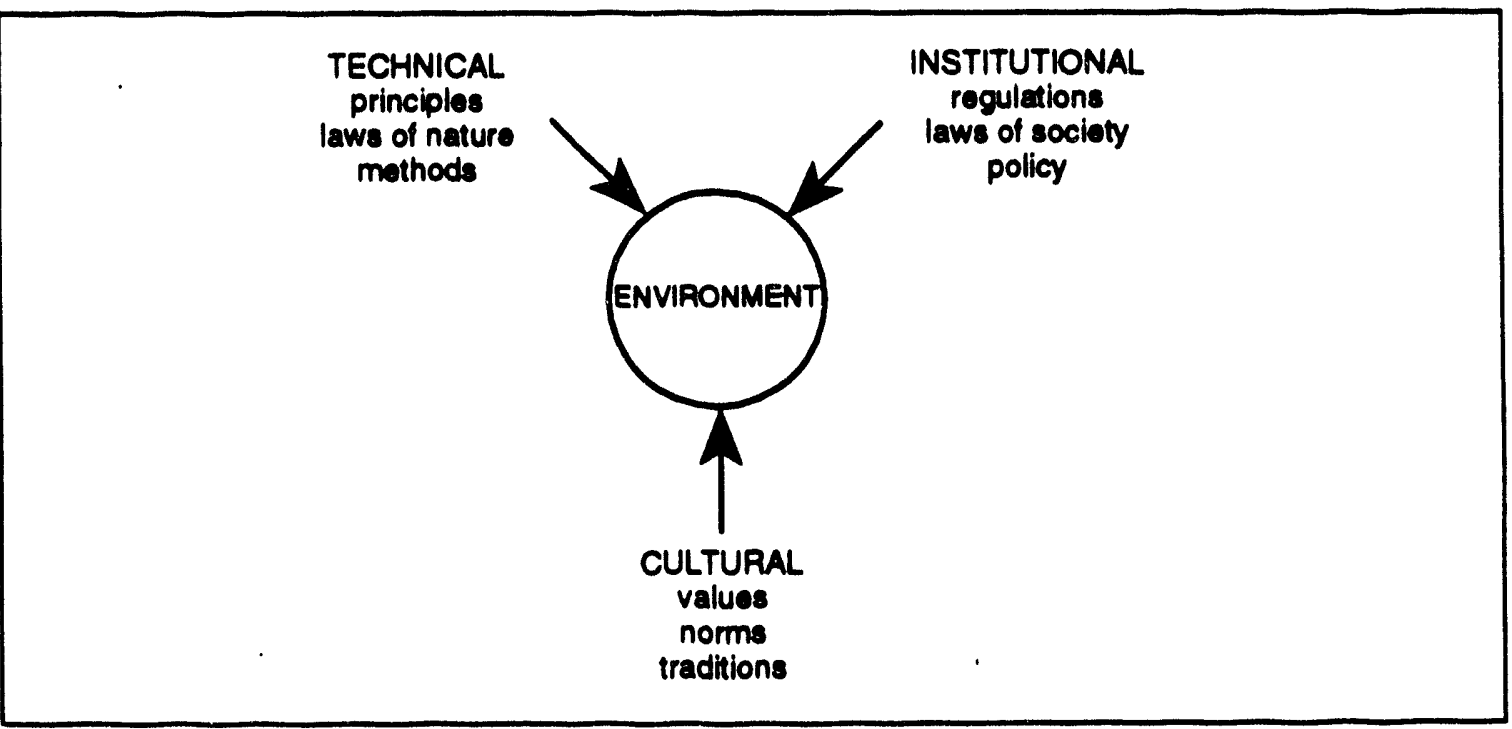

Figure 1. A balanced approach to the environment must include a cultural perspective. 
Today in environmental management, we have clashing value systems. So we specify clear-cut boundaries between what is and what isn't acceptable. We argue whether returning to a pristine condition or to some other condition represented by a quantitative standard represents what is acceptable. We'll continue to set boundaries until we establish a consensus on our overarching fundamental philosophy. We use the analytic approach to deal with different value systems by defining acceptability boundaries and working to specify tasks to just meet requirements. What we want is a unified value system so we can figure out what is desirable. In this holistic approach, we must put more effort into finding the common concept of desirability and put less effort into specifying tasks for acceptability.

\section{THE ENVIRONMENTAL TRILOGY PROJECT}

Based on the potential for contribution of the trilogy concept, Management Systems Laboratories (MSL) initiated a program to scope and operationalize the holistic approach to environmental management. The program starts with raising the issue of the holistic approach and then discussing the approach, defining its components, and modeling the components, all toward specifying areas for further study. The question, component, model discussion began with the October 1991 meeting in Blacksburg. A limited number of participants was invited to keep the atmosphere of a small informal grcup and to help us concentrate on a concept at the fringes of our understanding. Since the Blacksburg meeting, the participants and others have been studying issues, models, and perspectives stimulated by discussion in Blacksburg to prepare for a larger meeting to share study results with a broader representation of the environmental community. If the results of the broader meeting are as good as those from Blacksburg, I expect wide dissemination and discussion of the generated information.

\section{Participants}

The working group at the Blacksburg meeting included three groups of experts. The meeting organized, facilitated, and documented by six Virginia Tech administrative and research faculty. The three technical contributors included expertise in biology, chemistry, and engineering from university distinguished and endowed professors and a member of the National Academy of Sciences. The three institutional contributors included expertise in state and federal government for planning, water resources, and hazardous materials transportation from three Virginia Tech professors. The three cultural contributors included tribal, spiritual, and cultural leaders from the Umatilla, Yakima, and Shoshone-Bannock Indian Tribes. The facilitators contributed expertise in facilitation for consensus, environmental practitioner, organizational effectiveness, and program management.

\section{The Process}

The working group participated in five three-and-a-half-hour sessions during the Blacksburg meeting. The group also met together for all meals with lunches considered additional working sessions. One breakfast got so lively the group threatened not to go to the session room. For the first day, each person came prepared to present their perspective and how that perspective might connect with the other two perspectives. The participants individually and collectively considered how the perspectives might fit into a yet-to-be-defined model. During this session, the group realized holistic models might be different from analytic models. The group generated, listed, and discussed issues surfaced in the first two sessions. The 
group brainstormed a list of needs to increase holistic thinking and ways to improve holistic thinking. Finaily, the group discussed the value of continuing the study of the model and listed next steps.

\section{The Results}

The small size of the group clearly worked toward lively sessions with open and honest input and comprehensive opportunity for each participant to express their views. Several times participants lamented that more people weren't present to hear what they considered to be valuable considerations and revelations. In hindsight, a larger group could have not only heard the interactions first-hand but also could have affected the group interaction negatively in terms of the lively and open exchange of views.

The group came back often to the idea that the trilogy may, in fact, include more than three perspectives, thereby rendering the number implied in the word trilogy into something different from three. Given that the trilogy is holistic, we may never be able to define and analyze all perspectives. There may be a kind of glue among the perspectives that we can inversely determine but will have trouble operationalizing. Even considering this possibility, the idea I'm considering here is that more than one perspective must be integrated synergistically in environmental management. When using the Environmental Trilogy model, recognize two caveats: 1) there may be more than three perspectives and 2) the trilogy model is analytic. The trilogy model is an analytic means toward an holistic end. Whereas, analytically each of us may come at a given problem from one of these perspectives with its different image, holistically all of us must meld individual perceptions into one for the whole, a combined perception where each of the specific concerns and values are traded for one synergistic approach.

Reflecting on the basic premise of the working group efforts, environmental managers must balance Western analytic thinking with holistic thinking. In the analytic approach, if the individual parts of what we're considering are perfect, then the whole is perfect. However, in the holistic approach, if the individual parts are perfect, the whole may not be. W. Edwards Deming makes this idea stronger. He says, "If the parts are optimized, the system will not be. If the system is optimized, the parts will not be. (2)" The sign that the holistic approach is working is the resulting synergy. Synergy depends on the interfaces among the parts of the system. The performance of the whole results from the interactions, not from the addition of the parts. With good interaction, you get good performance of the whole. With bad interaction, you get bad performance of the whole.

Successful environmental management requires holistic thinking. For success, environmental managers need an overarching philosophy and a constancy and consistency of purpose (3). Philosophy and purpose come from participativelygenerated and universally-supported mission, vision, and principles statements. Holistic thinking requires ultimate understanding of the issue under consideration. The environmental manager must replace the logic of the uninformed with the logic of the informed. If we interpret Deming's concept of profound knowledge (4) as being properly informed, we'd have to include technical, institutional, and cultural understanding in the needed knowledge base. I believe we're indulging in uninformed analytic thinking; and we're not going to be successful until we do informed holistic thinking.

In the past, production of a good or service has often been the starting point and the 
continuous focus of our attention and effort. Figure 2 shows the traditional approach as starting with production and related resources (time and money). Based on production needs, we bring in the technical approach to determine technological constraints on more and better production and the risks associated with not having technology as good or as soon as our competition. Knowing what technology we need to meet production needs, we consider the institutional approach in terms of legal, regulatory, and policy constraints and the risks associated with changes in institutional forces. Given these technical and institutional considerations to meet production, we back fit goals and objectives, develop our guiding beliefs, and try to wring out environmental vaiues of some kind, hopefully all dovetailing with our production needs. This traditional approach may give us short-term production success and satisfaction; but we're finding the traditional approach leads to many long-term problems. We may, in fact, be mortgaging our future. The needed approach is to learn how to cope with the short-term impatience of those focusing on production needs, to work the progression in reverse to the traditional approach to gain long-term results our progeny will be proud to inherit.

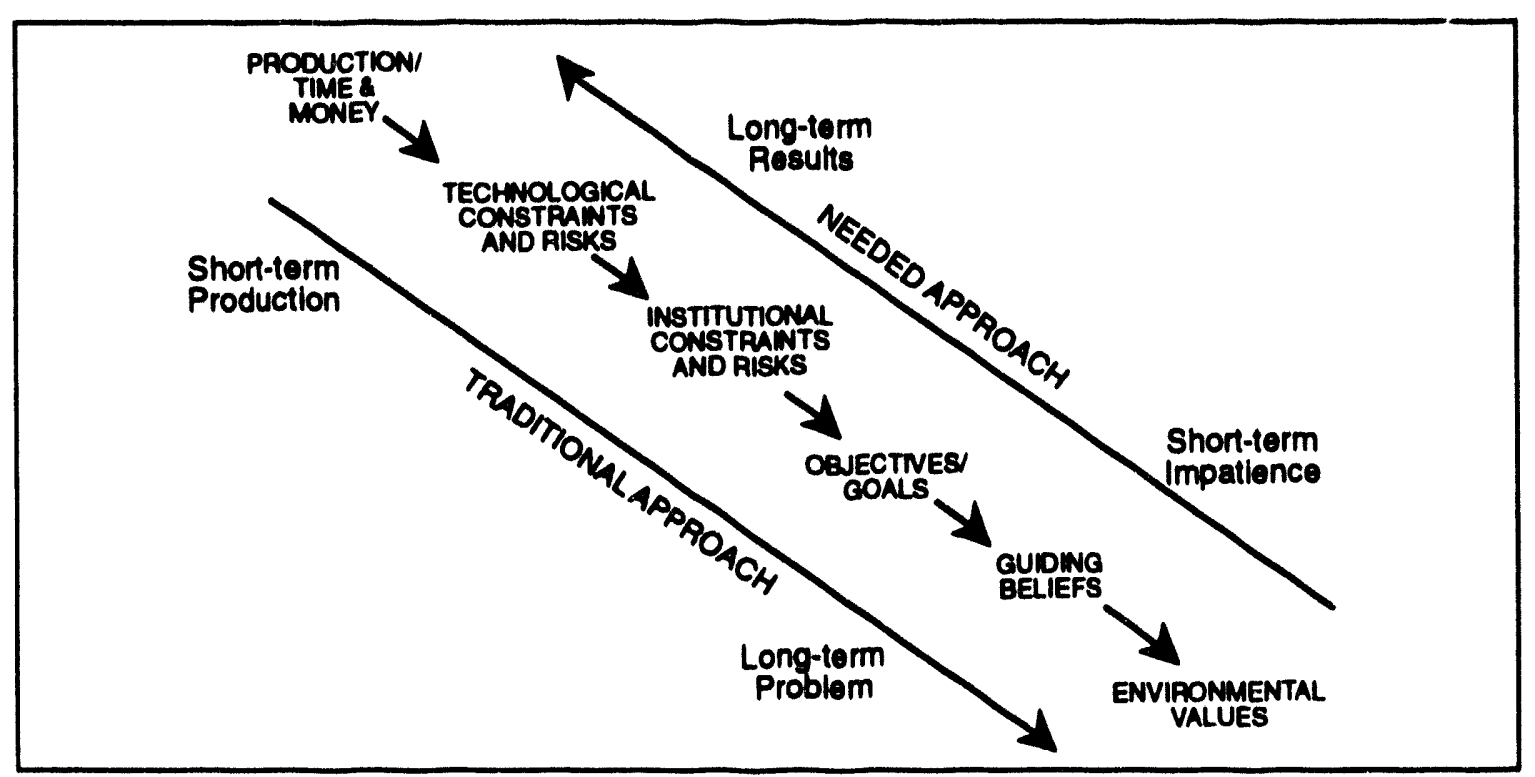

Figure 2. We've been starting with production.

Realizing that environmental managers must work the steps in Figure 2 from environmental values toward production needs (in opposite direction to the arrows of the traditional approach), Figure 3 emphasizes the players and their tools for environmental management. Figure 3 emphasizes the idea that stakeholders and the experts they choose must help set and evaluate standards and measurements for production and technological and institutional constraints resulting from the criteria and boundary conditions of the environmental values, beliefs, and goals and objectives. In Figure 3, the place of stakeholders and their effect on experts and criteria and boundary conditions leading to standards and measurements is intentionally aligned with the suggested approach to the steps from Figure 2.

Figure 4 expands on Figure 3 by suggesting some of the questions the stakeholders and experts must ask in continually monitoring the production system for improvement. The questions shown in Figure 4 are intended to represent more comprehensive and more penetrating questions in practice and to show the relative roles of the stakeholders, their experts, and measurements. Figure 5 emphasizes the 


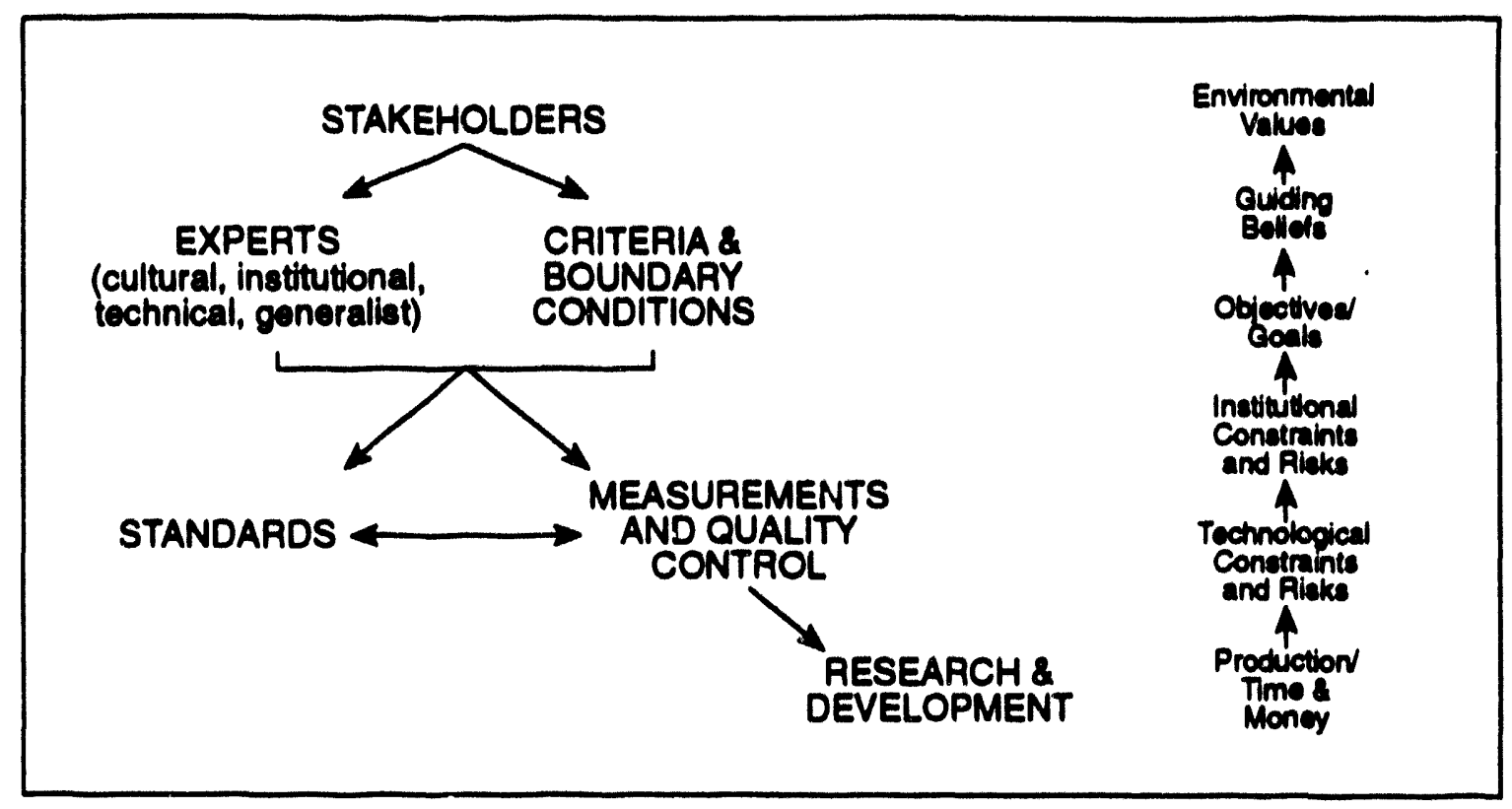

Figure 3. Together, stakeholders and experts set and evaluate standards and measurements.

alignment of the players and tools (stakehoiders, experts, standards and measurements, and research and development) from Figure 3 with the steps from Figure 2. By cross-walking between the steps, with arrows showing the suggested approach and the sources of indicators used to accomplish those steps, we see where we get and how we combine and compare indicators with the approach steps to carry the process from environmental values, through the cultural, istititutional, then the technological perspectives to get to the appropriate production. The working group called the model in Figures $2-5$ the idealistic model, because the model illustrates what we should aim for.

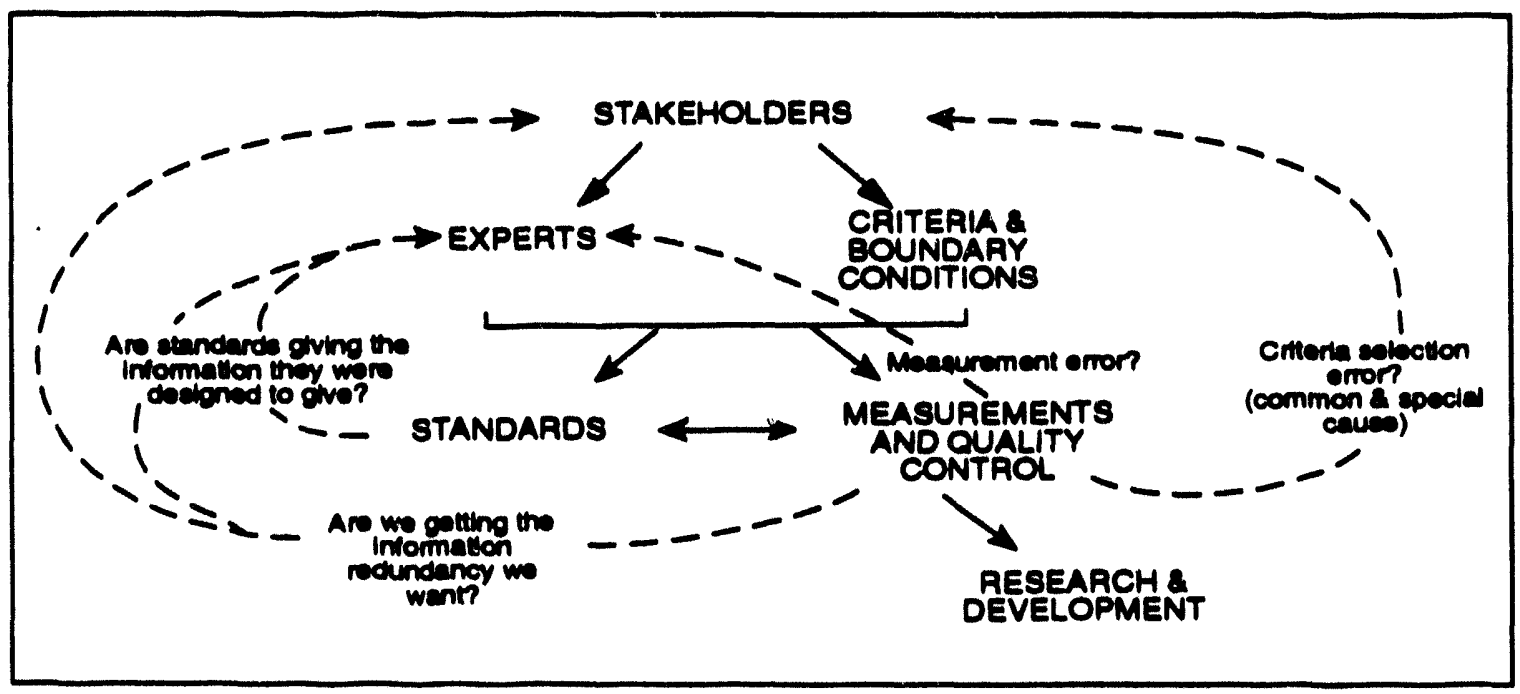

Figure 4. Stakeholders and experts must continually monitor the system for improvement. 


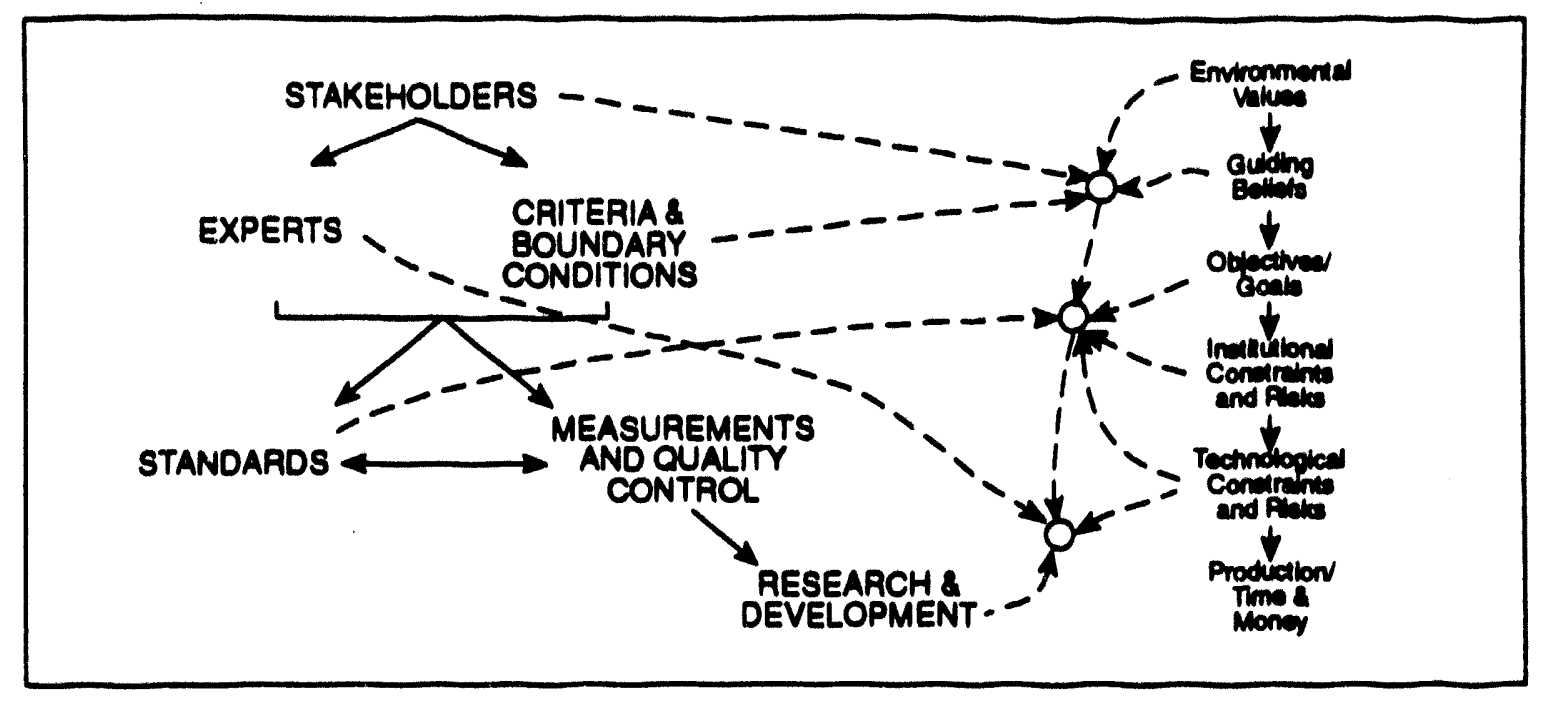

Figure 5. We resolve differences by comparing indicators and approach steps.

After the participants reviewed the perspectives and discussed models and the holistic approach, they collectively generated a list of rules for the holistic approach to environmental management. From these results, ['ve condensed six rules: 1) Consider relationships and interactions over components; 2) Get stakeholders' predecisional involvement and work Figure 2 in the direction of the needed approach; 3) Get a systems integrator in addition to a strategic manager; 4) Listen to what the environment tells us; 5) Break discipline barriers to eliminate parochial advocacy to a technology or one of the perspectives in Figure 1 (5); and 6) Consider permanency of the environment and those who evolve with it over transient needs and peoples.

We're interested in how the components, or parts, of a system are related and how they interact through that relationship. The interaction is the key to the gestalt (6) idea. We're probably not going to be able to separate out, define, and explain an interaction, but we must understand the interactions in total before we can apply the holistic approach.

The environment operates according to natural laws. We can allow our technical, institutional, and/or even our cultural laws to cause us to work against where the environment is heading, or we can go with the flow by listening to what the environment tells us. Obviously, we fight a flood because of the relative impact of the humans on the flood and its aims and objectives versus the impact of the flood on the humans and their aims and objectives. However, in most interactions of humans and the environment, the relative impact is reversed. In these cases we should flow with the environment because the long-term outcome is going to be best for everyone. So we should spend more effort figuring out where the environment is ultimately going and join in toward that direction rather than focusing on one instance in the environment's variation and reacting to it.

Toward the end of the Blacksburg meeting, the participants listed issues raised during the discussions they thought deserved more consideration. The issues serve as a starting point for study, presentation, and discussion in later gatherings on the holistic approach to environmental management. I've condensed the list to eight issues: 1) The unique and unexplored needs of and abilities for environmental communication; 2) Analytic versus holistic models as environmental management 
models; 3 ) The transference of principles of other holistic approaches like gestalt psychology and quality management to the Environmental Trilogy; 4) The translation of the idealistic model of Figures 2.5 and other models from the Blacksburg meeting into practice; 5) The difference in the time dimension for environmental management and its perspectives; 6 ) The number, identity, and characteristics of the perspectives in the Environmental Trilogy model; 7) The knowledge and skill environmental managers need to be system integrators; and 8) The removal of barriers that rob people of their ability to think holistically and work and communicate interactively, starting with discipline barriers in the university.

Techniques for environmental communication are new and difficult for environmental managers to do. In fact, environmental communication may be more sophisticated than risk communication, another new and difficult communication issue we're beginning to learn about (7). The complicating factor in environmental communication over the present understanding of risk communication is the need to incorporate holistic thinking both in the sender and the receiver of the communication.

The role of the integrator is one of the most difficult, important, and ill-defined roles in the holistic approach to environmental management because of the variety of perspectives and tasks and the diversity of information needing coordination at many levels of the organizational complex and among the many stakeholders concerned about an environmental activity. Environmental management requires a manager who knows how to integrate the many facets of a complex program. Top management must include a systems integrator.

Others have studied integrating multiple perspectives (8) and the holistic approach. We would be remiss if we didn't understand the work of others in these areas as we work on environmental management.

\section{Next Steps}

The participants are studying the issues from the Blacksburg meeting and are preparing papers for presentation or finding knowledgeable people on the issues for them to prepare papers for a larger follow-on meeting with broader participation. The information from the Blacksburg meeting is being discussed by the STGWG for their use and feedback to the Blacksburg participants. Some of the faculty involved in the Blacksburg meeting have used the results in their college classes. A detailed report of the Blacksburg meeting including more models, descriptions of rules and issues, and additional implications is available on request from the Management Systems Laboratories at Virginia Tech.

\section{SOME INTERPRETATIONS}

Those of us who are rooted in Western, or European, culture and are analytically trained (e.g., engineering, accounting, sciences, law, and medicine) have great trouble even conceiving of how to go about holistic thinking, let alone understanding holistic thinking well enough to model it. But, even the most devoted analytic thinkers may slip into holistic thinking a time or two, when dating or buying a house or a car, for example. As Dr. Kosaku Yoshida says when illustrating the holistic approach: "When you go out on a date, would you evaluate whether your date has intelligence: 95 points; Appearance: 96 points; Emotional stability: minus 20 points? Do you evaluate your partner like that? If you get a date, turn off the light and get the smell and get the total understanding. You [are] not going to analyze. You re 
going to capture the entire feeling. That I call ultimate understanding (9)." In holistic thinking, we strive for ultimate understanding. I believe environmental management requires holistic thinking and, the refore, ultimate understanding.

Have you ever ranked alternatives when buying a car or house or choosing a school to attend. You carefully list all the criteria important to your decision and even consider the relative importance of the criteria. By intuition or calculation, one car, house, or school clearly excels over the others. But you buy a different car or house or choose a different school instead, just because it feels right! Analyzing the date, car, house, or school doesn't work. You can't or don't list all the criteria and their importance. You don't want to admit a gadget in the car or the personality of the salesperson affects you. Or, for holistic thinking, you really aren thiding anything from yourself; you just, in your heart, prefer a particular car.

The top government or cumpany official for environmental management could turn off the lights (like in the Yoshida example) and come up with the priorities for the agency's or company's environmental management funding. That would be an holistic approach. He or she could either forget the analytic model for priorities or compare the results of the analysis to the holistic result. But, the top official's analytic-thinking constituents wouldn't accept the result of his or her individual holistic thinking. (The constituents drive different cars, don't they?) If any one constituent turned off the lights, he or she would come up with priorities somewhat different from the top official. So how can all the constituents collectively come up with holistic priorities? Not through an analytic model and not by tallying up all our individual results. The constituents need to come together and work as a group so they get synergy through the holistic approach and we get more for our environmental management money.

The only way I know how to come up with priorities as a group is to bring everyone, or their representative, together who has a stake in the priority decision and collectively derive holistic priorities. That's the holistic model. So far, only the human mind can come to a conclusion with incomplete or missing data. The human mind can come to a gestalt with some criteria and importances clearly defined and others only in their hearts, undefinable. A group of human minds can consider things no analytic model can and the group can render a synergistic answer. So, we have to figure out how to effectively bring stakeholders together to come up with the best answer through holistic thinking.

I believe the holistic model requires human minds rather than equations, algorithms, or computers because only the human mind and heart can bring together and relate all the issues, characteristics, nuances, alternatives, and importances needed for the holistic approach. The group of participating stakeholders must include everyone (or their representative) who will gain or lose by one alternative being selected or ranked over another. This holistic model allows the emphasis of perception over reality. Analytic models can't deal with perception. We must accept that in endeavors like environmental management a perceived barrier can be as effective as a real one. Until we include perception, we'll not get acceptable answers when solving environmental problems.

Consider a note here on how decision makers use the results of a model, whether it be analytic or holistic. The model results aren't answers, they're only information. However, information is the difference between a guess and a decision. The decision maker will have to use holistic model results just like they do analytic mcdel results-as input to their decision. The decision maker knows an analytic model's results are 
only as good as the variables and their relationships in the model, the boundary conditions, and the data put into the model. Likewise, an holistic model's results are only as good as the representativeness of the stakeholders thinking and feeling together, the data or information they have to work with, and the process through which they interact. The decision maker can use the holistic model as support for their decision just like he or she would use an analytic model. The decision maker can show the results of the model and describe the makeup of the model and how it works, describe the strengths and weaknesses of the model, and state the decision. The advantage of the holistic model duplicates the advantage of holistic thinking-synergy. Synergy is what you get when the holistic approach is working. In synergy the whole is greater than the sum of its parts.

A component of the holistic approach to environmental management is establishing an overarching fundamental philosophy. Once we have a long-term philosophy we set a constancy of purpose so everything can follow the philosophy, including goals, objectives, priorities, and task specifications. In analytic thinking, we can't function without clear-cut objectives and detailed task specifications. The holistic approach then asks us to first agree among all stakeholders on an overarching philosophy for environmental management--a philosophy for both the goals and the process of our management activities. A difficult requirement for using the holistic approach is the need for ultimate understanding and tor dealing with the logic of the informed. The significant value of this approach is that holistic thinking focuses us on what is desirable, whereas analytic thinking puts acceptability first. Figure 6 illustrates the difference between the acceptability and the desirability concepts (10). In analytic thinking, anything inside a given boundary is acceptable. In holistic thinking the most desirable is the center of the area of interest. As we get farther from the center, the result is less desirable. When common ground is limited, we reach for acceptability, not desirability. In environmental management, when stakeholders have different value systems (cultures) we tend toward analytic thinking. Therefore, trying to get holistic thinking from people of different value systems is difficult. Analytic thinking supports science, individualism, and discovery. Science and discovery certainly are critical to a 30-year clean-up goal. Holistic thinking supports management, consensus, and optimization. Management, consensus, and optimization are also critical to a 30-year clean-up goal. For environmental management, clearly we want to blend both holistic and analytic thinking in a situation where our differences force us toward analytic thinking.

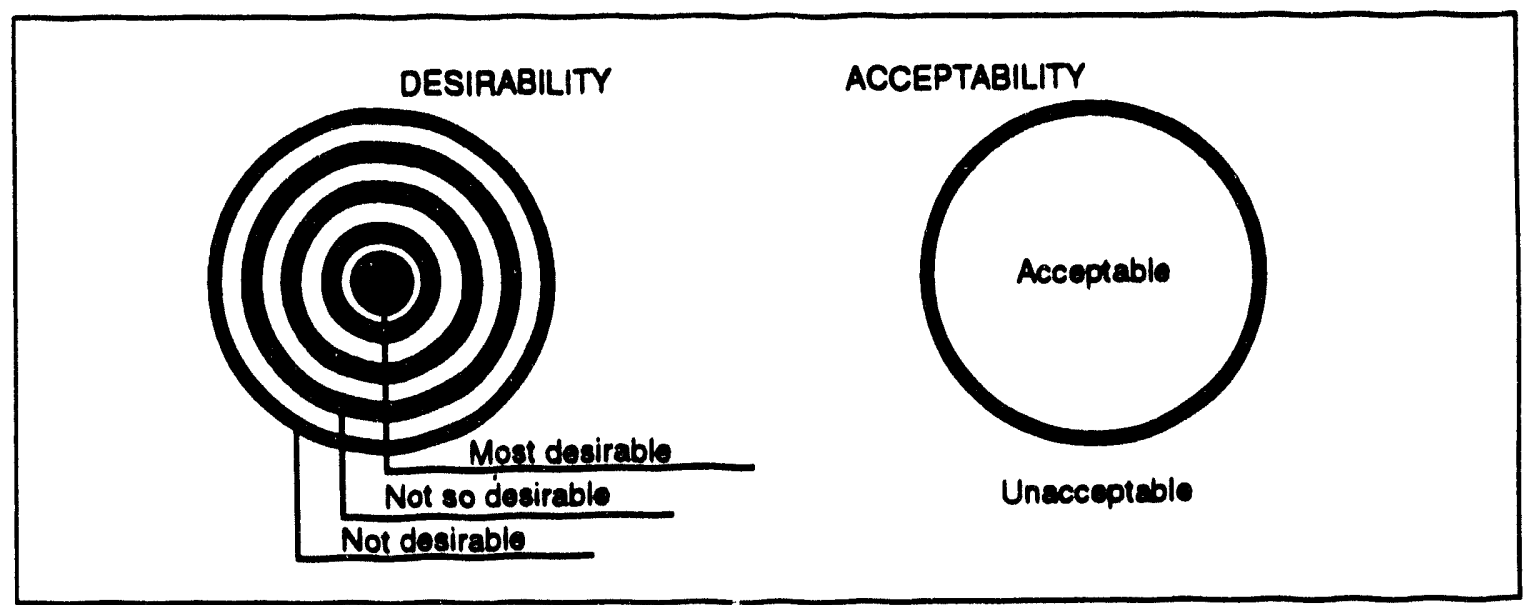

Figure 6. When common ground is limited, scciety reaches for acceptability, not desirability. 
We don't have to define desirability precisely. A rough estimate will do. So defining the area's center is easier than precisely defining the area's boundary. When judging whether an activity is acceptable or unacceptable we define the boundary exactly and argue over the definition and whether the activity in question meets the definition. So a rough definition of desirability is not only easier, it's better. Furthermore, when we define exact boundaries, people will focus on the boundary and meet lower requirements.

\section{THE BOTTOM LINE}

The answer is to optimally blend holistic and analytic thinking and to trade off individualism and technology against unified values and management. Holistic thinking is in itself oriented toward this blend. The environment deserves a profound understanding of the harmonious blend of science and management.

\section{ACKNOWLEDGEMENTS}

The pronoun "I" is used throughout to separate all of us affected by environmental management (we) from the authors (I). However, please recognize "I" is more than one contributor to this work. The preparation of this paper is funded by the United States Department of Energy (DOE) Grant No. DE-FG02-88DP48058. Management Systems Laboratories thanks the DOE's Office of Environmental Restoration and Waste Management for providing us the real-world laboratory for the research, development, and testing of state-of-the-art management tools, processes, and models for ur.derstanding how to make them successful.

\section{REFERENCES}

(1) The term environmental trilogy for the holistic approach to environmental management was coined by jeanette Wolfley. In Webster's Dictionary, trilogy defines a series of three dramas or literary works or sometimes three musical compositions that are closely related and develop a single theme. We borrow the word and concept to symbolize the holistic approach to environmental management.

(2) W. EDWARDS DEMING. Personal communication from notes taken at Instituting Dr. Deming's Methods for Management of Productivity and Quality, Washington, D.C., January 21, 1992.

(3) WILLIAM W. SCHERKENBACH, The Deming Route to Quality and Productivity, Washington, D.C., Ceep Press, 1991, pp. 13-14.

(4) W. EDWARDS DEMING. Personal communication from notes taken at Quality, Productivity, and Competitive Position, Atlanta, GA, September 9, 1991.

(5) W. EDWARDS DEMING. Out of the Crisis, Cambridge, MA, MIT Center for Advanced Engineering Study, 1982, p. 62. 
(6) In Webster's Dictionary, gestalt defines a structure, configuration, or pattern of physical, biological, or psychological phenomena so integrated as to constitute a functional unit with properties not derivable from its parts in summation. The concept of gestalt has been used to define gestalt therapy in psychology (c.f., Koffka, Principles of Gestalt Psychology, Harcourt, Brace \& World, Inc., New York, 1935.) and more recently in organizational effectiveness (c.f., Nevis, Edwin C., Organizational Consulting: A Gestalt Approach, Gardner Press, Inc., New York, 1987.). Gestalt involves interactions among the parts of the whole that we $d$ 7 understand. Likewise, the holistic approach to the environment must key on interactions, interactions among the minds, feelings, and perceptions of the stakeholders of the activity being managed.

(7) WILLIAM O. CONN AND RICHARD C. RICH. "Communicating with the Public about Hazardous Materials: An Examination of Local Practice," EPA 230-04-90-077 (April 1990).

(8) ERNEST R. HOUSE. "Three Perspectives on Innovation: Technological, Political, and Cultural," In Improving Schools--Using What We Know, edited by Rolf Lehming and Michael Kane, Sage Publications, 1981.

(9) Made in Japan "Whole"-istically (videotape transcript), Petty Consulting/Productions, Cincinnati, OH, 1990, p. 11.

(10) KOSAKU YOSHIDA. "Deming Management Philosophy: Does It Work in the U.S. as Well as in Japan?," Columbia Journal of World Business, Fall, 1989, p. 12. 


\title{
ARE EM'S COMMUNICATION TOOLS EFFECTIVE? EVALUATION RESEARCH OF TWO EM PUBLICATIONS
}

\author{
Evelyn Wight \\ Gene Gardner \\ Tony Harvey \\ Management Systems Laboratories \\ Virginia Polytechnic Institute and State University \\ Blacksburg, VA 24061
}

\begin{abstract}
As a reflection of its growing culture of openness, and in response to the public's need for accurate information about its activities, the U.S. Department of Energy (DOE) Office of the Assistant Secretary for Environmental Restoration and Waste Management (EM) has increased the amount of information available to the public through communication tools such as brochures, fact sheets, and a travelling exhibit with an interactive computer display. Our involvement with this effort has been to design, develop, and critique booklets, brochures, fact sheets and other communication tools for EM. This paper presents an evaluation of the effectiveness of two communication tools we developed: the EM Booklet and the EM Fact Sheets. We measured effectiveness using nonparametric testing. This paper describes DOE's culture change, EM's communication tools and their context within DOE's new open culture, our research, test methods and results, the significance of our research, and our plans for future research.
\end{abstract}

\section{DOE'S CULTURE CHANGE}

In 1989 Secretary of Energy James Watkins directed DOE to commit to cleaning up the environment and complying with the letter and spirit of environmental laws and regulations. To accomplish this, he mandated a new DOE culture of accountability and open communication with the public about past and present activities $(1,2)$. *

DOE and its predecessor agencies, created in the 1940's and 1950's, produced nuclear materials for weapons used in national defense activities. Since this work was secret, DOE shared information on a strict "need-to-know" basis, and DOE was largely a selfregulating agency. DOE technical professionals were often ill-equipped to communicate clearly or completely with the nontechnical public (2). This isolation allowed DOE production concerns to overshadow environmental ones. In addition, DOE lacked understanding of the effects its activities had on the environment. DOE's challenge is to mold itself into an organization whose highest priorities are maintaining a clean, safe environment, sharing information with and involving the public in decision making, and complying with federal, state, and local environmental laws.

* As stated in DOE-Richland's Draft Public Involvement Research and Planning Task Report, "the public" includes individuals and groups who might be affected by DOE's decisions, whether they know it or not. These groups are stakeholders, such as Indian Nations, state and local governments, environmental organizations, and others. We add to this definition every citizen and member of the public whether they have a demonstrated interest in DOE activities or not (1). 
DOE reorganized in 1989 to create an office focusing on cleanup and compliance, the Assistant Secretary for Environmental Restoration and Waste Management, or EM. Secretary Watkins said this office, confirmed by Congress in November 1991, "emphasizes the importance of our (DOE's) efforts to clean up radioactive waste resulting from 45 years of nuclear weapons production... To us at DOE, it is among our highest priorities" (3). DOE's new culture is being articulated at its sites across the country as well as at Headquarters. For example, the Richland Field Office describes itself as "a public agency, and members of the public have a right and need to access information" about it (1).

DOE is not completely successful at implementing this statement, as illustrated by a 1990 Office of Technology Assessment survey of 14 public interest groups. None of the groups felt they were getting the information they needed from DOE. However, this was before publications like the two we studied (the EM Booklet and EM Fact Sheets) were available. In order to meet its own and the public's needs without wasting taxpayer money, DOE must evaluate the effectiveness of its communication tools and public involvement programs (4).

\section{COMMUNICATION TOOLS}

EM produces booklets, brochures, and fact sheets; hosts public meetings; and solicits public review of its plans and policies. EM has learned from experience at public meetings that communicating with the public includes being open to vigorous criticism. According to a recently published study of international nuclear waste politics, listening to public complaints may help improve public opinion. Responding to public complaints with information helps even more (5). Failure to educate and provide accurate and meaningful information will likely result in a negative outcome (6). An untrusting public with more access to information from interest groups than information from DOE may choose to side with the interest groups' agenda. This could work to prevent EM from accomplishing its goals, as demonstrated by delays in starting the test phase for the Waste Isolation Pilot Plant in New Mexico.

EM's annually updated Five-Year Plan outlines DOE's cleanup and compliance goals and policies and incorporates public comment. EM's other communication tools also embody DOE's commitment to a new culture of openness and responsive communication with the public, but they are intended to provide a simpler overview of EM activities than the Five-Year Plan. They include the EM Exhibit; the EM Booklet, Environmental Restoration and Waste Management (EM) Program, An Introduction; numerous EM Fact Sheets; and other brochures.

The EM Booklet describes DOE's culture change, the Five-Year Plan, and EM's mission, functions, offices, and major activities including environmental restoration, waste management, and technology development. It also defines waste types, and treatment, storage, disposal, and transportation methods. The EM Fact Sheets describe waste types, environmental restoration and waste management activities at each of DOE's nine Field Offices, and environmental laws and regulations. 
EM's publications accompany the EM Exhibit, a portable multipaneled exhibit and interactive computer display designed to inform the general public about EM's program to clean up the environment and manage waste. The EM Exhibit is presented throughout the United States at professional conferences such as the American Bar Association, the National League of Women Voters, the National Conference of State Legislators, and the National Science Teacher's Association, as well as DOE-related meetings. The Exhibit is staffed by technical and nontechnical DOE profersionals who strive to provide citizens with information about DOE and offer a positive interaction with "the government."

\section{Communication Tool Design}

In many cases, EM communication tools and publications have been based on experience rather than systematic (but time-consuming) application of communication theory and technique. Many public affairs experts develop and conduct their programs based on intuitive guesses and experience. There is very little "hard" research defining what does or doesn't work (7). Although most of the studies that do exist focus on the commercial nuclear industry, research by the U.S. Council for Energy Awareness (USCEA) suggests that all nuclear-related activities are lumped together in the public mind. Thus, EM can learn from this research as well as its own.

Concern about the environment is high on the public agenda; but while nuclear waste is a national concern, it's far from the first thing on people's minds, according to public opinion polls. A 1990 USCEA study showed that although $12 \%$ of U.S. adults named waste as the most important environmental problem, only $1 \%$ named nuclear waste specifically (8). A series of 1990-1991 Gallop, Roper, and Opinion Research Corporation polls found air pollution listed most often as the greatest threat to the environment, with nuclear waste being mentioned significantly less (9). However, an August 1991 Roper Organization Survey found that $86 \%$ of adult Americans said they were somewhat or very concerned about nuclear or hazardous waste when choosing from a list of environmental problems (10).

Another USCEA study found that providing information about high-level waste disposal (in the form of a brochure) to an attentive public increased their confidence from $37 \%$ to $59 \%$ that nuclear waste can be disposed of safely. However, USCEA noted its research also showed that providing information to non-attentive audiences, or people who don't have some knowledge or context for nuclear issues already, may decrease their confidence by failing to address their concerns and bringing up any negative associations they already have (8).

EM's communication tools can benefit from amplifying the public's voice so EM can respond to public needs within a context that increases knowledge and improves perception. Cognitive learning theory suggests that the mind retains information categorically, or in context and reference to existing knowledge. Thus, new information can be easily memorized but not necessarily understood unless a context or knowledge base already exists (11). To take advantage of this theory EM could identify public contexts and present information within those contexts. Other improvements to increase cognition include grouping ideas, adding unique summaries, and clustering topics hierarchically from the general to the specific (12). EM's existing tools use some of these 
principles. For example, the EM Booklet presents a general overview with the EM Fact Sheets providing more detailed, specific data.

To produce effective communication tools it's useful to employ the formula: Audience + Purpose $=$ Design. . However, EM's public is made up of many audiences, and EM has had no time to survey and analyze them. EM's communication tools serve many different purposes, from illustrating DOE's new open culture to providing Congress and the public with technical and programmatic information about its activities. EM's audiences and purposes need to be better defined. For instance, how can EM assist the public in understanding highly technical and complex information? How much and what kind of information does EM want to provide? Whom does EM want to educate? What is the best kind of public involvement for DOE's activities?

A comprehensive study of EM's audiences and outreach methods would reveal which audiences are being exposed to information about EM and what they are actually learning from it. Eventually, we could provide a matrix describing which tools and what information would be effective for different audiences and purposes. Understanding the factors that affect communication tools will help EM describe its programs, policies, and goals to the public. It also will help reduce the costs of producing communication tools and the costs associated with responding to an irate, perhaps misinformed public.

\section{Publication Production}

In order to produce the best possible documents in the least amount of time, we gathered information by interviewing high-level DOE managers, talking with DOE'ers who have public relations experience, and conducting multiple content reviews with DOE nuclear experts, managers, and editors. EM managers at DOE Headquarters and Field Offices often modified content to accommodate national and local political or legal concerns.

We designed the 100-page EM Booklet to describe EM with minimal text and many illustrations. We translated highly technical and bureaucratic information into understandable terms and eliminated as much time-dependent information (like milestones) and jargon (including acronyms) as possible. We used many photos and figures with detailed captions to describe technical processes and boost an image of openness.

As an overview for such a large and complex organization, the EM Booklet left a lot of questions unanswered. So we designed fact sheets to provide more detail about EM activities and sites. Our goal was to answer as many questions as possible and include as much information as we could on a one-page fact sheet. Each fact sheet includes at least one picture or illustration with an accompanying caption, and in many cases, a map to geographically locate the facility or site for the reader. We gathered information and conducted reviews in the same way as for the EM Booklet. In both cases, the Five-Year Plan served as an information source.

Despite efforts to simplify the text, the politics and jargon associated with EM nuclearrelated information made it difficult to simplify these publications. Both the EM Booklet and EM Fact Sheets are written at about a 15th grade (junior in college) reading level 
according to the Flesch-Kincaid Readability Formula (13). This formula measures how easy a document is to read and comprehend based on sentence length and number of syllables per word (word complexity). The reading level often used by the news media for the general public is 8th grade (14). Although both the EM Bookdet and EM Fact Sheets are very difficult to read according to the above formula, word complexity (e.g. Environmental Restoration and Waste Management, Hanford Waste Vitrification Plant) was identified as a bigger problem than writing style or sentence length.

\section{TESTING EFFECTIVENESS}

In spite of its constraints--time, organizational inertia, negative public opinion--EM is actively pursuing the public trust. EM hypothesizes that educating the public by providing unbiased information about its program will improve public perception and encourage the interested public to learn more on its own about technical issues (15).

How much is the public learning from the communication tools EM provides? Is the public getting the information it needs? How does public opinion affect EM's effectiveness? What does the public think about radiation? About DOE activities? Does the information EM provides affect the public's perception, and if so, negatively or positively? EM needs answers to these and other questions.

In order to find these answers it's necessary to conduct tests. Test design impacts test results. It's as important to ask a relevant question as it is to design a test that answers that question. Otherwise, test results give false indications. It's also necessary to use applicable measurement tools. While it's difficult to assess how people feel about EM, it's relatively easy to test what facts they know about EM. Since EM's effort is to communicate with and educate the public, we need to measure how effective tools are for different audiences rather than comparing tools. For example, although EM and public response to the two communication tools we designed was very positive, we decided to explore their effectiveness by measuring how much different audiences learned from them. We wanted to find out whether these publications are good teaching tools--that is, whether after reading them, an audience remembered more correct information about EM than before. We used non-parametric testing to explore this question.

\section{EM Booklet Test Method}

Our first test group was 17 DOE interns participating in a training program. These were future DOE employees--college-educated professionals with a vested interest in learning the material presented to them. The training program introduced each office within DOE, including a session on EM. We tested the EM Booklet as text material for this session. All the students were given a pre-test to determine their knowledge before the EM session began. Some students received a pre-test on information unrelated to EM but included in previous lectures; the others received a pre-test exclusively on EM-related information. The instructor presented a three-hour lecture on EM that included information not covered in the EM Booklet. The instructor did not know an evaluation test was being conducted. The interns received the EM Booklet during the session on EM and were told to read it. 
We conducted a post-test one week after the interns received the EM Booklet and attended the lecture. The EM pre- and post-tests were designed to ask the same questions worded differently and in a random order. The test questions included specific material readers should learn from the EM Booklet. At least 25\% of the test questions were on material from the session that wasn't covered in the EM Booklet. This allowed us to determine the effect of previous knowledge about DOE activities. Of the 17 interns, 14 turned in both pre- and post-tests.

\section{EM Booklet Test Results}

We answered three questions: (1) Did we bias the interns' post-test scores by giving them a pre-test?; (2) Did the lecture and the EM Booklet teach the interns material they did not already know?; and (3) Did the lecture affect how much the interns learned from the EM Booklet?

The pre-tests did not affect the scores on the post-test. Taking the pre-test did not clue the interns in on what to study in the lecture and the book. The average post-test score was $75 \%$. This was significantly higher than the pre-test scores. We concluded that the lecture and the EM Booklet taught the interns materials they did not already know. The sample average improvement in their test scores was $20 \%$. Finally, test results showed that the lecture did not affect test scores and was not an effective tool for reinforcing the EM Booklet. The $20 \%$ improvement in test scores was $100 \%$ attributable to the EM Booklet. *

\section{EM Fact Sheets Test Method}

Our objective in testing the EM Fact Sheets was to explore their effectiveness as teaching tools, evaluate whether exposure time affected learning, and test whether attitude affected knowledge acquisition. We used a group of 15 undergraduate college students who had completed one or more semesters of college and did not have a vested interest in learning about EM. We tesied four of the 35 existing EM Fact Sheets: Resource Conservation and Recovery Act, Environmental Restoration Activities at Feed Materials Production Center, Waste Management Activities at Idaho Field Office, and Radioactive Waste. We measured the student's baseline knowledge about EM with a pre-test. The test questions reflected specific material readers should learn from the four EM Fact Sheets. The posttest asked the same questions differently and in random order. We also included opinion questions about radioactive waste and nuclear power.

The test was set up in a randomized complete block design with each block receiving one of two treatments. Group One was given five minutes to read each fact sheet in a mockexhibit environment (people talking, exhibit materials on the walls, TV, displays, etc.). They received a post-test four or five days later. Group Two took the four EM Fact Sheets home and were instructed to read them twice for 10 minutes each a few days apart. They received a post-test four or five days later.

* For question one, we applied the Smirnov two-sample test (16, Table 17) to the data with $\alpha>0.2$, $(T=0.4545<0.5917)$. For the second two questions, we applied the Wilcoxon Signed Ranks test (16, Table 7). Question two, $\alpha<0.005$, $(T=0<.6)$; question three, $\alpha>0.2$ (and $\alpha$ close to $0.4,18<\mathrm{T}=22<23$ ). 


\section{EM Fact Sheets Test Results}

We answered five questions with this test. (1) Did the two groups have different post-test scores (in other words did time exposure affect test scores)? There was no difference in test scores based on length of exposure time for this test with this audience. (2) Did the EM Fact Sheets teach the students information they did not already know? Test scores increased by $24 \%$ from the pre- to the post-tests for both groups. The average pre-test score was about $19 \%$ (11\%-34\% range) and the average post-test score was about $43 \%$ (11\%-65\% range). (3) Did attitude affect test scores? There was no difference in test scores based on attitude for this test with this audience. However, it appears that access to the information did not negatively impact this audiences' opinions about radioactivity or nuclear power. (4) Were test scores higher for different topic fact sheets? No. Based on this test, none of the four fact sheets was more effective than the others. (5) Were more questions answered correctly when based on information at the beginning and end of a fact sheet than the middle? We found no discemible pattern showing more information was remembered from the beginning and end of the fact sheets than the middle. *

\section{DISCUSSION}

EM is on the right track. Based on our test results and existing literature on public involvement, we can infer that EM is somewhat effective in meeting its goal of providing correct information to a select public.

The test groups in these experiments are comparable to the audiences for the EM Exhibit--mostly college-educated professionals, some of whom have never heard of DOE. Based on our test results, we can conclude that the EM Booklet and EM Fact Sheets are effective in communicating information to college-educated groups. We also can infer that EM Exhibit attendees learn from their experience. For the EM Booklet a $75 \%$ average grade and $20 \%$ improvement is acceptable. However, for the EM Fact Sheets, while a $24 \%$ increase in test scores is significant statistically, the average posttest score was $43 \%$. Thus, about 57\% of the information was not communicated to these groups.

EM publications need to be written at a level that is understandable by the public. DOE's written communication should be written at the simplest reading level possible, even for college-educated groups, because reading levels are

* For question one, we applied the Smimov two-sample test (16, Table 17) to the data with $\alpha>0.2$, $(T=0.214<0.482)$. For question two, we applied the Wilcoxon Signed Ranks test (16, Table 7) with $\alpha<0.005,(T=1<16)$. For question three, histograms of the conditional probabilities were compared. For question four, we applied the Friedman test (16, pp. 264-7) which is approximately chi-squared with $\alpha>0.25$ and three degrees of freedom (chi square $=2.8<4.108(16$, Table 2)). For question five, histograms of the conditional probabilities were compared. 
so low in the U.S. (17) * (The average reading level for college-educated people is said to be about 10th grade high school.) In addition, scientific literacy in the U.S. is low, and the public lacks basic scientific knowledge (18). These gaps make it even more difficult for DOE to communicate and for the public to understand highly technical and complex information. This is especially true with the topic of radiation, which is emotionally charged and misunderstood by the majority of the public (19).

In general, people remember only $10 \%$ of what they read, $30 \%$ of what they see, and $50 \%$ of what they read and see (20). Thus, pictures are invaluable aids in communicating and should be an integral part of the text to help people of all reading levels comprehend complex information. Our test result of about $20 \%$ improvement in test scores may be related to the plentiful photographs and illustrations used. However, this result also could be attributed to the reading level difficulty. For example, since our fact sheet test showed no difference in test scores based on time exposure, it's possible that the material is so hard to read that a saturation point is reached and test scores wouldn't improve no matter how much time the reader had. Further research is needed to determine which attributes should be changed to improve EM publications. For example, we could reduce the number of facts per page, add more illustrations, reduce information and word complexity, and add summaries.

Perhaps more important than reading level and even publication design is the distinction between credible information and authentic communication. Research in risk communication has found that credibility "depends less on the packaging of information than on the quality of the relationship" between the communicators. Information communicated by those in a relationship that nurtures mutual trust and respect is more likely to be believed no matter how it's packaged (21). Thus, EM's publications must work in concert with a public involvement program to create an authentic relationship with the public. EM also must consider that educating the public may improve public perception of DOE, but it does not guarantee public support of DOE's activities or the activities of the commercial nuclear industry (21).

\section{CONCLUSION AND FUTURE RESEARCH}

EM needs more research answers to improve its public outreach effort (15). Literature on learning and public participation supports EM's idea that educating the public is the best way to improve public perception and facilitate public involvement. By educating the public, EM is creating an initiated audienc. that can begin to understand the programmatic and technical aspects as well as the constraints of its program.

* According to the 1990 Digest of Education Statistics, a little less than half of college educated young adults (and only about 10\% of high-school graduates) are highly literate as measured by ability to comprehend prose, understand documents (such as maps, schedules, etc.), and calculate quantitative information (such as tips) (17). Ability to learn facts is related to reading level as well as education, interest, attitude, social factors, etc. 
EM communication tools can be evaluated to assess and improve their effectiveness. Some specific topics to investigate include: improving information portrayal, determining the best number of facts per fact sheet, and conducting an audience analysis to form a matrix of tools and audiences that will work well together. Another topic is whether and how proximity to a DOE site affects interest, understanding, and interpretation of EM-related information. Effective communication may differ significantly for this population compared to the general public. Another area that needs further investigation is whether and how attitude affects leaming rate. Additionally; EM's communication tools could be revised to reflect recent research findings that show the public responds better to scientific facts and information about safety than attempts to "naturalize" radioactivity or compare it to familiar or common things $(19,21)$.

EM needs to expand its outreach to the general public. The EM Exhibit is currently aimed at college-educated audiences. EM needs to inform the public at all levels, especially those who live near DOE sites. In addition to testing EM Exhibit audiences, a sample of the general public should be tested. These tests could be used to evaluate which audiences leam best from the EM Exhibit, and what kind of dialogue has a positive impact. These tests could also be used to determine how the information needs of the general public differ from those of EM Exhibit audiences. Research results would help EM evaluate what its audiences and purposes are, whether it is using the best communication tools, and how to improve its public outreach effort. 


\section{References}

1. U.S. Department of Energy-Richland, "Mission Plan Public Involvement Research and Planning Task Report (draft)," (January 7, 1992).

2. U. S. Department of Energy, Environmental Restoration and Waste Management Five-Year Plan, DOE/S-0070, (1989).

3. Nuciear Waste News, "Slants \& Trends," Vol. 11, No. 48, Business Publishers, Inc., (December 5, 1991).

4. U.S. Office of Technology Assessment, Complex Cleanup, OTA-0-484, (February 1991).

5. BLOWERS, ANDREW, DAVID LOWRY, AND BARRY D. SOLOMON, The International Politics of Nuclear Waste, St. Martin's Press, (1991).

6. BRANCH, KRISTI, CAROLYN E. ORIANS, AND GREG HOLTHOFF, "Literature Review and Analysis: Public Involvement To Support Hanford Mission Planning (draft)," The Hanford Mission Planning Project, U.S. Department of Energy-Richland Operations, (December 4, 1991).

7. PAVLIK, JOHN V., Public Relations, What Research Tells Us, Sage Publications, Beverly Hills, (1987).

8. BISCONTI, ANN S., "Public Attitudes About Nuclear Waste," Nuclear Plant Journal, (July-August 1991).

9. JACKSON, JACKSON, AND WAGNER, "Strategy and Plan for Coordinating and Focusing Outreach and Public Participation (draft)," (August 15, 1991).

10. PRICE, TOM, "The New American Way," Cox News Service, Roanoke Times and World News, (February 9, 1992).

11. DILLON, RONNA F., AND ROBERT J. STERNBERG, editors, Cognition and Instruction, Academic Press, Inc., (1986).

12. KOBALL, ELIZABETH, unpublished report and literature review on risk communication, (October 1, 1991).

13. PRICE, GAYLE DAWN, "The Easiest Way To Improve Your Writing," Grammatik IV User's Guide, Reference Software International, (1989).

14. MENCHER, MELVIN, News Reporting and Writing, Wm. C. Brown Publishers, (1984).

15. HELMINSKI, EDWARD, "On the ERWM PEIS Scoping Sessions, Pat Whitfield, DOE ERWM," interview, Weapons Complex Monitor, Exchange Publications, (February 25, 1991).

16. CONOVER, W. J., Practical Nonparametric Statistics, John Wiley \& Sons, New York, (1980). 
17. National Center for Education Statistics, Digest of Education Statistics 1990 , (February 1991).

18. MILLER, J., The Public Understanding of Science and Technology in the United States, 1990, A Report to the National Science Foundation, Northerm Illinois University, Dekalb, (1991).

19. BISCONTI, ANN AND ROBERT LIVINGSTON, "Let's Talk About Radiation," Nuclear Industry, (Fourth Quarter 1991).

20. SHORTLAND, MICHAEL AND JANE GREGORY, Communicating Science, A Handbook, Longman Scientific \& Technical, UK. (1991).

21. GWIN, LOUIS, Speak No Evil, The Promotional Heritage of Nuclear Risk Communication, Praeger, New York, (1991). 


\title{
Research and Development of a Method for Determining Strategic Information Needs of Managers
}

\author{
R. Martin Jones \\ Virdinia Polycectnic Institute and Stave University \\ Timothy J. Oreane \\ Otharom Stav University
}

\section{AEOTRACT}

Classical informedion ryserm dovelopment approaches have fallen shon of providing useful, informution suppon to menagers engazed in stravefic decision maleine. We need methodologies for studyins the stravegic information neads of manasens 10 determine If strate. sic information needs are predictuble and if enx mothodolosy could be successulu.

Using situation exerctses. we have developed a verbal protocol analysis cectrique besed on strategic problem formulation theories and LISP syntax atructures to identify and chanceserize the sintegic Informetion needs of managen. Wo briefly describe the elementary mental activitiea model theory developed a the besis for the methodolory. We describe the experimentel methodolosy and present examples and results of an experiment using the methodolozy 10 determine the strasegic information needs of Nuclear Covernment. Oversight-A pency managen.

\section{INTRODUCTION}

The broed question of Intereat is. "Is there ny methodology we can use 10 detign successful, strutegic decision support tools?" We begin by examining the clasedeal desien approuches to information systems development.

\section{Cleneleal Dedern Approsectre}

Classical design approsebes for information systems can be erouped into three besic approeches: architectural, systems modeling, and antificial ineelligence. The archilecturl approaches aim for the user to cell the systema developer precisely what information the user needs. Once the user and the information sysuems archilect have agreed on the precise output of the proposed information sysvem, syeterns people deaign and bulld the computer-besed package to specifications. The syotem modeling approsches provide infor. mation besed on andyals and modeling of the user's external processes. There are two hundamentel modaling activities within this group. Fira, there ere models of the user's physied processes such as a manufacturing operiotion. Then there are the models of the data resulting from either modellag the opention and/or data collection from the sctual opentioa. Duts or information modelling is an exercise of the system enaly to beild an information sysiem capable of providing all poselbls information formed from a siven set of date Artifictel intelligence eppronches focus on modeling the user's thinking or reseoning proceseses. The artificial invalligence approach implications for informution symem desizners is principally in the erea of expert syotems.

\section{The Problem of Andlelpating Stratejle Information Need.}

Architectural aps rosches place the burden of anticipating information needs on the user. An sasumption mode by the archilectural approaches is the manager can anticipate and verbalize information needs. The approach of prototyping addresses this problem by iteratively providing the manager with examples. A difficully with the architectural approach is the manager's strategic information needs appear to change repidy. Thus, the syeteme doveloper it condinually re-designing.

The system modalling approacties remove the bunden of extlctpa tion from the user by strempting to enticipen pacible intormeito needs through modeling the used's phyileal symem. One problem with systems modaling lends to be the mestive smount of laforma tion and size of compuser-bened syetems uned to genernes dection support information. The qualthatve physics cpipoeches anempt to address this (Falkenhainer a Forbus, 1988). The difficulty remaina determining what to model and to whit lovel of detell and eccurecy.

Artificial intelligence epproscties andicipan the user's need for information by atempting to cappure the uer's thataing in one form or another. The focus has been on repllexting twama chiaking using expen systems. In ex expen syesem, the problem spece doen't change. Therefore, this approsech is not directly appllesble to the manager fecins anategic problems.

\section{We Need Purther Study of Stratede Information Neede}

To determine if any methodology will rewh in accessully sup porting surategic decision maluing, we mad fursher study etrabegic information noeds. If the straceic informedion needs of a manacer vary significantly from problem to problem. then we should not spend any effor developine compuser-based decision support tools. If there is a cone set of informmion ecroes problems for a diven manager, then a computer-based decision suppon tool may be appropriate. If there is no cone set of information needs scross different manazen. then a computer-based decision suppont tool must be developed (or tilored) for each manaper. Such a situation may be cost prohibltive.

We need a methodology that enables us to study menagen' strate. sic information needs in enough detil to decermine if patems exint in informetion needs seross strulegic problams and/or ecross managers. To develop such a methodoloy, we must dovelop en under.

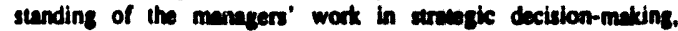
develop a theoretical basis, and develop the secocialed methodolo sies to determine stritegic information needs. Applying these methods should then provide the dets neaded to explore the charecteristics of the information needs. This peper presents research conducted to develop the theory and methods needed to study the stratesic information nects of muclear govemnen-over. sigh-agency (GOA) manaters (Jones. 1990). Wheress the results and conclusions of the study are renardizable only to mucleas. GOA manazers, many ere supported in the liverature as epplying 10 managers in general. The methods and theory developed should apply to any set of manapers faced with strategic problems.

\section{CONTEXT}

Figure I shows an overview of a coniext modal for strategic decision making. This context modal was adepted for strategic dectsion making from the Kursted et al. (1989) modal of decision making. The context model shows a manager faced with suruegic problems engaging in some mental acitvitios called strategic decision pro- 
cesses that result in a strategic decision. The manager's chnmeteristics, e.8. resources, imelligetice, experience, personality, and capability, affect the nature of the strategic decision processes. The manager may use external support in the form of decision tools during the strategic decision processes. The manager requests information from the decision tools and/or provides information to the decision tools. The decision tools collect, store, menipulate, and provide information to the manager to support the manager's strategic decision processes. The environment for straiegic decision making include the manager's domsin of responsibility and reality.

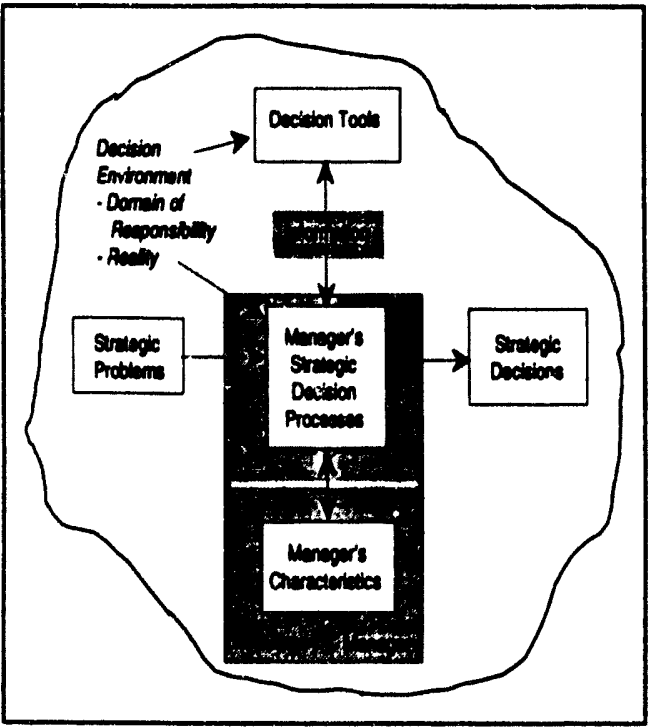

Figure 1. The Context Model for Strategic Decision Making

The stmtegic decision making context model provides a systematic means to break the global problem into smaller, related problems. Some of the elements are discussed in more detail in the following sections. This study is focused on a set of manasers' strateric decision processes in such a way as to determine the information the managers use when faced with a given set of strategic problems. Therefors, the managers' characteristics and how those characteristics might affect the decision processes is not within the scope of the study. Neither is the nature of the decision(s) made. For the experiment, there were no decision tools nor extemal inputs of information to affect the decision processes. For this discussion, we will only discuss the strategic problems and the decision processes elements.

\section{Stratede Problems}

Strategic problems are situations which have or are perceived by the manager to have the real or potential effect of altering the dlobal rules by which the manager's organization operntes. The characteristics of strategic problems are well documented in the literuture, e.s. Mason and Mitroff (1981). Many researchers agree on the eeneral definitions for strategic problems and have contributed additional characteristica of strategic problems. For example, Mintzberg (1973) adopted these characteristics in his work which documented that strategic problems reach managers as units of information.

\section{Manager's Strateete Dectalon Proceswes}

In the past, there have been no models of the strategic decision process that provide the level of understanding of information needs nec essary to research decision tools to support an individual manager's strategic decision making. A distinction is made in this research between supporting managers with the information they need and use and supporing managers by replicating or reprogramming how they make strategic decisions. This research does not attempt to replicate or reprogram how managers make strategic decisions.

The body of literature on strategic decision processes has been recently reviewed by Huff and Rezer (1987). This body of literature focuses primarily on orzmizational processes and group processes rather then the thinking proceseses of concem in this research. Process studies of people's thinking have grown out of the field of comitive psychology (Pinget, 1974) into the research of the decision theorists (Simon, 1960; Newell \& Simon, 1972). The dectsion theorists' works have focused on deccribine and replicaing the decision making processes used for well-defined problems (Simon, 1987). Strntegic decisions result principally from decision processes addreswing araiejic problems, which are typically ill-defined problems (Meson \& Mitroft, 1981). Strategic decision proceseses involve judement and intuition (Simon, 1987). A few researchen have linked the wotk of the decision theorists with strnietic decision processes (Pounds, 1969; Ramaprased \& Mitroff, 1984), but have not focused on linking the stratefic decision proceseses with the neture of information needs.

\section{THEORETICAL BASTS}

The elementary mental sctivities model wa developed as a theoretical basis for liniding struedic decision processes with information (Jones, 1990). The theory is briefly deweribed to provide a foundution for the development of the methods. A more complete description of the theory is presented by Jones (1990). The elementery mentel ectivities model is an extension of Pounds' (1969) model principally using Ramapresud and Mitrofi's (1984) model. The reevil in relaing the two views of strucale problem formulation is then it is posdble to describe the steps of struecic problem formulation sa iterations of a simple set of elementery mentel cetivities releted to the processing of besic iypes of informetion. The mental sctivities chosen are based primarily on Pounds' (1969) work.

The term elementary is used to distinguish these mental sctivities a building blocks upon which stracegic problem formulation is bused. Newell and Simon (1972) in their work on replicating humen problem colving and others in the fiald of expent systems (Greenwell, 1988) represent basic mental sctivity using set of primitive functions. Primitive functions are more detailed than the elementery mental activities proposed here. Primidive functions ere insended to replicate mental activity. Elementery mental sctivities are intended to represent the major mental activities in relation 10 information. As such, elo mentary mental sctivities represent eroups of primitive functions.

In the elementury mental setivities model, there are five types of information the manager uses in the manager's strntegic decision processes. They are entities, relationships, operators, desired outcomes, and model outcome predictions. Entities, relationships, end operators along with their atributes are propoed as the three besic lypes of information. These form the manarer's perception of external information. The manager chooses cervia values of entities, relationships, and operators as desired outcomes. The ateumption is the even though the model outcome predictions are generned within the manager's thinking procestes, they can also be represented by the three besic types of infortstion.

An entity is "something that has sepante and diatinct existence and objective or conceptual reality" (Webster's, 1988). Relationships se "Josical or causal connections" (Webster's, 1988) between entities. Operators ere special forms of relationahipe where one or more entities act upon one or more other entities to bring bout change. Attributes of entities, relationships, and operesors are inherent cheracteristics. Viewine reality $\mathbf{s}$ ensitics, relationships, operators, and their atributes has been the focus of syetems engineering (Blanchard \& Fabrycky, 1981) and systems andyeis and design lechniques (Chen, 1983).

\section{METHODOLOGY}

The overall approach closely perdlels the direct reserch spproach Henry Mintzbers (1973) used to study five chief executive oficees to derive his theory of the nature of manaperial work. The benic difference in Mintzbers's work and this research is that Mintebers studied the broad, visible activitics of managers. This reaench studies the more narrow, mental activities of manarers' thinking and the infor. mation needed to support decision makins. As such, this research addresses a criticism of Minzzberg's (1973) work. Carroll and Gillen (1987) suseest that manageried work is ments and not directly observable. There should be value in audying both mental activities and physical sctivities as managerial work. 
However, this difference in study objectives results in the application of different, basic, data collection methodologies. At the heart of Mintzberg's study wes the structured observation methodolosy to collect data about the visible setivities of the subjects. At the hean of this research is the verted protocol methodolony to elicit data from the subjects about the information they need to make strutegic decisions.

Verbal protocol methodologies dictave the design of antificial stimuli and of an antificial environment for elliciting the dau from the subject. i.e. the design of an experiment. This differs from structured observation methodolozy that Mintzbers (1973) used which allows study of the managers in their natural environment. Therefore, a methodology was developed to engage managers in atrategic problem formulation using scenarioe and role playing.

The experimental procedures were refined in a pilot study. Five man agers were chosen as subjects for the final study. Each subject was presented the same five strategic exercises consisting of a base doman description and a scenario of events. The subjects were asked to think aloud as they formulated the problem and arrived at decisions. The subjects' verbalizetions were recorded.

\section{Coding the Verbaj Protocol Date}

The verbal protocols recorded during the experiment were transcribed and then translated. Translation was necessary to; resolve incomplete or unclear references, resolve multiple references to the same thing using different words, simplify compound and complex sentence structures, and eliminate non-essential words and phrases. Translation required extensive domain knowledge. Transiation also introduced the greatest amount of subjectivity in the experimental process. Once translated, the protocols were coded using LISP-like syntax and coding rules. Figure 2 shows the three forms for a segment of a protocol.

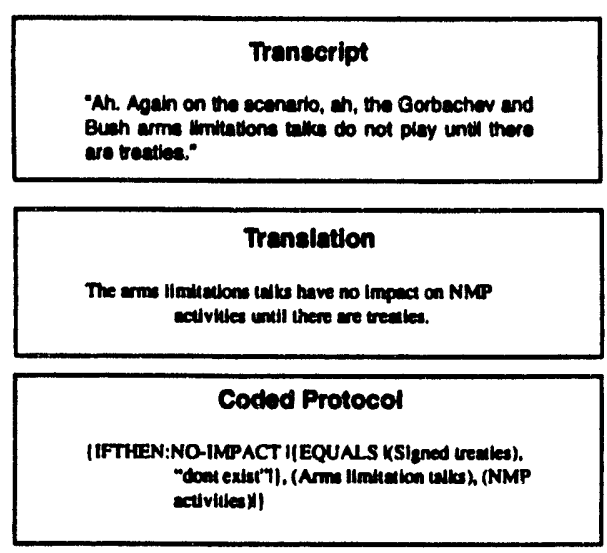

Figure 2. Portions of a Verbal Protocol.

Computer programs were developed to assist in the coding and validating of the date. Unique lists of each type of information were generated. The data base of coded protocols contains 1,230 coded sentences with unique lists of 276 values, 131 atributes, 270 entities, 870 relationships, and 1,011 operamors.

\section{Ascigning Information Characterbates}

A number of characteristics were assigned to each instance in each type of information. For example, entities were characterized by:

1. Source: where the entity originated--whether in the base description, one of the scenarios, or from a subject:

2. Level of detail: the grain size of the structural description necessary to yield the entity as a unit of information:

3. Class: the generic group of entities to which the specific entity belongs; and

4. Internal/extemal: whether the entity exists within or outside the laboratory domain of responsibility.

\section{RESULTS}

The results from the andyais of the exit interview showed the experimental scenarios were strategic and realistic. The subjects stated the scenarios represented the breadth of the atralegic problems they faced in their natural environment.

The data analyses performed showed a few paterns in tho use of units of information for a given subject. The analyeses filled to reved any significant patterns of the use of units of information scross all subjects. However, even with the limited number of information chancteristics assigned to the units of information, several useful patems of characteristics of information wexe devermined, not only for a eiven subject, but for all subjects.

Some examples of specific resules are:

1. A core set of entities was more consistent within subjects. The definition of use for this andysis was the binary messure since the concern was simply whether or not the subject ured the unit of information in a number of exercises. When the criterion for accepting a unit of information as pert of a core set was established as "used in three out of five scenarios," the avernge number of entities in the core set was 7.4. Only two entities appeared in a core set across all subjects.

2. Subjects used more level 1 and level 2 entities than other level entities and the greatest level of detailed endities used by subjects was level 5.

The definition of use for this analysis was the binary measure. Subjects used 564 level 1 and level 2 entities in the 25 exercises and only 131 emities more detailed than level 2

\section{CONCLUSIONS}

The following conclusions about nuclear COA managers engaged in strategic decision processes are drawn from the full set of results. The conclusions are stated in managerial terms. However, the scope of the research allows generalization only to nuclea GOA manazen.

1. Managers have individual approaches to strategic problems which can, in part, be charncterized by

- the amount of time they spend understending the situation versus the time spent considering managerial actions.

- the entities of concem to the manager in most atruegic decision situations, and

- the complexity of the relationships and operators they employ in the stratezic decision process.

This conclusion is supported by the work of Ramaprased and Mitroff (1984) to link strutezic decision processes with Plazet's (1974) theories of learning and the Jungian perzonality typology.

2. Units of information presented to the manager trizger the need for and use of more information that can be characterized as:

- lop-level.

- status information, and

- more extemal information than incernal information.

This conclusion is supponed by the work of Mintzbers (1973) and Gorry and Scolt Mortion (1986).

3. Managers focus as much or mure of their decision processes on dealing with internal information as extemal information.

There was no reference 10 this conclusion found in the literature reviewed.

4. Managers are more concerned with people, facilities, and products than with finances in all levels of detail and in the most detailed levels of information.

This conclusion differs from the central focus of financial information support for strategic decision making found in the liternture, e.8., Keen and Wagner (1979), Treacy (1986), and Schwenk and Thomas (1983).

5. Managers attempt to re-balance their domain of responsibility 
with the domain eavisonsmen by:

- determining the cumen situction tha exists between thetr domein of reaponsibility and the domita environment, and

- detemining ways to slier the siturion.

This conclusion is expported in the lleratare by a number of rescarchers in struegic dactsion makjing, e.e. Mintebers (1973), Gorry and Scok Monon (1985), and Simon (1960).

\section{IMPLICATIONB}

The results have the following general implications for designing and developing decision tools to suppon mucles GOA manavers engerged in strmegic decision mating:

I. Systems can be developed to collect, nore, manipulare. information in anticipation of struedic problems fecing strectic manazers;

2. With today's state of undertending of the manaper's information needs, the support exyetem will not provide all the information needed at the time the information is desired by the manazer.

3. Half or more of the information maintained and provided by suppon systems must be sbout entities outside the manager's domin of responsibility:

4. The primary focus of the information maintained should be on top-level status information bout people, facilities, and products;

5. The most detailed data should be level 4 or level 5, internal and externd facility date:

6. The suppont system munt have the copability to predict the impact of mangerial alcentions on people, facilities, and products; and

7. The support system must be cepable of dealing with fifth onder relationships and staributes of relationahips and operntors.

General Implicatlon:

The methods developed force the systems designer to focus on the manazer's domain and strutegic problems before any systems ansly. sis ectivity begins. The systems designer must understand enough about the manager's domain to develop stretejic scenarios the man asen agree are both atriegic and realiatic. These sctivities should be pert of any methodology for developing information systems to support managers. Ofien they are overtocked or execuled quictly.

The methods developed result in a large amount of detailed inform: tion using only two to three hours of each manager's time. Andyeis of this information points the systems deaimer in the rithe directions for design, development. and implementution of information syutems. In the case used in the experimen, it is obvious the manegers needs point to high-level facilities models of both insemal and extemal com plexes coupled with a system of incellizence stahering for external ortanizations 10 maintain sturus information. The andysis of the coded data points to spectfically which organizutions, people, and facilities. Considering the usase of the information scrose subjects and exercises provides the syotems designer with the relative imporince of developins systems to meintnin detn versus the one-time collection of data a the time of need.

\section{TURTHER RESRARCH}

Probably the most useful metivity would be to follow through with the actual design, development, and implementation of information sys vems based on the andyeis of the data reaulting from the application of the methods developed. The process of developing information systems would provide the necessery feedback 10 enhince the methods.

It may be pussible to revise the coding syntax used to allow direct input of the coded protocols into either entity-relationahip databases or LISP-type languages to capture and manipulace the data. The possible research objective should be to develop decision suppon advisor systems rather than expert systems to replicale the managers' thinking. The experimental methods capture valuable insights into strategic problem formulntion within the domain. It should be possible to use this data to begin to construct valuable databases and expen advisor systeras to help managen ensure they consider all the aspects of the situation at hand. Such systems could also be used in training managers to face real-world strategic problems.

\section{ACKNoWhedogmants}

The preperation of this peper was funded by the U. S. Depertuners of Energy (DOE) under erent nomber DE-FC02-88DP48058. The anthors thank DOE menaeen for providing the suadting and for perticipating in the experimeats 10 endy stranedic informetion neede.

\section{RETERENCXS}

1. Blancherd, Benjamin S., and Wolter J. Fabrycky. Smitm Ensincerine nnd Anelwit. Englowood Clifi: Preadice-Hall, Inc., 1981.

2. Carroll, Steptien J., and Dernis J. Gillen. "Are the Clasoical

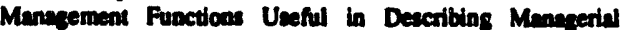

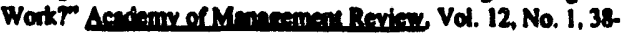
31.

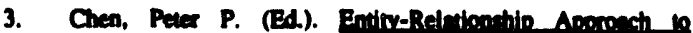
Informetion Modeline nd Antyris. Ameterdem: North Hollend Poblishing Compeny, 1983.

4. Falkentainer, Brion and Kenoeth D. Forbes. usenting up Large-Seale Qualitadive Models." Procedinne AAAls?

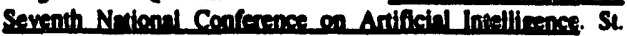
Peul, Americen Associadion for Artificial Intelligence. 1988. 301-306.

5. Gorry, O. Anthony, and Michnel S. Scoul Morton. "A Framework for Mannememen Information Synems." In John F.

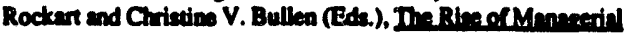
Comontin: Th. Bent of The Center for Informetion Swrem

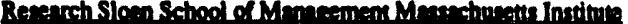
of Technoloses, Homewood: Dow Jones-Irwin, 1986.

6. Greenwell, Mike. Knoviled en Ensinerine for Brben Syntem?. Chinchester. Eltis Horwood Limited, 1988.

7. Jones, R. Martin. The Strategic Dectaion Processes and Informetion Needs of Nuclear Covernment-Oversichti-A gency Manazers." Ph.D. Dissertation, Depertment of Industrial Engineering and Opermions Reseanch, Virginin Polytectnic Institure and Since Univendity, Blactesbure. Virviaia, December. 1990.

8. Keen, Peter G. W., and G. R. Weener. "DSs: An Executive Mind-Suppon Sysiem." Denmmion, Noveruber, 1979, 117. 122.

9. Kurrled, H. A., Jr., Thomas E. Polk, and Janes E. Hughes. "A Modified Decision Strmery Selection Mecheniem" Proceedines of the Southern: TMMS, vol 19, October, 1989, 59 . 62.

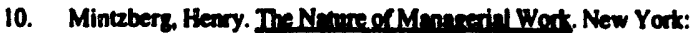
Herper \& Row, Publisters, Inc., 1973.

11. Newell, Anthony and Herber A. Simon. Himm Probitem Solvine. New Jerney: Prentice-Hall, Ine., 1972

12. Pinget, J. Undemindine Comelity. Now Yat: W. W. Nonion a Company, lac. 1974.

13. Pounds, William F. "The Process of Problem Finding." Industrill Menneement Review. Vol. 11. No. I, 1969, 1-19.

14. Ramaprased, Artelgud and lan 1. Mitroff. "On Formulating Strue dic Problems." Acedemy of Mmemmen Review, Vol. 9. No. 4, 1984, 597-605.

15. Schwenk, Charlea, and Howerd Thoones. Formulating the Mess: The Role of Decision Aids in Problem Formulecion."

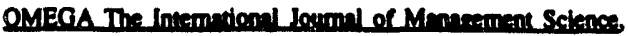
Vol. II, No. 3, 1983, 239-252.

16. Simon, Herber A. The New Sclence of Mmrnement Decirion. New York: Happer Row, 1960.

17. Trescy, Michnel E. "Supporting Senior Erecutives' Models for Planning and Control." In Joha F. Rockert and Christine V. Bulten (Eds.). The Rire of Menneredil Computins: The Bent of The Center for Information Syrtems Rezerch Sloan School of

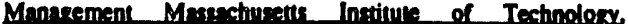
Homewood: Dow Jonee-Irwin, 1986.

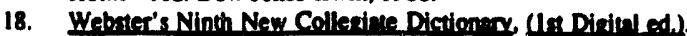
NeXT, Inc., and Merriem-Webecter, Inc., 1988. 


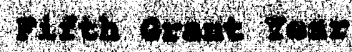

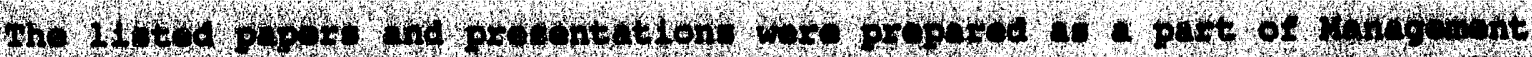

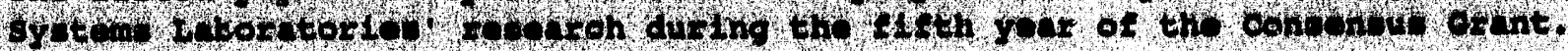

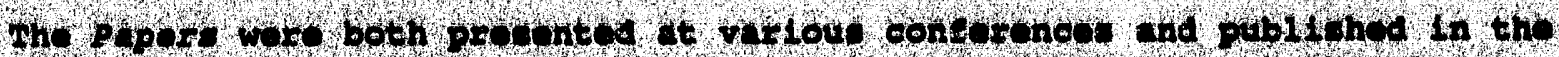

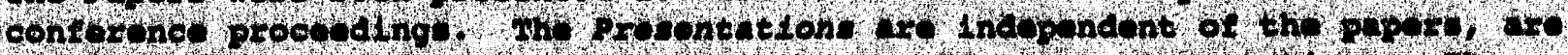

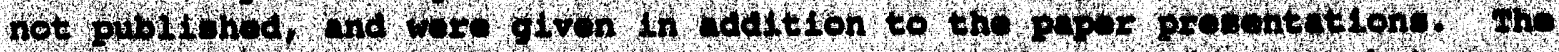

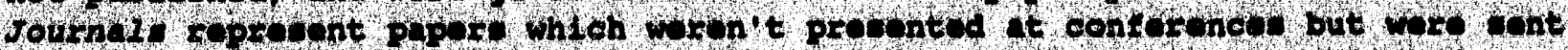

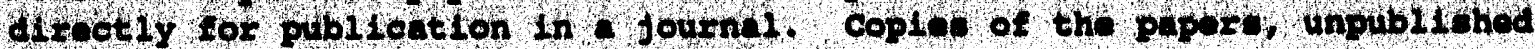
menued fot, And journal artlales follow.

titithe

Papors:

Multienltorla yodele for Analy is of the envitonmontel Rostoration and weoto Hanagemont Program. Computers and Induetrial Enginowelng.

Audience selection tor Interpersonal communication channolo In Publlc Involvament. Programe. Proodedinge of the wavte Hanegument 93 sympostum.

Curront Concepte and Vathodologios for Aeseseing and Communicating Riek in waste vanagement. Proceedings of Environmental Remediation 93 symposium.

Tool. and wethods for Involving the Public in Riek-Based Decleion vaking. Proceeding of Environmental Romediation 193.

Gleaning Information from opon-Ended Data to support Decision raking. proceodinge of the 29 th Annual reoting, southeastern chapter of the Institute of Management setonces.

Management syeteme Engineering: The Engineering Approach to guelity. southeaetern chapter of the Inotitute of Management sciences.

The Impact of Group Decleion support systems on Group conmonatu Procesees and outcomes. 1994 Annual keeting of the Human ractors and Ergonomics society.

Analyois of stakeholder concerns for the Environmental Restoration and waste Uanagement Program. Proceedinge of the Waste kanggement 194 symposium.

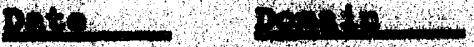

$3 / 93$

$3 / 93$

$10 / 93$

$10 / 93$

$10 / 93$

$10 / 93$

$3 / 94$

$3 / 94$
Corsentus: Procose coneonous proces:

Coneeneue Procese

consenmus Procese

Consoneue

Procens:
Fundamental 


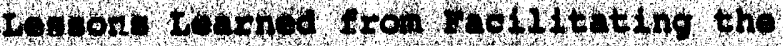

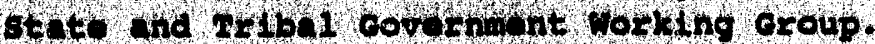
proekdinge of Whet Wongendite 94 .

Unpubilingd Hinuertpter

a Wetrle tor contonmus, dotign and allibeation.

Slmulation of consonous lotelo tost sconarlon.

Tho Effect of Expert knowledge on portormance in a small-croup Doctaton Kaking Taok.

Conder, Tralts, and Influenee in a small Group Doctelon Moking Teek.

Teohnical Roports:

Environmental Benchmarking for Performance Excellence.

\section{Presentation:}

Audience selection for Interperuonal Communication Channele in Public Involvemont. Waste Kanagemont 193.

Analyele of stakeholder Concerns for the Bnvironmental Restoration and waste Management Program. Waste Managnment ' 94.

Journales

Managing communication successfully in Your kanagemont system. Inducteriel Kansorment Vol, 35 .

Environmental Benchmarking as a Tool for Continuous Porformance Improvement. Total Ounlity Bnyirongental Journat.
$3 / 93$

$3 / 94$

Consensue Procene

contohtur Proche

conooneus

Drooen:

rundamontal

Pundomental

Conceneus

Procese

Conceneus

procese

\section{9-10/93 Consensus \\ Proceer}

Spring $\cdot 94$ Consensue
Procese 


\title{
Multicriteria Models for Analysis of the Environmental Restoration and Waste Management Program
}

\author{
Laurence J. Moore ${ }^{\dagger}$, Jack C. Davis $\ddagger$, Aubrey E. Harvey $\ddagger$, \\ John J. Imholz ${ }^{\ddagger}$, Robert J. Mcllwain ${ }^{\dagger}$, and Bernard W. Taylor, $\mathrm{m}^{\dagger}$ \\ tDepartment of Management Science, Virginia Tech \\ $\ddagger_{\text {Management Systems Laboratory, Virginia Tech }}$ \\ Blacksburg, VA 24061
}

\section{INTRODUCTION}

The purpose of this paper is to present research conducted to explore the potential for use of multicriteria math programming models for analysis of the DOE. Environmental Restoration and Waste Management Program (EM Program). The study is conducted by formulation of a prototype model of the problem/system as a mixed-integer multicriteria goal programming model. The research suggests ways to portray the system as a mathematical programming model with multiple goals. Examples of possible models are presented along with resulting solutions. The practicality of this modeling and solution approach is examined, and the solution results are assessed in terms of realism and usefulness.

The research results in the following: (1) a mixedinteger multicriteria math programming model of the problem/system, (2) several examples of applications along with the resulting solution results, (3) an evaluation of how realistic the solution results are, and an assessment of the practicality and usefulness of the approach.

\section{BACKGROUND}

In 1991 the Office of Environmental Restoration and Waste Maragement (EM) of the United States Department of Energy (DOE) developed a five-year plan for environmental management entilled Environmental Restoration and Waste Management: Five-Year Plan for Fiscal Years 1993-1997 [4]. EM was created out of recognition that a sienificant national effort had to be launched to clear up 45 year's worth of environmental pollution from the design and manufacture of nuclear materials and weapons [3, 4]. EM was charged with treating and disposing of the store inventory of radioactive and chemically hazardous waste [4, p. xxxviil.

The five-year plan deacribes the DOE's goals of bringing all DOE facilities and sites into compliance with applicable laws and regulations aimed a protecting public health and the environment, and cleaning up the inventory of contaminated insetive sites and facilities by the year 2019. An important part of this proeram conducted by EM is to treat, store, and dispose of hasardous, radionctive, and mixed waste in an environmentally sound and effective manner.
PROBLEM

An important part of the treatment, storage, and disposal problem described in the previous section is to consider the sites that generate (or contain) waste and the sites that could potentially serve as sites for treatment, storage, and/or disposal (hereafter referred to as "processing" sites). In many cases, sites that generate (or contain) waste could also serve as processing sites. Thus, an important part of the overall EM problem is to determine which sites should serve as processing sites and from which sites should they receive waste to be processed and in what quantities.

\section{MODELING APPROACH}

It appeared that the problem (and system) as described could be modeled as a version of the plane location model that is well known in the literature $[2,9,10,11]$. In this case, the waste processing sites would be the "plants," and the sites that either generate or contain waste that requires processing would be the "destinations" of the plant location model. However, the actual "now" of product (waste) would be in the reverse direction in this case, i.e., the waste would be shipped from the waste generating sites (destinations) to the waste processing sites (plants). This does not, however, detract from the potential for using this model for this problem. Since the plant localion model is a mixed integut piojsabroniting model, the problem requires a solution procedure for solving mixed integer programming models.

It was apparent almost from the beginning that the modeling approach needed to accommodate multicriteria analysis. Considerations that must be incorporated into the analysis of this problem include environmental, legal, technical, losistical, political, economic, and safety considerations. It was further obvious that not all of the considerations could not be reduced to rigid constrints and/or incorporated into a single objective function, such as cost. Thus, multicriteria (god) programmine was selected as the modeling approach of choice $[1,5,6,7$, 8, 12].

The resulting modeling and analysis approach is, therefore, a multicriteria mixed-inieger math programming model. The model formulated for the problem is given in the following section. 
In order to develop a prototype model for addressing the problem in a manageable frumework, the scope of this modeling effort was limited a follows. The category of waste considered is referred 10 by EM as "mixed waste." Other categories included in the EM program are: spent nuclear fuel, high level waste, iransuranic waste, hazardous waste, and low level waste [3]. Presumably the treatment, storage, and disposal of each category of waste would require a separate plan related primarily to the methods of handling required for the specific waste involved. Therefore, a separate modeling and andysis effort would be needed for each waste type. However, it is assumed that the problem to be addressed, in terms of processing site locations and shipping patterns, is approximately the same for each category of waste, and therefore, the modeling approach presented in this paper could be applied to each calezory of waste.

Another manner in which the scope of the problem was limited for this modeling effort is with respect to what is done with the waste at the processing site. The categories of "processing" include treatment, storage, and/or disposal. In this modeling effort, all three categories are incorporated into the single category called "processing." In a real-world application, the modeling approach proposed in this paper could be applied by formulating a separate model for each category of end result - treatment, storage, or disposal. An altemative approach would be to treat each category of processing as a different "site." For example, if site A could be used to treat waste, or store waste, and dispose of waste, then each case would be defined in the model as a distinct "end point," i.e., A-T, A-S, and A-D. This would not change the modeling approach. It would simple increase the size of the model in terms of total "processing" sites.

Thus, the model developed in this paper is for a system with a total of eight sites, of which five of the sites are generation sites, and all eight sites are potential processing sites. The system is illustrated in Figure 1 and Figure 2.
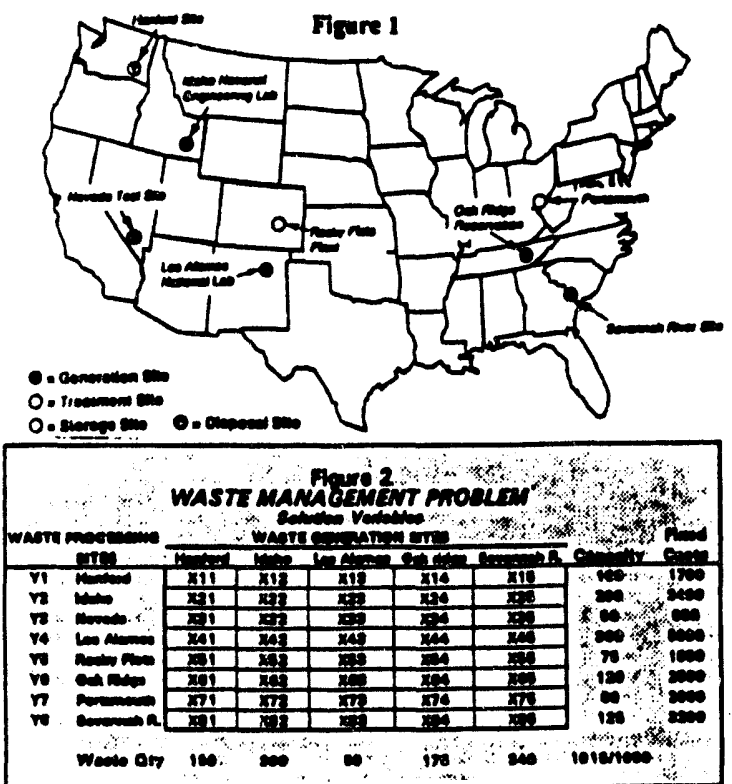

The modeling approach used for the research is a mixedinteger goul programming model with various modifications with regard to goals and priorities employed. The O.P. model is defined in terms of the problem examined, in which there are a total of eight sites, of which five are waste generating siles $(n=5)$ and all eight sites $(m=8)$ are potential waste processing sites. The number of waste generating and/or waste processing sites could be modified without loss of senerality in the modeling approsech. The specific number of sites was defined for this model in order to define the number of soul constraints in the model description below (i.e., $=\cdots$ ). A statement of the problem in terms of the general goal programming model is given as follows:

\section{G.P. objective function:}

Minimize $\mathbf{z}^{*}$

$$
Z=P_{1}\left(d_{8}^{-}, d_{8}^{+}\right), P_{2}\left(d_{8}^{-}, d_{8}^{+}\right), \ldots, P_{N}\left(d_{8}^{-}, d_{8}^{+}\right)
$$

where $Z^{*}$ is an achievement function in which $\mathbf{N}$ is the total number of different priority levels that are used in formulating the goals, and $P_{k}\left(d_{g}^{-}, d_{z}^{+}\right)$is a function of the deviation variables in the $g$ th goal constrint at the $k$ th priority level.

\section{G.P. goal constraints:}

As stated above, the G.P. goal constraints are defined in terms of the problem examined, in which there are a total of eight sites, of which five are waste generating sites and all eight sites are potential waste processing sites. The goal constraints in the model are identified by number below (i.e., $8=7$ ).

Total processing sites fixed cost + total cost of assigning waste from sites $j$ to processing at sites $i$ :

A. $\quad \sum_{i=1}^{8} f_{i} y_{i}+\sum_{i=1}^{8} \sum_{j=1}^{5} c_{i j} x_{i j}+d_{8}^{-}-d_{8}^{+}=b_{8}(g-1)$

Total waste at sites j requiring processine and capacity at sites i for procescing:

B. $\begin{aligned} \sum_{j=1}^{S} D_{j} x_{i j}-S_{i} y_{i}+d_{8}^{-}-d_{8}^{+}=b_{8} \quad(i=1 \text { to } 8) \\ (g=2 \text { t09) }\end{aligned}$

The sum of processing capacities at all 8 sites:

C. $\sum_{h=2}^{9} d_{b}^{+}+d_{8}^{-}-d_{8}^{+}=b_{8} \quad(g=10)$

Total waste at sites j requiring processing related to each potential processing site $i$ :

D. $\quad \sum_{j}^{g} D_{j} x_{i j}+d_{8}^{-}-d_{8}^{+}=b_{8}$

$(s=11$ (0 18) 
Total wate of each generating site assigned to at least one processing site (or more w/split assignments):

E. $\sum_{i=1}^{8} x_{i j}+d_{8}^{-}-d_{8}^{+}=1 \quad 0=1105 ; 8=19(023)$

Total waste at all siles to be processed:

F. $\quad \sum_{j \in a ; b=19}^{23} D_{j} d_{h}^{-}+d_{8}^{-}-d_{8}^{+}=b_{8} \quad(g=24)$

Individual assignment goals:

$$
\text { G. } \begin{aligned}
x_{i j}+d_{8}-d_{8}^{+}=b_{8} \quad(i=1108 ; j=1109) \\
(z=251064)
\end{aligned}
$$

Number of processing sites:

H. $\sum_{i=1}^{8} y_{i}+d_{i}^{-}-d_{i}^{+}=K$

\section{System Constraints:}

Force processing at site $i$ if assignment is made from site $j$ to site $i$ :

$$
\text { I. } x_{i j}-y_{i} \leq 0 \quad(i=1 \text { to } 8 ; j=1105)
$$

Variable definitions:

$$
\begin{array}{lr}
y_{i}=0 \text { or } 1 \text { integer } & (i=1 \text { 10 8) } \\
0 \leq x_{i j} \leq 1 & (i=1 \text { t0 } 8 ; j=1 \text { 10 })
\end{array}
$$

Where:

$y_{i}=1$ if sile $i$ is selected as a processing sile (incurring fixed cost $\left.f_{i}\right)$.

- 0 if site $i$ is not selected as a processing site

$x_{i j}=$ the fraction of $D_{j}$ (sice $j$ waste) to be shipped to site i for procesains

$m=8$ - number of sites at which waste sould be procesced

$n=5=$ number of sites at which waste is generaled

$K=$ number of sites, from among $m$ sites, that will be decignated as processing sites

$f_{i}=$ fixed cost associated with designating site $i$ as a procesesing site

$c_{i j}=$ cost of assigning all of site j's waste to site $i$ for proceasing

$D_{j}=$ quantity of waste at site $j$ requiring processing

$S_{i}=$ capacity of site $i$ for waste processing

\section{SAMPLE DATA}

The dat for the sample waste maragement problem is given in Figure 3. There are a lotal of eight sites, of which five are generation sites (listed across the lop), and all eight sites are potential processing siles (listed along

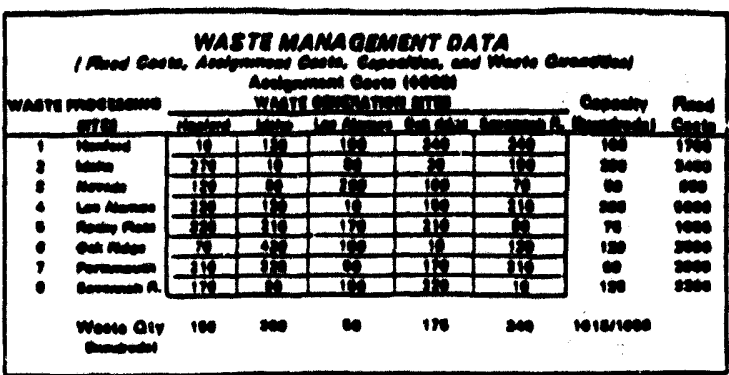

the lef side). The fixed costs represent capital costs and other fixed costs associated with designating a site as a sile to receive and process waste from one or more of the five generation sites. The assignment costs are the costs of assigning a zeneration site to a processing sile, including the costs of loading the indicuted quentity of waste at the senention site, transporting it from the generation site to the processing site, and unloading the waste at the processing site. The asignment costs are incurred when a particular assignment combination is selected. If split assignments are made, then the assignment cost is split accordingly among the processine sites.

The waste quantities needing procesed are given along the bottom for each of the generation siles listed seross the top. The capacity of each potential processing sile is given along the right side next to the fixed costs. The capacities are "potential" capacities, i.e., the processing capacity possible at the site given the various site features that place limitations on the size of a processing facility at the sile.

\section{G. P. MODEL VARIATIONS AND SOLUTION RESULTS}

Seven variations of the G. P. model were formulated and solved (models A through G). Each of the G. P. model variations is described in the complete paper (available from authors) in terms of the model goals and related priorities. In each case the solution is described in terms of meeting or not meeting the specified goals. Then each solution is given in table format, followed by a rapirsotk illustration of the solution results.

Following are illustrations of the information provided in the complete paper concernine the various models which were formulated and solved.

Model A:

Priority: Gonls:

P1: $\quad$ Don't exceed total processing capacity

P2: $\quad$ Meet total dema d for waste processing

P3: Minimize total cost

\section{Solution Reaults:}

Priority: Gols:

P1: $\quad$ Met; 35 hundred tons excess processing capecity at Portsmouth site

P2: $\quad$ Met; totil of 1015 hundred tons processed

P3: $\quad \$ 19,897.61(000)$ 
The solution results for modal $A$ are summarized in Pigure 4 and illuetreted in network form in Figure $s$.
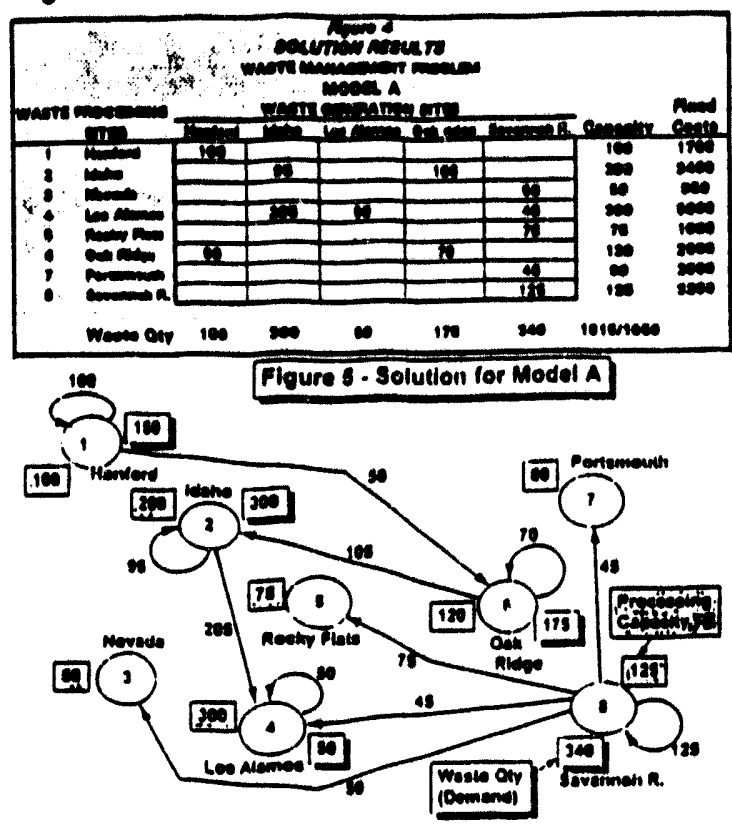

EVALUATION

One of the objectives of this research was to evaluate the modeling approach and the resulting solutions in terms of realism. Firs of all, the modeling variations that were used were limited to relatively few prioritized goals in order to make it easy to assess the validity of the model. It seemed rather apparent that the model solutions yielded results that were expected given the soals specified. Thus, the model and solutions were realistic (valid) as fur as the solutions to the formulated modets were concerned.

The model was, of course, limited to consideration of one callegory of EM waste, and the system definition was also limited to one catezory of "processing" at each site. Therefore, to be iruly realistic, a model (or , models) would need to be formulated for each of the calegories of waste. Also, to be truly realistic, the model would need to eccount for all three possible forms of "processing." including treatment, storage, and disposel. This might the the form of separate models for each type of waste, or is might whe the form of a single model, with separate "proceseing sites" defined for each end result (treatment, storage, and disposal). In either case, the result would be more models of a similar form, or a larger model of a similar form.

In terms of practicality and uefulness, the model is relatively eary to formulate and solve. Therefore, in this sense, it is relatively prectical. However, a critical element in making the approuch useful would be to obtrin timely and sccurate data concerning the parameters of the problem, and accurate stuternents of the reatrictions and coals of the system. The model would be useless without sccurate statements of constrints and goals. On the other hand, this could be an iterative leaming process in which the models would get better over time as the users leamed more about the modeling and analysis procedure and the system itself.
Another issue in aseacing the userulness of the approsech is the quations of whether if would be secepted by zovernment adminiatrators and others involved in makins decisions about EM waste processing. Since it involves the use of some rether esolerte lechniques, it might viewed with suspicion and skepticism by those responsible for making decisions concerning EM waste management.

\section{FURTHER RESEARCH}

Further research is needed to determine the value and usefulness of this modeling and analysis approach. For example, the model iteelf would need to be expanded and modified to include more soals (or, constraints). For example, soals with respect to budget levels would almost certinly need to be included. Another factor that would almost certainly need to be incorporated into the model is "risk." Goals with respect 10 risk associated with handling and transporting various types of hazardous waste would need to be included. If might also be necesury to include multiple alternate routes from each source to each destination in order to reflect altemate possibilities 10 choose from in getting the waste from a generation site 10 a processing site.

\section{REFERENCES}

1. Bozecon, Perer, and Prusan, Peter, Pinnnine with Multiole Critrexis, (Amseerdan: North-Holland), 1991.

2. Bradloy, Stcphea P., Hax, Arroldo C., and Magnand, Thomes L., Anolind Mothemetien/ Promernmins. (Reading MA: Addison-Wedoy Publiching Co.), 1977.

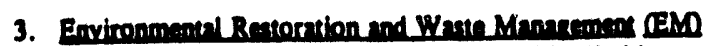
Prosermi An Imroduation, DOEJEM-0013P, Wechington, D. C.: U. S. Depertment of Enerty, Office of Environmeatel Reatoration and Wete Managrmen), June 1991.

4. Environmental Rextortion and Ware Mnnuement: Eive Yere Plan Firal Yera 1993-1927, FYP DOE/S-0089P, Weahingron, D. C.: U. S. Department of Enerty, Ontice of Enviroamental Reatoration and Waste Maragement), Augue 1991.

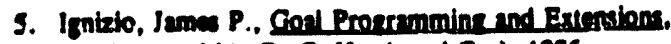
(Laxington, MA: D. C. Heath and Co.), 1976.

6. Intzio, Jane P., Ineroduction en linear $G_{0 \text { I }}$ Pronenmmins, (Beverty Hills: Sage Publications), 1985.

7. Ijiri, Y., Mnnazement Gonls and Accountine for Control. (Amuterdam: North-Holland Publishing Co.), 1965.

8. Lee. Sung M., Corl Prosrnmmine for Dexision Anslysis, Philadelphis, PA: Auerbach), 1972.

9. Schrage, Limus, LINDO: An Oxtimirntion Modelins Svrtem, 4h. ed., (Sen Francisco: The Scientific Preas), 1991.

10. Wagner, Harvoy M., Brinciolm of Onecotions Reanch, 2nd. ad., (Englowood Clifis, NJ: Prentice-Hall, Ine.), 1975.

11. Wiastoa, Wayos L., Introduction to Mrthemetical Pronermmine:Apolievtions ind Alrortithm, (Boston: PWS-Kene Publishing Co.), 1991.

12. Zeleny, Milan, Multiple Critedir Decision Makins, (new York: MeGraw-Hill Book Co.), 1982. 


\section{ACKN()WLE[X]MENT}

The preparation of this peper wes funded by the U.S. Department of Enaryy (DOE) (jmat No. DE.Fo02. 880P48058. Management Syterns Labontories thenks [XOE's Office of Environmental Restoration and Wath Menagement for providing us a real-world laboratory for the research. development. and teating of strete-of-the-ant management tools and the frameworts for unde runding how to make them successful. 
Audience Selection fior Interpersonal Communication Chanuels in Puhlic Involvement Prograll.

\author{
Gelle Gurdner \\ Evelyn Wight \\ Management Systems Lathoraturies \\ Virginia Polyteclunic Institute and Stute L'niversity \\ Blackshurg. VA 2t|k|
}

\begin{abstract}
As part of its counmitment to sharing information with the puhlic. the United States Deparment inf Energy's (DOE's) Otfice of Environmental Restoration and Waste Management (EM) has designed an exhihit which is presented at over 40 scientific. enviromment. education. and general cunterences every year throughout the country. The exhibit is stuffed hy people knowledgeable ahout EM programs. and features maps. photographs, interactive computer screens. and handout materials. Management Systems Laburatories (MSL) conducts research for DOE on improving the educational effectiveness of the exhitit materials.
\end{abstract}

The purpose of the EM Exhibit is to inform. involve. and get feedhack from the public. The design. although continually updated and modified, is largely fixed -. knowledgeable representatives talking in exhihit visitors. answering questions. and providing handout materials. That leaves only one major variable in the formula -- the audience. This paper focuses on MSL's research in developing criterid firr velecting audiences for the EM Exhihit.

\title{
INTRODUCTION
}

Until the 1980s. the United States Department of Energy (DOE) and its predecessur agencies were almost exclusively self-regulating. The environmental laws passed by Congress and the States during the $196(1)$ s and 1970s did not apply to DOE nuclear materials production operations. In the mid-1980s. several legal decisions required DOE to comply with these laws.* DOE's waste management and cleanup activities heciame suhject to a number of federal acts and state and local requirements. in addition to existing and new intemal DOE Orders. standards. and guidance documents. ** Many of these regulations mandated the public's right to know ahout activities affecting the envirunment and the right to he invilved with Jecisions conceming environmental restoration and waste management programs. DOE is faced with not "nly sharing information with the public and soliciting the public's input to its decision inaking hut alsu cleaning up its contaminated sites according to new standards.

* Fir ex.umple. a suit challengıng DX)E's self-regulatıng vatus in 1984. Legal Envuronmental Assistance Foundation i LE.tF , is. Hidel. was decided ayannst DC)E and requured it to comply with the Clean Water Act and the Resource Cunservatiun und Recuvery Act at the $Y .12$ Plant.

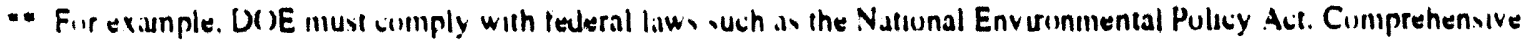

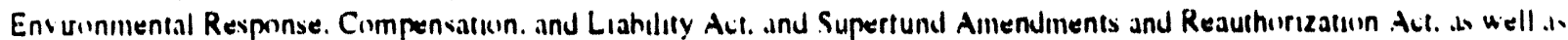

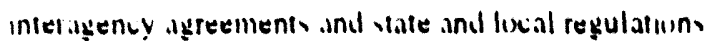




\section{DOE's Culture Change}

In 1989 DOE committed to cleaning up its sites and complying with all applicable laws. It also) inmmitted to changing from a cultural tradition of secrecy and distance frmm the public (which arove from a focus on national security and nuclear materials production) to a culture characterized hy proactive puhlic involvement. intemal and extemal accountability. and compliance with federal. state. and locial environmental laws. DOE is tringing its facilities into compliance with environmental laws. increasing independent scientists' aciess to DOE infurmation. reaching out to the public through meetings and review groups. and creating new intemal and extemal inonitoring and control inechods. DOE's culture change follows similar changes made hy many private industries heginning in the 1960s and $197(1)$ s. Organizations changed from passively to actively working with the public. That is. they went frum responding to citizens protests to anticipating requests for information and preparing outreach materials (1). Like inany managers in private industry, interacting with the public is completely new to most DOE ifficials and workers. It is likely that some managers in DOE are heginning to understand and apply public participation techniques. while others do not yet acknowledge its value.

DOE's hudget is derived from taxpayer dollars, so its approach to public outreach has constraints not experienced in private industry. DOE's outreach activities include holding public meetings. soliciting public input to its planning Jucument (the Five-Year Plan). and preparing informational materials to educiate the public. To coordinate and improve these efforts at its sites across the country. DOE created it headquarters office to oversee these activities.-the Office of Policy and Program Information.

The Secretary of Energy also estahlished an Advisory Board of independent environmental and inaluagement professionals to evaluate public trust and confidence in DOE and find ways to improve it. This Board found DOE has a long way to go hefore the public would have faith in DOE. The Board reciommended DOE commit to "consistent and respectful" outreach to state and community leaders to creste partnerships with the public. This outreach should be characterized hy "frequent contact. complete cundor. rapid and full response to questions. and assistance in increasing technical and oversight skills of the community (2)." The Board also found that "contradictory statements...undermine trust." In every cusse. the message content given to the public must he consistent. In addition. the Board stressed the need (1) e eluciate the public on the technical aspects of DOE's programs (2). DOE managers have also cincluded that tinding ways to interact meaningtully with the public must include educating the public ahout DOE's waste types. technologies, and constraints. and stimulating the public to leam more un its ("wn ahout DOE (3).

\section{Public Education and Outreach}

Eluciting the public is not easy. The volume of information makes it difficult even for insiders to keep up with everything there is to know ahout DOE. environmental restoration. and waste management. For cilizens. this is even harder. Absorhing this information takes more time and committed interest than inust people have available today, and aciess to the information has heen limited for decades. DOE's cleanup programs cun't he explained in a 60-second TV spot os even in a fealure aricle. The information DOE needs to communicate is technically complex and may not fit into a mass media approach using logans or tlashy visuals. such as the armed forces recruiting advertisements ("Be all that you can he .- in the Amy"). Even if it could. DOE doesn't have the funding for an extensive (and expensive) mass media campaign and must use taxpayer dollars frugally. People also have different reactions to DOE-related 
information. There is a strong need for clarification and discussion. In the past. citizens. puliticials. لدالـ

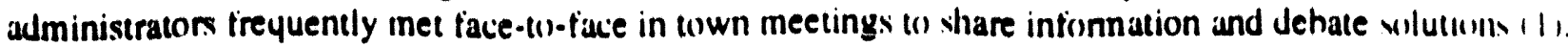
Whether an issue was complex or simple. face-(1)-face comınunication allowed a greater undentanding (1) topics involving govemment, husiness. and citizens. With today's media and technology urientation. interpersullal lone-on-one or tace-(1)-face) communication is rarer. However. as Clinton's presidential cumpaign demonstrated. interpersonal contact with people (like those town meetings) can have a positive impact on success. Today. citizens and administrators need to find ways to interact while discussing highly complex and technical information.

\section{Interpersonal Communication}

DOE must rely on interpersonal interactions and news coverage racher than mass media such is commercial advertising. Public relations research has shown mass communication is not the hest way (1) educiate the public. Publications. letters. and videos don't provide an audience the opportunity to ask questions or clarify information or a chance to establish human contact and understanding through vertial or nonvertal cues (4.1). Although interpersonal communication can the time consuming, logistically difficult. and costly, it provides invaluable opportunity for feedhack (4). Feedback makes it possible fior the public to tell DOE what its opinions and education needs are. Also. one-on-one interactions where DOE shures information and answers questions "on the spot" can help DOE build its credibility.

An interpersonal communication approach allows the audience to select whether they want to be cilnmunicated with or not and what information they want to receive. In self-selected audiences. a greater purtion of the message falls on receptive ears. and there is likely less confrontation. Audiences who join an interpersunal discussion probably have some interest in the topic. and "should therefinre reyuire less persuading. thus allowing the sender to devote more energy to information matters ( 4 )." While some audience members may approach a public outreach program with no desire to leam (and (cicasionally a Jesire to argue), most who make the effort to participate will be interested in hearing what the intended message is.

\section{E.M EXHIBIT BOOTH}

In urder to provide information to the public about its cleanup programs. DOE's Office of Environmental Reviluration and Waste Minagement (EM) created an interpersonal outreach tool--an exhibit both which is presented at uver 4) scientific. environmental. education. and general conferences every year throughuut the country. The exhibit informs the public about EM activities in three program areas: enviruninental restoration. waste management. and technology development. Thousands of people. including educators. civic and community leaders, state and tribal leaders. technical and industry professionals. interest groups, and the general public visit the booth. People knowledgeable atout EM programs staff the exhibit. which features maps. photographs, interactive computer screens. and handouts. The purpose of the hooth is to provide information about DOE to the public. not to promote anything (5). Staffers are directed to provide a positive interaction with "the govemment" and DOE so the public has a g(u)d experience and (hopetully) learns something. A lower priority goal is to develop public interest in science so DOE (and industry) will have the educated employee resources it needs in the future.

A common arsumption about public outreach and information sharing is that it can result in changed perceptions or heliets. Research by the U.S. Council for Energy Awareness shows that while 
communicating with and educating the public prohahly can't change opinions atwut nuclear power plalls, ur radiation. communication can reduce feur (6). DOE is operating under the assumption (which is validated hy literature in the field of public (upinion research) that educating the public will result in more ineaningtul public interactions. As early as 1947. public opinion researchers were asserting:

"[If] people in a democracy [are provided with] educational oppontunities and ready access (I) information. public opinion reveals a hard-headed common sense. The more enlightened people are to the implications of events and proposals for their own self-interest. the more likely they are (11) dgree with the mure ohjective upinions of realistic experts" (7).

But how can DOE know whether it successfully communicates with the public! Because it is difficult (1) quantity changes in feelings and hehavior and hecause DOE is focusing on educiating the public. Manugement Systems Lahoratories (MSL) has concentrated on measuring the educational effectivenesi of DOE touls. In one study, we compared audience ability to leam from different communication cowls and methods $(8)$. When studying DOE's environment, a prohlem solving approach incorporating fixed and flexible variables must he applied.

\section{Audience Selection}

One way to evaluate the effectiveness of communication tools is to apply the formula: Audience + Purpose $=$ Design. In DOE's case. this formula can he used to determine what is already defined and what . is tlexihle. The tlexible elements can then he evaluated and improved. The purpose of the EM Exhihit is established: to inform. involve. and get feedhack from the public. The design of the Exhibit. although continually updated and modified. is largely fixed -- knowledgeable representatives staff an Exhihit. talk (1) exhibit visitors. answer questions. and provide handout materials. That leaves only one flexihle variable in the formula -. the audience.

How should DOE choose its audience? Although DOE would like to reach the entire public. that goal is neither realistic nor practical. Public relations experts suggest it could he wasteful to target the entire public. Since the total public is complicated and heterogeneous. DOE must choose to communicate with groups within the public to be effective. While selecting which groups to focus on. DOE must he curetul init (1) luinp dissimilar organizations into the same category or choose too few groups to work with. For instance. puliticians don't always represent their constituents, and national environmental organizations may not represent locial agencies, grass mots activists, or citizens (9).

The number of people exposed to the information means little if the audience is inattentive or not very intluential. The public is divided into opinion makers and opinion holders. Opinion holders make up the vast inajority of a public. Inattentive opinion holders have neither the opportunity nor a strong inclination to hecome involved in the opinion-making process. Attentive opinion holders are inclined (I) participate. hut lack access or opportunity. It's the opinion makers who strongly influence, as well is articulate and represent. the opinion of a public (1).

Puhlic relutions expert Philip Lesly recommends concentrating on just 10 percent of the target public. According to Lesly's Paradigm. on any given issue approximately 5 percent of the public immediately 
agree with your position. 40 percent are leaning tavorably but won't do anything on their (Iwn. 4 () percent are leaning unfavorably. but won't do anything. and 5 percent immediately disagree. The remaining (I) percent are the interested. open-minded (attentive) opinion leaders who drive the ultimate resolution of the issue (10).

The audience needs to have not only the opportunity to hear hut also an interest in paying attention to the information presented. If the audience isn't interested in the topic. the result may in fact he damaging to the communication effor. The U.S. Council for Energy Awareness has found that providing intormation ahout nuclear waste to non-attentive audiences may bring out negative imagery that seems to lie heneath the surface without successfully addressing concems $(6,11)$. In other words, if you set up the exhibit in it shopping mall, and shoppers glance at the booth as they walk by, they may go home concemed about the nuclear waste problem without ever having obtained any information about DOE's approaches to solving the prohlem.

Individuals somewhat familiar with nuclear waste issues will often take the time to leam more. if given the upportunity. Involvement often leads to positive interactions (4). People with a technical background can usually understand technical issues hetter than the general public. Some groups. such as educators and govemunent workers, are good conduits to secondary audiences. For example, reaching one teacher may inean reaching many students. This "echo effect" multiplies the exposure of the message.

\section{Audience Research}

MSL first examined the Road Show (EM Exhibit) audiences while researching ways to improve the design of the exhibit. We considered a modular approach. where the booth layout would change depending on the audience. We examined the list of exhibit sites for 1992. DOE had selected goverument agencies. professional and technical societies, environmental groups. lahor and industry. ejucition conferences, and the general public. The selection criteria DOE used included size of organization for event ( 1.000 or more unless environment/public), meeting frequency and size. exhibit hill restrictions. and cost (registration fee. exhibitors' fee. travel). MSL concluded some of the (rgianizations would he inore interested in the puhlic involvement aspects of the cleanup effior. others would focus on the technical details or DOE's organizational structure, and educators would want information they could use in the classroom.

Figure 1 is a diagram we developed to help DOE determine what information would he appropriate and effective for various groups. Our goal was to design several distinct modules for the hooth that would change depending on the focus of the particular audience. The "public" modules would focus on environmental laws and citizen involvement in the program. The "education" modules would provide information that could he shared with students. The "technical" modules would provide more detailed Jescriptions of cleanup processes, and the "organization" modules would describe relevant DOE offices: and prograuns. For example. if the exhibit was taken to a Fernald Residents Group meeting. most of the infurmation would he ahout public involvement (e.g.. how to get involved in DOE's public participation programs) and the DOE organization, with a small amount of technical information about cleanup ativities. The modules for the American Nuclear Society would focus on the technical aspects of the cleanup effort. 


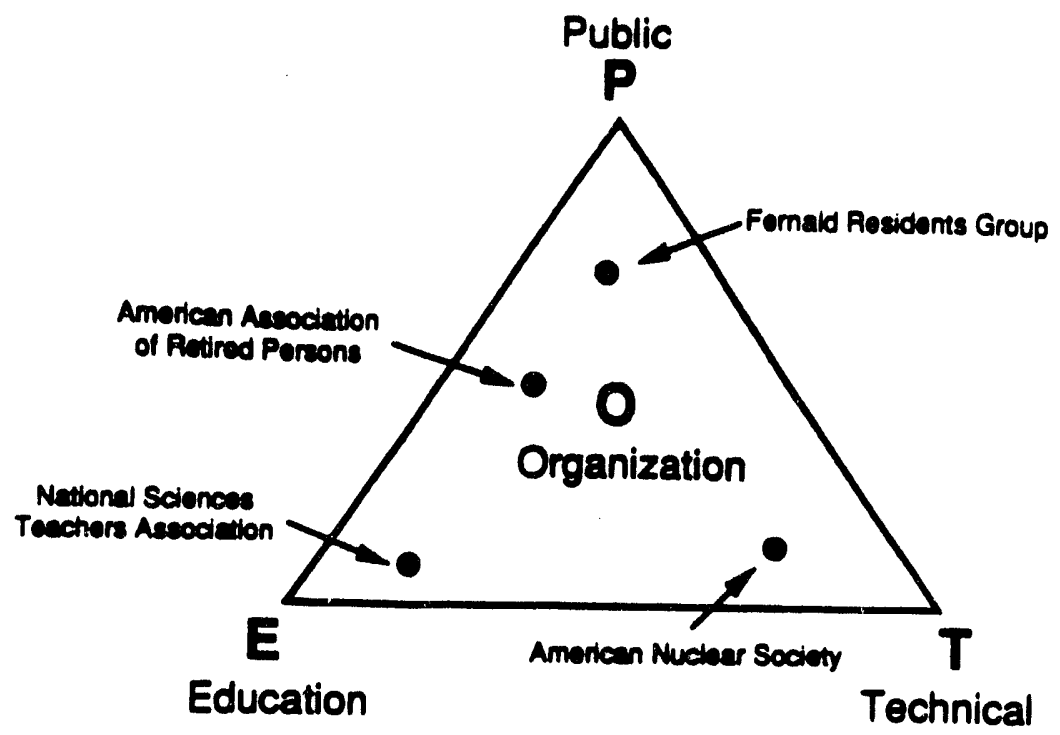

Audience Modules for the EM Exhihit

Figure 1

Although the eventual re-design of the full-size exhibit did not incorporate the module concept (due in part to the cost of recreating all the hooth materials). DOE developed smaller "table-top" exhibits focusing on a variety of issues. The examination of the needs. interests. and other characteristics of the organizations on the 1992 exhibit schedule later served as a hasis for our more comprehensive audience selection research.

\section{Selection Criteria}

To identify more potential audiences, first MSL surveyed lists of organizations and conferences. such is the 1992 National Trade and Professional Associations of the United States Directory. We used DOE's selection criteria described atove and the following key words in the search.

- environment (pollution control. recycling, waste management. cleanup, remediation, water)

- education (educators, higher education, science teachers, highly educated)

- engineering (mechanical, safety, sanitary, environmental, chemical. human factors, industrial)

- govemment (federal, legislation. quality control, govemment workers. public works. land use)

- health/medical concerns (cancer. health physics. radiation effects. public health. doctors)

- information transfer and distribution
- intemational organizations (distribute/transfer information among nations)

- justice or cause-oriented groups (unions, women's organizations, social concerns/issues, public good)

- nuclear (safety, energy)

- public opinion

- recruiting (women and minurities. career development)

- research organizations (if related t) government or science or environment)

- science and technology

- technology transter 
Then. MSL narrowed the selection criteria areas. hased on our assessment of criteria already in use und our key word search. We chose four areas: impact. receptivity, education, and physical factors. Each area was divided into two or more subcategories that could he scored on a scale of one to three (with three heing the highest). Because detailed information ahout each conference was not always available. scoring in some of the categories was, admittedly, somewhat subjective. Because using ranks of high. medium. and low in subcategories like influence and diversity was difficult. we provided examples of types of conferences DOE might attend to assist scoring. We ranked 129 conferences in the areas of husiness. education. engineering. environmental, general, govermment, science. and medical. The highest ranking possible was 39 points.

\section{IMPACT (15 points)}

Number - expected conference attendance (under $3000=1,3000-5000=2$, over $5000=3$ )

Influence - ability to affect public/govemment policy (American Library Association $=1$. National Academy of Sciences $=2$. National Conference of State Legislators $=3$ )

Echoes - extent group is conduit to secondary audiences (Society for Risk Analysis = 1, American Chamber of Commerce Executives $=2$, National Sciences Teachers Association $=3$ )

Diversity - variety of interests/hackgrounds in group (Association of Engineering Geologists = 1, National Education Association = 2. American Business Women's Association $=3$ ).

Length - duration of future relationship with DOE (Leave handouts in hotel room $=1$, May seek follow-up information $=2$. Ask for addresses of specific DOE officials $=3$ )

\section{RECEPTIVITY (9 points)}

Familiarity - extent of knowledge of EM issues (Low $=1$, Medium $=2$, High $=3$ )

Interest - extent of interest in EM issues (Low = 1. Medium = 2, High = 3)

Attitude - toward EM or DOE (Negative $=1$. Neutral $=2$. Positive $=3$ )

\section{EDUCATION (6 points)}

Degree - educational level of average participant (High School $=1$. College $=2$. Advanced $=3$ )

Technical - depth of understanding of such areas as radiation. waste types. environmental laws. risk analysis, etc. $($ Low $=1$. Medium $=2$. High $=3$ )

\section{PHYSICAL(2points)}

Exhibit - booth location, exhibit hours, cost. etc. (Poor $=1$, Average $=2$, Good $=3$ )

Site - proximity to EM site (No site in sight $=1$. In a state with one or more sites $=2$, Near a major site (Savannah River, Oak Ridge, Hanford. Waste Isolation Pilot Plant) = 3)

Location-geographic location of conference (West of the Mississippi $=1$. East of the Mississippi $=2$. Within 200 miles of Washington = 3)

When we applied this scale. the highest ranked conference was the National Academy of Engineering of the United States of America. This private organization meets annually in Octoher at the National Academy of Sciences Building in Washington. DC. It was established to share in the responsibility given the National Academy of Sciences under its Congressional charter to examine questions of science and technology at the request of the federal government; to sponsor engineering programs aimed at meeting 
rational needs; to encourage engineering research; and to recognize distinguished engineers. This meeting scored a 3 in all categories except number, familiarity, and site. In contrast, the lowest ranked conference was the National Employment Counselors Association (1993 meeting in Atlanta), which scored a $!$ in all categories except for a 2 in attitude. degree, and location.

We tested our audience selection model hy having DOE exhibit staffers review the list of sample conferences we created. Then we compared how they ranked the sites hased on their experience with the ranking produced hy our selection system. Although there were some slight variations in the order. in general. the selections matched. In other words. sites that ranked high from past experience scored high with the system. and sites that ranked low from past experience scored low with the system.

Using the selection model as a guide. DOE prepared a list of sites for 1993. Not all high scoring sites were included hecause of external factors. Sometimes the exhibit is taken to a conference to support a DOE official who is scheduled to speak. And often, conference dates conflict, and DOE must chouse among several conferences. The 1993 schedule reflects a shift from 1992 in some areas. The percentage of environmental and science conferences decreased slightly with a corresponding rise in education meetings. The percentage of engineering and government conferences stayed approximately the same.

DOE uses an evaluation form that is provided to all DOE and contractor employees who staff the exhibit. The form has sections for rating the reaction of attendees to the hooth. MSL will compare the ratings for 1992 and 1993 to determine if, in the opinion of the exhibit staff, the new audiences are more receptive.

\section{CONCLUSIONS AND FUTURE RESEARCH}

DOE operates in an environment with more fixed than flexible variables. Thus, its public outreach efforts must he improved within an existing framework. In the case of the EM Exhibit, hoth the purpose and design are largely unchanging. Since DOE is committed to educating the public as part of its outreach etfors, audience selection plays a vital role. Based on the literature review and results of this ranking system development. important factors DOE can consider in choosing audiences include: audience interest in and knowledge of topic area; message content and consistency; self-selection opportunity for audience memhers; audience status as opinion maker, leader, or holder, and feedback opportunity.

The audience selection model developed for the full-size exhibit could possibly he applied to the table top exhibits DOE is just starting to use. The table-top exhibits have a different purpose and use than the fullsize exhibit. Because less information can the portrayed, table-top exhibits can describe fewer topics. Physically smaller, they can he used at events with fewer participants, such as public hearings, smaller conferences, and meetings. DOE is still experimenting with which designs, topics, and locations work hest with this saialler interpersonal outreach tool. Different variables, however, may apply. A new audience selection model may be needed for smaller outreach tools and smaller audiences. MSL can apply the existing model to the table top exhibits and evaluate its effectiveness for that medium. MSL can also develop a new audience selection and evaluation model for the table-top exhibits.

Each time the full-size exhibit is sent to an event. a number of handouts are sent with it. Both the type and yuantity of handouts distributed at events varies. Some handouts are more effective with some 
audiences than others. For example. at some conferences all copies of the Environmental Restoration und Waste Management Program hooklet (or primer) are collected by audience members, while at another meeting it is largely ignored. With a tool to predict which handout style and content, as well as numbers of copies, work well with different audiences. DOE could save development. printing. and shipping costs. New handouts are developed for the exhibit on an ongoing basis. For example. a brochure was recently completed on the cleanup program, and a new brochure on risk is heing developed. If the way audiences select handouts was assessed and measured, new materials could be developed with these correlations in mind. Future research could include developing a model to predict and evaluate the effectiveness of different tools with different audiences. Also, a methodology could be developed to predict the right mix of tools for different audiences, in terms of style and content. as well as number of copies needed.

The preparation of this paper was funded in part by U.S. Department of Energy (DOE) Grant No. DE-FG0288DP48058. Management Systems Laboratories thanks DOE's Office of Environmental Restoration and Waste Management for providing us a real-world laboratory for the research, development, and testing of state-of-the-art inanagement tools and the frameworks for understanding how to make them successful. 


\section{REFERENCES}

I. GILBERT. WILLIAM H., editor. Public Relations in Local Government, Intemational City Management Association. (1975).

2. "DOE Still Lacks Public Trust. According to SEAB Task Force." Weapons Complex Monitor. Exchange Publications. (December 23, 1992).

3. HELMINSKI. EDWARD. "On the ERWM PEIS Scoping Sessions, Pat Whitfield, DOE ERWM." interview. Weapons Complex Monitor. Exchange Publications, (February 25, 1991).

4. SELNOW. GARY W. AND WLLIAM D. CRANO. Planning. Implementing, and Evaluating Targeted Communication Programs. A Manual for Business Communicators, Quorum Books, (1987).

5. WHITFIELD. R. PAT. Deputy Assistant Secretary for Environmental Restoration, comments recorded in minutes trom All Hands Road Show Meeting, (May 29, 1992).

6. BISCONTI. ANN S., "Public Opinion and Communication on Nuclear Waste." Thirteenth Annual Low-Level Waste Management Conference. (Novemher 19, 1991).

7. CUTLIP. SCOTT M., ALLEN H. CENTER, GLEN M. BROOM, Effective Public Relations. PrenticeHall. (1985). (Footnote--Hadley Cantril. Gaugine Public Opinion, (Princeton, NJ: Princeton University Press. 1947)|.

8. WIGHT, EVEL YN H., EUGENE J. GARDNER. AND A. TONY HARVEY, "Are EM'S Communication Tools Effective? Case Studies With Two DOE-EM Publications," Proceedings, Waste Manaqument '92. March 1992. pp. 191-195.

9. REICHER. DAN W.. Gaining Public Trust and Confidence, report prepared for DOE Task Force on OCRWM. (January. 1992).

10. Philip Lesly quoted in JACKSON, JACKSON, AND WAGNER. "Strategy and Plan for Coordinating and Focusing Outreach and Public Participation (unpublished draft)," (August 15, 1991).

11. BISCONTI, ANN S.. "Public Attitudes About Nuclear Waste," Nuclear Plant Journal. (July-August 1991). 


\title{
CURRENT CONCEPTS AND METHODOLOGIES FOR ASSESSING AND COMMUNICATING RISK IN WASTE MANAGEMENT
}

\author{
Eugene J. Gardner. Aubrey E. Harvey, and John B. Mitchell \\ Management Systems Laboratories \\ Department of Industrial and Systems Engineering \\ Virginia Polytechnic Institute and State University \\ Blacksburg. Virginia
}

\begin{abstract}
As part of its research on improving the effectiveness of DOE information exhibits. Management Systems Laboratories (MSL) is reviewing current concepts and methodologies for assessing and communicating risk issues to the public.
\end{abstract}

There are three types of what is commonly called risk: (1) ordinary risk. such as the chance a turbine will fail: (2) behavioral risk. such as the chance a smoker will get lung cancer: and (3) uncertainty, such as the chance of an unforeseen effect of low-level radiation on the molecular structure of a particular containment structure after a thousand years. Each type requires different analytical tools. This paper is a brief survey of some of the tools particularly adaptable to risk communications.

In communicating risk. it is important for authorities to add information about the risk assessment approach and its inherent scientific uncertainties. Presenting the benefits as well as the risks also can help the audience balance the issues involved in making decisions about technological risk.

\section{INTRODUCTION}

The odds of winning a lottery are about one in 10 million. but many people buy tickets. The odds of being injured in an automobile accident are about $50 \%$ over one's lifetime. yet many motorists do not consistently wear seat belts. The likelihood of properly disposed nuclear wastes creating environmental or health probletns are many times less than that incurred during temporary storage of wastes above ground. yet the public insists on zero risk. which may place unrealistic constraints on responsible waste management.
In general. public perceptions of risk are not founded on a scientific understanding of risk and uncertainty. Because the public as well as the media are not trained in mathematical tools, they both lack an objective framework to understand risk. Thus public opinion is vulnerable to being molded by sensationalistic and one-sided media portrayals exaggerating the risks associated with the nuclear industry (1). These portray. als help sell newspapers. magazines. and movies, but unfortunately create an environment of public hysteria surrounding the nuclear industry and suspicion of any program or spokesperson that could be construed to be "pro-nuclear."

Against this background of negative public opinion. waste managers must work with stakeholders to find ways to cooperatively address waste treatment. storage, and disposal issues, while addressing an ever-increasing regulatory burden. Some of these regulations and agreements may even be in conflict with each other. The more stringent reporting requirements and the proliferation of regulations are increasing the difficulty of finding feasible solutions to waste management problems. This is increasing the life cycle cost of waste storage sites.

Managers can improve their risk communication abilities by understanding scientific approaches to risk assessment and analysis. Informing the public about these approaches, and presenting the benefits as well as the risks involved in a proposed course of action, are ways managers can involve the public in the realistic management of technological risks associated with waste management.

\section{RISK PARADOXES}

Somewhere in space right now, rushing our way at 50.000 miles per hour, is a large chunk of rock. left- 
over from the formation of our solar system. on its way to a rendezvous with Earth and doomsday. This is the fear which is driving many in NASA and our defense establishment to propose a multi-billion dollar "star wars" - type solution to what is not even a quantifiable risk. but an unsolvable deterministic problem.

This problem is known in physics as the three body problem (2). There is a great deal of uncertainty as to what effect a collision or near miss with an asteroid might have. Depending on the interaction of several extremely precise variables. the result of intervention could be that the asteroid is safely deflected. an asteroid that would have missed collides with us, or that the Earth is knocked out of its orbit. Due to the uncertainty of this interaction. a space shield could be more dangerous than simply taking our chances with a cosmic collision (status quo option). These types of problems have become popularized under the term chaos (3). or more properly, mathematical chaos (4).

Even computer weather modeling has obvious limits to its accuracy. Ask anyone who has carried an unnecessary umbrella all day or wrung out his clothes in an airport terminal restroom when caught in an unexpected downpour. The problem with forecasting the weather is that uncertainty (chaos) can creep in to spoil the prediction. This is the public's most common encounter with uncertainty or what is known as chaotic systems (5).

\section{UNCERTAINTY}

Uncertainty of the type illustrated in the above examples can have one of two possible causes. The first cause is that the mathematical formulas which generate the forecast might represent a chaotic system. The impact of this is that a small numeric error can be greatly exaggerated as the solutions are iterated over time. Since such models can be solved only by computer approximation, they may appear to be random and unpredictable. The second cause is the simple lack of knowledge of the risk. Uncertainty from either of these causes is indistinguishable from the other.

When considering possible courses of action. risk managers must consider the status quo alternative. A case in point is the leaking HLW storage tanks at Hanford. At least one scientist (1) argues that no detrimental health effects can be expected from the leaking tanks, either now or in the future. All of the significant radioactive materials are absorbed in the soil within a few feet of the tank and the tank is more than 100 feet above the water table. Some radioactivity may be expected to eventually reach the water table after several hundred years. Only then can it move horizontally, toward the Columbia River, about 10 miles away. Best estimates of groundwater flow rates indicate 800 years would be required to cover that distance. The only significant radioactive material that would be left after that time would be a small amount of plutonium remaining after the filtration action of miles of travel through rock. Although plutonium. if inhaled. is one of the most toxic poisons known, the probability of any remaining plutonium becoming airborne is extremely remote.

It could be argued that the risks of a future leak at Hanford are minimal, and that present actions are adequate to avert risk to the environment and consequent health effects. Any cleanup alternative would generate additional risk factors associated with exposing unknown wastes to the air, the workers to the wastes, or moving wastes from one tank to another. These new risks could have negative consequences far greater than leaving well enough alone. Practically speaking, a rigorous monitoring program can identify problem areas such as leaking tanks that would require intervention.

Every technology has benefits and costs associated with it: its attractiveness depends on the probability and size of its possible gains and losses. Studies illustrate that people have difficulty thinking about and resolving the risks/payoffs involved even in simple gambles (6). When confronted with uncertainty, people often try to reduce the resulting anxiety by denying the uncertainty. This type of denial is illustrated by the case of people faced with the possibility of a severe flood at infrequent intervals. Some people deny (7) that a flood could recur in their area, despite experts assessment of another flood being likely within a statistically probable period of time (e.g., a "100-year flood"). Unfortunately, many take this to mean a major flood cannot recur before many years have gone by: however, a second "100-year flood" is equally likely during any of the years in that 100-year period. The problem is compounded when people place confidence in flood control dams and reservoirs. building their homes in flood plains at higher densities. which makes likely even greater losses in a catastrophic fail- 
ure of these systems than before the flood control was initiated.

The way some people deal with uncertainty is to attempt to outlaw the risk. Hence, we see "de minimus" standards for waste disposal systems, rather than objective approaches to the risks and benefits of all options under consideration. It must be remembered that risk can not be eliminated as a factor in technological decision-making. In trying to avoid a known. wellpublicized risk, we may expose ourselves to a greater. lesser known risk. The only way to achieve maximum societal benefits is to make objective. scientifically-informed decisions about issues involving technological risk trade-offs.

\section{DECISION THEORY}

Decision theory is one approach to try to objectify the human decision process. A way of dealing with uncertainty that does not use probabilities is to place the payoffs in an action consequence matrix (Figure 1 ). Payoffs can be either positive (gain) or negative (loss) depending on the actions taken and the states of nature. For example. the action of purchasing shares of a given stock can result in a loss or a gain depending on the stock market.

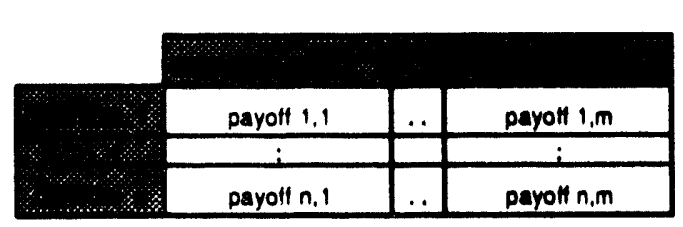

Fig. 1 -. Action-Consequence Matrix

Once the considerable task of building the matrix is completed. the decision maker uses a pre-determined rule reflecting his/her or society's ethical values to select the "best" action. Two of the most commonly used rules are to choose the action that (1) minimizes the minimum loss (mini-min), or (2) maximizes the maximum gain (maxi-max) (8).

In general. Rule (1) is used by environmentalists on the basis of their perception of equity (i.e.. spare no expense to achieve zero pollution and/or health risk).
Rule (2) is the perceived stance of industry (i.e.. wanting to maximize profitability of operations, often with little regard for the environment or public health).

A second wproach, often ignored, is to use a regret matrix which includes factors such as the cost of a lost opportunity and lives shortened as well as potential profits. The regret can be negative (representing a gain) or positive (representing a loss). Then the decision rule is to choose the action that minimizes the maximum regret (mini-max). In this way, the decision more closely resembles the public's approach to everyday decisions. ("...gee. I could have had a V.8").

An example of regret can be found in long-range planning for nuclear energy production. Lacking a cummitment to a breeder reactor program, our uranium reserves will be depleted in about fifty years. With breeder reactors we could have extended our nuclear fuel for thousands of years. This would have gained us the additional benefits of energy independence, as well as possible benefits affecting foreign policy in the mid-east (possible risk of war). A bonus would be the reduction of negative health effects from mining uranium since we have about a 200 -year supply of U-238 that is already mined and in storage (1).

There is no best decision rule, since it depends on the values of the decision maker. The way decision theory reflects the values held by the decision maker is to use probabilities to develop what are called utility functions. These are fit to the decision maker's preferences by the employment of lotteries. An example of a lottery is a weekly pool of 100 people, each contributing a penny with one person winning the dollar. Each participant has a one per cent chance of winning a dollar versus a 99 per cent chance of losing one cent. The expected value of this lottery is therefore zero. This means that this lottery (a truly fair lottery), would allow one individual each week to have a whole dollar to spend at a minimal cost to the others. Eventually, everyone would be expected to win the dollar once. This would be represented symbolically by Figure 2 . 


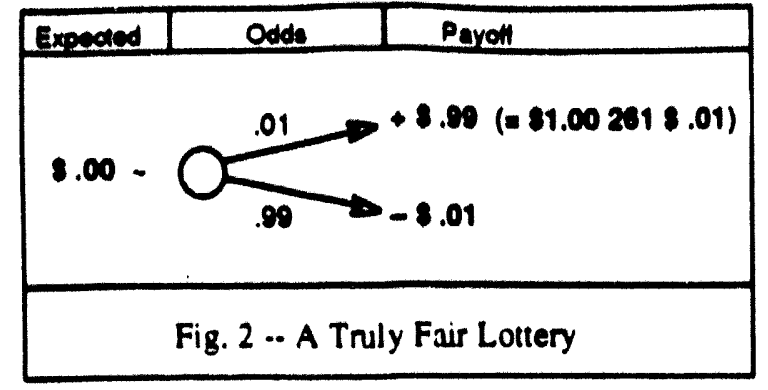

An interesting contrnst of two lotteries that express different ethical approaches to the same practical value are represented by figure 3 (9).

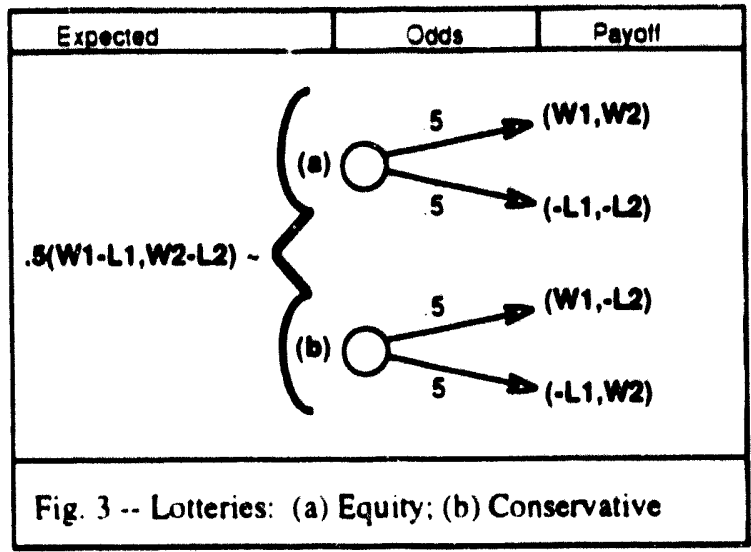

For both approaches. we assume an even chance (50/ 50) of a positive outcome for each of two people. In the first lottery. called the equity approach, it is agreed that either both will win or neither will win. This approach is mirrored in public thinking about technological processes. reflecting the public's insistence that a technology under consideration endanger no one. No technology for generating power or managing wastes is risk-free; choices between alternatives require balancing trade-offs between groups. If an equity approach to energy production is adopted then the loss (minus L1. minus L2) could result in a major decrease in the standard of living for everyone (13).

In the conservative or "hedging" approach, it is agreed that one party will win and the other party will lose under either circumstance. This approach is reflected in the concept of hazardous duty pay. From the perspective of making decisions using expected value. both these approaches are equal. It is the ethical per. ception which influences decision makers to choose one over the other. It is therefore very imporant to consider the whole problem in the process of evaluation.

The view of the whole problem can be accomplished by using vector valued utillty functions instead of single valued utility functions $(10,11,12)$ in decision trees. The vector valued approach allows the risk assessor to make decisions based on the effects the decision will have on the quality of tife. Another advantage is that the decision tree can be used as a communication tool showing the public the entire problem. not just an expected value result. An exam. ple of such a decision is depicted by the partial decision tree in figure 4 .

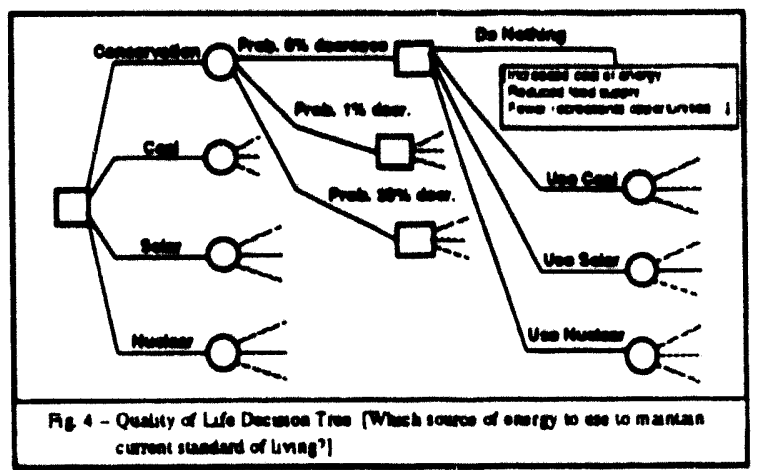

A difficulty in solving and communicating complex problems is fully understanding the problem. The decision-theoretic tools presented above are methods for gaining this understanding (even if the formal solution techniques are never applied). Communicating this understanding to the public is one of the major challenges of the risk analyst.

\section{RISK ASSESSMENT}

The way to determine the probabilities needed in a decision tree is through risk analysis. Risk is defined as the expected frequency of undesirable effects resulting from exposure to an agent. Risk assessment is the process of assigning magnitudes and probabilities to the adverse effects resulting from human activities or natlural events (13). 
There are three steps in a risk assessment: (1) bazard Ideatification. (2) hazard evaluatioa. and (3) risk evaluation. Before a risk can be assessed. someone must decide there is a risk. The domain of the risk communicator is the public perception of risk. The public perceives risk based on a number of factors that have been identified in risk communication literature (6.7). Among these are their personal experience. ac. counts of others' experiences. and what they most frequently hear about given risks. Further affecting public perception of risk is the public's desire for certainty.

An important factor in public perception of risk is that technological risk is perceived differently from an equivalent aatural risk. For example, pesticide residues. representing a technological risk. are viewed to be more dangerous than the identical hazardous chemicals which may occur naturally in some foods. To illustrate. FDA requires "de minimus" residue levels in foods treated with pesticides containing cyanide. while sudium cyanide occurs naturally in almonds and lima beans at higher levels than those standards.

Figures 5 and 6 depict the relationship between the public perception of technologically-derived risks versus its perception of naturally-derived risks. As the actual risk (quantifiable by experts) increases or decreases. the perception of risk assessment experts tends to correlate with actual risk. while the public perception tends to exaggerate technological risks while ignoring natural risks. For example. many people would forego a diagnostic chest $x$-ray to avoid radiation exposure, but would not give a second thought to the "natural" radiation incurred during a transalantic Night (6).

Another heuristic that helps explain differences in risk perception is called avallability (7). The idea is that an event is judged as more likely if instances of it are easy to imagine or recall. Availability also can be affected by a recent disaster or a vivid film such as the China Syndrome.

In the public arena the availability heuristic may have several effects. The public bias due to memorability or imaginability may create barriers to open, objective discussions of risk. For example. a risk communicator may present information on an underground waste stomge technology by using a fault tree depicting many low probability pathways that could lead to a system failure and environmental release. Despite the fact that compounded small probabilities further re. duce overall risk, the public may come away with the perception. "I didn't realize there were so many things that could 80 wrong." The very discussion of any lowprobability. high magniturte hazard may increase the public perception of its likelihood regardless of what the scientific evidence indicates (7). Figure 7 depicts the public misconception that high magnitude events are as likely to occur as low magnitude events. when in fact the opposite is true.

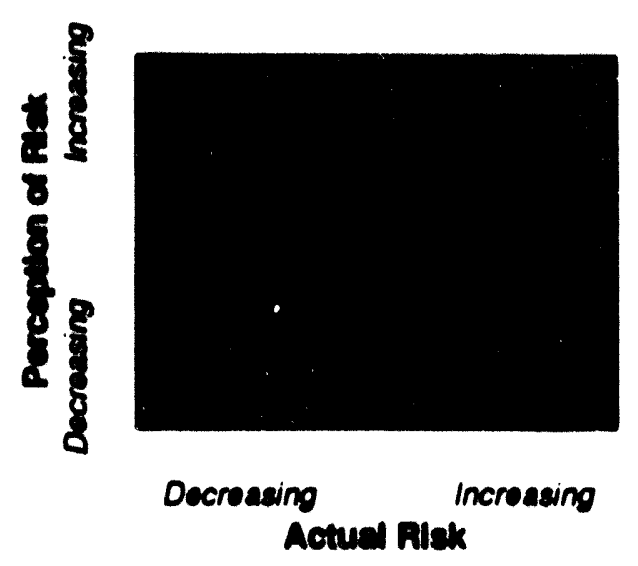

Fig. 5 .- Technological Risk

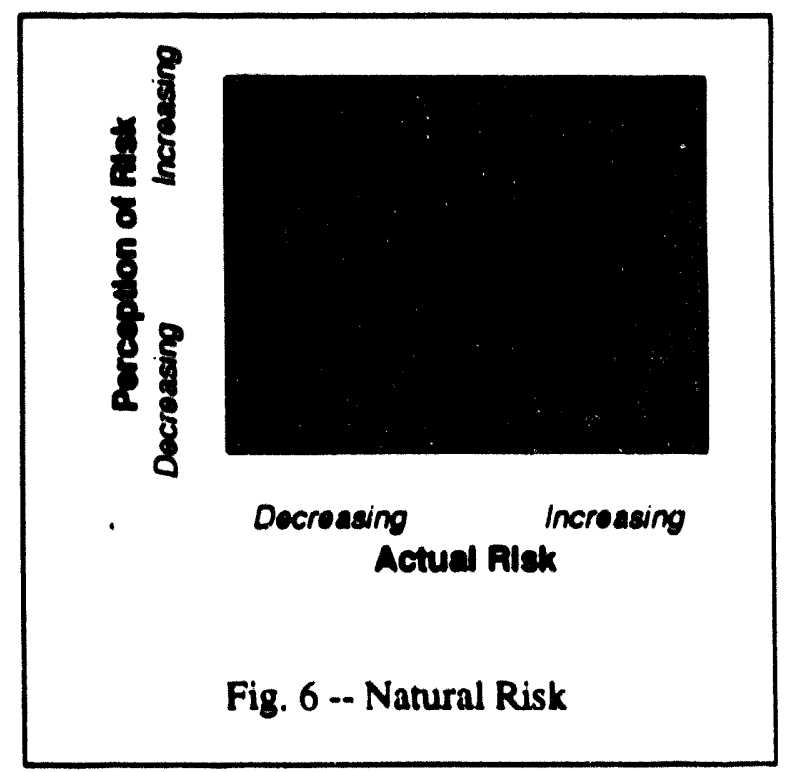




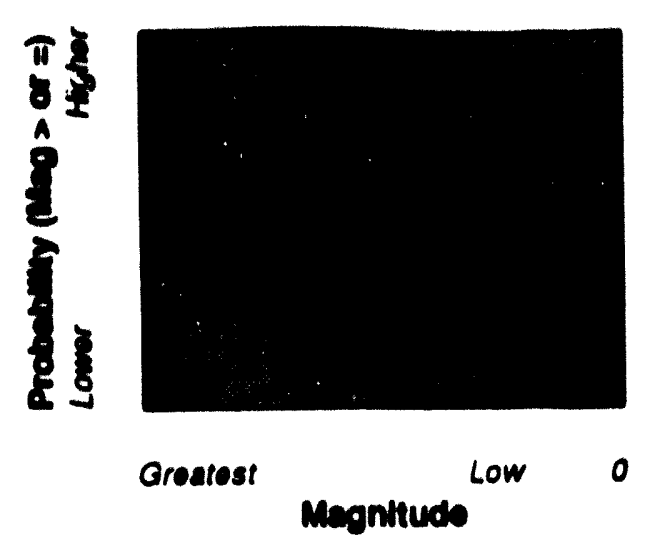

Fig. 7 -. Cumulative Prob. of Catastrophe

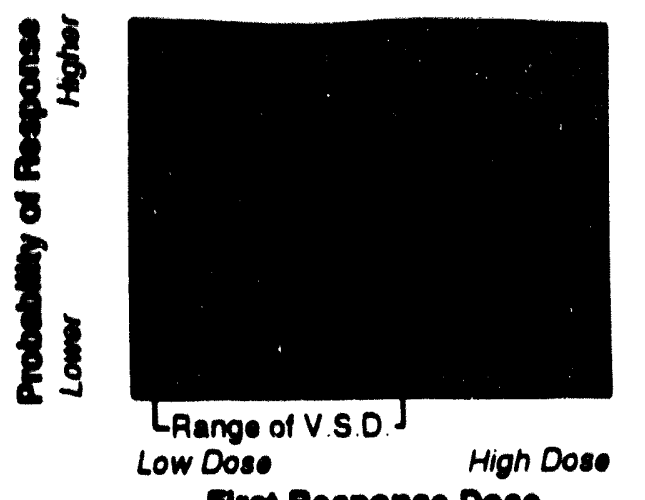

First Rosponse Dose

Fig. 9 .. Virtual Safe Dose

Fig. 10 .. View of Hazard
Fig. 8 -. Dose Response Curve

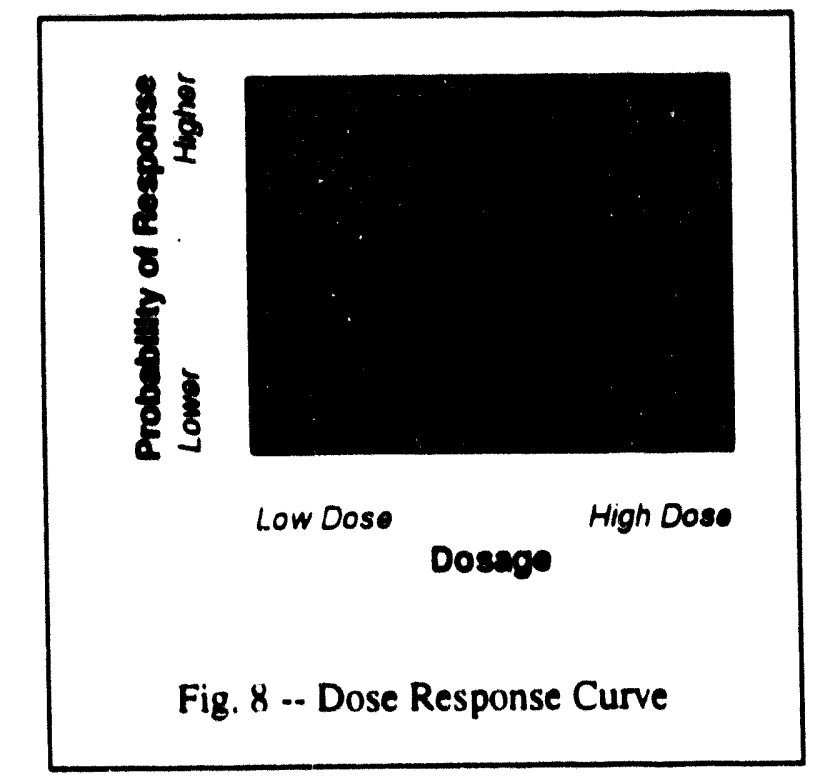

In evaluating an identified hazard. risk assessors cannot identify the effects of low dose exposures to haz. andous agents. They must use high dose animal experiments to determine the dose response curve as shown in Figure 8. This is then extrapolated for low doses using one of three models. The risk assessor uses the concept of acceptable risk to determine the range for a virtual safe dose (VSD) as shown in Figure 9. The risk assessor picks one of the models, frequently the sublinear curve (because the body can often repair minor damage resulting from exposure to an agent) and performs a curve fit to complete the high dose curve (see Figure 10).

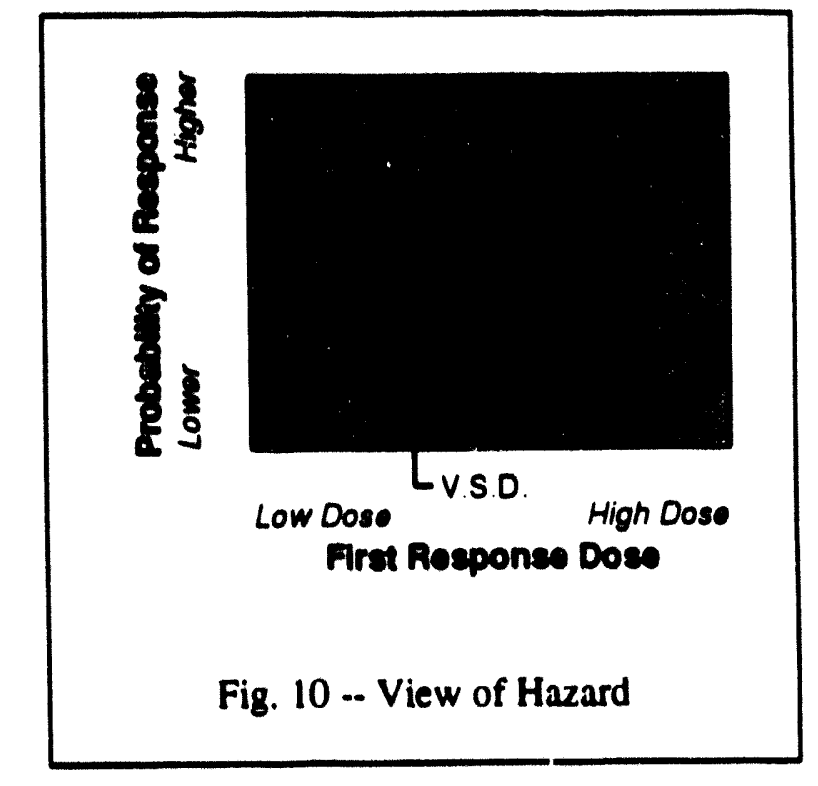

Figure 10 also depicts the public's perception of dose response. From news stories and observations it is ubvious that the public generally believes that if a negative response can occur at a high dose level, then it is equally probable at a low dose level. In a natural defensive reaction, the public does not understand the need for risk managers to employ the concept of acceptable riak if they are to make objective and balanced decisions regarding technological risks.

To complete the risk assessment, the risk of the hazard must be evaluated and compared to the risks of other 
alternatives. including the stans quo option, in order to allocase limited funds for courses of action which minimize the public's danger. Decision trees. although often overlooked by risk assessors. can be one of the most effective tools for presenting to the public a full understanding of the problem. The risk assessor can use such tools to see the problem more objectively and so obtain a better formulation. The challenge is to effectively communicate this formulation to the public.

One disadvantage of decision theoretic approaches is that the risk assessor and the public have different perceptions of the cost of risk reduction (see Figure 11). While the public believes zero risk is obtainable at low cost. the risk assessor realizes that costs often increase astronumically in attempting to reach even acceptable risk levels.

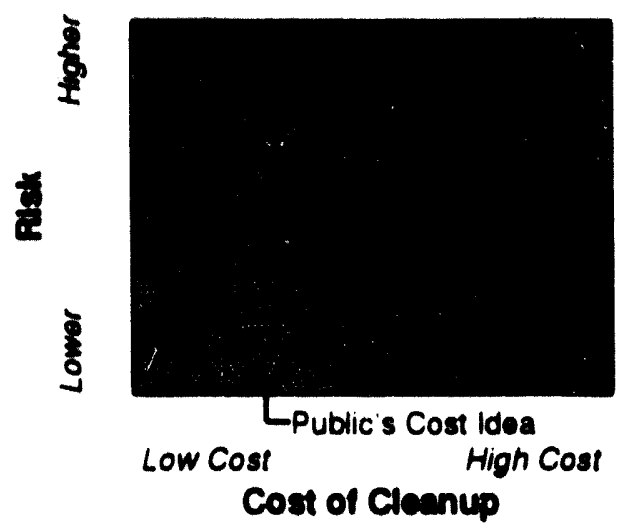

Fig. 11 .- Cost of Cleanup

Another disadvantage of decision theoretic approaches is that the public inherently distrusts risk comparisons that stress acceptability of risk (14). But risk comparisons are required to balance risk and opportunity objectively. There are always trade-offs in choosing between technological risks. The risk assessor sees the trade-off between reducing potential risk to the public and increasing the actual risk to those who must handle hazardous substances (see Figure 12). To resolve these conflicts. risk communicators should explain how cost approaches infinity as risk reduction is carried to "de minimus levels" and explain why appropri- ate risk comparisons (don't "compare oranges to apples") are essential to reach an objectively balanced decision.

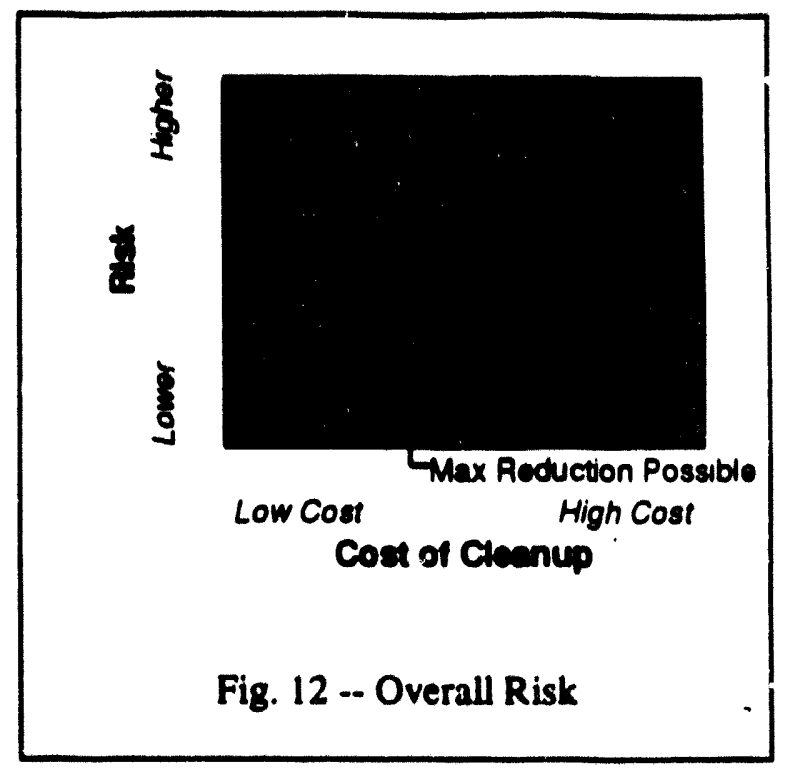

The risk assessor combines the probabulity of an undesirable event with the magnitude of that event. To do this he obtains a standard probability distribu. tion. Any statistical methodology can be used wo obtain a probability distribution if sufficient data is available. If data is sparse, the triangular distribu. tion may be useful (15). Here. the statistical analyst asks an expert to provide three points of likelihood: the minimum possible response, the maximum possible response, and the most likely response. Given these three points, there is only one triangular probability distribution which fits. In cases of great uncertainty, techniques such as Bayesian methods (16) have been used. These methods allow the statistical analyst to make a subjective guess of the distribution and iterate it through a series of empirical observations to obtain a valid distribution. Bayesian methods are the only statistical techniques which allow the analyst to use all previously gathered knowledge about the risks being studied.

Another family of statistical techniques for dealing with a known risk are stochastic processes. Stochastic processes are particularly well suited for deriving solutions to models of the reliability of technological systems. From these models. the analyst can deter- 
mine the risk of failure of a system. If failure involves the loss of life. then the analyst can estimate the cost of saving a life (a typical measure of risk in current literature). As a demonstration of this technique. let's examine the rollowing scenario.

There is an isolated tropical island which has sufficient minfall to provide water for its current 200.000 inhabitants. Census projections indicate that the population could grow to 300.000 in the near future if sufficient water could be found. The government plans to purchase a de-salinization and water purification system for $\$ 40,000,000$ which will provide water for the additional 100.000 inhabitants.

This system can be in one of three states. It can be in full operation (state 0 ), under minor repair (state 1), or under major repair (state 2). Under state 1 (minor repair), the water purification system is degraded with the result that some inhabitants may get sick from dysentery. Under state 2 (major repair), no water is produced with the result that 100,000 inhabitants will die if repairs are not made in a few weeks. (This problem assumes total isolation of the island.)

The specifications of the system are that a minor failure is expected to occur twice in a year, and a major failure is expected to occur once in five years. If the system is in minor failure, then the chance of a major failure increases to once a year. A minor repair is expected to take 14 days and a major repair is expected to take 90 days. Therefore. the chance of a major failure must be minimized. An alternative is to buy a second "stand-by" system at a total cost of $\$ 80,000,000$. reducing the risk by increasing the probability that one system will always be operating.

These problems can be modeled by what is known as a Markov process (17). The single system problem is expressed in the trassition matrix shown in Table 1.

\begin{tabular}{|cc|c|c|}
\hline \multicolumn{1}{c}{} & \multicolumn{1}{c}{ State 0 } & State 1 & State 2 \\
\cline { 2 - 4 } State 0 & $1 \cdot .00603$ & .00548 & .00055 \\
\cline { 2 - 4 } State 1 & .07143 & $1-.07417$ & .00274 \\
\cline { 2 - 4 } State 2 & .01111 & 0 & $1-.01111$ \\
\hline Table I -- Single System Transition Matrix \\
\hline
\end{tabular}

From this transition matrix, a set of differential equa. tions (measures of change) can be derived describing the probability of the system moving to a different state at any instant in time. Since the decision makers are interested in the long-range behavior of the system. the differential equations are solved in stady state (simply put, the change in the probabilities stops. that is, the differential equations equal zero) (18). The chance at any given moment in time of finding the system in each state is found in table 2.

\begin{tabular}{|c|c|}
\hline State & Probability \\
\hline 0 & .87596 \\
1 & .05932 \\
2 & .06472 \\
\hline Table II -- Chance of Finding Single \\
System in a Particular State \\
\hline
\end{tabular}

This means that the expected number of deaths at any time is 6.472. (Note that the system is slightiy more apt to be in state two than state one due to the longer repair time for complete failure and also the compounding of probabilities.) The alternative problem involving two systems has six states as shown in table 3.

\begin{tabular}{|c|c|}
\hline State & Condition \\
\hline 0 & 1 unit working, 1 unit standby \\
1 & 1 unit working, 1 unit minor repair \\
2 & 1 unit working, 1 unit major repair \\
3 & 2 units minor repair \\
4 & unit minor repair, 1 unit major repair \\
5 & 2 units major repair \\
\hline \multicolumn{2}{|c|}{ Table III -- State Definitions } \\
For Two Systems
\end{tabular}

State 5 is the only state that could involve fatalities. The problem is expressed by the transition matrix in table 4. 


\begin{tabular}{|c|c|c|c|c|c|c|}
\hline & State 0 & State 1 & State 2 & State 3 & State 4 & State 5 \\
\cline { 2 - 7 } State 0 & $1-.00603$ & .00548 & .00055 & 0 & 0 & 0 \\
\cline { 2 - 7 } State 1 & .07143 & $1-.07746$ & 0 & .00548 & .00055 & 0 \\
\cline { 2 - 7 } State 2 & .01111 & 0 & $1-.01714$ & 0 & .00548 & .00055 \\
\cline { 2 - 7 } State 3 & 0 & .14286 & 0 & $1-.14834$ & .00548 & 0 \\
State 4 & 0 & .01111 & .07143 & 0 & $1-.08528$ & .00274 \\
State 5 & 0 & 0 & .02222 & 0 & 0 & $1-.02222$ \\
\hline & \multicolumn{6}{c}{ Table IV -- Two System Transition Matrix } \\
\hline
\end{tabular}

Table 5 gives the steady state solution to the two machine problem.

\begin{tabular}{|c|c|}
\hline State & Probability \\
\hline 0 & .87959 \\
1 & .06735 \\
2 & .04530 \\
3 & .00250 \\
4 & .00358 \\
5 & .00168 \\
\hline \multicolumn{2}{|c|}{ Table V -- Chance of Finding Two } \\
Systems in a Particular State \\
\hline
\end{tabular}

This means that the expected number of deaths in the dual system problem is 168 . Therefore, the alternative system has an expected value of 6,304 lives saved. The total cost per life saved would be $\$ 6,345$. Over the thirty year system life, this comes out to $\$ 212$ per life saved, a very small investment in risk avoidance.

To put risk avoidance costs in perspective, contrast this example with industry plans to spend upwards of $\$ 1$ billion to vitrify HLW at West Valley, New York to avert an estimated .01 deaths that could be eventually expected if the wastes stored there were simply converted to concrete in situ (at a cost of only $\$ 20$ million). This corresponds to spending $\$ 100$ billion per life saved. At the same time, our government is tuming down other risk reduction projects that could save a life for every $\$ 100,000$ invested (1). The public does not realize there are such inconsistencies in our public policies on technological risk. If the public receives objective and complete infermation on the true costs of risk reduction, they might well decide to pursue a very different course on issues involving energy production and waste management.

\section{SUMMARY}

This paper has attempted to present some of the statistical, decision theoretic, and modeling techniques which can enhance the objectivity of decision making affecting technological risk management. These techniques are not only recommended for analysis and assessment. but also have value as communication tools enhancing the public's understanding of complex issues. The public should be educated to understand mathematical approaches to balancing risks and benefits so that informed consent can be given to the policies of the nation. The challenge to risk communicators is to present these complexities in ways that are accessible to the average citizen. Only then, can the ideals of a democratic society find their true expression in a realized national policy which ensures the most equitable and efficient use of national resources.

\section{REFERENCES}

1. B. COHEN, "Before It's Too Late - A Scientist's Case FOR Nuclear Energy," Plenum (1983) 
2. M. SCHROEDER, "Fractals, Chaos, Power Laws. Minutes from and Infinite Paradise," W.H. Freeman and Company, New York (1991)

3. J. GLIECK. "Chaos - Making a New Science," Penguin Books, New York (1987)

4. I. STEWART. "Does God Play Dice? The Mathematics of Chaos." Basil Blackwell, Inc., Cambridge. MA (1989)

5. R. DEVANEY. "An Introduction to Chaotic Dynamical Systems." Second Edition. Addison-Wesley Publishing Co.. New York (1989)

6. B. FISCHHOFF, "Improving Risk Communications." Commission on Risk Perception and Communication, National Research Council, National Academy Press (1989)

7. P. SLOVIC, B. FISCHHOFF, S. LIECHTENSTEIN. "Rating the Risks: The Structure of Expert and Lay Perceptions," Risk in the Technological Society (ed. C. Hohenemser, J. Kasperson), American Association for the Advancement of Science Symposium 65. Westview Press (1982)

8. W. MORRIS. "The Analysis of Management Decisions," Revised Edition. Richard D. Irwin, Inc., Homewood. IL (1964)

9. R. KEENEY, "Siting Energy Facilities," Academic Press, New York (1980)

10. S. FRIEDMAN, A. HARVEY, "A Technique for the Selection of Hardware in Automatic Control Systems." Proceedings ISA International Conference, October 11-14, 1976. Houston, TX

11. S. FRIEDMAN, A. HARVEY, "Application of a Vector-Valued Decision Model," Proceedings of the
First International Conference on Mathematical Modeling, August 29 - September 1, 1977. St. Louis, MO

12. R. BROCKWELL, S. FRIEDMAN. A. HARVEY. "Decision Analysis for Environmental Management. An Approach wo Choices Among Multiatuributed Alternatives with Intangible Cost of Benefits," Proceedings of the Second International Conference on Mathematical Modeling, July 11-13, 1979, St. Louis. MO

13. S. RAMAMOORTHY, E. BADDALOO, "Evaluation of Environmental Data for Regulatory and Impact Assessment," Elsevier, Amsterdam (1991)

14. E. ROTH. M. MORGAN, B. FISCHHOFF, L. LAVE, A. BOSTROM, "What Do We Know About Making Risk Comparisons?" Risk Analysis, Vol 10. No. 3. (1990)

15. R. MEGILL, "An Introduction to Risk Analysis," Petroleum Publishing Co., Tulsa, OK (1977)

16. G. IVERSON, "Bayesian Statistical Inference." SAGE Pub., CA (1984)

17. E. FRANKEL, "Systems Reliability and Risk Analysis," Martinus Nighoff Pub., Boston (1984)

18. H. TAHA. "Operations Research, An Introduction," Third Edition, MacMillan Pub. Co., Inc., (1982)

The preparation of this paper was funded by U.S. Department of Energy (DOE) Grant No. DE-FG0288DP48058. Management Systems Laboratories thanks DOE's Office of Environmental Restoration and Waste Management for providing us a real-world laboratory for the research, development, and testing of state-of-the-art management tools and the frameworks for understanding how to make them successful. 


\title{
TOOLS AND METHODS FOR INVOLVING TBE PUBLCC IN RISK-BASED DECISION MAIING
}

\author{
Aubrey E. Harvey and Joho B. Mirchell \\ Mningemeat Systems Lesbartories \\ Deperment of Industrial and Sywem Encincering \\ Virginia Polytochnic Institute and Sum Univenity \\ 1900 Krat Dive \\ Blectreurg, Virginia 24060
}

\begin{abstract}
In this peper, MSL will present reaults of its research on communicating risk iseves to the public. We will explain bow pitfills in meny risk communications programs and public misconceptions and mis-education about risk secearment canse problems in gaining public appon for and involvement in risk-baned decision miking. We will then show bow beat to employ rick ascesamen conceptes and methodologies for public edveation progrom. Finally, we will report on MSL's wotk for the Depurtment of Energy to develop risk communications proeram the ealix the public as a willing and interested participent in its own ectucation about riak trade-ofis and decision making in the public sector.
\end{abstract}

\section{INTRODUCTION}

As the nution continues to wreatle with the complex and interdependent problems ascocinted with cleaning up the wastes from our nuclear legacy, we are becoming increasingly awere of bow the public's perception of risk affects the cleanup procese. Lecking $m$ objective framewort to approsch the concept of ecceptable risk, public opinion is pulled this way and that by modia reporting and vocal epecial intereats to the point where cleanup projects are often atalled by conflicting priorities and intereats, thereby increasing the final costs. Breaking the geidlock on planaing and implomenting clesnup projects requires involving the public in their own education sbout risk so they will see clearly for themselves the necescity to mike intelligent trade-offe between risks and opportunities when making decisions affecting our quality of life.

As part of its ongoing research for the Department of Energy on building and measuring consensus, Management Systems Laboratories (MSL) is studying and cating efrective rools and methods for educating and communicating with the public on the subject of risk Uaing atvenced informosion proceasing and display rechnolosy to apport collabornive wott, MSL etrives

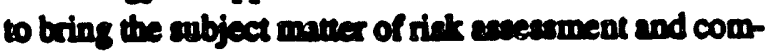
munication to a level eccenaible to the layman, and opens up rew sppronches to involving the public in rist-besed decision mating. One sproech to building excetive consenens is to wats with stakeholders to develop a common definition and undertending of the problems the andertis the iemes being discrened. MSL is wodting with poblic participation groups to build shered models of anch problem to asint this proceses of definition and underumenting between the public and government decition makess.

To acceatully exteblich a public forum for risk communication, we believe ceveral things must be accompliched. If is necescary firt to underetand the reasons ecceasful rist communication hes eluded many practitioners. It is impartant to underetand public mieconceptions sbout rist, and the reseons for public revistance to may efforts to communicate risk conceptes related to decisios making. It is important to underetand why unditionsl forums for public involvemeat inadequately deal with the rist ectucation neceasery to sada in docision maling where stermstives involve verying degrees of societal risk and beoeft

Finally, int commonicatione practitioners oeed to consider new outreach sppronches for involving the public in rist education. Theed sew spprosches include multienge risk education progroms for members of public involvement groups, so well as risk tutorials for medin representatives and public school teachers. MSL hes also inveatiganed uaing Group Decision Support System (GDSS) as tools to enhance objectivity in stakebolder consensus-building efforts. GDSS show 
promine thet if ellectively dealened and presented. they can main in formulnting and objectifying riskbesed probleme, while colucating participanes in besic risk aseasement concepes and rist-besed decision mnting methodologies.

\section{PUBLIC MISUNDDRSTANDING OR RIST}

The risk communication literuture contrins a multwode of sudies of public percepdions of rins and public reaction to disk communication efforts. $(1,2,3,4,5,6,7)$ MSL has been researching thowe ares where public misunderatunding of rick aseasment concepts and riak communications methodologies impaire the public's effective participation in risk-baed decision making. Some of these typical public misunderetandinge are summarized in this rection.

The public distousts risk comporisons the atress ace ceptability of the risk.

Many risk communications practitionens argue thet the risks of a new mbetance, ectivity, or technology cm best be understood by comparing these risks with the risks of more common or familier substances, sctivities, or technologies.(5) Aseumptions thet undertie that argument include the following:

- Comparisons help put ristes in poychological perupective, providing a "conceppual yurdetick" for understanding relative risk

- Comparisons improve underctuncting of risk mamitudes, providing more intuitively meaning ful informstion than mw numerical probabilities.

While appropriate risk comparisons can indeed clarify public understanding of insves, meny manuficturess of technologies that creste risks look 4 rick communicstion as a way to paruade the public that the riak from a given technolory is anall ecough to be iprored. Sheila Jasanoff bes meseated that "rist communicstion is often a code wond for brinwahing by experts or industry." (8)

Risk communications prectitioness have ofteo relied on risk comparisons such as: "If you would be willing to (eat peanut butter, fly on transcontinental alghw, etc.) then you should be willing to (live near a nuclear power plant, sccept the risks aseociated with operating nuclear reactors, etc.)." Wileon (9) identified a set of ectivities with eproximaty equal riks: smoking 1.4 cirarettea, riding wen miles by bicycle, enting 40 tablespocons of pesnue buter, driniting 30 12-ounce cans of diet sodh and living within tive miles of a nuclear re sator for 50 year.

This line of reaconing is sot ecceped by the public (25), which mepects commonicaters uaing exch risk

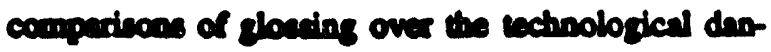
gase and tying to moks them cound like everyday risk decivione The public sleo reates comperieons bo tween qualitudively different cregories of risks (S) For exnmples while mox people concur the cigerette smoking is hermful, there is a qualitative difference between celf-inficted tict lite the incurred from moking, and involuntery risk, mch os being expoeed to harandous contaminants in the sir we breathe.

Nocetheleas, eppropriate rist comperiecons are deceet any for decinion makes to bulance rick and opportur nity objectively(10). There ace always undo-0ate in choosing between wehnological riste, including the risk of lowering the anderd of living by not employing a given wacholoyy. The challenge to the riak communicator lies in alocting ppropriate risk comperisons and presenting them in ways that are accoptable to the public.

The mblic nerceive: rechnoloricel rist differently from "nnturlenters.

The rist communications literature contain mny examples of public misperception of equivalent natural and technological risto (1). Covello determined that people give more weight to risks brought on through bumen ection then thoce caused by sets of neture (5). Gendrex, Hervey and Mitchell (10) cite the relationship between the public perception of technologicallyderived risks verus its perception of asturally-derived risks. (See Figures 1 and 2). As the sctuld risk increas es or decresuct, the perception of rist encensmeat experts leads 10 cocrelme with the sctural risk, while the public perception eods to exnszernte rechnological ricks while igmoring antural risks. 

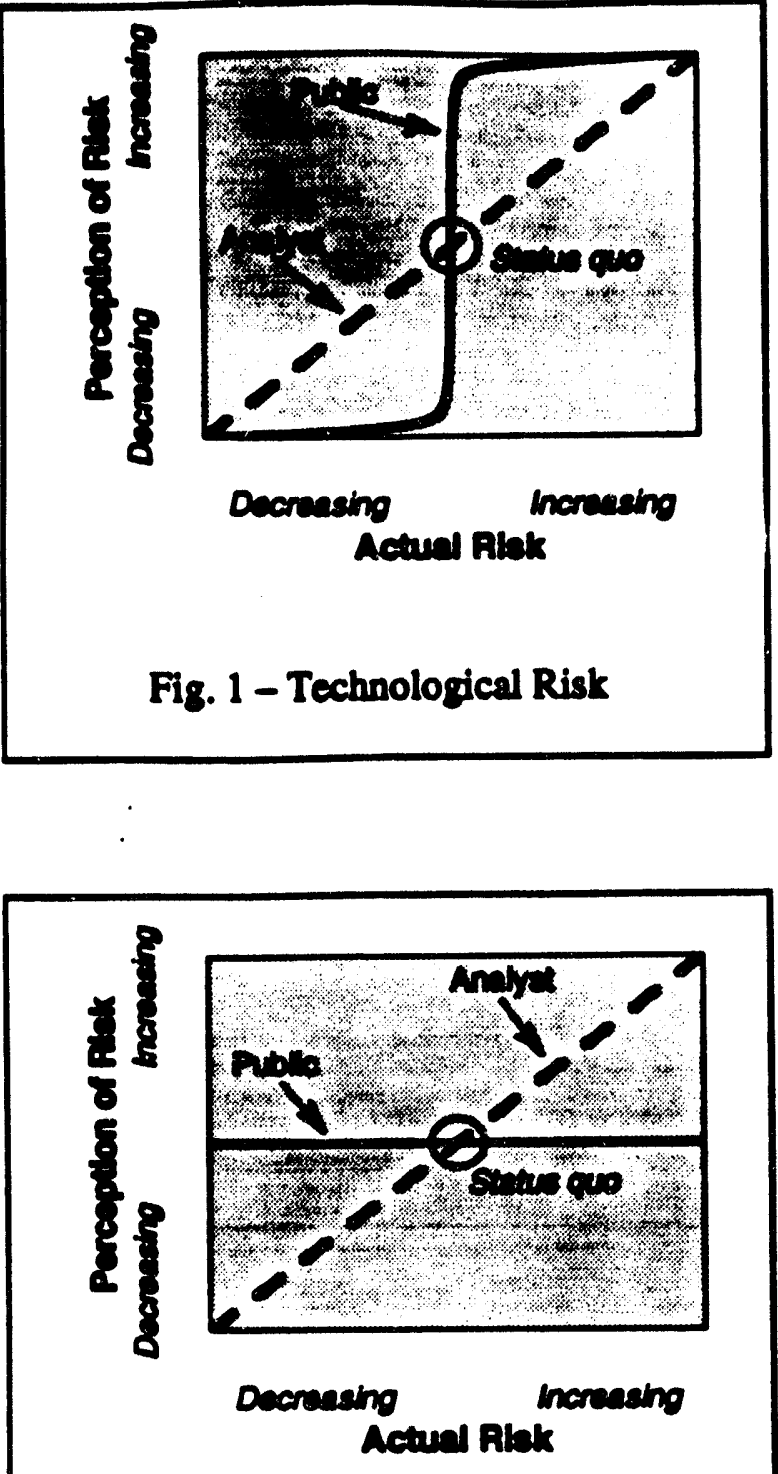

Fig. 2 - Natural Risk

As an example, come people might forego a diagnoctic $x$-ray 10 avoid the coccomitunt redietion exposure, while willingly exposing themedves to even higher levels of "nntural" adintion incurred during high-eltitude jet flights. Another example of the public perception thet netural risk is benign is the cendency for the public to rely on tood control syutems in their selection of a bomesite. As we all aw during the Midweat Hooding this past summex, flood control syatem $\mathrm{cm}$ actually execerbate the effects of floodine, yet man people rely on dame and levees, or the reasuring but statistically fallacious interpretations of "100-year toods" 2 promiding a mardin of cecurity for the "bext 99 yeer." (10)

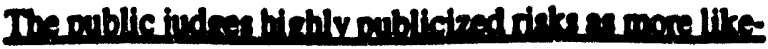
Ir.

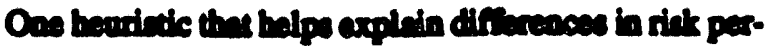
ception is called avellobility (3). This maen the a hichly-publicined rist or poceotial even is jodged by

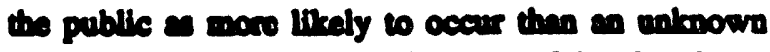
tilk a evene For exmole, becanse of the sbund nnce of public tinformiba on sucles reactor ristes, beludins vivid movies ach a The Chine Syndrome, the public oveceatimeses the denger of a resctor melidown. of other cennerophic oncleer cecident like the scoident at Chernobyl This misperception continues despile public rocen to informmion sbout U.S. reactor design.

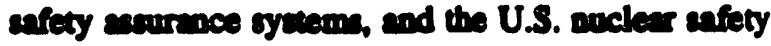
recond.

Masur (11) anded thrt the mere increane in prese coverige of en event or bechnology contributes to increas. ing the public's perception of dengex, even if the covernge is sox perticulatly negrive. The pablic too-

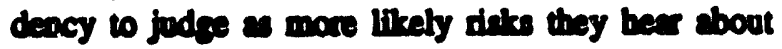

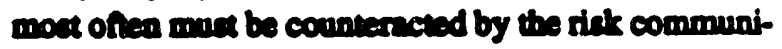
cetor by cerefully laying out the beat avilable scientific informution sbout the rithe involved If is important to avoid eppearing a "pertisl" to the ecientiflc side; better the the poblic weigh all the informston from all sources and reach their own conclusions.

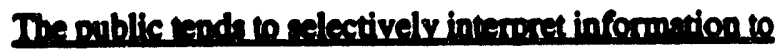
meintrin consistency with guroent belief:

Human beinge have a fundamental readeacy to reek information consintent with their current attitudes and beliefe, and to imore informmion which potentially controdicts these suitudes and beliefe. This is commonly known in the liternture as the confirmation bias. (12) For exemple, there hes been a greal deal of modia attention directed to very low probsbility risks susociand with childhood pertuseis veccinations. Many popaler daytime television shows have covered this is we in a senacionalintic menner, concentrating on the very low probability rises of serjous side effects in children receiving the veccine. Ore result of this publicity hes been thet fewer perents now vecoinnte their childrea for pertucie. This hes reulted in a comebeck of the very dengerous childhood disesese of whooping cough. 


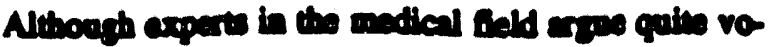
celly the the veceinemion rists are may times lower then the (now evidede) rists of the dicence, many people choove to foces on the emall probubility of en ed verese resction to the vecise, rether then on the demonetrebly inger probability of cucions illneas iner. Confirmation bies bere implies the coms people berbor aupicion of oflecial or expert epaterpencoen and "went to believe" the more reasedionalietic bedimooy. Public distrue of enthority figures promineatly in many rick communications billurea.

The public does nos underend form-l reanoningets pecinlly when number me involved.

To hnve en effective role in tiak-bened decision making, the public must perticipete in decision proceseres involving bighly technical information. Underetunding much of this informntion requires the sbility to deal with bw and high probebilities and underctend and apply besic principles of logical and ataintical in ference. The public misunderetanding of wch formal reasoning procesces hes been termed "innumercy." (13)

The problem of the public lack of proficiency in onmerical and logical reasoning is compounded when instiwtion for informing the public thil to addrese public misconceptions sbout small probabilities. Consider stories involving the chance of a metecr impact destroying life on errth. Often sensationsliatic stories are transmitued concerning such events with probabilities so low as to be negligible. Mnny in the public, for example, now believe the expendiure of billions to construct a (posaibly ineffective) sateroid detector system is money well spent, despite the very low probability of a catastrophic collinion. Here, given the premice that we have limited public fundes, the logical conclusion would be the the money would be better spent on more preasing probleme.

Another example of the trilure of the public to mate proper logical inference invalves decivion-making in the bealth industry. We spead a vast amount of our an cional bealth care funds on whes hes become known 2 "futile care." Futile care is characterized as that readered during the last two weeks of a terminal patient's life, when beroic and expensive meanures me taken to attempe to prolong life, with results that 100 often add only days or weeks to the patient's life. Many families confer together 10 make the difficult decision to forego

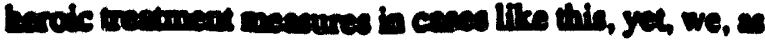

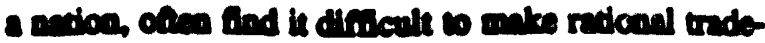

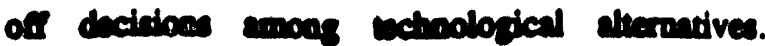
Wheoserer undo-ofe involve bomen eafery, the typical reaction is wo eat of if funds seo nollimited.

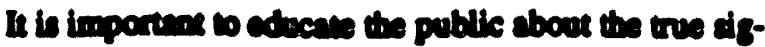
nificances of bow probubilitioes and the need to foeve st. bation and limiled recources on projects that have the

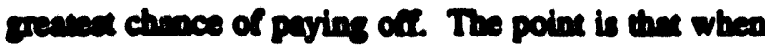
you dover recoureas to projects with a low probability of moceses, those secources cenact be eppent on other projects which might bring greater weceses. The pub-

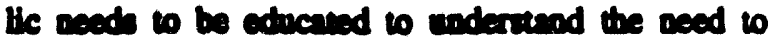

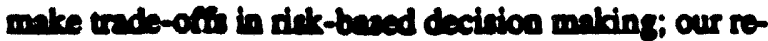
sources exe not infinite.

\section{FUBUC RDSTSTANCE TO TRATIONAT PISE COMMUNICATION}

Poople cen only procen sew information through epplyins is to thetr exinting conceppend tremewod or

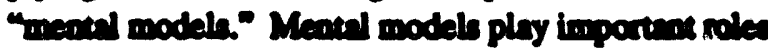
in bow people build their boowledes bees end me decisions (14). If they boow nothing about a risk probben, then eny measenes communicered sbout it will cary bith meaningful informmion for them. If the pablic the beliets the re inccurnte, then they may mircocentrue the mearage.

Eflective tist communication presupposes that the cender and receiver both shere a common concepts: frumevort or mentel model sbouk what is commi." cred. Unfortunately, mot treditional risk communcenion bes involved neing "expert" cources to "convince" a lay public thet rists incumed with a given courve of ection are "eccepteble." This one-way atyle of rist communication shows litule regend for the menil models of the andience. When the audience perceives the their views are beins divegurded, they adope a more enengonistic relationehip with the communicator. The dimne of dintruat the has come to exin between induetry epokeapersons and the public represents a sionificant bearier to the development of tur neceasiry for consensus between otakeboldens on riak-beaced decision.

There is usually litile in common between the mental models of industry experts and the lay public; this makes it all the more important to sctively solicit the 


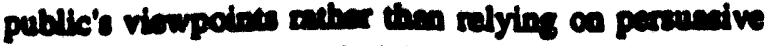

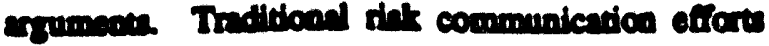

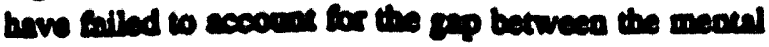

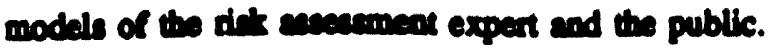
Efiors to communicene rits without fru bridging this

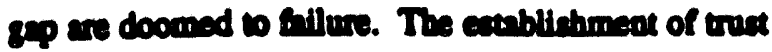
benween poblic policy meteres and the public to rickbesed decivion moling censol oceur undil efforts axe

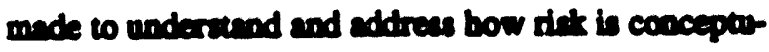
ally defined by the pablic.

To quote O. Rean, in Strite gies of Risk Communicer tion, (15) "The principel objective of risk communicer tion should therefore sol be 10 infuesce or slta people's atritudes lowade risk sources, but 10 provide easily comprehensible cools to laypersons to eanble them to underrand informetion about ritks, to proceses probabilitatic informetion, and to evaluate the litrely impacts for oneself and cociety... [thus,] rick communication must incorpornie a broed conception of rist, and operate with the realization thr communication is a two-way proceas in which both aides should bam from each other."

\section{NEW APPROACEISS TO PUBLC PARTICTPA- TION IN RISK COMMUNICATION}

The Departmeat of Energy bas as a atrod god to croate a forum for risk discuscion to effectively involve the public in risk-baeed docision making (16). MSL believes this goal can be eccomplished by intelligendly developing new risk communication progrm and public outreach ectivities thes approech risk education from a variety of innovetive platfarm. The key to risk education is active solicitution of the mental models of the public (17). This easublishes an initial level of truat in the audience. They realize they are not receiving a canned precentation, with all the nowers predetermined.

Once the mentul modals of the poblic heve been solicited, the risk communicrer cen begin providing the audience with information needed to etructure the problem at hand. This information includes beat available extimates of the risks and benefits involved in each decision oode of the problem. The audience can suidy the problem and refure expert asesesmeats, but they must record their ressons why, and eater them into the record of the proceedings.
This procens allows the sudience to becoms self-criti-

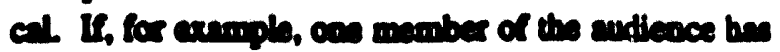

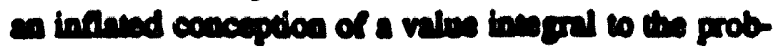
bon, the rex of the sudience cen cerve a a reality chect By theneting trough the vedous poedbilities.

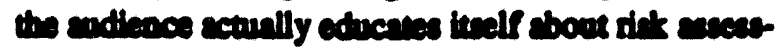

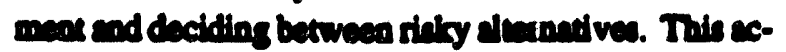
ive involvenon of the endience counderects the

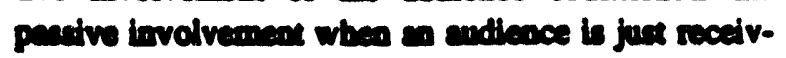
ing infoconstion twe wo edopt en enengoniatic atritude withon merpeding a conetructive subetibute course of ection.

The role of the fecilionior is crucial. His or sbe muat eawre the the andieace naye within the bounde of the risk problem. The andience muat aleo be reminded of the rules catablisbed for the risk problem, and guided

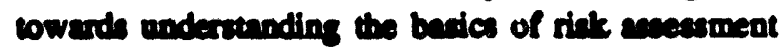
methodologied. This is the self-chention festure of this epproech. While meny laypersons would not ecively purave en educadon in rist conceppes and methodolosicen they ectively puricipare in thoir owo chacution when they ave inveated in a grided problam, weh en propoed.

\section{A SAMTLE RISE DOUCATION FORUM}

We have constructed a enmple tiak chucation forum and outlined a multientere procese for involving a aroup of etrkebolden in the decision miking process. The problem is decigned to solicit a wide range of viewpoincs trom the andieace, and to encble the andieace to wats through isenes to begin to develop concensus on bow to solve riat-baned problems affecting netional policy on wase menagement and environmental reviontion. The entire process is scheduled to whe three daye.

On the fint day, the group is tonght sbout rick asees ment lechnigues, including uning probebillities and do cirion treen. This ceasion is supplemeoted by simple exnmples uaing bovecies and earily comprebensible problems anch a whether to drive a cer or ay a plane for a given journey. Included in this problem are probabilities for eccideste, calculations for time raved, etc. The puppose of this fira day is to carbblich a $800 d$ foundation in the group about the role of risk in everyday decisions, and to begin to think in terms of making risk trob-otis to meximize expected value and gain opportunities. 
On the recond day a lezer problem is presented to the

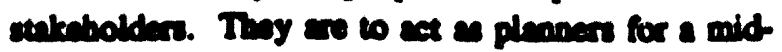
sised ciry beated cloas a mijor river which is prove to roodine. Sundetically, evary 100 yeen, a flood cen be

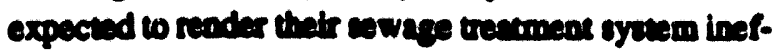
fective. This will reoult in public bealth tists, incoor venience, and added expeave. Thoy man enceas the risks of the trous quo verens the benelits to be goined in the decision to build a cot to build a beckup seware trestrenter system on higher ground.

Throughout the eccond day the strkebolden practice modeling this problem as a decision tree. They muax agree on the velues to be input for each brench of the tree. Aner iteruting this procees to coocludion, they have gained an undertending of bow more complex risk problems are etructured and the relevicashipe be tween the variables and values of the problem. This second day aleo brings bome the finite anture of ro sources and the fect that all risk-based decision making involves unde-off between rists and opportunities.

On the thind day, the group is given fundamental information on a national cleanup problem, such a wing incineratore to dispore of toxic wactes. The group must then take all the information availsble on were incineration to build a model of the problem, incorporating all boown risks and benefits. Computer models can be designed to assiat this proceses. At the end of the three days, the group should be able to recommend a course of setion for using toxic wate incineration based on a thorough analyuis of the riths and benefits of using the technology.

\section{USING GROUP DECISION SUITORT SYS- TEMS TO SUFPORT IAI GROUP PROCDSS}

Over the lant tea yeens or 20 , behnological advances in computer-generned enphicel informntion diplays and bigh-capacity deciaion expont symem (DSS) have made it prectical to integrite and reprecent vast amounts of complex informetion. As promising and successful as eraphical portrayal methods have provea to be in some domains, the use of ruch nootraditional communication medin and information partrayl formats for risk communication has gore unexplored (19)
Mat the devaloped a Croup Dection Support

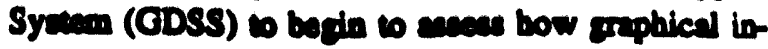

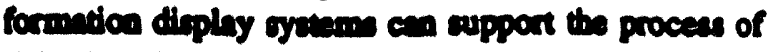
tink edreation end public involvement The GDSS we

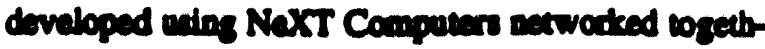

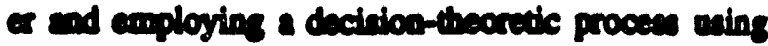
modeled docilioa tron th a lyyed diplay covtron-

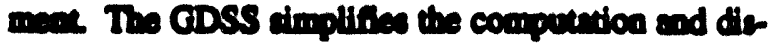
play of riektread problem with multiple decition sodin. Meny of the methemedical culculations neceen ery for decinton tre problams ere antomntically cerried out by the ODSS, readering the decition tree colvtione ingely trenpercaet to the croup.

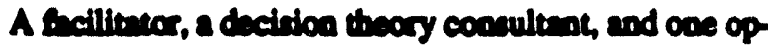
entor for the ODSs ere required to the the GDSS. The fellitertor is reponalbie for acdeting the group in ariving $a$ vilues and probabillities to be need in iterntins the problem; the opernecr inputs velved arived at by the croup inso the GDSS; and the decivion theory

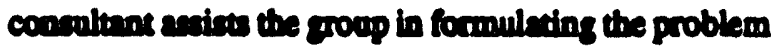
to rum in the decteica tree model.

\section{Rerenlin of the res of adoss}

MSL coavened a bat group of employeen to simulate a citisen's comminas cherged with providing input to

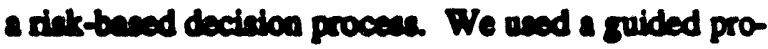
ceas to contruct decision trees to eruphically eupport the group's decivion moling proceses.

The enp involved neing four NeXT computer wortention linked topether and controlled trom a centrul worbetution where the cpercilor eatered information and built the decidion trees. Bech member of the bex group en at a NeXT difplay terminal and we able to weth the decision trees 25 they were buill.

To belp the roup anderenend how to employ the GDSS, the decicion theary consultane conducted a bief demontention ceacion, using the Virginia State Lowery as an example. The group was shown bow to fructure the Lotery problem into a decision tree to model expected value. This demondtration we do signed to provide the teat group eome familizity with the GDSS uxing en example thet mode of them had come bnowledpe of and to focter group truat in the objectivity of the GDSS.

We then presented the teat problem to the group. The problem was etructured as follows: 
"As a group of cilizene emponved by the govenumear to decide bow to allocele enery recourch fundh you muxt docide between the following four dininct belds of rewarch:

\section{Soler Enery}

\section{Nucler Enery}

\section{Enerey Conservation}

Coal and other Focail Fuels (Strus Qwo Op(ba)

"You mux decide bow to epend available funds of $\$ 10$ Billion on reserech, but ell of the funde mut go into only are of the four fiald of resenrch. Yos mont decide whnt is the beat option based on your underunating of the relative marits and drewbecks of each energy rechnology.

"It is eugrested you ant with one of the for options as the rook node, end build a decision tree for that option. You will reed to come to agreement about the outcomes of each branch of the decision tree, and about the values you assign to each decision. Surgeated values in clude but are oot limited to economic corth waste generated, and quality of life effects. including diminished standard of living."

The group then begen identifying outcomes and values to be modeled for the decivion procese. A sample do cision tree for weh a problem is portunyed in Figure 3. After using the bleckboard for froilinting this diecuer sion, the group we told to ty wo conetruct a decieion tree based on their diccusion. The group etarted with the Nuclear Reaench option.

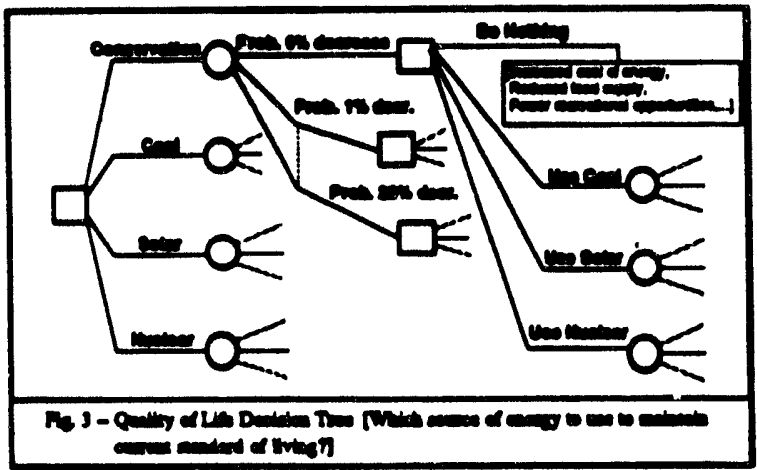

Altrough the grop anceceded in providing informs.

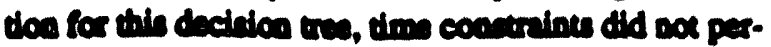

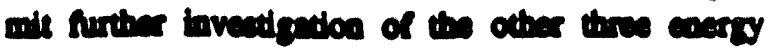

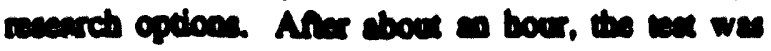
caded and commenter were coliciled trom the group shout their apperibece. From the commeath, we have shetrected the following caltique of the ODSS.

\section{Crition of the ODSS}

One mirecosopption the became evideat wes the the ODSS docition twe was meand to represent cane and

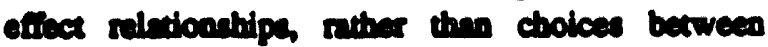
weinged alvernetives. This minconception remmed trom indegane group treining/bmilierity with the ODSS. It strould not be expected thet a group of nominnlly "tay" pereacs should be able to repidly develop en undercunding of the principlas of decthion trees. We have edded more trining in the inroductory nee sion on naing docition trees for modeling ricktaned problem.

Another inove relmed to ODSS fomillintity was the of true of the procees and the freilituton. These is $a$ in-

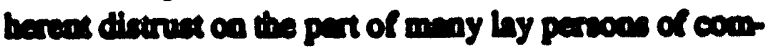
puradzed projaction aymeme, and of "axperts" in renerel. The perception is the there is a sales job bo ins perpetrinedi then the whthorities conducting the for cilintioa procees mxy not be uobiased or without their own apeoden. Technical people involved in such croup efroxts wad to distrut the emotionalism menifeoted by econe of the lay public. This distruat on both pers is extremely deatructive to the consensus-buildins proceas. Fecilintons must prosetively adtreas if wes of trut when using a GDSs to mpport group decielon miling proceseces. We have found thet truat is enhanced when the endiecen thoroughly underronds the cools and methode oned to ameas rist and choose between sliernetives.

Pethep the mont significme ieve to come trom the ceitique was the the ODSS rended to drive the group discuscion rather then the group discuesion dive the GDSS. This cecurred when much time was spent at dreasing ways and meens to make the information coming from the discusdion at the decivion tree model. It is permount the the ODSS be a transparent 2 poceible to the group during the diecusaion and modelins proceen. This traneperency could be improved by a combinntion of a more uner-friendly interfece and more training before the eascion. The operator of the 
ODSS could ecomplith ell seceecery functions roles.

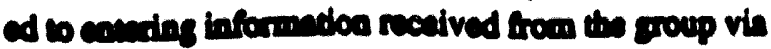

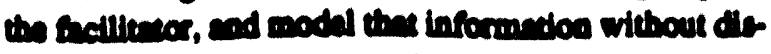
rupting the group procene The ODSS could then be und to padodically porray to the group the then wotk for the informention baing diccuesed es an objece-

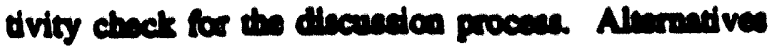
being conaldered could be projected for the group. alons with objective incerpretations vin the ODSS of outcomes for each allementive, based on the values bo ing furnishod by the group.

Wo believe the roole weh as the ODSS can be valu able in belping to objectify the decteico-muling procese where choices involve risk These tools alo provide reeded education to the public in decision-the oretic principles. The reed to schieve trenepareacy in the modeling of information and to wodk clocely with the audience for facllitating the group proceas hes been eatablished, and future efforts to employ ODSS in group proceseses will incorparate these besons.

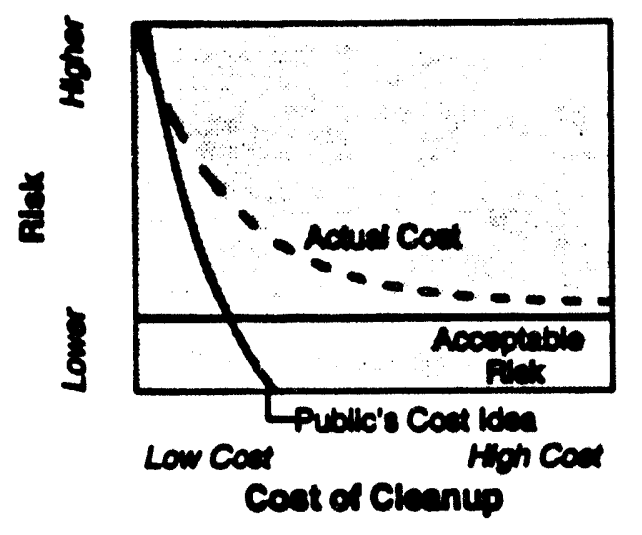

Fig. 4-Cont of Cleanup

\section{SUMMARY AND CONCLUSIONS}

The problem of afectively communicating risk and soliciting positive contributions trom the public for risk-based decision making bas been shown to be complex and multidimenaional. Nong with being knowledgeable of and addreasing public misperceptions of risk, it is very important for the risk communicator to coaduct a thorough and careful analyais of the audience's mental models.

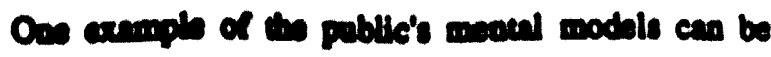

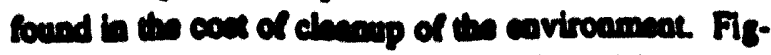

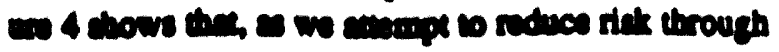

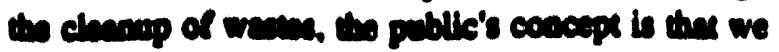

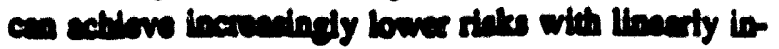

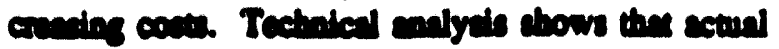

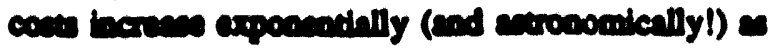

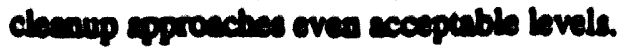

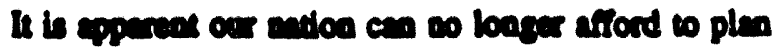

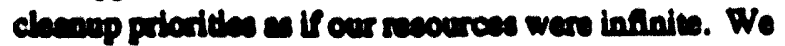

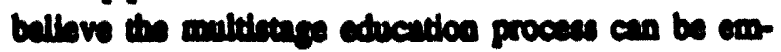

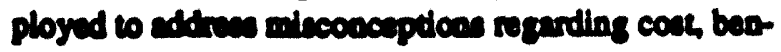

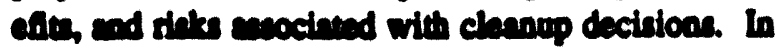

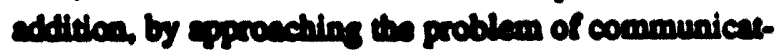

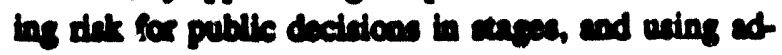
veaced roole ench o ODSs, we and to gain boch

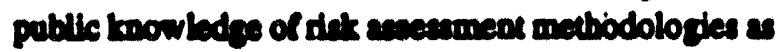
well es pablic true in and commitment to the dectition moning procesea.

\section{DXIDTNCE}

1. B. Fiecthot, "Improving Rint Communicatons," Commieaion on Rist Perception and Communication, National Reaench Councll, Netional Academy Press (1989)

2. B Roth, M. Margen, B. Flechbot, L. Lave, A. Bor trom, Whes Do We Know About Miring Rikk Comparicons? Rick Annlyrie, Vol 10, No. 3, (1990)

3. P. Slovic, B. Finchboft, S. Lbecheontein, Rating the Rinte: The Structure of Expent and Lay Pexceptione, Rirt to the Techoological Sociony (ed. C. Hobenemex, J. Reppersoa), Amedicen Areocintion for the Advencemeat of Science Sympocium 65, Weatviow Proes (1982)

4. O. Kreen and H. Elfrelhof, Prior Koowledge and Rink Communication: The Case of Nuclear Rediation and X-Rayr," Communicating Risks to the Public (R. Kappescon and P. Strllen, eds.), Nuwer Academic Publinters (1991)

5. V. Covello, Rink Comperieons and Risk Communication: Iever and Problem in Comparing Health and Environmental Riste," Communicating Riske to the Public (R. Kenperion and P. Strllen, eds.), Nuwer Academic Publishen (1991) 


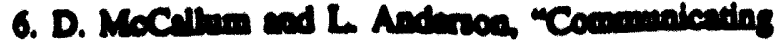

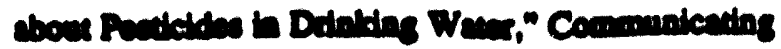

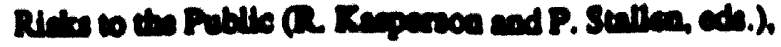

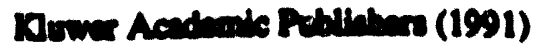

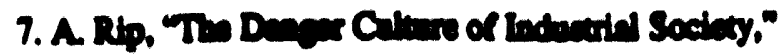

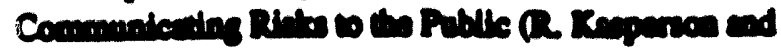

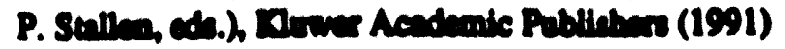

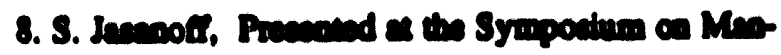

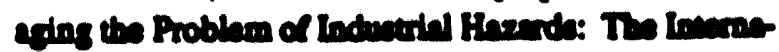

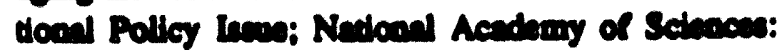
Wahington D.C. Fobrumy 27, 1999 (1989)

9. R. Wiecen, "Analycing Dedly Rirtes of Lifo," Technoloyy Reviow 81 (1979)

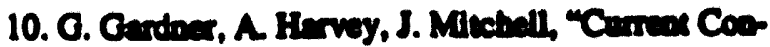
cepes and Methodologies for Accescing end Communt-

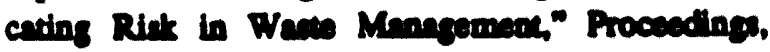
Wase Mansgemere 93, (1993)

11. A. Mersur, The Dynemics of Tecturied Controversy," Commuaicadoos Preas (1989)

12. J. Bercen Redonality and Imelligance," Cm bridpe University Preas (1985)

13. J. Pullow, Inoumerecy," Hill-Wang (1988)

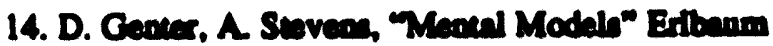
(1983)

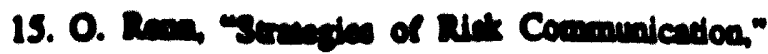

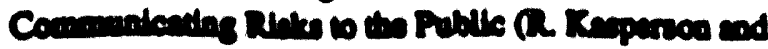

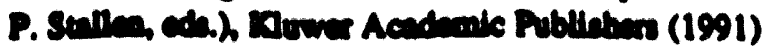

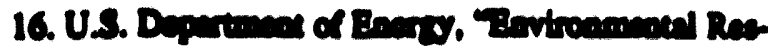

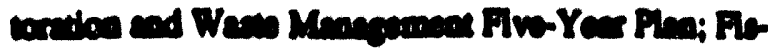

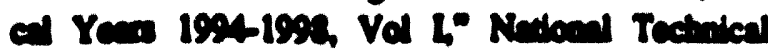

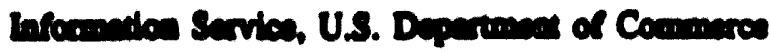
(1993)

17. M. Magen, D. Feched, A Bowou, L. Love, C. Amm, "Commmicating Rite to to Public," Bovt-

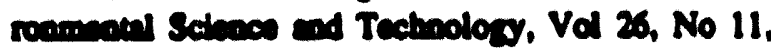
(1992)

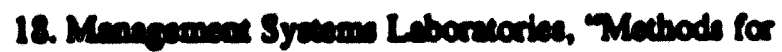
improving the Quality of Public Involvemeat is Rist.

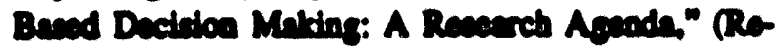

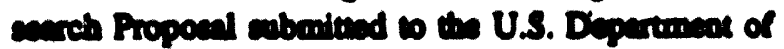

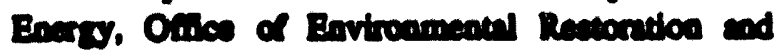
Weas Menaponsa) (1993)

The prepention of this peper we funded by U.S. Deperimed of Bnery (DOZ) Crm No. DEFON2.

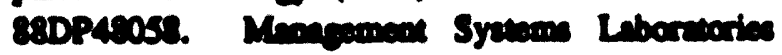

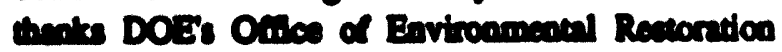

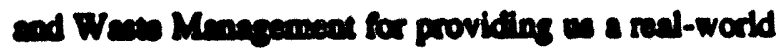

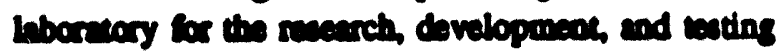

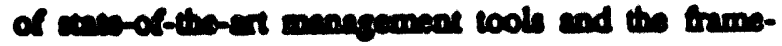

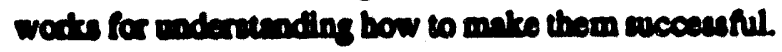




\section{Gleaning Information from Open-Ended Data to Support Decision Making Timothy G. Kotnour \\ Betty Koball \\ George Batrouny \\ Melisea Shaw \\ Tony Harvey \\ Management Systems Laboratories \\ Virginia Polytechnic Institute and State University}

\begin{abstract}
The contribution of this paper is a content analysis process for gleaning descriptive information and summarization from data to be used in public management decision making. In quantitative analysis of data we emphasize reliability, validity, generalizability, and replicability as we judge the quality of our research. Correspondingly, in qualitative analysis (e.g., content analysis) we emphasize dependability, credibility, transferability, and confirmability [1] to judge the quality of the information we obtain from a study.
\end{abstract}

From our study, we will be demonstrating to managers four viable criteria for judging information. Our study will use a case study of the analysis of over nine thousand (9000) comments from the scoping meetings of the Implementation Plan for the Prorammatic Environmental Impact Statement for the Department of Energy Environmental Restoration and Waste Manarement Proram.

\section{INTRODUCTION}

The contribution of this paper is a content analysis process for gleaning descriptive information and summarization from data to be used in public management decision making. A process is presented to use in gleaning information from responses to open-ended questions. The process is discussed in terms of four criteria: dependability, credibility, transferability, and confirmability. Both the process and criteria are useful to managers and suppliers of information to managers because they can be used in the production and evaluation of the information managers use in decision making.

This study was completed to describe a process we can use to glean tristworthy and useful information for managers from reaponses to open-ended questions. Three sub-questions were used to answer the research question: What is the process? What are the criteria to assess the process? How does the process meet these criteria? The objective was to develop a process within a set of criteria to produce and assess management information. The purpose was to provide a means for managers to assess the information they use in decision making.

This paper describes 1) a connection between management information and research, 2) measures for evaluating information, 3) a content-analysis process, 4) the process in terms of the four qualitative criteria, and 5) lessons learned.

\section{MANAGEMENT INFORMATION AND RESEARCH}

The link between management information and research is made through the common goal of reducing uncertainty about a phenomena. I define management infurmation as information used by managers to make decisions. Galbraith [2] defines uncertainty "as the difference between the amount of information required to 
perform the task and the amount of information already possessed by the organization" (p. 5). Huber [3] defines information as "data that give meaning by reducing ambiguity, equivocality, or uncertainty" (p. 89). The role of management information systems is to provide the information to reduce a manager's uncertainty about a decision. According to Brinberg and McGrath [4] research attempts to reduce uncertainty about a phenomena by producing information about the phenomena. The connection between management information and research is important because we can use the concepts of research to help us understand both the production and evaluation of management information.

\section{MEASURES FOR EVALUATING INFORMATION}

Managers deal with information that can be characterized as quantitative (e.g., production or inventory levels, downtime) or qualitative (e.g., stakeholders' or customers' desires). The qualitative information is accumulated over months from a diverse set of sources (e.g., customer surveys, focus group interviews, trade journals, newspapers, memos, word-ofmouth). In the management information literature many measures have been suggested for evaluating management information. McKinnon and Bruns [5] suggest timeliness; accuracy; relevance; and corroboration, redundancy, and data presentation. Hill, et. al. [6] operationalize measures of good, bad, or useful information. The quantitative research world emphasizes reliability, validity, generalizability, and replicability.

The "quality" of qualitative information needs to be evaluated differently than the quantitative information. The qualitative research world and in particular Lincoln and Guba [1] suggest dependability, credibility, transferability, and confirmability to judge the trustworthiness of findings. Establishing the trustworthiness of information is important because managers use information they trust [5]. Also, the qualitative measurers are more global and overarching than the measurers suggested in the management information literature. For example, accuracy, corroboration, and redundancy are subcategories of credibility.

This paper focuses on applying the four criteria of Lincoln and Guba [1] to a content analysis process used to produce management information. Lincoln and Guba provide suggestions to meet each criterion. Definitions relating to these criteria and the content analysis process are given next.

\section{Definitions}

Credibility refers to the "truth" of the results. Credibility can be achieved by 1) prolonged engagement in the domain to understand the context of the research; 2) persistent observation to identify the most relevant characteristics relating to the focal problem; 3) use of triangulation of sources, methods, researchers, and theories; 4) peer debriefing; 5) continual refinement of hypothesis until no exceptions exist; 6) archiving the data for future examination, and 7) member review of the complete research process. Triangulation is a process of using a diverse set of methods, sources, and interpretations.

Transferability refers to the applicability of the results from one domain to another. Transferability can be achieved by 1) providing a description of the context in which the research occurred to allow one who wishes to apply the results to a new situation the ability to determine if the old and new context are similar enough for a possible transfer and 2) making the database available to the appliers. 
Dependability refers to the repeatability of the results in the same or similar contexts. Dependability can be enhanced by 1) using triangulation techniques, 2) using multiple team members to independently review the data set and communicate with each other, and 3) conducting a formal audit process.

Confimability refers to the notion of "bias" in the results due to the research process. Confirmability is enhanced by the use of an audit process, triangulation, and a journal to capture decisions made about the research process.

An audit process is a formal examination of the complete research process. The audit process used to establish dependability and confirmability ensures research process components (e.g., the research question; the raw data; data reduction and analysis procedures and products; data reconstruction and synthesis products; process notes; and instrument development information) are aligned.

\section{SAMPLE USE OF QUALITATIVE MEASURES CASE STUDY.-CONTENT ANALYSIS PROCESS FOR RESPONSES TO OPEN-ENDED QUESTIONS}

We chose content analysis to help us answer: What do the stakeholders of the Department of Energy's (DOE) Office of Environmental Restoration and Waste Management (EM) expect EM to do with the environmental restoration program? The purpose was to reveal the focus of attention (i.e., goals and definable actions or plans for achieving the goals) as defined by the stakeholders that EM should be pursuing. "Content analysis is a research methodology that utilizes a set of procedures to make valid inferences from text. These inferences are about the sender(s) of message, the message itself, or the audience of the message" $[8, p .9]$. The idea is to classify the diverse set of words of a text into much fewer content categories. This section describes the content analysis methodology used to answer the above question.

\section{Data Sources}

The two main sources for our data were a database of comments from scoping meetings conducted by DOE for the Implementation Plan for the Programmatic Environmental Impact Statement (PEIS) for the Department of Energy Environmental Restoration and Waste Management Program and newspaper articles. The PEIS database of comments from the twenty-three scoping meetings was used as a primary source of stakeholders' comments on waste management. Newspaper articles were searched through the on-line service Lexis/Nexis. We conducted the search with the waste streams (i.e., high-level, low-level, transuranic, and hazardous) used as keywords for the time period from January 1, 1991 to January 31, 1993. We chose these dates to gather the most recent stakeholder concerns. The reviewed newspaper articles provided a list of stakeholders and their comments on waste management issues. This search provided an initial understanding of the range of stakeholders and issues. Other sources of data used, especially in "triangulation," were domain experts, a report prepared by DOE on the PEIS meetings [8], and EM's strategic plan. The report summarized the range of comments from the meetings. The strategic plan provided an initial listing of issues.

\section{Data}

The data elements for this study were: 1) stakeholder as defined by its name and type (i.e., Coiporation; Education; Health; Interest Group; Non-Profit; Religious; Trade; Tribe; Federal, State, or Local Government; State and Tribal Government Working Group); 
2) stakeholders' comments on specific issues pertaining to waste management; 3) EM's strategic planning issues identified in the draft strategic plan; and 4) the issues identified in the PEIS database.

\section{Data Conversion}

We converted the original data from the PEIS database to allow more direct analysis. The conversion had two steps. In the first step, we converted the text based comments to database files. Two data base files were created: list of stakeholders and list of comments made by each stakeholder on an issue. The data elements in the stakeholder database are stakeholder and stakeholder type. The data elements in the issues database are: stakeholder, PEIS issue identification number, comment identification number (from PEIS), and comment. (Note: In the PEIS database a comment may have been associated with more than one issue and stakeholder. In the stakeholder analysis database, each stakeholder-issue-comment has its own record, that is, a record for each issue and stakeholder the comment is associated with.)

The second data conversion step was to group the individual comments into EM's issues. A table associating a PEIS issue to an EM issue was used in mapping the conversions. This conversion was necessary because an EM issue was composed of more than one PEIS issue. The records in the database files were filtered and printed into a separate file for each EM issue. The EM issue files contained the stakeholders' comments for the given issue. These files were used for the analysis. This resulted in fourteen issue files.

\section{Data Analysis}

Content analysis was the method used to analyze the stakeholder comments on a given $E M$ issue. The set of procedures to convert the text into categories is the coding scheme process. This process is the most important step of content analysis. Through this process we determine how a unit of text (e.g., word, sentence, theme, paragraph) is categorized. Weber [7] defines steps to develop the coding scheme: 1) define the recording units (basic unit of text to be classified), 2) define the categories, 3) test coding on sample of text, 4) assess accuracy or reliability, 5) revise coding rules, 6) return to step 3 if necessary, 7) code all the text, and 8) assess achieved reliability and accuracy. In our case, the explicit analysis of reliability and accuracy is this paper and the analysis is in terms of the four qualitative criteria. Through the process we developed a hierarchy of issues, categories, and themes from the comments for the needs of stakeholders from DOE (Figure 1.)

\section{FIGURE 1. CONTENT ANALYSIS} HIERARCHY.

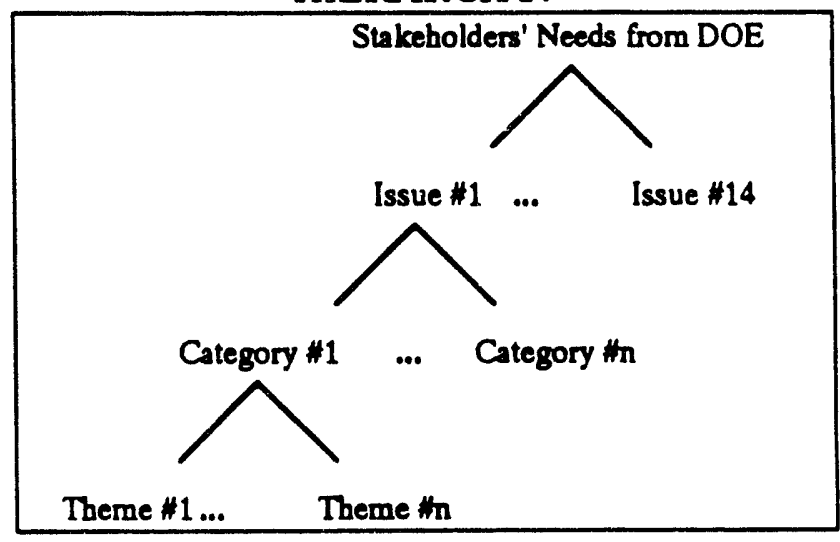

The first data analysis step was to determine the basic unit of text to be classified. A stakeholders' comment was the basic unit of text analyzed and classified. Each comment was analyzed for the theme(s) a stakeholder wanted EM to address (i.e., goals and definable actions or plans for achieving the goals). A 
comment may have contained none, one, or more than one theme.

The second step was to develop categories for each issue. All comments for a given issue were read. The comments were tagged (i.e., classifying words of the text) for each theme, if one existed, by assigning meaning to the comment. If a theme(s) was identified, the theme was added to the list of themes for that issue and given a number. The associated number was then placed next to the comment. After all comments were read for the given theme, the themes were organized into categories. The categories were developed based on similar concerns or desires. The comments were filtered by keeping only the comments relevant to the issue in the file. Comments related to other issues were eliminated. However, there are some comments common to all issues and these comments were retained. The complete text was reviewed during this step.

The third step was to verify the themes and categories. The comments were reread by the same researcher and checked for correct theme and category assignment. Any changes to theme assignment or category assignment of themes was conducted at this time. The complete text was reviewed during this step. The third step implicitly covers steps 3-8 of the process outlined by Weber [7].

The whole process was pilot tested using a stakeholder as the primary unit for dividing the database of comments. All the comments made by a stakeholder were read independent of any issue. It was difficult to develop a coherent set of themes and categories for a given issue using this approach. Therefore, the process was conducted with the issue being the primary data element to divide the comments on.
The fourth step to data analysis was to count the number of stakeholders in a stakeholder group who commented on a given sub-issue. If a stakeholder made more than one comment in a category, then the stakeholder was counted only once on that given category or sub-issue. This count provided a frequency to use in determining the "significant" sub-issues of an issue.

\section{Data Summary}

The data was summarized by producing a "paper" on each issue. The issue paper contains a description of the issue and the subissues (i.e., category). The sub-issues are grouped in order of their relative importance to the stakeholders based on the frequency of stakeholders commenting on the sub-issue.

\section{CRITERIA AND CONTENT ANALYSTS}

The four criteria of credibility, transferability, dependability, and confirmability are used to assess the preceding content analysis process. This assessment explicates step 8 of the Weber coding scheme process.

Seven activities were given to enhance the credibility of the results. The first three activities, prolonged engagement, persistent observation, and triangulation, were completed by reviewing the Lexis/Nexis newspapers, strategic plans of DOE, and a PEIS report and talking with people who have had direct contact with the stakeholders. The results from the study were in alignment with the documents and the opinions of those with direct exposure with the stakeholders. Peer debriefing and member review of the research process was completed on a daily basis. There were three specific meetings with a research scientist to verify the approach we were taking. Finally, the data has been archived for verification. 
Transferability was enhanced by providing in the final report a description of the process and source for the data. The original database can be acquired from the Department of Energy. The comments used in the content analysis are on an electronic, indexed file. The analysis was split into fourteen issues by stakeholder groups, that is, each issue was further divided and analyzed according to stakeholder group. A list of the specific stakeholders in each group was provided.

Dependability of the results was enhanced by using triangulation of sources: newspaper articles, PEIS report, and review by people familiar with stakeholders. The people familiar with stakeholders have been involved in formal government-stakeholder interactions. Dependability was also increased by having each of the three primary researchers review separate issues. Comments and themes were common across issues because the original data set classified each comment according to multiple issues. The interpretations of the same comments across issues were confirmed by daily meetings of the researchers. A formal audit process was not conducted. However, the daily meetings of the researchers did serve as an informal audit process.

Confirmability was enhanced by all of the above interventions made by the researchers. The review of the research process with the research scientist and daily meetings added to meeting this criteria. The notes and decisions about the research process were captured. A formal notebook was not used.

\section{LESSONS LEARNED}

The overall research process could have been improved by:

- Increasing transferability by describing the stakeholders, DOE sites, and relationship between a stakeholder and site.
- Increasing transferability by delaying data aggregation. The frequency counts were made by stakeholder group and not to a specific stakeholder. Also, the connection between a given stakeholder group and DOE site would increase the transferability. We could have started with the most basic data elements and then aggregated up.

- Increasing credibility by collecting and storing the original themes to categories sheets. These sheets would have made the rules for converting the comments to issues explicit.

- Increasing dependability and confirmability by implementing a formal audit process with a reflexive and methodological log. We did keep track of methodological issues with agendas and minutes to meetings discussing the methodology.

The process did these things well:

- Dependability was increased by each researcher reviewing all comments associated with an issue, even thought comments did cross many issues. The overlapping themes and categories were discussed by the researchers.

- Credibility was increased by classifying themes into higher-level categories. These categories were developed by reviewing the comments before the assignment of a comment theme to a category.

- Credibility, dependability, and confirmability were increased by triangulating with Lexis/Nexis searches, stakeholder group information, and PEIS published report.

- Credibility was increased by using purposive sampling because these groups represented the most vocal. These groups may not represent the general public but are the ones managers most often try to satisfy. 


\section{CONCLUSIONS--SUGGESTIONS TO MANAGERS}

This paper presented a content analysis process for managers to use in producing and evaluating qualitative information. Four qualitative criteria and specific actions to meet these criteria were presented and applied to the content analysis process.

From this study, suggestions are to made to managers on how to implement the concepts presented in this paper to their daily activities:

- Use multiple sources for data and information. The double analysis of comments across issues does add time but allows multiple interpretations into the process.

- Use a matrix of stakeholder group versus issues with the cell being the specific theme or desires on the issue. The matrix will allow a manager to see the trends across the groups and issues, as well as the specific concerns of a stakeholder.

\section{REFERENCES}

[1] Lincoln, Y. S. and Guba, E. G., Naturalistic Inquiry. (Newbury Park, California: Sage Publications, Inc., 1985).

[2] Galbraith, J., Designing Complex Organizations. (Reading, Massachusetts: Addison-Wesley Publishing Company, 1973) .

[3] Huber, G. P. (1991). Organizational learning: The contributing processes and the literatures. Organization Science, 2(1), 88-115.

[4] Brinberg, D. and McGrath, J. E., Validity and the Research Process. (Newbury Park, California: Sage Publications, Inc., 1985).

[5] McKinnon, S. M. and Bruns, W. J., The Information Mosaic. (Boston: Harvard Business School Press, 1992).
[6] Hill, D. T., Kurstedt, H. A., Koelling, C. P., Doss, A. R., and Cavel, M.S. "Measuring Information -Oriented Performance."

Proceedings of the 25th Annual Meeting,

Southeastern Chapter of the Institute of Management Sciences, (October 1989), pp. 253-256.

[7] Weber, R. P., Basic Content Analysis. (Beverly Hills, California: Sage Publications, Inc., 1985).

[8] The United States Department of Energy, Programmatic Environmental Impacts

Statement for the Department of Energy

Environmental Restoration and Waste Management Program. (Washington, D. C.:

Department of Energy, 1992).

\section{ACKNOWLEDGMENTS}

The preparation of this paper is funded by U.S. Department of Energy (DOE) Grant Nc. DEFG02-88DP48058. Management Systems Laboratories thanks the DOE's Office of Environmental Restoration and Waste Management for providing us a real-world laboratory for the research, development, and testing of state-of-the-art management tools and the frameworks for understanding how to make them successful. 


\title{
MANAGEMENT SYSTEMS ENGINEERING: THE ENGINEERING APPROACH TO QUALITY
}

\author{
by \\ Harold A. Kurstedt, Jr. \\ Virginia Tech
}

\begin{abstract}
Management systems engineering applies the engineering process to the management process under the purview of the systems approach. The recipient of that application is an organization of one or more people making decisions about a set of responsibilities. Management systems engineering is a structured approach for management and for building and using management tools, which convert data into information to support decision making. Management systems engineering focuses on closed-loop engineering and management processes and views the organization from a broad enough perspective to get the most out of the systems approach. Applying the engineering process requires knowledge of fundamentals that mix methodology, structure, and science with human interaction, dignity, and meaning. Through engineering constructs, a number of analogies and models provide relationships for principles of good management. Management tools support the decision maker as he or she works within the nine functions of the management process, which corresponds to the Plan-Do-Study-Act Cycle for continuous improvement. The interfaces of the Management System Model lead to the enablers of quality, which are together used as a guide for providing quality leadership through measurement and trust.
\end{abstract}

\section{INTRODUCTION}

"Quality is the inevitable result of good management." W. Edwards Deming (1900-1993)

In management systems engineering, we deal with good management and its inevitable consequence by applying the engineering process to a domain of responsibility I call a management system. To scope a discipline around which we've built an academic option within industrial engineering and a supporting research laboratory employing 200 people, I had to renew my understanding of engineering, the engineering process, a system, the systems approach, management, and the management process. I've had to understand these concepts in a way so I could apply engineering principles to managing organizations.

Management systems engineering is concerned with researching, designing, building, operating, and improving a management system, and draws upon knowledge of the natural laws of the physical, social, and life sciences. A management system comprises any person or group of people making decisions about and taking action on a set of responsibilities, the work process for meeting those responsibilities, and the management tools for converting. data from measurements of the work process into information for decision making. Management systems engineering applies the fundamentals of the engineering process, which centers on design, to the management process, which centers on decision making and leads to continuous improvement. Management systems engineering integrates principles of human interaction with principles of processes, problem solving, and systems under the purview of the systems approach. 
Much of our effort in understanding management systems engineering will focus on understanding and integrating concepts in addition to engineering, systems, and management, like natural laws, principles, tools, work process, decision making, measurement, data, information, knowledge. processes, systems approach, problem solving, motivation, empowerment, trust, and responsibilities with enough depth and precision that we can understand the interplay of these concepts within the framework of a way of thinking. The integration of these concepts into an approach to the world makes management systems engineering unique.

People can delimit their responsibilities into domains, some simple and others more complex, involving one person or many people. I consider any organization or subunit to be a domain of responsibility, which I approach as a management system. The organization is a person-made system, like a bridge or a turbine. We can apply the engineering process to the organization, or management system, which is a domain of responsibility. Like all person-made systems, the management system converts inputs into outputs resulting in throughput to meet a common aim with measures of performance to see how well we meet the aim.

I believe management systems engineering is either a new discipline or the rediscovery of the origins of an old discipline. The updating of the old discipline factors in what we're learning about motivation and empowerment and about measurement and information to produce quality, continuous improvement, and leaming systems, especially learning organizations. The question is whether the new discipline should stand alone or should influence other disciplines to broaden their scope to include challenges from ongoing societal change. I prefer to think management systems engineering should influence the traditional disciplines in many professions, including engineering, to blend the quantitative with the qualitative. Management systems engineering has the advantage of looking anew at the fundamentals of engineering, management, and systems to discover new connections and opportunities to serve humankind.

I'll outline the philosophy and concepts that make up management systems engineering. I've chosen a bottom-up approach for assembling the concepts of management systems engineering and how they affect quality. I'll start with the concepts of engineering and its associated application system. Then, I'll describe a system and management before I define management systems engineering. With an understanding of management, system, and engineering. I'll focus on a management system and the engineering and management processes for producing a successful management system and for achieving quality.

I've found that researching the basic philosophies and concepts of engineering, the engineering process, management, the management process, and systems has caused me to look at them anew and to focus on their relationships and differences. An advantage of studying these relationships is the effect they have on organizational culture, quality, and changing to a quality culture. Through the systematic understanding of good management, I find an understanding of quality. The documentation of this research is exceedingly long. To meet the constraints of this paper, I'll be curt to excess.

\section{ENGINEERING}

Engineering has been defined as "... the profession in which a knowledge of the mathematical and natural sciences gained by study, experience, and practice is applied with judgment to develop ways to utilize, economically, the materials and forces of nature for the benefit of mankind." [1] For my purposes, I'm oriented toward the engineering process rather than the engineering profession and the 
genderless user of the application system rather than mankind. The application system is the object of the engineering process. Engineering centers on design, which is a closed-loop process. The design process constitutes a function in the engineering process, which is also a closed-loop process. To do the design process you must do activities that come before and come after the layout of related components for the design.

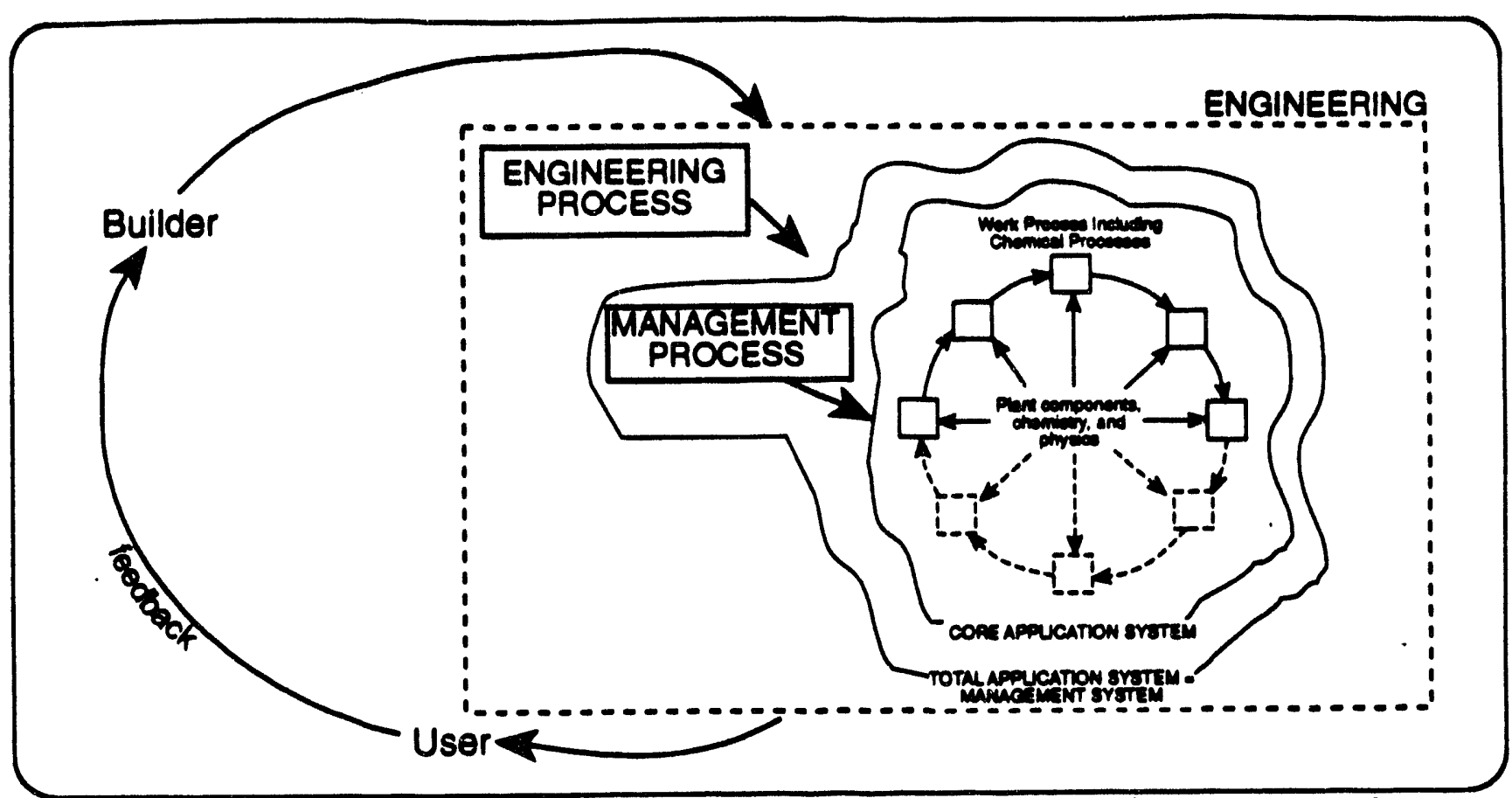

Figure 1. Chemical engineering applies the engineering process to an application system that includes a work process with chemical processes.

As shown in Figure 1, in engineering, we apply the engineering process to an application system and its associated work process, which should be a closed-loop process. Figure 1 implies that the engineer is a problem solver and builder who practices engineering for the benefit of a user. The engineering process connects the builder to the user's application, which is designed to serve the user. The engineering process succeeds because of the feedback loop from the user to the builder. Figure 1 emphasizes the roles of both the engineering process and the application system in engineering and the overlay of the management process on the core application system. When we apply the engineering process to the total application system, we're applying the engineering process to the management system. The management system includes the management process as it overlays the work process, or the core application system.

\section{THE APPLICATION SYSTEM}

Here's a key issue. In Figure 1, I separate the engineering process from that which is designed or the thing we apply the engineering process to. For example, I separate the engineering process from the bridge we engineer. My purpose is to focus on the engineering process and its fundamentals and how that process and those fundamentals apply to a particular application system; e.g., the bridge.

When we practice engineering, how much time do we devote to understanding the bridge and the natural laws and principles that govern the bridge's operation and how much time do we devote to understanding the engineering process? Which do we truly understand, the bridge or the engineering process? By applying the engineering process to an organization, which is a total application system 
governed by social science as well as physical science natural laws, I've had to renew my understanding of engineering, the engineering process, and the fundamentals of the engineering process. I've found that to do engineering well, we need to pay as much attention to the engineering process and its fundamentals as we do the application system and its natural laws and principles.

In Figure 1, I assume the application system is a chemical plant, with its particular structure and aim and the associated natural laws, science, and math. The work process associated with the chemical plant largely involves chemical processes. I call the plant and its work process the core application system. I call the core application system together with the overlying management process for the work process the total application system. The total application system serves the user. We apply the engineering process to the core application system, the management process, or both-the total application system.

In traditional engineering, we focus on applying the engineering process to the core application system-in the figure, the chemical plant or its operations (functions) within the work process. We tend to ignore the management process that overlays the work process. Often, we focus so much on the application system, we even forget the engineering process.

In management systems engineering, we must focus on both the engineering process and the management process, while having to understand the application system being governed by the management process and knowing how the application system follows the natural laws and principles of its work process. Management systems engineering requires a larger frame-the understanding of the natural laws of social science and life science as well as physical science. The larger frame conteins the engineering process and the total application system, where the total application system contains the core application system (in Figure 1, that of the chemical engineer) and the management process and the interactions between the two.

As we'll discover when I describe a management system, the total application system shown in Figure 1 is the management system, or our domain of responsibility, which includes the physical responsibilities, or work process, and the decision maker and the management tools for converting data about the work process into information to support decision making. The work process is the process associated with the core application system in Figure 1. The decision maker and management tools are associated with the management process in Figure 1. The core application system is what is managed when we do the management process.

We engineer a management system like we do a chemical system (or a mechanical or electrical system). We must have profound knowledge of the application system-both the work process and the management process, which are what we engineer. In Figure 1, a chemical engineer must understand chemistry, chemical processes, and the tools and facilities for operating the chemical processes-all part of the work process in the application system. A management systems engineer must understand this work process enough so his or her understanding of the management process and the tools for managing the work process will meet the needs of the user. Together, as supported by the appropriate natural laws (based heavily on social science) and principles, the objects of this understanding make up the application system of the management systems engineer.

I worry that we spend more effort on what we engineer than we do on how we engineer. A successful chemical engineer must understand both the chemical process and the engineering process.

Similarly, a successful management systems engineer must understand both the management process and the engineering process. But, the management systems engineer must understand the chemical 
process (or whatever core application system) enough so he or she can walk the workplace and see management principles at work in that particular setting.

\section{A SYSTEM}

When considering an organization to be a management system, the management systems engineer distinguishes between and blends the concepts of a system as a thing and a system as meaning. Blanchard and Fabrycky [2] describe a system as "composed of components, attributes, and relationships"- therefore, a thing. Weinberg [3] describes a system as "a point of view"-therefore, meaning. I accept the complementary notions of two radically different definitions for a system. I use the word system when thinking of a system as a thing. I use the words systems approach when thinking of a system as a point of view. I can diagram a system as a thing using the Management System Model [4] with its components and relationships shown at the top of Figure 2. A system as meaning is exemplified by the systems approach, which I'll describe later.

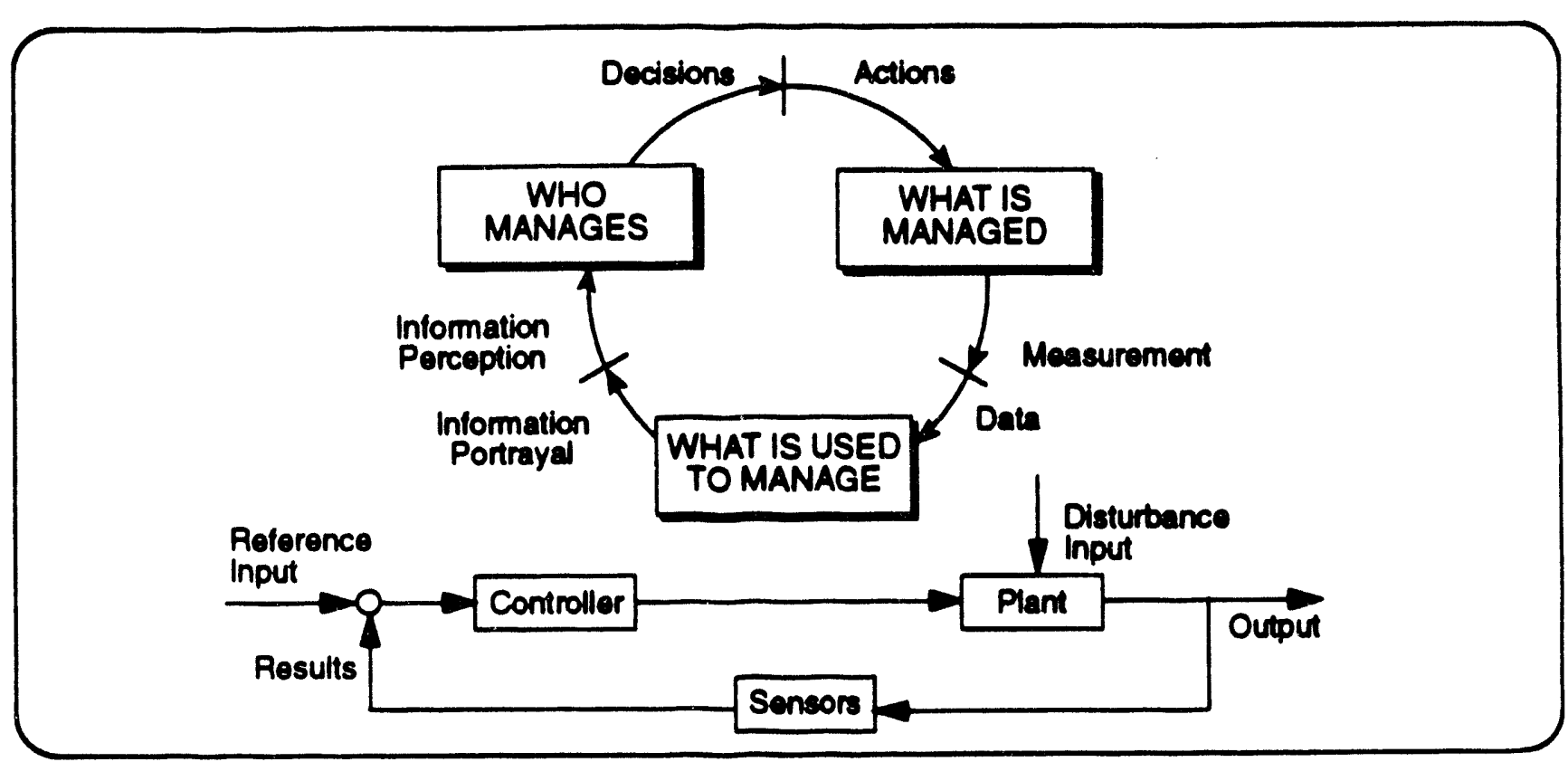

Figure 2. The control loop analog helps make the Management System Model predictive.

\section{MANAGEMENT}

For applying the engineering process to the management process, I use Simon's [5] and Forrester's [6] definition of "management as being synonymous with decision making." [italics added] That's it! Management equals decision making, nothing more, nothing less. Therefore, any person does management whenever he or she makes a decision. This definition of management makes the act of decision making the behavior that embodies management-an act we can model and design into a management system.

As design is a closed-loop process within the engineering process, decision making is a closed-loop process within the management process. To make a decision, you must do activities that come before and come after the choice of alternatives for the decision.

Managers make decisions within any of a number of roles described by people like Mintzberg [7], including leadership, negotiator, and resource allocater. Therefore, comparing management and 
leadership is like comparing apples and oranges. All people manage when they make decisions affecting their domain of responsibility. When people make decisions about vision, empowerment, and other leadership concepts, they're managing within their leadership role. Neither position nor supervisory responsibility is the sole jurisdiction of decision making. Likewise, neither position nor supervisory responsibility is the sole jurisdiction of leadership. Anyone is a manager when they make decisions. A leader makes decisions about vision and empowerment. A supervisor makes decisions about the one or more people reporting to them. Supervision is different from management and from leadership.

The management systems engineer applies the engineering process to decision making and to the work process, which is the object of the decisions. The avenue to decision making is through the management tools decision makers use to get information based on data from the work process.

\section{MANAGEMENT SYSTEMS ENGINEERING}

Management systems engineering constitutes a different way of thinking from other engineering and management disciplines. To differentiate management systems engineering, I'll cail engineering management the study of how to manage technological enterprise, which in the business college is called management of technology. For management systems engineering, I'm suggesting the opposite approach to the interplay between engineering and management. Instead of a type of management. I'm suggesting a type of engineering. I'm suggesting we study how to engineer the management of any enterprise, which I'll call management engineering.

Perhaps management engineering says it all. However, I like to emphasize the systems nature of the organization. Those who profess quality today insist on understanding and acting on the systems nature of the organization. Therefore, I'll stick with the term management systems engineering-the engineering of systems of management.

Management systems engineering is concerned with researching, designing, building, operating, and improving a management system, and draws upon knowledge of the natural laws of the physical, social, and life sciences. A management system comprises any person or group of people making decisions about and taking action on a set of responsibilities, the work process for meeting those responsibilities, and the management tools for converting data from measurements of the work process into information for decision making. Management systems engineering applies the fundamentals of the engineering process, which centers on design, to the management process, which centers on decision making and leads to continuous improvement. Management systems engineering integrates principles of ruman interaction with principles of processes, problem solving, and systems under the purview of the systems approach.

Management systems engineering is an engineering discipline, where the engineering process centers on design and involves fundamentals often practiced outside traditional engineering disciplines. This new discipline requires an approach to the world, or a way of thinking, that goes beyond problem solving, quantification, and physical science into human feelings and interactions, the immeasurable, and social science. Deming would call the immeasurable the unknown and the unknowable, which tum out to be the important things in management.

We're comfortable with the idea of engineering a chemical system, or process. Now, I want us to become comfortable with engineering a management system and its associated management process. Management systems engineering is a structured approach for management and for building and 
using tools to support management. Management systems engineering focuses on closed-loop processes and draws frames large enough to get the most out of the systems approach. In management systems engineering, you must not only have profound knowledge of systems, engineering, and management you must understand how to blend the three to get the harmony that makes the mix work and the robustness that makes the mix work well.

\section{THE SYSTEMS APPROACH}

The systems approach is different from a system, but works hand-in-hand with a system. The systems approach includes at least three perspectives, or viewpoints: 1) the systern perspective, 2) the holistic perspective, and 3) the generalist perspective. I'm about to analyze the systems approach. Analyzing an holistic concept is an oxymoron, but, in this case, still informative.

In the system perspective, we emphasize the necessity of each of the components of the system and its importance in moving toward the system's aim. If a system component changes, the combination of components changes, the system isn't the same, and the person-made system can't meet its aim. In a management system, the system perspective says each person is vital to the organization in his or her role for supporting the organization's aim.

In the holistic perspective, we get a gestalt. The heart of the system rises above the individual components. In a management system, the holistic perspective says the spirit or soul of what the organization stands for drives the system. The organization is more than its components and its aim. The organization has meaning.

In the generalist perspective, mother nature is consistent. The principles and lessons learned from one system transfer to another system in as much as the two systems are similar. In a management system, the generalist perspective says we can learn from theory and experience in the organization. in other organizations, and from other systems, like mechanical and living systems. We can transfer that leaming by exercising similitude.

As a way of looking at the world, the systems approach affects behavior. The combination of the perspectives blends the culture of the organization with the need for measurement of components, relationships, and their workings. We need a learning organization that balances measurement and meaning. This type of learning organization practices continuous improvement, which leads to quality. Management systems engineering must balance cultural values, norms, and traditions with measurement of work process and management tool functions, operations, and output.

\section{A PROCESS}

A process is different from both a system and the systems approach. Classically, a process is a series of actions or operations, or functions, conducing to an end. We usually see once-through, linear processes. In management systems engineering, we focus on closed-loop processes working closely in association with corresponding management systems. The difference is that the end of a process occurs after the last function in a linear process and occurs when you stop cycling in a closed-loop process. A closed-loop process implies we never complete, only abandon, the process. However, we garner the benefits of continuous improvement, organizational learning, information-rich feedback, creativity, and empowerment. The important connection between a process and the associated system is that for person-made systems, the end the closed-loop process conduces to is the aim of the system. 
A system includes components, or things (nouns in boxes in a system diagram). A process includes steps, or functions (verbs in boxes in a flow chart). A system and a process work hand-in-hand, but there's no necessary one-to-one correspondence between a component and a function.

As systems have subsystems, processes have subprocesses. For example, the analysis process is a subprocess of the engineering process. The products and services of a management system result from an interdependent network of processes. [8] When we remove the typical cross-flow structures of supervision and information gathering (e.g., the engineering department structure and financial information), we find the fundamental work flows in an organization. When we undertake quality efforts, we focus on these work flows, or processes, which cross typical organizational boundaries. In those quality efforts, we produce cross-functional teams of people to work on continuous process improvement. Problem solving, performance improvement, and process improvement are all valuable but use different implementation approaches. Since most organizations are organized functionally rather than cross-functionally by process, which is a pity, I look at improving domains in addition to improving processes.

\section{THE ENGINEERING PROCESS}

When we approach the engineering process, we consider the word engineer to be a verb rather than a noun. The application system is the object of the verb. We always engineer a system. The question is: What kind of system are we interested in, how does it differ from other systems, and what does the difference mean in terms of natural laws and applicable science (e.g., physical, life, and social sciences)?

Figure 1 tells us 1) we have systems within systems; 2 ) the core application system specifies the structure, natural laws, science and math, and aim of the object of the engineering process; 3) the example chemical system has one or more chemical processes and, like other systems in other disciplines, can, and should, include environmental system processes; 4) the example chemical system is a component of a management system, which has a management process; and 5) you apply the engineering process to the core application system, to the management process, or to the total application system, depending on what you want to improve.

Figure 3 expands the engineering process part of Figure 1 and de-emphasizes the total application system. I've emphasized the engineering process by indicating five groups to represent the 22 functions in the engineering process framework. The framework doesn't show the fundamentals or the philosophy behind the process. We see the process here as a series of steps. Since the steps are shown as a cycle, we see that we never finish the sequence. We continually improve. We stop when we abandon the process.

Guided by fundamentals and governing natural laws and the results of basic and applied research, the engineering process aims to convert matter and energy into beneficial orientations within the application system to serve a user by supporting, joining with, and assuming the nature of the application system.

Just as the chemical process associated with the application system in Figure 1 includes a number of operations, which we can neatly categorize into functions, the engineering process in Figure 3 also includes functions. The engineering process functions are shown in Figure 3 as groups of functions. Learning the functions of a process is a necessary but not sufficient condition for becoming good at the process. To complete our understanding of these processes, we must learn about ways of think- 
ing, like the systems approach, and we must leam the rules for conducting the functions. Processes include functions and rules guiding the functions and a way of thinking oriented to the aim of the process.

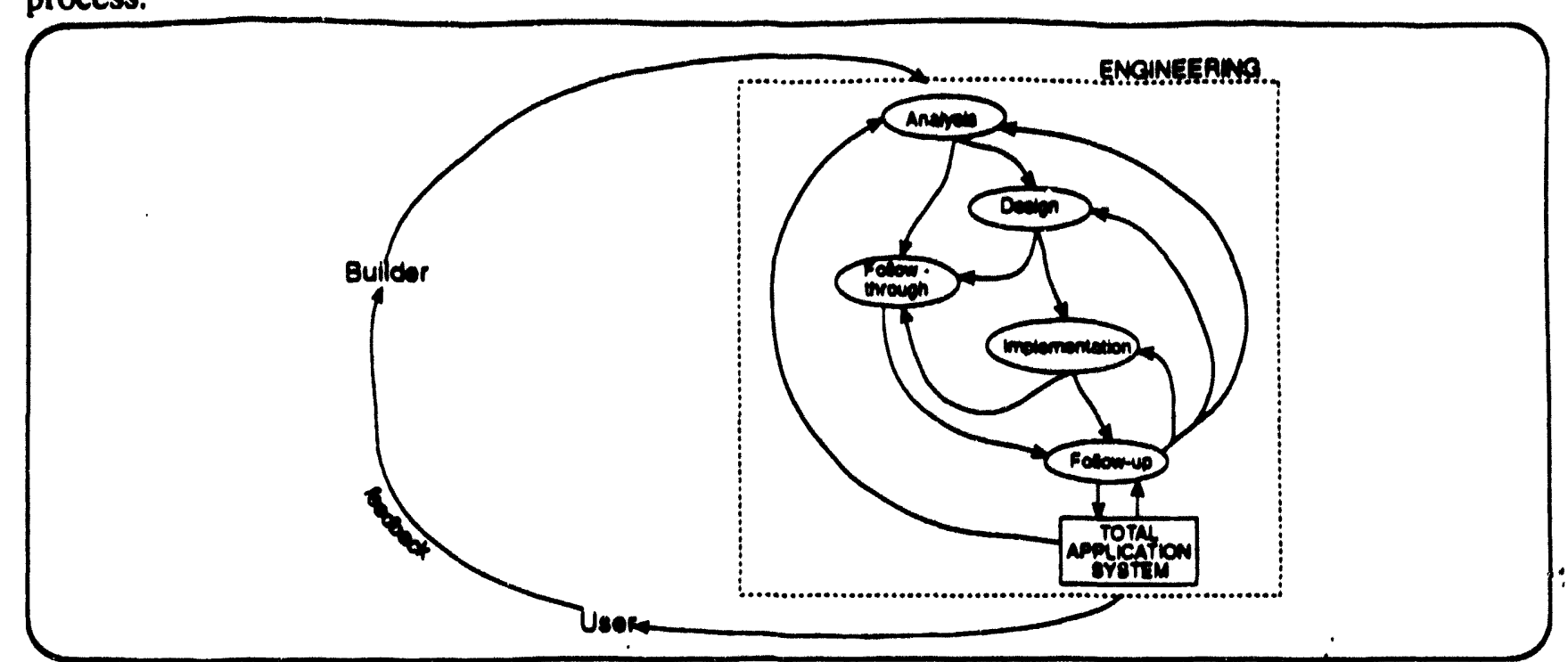

Figure 3. You never finish the engineering process, you only abandon it.

I claim there's a difference between the engineering process and the engineering profession. Many people not in the engineering profession practice the engineering process very well and many people in the engineering profession don't. Of course, I feel this situation is unfortunate. Engineers ought to be very good at the engineering process. The closed-loop design process is central to the engineering process. Many disciplines are based on processes revolving around the design process and what must precede and follow the design. Example disciplines include education, psychology, art, and dentistry. Perhaps the key to whether we practice what I call the engineering process is whether or not we understand the fundamentals of that process. To apply the engineering process to the management process, we must understand the fundamentals of both processes.

\section{ENGINEERNG PROCESS FUNDAMENTALS}

The fundamentals of the engineering process are: 1) language for communication; 2) problem solving and the scientific method; 3 ) the systems approach as a way of thinking; 4) walking the workplace (application system); 5) the system life cycle as the framework of the engineering process; 6 ) envisioning and imagination; 7) the collecting, converting, and conserving of energy; 8) knowledge of the lessons of the past; 9) the philosophy of the aims of the engineering process and the application system; 10) teamwork, professionalism, and empowerment; and 11) dedication to continuous vigorous, lifelong learning. These fundamentals provide a balance of science (physical, life, and social sciences) and art and of the technology, human, and economic subsystems of the organization.

One engineering process fundamental is devoted to the system life cycle, which is the structure, or framework, of the process; that is, the steps or functions of that process. System life cycles apply to everything, including organizations. [9] The other fundamentals are more or less increasingly more qualitative-and correspondingly more or less increasingly more powerful. The system life cycle includes 22 functions for analyzing, designing, implementing, and following-up (e.g., maintenance, restoration, and decommissioning) after organizations with their work processes and management processes and for following-through (e.g., documentation and evaluation) on the other functions. 
The 22 functions are additional detail for the five function groups of the engineering process as shown in Figure 3. In Figure 3, the total application system includes both the core application system (e.g. the chemical process, the banking process, or the manufacturing process) and the management process, which must overlay the core application system, as shown in Figure 1. The management process includes decision making, converting data into information, and working with and empowering people. The engineering and management processes show similarities because they both stem from the scientific method.

Consider the other engineering process fundamentals in the sequence I used earlier. We leam language through mathematics and English. We also leam graphics (engineering drawing) and computer languages for communication. In communication, you must be able to "write it down and write it up." When you use words (lext) or symbols (math) to think through a situation, you're "writing it down." When you use words or symbols to communicate a situation, you're "writing it up." Because you're making things better by taking action, you must know how to diagnose and solve a problem, know how to use the scientific method and design experiments, and practice and balance deductive and inductive reasoning (including strong inference). Because all applications are systems, you must know how to think in systems terms, including the system, holistic, and generalist perspectives. You must be able to walk the workplace and see the natural laws and principles at work so well you can feel the cause-and-effect relationships of the application processes. Because the engineering process requires ingenuity, innovation, and creativity, you must be able to envision and to imagine.

All application systems function by collecting, converting, and conserving some form of energy. For example, in the engineering process, you need to transfer the idea of entropy and the First Law of Thermodynamics to biological and organizational systems. You perform the functions of the engineering process better because of knowledge of lessons of the past. If you don't know the purpose. aim, or meaning of the application system, you can't improve it. Because the engineering process seldom is an individual activity, you must know and practice teamwork, professionalism, and empowerment. A successful team functions as a cooperative community exhibiting team spirit and mutual goals. Because the engineering process must be cyclic to be successful through continuous performance improvement, you must have a motivation and capability to continue the learning experience on the job and through extracurricular readings, meetings, workshops, and degree programs.

This crisp summary of the eleven engineering process fundamentals shows the breadth of abilities needed by the management systems engineer and all those who design solutions for the benefit of a user. These abilities must be blended to get the full power of each fundamental. Taken together, the engineering process fundamentals mix methodology, structure, and science with human interaction, dignity, and meaning. We won't truly help a user until we mix these ideas well.

\section{THE MANAGEMENT SYSTEM}

The upper part of Figure 2 shows a diagram for the management system as a thing. The Management System Model includes the system components and their relationships. The model doesn't show the attributes of the components or the interactions of the system with its environment. I'll discuss the issue of the closed-system perspective of The Management System Model when I discuss the control loop analogy. I'll discuss component attributes now.

One component of the management system (who manages in Figure 2) is the person who makes 

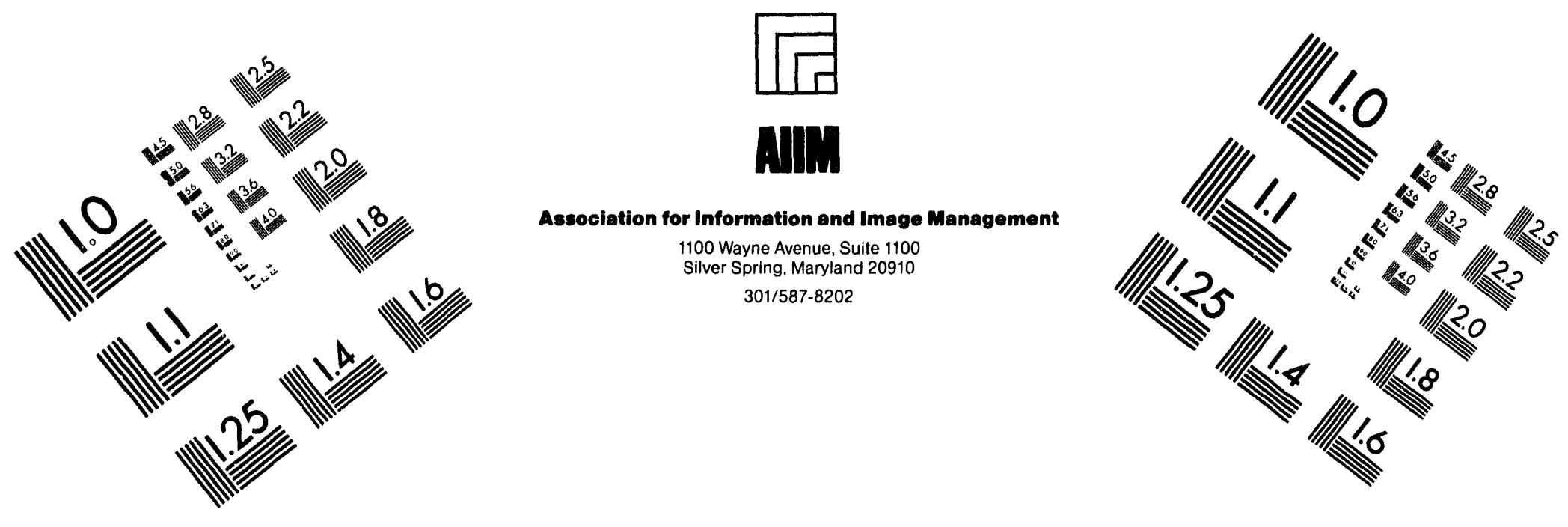

\section{Centimeter}

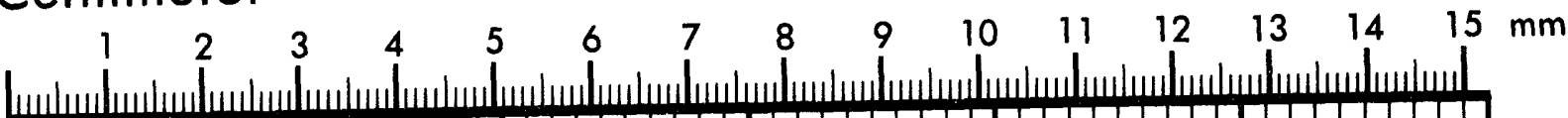

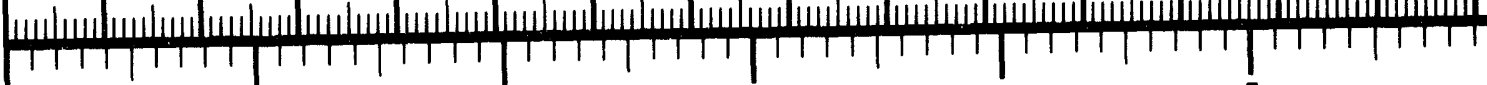

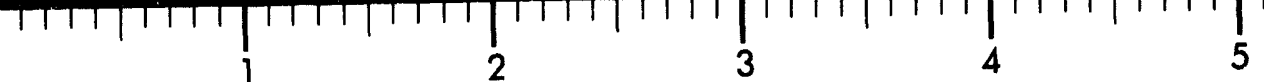

Inches
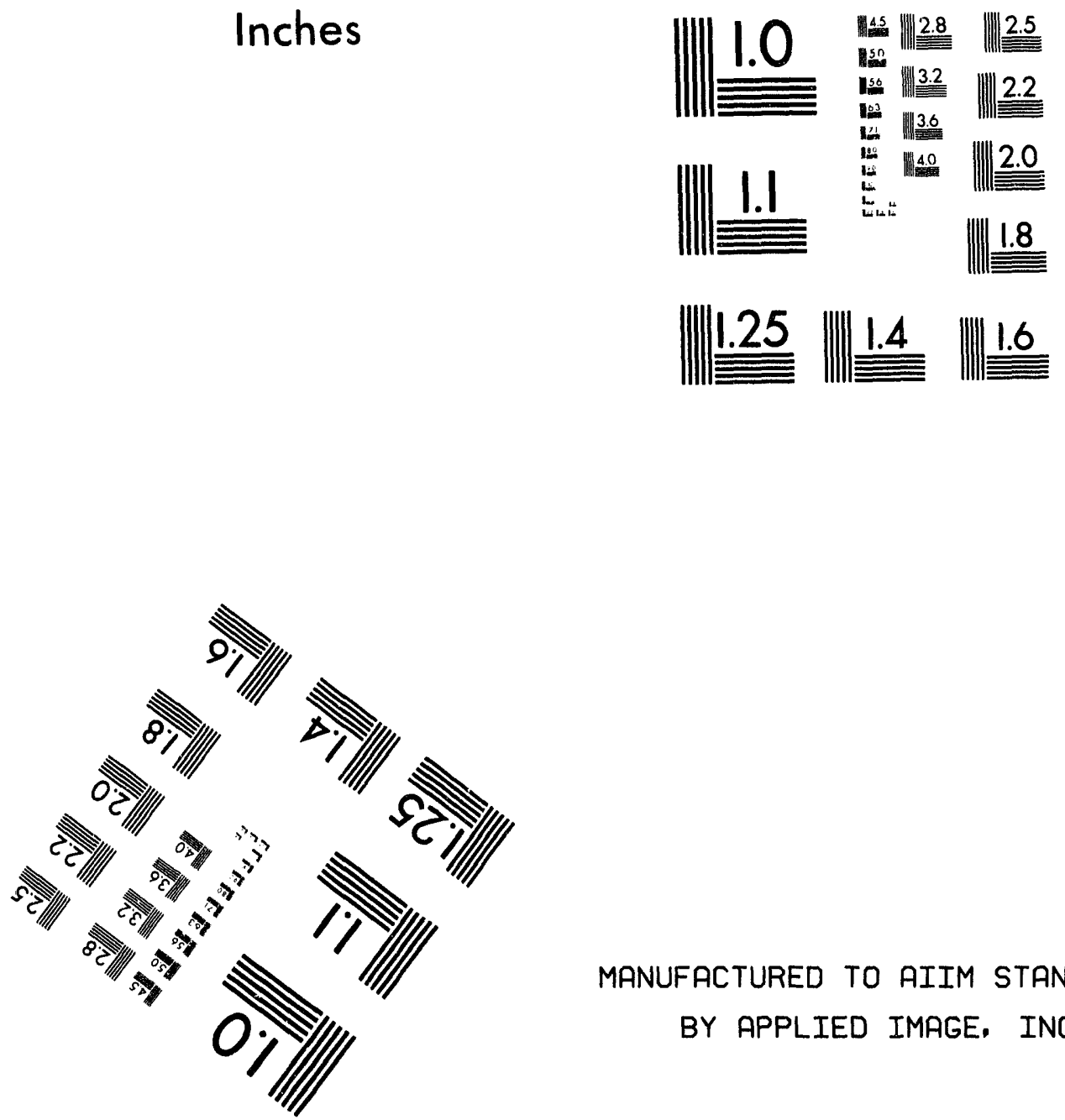

MANUFACTURED TO AIIM STANDARDS

BY APPLIED IMAGE, INC.

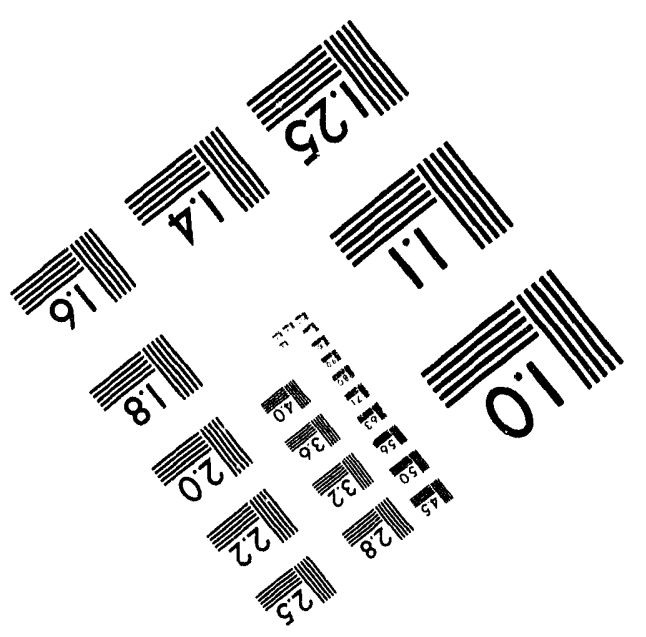



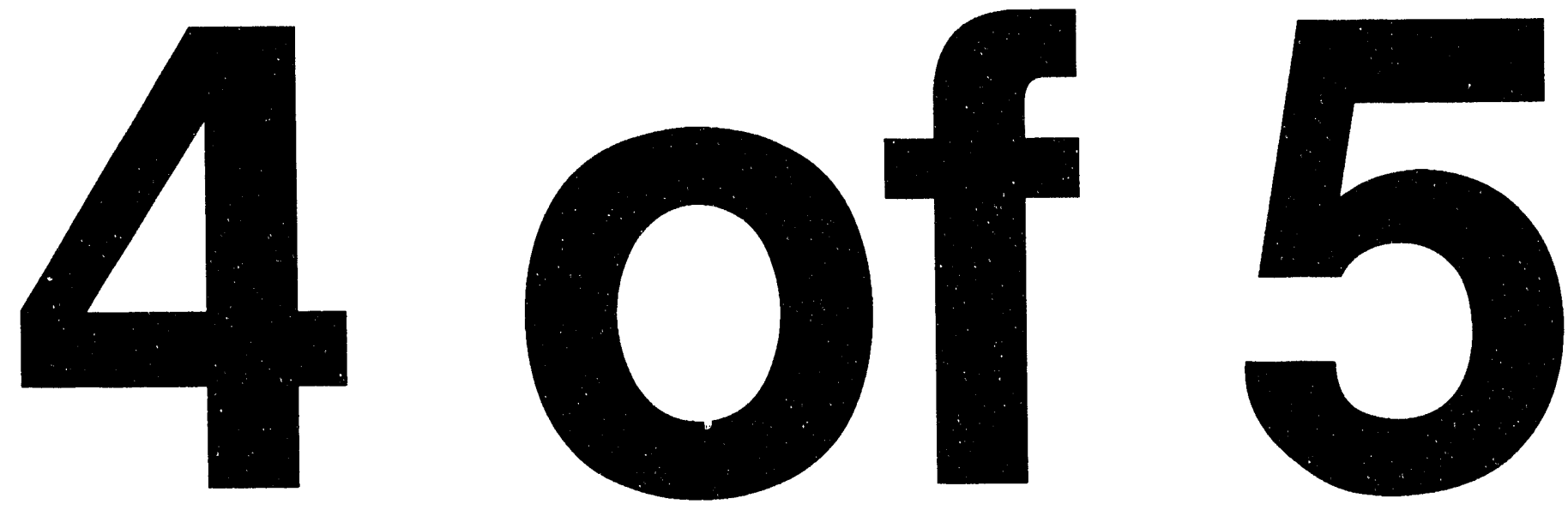
decisions that should lead to actions [10] affecting the work process (what is managed in Figure 2) of the management system. But, as we learn from Forrester [11], the manager needs information to make decisions. The information-providing component is the management tools (what is used to manage in Figure 2). A danger in analyzing a system is taking the components-who manages, what is managed, and what is used to manage-that in reality are mixed within the system and separating them apart for study, observation, and explanation. The components are one within the system.

Consider a classroom as a domain of responsibility, or a management system. The who manages is the professor. The what is managed includes students, the room, books, and other physical responsibilities. The what is used to manage is the data and information in the grades, homework, rolls, and other information-oriented tools.

In Figure 2, the decision maker converts information into action, the management tools convert data into information, and the work process converts action and resources into products and services and data about the work process and associated products and services. We get the data by measuring the work process functions and the results of the actions the manager takes.

The attributes not shown in the figure include 1) the capital, labor, equipment, materials, and energy resources in, the functions within, and the products and services out of the work process; 2) the ability, experience, and cognitive style of the decision maker; and 3) the accuracy, timeliness, and relevance characteristics of the management tools. The physical input of resources and output of products and services aren't part of a closed-system model for an organization. I'll discuss the limitations of a closed-system model for an organization, which is an open system, and extensions of the Management System Model into the control loop analogy shown at the bottom of Figure 2 when I discuss the control loop. Shortly, I'll discuss the strengths of the simple, robust closed-system model.

The arrows in the Management System Model show sequence, or relationships, not flow. The arrows in the model show 1) the who manages component uses information and makes decisions resulting in actions affecting the what is managed component; 2 ) the what is managed component is measured to yield the data provided to the what is used to manage component; and 3) the what is used to manage component comprises tools we could keep in our head, like organizational structures, plans, and information systems and portrays information to be perceived by the who manages component. The Management System Model explicitly separates the means of the manager, or what is used to manage, from the ends of the manager, or what is managed. A common source of management tool failure is emphasizing what is used to manage as the ends rather than the means. The Management System Model implies the focus of management attention is the tangible operation, or work process, that constitutes what is managed.

The Management System Model illustrates many important management principles, including: 1) management is decision making; 2) the decision maker converts information into decisions; 3 ) decisions are valueless unless they result in appropriate actions; 4) actions cause your operation to change; 5) you must know what to measure before you get useful data; 6) all management tools convert data into information; 7) when you portray information, you must consider how the information will be perceived; and many more.

\section{STRENGTHS AND WEAKNESSES OF THE MANAGEMENT SYSTEM MODEL}

A successful domain of responsibility balances the three components in the Management System 
Model. Without balance, you and your domain fail. I've found that management tools fail when they're not in balance with (don't fit) [12] who manages and what is used to manage. Conversely, those tools that succeed do achieve the required balance. If a management tool is too sophisticated for a manager, the balance is lost. If a management tool doesn't reflect the operation, the balance is lost. If the manager doesn't provide the right intervention to the work process, the balance is lost. A system out of balance is out of control. The question is: How do we identify, achieve, and maintain balance among the components of a management system? The answer is to focus on the interfaces between pairs of components in the model: the decision-to-action, the measurement-to-data, and the information-portrayal-to-information-perception interfaces.

The Management System Model meets two of the three goals of a model: simplicity, accuracy, and generality. The model is simple and general. The Management System Model lacks accuracy in that it doesn't include a representation of the environment around the domain of responsibility. Since organizations are affected by their environment, the model is a closed-system look at an open system. We use the Management System Model to look at balance or equilibrium and the strength of the system components-much like we use the simple and general but inaccurate sum of the forces equal to zero in statics. The model is good for what it does, but it contains an approximation that makes the model inaccurate for some considerations. In mechanics, when we want to see the effects of the environment on changing an object over time, we must look to dynamics where the sum of the forces aren't equal to zero. The management system model gives us a snapshot of the organization, not a movie.

\section{THE CONTROL LOOP ANALOGY}

The control loop representation at the bottom of Figure 2 is an open-system model [4]. I place the control loop together with the Management System Model in Figure 2 to show their analogous components and relationships. The controller in the control loop is the who manages in the Management System Model, the plant is what is managed, and the sensors are what is used to manage; the interfaces are intact.

We need a repertoire of simple models, each contributing to our understanding of good management. The Management System Model is a descriptive model. The control loop is predictive. The difference between the models is that the control loop shows the interactions with the environment as inputs (reference points), disturbances, and outputs. Closed-system models highlight balance and strength of components. Open-system models highlight environmental effects and change. The difference between the Management System Model and the control loop analogy is similar to the difference between statics and dynamics in mechanics. Since all models are approximations based on assumptions, the right model is the one where the assumptions apply.

The analogy between the Management System Model and the control loop can be explored further. When something goes wrong and a manager is unable to control the operation as desired, either the manager is replaced, the organization changes, or both. That corresponds to either replacing the controller or facing an unstable system. Another altemative is to change goals, but that corresponds to changing the inputs, the desired system behavior. Similarly, changing a manager's attitudes is equivalent to tuning the controller, and improving the tools is like calibrating the sensors.

The control loop stands on its own in modelling the organization. The Management System Model serves its purpose by giving us confidence that the analogy works. Now we have two different models, each with its strengths. One strength of the control loop analogy is that by imbedding other 
control loops in series or in parallel within the plant component of the control loop at the bottom of Figure 2, we can study information sharing and consensus. Communication, information sharing, consensus, and conflict are crucial in understanding the working of cross-functional teams, so important in continuous process improvement.

\section{OTHER ENGINERING ANALOGIES}

The control loop is one of many analogies from physical science and engineering principles we can use as a starting point for testing similarity and provoking insight into connections among management principles. Compare Lewin's force field analysis [13] to free body diagrams in mechanics. Organizations experience force, work, energy, friction, momentum, inertia, and culture strength. For example, we can compare culture strength to strength of materials, where members change as a result of forces. When the force is removed, the member may return to its original configuration. Or, the force may cause the member to yield or fail, depending on the strength of the material. When a force is applied to an organization, the organization experiences stress. Stress generates a corresponding deformation or change in the organization. Depending on the type of force and the way the forces are loaded on the organization, the organization may recover fully, recover in a changed state, or fail. We can define culture strength as "Culture strength is a property of an organization's cuiture as defined by the forces acting on the organization, the effects on the organization from the forces, the organization's values, and the relationships among the forces, the effects, and the organization's values." [14] The strength of materials analogy leads us to a new definition of organizational culture-a definition more conducive to measurement.

Morris indicates that analogies are good starting points for new models. [15] He says that analogies between the problem at hand and some previously well-developed logical structure help us make intuitive leaps. Analogies are effective when dealing with an emerging science in that they stimulate premises to be proven or disproven through further study. Engineering analogies help us with management systems engineering and management, which are emerging science.

\section{MANAGEMENT TOOLS}

Management tools include 1) relationships and structures, like the organization structure; 2) methods. like operations research methods and expert systems; 3) guides and rules, like policies and procedures; 4) precedents, like culture management tools; and 5) the data-to-information chain, which is the regular and frequent accessing, storing, retrieving, and analyzing data in preparation for making information.

Management tools convert data into information. Data and information aren't the same. (Note for reference later that information isn't knowledge.) A datum contains fact plus meaning. [16] The number 72 is a fact. Degrees Fahrenheit provides meaning. Then, 72 degrees Fahrenheit is a datum. You can't make a decision based on 72 degrees Fahrenheit, the datum. You need information to make a decision. I prefer a 68 degree Fahrenheit environment and have therefore added another datum, a reference datum representing the voice of me, the customer. [17] The 72 degree Fahrenheit environment is an indicator datum representing the voice of the process. The information I can use to make decisions with is that it's four degrees too hot. The decision is to turn on the air conditioner. I've compared the indicator datum to the reference datum, or matched the voice of the process to the voice of the customer, to make information for decision making. Aligning the voice of the process and the voice of the customer is the focus of our total quality management efforts. 
View the Management System Model from three orientations. My laboratory views the organization from the perspective of the what is used to manage component in terms of how the management tools fit the manager and reflect the work process. We focus on information-oriented performancehow well the tools work. Most productivity centers view the organization from the perspective of the what is managed component in terms of how well the work process performs in converting inputs into outputs. They focus on operational performance-how well the work process works. I believe business schools consider organizations from the perspective of the who manages component in terms of how the manager does his or her job. They focus on personal, or professional, performance. Ultimately, we want to improve organizational performance, which represents a holistic combination of the other three orientations. In management systems engineering, we want to work toward organizational performance.

\section{THE MANAGEMENT PROCESS}

Consider Figure 4. In the management process, we apply interventions to organizations (models) to get performance (criteria sets). We feedback our measurement of performance to make better interventions. Decision makers internally apply interventions to the work process. Their organizational model represents the core application system shown in Figure 1. Consultants externally apply interventions to organizations. Their organizational model represents the total application system shown in Figure 1. For example, the consultant could use the Management System Model or the control loop shown in Figure 2 to represent the total application system in the center box in Figure 4. We can represent the organization or the performance criteria through many different models. No model represents the organization perfectly. Each model focuses on some aspect of the organization and its associated components and processes. Much of what is written in management relates to developing or using one or more of these kinds of models or the procedures coming out of the models. The performance models represent either operational or organizational performance, depending on whether the work process, what is managed, or the domain of responsibility, the Management System Model, is in the center box, respectively. In the first case, I'm looking at Figure 4 like the who manages would. In the second, I'm looking at Figure 4 like a consultant would.

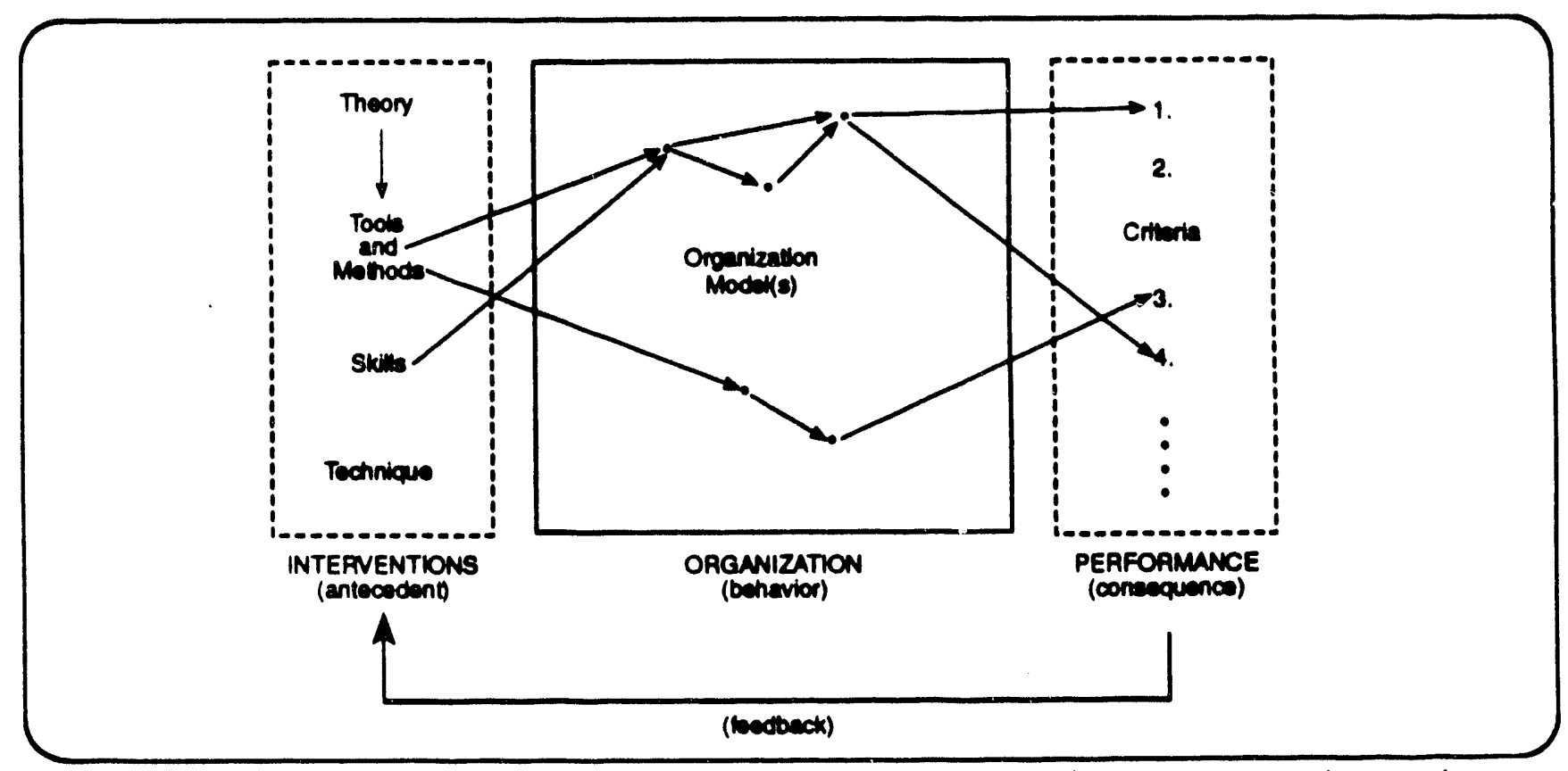

Figure 4. View the management process as a traditional system model with interventions as input and data on performance as output. 
The framework for the management process represents one organizational model for the center box in Figure 4. This framework includes 14 functions for building and using management tools. The five management process functions for building management tools, in sequence, are: delimit your domain, determine decisions and actions, define information for decisions, outline data for information, and list measurements for data. These five functions constitute what I call management system analysis. The nine management process functions for using management tools are shown, in sequence, against the Plan-Do-Study-Act (PDSA) Cycle in Figure 5. These nine functions constitute what I call management system synthesis.

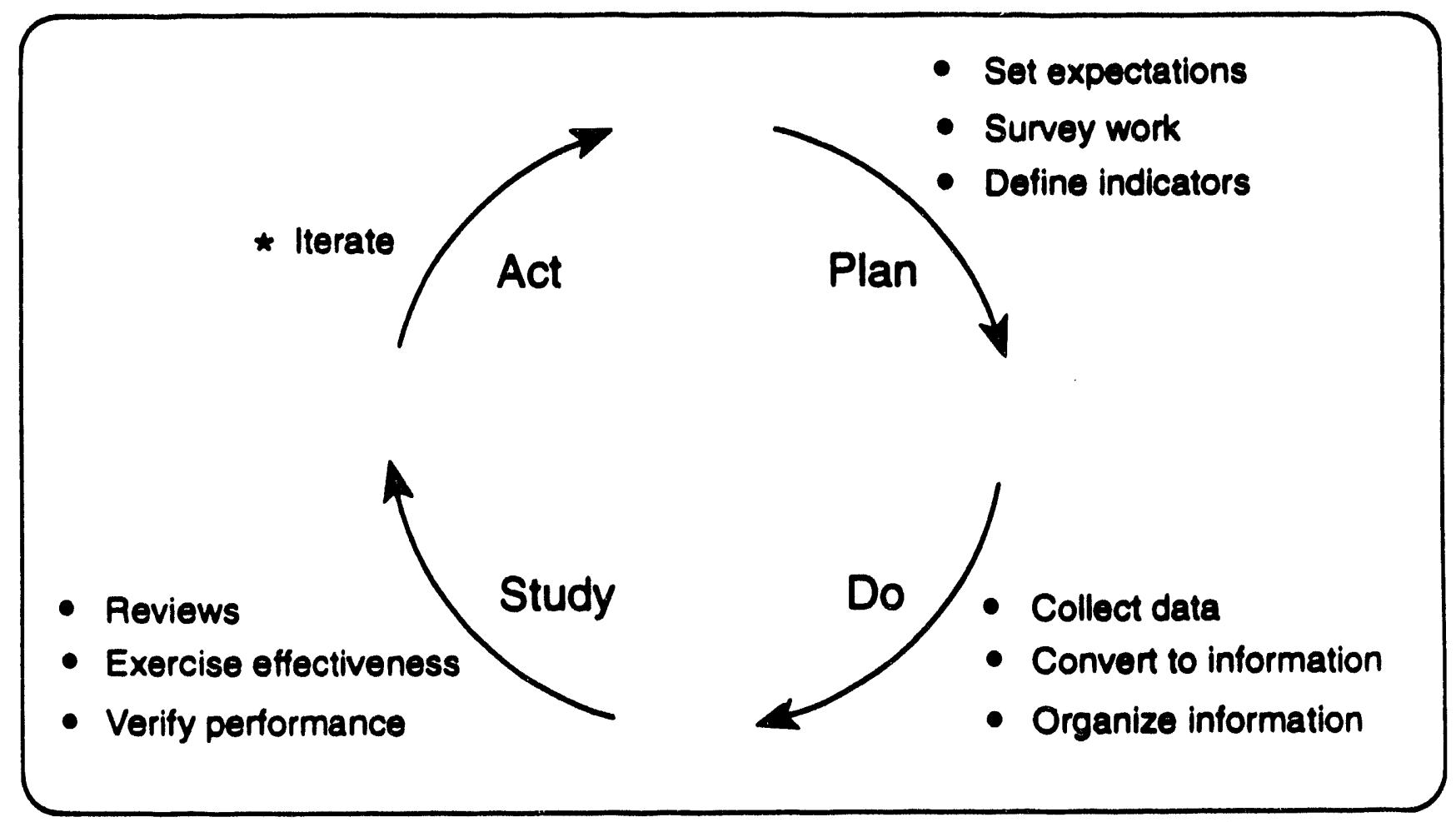

Figure 5. Functions for using management tools emphasize PDSA Cycle closure and iteration.

In analysis, we partition the whole into its parts for understanding (partition the intervention's action on the domain into the decision and action of the intervention, partition the decision into the information and alternatives for choosing, and so on). In synthesis, we gather the parts into the whole for meaning (develop measures to get data, compare data to make information, and so on). Both analysis and synthesis work together in the management process.

The sequence of functions of management system synthesis fall naturally into three groups. The first group includes three functions for planning what the manager will do with his or her operation using the management tools (decide what to do). These functions relate to the Plan part of the PDSA Cycle. The second group includes three functions for executing the plan using the management tools (do what you decided to do). These functions relate to the Do part of the PDSA Cycle. The third group includes three functions for comparing the plan with the execution to determine status and progress (see how well you did what you decided to do). These functions relate to the Study part of the PDSA Cycle.

The groups of functions, and therefore the functions themselves, are cyclic. So, after seeing how well you did what you decided to do, you start all over again by planning what to do to improve 
based on what you learned in the preceding cycle. The closing of the cycle and the implications for recycle relate to the Act part of the PDSA Cycle. In this way, you execute the cycle for continuous improvement and organizational learning by iterating through the cycle.

The nine functions provide a map for continuous improvement. The map is an embellishment of Deming's Plan-Do-Study-Act Cycle, which is an embellishment of Shewhart's Specification-Production-Inspection Cycle, which is an embellishment of the scientific method. [18] The map contributes steps for telling us how to do each part of the PDSA Cycle. Within each function, we can identify a range of tools and techniques for carrying out the function. For example, to collect data, we can tally tick marks in a check sheet, plot dots on a run chart, or use other tools. One or more of the tools and techniques will work well in a given organization. A particular tool isn't right for all applications. However, each function is necessary for all applications. For my example, you always have to collect data; but you may find that the check sheet is the right tool for this particular application but not the next application.

The PDSA Cycle of Figure 5 explains the potential for any closed-loop process. The potential is continuous improvement, learning, and information-rich feedback. I've shown closed-loop processes in Figures 1, 2, 3, and 4.

The aim of management tools working within each of the nine management system synthesis functions is to support decisions designed to take control over disturbances (mostly of our own making) and enhance the decision makers's ability to be creative in building the business and in improving the quality of work life. (See the control loop at the bottom of Figure 2.)

\section{MANA GEMENT SYSTEMS ENGINEERING IN THE FUTURE}

Imagine a management systems engineer in the future addressing a set of responsibilities that needs to be designed or that needs improvement. By asking the right questions and observing the right indicators, he or she scopes the situation and classifies the organization according to a number of parameters. The parameters characterize the organization in terms relating to management tools and their use. Based on the classification of the organization and its decision maker, the management systems engineer identifies which management tools are working well and which aren't and why. Then he or she determines how to improve existing management tools or which new management tools can be selected and which need to be built to help make the needed improvement. The management systems engineer helps select the right person to build the new tools or builds them himself or herself. The management systems engineer predicts the resulting organizational performance from using the tools and the extent of other interventions directly or indirectly affecting performance. The management systems engineer either helps find or performs training and advice to identify and teach the needed skills and techniques to get the most out of the new and continually improving set of management tools and the organization.

\section{THE CONNECTIONS TO QUALITY}

I can't define quality to everyone's satisfaction; so, I won't. What's important is defining the process by which we get quality. Deming says, "Quality is the inevitable result of good management." [19] Now, I can approach quality from the perspective of the results from good management. Even though each of us may have a different idea of what the results from good management are, I'll suggest you need three necessary but not sufficient enablers to get those results. 
As shown in Figure 6, the enablers of quality are 1) measurement and data, 2) information and knowledge, and 3) empowerment and trust. To get quality, you must integrate these enablers. You can't do measurement on Monday and trust on Tuesday and be successful. The measurement and data enabler includes the fact-based view of quality and the seven old and seven new quality tools. [20] The information and knowledge enabler includes the need for intellectual and emotional intimacy with the work place, your customers, your staff, and the theory behind variation, systems, knowledge, and psychology professed by Deming. [21] The empowerment and trust enabler includes competency in human interaction and emphasizes personal values and personal change, or self-awareness and self-renewal professed by Covey. [22] You drive fear out of an organization by bringing trust into an organization. Figure 6 also shows that tangibility increases toward the measurement and data enabler and power increases toward the empowerment and trust enabler. Figure 6 suggests a new meaning for leadership. [23]

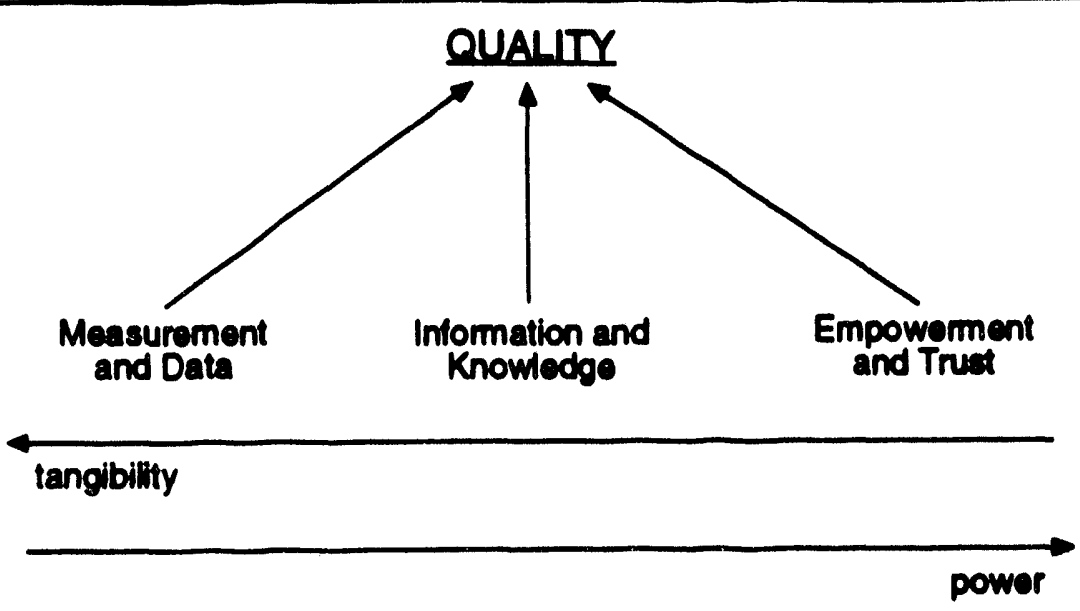

Figure 6. Each enabler is necessary but not sufficient for quality.

To implement and blend the enablers, I use the functions of the management process framework. The management process 1 ) defines what to do and how to do that, 2) spreads the tangibility of the enablers to the right, and 3) spreads the power to the left. The functions emphasize the tangible side of Figure 6. Eight rules guide the functions and draw in the principles emphasizing the integration of the powerful side of Figure 6 with the tangible side. The eight rules are: 1) focus on what you can do, 2) give physical evidence of your progress, 3) pay attention to detail, 4) practice inquisitiveness with everything you touch, 5) review progress routinely and frequently, 6) face up to your commitments squarely, 7) conduct honest and open interactions, and 8) communicate crisply.

The enablers for quality shown in Figure 6 are related to the interfaces in the Management System Model shown at the top of Figure 2. To help see the relationship, consider that Juran defines empowerment as "conferring the right to make decisions and take actions." [24] Others strengthen Juran's idea by expanding the idea of conferring the right to conferring the freedom and resources to make decisions and take actions. By working the enablers, we balance the components of the Management System Model. Through the systems approach, the organization achieves its aim.

I claim success of a management system depends on balance in the system resulting from matching the two sides of each interface of the Management System Model shown at the top of Figure 2. In Figure 6, I say that quality depends on the three enablers, which relate to the interfaces of the Management System Model. I describe balance as a matter of fit [12] and not a matter of equal emphasis. When perturbed, a balanced system will return to an equal or improved state. 
Success, balance, and quality are closely related through concepts and processes for good management. We achieve good management through processes and tools to match both sides of each Management System Model interface, thus bringing the system into balance. The management system achieves success and quality by dealing with perturbations, or change, in ways to achieve elevated states of performance to delight the customer, or user, as shown in Figures 1 and 3.

\section{TEACHING, PRACTICING, AND RESEARCHING MANAGEMENT SYSTEMS ENGINEERNG}

In 1981, we founded the Management Systems Laboratories_a research laboratory at Virginia Tech. Management Systems Laboratories is heavily involved in public service; and gains synergy between its research and its public service activities. Through our instruction, research, and public service activities, we test, evolve, and improve our understanding of the concepts of management systems engineering, management tools, data and information, communication, continuous improvement, and their application. Management Systems Laboratories is highly cross-disciplinary, blending many disciplines from within the College of Engineering and from many colleges throughout the University, including education, arts and sciences, architecture, and business. Each discipline contributes to the design of management tools to support decision making. Management Systems Laboratories conducts training to help practitioners learn about and apply management systems engineering.

In 1986, based on concepts and principles discovered through Management Systems Laboratories' activities, we founded the management systems engineering curriculum at Virginia Tech. The Industrial and Systems Engineering Department offers M.S. and Ph.D. degrees in management systems engineering. We also offer a two-semester management systems engineering sequence at the senior level. In recent years, the classes have grown to include students from other departments in the college and other colleges in the university.

\section{ACKNOWLEDGMENT}

The preparation of this paper is funded by U.S. Department of Energy (DOE) Grant No. DE-FG0288DP48058. Management Systems Laboratories thanks DOE's Office of Environmental Restoration and Waste Management for providing us a real-world laboratory for the research, development, and testing of state-of-the-art management tools and the frameworks for understanding how to make them successful.

\section{REFERENCES}

[1] 1992 ABET Accreditation Yearbook, Accreditation Board for Engineering and Technology, Inc., back cover.

[2] Blanchard, Benjamin S. and Wolter J. Fabrycky, Systems Engineerine and Analysis, Prentice-Hall, Inc., 1990, p. 2.

[3] Weinberg, Gerald M., An Introduction to General Systems Thinking, John Wiley \& Sons, 1975, p. 52.

[4] Kurstedt, Harold A., Jr., Pedro M. Mendes, and Kwang S. Lee, "Engineering Analogs in Management," Proceedings of the Ninth Annual Conference, American Society for Engineering Management. 1988, pp. 197-202. 
[5] Simon, Herbert A., The New Science of Management Decision, Harper and Brothers, 1960, p. 1.

[6] Forrester, Jay W., Industrial Dynamics, MTT Press, 1961, p. 93.

[7] Mintzberg, Henry, "The Manager's Job: Folklore and Fact," Haryard Business Review July-August 1975, pp. 49-61.

[8] Scherkenbach, William W., Deming's Road to Continual Improvement SPC Press, Inc, 1991, p. 10.

[9] Adizes, Ichak, Corporate Lifecycles: How and Why Comporations Grow and Die and What to Do about. It. Prentice Hall, 1988, p. 88.

[10] Drucker, Peter F., The Effective Executive, Harper and Row, 1967.

[11] Forrester, Jay W., Industrial Dynamics, MTT Press, 1961, pp. 93-94.

[12] Tushman, Michael L., and David A. Nadler, "Information Processing as an Integrating Concept in Organizational Design," Academy of Management Review July 1978, p. 622.

[13] Weisbord, Marvin, Productive Workolaces: Organizine and Managine for Dienity Meaning and Community Jossey-Bass Publishers, 1991, p. 79.

[14] Mallak, Larry A., The Development and Application of a Procedure to Meacure Culture Strength in Organizations, an unpublished Ph.D. dissertation in Industrial and Systems Engineering, Virginia Tech, April 1993, p. 33.

[15] Morris, William T., “On the Art of Modeling," Management Science, 13 (12), August 1967, p. B-709.

[16] Berube, D. Steven, Harold A. Kurstedt, Jr., and R. Martin Jones, "A Framework for Data and Information," Proceedines of SETUMS, 1989, pp. 266-268.

[17] Scherkenbach, William W., Deming's Road to Continual Improvement SPC Press, Inc, 1991, pp. 14-15.

[18] Shewhart, Walter A., Statistical Method from the Viewpoint of Ouality Control, Dover Publications, 1986, p. 45.

[19] Deming, W. Edwards, Ouality. Productivity. and Competitive Position, notes from the September 1992 workshop.

[20] Imai, Masaaki, Kaizen: the Key to Japan's Competitive Success, McGraw-Hill, Inc., 1991, pp. 239-242. 
[21] Deming, W. Edwards, The New Economics, MIT Center for Advanced Engineering Study, 1993, p. 96.

[22] Covey, Stephen R., Brinciple-centered Leadership, Simon and Schuster, 1990.

[23] DePree, Max, Leadership is an Art, Dell Publishing, 1989.

[24] Juran, Joseph M.. The Last Word Lessons of a Lifetime in Managing for Quality, notebook from the August 1993 workshop, p. 43. 
Title: The Impact of Group Decision Support Systems On Group Consensus Processes and Outcomes

Authors: Suvit Nopachal

Department of Industrial and Systems Engineering

Virginia Polytechnic Institute and State University

Blacksburg, VA 24060

and

Sherry Perdue Casall

Department of Industrial and Systems Engineering Virginia Polytechnic Institute and State University

Blacksburg, VA 24060 


\title{
The Impact of Group Decision Support Systems On Group Consensus Processes and Outcomes
}

\begin{abstract}
In recent years, there has been a growing interest in the application of computerbased technology to support multiple individuals jointly engaged in a single task. One form of such technology is that which is designed to assist the communication and information coordination within a group meeting (often referred to as electronic meeting support systems (EMSs) or group decision support systems (GDSSs). While field reports are beginning to emerge which demonstrate successful applications of some systems (Dennis, Nunamaker, Paranka, and Vogel, 1990), empirical research is just beginning to emerge to offer guidance in terms of successful use of current systems or the development of future systems.
\end{abstract}

A laboratory experiment was conducted to examine how the use of a group decision support system (GDSS) influences the formation of group consensus. In a task requiring group members to jointly prioritize a list of items, 12 groups of 8 members each ivere randomly assigned to one of three experimental conditions: (1) a group decision support system, (2) a manual counterpart to the structure imposed by the GDSS, and (3) no structured support. Dependent measures of consensus and perceived consensus, decision quality and perceived decision quality, and perceived opportunity to express views were taken.

Results found few significant differences between the three levels of technology in the six dependent measures. Objective measures of consensus, decision quality and perceived decision quality, and perceived opportunity to express views were all similar for each of the three levels of technology investigated. Only perceived consensus was found to vary across conditions. The practical implications of these results are discussed. 


\section{The Impact of Group Decision Support Systems On Group Consensus Processes and Outcomes}

\section{Background}

Information technology has long been employed to aid in individual work. Recently however, there has been a rapidly growing interest in the use of information technology to support collaborative work (i.e., computer-supported collaborative work (CSCW), groupware, group decision support systems, etc.). One form of such technology is that which is designed to assist the communication and information coordination within a group meeting (often referred to as electronic meeting support systems (EMSs) or group decision support systems (GDSSs). Because managers and other information workers spend a significant portion of their time in meetings, where a large portion of time is essentially unproductive due to poor meeting management, the potential impact of a technology which makes meetings more efficient is tremendous. Despite a growing number of reports of successful use of GDSSs in industry (Dennis, Nunamaker, Paranka, and Vogel, 1990), little empirical evidence is available to guide the successful application of existing technologies (e.g., when to use a system, how to implement the system), or direct the development of future systems.

\section{GDSSs and Groun Consensus}

GDSSs may potentially affect group decision making, conflict management, and ultimately consensus attainment, in a number of ways. Some effects may positively facilitate the process and outcomes while others may exhibit a negative influence. The balance of the different opposing effects should determine the net result of electronic meeting support on consensus attainment. As described by Poole, Holmes, and DeSanctis $(1988 ; 1991)$ and summarized in Figure 1, a review of the current literature suggests at least seven distinct potential effects:

1) "There is a greater expression of affect, both positive and negative, in computer-mediated communication than in face-to-face modes". In addition, evidence suggests that negative affect is portrayed more strongly than positive. Such increase in the expression of negative feeling may make productive work more difficult since attention is focused on feelings rather than issues, and therefore may result in lower consensus.

2) "GDSSs emphasize written media over spoken communication". Evidence suggests that once an individual puts a position in writing, $s /$ he tends to become more committed to that position, thereby discouraging compromise and resulting in lower consensus.

3 ) "Computer-mediated communication may de-emphasize personal relations". Because computer-mediated communication is less personal than face-to-face encounters, conflicts may not tend to be taken as personally in electronic supported meetings. As a result, not only should groups tend to fully address issues which need to be addressed (rather than avoiding them to reduce conflict), but individuals should be able to focus more on the issues rather than personal antagonisms. As a result, integrative behavior will be encouraged and consensus should be higher. 


\section{Impact on Consensus}

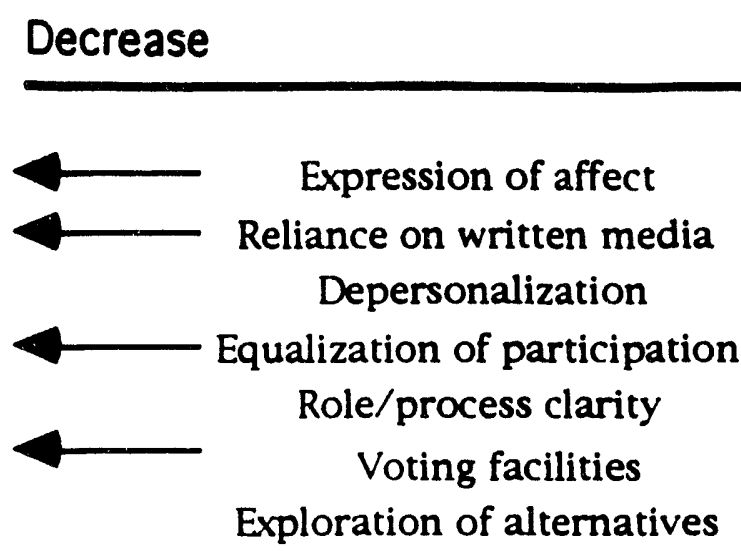

Increase

Figure 1. Possible outcomes of GDSS features effects on group consensus (from Poole, Holmes, and DeSanctis, 1988).

4) "GDSSs equalize member participation". A number of studies suggest that GDSSs tend to more equally distribute member participation; promoting the participation of those who tend to be less vocal in traditional meeting, and subduing individuals who have a tendency to dominate. This, too, may tend to ensure that all pertinent issues are addressed if every individual feels free to express his/her views. While this may decrease consensus by making conflict more difficult and increasing the time to reach a decision, if members can resolve the conflict, consensus may be increased since all group members can feel that they have had an opportunity to contribute.

5) "GDSS procedures make processes and roles in conflict management clearer". Because of the structure imposed by GDSSs, individuals are clear of their roles and the procedures to be employed, thus the group and the process can easily be organized. As a result, one of the key sources of conflict, tension created by social uncertainty is eliminated, making conflict less extreme and consensus higher.

6) "When GDSSs incorporate decision rules, these can influence the course of the conflict". As discussed previously, scme GDSSs include voting or other decision procedures which have been shown to serve as disincentives to conflict resolution. When members know that a decision will come to a vote, they have little incentive to compromise, thus lowering consensus. On the other hand, voting may be used to raise conflict for the purpose of spawning group discussion and problem-solving. Used in this way, voting may serve to increase the consensus on the final decision.

7) "When GDSSs have procedures for brainstorming or defining solutions, they will stimulate the group to explore a wide range of alternatives". The more 
alternatives explored by a group, the higher the likelihood a solution will be discovered that fulfills the needs of all parties, thus leading to higher consensus.

Although these hypothesized effects are based, in some cases, on sound theoretical principles and in other cases, on empirical evidence, there is little information to suggest which effects apply for a given situation, or where a particular effect does apply, what the resulting magnitude may be. Only a limited portion of the small body of behavioral research concerning GDSSs have included a dependent measure relating to group consensus. Watson, DeSanctis, and Poole (1988) investigated the impact of computer-based meeting support on group consensus. This data were later re-analyzed to study more extensively the conflict management processes employed in GDSS-supported groups and the resulting effect on group consensus (Poole, Holmes, and DeSanctis, 1988; 1991). While this study offers a significant step in discovering the potential impact of meeting management technology on the consensus process and outcome, the authors themselves conclude that the study had several limitations that prohibit generalization of the findings to other populations or settings and should serve only as a starting ground for exploration. The influence of a GDSS on the consensus process and resulting outcome no doubt depends on the particular features of the GDSS, the nature of the group's task, the composition of the group, and how the group uses the GDSS.

\section{Method}

Subiects. Ninety-six subjects from the university community participated and were compensated for their time. Subjects were randomly assigned into the three experimental conditions. Efforts were made to ensure that individuals assigned to a single group ivere not previously acquainted with one another, and that equal or ncarly equal numbers of male and female participants were assigned to each group.

Task. Groups performed the "Lost on the Moon" exercise, developed by Hall (1971), in which participants assume they are the crew members of a spaceship which has just crash-landed on the moon. Group members are required to rank order (individually and as a group) a list of 15 items which survived the crash landing in terms of the importance of each to the group's survival. When the task was completed as a group, team members were tasked with reaching consensus on the decision, and were given a set of guidelines for reaching consensus (Hall, 1971) for assistance.

Experimental Design. The type of technological meeting support was the primary independent variable, with three levels: baseline, manual, and computer-supported. Four groups, each consisting of eight randomly-assigned participants, were randomly assigned to each of the three technology-type conditions. To control for spurious effects due to the compositions of different groups, group was considered a second independent variable nested within technology type; hence a $3 \times 4$, complete hierarchical, between-subjects design was used for data collection.

Evperimental Apparatus and Procedures. The computer support system employed in this study, GroupSystems V'm , was developed by a research team from the Department of Management Information Systems at the University of Arizona's Eller School of Management. This system has been employed extensively in their laboratory and field research and is sold and used for commercial purposes. (See Nunamaker, Dennis, Valacich, Vogel, and George, 1991 for a detailed description of the system and a summary of the University of Arizona research program). The software system ran 
on a network of 10 workstations arranged in a large two-tiered meeting room. The system offers a wide variety of electronic meeting system (EMS) tools to support typical meeting tasks. The tool set selected for use in the present study allows group members to simultaneously enter comments associated with each of the 15 items (however, only one person is able comment on a given item at one time). Participants "toggle" between viewing the brief list of items, and opening one of 15 windows to view/add to the comments associated with each given item. Every group member's screen is updated each time another participant adds a comment to an item and exits the item window.

Participants first spent approximately 30 minutes considering the items, entering comments, reviewing the comments of others, posing questions to the group, and responding to comments and questions. While most of this period was spent commenting on the usefulness of individual items, some dialogue regarding the relative importance of items also emerged. When the "discussion" began to wane, the experimenter then led the group to begin actually ranking the items. Because of limitations in the software tool, only the group leader (i.e., the experimenter) had the ability to physically arrange the order of the 15 items. His role, however, was one of "technology chauffeur", in which he provided technical support and task instructions, but v/as not an active participant in the group discussion. As he made modifications to the list, the results were reflected on each group member's screen. The dialogue during this portion of the study was oral rather than electronic, however, each individual was able to open individual item windows as $s /$ he chose to review the group's comments entered earlier.

For the manual condition, paper and pencil methods of structuring the meeting/decision process as analogous as possible to those imposed by the computersupport were developed. This consisted of mounting 15 large pieces of paper along the front wall of the meeting room, labeling one for each item to be ranked. As group members commented orally on an item, the experimenter/chauffeur printed a summary of the comment on the appropriate page. To rank the items, the experimenter/chauffeur used a white board which had the 15 items printed in a column on the left-hand side. As group members rearranged the items, the experimenter wrote the ranked list along the right-hand side of the board.

The baseline groups were not provided with any structured support, however, paper and pencils, a flip chart, and a white board were available if the group itself chose to use them; their use was neither encouraged nor discouraged. Task instructions were identical to those employed in the other two conditions.

Dependent Variables. The effects of the three forms of technology on consensus were assessed by three separate measures. In addition, the effects on decision quality, perceived decision quality, and opportunity to express views were also measured.

Consensus/Perceived Consensus. The first measure of consensus was derived by calculating the sum across items of the absolute difference between an individual's final ranking (post-discussion) of each item and the group ranking:

where

$$
\sum_{j=1}^{15}|F j-G j|
$$

$$
\begin{aligned}
& F_{j}=\text { final individual ranking on item } j \\
& G_{j}=\text { group ranking on item } j
\end{aligned}
$$


This measure resulted in an individual-level measure of consensus.

The second measure of consensus was derived by computing Kendall's coefficient of concordance for the eight post-discussion individual rankings within each group. This resulted in a group-level measure of consensus.

The third measure of consensus, perceived consensus, was derived from the responses to the post-discussion questionnaire. The consensus measure was formed by summing individual responses to six 7-point Likert-scale questions addressing three components of consensus (acceptance, agreement, and commitment), and which have been shown in previous research to measure a single construct (Cronbach's alpha $=0.68)$ (Polk, 1991). This resulted in an individual-level measure of consensus.

Decision quality/Perceived Decision Quality. Decision quality was calculated by summing the absolute difference between a group's ranking of each item and the NASA expert ranking:

where:

$$
\sum_{j=1}^{15}|E j-G j|
$$

$E_{j}=$ expert ranking on item $j$

$G_{j}=$ group ranking on item $j$

This resulted in a group level measure of decision quality.

Individual's perceptions of the quality of the decision reached by their group was measured by summing individual responses to four 7-point Likert-scale questions which have been shown in previous research to measure a single construct

(Cronbach's alpha $=0.79$ ) (Polk, 1991). The resulting measure was at the individual level.

Perceived opportunity to express views Individual's satisfaction with opportunity to contribute to the discussion and influence the group's decision was measured by summing individual responses to eight 7-point Likert-scale questions which have been demonstrated to measure a single construct (Cronbach's alpha = 0.81 ) (Polk, 1991). This resulted in an individual level measure.

Protocol. Upon arrival, subjects first listened to a standard introductory script read by the experimenter, and then read and signed an informed consent form. Subjects individually completed the "lost on the moon" task. Next, subjects introduced themselves to the other group members and completed a 15 minute training exercise as a group, led by the experimenter, to acquaint them with the specific meeting support technology to be used by their group. The training exercise required the group to rank five local restaurants in terms of the appeal of each to college students. This task was similar in structure to the experimental task and thus acquainted the participants with one another, the task structure, as well as the procedures and technology particular to the type of support to be used by their group. Participants next completed the "lost on the moon" exercise as a group. Finally, participants again completed the experimental task individually, and then completed a post-session questionnaire measuring the perceptions of the decision-making process. The experimenter debriefed and dismissed the participants. 


\section{DATA ANALYSIS AND RESULTS}

Consensus. Each of the two objective measures of consensus was analyzed individually. The first measure was analyzed using a one-way analysis of variance. The analysis failed to find a significant difference between the levels of technology $(F(2,84), p>0.05)$. The second measure (Kendall Coefficient of Concordance $(W))$ was analyzed using a Kruskill Wallis one-way analysis of variance. The results $(\mathrm{H}(2)=$ $2.14, p>0.05$ ) show that no significant differences were found between levels of technology.

Despite random assignment to groups, there would potentially be differences between groups in terms of their "pre-existing" consensus, or likelihood that group members tend to respond to the exercise in similar fashion to one another. To ensure that the lack of significance for the two objective measures of consensus was not a result of pre-existing consensus, potential for consensus (or conflict potential) was calculated in a similar manner as the second measure of consensus (described above), but using individual rankings from the pre-discussion administration of the exercise. An ANOVA found no differences between groups in terms of pre-existing consensus, or consequently, conflict potential.

Perceived Consensus. The measure of each individual's perception of the consensus their group reached was attained from the six questions on the post-study questionnaire that related to consensus. An analysis of variance was performed on the hierarchical model. The results showed a significance difference between the three levels of technology support $(F(2,84)=0.45 ; p<0.05)$. Post-hoc comparisons using the Tukey test found that no support and manual support resulted in similar ratings, as did manual support and GDSS conditions; where perceived consensus was higher as the level of technology available was greater.

Decision Ouality. The group-level measure of decision quality, as calculated by summing the differences between group and expert rankings of each of the 15 items, was analyzed using a Kruskill Wallis one-way analysis of variance. This analysis failed to find a significant difference between levels of technology $(\mathrm{H}(2)=0.269$, $\mathrm{p}>0.05)$.

Perceived Decision Quality. The measure of each individual's perception of the quality of their group's decision was attained by the four items of the questionnaire pertaining to decision quality. An analysis of variance performed on the complete model also failed to find a significant difference between levels of technology $(F(2,84)=0.62 ; p>0.05)$.

Perceived Opportunity to Express Views. This individual-level measure derived from the questionnaire data was analyzed by performing an analysis of variance on the 3 $x 4$ hierarchical model. The results indicate that no significant differences were found among the three levels of technology $(F(2,84)=1.991 ; p>.05)$.

\section{DISCUSSION}

The results of the present study fail to support the speculations of many individuals, certainly of those individuals involved in the development and marketing of decision support systems, that automated meeting systems will lead to better decision quality, easier consensus, and more opportunity for participants to participate in the decision 
making process. Of the six dependent measures taken, differences between the three levels of support tested were found in only one: perceived consensus. However, these results should not be interpreted as suggesting that GDSSs do not offer any advantage to groups.

The lack of differences between the baseline and manual process group would have been expected having seen the processes that groups within the two conditions adopted. Although not instructed to do so, most groups in the baseline condition adopted a very similar strategy for discussing the exercise and reaching a conclusion as those in the manual condition. In this particular task and experimental protocol, it was possible to closely simulate the process imposed by the GDSS in the manual condition. The results presented here suggest that under these conditions, automation may not offer a significant advantage over traditional approaches. However, for more complex tasks, GDSSs offer features which can not easily be replicated with traditional tools. Under these conditions, larger differences may be found. Other details specific to this study may have accounted for the results found, and therefore the results should be generalized to other situations with care. These limitations will be discussed fully in the complete paper.

The practical implications of the one significant finding must also not be underestimated. Perceived consensus without actual consensus obviously can be detrimental. However, consensus without perceived consensus is equally counterproductive. Perceived consensus is essential in order for individuals to feel committed to a group's decisions, and for the group to build a stronger sense of teamwork.

\section{REFERENCES}

Dennis, A., Nunamaker, J., Paranka, D., and Vogel, D. (1990). A new role for computers in strategic management. Journal of Business Strategy, September/October, 38-43.

Hall, J. (1971). Decisions, decisions, decisions. Psychology Today, November, pp. 51 $54,86-88$.

Poole, M.S., Holmes, M., and DeSanctis (1988). Conflict management and group decision support systems. Proceedings of the Conference on ComputerSupported Cooperative Work. Sept. 26-29, Portland Ore., (pp. 227-242). New York: Association for Computing Machinery.

Poole, M.S., Holmes, M., and DeSanctis (1991). Conflict management in a computer-supported meeting environment. Management Science, $37(8), 926-953$.

Polk, J. (1991). The effects of an expert on the small-group consensus process. Unpublished Masters thesis. Blacksburg, VA: Virginia Polytechnic Institute and State University.

Watson, R.T., DeSanctis, G., and Poole, M.S. (1988). Using a GDSS to facilitate group consensus: some intended and unintended consequences. MIS Quarterly, Sept., 463-477. 
Analysis of Stakeholder Concerns for the

Environmental Restoration and Waste Management Program

Timothy G. Kotnour, Management Systems Laboratories, Virginia Tech Joseph A. Coleman, Office of Waste Management, Department of Energy

George Batrouny, Management Systems Laboratories, Virginia Tech

Melissa Shaw, Management Systems Laboratories, Virginia Tech

Harold A. Kurstedt, Management Systems Laboratories, Virginia Tech

\begin{abstract}
The Office of Waste Management, Department of Energy (DOE) directed this study to improve understanding of the concerns of their stakeholders. The data was gathered from the open-ended comments made during the Office of Environmental Restoration and Waste Management (EM) Programmatic Environmental Impact Statement (PEIS) Scoping Meetings in 1990-1991. A content analysis process was applied the comments to develop a list of issues and sub-issues the stakeholders want DOE to address. From these issues, a set of recommendations were made.
\end{abstract}

This paper contributes a set of stakeholder concerns grouped according to issues for the Environmental Restoration and Waste Management Program (ERWM). These stakeholder concerns can be translated into strategic issues for EM to consider in its planning efforts. The description of major issues and sub-issues helps identify the strengths and weaknesses of EM a defined by its stakeholders. Opportunities and threats for EM can also be identified through the study of these issues. Finally, a set of recommendations for DOE from these concerns is described.

\title{
INTRODUCTION
}

EM's strategic plan states EM will complete its mission in a "manner that provides appropriate opportunities for public involvement" and will achieve its vision through "...openness to public input, and overall accountability to the Nation for its actions" [1, p. 1]. This statement clearly identifies EM as a stakeholder-oriented organization that values public acceptance of its program. A stakeholder is recognized as any person, group, or organization that can place a claim on or is affected by an organization's attention, resources, or output. They are all vested interest groups (institutions and individuals) who either affect or are affected by an organization's policies and behaviors. To fully understand the strategic planning environment, the organization should develop a precise picture of the stakeholders and the potential effects they have on the organization.

The key to success in a public or nonprofit organization is the satisfaction of key stakeholders. Public organizations are usually established to serve a public need. The organization's performance is measured through its ability to satisfy this need. If an organization doesn't recognize who its stakeholders are, and how they measure the organization's performance, there is little chance of the organization knowing how to operate successfully. Over the last decade, states and public interest groups increasingly demanded, often through the courts, access to 
information about and regulation over an organization's activities. These demands were heightened when an organization's policies and decisions potentially affected public welfare and/or the environment. For example, government agencies often find themselves accused of breaching public trust and leaving the "world" they were assigned to service in worse shape than they found it. A responsible government agency should understand the reasoning behind such accusations and address the issues raised. An extra effort should be taken to understand why stakeholders feel the way they do. EM recognizes the importance of improved communication with external parties to improve its decision-making process [2]. The objective of this study was to develop a list of stakeholder concerns for the ERWM.

\section{METHODOLOGY}

A content analysis process was used answer the question: What do the stakeholders of DOE's EM expect EM to accomplish with the ERWM? The purpose of the study was to reveal the focus of attention (i.e., goals and definable actions or plans for achieving the goals) that EM should be pursuing as defined by the stakeholders. "Content analysis is a research methodology that utilizes a set of procedures to make valid inferences from text" [3]. The idea is to classify the diverse set of words of a text into fewer content categories. This section briefly describes the content analysis methodology used to answer the above question. A more detailed description and analysis of the process can be found in Kotnour, Koball, Batrouny, Shaw, and Harvey [4].

The main source for our data was a database of comments from scoping meetings conducted by DOE for the Implementation Plan for the PEIS for the ERWM. The comments from the PEIS database were grouped into issues based on the issues identified in EM's and WM's strategic plans [1][5]. Each comment in the PEIS database had one or more assigned issue numbers. The PEIS issue number was matched with an EM issue. This grouping resulted in fourteen issue files. Some comments were placed in multiple issue files because they had multiple PEIS issue numbers.

Through the content analysis process a hierarchy of issues, sub-issues, and themes from the comments was developed. (See Figure 1.) A stakeholder's comment was the basic unit of text analyzed and classified. Each comment was analyzed for the theme(s) a stakeholder wanted EM to address (i.e., goals and definable actions or plans for achieving the goals). A comment may have contained none, one, or more than one theme. All comments for a given issue were read. The comments were tagged (i.e., classifying words of the text) for each theme, if a theme existed. After all comments were read for the given issue, the themes were organized into sub-issues. The sub-issues were developed based on similar themes. The comments were reread by the same researcher and checked for correct theme and sub-issue assignment. Any changes to a theme-tosub-issue assignment were conducted at this time. The complete text was reviewed during this step. The number of stakeholders who commented on a given sub-issue were counted. If a stakeholder made more than one comment in a sub-issue, then the stakeholder was counted only ince on that given sub-issue. This count was used to determine the "significant" sub-issues of an issue. The data were summarized by producing a "paper" on each issue. Each issue paper contained a description of the issue and the sub-issues. The sub-issues were ranked by relative importance to the stakeholders based on the frequency of stakeholders commenting on the subissue. 


\section{FIGURE 1. CONTENT ANALYSIS HIERARCHY.}

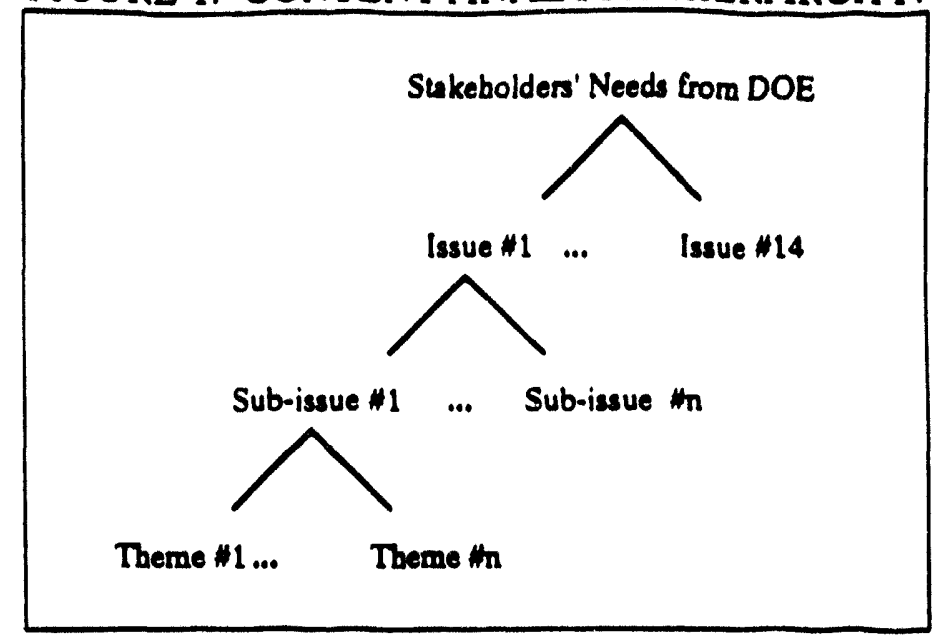

\section{RESULTS-.STAKEHOLDER ISSUES}

In this section the analysis results are described. These results support the findings published by DOE [6] but also extend the earlier findings by providing more than a range of comments. The comments are categorized, ranked, and related to EM's strategic plans. These results are the views and opinions of the stakeholders at the PEIS scoping meetings, that is, the results are limited to the stakeholders who commented on the PEIS. Furthermore, not all stakeholders share the same views. Table 1 provides a list of issues and sub-issues the stakeholders commented on. The bold titles represent the issues and their respective sub-issues are listed below the issues.

The issues provided a means of grouping the comments. Due to the paper length limits each issue cannot be discussed. However, a copy of the complete report can be obtained from Management Systems Laboratories (MSL'). In this paper, the comments and sub-issues most common across the issues are discussed: State, Local, and Public Involvement in Decision Making and Information Sharing; Environmental, Public, and Worker Health and Safety; Technologies Used to Manage Waste; Monitoring; and Standards. The reader should remember these issues are not isolated. The waste management problem is complex with many interrelated issues and concerns.

\section{State, Local, and Public Involvement in Decision Making and Information Sharing}

State, local, and public representatives want their input to be considered in decisions made at DOE on issues such as: land use, siting, transportation, and technologies used. DOE is expected to make public all past, present, and future information related to its cleanup activities. In the face of public opposition to many of DOE's activities, openness and honesty in the release of this information will do much to restore public confidence in DOE. The "legacy of secrecy" is still a major problem in the eyes of DOE's stakeholders. Also, stakeholders want independent oversight and monitoring of DOE activities supported by DOE with funding to the interested parties.

\footnotetext{
'Requests for the final report should be sent to: Management Systems Laboratories, Virginia Polytecbnic Inatitute and Stalc Univeristy, 1900 Kraft Drive, Blacksburg, Virginia 24061.
} 
Stakeholders appear to have little faith that DOE can be dependeo un to complete its mission in a manner acceptable to the stakeholders.

Specific actions the stakeholders want DOE to take on the issues of State, Local, and Public Involvement in Decision Making and Information Sharing include:

1. Establishing techniques to better inform and involve citizens and local and state govemments in the decision-making process regarding oversight of facilities, planning, and monitoring;

2. Educating the public about the health risks of disposal at sites;

3. Holding public forums and hearings to inform the public and workers of the health risks and environmental damage;

4. Involving citizens in siting since there is concem with locating facilities close to heavily populated centers;

5. Making available to independent reviewers for analysis all DOE data regarding: violations, health studies, community and worker health records, waste release and contamination, and hazardous materials;

6. Informing and involving the public in inter-site transportation issues such as transportation routes. safety, and the presence of hazardous waste in the community where transporting waste inrough a state;

7. Giving all interested parties input into decisions on how clean "clean" means; and

8. Giving all interested parties a chance to express their views on appropriate technologies and the implementation of these technologies.

\section{Environmental, Public, and Worker Health and Safety}

State, local, and public representatives voiced their concern over what they see as DOE's apparent disregard for environmental, public, and worker health and safety. Health and safety at and around sites should be the primary concern. DOE was described as lacking in programs that support worker safety at its sites. Private industry was described as superior in this area. Stakeholders said DOE needs to develop such programs. Concerns over DOE's intolerance of whistle blowing were voiced and DOE was asked to devise a program to encourage concerned workers to speak out. Health care for workers was a concern, especially since workers could not get second opinions and must use DOE-designated physicians. Also, the stakeholders want DOE to consider the cumulative effects of waste and production activities. "DOE's ability to meet health, environmental, and legal criteria cannot be evaluated adequately unless it considers the cumulative exposure resulting from all activities at a given site" [7].

Public health and safety was another concem especially for people living around nuclear facilities. Stakeholders are concerned about the extent of on-site and off-site contamination. The contamination must be known, minimized, and contained. Stakeholders are concemed about water, soil, and air contamination. Stakeholders are concemed about the long-term impacts the waste poses. Future generations should be protected from the dangers of this waste, DOE is "playing with the future of the human race" [7].

Stakeholders are concerned about the socioeconomic costs of DOE activities. They are concerned about the workers and communities of DOE sites. They support economic 
development of non-production activities such as using the sites and resources for waste management technology development. Also, communities want compensation for adverse impacts of DOE activities on the community. Stakeholders suggested DOE consider workers' economic state by setting up a "superfund" and retraining them.

Specific actions the stakeholders want DOE to take on the issue of Environmental, Public, and Worker Health and Safety include:

1. Minimizing the risk to workers;

2. Isolating the waste to protect health and safety;

3. Preventing accidents and having evacuation plans;

4. Complying with the worker safety laws at federal facilities the way private industry must comply;

5. Training and re-training workers in safety techniques and providing them with adequate medical care;

6. Protecting workers who report problems and preventing retaliation;

7. Independent assessment of contamination effects;

8. Disclosing accurate contamination data and assuring that existing wastes are contained properly;

9. Protecting the health and safety of current citizens and workers and minimizing the effects;

10. Conducting independent health studies to determine the effects on the public; and

11. Providing educational and training support to state and local govemments, as well as the public, to better understand the cleanup problem and how to deal with it.

\section{Technologies Used to Manage Waste}

Current technologies are considered primitive and are viewed as insufficient to deal with wastes.

DOE's lack of adequate technology and ability to handle the cleanup has been called the "dark ages of waste treatment" [7]. Stakeholders feel DOE underestimates the seriousness of the problem. "DOE must commit to shitting its research efforts, focus, and resources towards developing new cleanup and waste technologies" [7]. Treatment, storage, disposal, and minimization technology development is essential for the cleanup and waste management efforts at DOE. Major issues related to technology involve DOE's ability to characterize its waste and measure the extent of contamination, and to develop the appropriate technologies to deal with this waste.

Specific actions the stakeholders want DOE to take on the issue of Technologies Used to Manage Waste include:

1. Not using incineration and shallow land burial for treatment and disposal;

2. Developing a comprehensive understanding of the current levels of contamination and needed technologies as the first step in developing the appropriate solutions;

3. Setting priorities for which waste types and sites need to be cleaned up;

4. Developing a master technology plan that will lay out cleanup requirements, how to develop required technologies, and a timetable for development (Immediate investigation of the viability of waste repositories and development of alternative plans if the repositories are not viable is needed.); 
5. Developing interim technologies intended to contain and stabilize the waste while searching for long-term solutions;

6. Using on-site treatment whenever possible since transportation still has risks associated with it;

8. Developing new, less-expensive technologies and researching solutions for cleanup;

9. Considering interim technologies to contain the waste while permanent disposal of the waste is the ultimate goal;

10. Seeking innovative and radical technologies, but taking caution to ensure environmental and public safety is the top priority;

11. Taking a scientifically credible approach in which technology research and development is shared among sites and private industry;

12. Obtaining through Congress sufficient funding to ensure the proper development and testing of technologies;

13. Considering environmental laws so the technology complies with all federal, state, and local laws;

14. Making future technologies more flexible and transferable than current ones; and

15. Testing and demonstrating future technologies without any harm to the environment and human life.

Monitoring

Stakeholders have concerns over who monitors DOE and problems the Environmental Protection Agency (EPA) may have enforcing regulations with DOE. Stakeholders believe they can improve DOE programs and can better protect humans and the environment through public monitoring of DOE to make sure DOE has public safety as its first priority. Stakeholders believe restoration, monitoring, and evaluation of contamination and oversight of cleanup activities may be handled best by an agency independent of DOE. Current monitoring and evaluation lack elements necessary for accurate monitoring and are inadequate to achieve as low as reasonably achievable (ALARA) standards. Stakeholders ask: What are the inspection and monitoring practices?

Specific actions the stakeholders want DOE to take on the issue of Monitoring include:

1. Funding long-term, independent monitoring of health, air, water, and soil contamination, and of regulatory compliance (DOE should not be allowed to be self-enforcing.);

2. Funding state and local assessments of waste activities;

3. Sponsoring independently conducted dose studies and research into health studies of effects of long-term exposure;

4. Putting the EPA in charge of the cleanup (Cleanup priorities should be set by EPA and the states.);

5. Establishing public health safeguards to monitor risk of low-level radiation to fetuses and children near the sites and looking at the effects of radioactive materials on reproduction;

6. Reevaluating the effects of radiation;

7. Considering the cumulative impact from exposure from all activities to meet health, environmental, and legal criteria;

8. Opposing "below regulatory concem" (BRC) as jeopardizing public health and safety; 
9. Developing and using data bases for long-term monitoring of the risk from past and future nuclear operations and the effects of exposure on humans, animals, and the environment;

10. Funding independent, comprehensive dose/epidemiological studies and analysis conducted onsite and off-site to measure the health effects of exposure and contamination; and

11. Setting up reviews by an independent agency/scientists at each facility to test if populations have been exposed.

There is concern that DOE has rejected ALARA levels and over the accuracy of dose/level exposure information. Stakeholders want DOE to adopt a zero-release goal. There must be explicit and low limits on ionizing radiation dosage to exposed persons at all times and from all operations.

\section{Standards}

The stakeholders' main concern with standards is for drinking water. Citizens are concerned about the sources of their water being contaminated. Stakeholders want standards defining "how clean is clean." More specifically, they want health-based standards that adhere to federal, state, and local laws. Also, they said states should have input and regulatory authority in developing these standards. Stakeholders believe there are no comprehensive cleanup standards, and what exists is inadequate and results in unnecessary exposure. Stakeholders ask: How do policies and standards of DOE conform to federal, state, and local regulations and laws? Stakeholders want standards and regulations established by entities external to DOE.

Specific actions the stakeholders want DOE to take on the issue of Standards include:

1. Overhauling radiation protection standards to take into account new information on the hazardous effects of exposure;

2. Meeting and upholding the most stringent standards across the complex, e.g., use Occupational Safety and Health Administration to enforce worker safety compliance;

3. Giving states an input and regulatory authority in developing these standards (Public and state involvement must be allowed in setting more stringent standards than EPA or the Nuclear Regulatory Commission.);

4. Eliminating DOE's sovereign immunity;

5. Complying with Resource Conservation and Recovery Act (RCRA) and Comprehensive Environmental Response, Compensation, and Liability Act (CERCLA), and holding DOE to the same standards as private industry;

6. Considering the cumulative exposure of all activities from any given site (Consider a low limit on ionizing radiation. Zero emissions, or the lowest achievable emission rate should be the only acceptable standard.); and

7. Not implementing "below regulatory concern" (BRC) characterization of waste. 


\section{CONCLUSION}

The major issues discussed by stakeholders at scoping meetings for the Office of Environmental Restoration and Waste Management Programmatic Environmental Impact Statement were identified. The results of this study identify for EM the important issues EM's stakeholders want addressed. To be "successful" in the eyes of their stakeholders, EM should demonstrate concern and action on these and on other issues. DOE's goals, objectives, actions, and measures should be developed in balance with stakeholder concerns and the requirements of its mission.

The analysis of these issues suggest a shared understanding of the issues facing the waste complex between DOE and its stakeholders is lacking. The public may not fully understand the complexity of the cleanup problem and its implications, while at the same time, DOE may not have a full understanding of the public's concerns. State, local, and public representatives expect a commitment from DOE to develop better programs to deal with the waste problem. DOE should report in a form the public can understand. The public questions the scientific integrity of DOE studies and claims there is too much analysis and no action being taken.

\section{RECOMMENDATIONS}

The stakeholders are asking DOE to help them understand the situation, share information with them, and to make them part of the decision-making process. The following recommendations are driven by the need for DOE to understand stakeholders' concerns and stakeholders to understand the complexity of the waste management problem:

- Establish techniques to better inform and involve citizens and local and state governments in the decision-making process regarding oversight of facilities, planning, and monitoring. For example, state and local governments, as well as the public, want educational and training support to better understand the cleanup problem and how to deal with it. The issues identified in this paper provide insight into the breadth and depth of the educational objectives and content.

- Demonstrate DOE's concern for public, worker, and environmental safety.

- Demonstrate progress on environmental restoration and waste management technology development.

- Determine and manage the risks in the complex for the general public, those living around sites, the workers, and future generations.

The recommendations in the "Keystone Report" [8] and EM Program Goals [9] begin to address the stakeholders concerns and the above recommendations for DOE.

\section{REFERENCES}

1. U. S. DEPARTMENT OF ENERGY, "Environmental Restoration and Waste Management Strategic Plan: Draft," (1992b).

2. U.S. DEPARTMENT OF ENERGY, "DOE Endorses Keystone Report Recommendations (R-93-068)," (April 20, 1993a).

3. R. P. WEBER, Basic Content Analysis, (Sage Publications 1985).

4. T. G. KOTNOUR, E. KOBALL, G. BATROUNY, M. SHAW, and T. HARVEY, "Gleaning Information from Open-Ended Data to Support Decision Making," Proceedings 
of Twenty-Ninth Annual Meeting of the Institute of Management Sciences, Southeastern Chapter, (October 7.8 1993).

5. U.S. DEPARTMENT OF ENERGY, "The Office of Waste Management Strategic Plan: Draft," (December 16, 1992c).

6. U. S. DEPARTMENT OF ENERGY, "Implementation Plan for the Programmatic Environmental Impact Statement for the Department of Energy Environmental Restoration and Waste Management Program," (January 1992a).

7. Stakeholder, Comment presented at the Scoping Meetings on the Implementation Plan for the Programmatic Environmental Impact Statement for the Department of Energy Environmental Restoration and Waste Management Program, (1990-1991).

8. U. S. ENVIRONMENTAL PROTECTION AGENCY, "Interim report of the Federal Facilities Environmental Restoration Dialogue Committee: Recommendations for Improving the Federal Facility Environmental Restoration Decision-Making Process and Setting Priorities in the Event of Funding Shortfalls," (February 1993).

9. U. S. DEPARTMENT OF ENERGY, "Environmental Management Program Goals (version 11)," (November 4, 1993b).

\section{ACKNOWLEDGMENTS}

The preparation of this paper is funded by U. S. Department of Energy (DOE) Grant No. DEFG02-83DP48058. Management Systems Laboratories thanks the DOE's Office of Environmental Restoration and Waste Management for providing us a real-world laboratory for the research, development, and testing of state-of-the-ant management tools and the frameworks for understanding how to make them successful. 
Table 1. LIST OF ISSUES AND SUB-ISSUES

\begin{tabular}{|c|c|}
\hline $\begin{array}{l}\text { Public Particlpatton and Oversight } \\
\text { State, local, and public involvement in decision making } \\
\text { Public perception of DOE activities } \\
\text { Environmental, public, and worker health and safety } \\
\text { DOE management of cleanup } \\
\text { Compliance with applicable laws and agreements } \\
\text { Occupational and Publlc Health } \\
\text { Monitoring and exposure } \\
\text { Hcalth and safety } \\
\text { Public information and disclosure } \\
\text { Standards } \\
\text { Risk } \\
\text { Waste Disposal } \\
\text { Health risk characterization } \\
\text { Indian health } \\
\text { Burden of contamination } \\
\text { Impacts.Economic and Environmental } \\
\text { Contamination } \\
\text { Standards } \\
\text { Concern for current and future generations } \\
\text { Impacts on communities and workers } \\
\text { Effect of waste management activities } \\
\text { Release waste management related dat } \\
\text { Monitoring } \\
\text { Waste Management } \\
\text { Concerns about current technology } \\
\text { DOE waste management strategies } \\
\text { Current waste situation and future impacts } \\
\text { State, local, and public involvement in decision making } \\
\text { Public and worker health and safety }\end{array}$ & 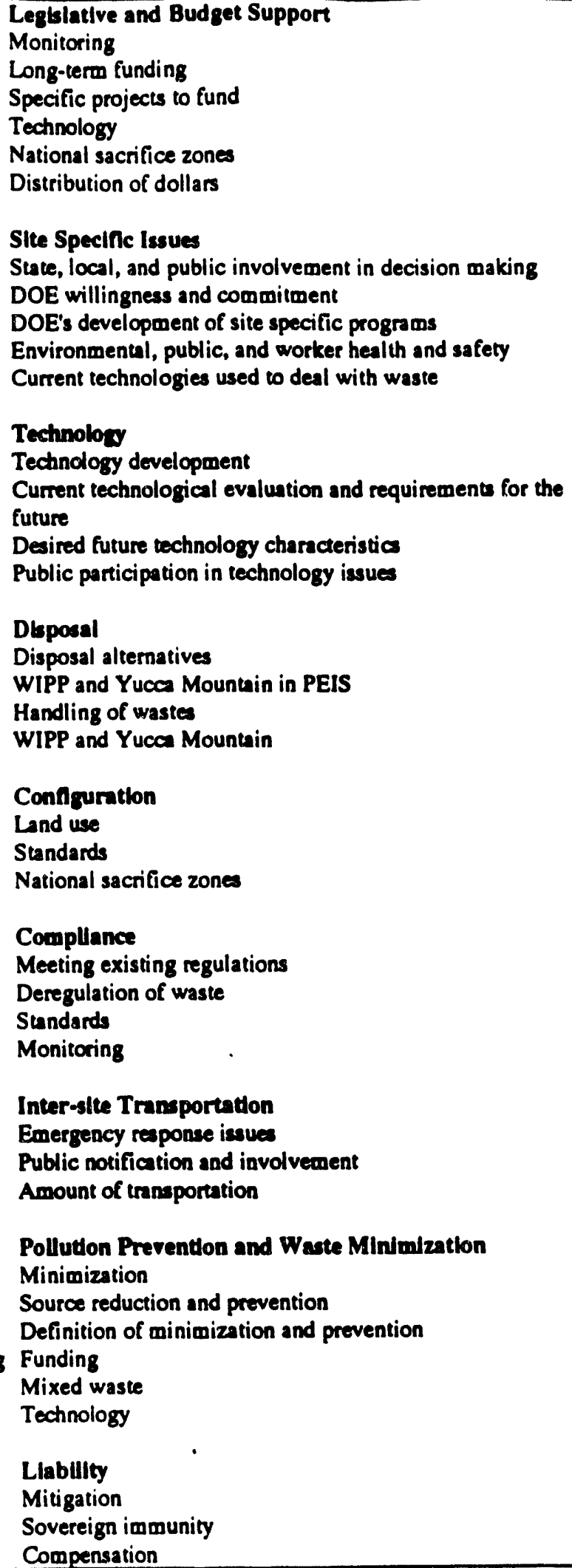 \\
\hline
\end{tabular}




\section{LESSONS LEARNED FROM FACILTTATING THE STATE AND TRIBAL GOVERNMENT WORKNNG GROUP}

Harold A. Kurstedt, Jr.

Management Systems Laboratories, Department of Industrial and Systems Engineering, Virginia Polytechnic Institute and State University

\section{ABSTRACT}

I discuss thirteen lessons leamed from my experience in faciitating the State and Tribal Govemment Working Group for the U.S. Department of Energy. I develop the conceptual base for supporting the veracity of each lesson. I believe the lessons are transferable to any stakeholder group. The crux of stakeholder group success is the two-directional, two-mode empowerment required in this case. Most of the lessons leamed deal with the scope of that empowerment. A few of the lessons leamed deal with the operations of the group.

\section{INTRODUCTION}

Facilitating the State and Tribal Government Working Group for the U.S. Department of Energy (DOE) has revealed and defined lessons leamed I believe should become principles for organizing and facilitating public involvement groups. The State and Tribal Government Worting Group (STGWG), formed in 1989 as DOE's first national public involvement group, includes gubernatorial representatives from states with DOE facilities, affected Indian Nations, and state government associations. STGWG has met quarterly to provide input to the DOE-EM Five-Year Plan for Environmental Restoration and Waste Management and associated policy issues potentially affecting the health and safety of workers, the public, and the environment.

As a neutral, third-party facilitator of these meetings since 1989, Ive identified lessons learned and listed them below as observations followed by discussions explaining each one. STGWG members have reviewed the first ten lessons and their conceptual justifications and have tacitly accepted them. I've developed the last three lessons recently. Tve written the lessons to be understandable and applicable to all DOE stakeholder/public involvement activities. These lessons are corroborated by my experience developing or facilitating two other DOE public involvement programs: the Environmental Management Advisory Committee (EMAC) and the annual Stakeholders' Forum.

I believe you can substitute the name for any stakeholder group for STGWG and substitute the name for any convenor, or sponsoring agency, for DOE, and the leasons leamed will transfer. Other substitutions also apply, such as substituting a state system for lessons about the federal system. Therefore, this paper isn't about STGWG or DOE. Instead, this paper is about improving stakeholder group activities based on fundamental, generalizable lessons learned from the STGWG experience.

\section{OBSERVATIONS FROM FACILTTATING STGWG}

1. If DOE brings organizational results rather than organizational processes to STGWG, the best DOE can expect is criticism.

2. If DOE brings the urgent instead of the important to STGWG, the best DOE can expect is frustration.

3. A person outside the federal system sees any insider as speaking for the entire perceived system. 
4. The need for stakeholders to feel informed doesn't necessitate discussion of or response to all issues.

5. If STOWG members seek individual aims before system-wide clean-up aims, STGWG fails.

6. The fecilitator must focus on the meeting process not the meeting content.

7. Public involvement and consensus require leadership and training from everyone.

8. Without specific action items, participants feel non-productive.

9. Each participant remembers his or her uncompleted perceived action item as a failed promise of the group and/or its convenor (DOE).

10. When stakeholders are involved, their feedbeck turms constructive.

11. Without constancy and consistency of purpose, STGWG flounders for meaning and existence.

12. STGWG doesn't feel empowered just because DOE says so or even because DOE's intentions are good.

13. If DOE doesn't recognize the inherent variation in stakeholders' descriptions of DOE, they'll feel maligned.

\section{DISCUSSION OF OBSERVATIONS}

1. If DOE Brings Organizational Results Rather Than Organizational Procesees to STOWG, the Best DOE Can Expect Is Criticism.

Through the famous funnel experiment. W. Edwards Deming (1) shows that if you act on results your efforts will move you farther and farther away from your target. However, if you act on the process and improve the process you can improve your results and get closer to your target. Results are necessary only as a window into the process. Mathematically, there's no way around this issue.

People involved in organizational processes are forward thinking. People involved in organizational results are backward thinking. If the objective is to improve, then the only ways available to STGWG for acting on organizational results most likely will make matters worse; and if the acting on the results is advice, the only possible advice is criticism.

If DOE brings an organizational reault to STOWG like a plan (such as the Five-year Plan), method (such as a prioritization system), policy (such as the Indian policy), and 30 on and astas for input, the input they'll gat is what's wrong with the plan, method, or policy, not what's right with it. Bringing a settled-on process isn't much better. For positive input, DOE must bring a process in the formulation stage and supply only the aim of the process and the constraints on the process. The process begets the result; so, if a group worts the process cooperatively, the group members own the results together.

To make a contribution, STGWG can make suggestions toward a good or better process and can participate in the process. They will own the results as much as DOE does. Given these results, the only thing left to do is to figure out a way to improve the process to get better results.

2. If DOE Brings the Urgent Instead of the Important to STGWG, the Beat DOE Can Expect Is Frustration. 
Purticipative interaction and involvernent is heavily front-end loaded, time-wise. In other words, you can't puch participative internction faster than the ability for a large group to give each person a chance to express his or her views and to wort on gaps between suggestions.

Typically, DOE comes to STOWG in a perpetual condition of being up to their ears in alligators. Anyone in this situntion would want to share any alligator with any sympathetic ear. While these issues are important and STOWG needs to be fully informed, no participative process can act quickly-relative to individual decision making and action taking. Issues with a reasonable lead time can be affected and improved through public involvement. Stephen Covey (2) and Peter Drucker (3) say we must learn to put the important before the urgent.

People who're working together on a process for a long-term solution accept expediencies needed to keep up with day-to-day situations while crafting the solution.

3. A Person Outside the Federal System Sees Any Insider as Speaking for the Entire Perceived System.

Outsiders don't delinit responsibilities of the insider the way insiders do. Generally speaking. STGWG members feel they're outside the federal government system. So, to them, anyone in the federal government represents and is responsible for everything the federal government does.

There are two ways to deal with this untenable situation. In the short term, the federal government representative can make connections between the outsider and the person inside the federnl government appropriate to the outsider's concem. In this way, the insider represents the parts of the perceived system for which they aren't responsible as a broker or match-maker. In the long term, when the outsider participates enough in developing and contributing to the processes of the perceived system, they'll be able to divide the larger system into its subsystems until they see the insider as responsible for what the insider feels they're responsible for.

This misunderstanding of extent of responsibility and authority clears up when the stakebolders gain ownership of the processes in the ominous system they feel outsiders of.

4. The Need for Stakeholders to Feel Informed Doesn't Necessitate Discussion of or Response to all Issues.

Informed people are more comfortable with each other and make better decisions both for themselves and for the group. People who feel uninformed or misinformed feel threatened. However, there are more efficient ways than formal STGWG meetings to inform STOWG and DOE of each other's situation and needs. Document distribution, news dissemination (immediate happenings), and workshops in coordination with formal STGWG meetings can help the formal meetings be more productive.

\section{If STGWG Members Seek Individual Aims before System-wide Clean-up Aims, STGWG} Fails.

Deming says, "If the parts are optimized, the system will not be. If the system is optimized, the parts will not be." (4) STGWG members must be eternally vigilant of issves carrying the potential of distracting them from the national clean-up effort.

If STGWG members focus on the common aim of the group (the common system), they can achieve cooperation. However, if STGWG members begin to focus on the aims of their individual state or tribe (the parts of the system), they get wrapped up in competition--usually for a limited resource, such as funding. 
Eech STOWO member represents a part (subsystem) of the madional clean-up effor (common system). Any ieve having the potentid to cause STOWG members to refocus from national clean-up to a parochial need ropresents a threat to STGWG's contribution.

When penceiving multi-million-dollar funding as potentially available to STGWG, members become distracted into a zero-sum game for optimizing the parts (each Stnte's portion of the funding) at the expense of the whole (National clean-Up). As STOWG matures, it will increace its potential energy and power. Everyone will be templed to focus that power on their specific needs. Doing so will hurt STOWG. STOWG must always focus on the nationdl clean-up effort.

\section{The Fecilitator Must Focus on the Meeting Process not the Meeting Content.}

The facilitator must be an honeat broler. The facilitator is responsible to balance moving the meeting process forwand to the needed conclusion with ensuring each stheholder gets ample opportunity to express their views. When the quality of a decision can't be teated, group members perceive information sharing, resolution of conflict, and opportunity to expreas their views as decision quality or consensus. (5)

In any group (and especially a group as large as STGWG), a facilitator's full-time job is scrupulously watching for hesitation in participating by group members, concem that queations aren't addresced or answered, desire that the sequence of generuted ideas be followed, all agenda items are firly addressed, action items are recognized and followed-up, and many more. This full-time job is not concerned with logistics like seating and writing ideas on paper. Instead, the job is to eneure stakeholders get to express their views, are heard or know the reason why not, and that diverse ideas get a fair hearing.

The facilitator must ensure meeting process expectations are set and met. For example, when consensus isn't needed or sought, the worst iden is to expect consensus. The fecilitator must make sure the group sets the expectations they need and can produce.

7. Public Involvement and Consensus Require Leadership and Training from Everyone.

Leadership for participative groups can come from the facilitator in terms of the meeting process, from the convenor in terms of needs and constraints, and from the group members in terms of meeting content and products. Max DePree says the art of leadership is "liberating people to do what is required of them in the most effective and humane way possible." (6) In STGWG, DOE, STGWG members, and the facilitator have the opportunity to practice the art of leadership.

To come to worthwhile group results, participants must learn about the system they're addressing, current and long-term issues, and group process implementation.

8. Without Specific Action Items, Participants Feel Non-productive.

People like uncible evidence of accomplishment-especially in time-consuming meetings.

Documented decisions made, items for action, and action items addressed provide tnngible evidence. When an issue is raised that needs a decision or action, the facilitator or any group member needs to make sure a specific action item is identified and assigned and that a date and method for bringing the resolution to the group is documented for later reference and check off. Without regular and trequent tangible evidence of accomplishment, group members feel they're bringing up old issues ignored in the past so they can be ignored in the future.

9. Each Participant Remembers His or Her Uncompleted Perceived Action Item as a Failed Promise of the Group and/or its Convenor (DOE). 
Sooner or inter, a person remembers any isuve for which they thought an sction was to be tiken. To him or bex. the perceived cotion item becomes an ectual sction item that ien't carried our. Then trust is weakened. I's eavier in the long run to track all sction items and confront forgoten or low-priority action items with the pereon who cares.

Stakeholder groups and government convenors are reaponsible for a public truet Neither the group nor the convenor deserves or achioves the public truxt until thoy cen echiove mutunl truet within the group and between group rembers and the convenor. Mutual trut is paramount-the foundation on which everything elee is built As priorities compete for attention during periods of rapid change and the surfacing of important iseves, mutul trux must come first.

\section{When Stakcholders Are Involved, Their Feedback Turns Constructive.}

Stakeholders involved in process iserves nther then result issues contribute by improving the process rather than criticiaing the results. Faced with procese-formation ineves, the tendency is to improve the process-resulting in suggestions for what is 10 come. Feced with results iseves, the tendency is to improve the results--resulting in criticism of what is past.

I've adapted an old Chinese proverb to read: Tell 'em and they'll forget; show 'em and they'll remember; involve 'em and they'll understand. III reathe this ides in atrebolder meeting terms: Oive 'em a briefing and they'll forget; take 'em on a cour and they'll remember, listen sctively and integrate their ideas (III discuss empowerment later.) and they'l undersennd. When a member of the group understands the purpose, constraints, needs, isves, status, and progrese, he or she wants to balp. His or her response to any associated occurrence is largely constructive.

Don't get into the stakeholder group business unless you're after underwanding. People who understand feel a sense of ownership. Ownership leads to cooperntion and wanting to improve rather than to situing and criticizing.

The convenor needs to recognize what's constructive. When STOWO's agenda includes results and urgent issues--things most conducive to criticism and frustration-constructive involvement can seem critical.

\section{Without Constancy and Consistency of Purpose, STOWG Flounders for Meaning and Existence.}

STGWG must develop and preferably get ownership through participation in developing STOWG's purpose. The purpose of STOWO is the purpose of the group, not necesentily the purpose of DOE. While STOWG must focus on the nationd cleanup aim, they must have a pupose for their interaction, or a group aim. The purpose, or aim, of the group can't be limited or capricious, because the purpose won't be maintnined long enough for the group to be successful. When the purpose is met or becomes superfluous, a now purpose must be developed immediately for the group to continue.

STGWG was extablished ostensibly to review the Five-year Pian, but DOQ couldn't meah the review process with the production process with any reasonable lead-time due to gliches in releasing budget numbers and in getting internal approvals on plan content. Although STOWO has commented extensively on the Five-year Plan and has sugsested improvements in the process, STOWG has felt frustrated with the stated purpose and hasn't yet found another. The oaly way to improve this situation is to face up to the empowerment iseve in leseon 12.

Bill Scherkenbach says that constancy of purpose is necessery for success. "As Dr. Deming says, Do you know that doing your best is not good enough? You have to know what to do. Then do your best.' These are profound words because they summarize the two important mesages in [Deming's] 
first point: thet of lnowing what to do-establishing the constancy of purpose and then doing your best--maintrining consistency of purpoce." (7) STOWG and DOZ are both doing their best but with no consent and consistent pupose for the group, STOWO flounders. Who is responsible for STGWO's purpose?

\section{STOWO Doesn't Feel Empowered Just Because DOE Says So or Even Because DOE's Intentions Are Good.}

Neither DOE nor STOWG, nor perhaps anybody else, lnows exactly what empowerment means or how to do empowerment. In empowerment we don't know what it tikes to fot the behavior we consider to be empowered behavior. We think that if we say "You're empowered." the percon or people we said it to will behave the way we expect. We believe thnt if we go beyond words and give the person or people spece and recources, thoyll set empowered. Can we give them the responsibility and authority? How about cccountebility? Even if wo could dive them all thet what about their empowered behavior? We must give them something more fundhmental. We muxt give them in attitude. An attitude is a belief thrt influences emotions, behavior, and what the members of the group think.

To me, empowerment means more than the idea you have the right to mike decisions and tale actions. I believe empowerment means you have the 1) self-confidence, 2) optimism, 3) perceived coatrol, 4) purpose, 5) trust, 6) selfesteem, 7) eccountability, 8) causality, 9) loydity, 10) stewardhip, and 11) ownership to do what needs to be done. Without these eleven atuributes, can you really fulfill your responsibilities for contributing to a group? These eleven atributes mean that, through empowegmenth a person feels: 1) "I can do it" from self-confidence; 2) "I expect the beet " from optiminem; 3) "I can make a difference," from perceived control; 4) "Im doing something meaningful, from purpow; 5) "I feel safe," from trust; 6) "I believe in myself," from self-eteem; 7) "I care, "tom eccountability; 8) "I have a choice," from caunality; 9) "I belong," from loyalty; 10) "I want to serve," from stewardship; and 11) "I get joy from what I do," from ownership.

Part of perceived control are the freedom and resources to matre decisions and tate ections. Only this freedom and these resources come from an external locus of control. Everything elee comes from within the person. If you buy any of my eleven atributes and associated feelings in addition to the issue of freedom and resources, you must then believe a person can't empower another person. A person can only empower himself or herself. Empowerment ien't a state of being--"I am empowered." Empowerment is a state of feeling-"I feel empowered."

You can't empower a group any more than you can empower an individual. DOZ can't empower STGWG, no matter what their intent is. DOE can only support the group as the group empowers itself. We're in a new paradion here. Empowerment ien't for the impatient or the uncaring.

STGWG-type groups need to find empowerment in two directions: from their states, tribes, or associations to ect as individusls and from the DOE to act as a group. Empowerment of a group is different from empowerment of an individual. For example, the individuals each may have selfconfidence, but the colloctive group may not. Or, for another example, a person may trust his or ber state, but not DOE or the other members of the group. I believe the atributes of an empowered group are the same as those for an empowered person. But, we're looking at collective (additive or multiplicative) variables, not individual ones.

\section{If DOE Doesn't Recognize the Inherent Variation in Stakeholders' Descriptions of DOE, They'll Feel Maligned.}

The key word here is inherent. All actions carry variation. Any stakeholder group will voice opinions ranging from the negative to the positive, across a fairly wide range. Therefore, there will always be the most negative opinion and the most positive opinion. Variation is part of nature and we have to 
eccept verintion. Focuning on only the regntive opinion to the poine of being cloced to bearing an

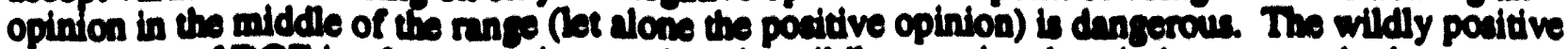
description of DOZ is of no more impon then the willdy negative deacription, except both extremes are neceeseny 80 we cen get a middle. Both the middle of the runge and the sibe of the rnnge are viluble bits of informsion. You have to be able to set exide your ego to receive both the negative ead positive comments equilly and in such a way they both point coward the middle.

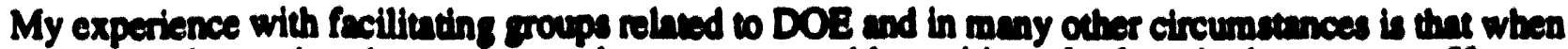
groups work together they ead up at the most reasonble pocition-far from both extremes. If you don't trust the stakeholder group to obthin the bast reall, your oven or coven reaction to the negative end of the range of opinion will trint the group's wort. DOE's culture and hivtory come from an egobased approsch to the world.

Stakeholders' comments don't necesenrlly reflect anitude or pocentinl behwvior but are many different color threads woven into a multi-color fabic of perticipation in and underatending of the iences. DOE must deal with the fabric, not with individual theads, and eapecinlly not with threads of a color they don't like.

\section{CONCLUSIONS}

STGWG is one model for stakeholder involvement; and represents an important lint in the chin of history for the way civilized people must addreas multifnceted antiond or internationd problems. We must leam all we can from the STOWO experience if we are to move into a government that recognizes its customers and knows how to serve them.

STGWG hes made major contributions to the success and underntending of anizeholders groppe. They initiated key advances on the part of DOZ by simulating the 30-yoar un? engendering DOE's recognition of the sovereignty of the Indinn Nations. They brotre ground in leaming leseons about stakeholder groups.

The crux of a successful stakeholder group is resolving the incus of empowerment. For a STOWOtype stakeholder group, we have four degrees of fieedom: a two-directiond, two-mode empowerment. One direction is from the state, tribe, or associntion; the other direction is from DOZ. One mode is to feel empowered as a person; the other mode is to feel empowered as a group.

Techniques for empowerment are elusive. However, I beliove a clnsic exnmple technique is sctive listening. Until STOWG members and DOE become stalled at sotive listening, they'll prictice ective talking to nobody's benefit.

The rest of whnt is needed for a succestul striteholder group is the scope of empowerment. Is STGWG empowered to critigue results or influence a process within DOE? Whnt is the puppoce of STOWG? HOW can the facilitator support the group as each member expreaces his or her views and as the group earerates inngible evidence of eccomplishment?

Many of the leacons are intertwined. For example, can you be empowered in a group where there's no constancy and consistency of purpose?

\section{ACKNOWLEDGEMIENT}

The preparation of this paper is funded by U.S. Deparument of Energy (DOE) Ornnt No. DE-FG0288-DP46058. Management Systems Laboritories thantes the DOE's Oitice of Bavironmental Restoration and Waste Management for providing us a real-world liborntory for the research, development, and testing of sthte-of-the-art manafement tools and the framewortes for understanding how to make them successful. 


\section{REFERENCES}

1. W. EDWARDS DEMMNO, Out of the Crisis, MIT Center for Advanced Engineering Sudy, pp. 327-328 (1986).

2. STEPHTEN R. COVEY, The Seven Habits of Highly Effective People, Simon and Schuster, pp. $149-162$ (1989).

3. PETER F. DRUCKER, The Effective Execudive, Harper and Row, (1967).

4. W. EDW ARDS DEMNN, notes from Inetituting Dr. Deming's Methods for Management of Productivity and Quality, (January, 1992).

5. Building Small Group Concensus: A Handbook for Managers, a research report to DOE by Management Systems Laboratories of Virginia Tech, (1994).

6. MAX DEPREE, Leadership is an AT, Dell Publishing, p. 1 (1989).

7. WILLAM W. SCHERKENBACH, The Deming Route to Quality and Productivity, Ceep Press, p. 13 (1991). 


\title{
A METRIC FOR CONSENSUS - DESIGN AND CALIBRATION
}

by

\author{
Aubrey E. Harvey \\ Research Associate \\ Management Systems Laboratories \\ Virginia Tech \\ Michael C. Kleder \\ Graduate Researcher \\ Management Systems Laboratories \\ Virginia Tech
}

Submitted to:

Computers \& Industrial Engineering

Dr. Hamed Kamal Eldin, Editor

Industrial Engineering

College of Engineering

The University of Iowa

Iowa City, IA 52242-1527 


\section{Purpose and Scope}

This paper presents the methods and results of the authors' testing and analysis of the metric for consensus ${ }^{1}$ proposed in 1992 by Dr. Aubrey E. Harvey of Management Systems Laboratories in Blacksburg, Virginia. ${ }^{2}$ This will entail: (1) a review of the algorithm for computing the consensus metric from questionnaire results, (2) the authors' recommendations for interpreting the metric as derived from simulation and observation, (3) an assessment of the validity of the metric, and (4) recommendations for further research.

\section{Computation of the Consensus Metric}

The consensus metric is a mathematical tool which places a numerical value on the closeness of the opinions held by the members of a group. The application of the consensus metric requires the administration of a questionnaire with an interval response scale ${ }^{3}$ for each question (rather than merely an ordinal scale). The responses are given numerical values; for example, a Likert ${ }^{4}$ scale with eleven responses in its range could be encoded into a zero to ten scale. These values are used as coordinates in an opinion space having as many dimensions as there are questions in the questionnaire. Each questionnaire respondent is thus represented by a point in the opinion space having a set of coordinates corresponding to that respondent's answers to the various questions.

After the respondents are represented by this set of mathematical points in space, a lower-diagonal matrix is created which tabulates the distance between every possible pair of points, so that for a population of $\mathbf{N}$ members, an $\mathbf{N} \times \mathbf{N}$ matrix must be constructed..$^{5}$ The two members which are spatially closest are clustered; 6 that is. removed and replaced by a single point at the average of the clustered respondents' coordinates. A second lower-diagonal matrix is then constructed which includes both the unclustered points and the clusters, each cluster as if it were a single point. The closest two members in this new matrix are again clustered, and the process is iterated until only a single cluster remains comprising the entire population of respondents. The clustering algorithm is shown pictorially in Appendix A.

After the cluster analysis, every cluster - from those first formed to the final overall cluster - can be measured by the consensus metric. A cluster's consensus score is computed using the formula

$$
C=m-d
$$


where $\mathbf{C}$ is the consensus score; $\mathbf{m}$ ("membership") is the fraction of the population composing the cluster; and $\mathbf{d}$ ("dispersion") is computed by dividing the radius of the smallest "sphere" which can contain the cluster (while being centered on the average coordinates of the cluster's respondents) by a predefined standard radius $\left(\boldsymbol{R}_{\max }\right)$ equal to $40 \%$ of the width of the response scale times the square root of the number of dimensions in the opinion space. ${ }^{7}$ Replacing $\mathbf{m}$ and $\mathbf{d}$ with their respective formulae, and allowing the Likert response set to have been encoded into a 0-10 scale, the equation becomes

$$
C=\frac{n}{N}=\frac{r}{4 \sqrt{D}}
$$

where $\mathbf{N}$ is the size of the population, $\mathbf{n}$ is the number of points in the cluster, $\mathbf{r}$ is the radius of the cluster (or specifically that of its "spherical" boundary as specified above), and $D$ is the number of dimensions (questions) in the opinion space.

Each cluster, with its contained questionnaire respondents, is recorded in a table together with the cluster's consensus score. The cluster with the highest consensus score is termed the consensus group, and any excluded respondents are termed outliers. The consensus of the population is taken to be that of the consensus group, but the term overall consensus is reserved for the consensus score of the cluster comprising the entire population. ${ }^{8}$ (The overall consensus may be computed without first applying the clustering algorithm, but ouliers will not be excluded and partitions will not be identifiable, as described below.) Each respondent to a questionnaire has a consensus value equal to the inverse of the number of respondents (simply membership of one respondent minus a dispersion of zero). If this value is the highest consensus for the population, then the population has a consensus score equal to this inverse of the number of respondents; thus, the population's consensus score must be a positive real number. (It can be shown that the metric also conforms to the other strictures placed upon a mathematical metric.)

\section{Interpretation of the Consensus Metric}

For the consensus metric to be useful to group administrators, it must be presented together with a description of a systematic method of interpretation. To begin, hereafter are presented the meanings of the consensus score (also denoted "highest consensus" in accordance with its derivation) and overall consensus score. 
The highest consensus represents one of the following, depending on the nature of the population to which it is applied:

(1) the consensus of the overwhelming majority of a population, exclusive of a few outliers;

(2) the consensus of the strongest (i.e., tightest and/or most populous) subgroup in a factionated population;

(3) the consensus of the few members that happen to be nearest to each other in a widely scattered population.

In each case, this highest consensus score represents what can be considered to be the population's veritable consensus, since it represents the degree to which the population is banded together toward a uniform opinion. In the case of an overwhelming majority, the validity of this observation is intuitive. In the case of factional or dispersed population, this observation is again intuitive if one considers that the strength of the strongest faction or coalesced group presents an upper bound on what unity can be expected of the population without intervention directed at generalizing agreement.

The overall consensus score, by contrast, represents the degree of unity inclusive of outliers, across factional boundaries, or among all members of a dispersed population. This score presents itself as less indicative of the population's capacity for decisive action when one considers the observation that outliers and weak factions generally have little power to effect a significant change in the population's eventual disposition.

Information on factionation in the population can be obtained from an inspection of the cluster table mentioned in the review section above. For this analysis, the authors determined that any cluster which has a higher consensus than some other cluster which contains it should be labeled a partition. (The term partition was chosen because it implies the existence of an aggregation which is tighter than some larger grouping that the partition is a part of. A partition may be "looser" than the population, but tighter than some other group of which it is a part. The same cannot definitively be said regarding factions; however, information regarding factionation can be inferred from partition data. Note that the entire population can never be considered a single partition because it can never be a part of a larger cluster. Thus, if a population has no clusters of higher consensus than their containing clusters then there are no partitions in that population.) The authers suggest that partitions comprising $25 \%$ or more of the population be considered major partitions, those that are smaller be termed minor partitions, ${ }^{9}$ 
and any partition which is wholly contained within a larger partition be termed a subpartition (note that the larger partition must have a lower consensus than that of the subpartition or else the subpartition would not have been selected as a partition at all). Separate partitions are those which share no members; that is, neither is a subpartition of the other. (Because partitions are clusters derived from the given clustering algorithm, they cannot overlap unless one is a subpartition of the other.) Any minor partition which constitutes less than $5 \%$ of the population is duly omitted from consideration except perhaps as a small interest group. (Note that the consensus metric presumes that all members of the population have equal weight.)

A guide to the interpretation of a population's consensus score ${ }^{10}$ (not its overall consensus) is presented in Appendix B. Two sources provided information for the construction of the table: firstly, an examination of actual and simulated population measurements, and secondly, the construction of surface graphs (appendix C) which show the behavior of the metric when applied to a population consisting of two equally sized subgroups (figure 1) or three equally sized subgroups (figure 2) of varying cohesiveness (standard deviation) and varying disagreement (distance between the means of the groups). The actual descriptions in the table are qualitative and are founded in the observations made by the authors.

To further understand the behavior of the consensus metric, the authors looked at one-, two- and three-subgroup populations to determine the indifference ${ }^{11}$ that a consensus score implies between populations with the same consensus score, but differing numbers of subgroups. Two examples of this indifference are (1) indifference at the 0.5 consensus-score level between a unanimous subgroup (or "singularity") consisting of half of the population, or a single-subgroup population with a standard deviation such that the consensus score is 0.5 ; and (2) indifference at the 0.333 consensus-score level between a singularity consisting of one third of the population, a two-subgrcup population with the subgroups far enough apart so that the subgroups are distinct and each has a consensus of 0.333 , and a threesubgroup population with the subgroups equally spaced and distinct, each having a consensus of 0.333 (three singularities). The specific indifference-point data are presented in appendix D. Appendix E shows indifference curves for two- and three-subgroup populations, the similarity between the two graphs illustrating the robust nature of the metric.

\section{Validity of the Consensus Metric}

A test of the ability of the metric to measure what it purports to measure must necessarily address the following issues: (1) the consistency of the metric with 
professional evaluations, (2) the constancy of the metric across similar but not identical populations, (3) the discerning power of the metric relative to that of a standard statistical technique.

The first issue, that of agreement with professional opinion, is most readily addressed by a comparison of the assessments of an actual population by the metric and by professional advisors. This was undertaken in a study of the State and Tribal Government Working Group, a body composed of representatives from geographically dispersed regions in the United States, organized for the purpose of advising the Department of Energy (DOE) on issues concerning hazardous waste. The opinions in this group were obtained through a Likert-scale questionnaire and tracked through time. In both accuracy of assessment and trend analysis reliability, the consensus metric was in agreement with both the observations of the group administrators and the observations of multiple external analysts at Management Systems Laboratories with regard to the population's opinions. The assessed opinions ranged from issues of meeting procedure and faimess of individual contribution, to issues as tenuous as anticipated consideration by the DOE, and even personal opinion of the DOE itself. It was the metric's admirable success in evaluating even these tenuous personal opinions that justified further analysis in the form of the present research. A graph of some of the metric's evaluations of these meetings are presented in appendix $\mathrm{F}$.

The second issue of validity, that of the metric's constancy across similar but not identical populations, was addressed by the authors in an extensive series of simulated scenarios involving hypothetical populations generated from statistical distributions. The authors discovered that populations generated from identical normal probability distributions ${ }^{12}$ (creating populations that were similar but not identical) generate consensus scores within a narrow distribution of normal shape. Thus a normally distributed population generates a normally distributed consensus metric frequency distribution. A graph of the range of consensus scores generated from a normally distributed population of varying standard deviation can be found in appendix G. A histogram showing the normal shape of the metric's distribution can be found in appendix $\mathrm{H}$.

The third issue of validity, that of comparing the metric's ability to discern subgroups in a population to the ability of a standard statistical technique to do the same, was addressed in additional simulated scenarios. To assess the validity of the measurement concept, the authors chose to compare the consensus metric to the Kolmogorov-Smymov two-sample test (or Smymov test ${ }^{13}$ ) to ascertain which was more capable of distinguishing two known, distinct groups at close proximity to each other in the opinion space. ${ }^{14}$ The consensus metric distinguished separate 
groups (if the two groups when combined produced a higher consensus score than that of either group alone, then they were considered indistinguishable; if a lower score was produced, then they were considered to be separate) with an average $86 \%$ reliability when the two groups were separated by two response intervals in each of five dimensions, while the group members were distributed within each group with standard deviations ranging from zero to five intervals. The Smymov test could not discern the two groups with a similar level of reliability (as averaged across the varying standard deviations from zero to five) until the groups were separated by eight response intervals. The Smymov's reliability was then $80 \%$. (The simulation for these tests involved five dimensions, with a Smymov test done in each dimension at a $2 \%$ alpha level. Rejection of the null hypothesis in any dimension indicated inter-group dissimilarity, for an overall alpha level near $10 \%$.) The consensus metric resolved subgroups with more regularity - especially subgroups that were close together (less discemible) - than did the Smymov test.

It is the authors' conclusion that there is sufticient evidence to accept the consensus metric as a valid numerical description of the degree of consensus existing in a population and any subgroups thereof. There are, however, some restrictions in the application of the metric which result from its method of computation. These are listed below:

(1) The metric requires a Likert scale or other interval scale, rather than merely an ordinai scale, for all measured questions.

(2) The metric presumes equal weights for all questions and for all respondents.

(3) It may be possible for the highest possible consensus in a population to result from a cluster that is not chosen by the given clustering algorithm (endnote 8).

(4) The metric's "highest consensus" score, if presented alone, diminishes the significance of outliers and small partitions. It is problematical whether this emphasis would reflect reality in the context of any given population to which the metric may be applied.

(5) When the consensus metric and clustering algorithm are applied to a questionnaire addressing two (or more) unrelated or sparsely related issues, partition information becomes less reliable. An individual may be a member of two different clusters with regard to the two issues, but is artificially confined to a single cluster by the clustering algorithm. To obtain issuespecific partition information, a user of the metric is advised to separate the unrelated issues into separate analyses. Information on the population's general cohesiveness and partitioning across issues may still be obtained by an analysis of all questions together. 


\section{Recommendations for Further Research}

The following topics present themselves as candidates for further research into the consensus metric:

(1) the incorporation of algorithms for accommodating unequally weighted questions and unequally weighted respondents; and

(2) the generation of expanded prose descriptions of the consensus interpretations, including recommendations for the best steps to improve consensus available in each indicated situation.

\section{The Authors}

Dr. Harvey is a Research Associate at Management Systems Laboratories in Blacksburg, Virginia, and a member of the research faculty of Virginia Polytechnic Institute and State University ("Virginia Tech"). Mr: Kleder is a researcher at Management Systems Laboratories and a Master's Degree candidate at Virginia Tech.

The research herein described was funded by the United States Department of Energy under agreement number DE-FG02-88DP48058. Management Systems Laboratories thanks the Department of Energy for providing a real-world laboratory for the research, development, and testing of state-of-the-art management tools and the frameworks for understanding how to make them successful. 


\section{Appendix A}

Below is a set of diagrams which illustrate the clustering procedure in two dimensions for a simulated population:

Questionnaire Responses

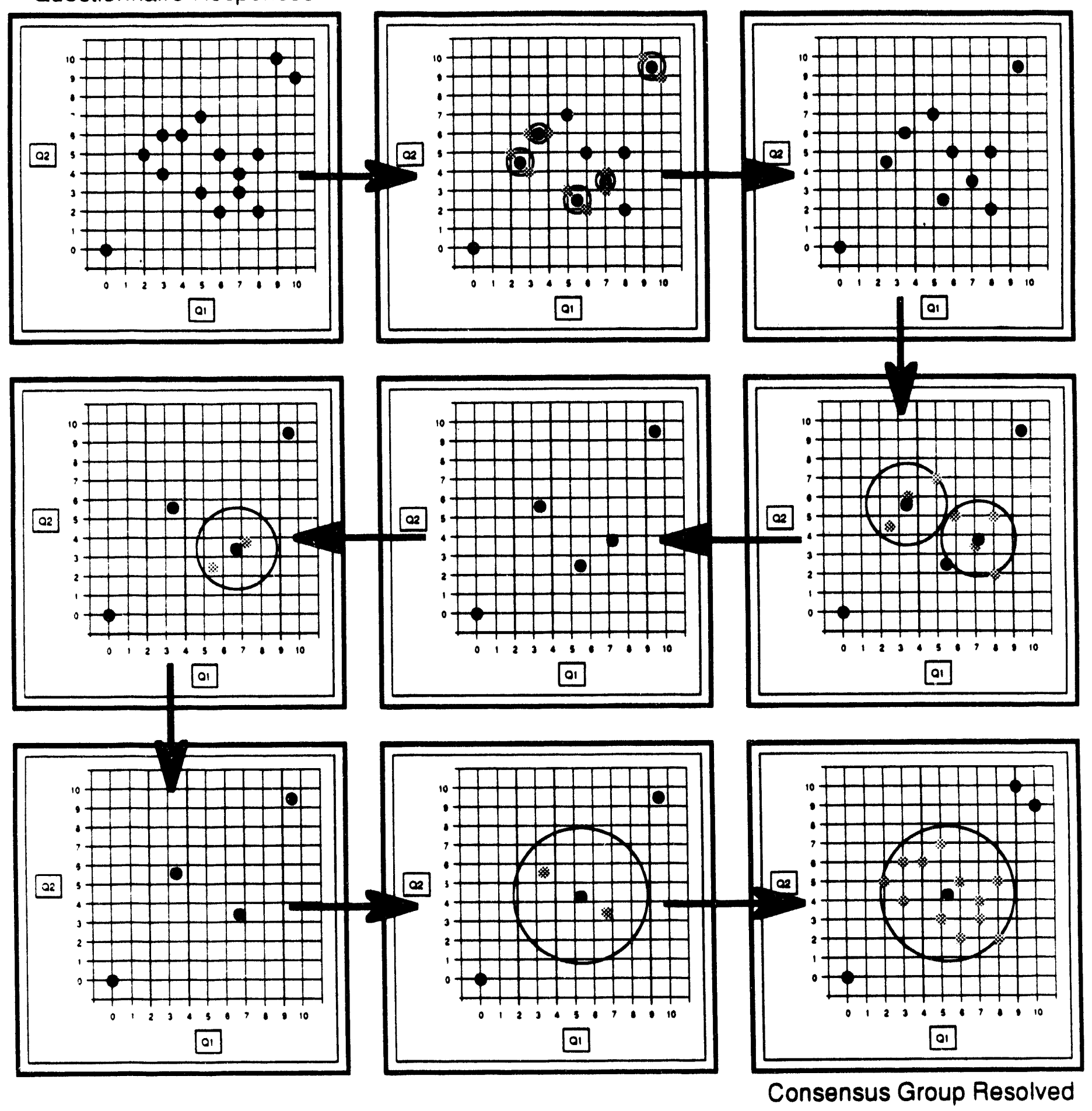




\section{Appendix B}

The Harvey consensus score may be interpreted according to the following table. If a questionnaire administrator has information about the degree of partitioning in the population, he or she may apply the interpretations in columns other than the "partitioning unknown" column.

\begin{tabular}{lllll} 
Consensus & Partitioning & Single Powerful & Multiple & Dispersed \\
Score & Unknown & Partition & Partitions & Population \\
\hline
\end{tabular}

1.0 Absolute unity Absolute unity Absolute unity Absolute unity

0.9 Near Unanimity Negligible outliers Negligible Negligible disagreement spread

$0.8 \quad$ Near Unanimity Very few outliers Very close Very tight partitions spread

$\begin{array}{llll}0.7 & \text { Tight agreement } & \text { Few outliers } & \text { Close partitions }\end{array}$

$0.6 \quad$ Strong agreement Small segment Close partitions $\quad \begin{aligned} & \text { Slightly } \\ & \text { scattered }\end{aligned}$

$0.5 \quad$ General Small segment agreement of outliers

Slight

General disagreement agreement

$0.4 \quad$ Some disagreement outlier segment

Some

Some disagreement disagreement

0.3

General disagreement

Large outlier segment

Discordant partitions

Dispersed population

Partition not

0.2 or lower Anarchy or severe discord significant

Small, weak, disWidely scattered cordant pastitions population 


\section{Appondlx C}

The Harvey consensus score follows the surface curvature below for a population composed of equally sized subgroups with the indicated standard deviations and distances between means.

For two subgroups (figure 1):

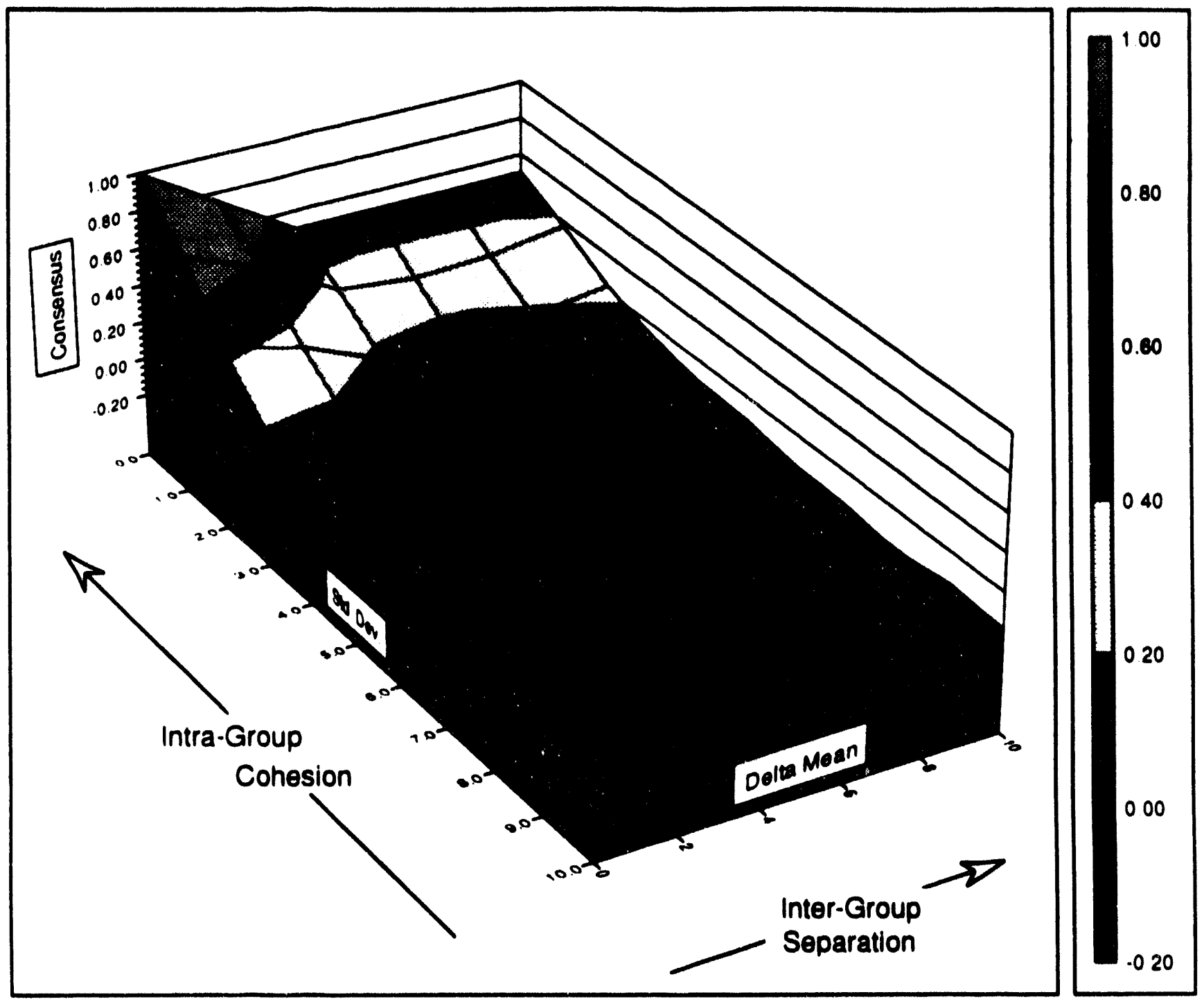




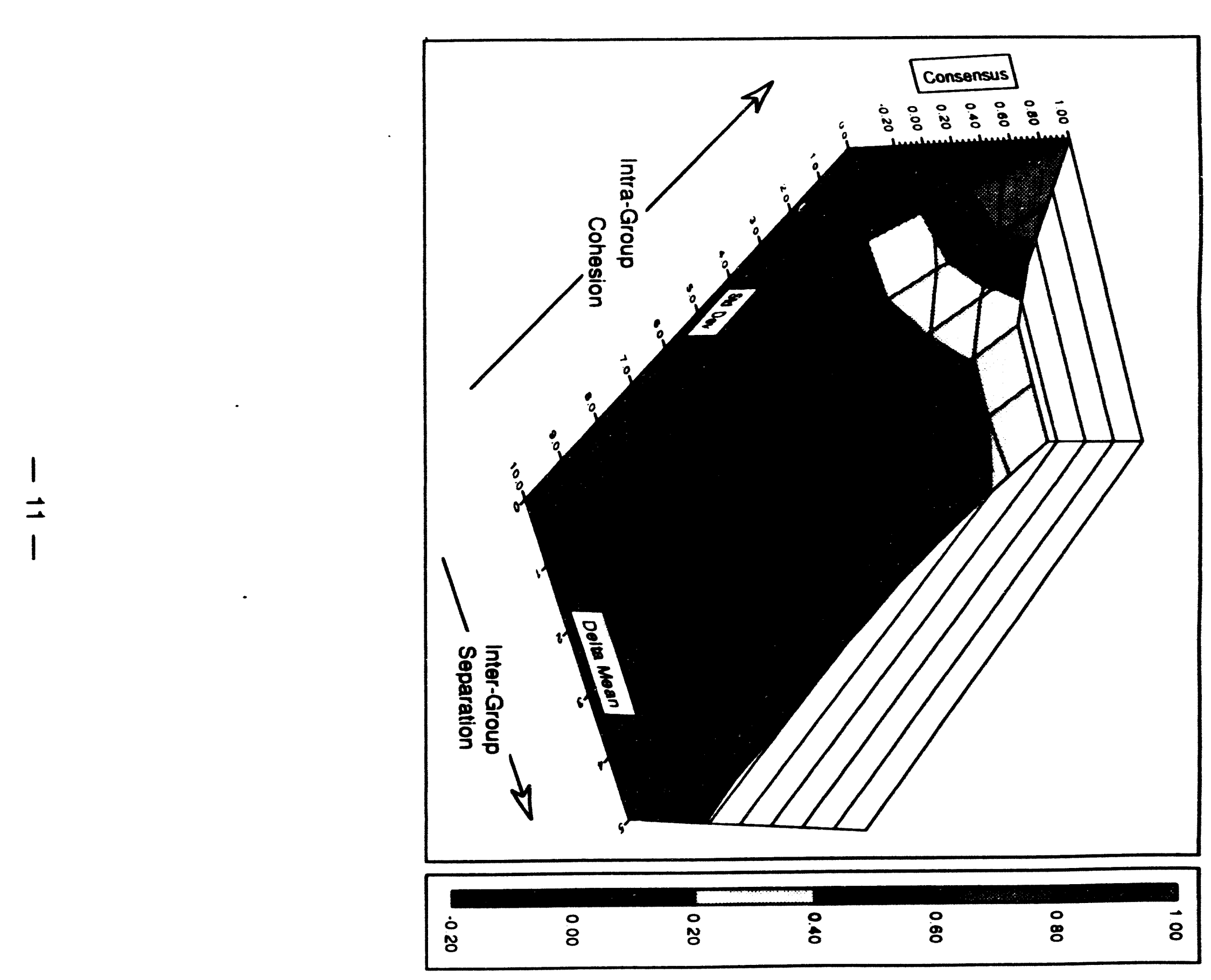

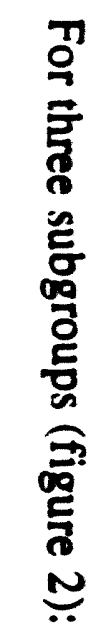




\section{Appendix D}

Below are presented data illustrating various indifference arrangements as described in the body of this text. Standard deviaitons and separations are measured relative to the interval between two adjacent responses to the questionnaire questions.

Indifference exists at the 0.5 consensus level among:

(1) a singularity comprising exactly half of the population:

(2) a single subgroup with a standard deviation of 1.8; and

(3) two subgroups each having a consensus of 0.5 (this restricts the standard deviations to 0.0 ) at a separation of 4.0 .

Indifference exists at the 0.333 consensus level among:

(1) a singularity comprising exactly one third of the population;

(2) a single subgroup with a standard deviation of 2.8;

(3) two subgroups each having a consensus of 0.333 (this restricts the standard deviations to 1.2) at a separation of 5.0. The separation can vary from about 4 to about 8 and still generate a consensus score of about 0.33 , because as the groups separate, truncation severely frustrates the decrease in consensus; and

(4) three subgroups each having a consensus of 0.333 (this restricts the standard deviations to $\mathbf{0 . 0}$ ) at a separation of $\mathbf{3 . 0}$ or greater between each subgroup and its neighbor(s). (Increasing the separation beyond 3.0 does not effect the score for these singularities.)

Indifference is presented graphically in appendix $\mathbf{E}$. 

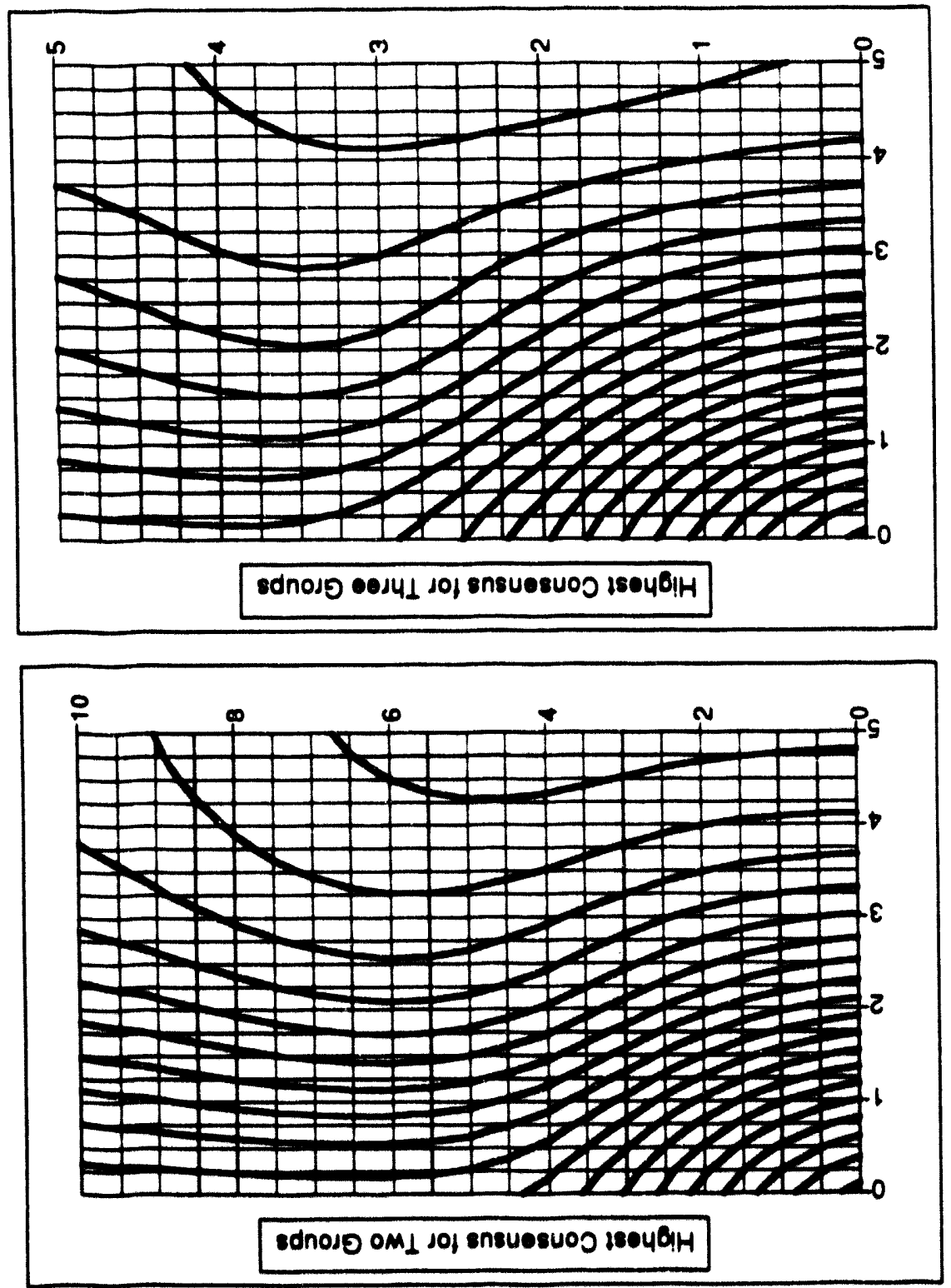

'S0'() jo sjuวursou!

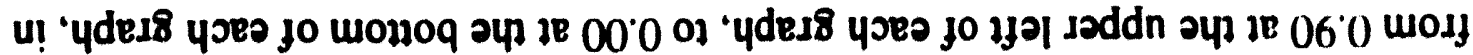

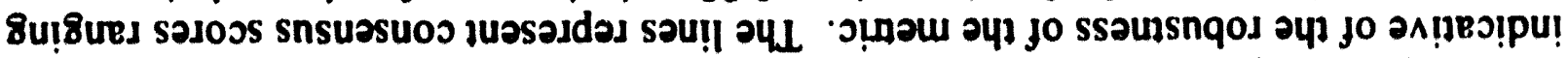

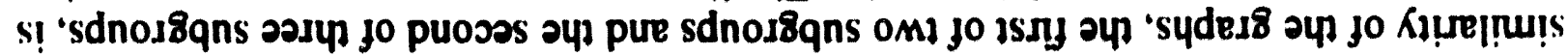

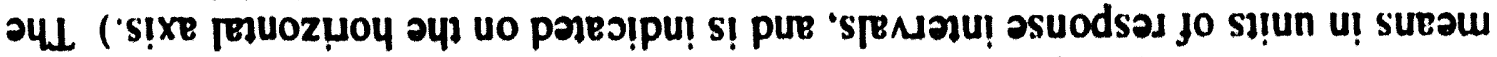

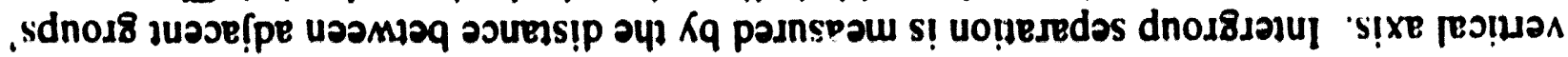

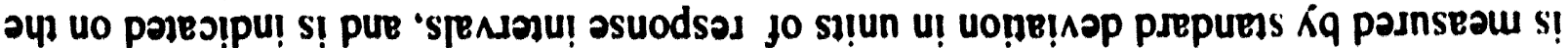

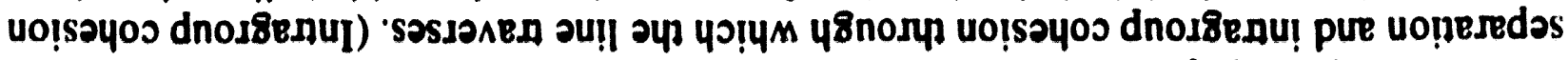

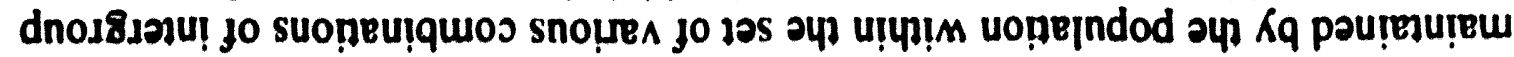

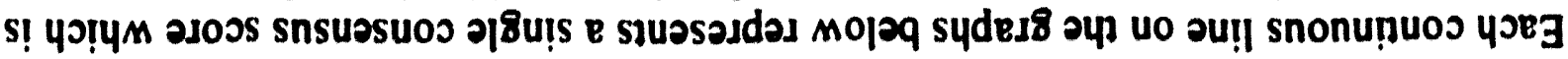




\section{Appondix F}

Below is a time-series graph of the consensus points (in four dimensions, reduced from sixteen questions) from the State and Tribal Government Working Group questionnaires. The four dimensions are areas of opinion that were investigated during the research.

\section{Questionnaire Responses Through Time*}

Data Smoothed With Cubic B-Spline - Exploded View

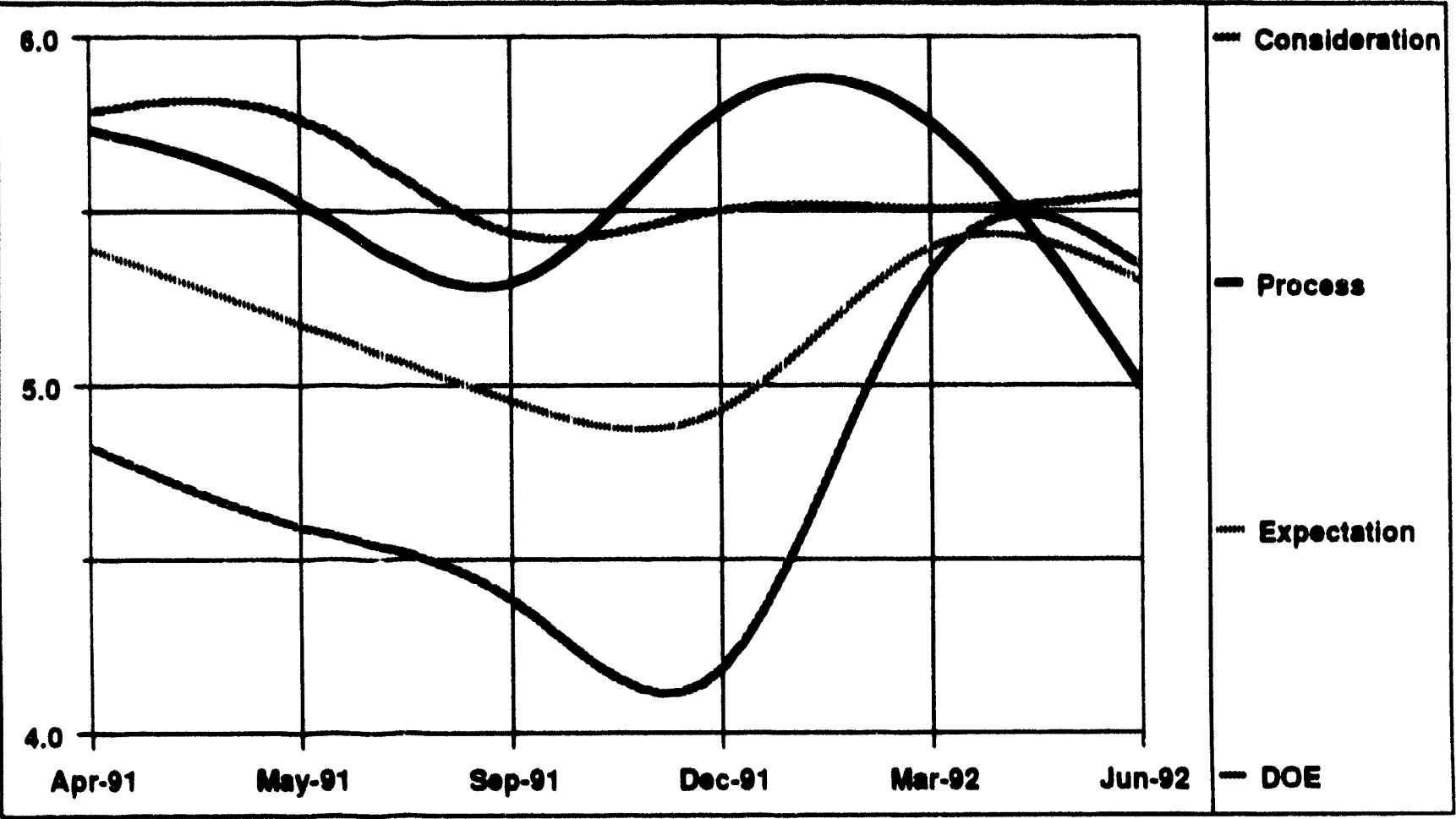

- March '92 and June '92 Responses Adjusted to 7-Point Scale 


\section{Appendix G}

Below are graphs of the $95 \%$ confidence bands for the Jistribution of the Harvey metric (ordinate) given various standard deviations of a single-subgroup population (abcissa).
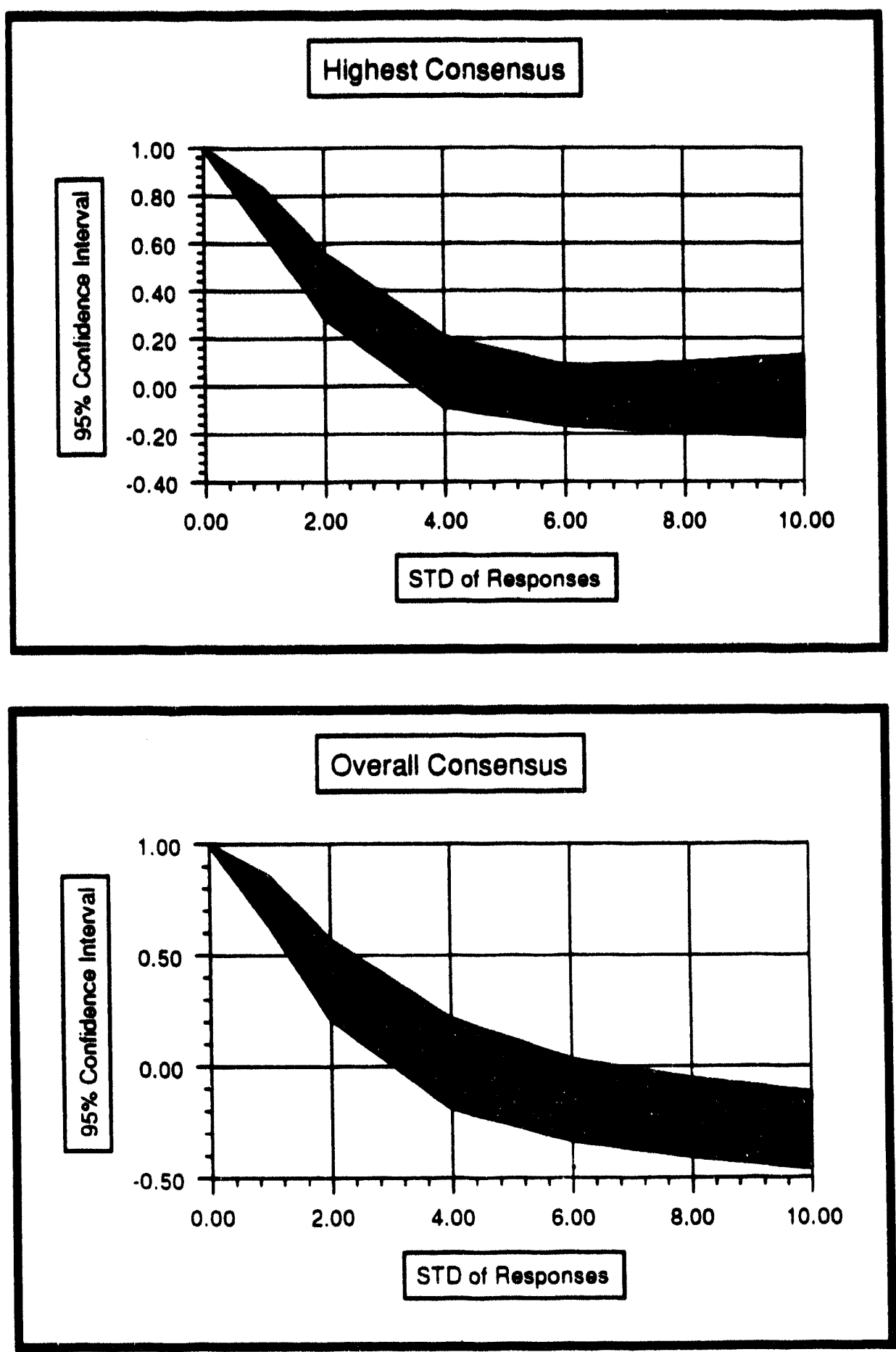


\section{Appendix H}

Below is a graph of the frequency distribution of Harvey metric scores for many sample populations having the indicated parameters. The graph displays the metric's normal distribution, the normality of which was also verified with the Kolmogorov test at the $20 \%$ alpha-level. (A cubic B-spline smoothing algorithm has been applied to increase clarity.)

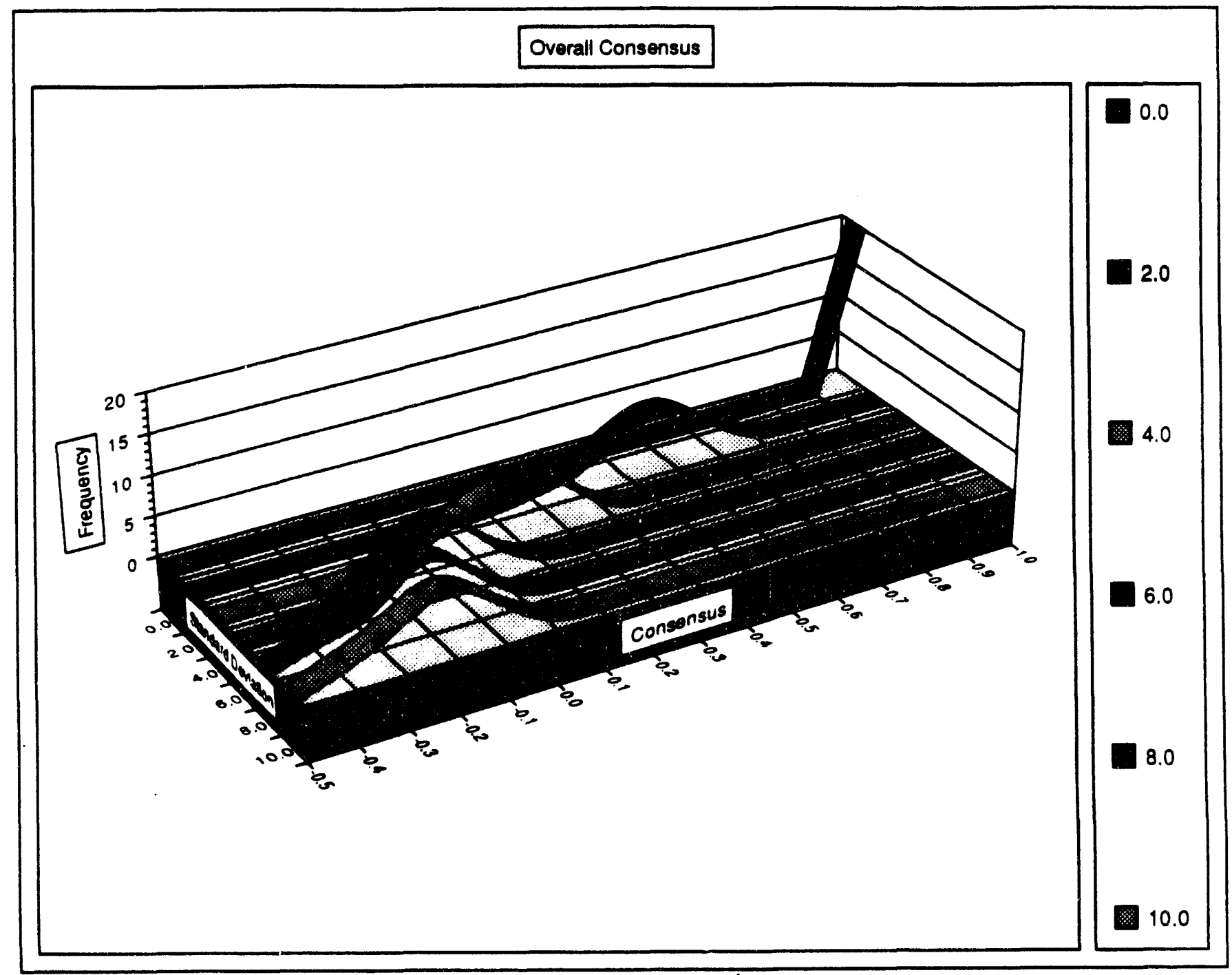




\section{Notes}

1. Webster's Ninth New Collegiate Dictionary defines consensus as "general agreement: unanimity" and as "group solidarity in sentiment and belief."

2. Harvey, Aubrey E. An Introduction to the Study of Consensus Measurement Tools. (Management Systems Laboratories, 1992).

3. An interval scale is one in which the distance between any two adjacent demarcations is constant; e.g. the difference between the the first and second responses on an interval answer scale is the same as the difference between the third and fourth responses. (This alone is insufficient for a ratio scale, since the zero point "anchor" is arbitrary.) [Gulliksen, Harold, Ed. Psychological Scaling: Theory and Applications (John Wiley \& Sons, 1960), p. 53]

4. A Likert scale is a selection of responses ranging from strong disagreement to strong agreement with a given statement. The response intervals are designed to represent equal spacing in the "attitude continuum." [Torgerson, Warren S. Theory and Methods of Scaling (John Wiley \& Sons, 1967), p. 47]

5. The rows of the matrix each represent one of the points (in some sequence), and each column also represents a point (in the same sequence). In the cell intersecting each row and column is placed the spatial distance between the two points represented by the row and column. In this fashion, all pairs of points are considered. For the "lower diagonal" matrix, only the cells below the diagonal stretching from the upper-left of the matrix to the lower-right have values. The cells on the diagonal itself will necessarily contain zero (the distance between each point and itself) and the cells above the diagonal will be redundant, as all possible pairs are considered in the lower half.

6. Clustering algorithms see frequent use in psychological scaling. For insight into various clustering algorithms so employed, see [Baird, John C. Fundamentals of Scaling and Psychophysics, (John Wiley \& Sons, 1978) p. $206 \mathrm{ff}]$. Noteworthy is the coverage of the Euclidian multi-dimensional distance metric, which is identically the "spatial distance" mentioned in the present paper. 
7. The width of the interval response scale is simply the number of options it contains minus one. The given value of $\boldsymbol{R}_{\max }$ represents an amount of disagreement among cluster members equal of $40 \%$ of this response scale width in either direction from the group's mean, simultaneously in all dimensions. Dr. Harvey felt this to be the maximum permissible disparity that could still marginally be considered a consensus. (A cluster with its radius beyond this limit, even if it contained the entire population, would have a negative consensus score which would have to be adjusted up to the minimum allowed score of zero. The consensus score for the population would then be determined by the scores of the individual members alone.) The numeric value of the $40 \%$ parameter is otherwise academic, since a change in its value would merely result in a recalibration of the metric's interpretation.

8. A prudent reader may inquire why a clustering algorithm is employed prior to the application of the Harvey metric. It would seem intuitively that all possible clusters of respondents should be tested for their consensus scores, and the cluster with the highest score then be chosen to represent the population. This assertion would be theoretically accurate, but entirely impracticable. The number of possible clusterings of points rises explosively as the population increases, according to the formula

$$
\sum_{n=2}^{N} \frac{N !}{(N-n) ! n !}
$$

where $\mathbf{N}$ is the size of the population and $\mathbf{n}$ represents the number of points to be clustered together for each term of the summation. As the population increases from two respondents to ten to twenty, the number of necessary clusterings increases from 1 to 1,013 to $1,048,555$. The utilization of the clustering algorithm eliminates such combinatorial explosion while creating a justifiable set of groupings. The inspiration for the use of the particular algorithm described in the body of this paper is the application of a similar algorithm in the taxonometric classification of biological organisms. Skeletal remains can be clustered into species by a comparison of the lengths of various structural components: it is a distance in space which determines the groupings.

9. The $25 \%$ delineation between major and minor partitions is intuitive and may 
be adjusted. With the $25 \%$ delineation, however, note that no more than 4 major partitions may coexist in the same population - a reasonable, easily handled figure. The coexistence of five ostensibly "major" partitions seems untenable, as one or more would likely be much smaller than the others.

10. In view of the scarcity in the literature of standardized terminology for the description of the degree of consensus in a population, the author has chosen to utilize instead phraseology which would (1) be understood by an uninitiated user, and (2) be interpreted with an acceptable degree of constancy across geographical, cultural, and educational boundaries. Further descriptive definition can be devised to further limit ambiguity, and will be undertaken in later research.

11. Indifference is a quality of two or more possible arrangements of subgroups in a population which produce the same populational consensus score. The metric's indifference means that "you have just as much work to do" to unify the population under any of those arrangements.

12. The normal distribution was chosen because the Dr. Harvey felt it to adequately describe the shape of the variability of human opinion within a defined subgroup. The standard deviation was adjusted to simulate varying amounts of agreement within the subgroup.

13. This follows the convention of W. J. Conover's Practical Nonparametric Statistics (John Wiley \& Sons 1971), in discussion about tests of the Kolmogorov-Smymov type.

14. The purpose of the Smymov test is to determine whether or not two samples can be considered to belong to the same population, and can therefore be combined into a single, larger sample. Thus, the Smymov tests whether or not two samples are "the same" or "different." 


\title{
SIMULATION OF CONSENSUS METRIC TEST SCENARIOS
}

\author{
Aubrey E. Harvey and Michael C. Kleder \\ Management Systems Laboratories \\ Department of Industrial and Systems Engineering \\ Virginia Polytechnic Institute and State University \\ Blacksburg, Virginia
}

\begin{abstract}
The behavior of the quantitative measure of group consensus proposed and calibrated previously for a single instant in time by the authors is extended to an analysis of the behavior of the measure through time in simulated group-dynamic scenarios.
\end{abstract}

\section{INTRODUCTION}

In accordance with its objective of continuously improving the effectiveness of its meeting facilitation activities, Management Systems Laboratories required in mid-1992 a quantitative measure of group consensus. ${ }^{\dagger} \mathrm{A}$ metric was proposed by Dr. Harvey at that time ${ }^{1}$ and has since been calibrated by the authors for the case of measurement at a single instant. $^{2}$ The present paper describes the authors' design and operation of a group-dynamic process simulator and the conclusions regarding the consensus metric that can be drawn from observations of the metric's behavior in those scenarios.

The computation of the consensus metric is summarized in the previous paper, "A Metric for Consensus - Design and Calibration." In brief, the consensus metric is a measure of the closeness of the opinions held by the members of a group, as indicated by their responses to a questionnaire with an interval re-

†. Webster's Ninth New Collegiate Dictionary defines consensus as "group solidarity in sentiment and belief" and as "general agreement: unanimity." sponse scale. A person's responses are numerically encoded (for example, onto a zero to ten scale) and interpreted as that person's coordinates in an opinion space having as many dimensions as there are questions on the questionnaire. The points in the opinion space are then hierarchically clustered using the Euclidian distance metric, using standard cluster analysis with one modification: the distance of a point from an existing cluster is calculated as its distance from the average of the points in the cluster rather than the average of its distances from each point in the cluster (i.e., distance from the mean instead of mean distance). This modification was chosen because it is the exterior point's conformity to a cluster's potential compromise position that is of interest.

After the questionnaire respondents have been hierarchically clustered, each cluster in the hierarchy is assigned a consensus score according to the formula:

$$
C=\frac{n}{N}-\frac{r}{4 \sqrt{D}}
$$


where $\boldsymbol{C}$ is the consensus score, $\boldsymbol{n}$ is the number of people in the cluster under consideration, $\boldsymbol{N}$ is the number of people in the population to which the questionnaire was administered, $\boldsymbol{r}$ is the spacial radius of the cluster, and $D$ is the number of dimensions in the opinion space (i.e., the number of questions on the questionnaire). The radius $r$ of a cluster is equal to the distance from the center of the cluster to its most remote member; that is, it is the maximum distance from any point in the cluster to the location specified by the average of the coordinates of all of the members of the cluster. The term radius was chosen because the length described is equal to the radius of the smallest circle or sphere (if there are two or three dimensions in the opinion space, for example) which can contain all of the members of the cluster while being centered at the average of the coordinates of those members.

Each cluster, with its contained questionnaire respondents, is recorded in a table together with the cluster's consensus score. The cluster with the highest consensus score is termed the consensus group, and any people not in that cluster are termed outliers. The consensus metric of the population is taken to be the score of the consensus group, but the term overall consensus score is reserved for the consensus score of the cluster comprising the entire population.

Each individual respondent to a questionnaire has a consensus score equal to the inverse of the total number of people in the population, by direct application of the above formula for a cluster with one member and zero radius. If this value is the highest consensus score in the cluster hierarchy, then the population has a consensus metric equal to this inverse of the population's size; thus, the population's consensus metric must be a positive real number.
In addition, the limiting case of everyone in a population providing identical questionnaire responses produces a consensus metric of one. Therefore, the consensus metric for a population will always be a real number in the range between the inverse of the population size and one. Note that every person and every cluster has a consensus score, but because the selection of the highest consensus score is a necessary step in its computation, there is only one consensus metric for a population. The preceding paper presented the test data and conclusions resulting in the following simple-language table of consensus metric interpretations for the case of a population consisting of a single powerful group and scattered outliers, and also for the case of a population with two or more faction-like groups:

\begin{tabular}{|c|c|c|}
\hline $\begin{array}{l}\text { Consensus } \\
\text { Metric }\end{array}$ & $\begin{array}{l}\text { Single } \\
\text { Group }\end{array}$ & $\begin{array}{l}\text { Multiple } \\
\text { Groupe }\end{array}$ \\
\hline 1.0 & $\begin{array}{l}\text { Abeolute } \\
\text { Unity }\end{array}$ & $\begin{array}{l}\text { Absolute } \\
\text { Unity }\end{array}$ \\
\hline 0.9 & $\begin{array}{l}\text { Negligible } \\
\text { Outliers }\end{array}$ & $\begin{array}{l}\text { Negligible } \\
\text { Inter-Group } \\
\text { Disagreement }\end{array}$ \\
\hline 0.8 & $\begin{array}{l}\text { Very Few } \\
\text { Outliers }\end{array}$ & $\begin{array}{l}\text { Very Close } \\
\text { Groups }\end{array}$ \\
\hline 0.7 & Few Outliers & Close Groups \\
\hline 0.6 & $\begin{array}{l}\text { Small Outlier } \\
\text { Segment }\end{array}$ & Close Groups \\
\hline 0.5 & $\begin{array}{l}\text { Small Outlier } \\
\text { Segment }\end{array}$ & $\begin{array}{l}\text { Slight Group } \\
\text { Disagreement }\end{array}$ \\
\hline 0.4 & $\begin{array}{l}\text { Significant } \\
\text { Outlier } \\
\text { Segment }\end{array}$ & $\begin{array}{l}\text { Moderate } \\
\text { Group } \\
\text { Disagreement }\end{array}$ \\
\hline 0.3 & $\begin{array}{l}\text { Major Outlier } \\
\text { Segment }\end{array}$ & $\begin{array}{l}\text { Discordant } \\
\text { Groups }\end{array}$ \\
\hline Lower & $\begin{array}{l}\text { Group Not } \\
\text { Significant }\end{array}$ & $\begin{array}{l}\text { Small, Weak } \\
\text { Discordant } \\
\text { Groups }\end{array}$ \\
\hline
\end{tabular}


In practice, a meeting facilitator would obtain the consensus metric for the population and then, having ascertained whether or not multiple groups exist via an analysis of the cluster table (or by intuition, if pressed for time), the facilitator would simply locate the appropriate cell in the above table and immediately find a qualitative description of the level of consensus in the population. Note that the number of groups in a population can be estimated by observing the number of distinct (non-overlapping) clusters in a population that have a higher consensus score than that of the cluster comprising the entire population (i.e., a consensus score greater than the overall consensus score).

\section{SIMULATION BACKGROUND}

In order to test the behavior of the consensus metric through time, the authors created a group-dynamic simulator with several variable parameters. Initially, the topics of interpersonal influence and momentum (viewed as self-feedback) suggested a neural network algorithm for simulating the evolution of opinions within a population. The opinion of each person on each issue becomes a node in the neural network capable of influencing other nodes and itself. The activation level of each node corresponds to the quantified opinion of a particular person regarding a particular issue, and each influence weight corresponds to a quantification of the influence that one person's opinion about an issue has on that same or another person's opinion about that same or another issue. For example, our simulated questionnaire with 5 issues (questions) queries a simulated population of 12 people, for a total of 60 nodes. Total twoway interconnectivity among opinion nodes, plus self-feedback influences, requires 3,600 influence links, ${ }^{\dagger}$ so to reduce complexity we allow influence between people only within the same issue ("interpersonal influence"), and influence between issues only within the same person ("cognitive process"). The resulting neural network contains the same sixty nodes but only 960 influence links. ${ }^{\ddagger}$ For the purpose of viewing the neural network graphically, the reader can picture the "cognitive process" links as an information "bus line" among the opinion nodes within each person and omit explicit visualization of each node's feedback influence, so that the interconnectivity is visualizable as in the diagram below. Each of the five issues is represented

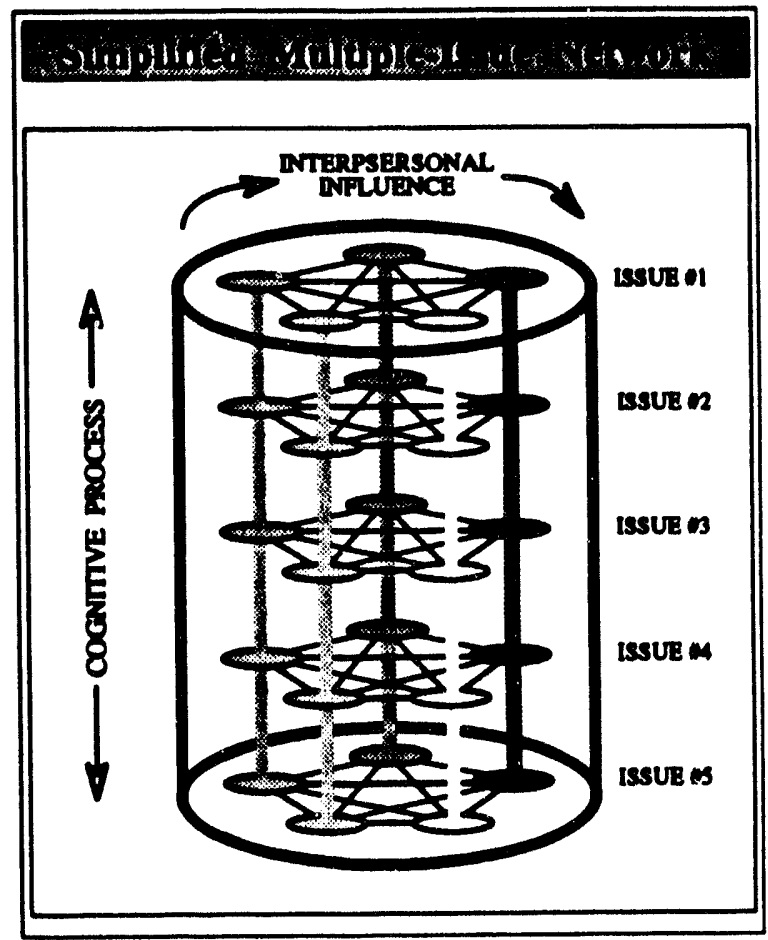

t. Each of the 12 people has 5 opinion nodes, for a total of 60 nodes, each of which sends outgoing information to itself (feedback) and to the 59 other nodes: $12 \times 5 \times(1+59)=3,600$

F. Each of the 60 opinion nodes now sends outgoing information to itself (feedback) and to 11 other nodes within the same issue, as well as to the four other nodes within the same person. The node provided its feedback as part of the interpersonal influence; it does not need to do so again as part of the cognitive process:

$60 \times[(1+11)+4)]=960$ 
by a set of five interconnected nodes lying in a horizontal plane, and each of five people (for simplicity) is represented as a vertical stack of nodes with an interconnecting influence "bus line".

For our study, we chose to simulate a questionnaire with properly formed independent questions, so that a person's opinion on one issue is independent of his or her opinion on any other issue. Thus, the "cognitive process" influence weights vanish. Additionally, the neural network is not required to "learn" anything, but rather merely to reach a stable equilibrium (to allow measurement of consensus attainment); therefore, it was not necessary to choose any particular transfer function to optimize a learning process. So long as any node's incoming influence causes its activation level to become a weighted average of the activation levels of other nodes and its own feedback, no transfer function is necessary to keep the node's activation level within the bounds of the opinion space (i.e., within the questionnaire response range).

With the above considerations, a particular person's opinion about a particular issue changes during an interval of time via the group process according to the following equation:

$$
a_{i j}^{\prime}=\sum_{k=1}^{N} w_{i k} a_{k j}
$$

where $a^{\prime} i$ is the $i$ th person's opinion about issue (or question) $j$ after the time interval has elapsed, $N$ is the number of people in the population, $w_{i k}$ is the weight of influence effected on person $\boldsymbol{i}$ by person $\boldsymbol{k}$ per time interval, and $a_{i k}$ is the the person's opinion about issue $k$ at the beginning of the time interval. It is apparent that a person's opinion about an is- sue after an interval of time is simply the weighted average of the opinions of everyone in the population prior to the time interval. ${ }^{\dagger}$ Because of this, we can define the weight of influence from one node to another as that portion of the influenced nodal opinion which we wish to bring into conformity with the opinion of the influencing node, during a standard time interval. Self-feedback is simply the weight of influence a person has on himself or herself, so if person $i$ is slow to change opinions, then $w_{u}$ would be quite high, and $w_{y}$ where $i \neq j$ would be low. Further, since the set of $w_{y}$ where $t$ is held fixed but $j$ ranges from 1 to $N$ represents the weights for the weighted average of incoming influences to person $i$, they must add up to exactly one. Outgoing influences do not have to be similarly normalized; one person may simply be much more influential than another.

Clearly, the above equations for $i h j$, and the index variable $k$ can be compressed into the following matrix equation:

$$
\mathbf{A}_{t+1}=\mathbf{W} \mathbf{A}_{t}
$$

where $t$ represents an iteration count. Below are examples of a properly normalized influence matrix $W$, and an initial opinionation matrix $A_{0}$. Notice that the rows in $\mathbf{W}$ each sum to one (each row specifies the weights for the weighted average influx of influence) but the columns do not (arbitrary outgoing influence). The highlighted diagonal entries are the self-influence feedback weights. With $100 \%$ feedback, persons $\# 1$ and $\# 12$ are zealots - they will never deviate from their initial opinions. For this particular hypothet-

\footnotetext{
t.This weighted average result is characteristic of neural network algorithms with normalized influence weights and no transfer function.
} 
SAMPLE INTLUENCE MATRIX:

\begin{tabular}{|c|c|c|c|c|c|c|c|c|c|c|c|c|c|}
\hline \multirow[b]{2}{*}{ Infiuenced: } & \multicolumn{4}{|c|}{ Influencer: } & \multirow[b]{2}{*}{5} & \multirow[b]{2}{*}{6} & \multirow[b]{2}{*}{7} & \multirow[b]{2}{*}{8} & \multirow[b]{2}{*}{9} & \multirow[b]{2}{*}{10} & \multirow[b]{2}{*}{11} & \multirow[b]{2}{*}{\begin{tabular}{|l|}
12 \\
\end{tabular}} & \multirow[b]{2}{*}{ Sum of Rows } \\
\hline & 1 & 2 & 3 & 4 & & & & & & & & & \\
\hline 1 & 1 & 0 & 0 & 0 & 0 & 0 & 0 & 0 & 8 & 0 & 0 & 0 & 1 \\
\hline$\overline{2}$ & .10 & .62 & .02 & .02 & .02 & .02 & .02 & .02 & .02 & .02 & .02 & .10 & 1 \\
\hline$\overline{3}$ & .10 & .02 & .62 & .02 & .02 & .02 & .02 & .02 & .02 & .02 &.$\overline{02}$ & .10 & 1 \\
\hline 4 & .10 & .02 & .02 & .62 & .02 & .02 & .02 & .02 & .02 & .02 & .02 & .10 & 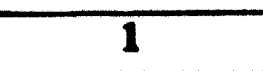 \\
\hline$\overline{5}$ & .10 & \begin{tabular}{|l|}
.02 \\
\end{tabular} & .02 & .02 & .62 & .02 & .02 & .02 & .02 & .02 & \begin{tabular}{|l|}
.02 \\
\end{tabular} & .10 & 1 \\
\hline$\overline{6}$ & .10 & .02 & .02 & .02 & .02 & .62 & .02 & .02 & .02 & .02 & .02 & .10 & 1 \\
\hline 7 & .10 & \begin{tabular}{|l|}
.02 \\
\end{tabular} & .02 & .02 & .02 & .02 & .62 & .02 & .02 & .02 & .02 & .10 & $\overline{1}$ \\
\hline 8 & .10 & .02 & .02 & .02 & .02 & .02 & .02 & .62 & .02 & .02 & .02 & .10 & 1 \\
\hline 9 & .10 & .02 & .02 & .02 & .02 & .02 & .02 & .02 & \begin{tabular}{|l|}
.62 \\
\end{tabular} & .02 & .02 & .10 & $\mathbf{1}$ \\
\hline 10 & .10 & .02 & .02 & .02 & .02 & .02 & .02 & .02 & .02 & .62 & .02 & .10 & 1 \\
\hline 11 & .10 & .02 & .02 & .02 & .02 & .02 & .02 & .02 & .02 & .02 & .62 & .10 & 1 \\
\hline$\overline{12}$ & 0 & 0 & 0 & 0 & 0 & 0 & 0 & 0 & 0 & 0 & 0 & 1 & 1 \\
\hline Sum of Columns: & 2 & .80 & .80 & .80 & .80 & .80 & .80 & .80 & .80 & 80 & .80 & 2 & \\
\hline
\end{tabular}

ical scenario, those two people also have extremist viewpoints, as can be seen from the sample initial opinionation matrix below:

\begin{tabular}{|c|c|c|c|c|c|}
\cline { 2 - 5 } \multicolumn{1}{c|}{} & \multicolumn{2}{c|}{ Issue } & \multicolumn{3}{c|}{} \\
\hline Person & 1 & 2 & 3 & 4 & 5 \\
\hline 1 & 0 & 1 & 2 & 1 & 3 \\
\hline 2 & 6 & 5 & 4 & 7 & 8 \\
\hline 3 & 4 & 8 & 4 & 4 & 5 \\
\hline 4 & 4 & 6 & 3 & 6 & 5 \\
\hline 5 & 6 & 5 & 7 & 3 & 4 \\
\hline 6 & 8 & 5 & 7 & 7 & 8 \\
\hline 7 & 4 & 7 & 8 & 4 & 6 \\
\hline 8 & 4 & 6 & 5 & 7 & 9 \\
\hline 9 & 8 & 2 & 3 & 2 & 5 \\
\hline 10 & 8 & 7 & 4 & 5 & 4 \\
\hline 11 & 4 & 6 & 5 & 7 & 9 \\
\hline 12 & 9 & 10 & 8 & 8 & 9 \\
\hline
\end{tabular}

The actual consensus simulations allow noninteger opinions, to allow simulation of a continuous distribution of actual attitude rather than merely a simulation of integer questionnaire response data.

For the neural network group-dynamic process simulator described above, because a node's activation level conforms to a weighted average of it's feedback and it's received influence, a node will be more influenced by distant members in the opinion space and less influenced by close members. Thus, the population will converge rapidly at first and then more slowly as the members get closer together. This concave convergence pattern is illustrated below, and is inescapable under the above-described neural network paradigm.

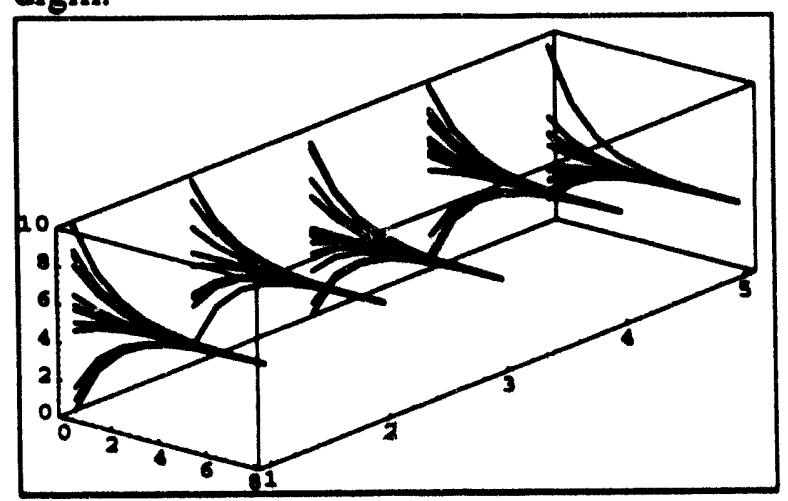


In the graph, the issues are numbered 1-5 on the receding axis, the iteration count is on the approsching axis, and the opinion range 0-10 is marked off on the vertical axis. Each line in the plot region is a trace of the opinion of an individual changing with time. The above neural network convergence produced the following typical behavior of the consensus metric, as plotted against the iteration count:

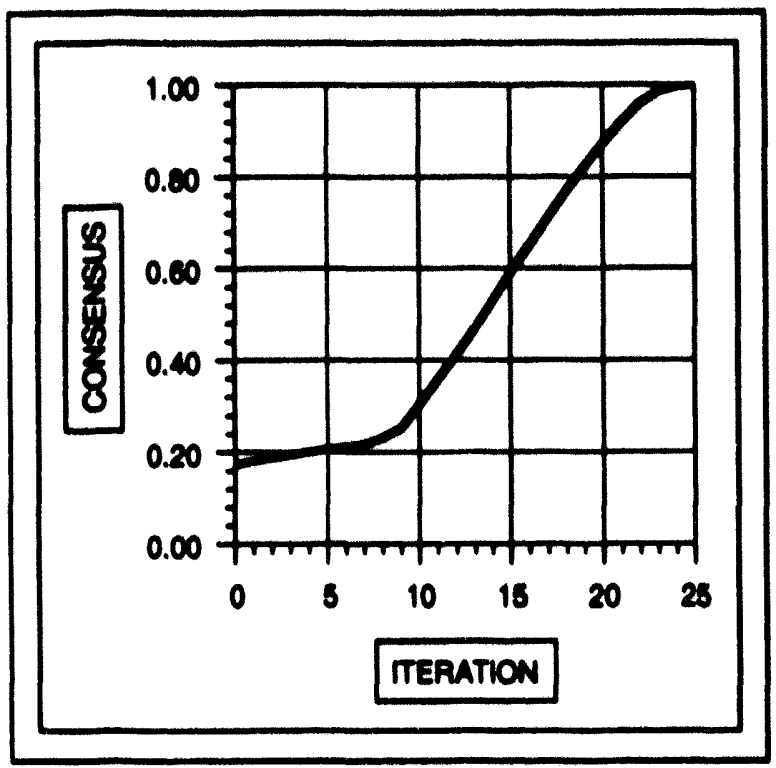

The reader will notice from the table of consensus metric interpretations presented earlier that the consensus metric is "tuned" to be very sensitive to slight improvements in a closely agreeing population, but less sensitive to the precise degree of disagreement in a discordant population. The metric is designed to present itself as most useful to a group administrator precisely at such time as improvements in the disposition of the consensus attainment process become delicate. This is illustrated here. The two preceeding graphs are drawn from different $W$ and $A_{0}$ matrices with different convergence times (the iteration counts reached before the consensus metric approached one) to illustrate that under the neural network paradigm the group's behavior always followed a consis- tent pattern: rapid initial convergence while in a state of discord, to which the consensus metric is tuned to respond slowly, and then slower convergence once a semblance of agreement is reached, when the consensus metric becomes very sensitive to small improvements. This behavior was evident under all of the following simulated scenarios:

(1) Random Ao matrices and random but normalized $W$ matrices;

(2) One, two, or three factions within the Ao matrix where a faction was simulated as a subgroup of four members of the population wherein the probability distribution from which the opinions were stochastically generated had a standard deviation of 1 unit on a zero to ten scale, and the remainder of the population was randomly distributed;

(3) One, two, or three uninfluenceable members of the population with random or deliberately diametric (zero vs. ten) initial opinions, and the remainder of the population randomly distributed.

From the consistent agreement of the behavior of the consensus metric through time in scenrios of random group disposition, factionation, and zealotry with that behavior for which it was desiged we conclude that under this neural network paradigm of group dynamics, the consensus metric performs exactly as expected with regard to its sensitivities to group cohesion under discordant and concordant conditions. Specifically, the metric has demonstrated itself fit and useful for concavely convergent populations. It still remains to be determined whether or not the metric performs as expected for convergences of linear or convex shapes or for divergent populations.. 
(Divergences will be handled by parity symmeaty of time and the independence of the metric of the order in which the measuremeats are taken; convex convergences will be handled with the modified group-dyanmic simulator..)

SUMMARY

REFERENCES

1. Harvey, A. E., An Introduction to the Study of Consensus Measurement Tools. Mrangement Sycee Laboratories (1992).

2. Harvey, A. E. and M. C. Nader, "A Metric for Consensus - Design and Celibrudoa." Computers and Industrial Engineering, Vol. 25, pp. 179-182. (1993).

3. Ibid. 
The Effect of Expert Knowledge on Performance in a Small Group Decision Making Task

\author{
John W. Polk, Jr. \\ Thomas F. Carolan \\ Elizabeth H. Koball
}

Consensus groups are created when individuals who may or may not have a stake in a mutual problem are brought together to find a solution. These individuals often possess knowledge or expertise on one or more aspects of the problem. The group interaction results in a group decision and a level of individual consensus for each member. The primary reasons for decision-making by consensus group are to increase the acceptance and quality of the group's decision. Each group member's apreement with, acceptance of, and commitunent to the decision is considered an important outcome of the decision process. The role of an expent is to provide information to improve decision quality without hindering consensus. Like any other individual the expert must be able to get his or her knowledge across to the group. If the expert dominates the group interaction, other menibers may perceive they had litule opportunity for input. If the expent does not effectively communicate, his or her information may nor be adequately considered and the quality of the group decision may suffer.

This research investigates the effects of an expert on the strength of consensus and decision quality when the expert is an integral part of a consensus group trying to reach a shared ranked-list decision. Strength of consensus is the degree to which the group members accept and agree with the decision, and decision quality is the degree to which the decision meets an external evaluation criterion. The effect on group performance of including an individuals with one of two types of knowledge is compared. A content expert is an individual with rechnical knowledge that is relevant to the problem. A process expert is an individual with knowledge of methods to use to solve a particular type of problem.

\title{
GROUP DECISION MAKING
}

\section{Consensus and Decision Quality}

In the context of small group decision making, the concept of consensus can refer to either a state of agreement to be achieved (e.8.. Shils, 1968) or a process of achieving group objectives (e.g., Hall, 1971). Consensus as a group state is said to exist when most of the individuals concerned have reached a common judgment for which they are willing to show some level of acceptance, agreement, and commitment. Some authors define consensus as unanimous agreement (Forsyth, 1983; Hirokawa, 1984; Rawlins, 1984). Other authors recognize degrees of consensus (Kaprzyk \& Fedrizzi, 1988; Price, 1972). The level of individual consensus can vary from conformity, where the individual only agrees publicly, to private intermalization, where the 
individual has a strong belief in and is committed to the group decision. Public agreement without private commitment is a false consensus (Scheff, 1967).

Consensus as group process refers to a method or strategy that encourages active participation from all members of the group and has as its objective a group decision that reflects the views of all the members (Wood, 1984). Various authors have developed guidelines for groups to follow to gain consensus (e.g., Hall, 1971; Hare, 1952; Johnson, 1975). Most consensus guidelines stress the importance of group members expressing their views and being listened to and understood. A group can achieve a state of relative consensus without using a consensus building process. Altematively, a group can use a consensus building process and never achieve a state of consensus. However, the goa of using a consensus decision making process is to reach a state of consensus about a decision and also to reach a higher quality decision than would be achieved by other methods. The more individual group members perceive they had input into the group process, the stronger the consensus. Individuals who have input into a decision tend to identify with it and perceive it to be "their decision" (Yukl, 1989).

Research supports the view that a consensus process affects both the group's decision and group members' satisfaction. Effmeyer and Lane (1984) compared the quality and the acceptance of group decisions across four decision-making formats and found consensus groups had the highest levels of acceptance while still maintaining a high decision quality. Decision quality is a measure of how close a decision is to some external evaluation criterion. The evaluation criterion can be a correct decision or solution, a best decision as defined by experts, or by the consequences of the decision. The relationship between decision quality and consensus process has not been consistently validated by research (Hirokawa, 1984; Hare, 1980). It is possible for groups to agree on a poor decision. However, for tasks that are dependent upon individual sharing specific information to solve a well defined problem consensus groups generally perform better than the average individual.

Effective communication and conflict management are considered important components of the consensus process. Groups with little communication will likely have low consensus and quality. Therefore, opportunity to express views is an important facet of a group's communication. Good ideas won't improve decision quality without being communicated to the rest of the group. Conflict within groups when not managed properly, may also decrease both consensus and decision quality (Katz \& Kahn, 1972). However, conflict does not necessarily detract from group goals. When skillfully managed, conflict can contribute to effective performance by improving both decision quality (by considering more ideas) and consensus (by increasing understanding of issues)(Tjosvold, 1987).

When the group's goal is to reach a best possible decision the immediate task of each individual group member is to share opinions, preferences, and knowledge to achieve a consensus through mutual influence. Each individual has the task of either changing their own position, influencing others to change their position, or a litule of both (Stasser, Kerr \& Davis, 1989). We can characterize this process for each individual as converting knowledge, beliefs and preferences to influence. 


\section{Influence}

Influence, considered as the attitudinal and behavioral effects of one person on another (Hopkins, 1964) is a central construct in group decision making research. In many ways the study of group decision dynantics is the study of influence processes among individuals in groups. In a small group process, each group member affects the other group members' opinions. These murual social influence processes change the distribution of opinions over time and allow groups to reach consensus (Pauius, 1989). The interaction resules in the group decision and a level of individual consensus for each group member

The type of influence can affect the quality of the decision and each group member's degree of individual consensus with the decision (Jackson, 1975). The dominant theory of social influence suggests that shifts in group members' opinions are a result of a combination of informational and normative influence processes (Deutsch \& Gerard, 1955). Informational influence refers to influence based on reducing subjective uncertainties by sharing facts or persuasive arguments about the issue. Informational influence leads group members to change their opinion on the belief that another alternative is a better solution. Therefore, informational influence leads to both public and private decision support. Normative influence refers to conformity to implicit decision norms and others' preferences (Kaplan, 1987). Normative influence involves group members conforming to others' ideas leading primarily to public decision support. Based on this framework the informational dependence on others in a primary source of influence that leads to the group structure. Other theorists however, view group formation as a prerequisite to influence. From this perspective informational influence can only come from individuals with whom one expects to agree. An individuals confidence in the validity of his or her opinion is a function of the degree to which similar individuals would agree with that opinion (Tumer \& Oakes, 1992).

Research has suggested that minority and majority influence may involve different underlying processes. Moscovici and Lage (1976) found minorities' influence caused both behavioral changes and opinion changes. Majorities, however, primarily exert influence on behavior, not opinions. From this perspective minority influence requires the majority to consciously consider the minorities' opinion and decide the opinion is better. Since this is more difficult than conforming to the majority, minorities can only exert influence in some situations. The majority will often discredit the minorities' opinion if the minority isn't consistent, it consists of only one person, or the minority differs from both the group's opinion and their social categorization (Maass \& Clark, 1984, Moscovici \& Lage, 1970).

A number of factors have been found to affect influence in groups. The status and knowledge of the source of the influence and its proximity in time and space affects the amount of influence it has on the group decision (Latane, 1981). Social influence has also been found to be affected by the number of individuals that make up the influence source and individual differences in susceptibility to persuasion (Tanford \& Penrod, 1984). Some strategies are more effective than others for increasing influence. Assertiveness, ingratiation, coalition building rational persuasion and inspirational appeal have been found to be effective influence tactics (Kipnis, Schmidt, and 
Wilkinson, 1980; Yukl and Tracy, 1991). Individual differences in communication skills and personality characteristics have been shown to affect how much influence a member has over the group (Hoffman \& Smith, 1960; Bouchard, 1969; Haythorn, 1953). Characteristics such as maturity, adaptability, and acceptance of others have also been positively related to effective group functioning (Vroom, 1959). Bottger (1984) investigated the relationship among quality of verbal contributions, quantity of verbal contributions, and group member's influence. He found the while quantity of a group member's contributions increased the other group member's attributions of influence, the individuals expertise was a better predictor of the actual influence on the group decision.

Factors that affect the structure of a group also affect the group dynamics and the resultant distribution of influence (e.g., Hackman and Morris, 1975). The type of structure the group develops, including role differentiation and attraction patterns can influence decision quality, and strength of consensus (Doreian, 1986; Forsyth, 1983). Group size and level of cohesiveness affect group performance (Forsyth, 1983; Yukl, 1989). Highly cohesive groups can have both positive consequences and negative consequences (e.g., Janis, 1972). Group homogeneity with respect to personality is another group factor that has been found to affect performance (e.g., Hoffman, 1959; Hoffman \& Claggett, 1960; Wanous \& Youtz, 1988). Hoffman (1959) found heterogeneous groups perform better on problems where quality was important but found no difference in decision acceptance.

Individual consensus may be more a function of how individuals perceive the group process and their influence in the decision. Research has shown that perceived decision quality and perceived influence in the decision increases an individual's private strength of consensus (Hoffman, 1959). A group member's perception of influence has been shown to be a positively relationed to acceptance of that decision (Effmeyer \& Lane, 1984; Hoffman \& Maire, 1961). Silk and Kalwani (1982) found people aren't good judges of the amount of influence they have in a decision. Differences between perceived and actual influence can result from individual cognitive skills and biases and from the joint information sharing process (Corfman, 1991). Group members opinions evolve as information is made available through group discussion and mutual influence. This mutual process may affect each individuals ability to accurately judge their influence. In addition people are inclined to perceive themselves to be similar to their self-image. For example, people who consider themselves to have some expertise might overestimate their influence to support their self-image.

\section{Task Type}

The type of task has an impact on the type of influence group members will use and the possibilities for consensus. Laughlin (1980) distinguishes between intellective and judgmental tasks. With intellective tasks, group members try to reach agreement on a correct answer, with judgmental tasks, group members try to reach consensus on a preferred position. Kaplan and Miller (1987) found on intellective tasks, group members relied on more informational influence, whereas on a judgmental task, group members relied more on normative influence. In an 
intellective task, individuals with greater knowledge about the subject should be in the best position to convert that knowledge to influence; research supports this argument (e.g., Kirchler \& Davis, 1986). Intellective tasks have solutions that can be evaluated against some external criterion, and therefore a quantitative measure of decision quality can be assigned. Judgmental task typically do not have an external evaluation criterion; therefore, the decision is evaluated by the solution's ability to satisfy group members. In consensus decision making the objective is usually to maximize both of these measures [decision quality and acceptance] even though the criterion are often qualitative (Miner, 1972).

Using Steiner's $(1972,1976)$ task typology, tasks that are disjunctive (individual members contribute different pieces of information), discretionary (the group decides how to integrate the different pieces of information) optimizing (there is a best solution) and compensatory (group product is a compote of individual inputs) provide a way to assess the contribution of each individual's knowledge and influence to the final decision. Groups perform better on disjunctive tasks because groups stand a better chance of having a member who knows the correct answer (Marquart, 1955). This conclusion assumes that "truth wins." In other words, the individual with the correct answer must convince the other members he or she is correct. Shaw (1981) concludes that groups perform better than individuals on compensatory tasks. The averaging process tends to remove extreme answers.

\section{Expertise and Group Interaction}

In a consensus group process one individual may emerge as a leader, perhaps because the individual has more task competence or communication skills than others. The emergent leader gains status in the group and is allowed to have greater influence over group decisions. The amount of influence is proportionate to the group's evaluation of the person's potential contribution to the group. These contributions may take the form of access to information, or skill at group problem solving or interpersonal relations. Hollander (1960) proposed the concept of idiosyncrasy credits to explain emergent leadership. A person gains idiosyncrasy credits by showing competence and by conforming to group norms. These credits can then be used to deviate from nonessential group norms and to be more innovative.

In knowledge-based tasks, emergence can be explained largely based on subject expertise. Expertise in a particular task or subject area can be defined in terms of knowledge, skill, or experience relative to other individuals in that area. Perceptions of expertise can vary based on credentials, years of experience, past performance, or various personal characteristics of the expert. Persuasive skills, combining logical arguments with good communication, affect the expert's ability to influence others, and therefore affect others" perception of his or her expertise.

One way of characterizing the potential influence an individual has over the attitudes and behavior of other individuals is through the concept of power. Research that uses power as an explanatory construct (e.g., Yukl, 1989) use the taxonomy of power bases developed by French \& Raven (1959). An expert has a base of power over other group members to the extent that group 
members need the expert's knowledge or information to solve their problem. Hinkin and Schriesheim $(1989$, p. 562) define expert power as "the ability to administer to another information, knowledge, or expertise." Expert power is commonly exercised as rational persuasion (Kipnis, Schmidt, Price, and Stitt, 1981). The expert uses logical arguments with supporting evidence to convince others of the "correctness" of a decision. For expertise to be a source of power the expertise must be recognized and the expert perceived as reliable (Yukl, 1989). The judgment of the reliability of the expert's information depends on how convincing the expert's arguments are. For certain tasks, group members need an expert's information to perform the task effectively and efficiently. The use of expert power can, however, have a negative influence on quality and consensus if the expert misuses his or her position (e.g., Podsakoff \& Schriesheim, 1985). Individual group members may resist influence even if they agree with the expert's position when they believe they have relevant information or ideas that are not being considered (McClelland, 1975). Successful use of expert power depends on the expert's logical ability and communication skills (Yukl, 1989). In a knowledge oriented task, an individual with expertise is more likely to have ideas that match some ideas of each group member and therefore be perceived as the person who has the most in common with each individual group member. Conceptualizations of emergent leadership and expert power suggest that in intellective or knowledge oriented decision making tasks, the individual with expertise combined with the ability to communicate it effectively will emerge as a leader and therefore have the most influence on the group decision.

\section{Presence of an Expert and the Perception of Decision Quality}

The more experts use their expert power, the more their information is injected into the group decision. If group members perceive the expert's knowledge as accurate, they should perceive that using this knowledge will result in a higher quality decision. Perceptions of accuracy will depend on how salient the expertise is and whether or not the expert's information conflicts with other group members' knowledge. Whether or not the expert's knowledge conflicts with other group members' knowledge will depend on the accuracy of both parties' knowledge. Hovland and Weiss (1951) found that subjects' opinions were changed in the direction advocated by a communication to a significantly greater degree when the material was presented by a credible source than when presented by a non-credible source. Members of groups with an expert should have a higher perception of decision quality than members of groups with no expert.

\section{Methodology}

\section{Hypotheses and Design}

An experiment was designed to evaluate the effect, on decision quality and consensus, of including an individual with either content or process expertise in a small group decision task. The experimental study discussed in this paper focused on group level effects of the experts. A relational study focused on the individual unit of analysis and is discussed in another paper 
(Walsh, Carolan \& Koball, 1994). Since the focus is on group consensus and decision quality, not individual consensus and decision quality, a group level of analysis was used to test hypotheses.

A one factor (expertise), three level (content expert, process expert, no expert) design was used to evaluate the following hypotheses:

(1) The presence in the group of an individual with either task specific content or process knowledge will increase the quality of the group decision. Content expertise is expected to contribute more to decision quality than process expertise.

(2) Members of groups with either a content or a process expert will have a greater perception of decision quality than members of groups with no expert and will therefore have a higher rating on individual consensus.

(3) Group members will be more likely to accept a decision if they believe their views were considered by the group, therefore scores on a measure of perception of opportunities to express views should be positively related to scores on a measure of individual consensus.

\section{Subjects}

Three hundred and eight undergraduate students from a large land grant university were randomly placed into 77 four-person groups. Each group consisted of three members and one expert. There were 231 subjects recruited from an introductory psychology class who served as the group members. The 77 experts were recruited from the sociology pool. The 231 psychology students received three points toward their final class grade for participation; sociology students received \$20. Performance in the task had no bearing on either reward.

\section{Task \& Procedures}

The experimental task for this experiment was the Lost on the Moon exercise. This exercise was developed by Hall (1971) to study consensus groups and is characterized as a decision-making task (choosing among several alternatives) rather than a problem-solving (generating new solutions) task (Hoffman and Smith, 1960). The exercise has subjects assume they are crew members who have just crash landed on the moon. They are asked to rank (individually and as a group) fifteen items according to their importance for survival. When groups discuss the problem, they are told they must reach a consensus because they must stick together for survival. The groups' task is to reach the highest quality solution to a ranking exercise and to reach the strongest consensus on that solution. Each subject completed the ranking exercise three times: initial individual ranking, group ranking, and final individual ranking. The Crew Equipment Research Section of the NA.SA Manned Spacecraft Center at Houston, Texas generated an expert answer for the exercise. A quality score can therefore be computed for each individual and for each group based on how close a ranking matches this expert ranking. A high quality score implies that specific knowledge about the moon was available to the group and that the group was able to access and use that knowledge. The task is therefore an appropriate task on which to investigate factors which mediate the convergence of individual knowledge on a consensus 
decision.Other researchers who have used the Lost on the Moon exercise or similar survival exercises include Hall and Watson (1970), Herbert and Yost, 1979), Tjosvold and Field (1983), and Wanous and Youtz (1986).

Approximately one week prior to the experimental session, the sociology students received one of three types of training. In the subject or content expert condition, sociology students were tested on their knowledge of the moon, given a lecture on the moon, and then retested on their knowledge. This training was derived from NASA's rationale for the correct ranking of the fifteen items. This information was also used to develop the test given to experts. The questions on the test included information useful for completing the exercise and additional information so the test wouldn't directly prompt responses on the exercise. In the task or process expert condition, sociology students were led through two survival exercises similar to the NASA moon survival exercise and given tips for solving these types of exercises. In the control condition, sociology students were given no training. In all three conditions, they were told the other members of their group were psychology students who wouldn't have participated in a pre-experimental session. The initial individual quality score on the Lost on the Moon exercise was used as a manipulation check for expert knowledge.

During the experimental sessions, the experimenter explained the task instructions and told the subjects their goal was to reach the best decision possible. Participants signed a consent form and were given ten minutes to perform the individual-ranking step. They were asked to work silently prior to the group-discussion step. Groups were formed with one expert (trained or untrained) and three randomly placed participants. Before performing the task, group members introduced themselves by giving their name, major, and any classes or hobbies they believe would help them solve the problem. This was intended to let other group members know one member of their group had some knowledge of the moon without labeling that person an expert. A proctor collected the individual ranking and explained the task to the group. Groups were allowed 30 minutes to interact and rank items. All groups reached consensus within 30 minutes. Participants were then given ten minutes to perform a final individual ranking. After the final individual ranking, they filled out the post experiment questionnaires and the personality test. The subjects were asked not to discuss the exercise with anyone since the experiment was completed over four days.

\section{Dependent Measures}

The dependent variables for this study are strength of consensus and decision quality. Two variables are proposed that intervene in the relationship between presence of an expert and strength of consensus: group members' perceptions of opportunity to express views and group members' perceptions of information sharing.

Decision quality is defined as the closeness of the group's ranking to the ranking developed by NASA experts. "Closeness" was computed by summing the absolute difference between the group's rank for an item and NASA's rank for that item. Lower scores indicate higher quality. Individual decision quality was also computed by comparing individual rankings to the NASA 
ranking. The group member's initial individual quality score was used as a measure of the initial knowledge the individual brought to the group. A low score indicates high initial knowledge. Group quality scores were used to see if the presence of an expert increased decision quality.

A post-interaction questionnaire was used to measure individual consensus, perceptions of opportunity to express views, and perceptions of information sharing. The questions were generated based on questionnaires found in the literature (Bouchard, 1969; Hoffman, 1959; Knutson \& Holdridge, 1975; and Mahler, 1987). Perceived opportunity to express views and therefore influence the final decision was measured with a scale derived using nine questionnaire items. Through a factor-analytic study using least squares and maximum likelihood estimation and varimax and promax rotations, a 4-item questionnaire was derived. The questions used to measure the construct of influence focused primarily on the individual's perception of opportunity to express views. So while we originally conceived of this variable as perceived influence, it is, more accurately, perceived opportunity to influence. Individual consensus was measured with a 10-item scale also derived from the factor analysis. This scale contains both the items from a consensus scale and from a perceptions of decision quality scale and addresses acceptance of, agreement with, and commitment to the group decision by group members. For all questionnaire measures, individual ratings were summed to get group measures.

\section{RESULTS}

\section{The Effect of Type of Expert on Group Decision Quality}

A single factor, three condition (subject expert vs. process expert vs. non expert) multivariate analysis of variance (MANOVA) was computed on the three group level dependent variables (group quality score, group gain in quality, and quantitative group consensus). The overall Wilks' Lambda was significant, $F(6,144)=2.67, p<.05$. The analysis for the individual dependent variables revealed a main effect of type of expert on the group decision quality score, $F(2,74)=7.54, p<.01$; a main effect of type of expert on the group gain in quality, $F(2,74)=$ $3.16, p<.05$; and no effect for the quantitative consensus score. Post hoc comparisons (using Fisher's LSD) indicated that the effect on group decision quality score reflected a difference between the content expert condition and each of the other two conditions $(p<.05)$. The effect on group gain in quality reflected a difference between the means of the two extreme groups, content expert condition and process expert condition $(p<.05)$. The group means for each of the two dependent variables are presented in Table 1. 
Table 1: Group Decision Quality and Group Gain in Quality Means and Standard Deviations for the three expert conditions.

\begin{tabular}{llrll}
\hline Type of & \multicolumn{2}{l}{ Group Decision Quality } & \multicolumn{2}{c}{ Group Gain in Quality } \\
Expert & Mean & SD & Mean & SD \\
\hline Content & 23.48 & 6.34 & 19.63 & 8.30 \\
Process & 31.00 & 10.22 & 14.04 & 9.09 \\
None & 31.08 & 7.41 & 15.51 & 7.66 \\
\hline
\end{tabular}

The Effect of Type of Expert on Individual Decision Quality

A three (subject expert vs. process expert vs. non expert) by two (experts vs. group members) analysis of variance (ANOVA) was computed for both the individual initial decision quality scores and the final individual quality scores. The analysis of the initial decision quality scores revealed main effects for type of exper, $F(2,302)=8.08, p<.001$, and between experts and group members, $F(1,302)=30.62, p<.001$. In addition, there was an interaction between type of expert and the expert/member distinction, $F(2,302)=4.17, p<.05$. Post hoc comparisons (using Fisher's LSD) indicated that the main effect for condition on the individual initial decision quality scores reflected a difference between the content expert condition and each of the other two conditions $(p<.05)$. The initial individual quality score means and standand deviations for the expert condition by exper/member analysis are presented in Table 2 and illustrated in Figure 1. Post hoc comparisons indicated the effect on group gain in quality reflected a difference between the means of the two extreme groups, content expert condition and process expert condition, ( $p<$ $.05)$.

The analysis of the final individual decision quality scores revealed only a main effect for type of expert, $F(2,302)=19.03, p<.001$. Post hoc comparisons indicated that the main effect for type of expert on the final individual decision quality scores reflected, as they did for the initial individual decision quality scores, a difference between the content expert condition and each of the other two conditions $(p<.05)$. The final individual quality score means and standard deviations for the expert condition by expert/member analysis are presented in Table 2 and illustrated in Figure 2. A three (subject expert vs. process expert vs. non expert) by two (experts vs. group members) by two (initial quality vs. final quality) repeated measures MANOVA revealed a main effect for quality, $F(1,302)=241.21, p<.0001$, and a quality by expert/member interaction, $F(1$, $302)=24.39, p<.0001$. 
Table 2: Individual Initial and Final Decision Quality Mea:- and Standard Deviations for the experts and group members in the three type of expert conditions.

\begin{tabular}{llrrrr}
\hline & \multicolumn{3}{c}{ Initial Quality } & \multicolumn{2}{c}{ Final Quality } \\
Condition & N & Mean & Std Dev & Mean & Std Dev \\
\hline Content Exp.-Experts & 26 & 32.69 & 8.79 & 25.92 & 8.21 \\
Content Exp.-Members & 81 & 46.20 & 10.35 & 26.57 & 7.72 \\
Process Exp.-Experts & 27 & 44.04 & 10.35 & 36.07 & 9.50 \\
Process Exp.-Members & 78 & 47.67 & 11.79 & 33.60 & 10.76 \\
Non Experts -Experts & 24 & 41.79 & 12.29 & 31.00 & 7.60 \\
Non Experts -Members & 72 & 48.65 & 11.20 & 33.03 & 8.43 \\
\hline
\end{tabular}

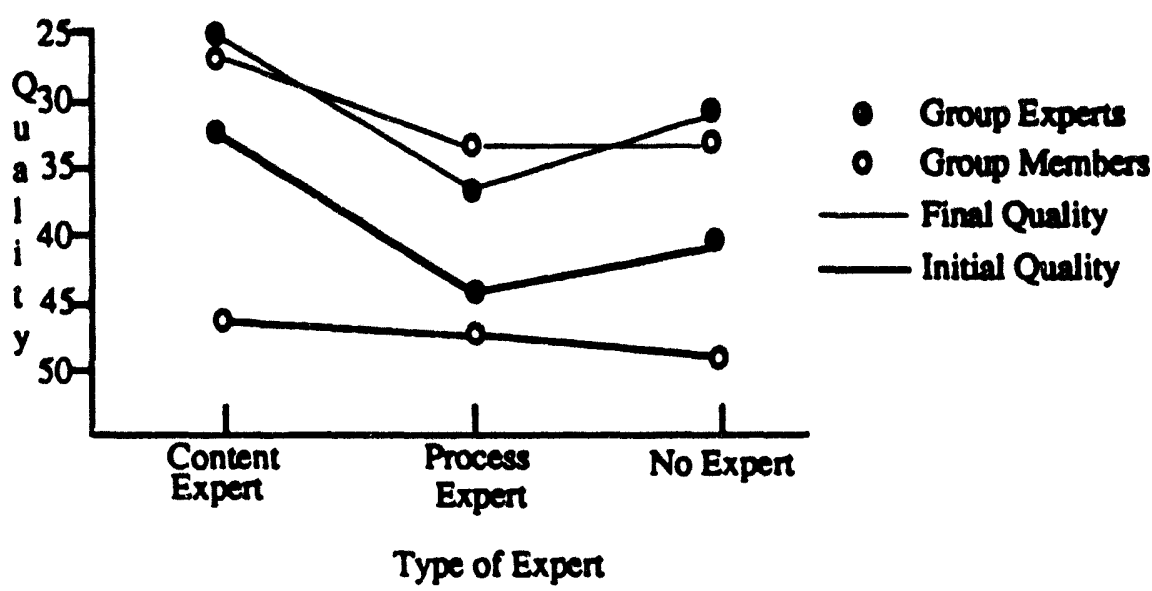

Figure 1: Mean initial and final individual decision quality for the group experts and group members in each of the three type of expert conditions

The Effect of Type of Expert on Perceived Opportunity to Express Views, Perceived Group Information Sharing and Individual Consensus.

Reliability of the three questionnaire measures were assessed using Cronbach's alpha, a measure of internal consistency. Alphas above .70 are considered acceptable. The reliability for Perceived Opportunity, Individual Consensus and Perception of Information Sharing were in the acceptable range.

A three (subject expert vs. process expert vs. non expert) by two (experts vs. group members) MANOVA was computed for the three dependent variables (perceived opportunity to express views, perceived group information sharing, and individual consensus). The analysis of the perceived-opportunity-to-influence-the-group scores revealed no main effects, but an 
interaction between type of expert and experts/members, $F(2,296)=3.91, p<.05$. The group means and standard deviations are presented in Table 3. An inspection of the group means indicates that the process group experts had lower perceived-opportunity-to-influence-the-group scores than any of the other five groups. The analysis of the perceived-information-sharing scores revealed main effects for type of expert, $F(2,296)=3.25, p<.05$, and for experts/members, $F(1$, 296) $=9.39 \mathrm{p}<.01$, and no interaction. Post hoc comparisons (using Fisher's LSD) indicated that the main effect for type of expert reflected a difference between the process expert condition and the non-expert condition $(p<.05)$. The group means and standard deviations are presented in Table 3. An inspection of the group means indicates that those individuals that received some training (content and process group experts) had lower perceived-information-sharing scores than the group members or the nontrained "experts". The analysis of the individual consensus scores revealed main effects for type of expert, $F(2,296)=7.27, p<.001$, and for experts/members, $F(1,296)=12.16, p<.001$, and an interaction between type of expert and experts/members, $F(2$, $296)=8.12, p<.001$. Post hoc comparisons indicated that the main effect for type of expert reflected a difference between the process expert condition and the non-expert condition ( $p<.05)$. The group means and standard deviations are presented in Table 3. An inspection of the group means indicates that the process group experts had lower individual consensus scores than any of the other five groups.

Table 3: Perceived Opportunity to Influence (PO), Perceived Information Sharing (IS) and Individual Consensus (IC) means and standard deviations for the experts and group members in the three type of expert conditions.

\begin{tabular}{lccccccc}
\hline & \multicolumn{3}{c}{ Perceived Opportunity } & \multicolumn{2}{c}{ Information Sharing } & \multicolumn{2}{c}{ Individual Consensus } \\
Condition & N & Mean & Std Dev & Mean & Std Dev & Mean & Std Dev \\
\hline Content Exp.-Experts & 26 & 5.74 & 0.83 & 5.20 & 0.52 & 5.17 & 0.80 \\
Content Exp.-Members & 79 & 5.64 & 0.85 & 5.50 & 0.57 & 5.38 & 0.85 \\
Process Exp.-Experts & 26 & 5.12 & 1.51 & 5.01 & 1.02 & 4.27 & 1.35 \\
Process Exp.-Members & 78 & 5.75 & 0.69 & 5.50 & 0.06 & 5.37 & 0.84 \\
Non Experts -Experts & 23 & 5.64 & 0.82 & 5.52 & 0.78 & 5.35 & 0.89 \\
Non Experts -Members & 70 & 5.74 & 0.70 & 5.54 & 0.57 & 5.36 & 0.80 \\
\hline
\end{tabular}

\section{DISCUSSION}

\section{Decision Quality}

As expected, the individuals given subject matter training scored significantly higher on the first individual ranking than anyone else. In addition, these individuals were able to translate their knowledge into a higher quality group decision and higher individual quality decisions for their 
team members. However, training in, and experience using, a process for solving this type of problem did not provide any advantage over no training. Those individuals provided process knowledge did not have higher quality initial individual rankings. And apparently the group process did not help to activate that process knowledge; performance for these groups was no better than for groups without a trained group member. In fact, although the members of these process knowledge groups improved as much as the individuals in groups without expertise, the process "experts" showed the least gain in quality from initial to final ranking. It is as though they were committed to a process at the expense of content knowledge.

Of most interest for advocates of group process is the overall gain in quality across all groups. The quality of the group decisions subtantially exceed the average initial decision of individual group members irrespective of the presence of a trained expert. The gain in quality of the groups that included an individual with subect matter knowledge is not statistically different from the average gain in quality of the groups without an expert. The process of mutual information sharing and influence led to high quality decisions even for groups that were.not provided with any prior knowledge.

\section{Consensus}

Clearly for the individual group members, there were no differences in how they perceived the group process that can be attributed to the presence of a group member with either subject matter or process expertise. Regardless of the type of knowledge piovided to the presumed group leader, the group members felt similar about the information sharing and opportunity to express views within the groups. In addition, the strength of agreement and acceptance of the decision was similar for groups with and without an expert.

The principal finding with respect to perceived group process and stregth of consensus centered around the process experts. Those individuals trained in a general problem solving process felt there was less information sharing and less opportunity to express views than any other group of experts or group members. In addition, they expressed less agreement with the group decision. Lower perceived information sharing and opportunity to express views scores implies that they felt group members did not accept their input. Lower consensus scores implies they did not emerge as group leaders and did not agree with the group decision. The lower individual quality scores for these individuals implies that they were trying to steer the group in a direction dictated by process considerations and conflicting with some content knowledge. The strength and extent of this effect points to something in the training these individuals received. Perhaps a particular general strategy or technique that they were taught that was contra indicated in this instance.

\section{Conclusion}

Successful performance on problems like Lost on the Moon requires specialized subject area knowledge and the ability to apply both common and specialized knowledge situationally to accomplish a task. In the small group task, this knowledge is distributed over the different group 
members and is accessed and integrated through the group interaction. Each individual's initial ranking is a measure of the knowledge they bring to the group interaction. As the group interaction progresses, information sharing provides each member of the group with pieces of information missing from their initial analysis. Group members move toward a shared understanding of the situation, in this case the lunar environment. Once there is a shared understanding of the problem, the group interaction can progress towards applying common and specialized lonowledge to structure a plausable solution. While these information sharing and solution structuring phases progress concurrently, it is more likely that, at least in this task, that the influence process shifts from informational to include more normative influence during the later solution structuring phase.

The performance of the groups without additional expert knowledge provides the baseline for comparison. The finding of most interest is how well these baseline groups performed. The group information sharing and mutual influence process resulted in substantial improvement in the quality of the group decision over that of the individual members. As hypothesized, the presence in the group of an individual with task specific subject knowledge increased the quality of the group decision. However, while the presence of a subject matter expert did not decrease the strength of group consensus, it also did not increase the perception of group quality nor the strength of group consensus. This is not an unexpected result. While the subject matter experts identified themselves as having some relevant knowledge, they were probably not considered by the other group members to be true "experts" whose ideas would quarantee a high quality decision. They also did not appear to have dominated the discussion at the expense of other group members. The best interpretation of the pattem of results is that the group interaction process worked well to integrate this individual's expert knowledge into the group decision.

The process experts, in this study, seemed to bring an approach that was counter productive to the problem objectives. However, since these individuals were not designated as group leaders, the groups were apparently nevertheless able to proceed effectively. Only the "process experts" suffered in terms of quality, perception of influence and individual consensus. Perhaps, however, if they had been given group leadership status, the group members' perception of the group process may have been different. 


\section{REFERENCES}

Bouchard, T. J. (1969). Penonality, problem-solving procedure, and performance in small groups. Journal of Applied Psychology Monograph

Botrger, P. C. (1984). Expertise and air time as bases of actual and perceived influence in problem-solving groups. Joumal of Applied Paycholosy, 62, 214-221.

Corfman, K. P. (1991). Perceptions of relative influence: Formation and measurement. Iournal of Marketine Research, 28(2), 126-136.

Deutsch, M., \& Gerard, H. B. (1953). A study of normative and informational social influences upon individual judgment. Ioumal of Abnomal and Social Psychology, 52, 629 - 636.

Doreian, P. (1986). Measuring relative standing in small groups and bounded social networks. Social Psychology Quarterly, 49, 247-259.

Effmeyer, R. C., \& Lane, I. M. (1984, December). Quality and acceptance of an evaluative task: The effects of four group decision-making formats. Gmup and Organizational Srudies, 4(9), 509-529.

Forsyth, D. R. (1983). Group dynamics. Pacific Grove, CA: Brooks/Cole Publishing Company.

French, J.R.P., \& Raven, B. (1959). The bases of social power. In D. Cartwright (Ed.), Studies in social power. Ann Arbor, MI: Institute for Social Research.

Hackman, J.R., \& Morris, C.G. (1975). Group tasks, group interaction process, and group performance effectiveness: A review and proposed integration. In L. Berkowitz (Ed), Advances in experimental social psychology (Vol. 8, 47-49). New York Academic Press.

Hall, J. (1971, November). Decisions, decisions, decisions. Psychology Today, 51-54.

Hall, J., \& Watson, H. (1972). The effects of a normative intervention on group decision-making performance. Human Relations, 23(4), 299-317.

Hare, A.P. (1952). A study of interaction and consensus in different sized groups. American Sociological Review, 17(3), 261-267. 
Haythorn, W. (1953). The influence of individual members on the characteristics of small groups. Joumal of Abnomal and Social Psycholosy, 48, 276-284.

Herber, T.T., \& Yost, E.B. (1979). A comparison of decision quality under nominal and interacting consensus group formats: The case of the structured problem. Decision Sciences, 10, 358-370.

Hinkin, T.R. (1989). Developement and application of new scales to measure the French and Raven (1959) bases of social power. Ioumal of Applied Psycholory, 24, 561-567.

Hirokawa, R.Y. (1984). Does consensus really result in higher quality group decisions? In G.M. Phillips \& J.T. Wood (Eds.), Emergent issues in Human decision makine. (pp. 40-49). Carbondale, IL: Southem Illinois University Press.

Hoffman, L.R. (1959). Homogeneity of member personality and its effect on group problemsolving. Loumsl of Abnormal and Social Psychology, 58, 27-32.

Hoffman, L.R., \& Claggett, G.S. (1960). Some factors affecting the behaviors of members of problem-solving groups. Socimetry. 23, 273-291.

Hoffman, L., \& Maier, N. R. F. (1961). Quality and acceptance of problem solutions by members of homogeneous and heterogeneous groups. Abnormal and Social Psychology, 62(2), 401 407.

Hoffman, L.R., \& Smith, C.G. (1960). Some factors affecting the behaviors of members of problem-solving groups. Sociometry, 23, 273-291.

Hollander, E. P. (1960). Competence and conformity in acceptance of influence. Loumal of Abnormal and Social Psychology, 61, 365-369.

Hovland, C.I., \& Weiss, W. (1952). The influence of source credibility of communication effectiveness. Public Opinion Ouarterly, 15(4), 635-650.

Hopkins, T. D. (1964). The Exercise of Influence in Small Groups. Totowa, NJ: The Bedminster Press.

Janis, I.L. (1972). Victims of groupthink. Boston, MA: Houghton-Mifflin.

Jackson, J. (1975). Normative power and conflict potential. Sociological Methods \& Research, 4(2), $237-263$. 
Johnson, D.W. (1975). Joining together. Englewood Cliffs, NJ: Prentice Hall.

Kaplan, M.F., \& Miller, C.E. (1987). Group decision making and normative versus informational influence: Effects of type of issue and assigned decision rule. Joumal of Personality and Social Psychology, 53(2), 306-313.

Kaprzayk, J., \& Fedrizzi, M. (1988). A soft measure of consensus in the setting of partial (fuzzy) preferences. European Joumal of Operational Research, 34, 315-325.

Katz, D. and Kahn, R. L. (1972). The Social Psychology of Organizations (2nd ed. New York: John Wiley \& Sons, Inc.

Kipnis, D., Schmidt, S.M., Price, K., \& Stitt, C. (1981). Why do I like thee: Is it your performance or my orders? Joumal of Applied Psychology, 66(3), 324-328.

Kipnis, D., Schmidt, S. M., \& Wilkinson, I. (1980). Intraorganizational influence tactics: Explorations in getting one's way. Journal of Applied Psycholosy, 65, 440-452.

Kirchler, E. \& Davis, J.D. (1986). The influence of member status differences and task type on group consensus and member position change. Joumal of Personality and Social Psychology, 51, 83-91.

Knutson, T.J., \& Holdridge, W.E. (1975). Orientation behavior, leadership, and consensus: A possible functional relationship. Speech Monographs, 42 107-114.

Maass, A., \& Clark, R. D. (1984). Hidden impact of minorities: Fifteen years of minority influence research. Psychological Bulletin, 25, 428-450.

Mahler, T. (1987). Homogeneity of member personality and its effect on group problem-solving. Joumal of Abnormal Social Psychology, 58, 27-32.

Marquart, D.I. (1955). Group problem solving. Joumal of Social Psychology, 41, 103-113.

McClelland, D.C. (1975). Power. The inner experience. New York: Irvington.

Miner, F.C. (1979). A comparative analysis of three diverse group decision making approaches. Academy of Management Joumal, 22(1), 81-93. 
Moscovici, S., \& Lage, E. (1976). Studies in social influence III: Majority versus minority influence in a group. European Journal of Social Psychology, 6, 149-174.

Paulus, P. B. (Ed.). (1989). Psychology of group influence. Hillsdale, NJ: Lawrence Erlbaum Associates.

Podsakoff, P.M., \& Schriesheim, C.A. (1985). Field studies of French and Raven's bases of power: Critique, reanalysis, and suggestions for future research. Psychological Bulletin, 27(3), 387-411.

Price, J. L. (1972). Handbook of organizational measurement. Lexington, MA: D. C. Heath \& Company.

Rawlins, W.K. (1984). Consensus in decision-making groups: A conceptual history. In G.M. Phillips \& J.T. Wood (Eds.), Emergent issues in Human decision making (pp. 19-39). Carbondale, IL: Southern Illinois University Press.

Scheff, T.J. (1967). Toward a sociological model of consensus. American Sociological Review, 32, 32-46.

Shaw, M.E. (1981). Group dynamics: The psychology of small group behavior (3rd ed.). New York: McGraw-Hill.

Shils, E. (1968). Consensus. In D. L. Sills (Ed.), International Encyclopedia of the Social Sciences (Vol. 3, pp. 260-266). New York: The Macmillan Company \& The Free Press.

Silk, A.\& Kalwani, M. O. (1982). Measuring influence in organizational purchase decisions. Joumal of Marketing Research, 19(2), 165-181.

Stasser, G., Kerr, N.L., \& Davis, J.H. (1989).Influence processes and consensus models in decision-making groups. In P. Paulus (Ed.) Psychology of group influence (2nd ed., pp. 279-326).

Steiner, I.D. (1972). Group process and productivity. New York: Academic Press.

Steiner I.D. (1976). Task-performing groups. In J.W. Thibaut, J.T. Spence, \& R.C. Carson (Eds.), Contemporary topics in social psychology (pp. 393-422). Morristown, NJ: General Learning Press. 
Tjosvold, D. (1987). Making conflict productive. In R.W. Beatty, H.J. Bernardin, \& J.E. Nickel (Eds.), The productivity sourcebook (pp. 184-189). Amherst, MA: Human Resource Development Press.

Tjosvold, D., \& Field, R.H.G. (1983). Effects of social context on consensus and majority vote decision making. Academy of Management Joumal, 26(3), 500-506.

Tanford, S., \& Penrod, S. (1984) Social Influence Model: A formal integration of research on majority and minority influence processes. Psychological Bulletin, 25, 189-225.

Turner, J.C.\& Oakes, P.J. (1986). The significance of the social identity concept for social psychology with reference to individualism, interactionism, and social influence. British Journal of Social Psychology, 25, 237-252.

Vroom, V.H., (1959). Some personality determinants of the effects of participation. Joumal of Abnormal and Social Psychology, 59, 322-327.

Walsh, C. M., Carolan, T. F., Koball, E. H., (1994). Gender, traits, and influence in a small group decision making task. Technical Report.

Wanous, J.P., \& Youtz, M.A. (1986). Solution diversity and quality of group decision. Academy of Management Journal, 29(1), 149-159.

Wood, J. T. (1984). Alternative methods of group decision making. In G. M. Phillips, \& J. T. Wood (Eds.), Emergent issues in human decision making (pp. 3-18). Carbondale, IL: Southern Illinois University Press.

Yukl, G.A. (1989). Leadership in orgainzations (3rd ed.). Englewood Cliffs, NJ: Prentice Hall.

Yukl, G., \& Tracy, J. B. (1991). Consequences of influence tactics used with subordinates, peers, and the boss. Journal of Applied Psychology, 77,(4), 525-535. 


\title{
Gender, Traits, and Influence in a Small Group Decision Making Task
}

\author{
Christine M. Walsh \\ Thomas F. Carolan \\ Elizabeth H. Koball
}

As industry shifts toward a more participative approach to management, research into group dynamics is increasingly important. Working groups are often convened to gain consensus on a high quality decision with the highest level of agreement and acceptance possible. The members of a working group typically bring different sources of knowledge to the group decision making process. Arriving at the highest quality decision that all members of the group can agree on, implies that each group member uses his or her task-related knowledge to influence the group decision and that each group member is also open to considering (i.e., being influenced by) information from other group members.

Our interest is in understanding the individual and social factors that mediate and shape the processes by which individuals in consensus groups share information and arrive at a group decision. The primary reasons for decision-making by consensus are to increase the quality of the decision and to insure a high level of acceptance of that decision (Wood, 1984; Hirokawa, 1984). Understanding the factors that limit either the quality of group decisions or the degree of consensus about a group decision is a first step towards developing strategies for improving group decision making performance. The research that we report on in this paper investigated both individual factors and grouplevel factors. We focus here on individual factors that may affect performance in a group and satisfaction with a group decision. Group-level factors and their affect on performance are discussed in another paper (Polk, Carolan, Koball, 1994). The primary research objective was an investigation of the role of specific personality traits on an individual's influence on the group decision and on individual consensus with the group decision. Are there particular dispositional characteristics of individuals that affect the influence they have in groups? From a management perspective the question might be framed differently. Can group performance be improved by: a) selection of group members on the basis of personality characteristics, or b) consideration of individual characteristics during the group process. 


\section{GROUP DECISION MAKING}

\section{Consensus and Decision Quality}

In the context of small group decision making, the concept of consensus can refer to either a state of agreement to be achieved or a process of achieving group objectives. Consensus as a group state is said to exist when most (some authors argue all) of the individuals concerned have reached a common judgment for which they are willing to show some level of acceptance, agreement, and commitment. The level of individual consensus can vary from conformity, where the individual only agrees publicly, to private internalization, where the individual has a strong belief in and is committed to the group decision. Public agreement without private commitment is a false consensus (Scheff, 1967).

Consensus as group process refers to a method or strategy that encourages active participation from all members of the group and has as its objective a group decision that reflects the views of all the members (Wood, 1984). A group can achieve a state of relative consensus without using a consensus building process. Alternatively, a group can use a consensus building process and never achieve a state of consensus. However, the goals of using a consensus decision making process are usually to reach a state of consensus about a decision and to reach a higher quality decision than would be achieved by other methods.

Research supports the view that a consensus process affects both the group's decision and group members' satisfaction. Effmeyer and Lane (1984) compared the quality and the acceptance of group decisions across four decision-making formats and found consensus groups had the highest levels of acceptance while still maintaining a high decision quality. Decision quality is a measure of how close a decision is to some external evaluation criterion. The evaluation criterion can be a correct decision or solution, a best decision as defined by experts, or by the consequences of the decision. The relationship between decision quality and consensus process has not been consistently validated by research (Hirokawa, 1984; Hare, 1980). It is possible for groups to agree on a poor decision. However, for tasks that are dependent upon individuals sharing specific information to solve a well-defined problem, consensus groups generally perform better than the average individual.

When the group's goal is to reach a best possible decision the immediate task of each individual group member is to share opinions, preferences, and knowledge to achieve a consensus through mutual influence. Each individual has the task of either changing their own position, influencing others to change their position, or reaching a compromise (Stasser, Kerr \& Davis, 1989). We can characterize this process for each individual as converting knowledge, beliefs or preferences to influence. 


\section{Influence}

Influence, considered as the attitudinal and behavioral effects of one person on another (Hopkins, 1964), is a central construct in group decision making research. In many ways the study of group decision dynamics is the study of influence processes among individuals in the group. In a small group process, each group member effects the other group member's opinions. These mutual social influence processes change the distribution of opinions over time and allow groups to reach consensus (Paulus, 1989). The interaction results in the group decision and a level of individual consensus for each group member

The type of influence can affect the quality of the decision and each group member's degree of individual consensus with the decision (Jackson, 1975). The dominant theory of social influence suggest that shifts in group members' opinions are a result of a combination of informational and normative influence processes (Deutsch \& Gerard, 1955). Informational influence refers to influence based on reducing subjective uncertainties by sharing facts or persuasive arguments about the issue. Informational influence leads group members to change their opinion on the belief that another alternative is a better solution. Therefore, informational influence leads to both public and private decision support. Normative influence refers to conformity to implicit decision norms and others' preferences (Kaplan, 1987). Normative influence involves group members conforming to others' ideas leading primarily to public decision support. Based on this framework the informational dependence on others in a primary source of influence that leads to the group structure. Other theorists, view group formation as a prerequisite to influence. From this perspective informational influence can only come from individuals with whom one expects to agree. An individual's confidence in the validity of his or her opinion is a function of the degree to which similar individuals would agree with that opinion (e.g., Turner \& Oakes, 1992).

Research has suggested that minority and majority influence may involve different underlying processes. Moscovici and Lage (1976) found minorities' influence caused both behavioral changes and opinion changes. Whereas majorities primarily exert influence on behavior, not opinions. From this perspective minority influence requires the majority to consciously consider the minorities' opinion and decide the opinion is better. Since this is more difficult than conforming to the majority, minorities can only exert influence in some situations. The majority will often discredit the minorities' opinion if the minority isn't consistent, it consists of only one person, or the minority differs from both the group's opinion and their social categorization (Maass \& Clark, 1984, Moscovici \& Lage, 1976).

A number of factors have been shown to affect influence during the group process. The status and knowledge of the source of the influence and its proximity in time and space affects the amount of influence it has on the group decision (Latane, 1981). Social influence has also been found to be affected by the number of individuals 
that make up the influence source and individual differences in susceptibility to persuasion (Tanford \& Penrod, 1984). Some strategies are more effective than others for increasing influence. Assertiveness, ingratiation, coalition building, rational persuasion and inspirational appeal have all been found to be effective influence tactics (Kipnis, Schmidt, and Wilkinson, 1980; Yukl and Tracy, 1991).

Individual agreement with the group decision may be primarily a function of how individuals perceive the group process and their influence in the decision. Research has shown that perceived decision quality and perceived influence in the decision increases an individual's private strength of consensus (Hoffman, 1959; Polk, 1991). A group member's perception of influence has been shown to be a positively related to acceptance of that decision (Effmeyer \& Lane, 1984; Hoffman \& Maire, 1961). Silk and Kalwani (1982) found people aren't good judges of the amount of influence they have in a decision. Influence between perceived and actual influence can result from individual cognitive skills and biases and from the joint information sharing process (Corfman, 1991). Group members opinions evolve as information is made available through group discussion and mutual influence. This mutual process may affect each individuals ability to accurately judge their influence. In addition, people are inclined to perceive themselves to be similar to their self-image. For example, people who consider themselves to have some expertise may overestimate their influence to support their selfimage.

\section{Task Type}

The type of task has an impact on the type of influence group members will use and the possibilities for consensus. Laughlin (1980) distinguishes between intellective and judgmental tasks. With intellective tasks, group members try to reach agreement on a correct answer; with judgmental tasks, group members try to reach consensus on a preferred position. Kaplan and Miller (1987) found on intellective tasks, group members relied more on informational influence. Whereas on a judgmental task, group members relied more on normative influence. In an intellective task, individuals with greater knowledge about the subject should be in the best position to convert that knowledge to influence; research supports this argument (e.g., Kirchler \& Davis, 1986). Intellective tasks have solutions that can be evaluated against some external criterion, and therefore a quantitative measure of decision quality can be assigned. Judgmental task typically do not have an external evaluation criterion; therefore, the decision is evaluated by the solution's ability to satisfy group members. In consensus decision making the objective is usually to maximize both of these measures decision quality and acceptance even though the criteria are often qualitative (Miner, 1972).

Using Steiner's $(1972,1976)$ task typology, tasks that are disjunctive (individual members contribute different pieces of information), discretionary (the group decides how to integrate the different pieces of information), optimizing (there is a best solution) 
and compensatory (group product is a composite of individual inputs) provide a way to assess the contribution of each individual's knowledge and influence to the final decision. Groups perform better on disjunctive tasks because groups stand a better chance of having a member who knows the correct answer (Marquart, 1955). This conclusion assumes that "truth wins." In other words, the individual with the correct answer must convince the other members he or she is correct. Shaw (1981) concludes that groups perform better than individuals on compensatory tasks. The averaging process tends to remove extreme answers.

\section{INDIVIDUAL CHARACTERISTICS OF GROUP MEMBERS}

Individual group members may contribute differently to the consensus decision due to differences in individual characteristics (Kirchler \& Davis, 1986). Individuals may contribute differently because of different roles, social status, task knowledge, communication skills or personality traits. Personality refers to the "structures, dynamics, processes, and propensities" that explain why a person behaves in a characteristic way (Hogan, 1991). Traits are descriptive constructs that refer to relatively stable regularities or dispositions that mediate the influence of situational factors on behavior (Hogan, 1991; Allport, 1937). From the perspective of trait theory, although behaviors change from situation to situation, core behavioral dispositions remain the same (e.g., Hogan, 1982). Critics of trait theory stress the importance of situational variables in accounting for the variance in social behavior (e.g., Mischel, 1968). They question the stability of traits over time and situations.

The recent resurgence of trait theory and trait oriented research has emphasized the interplay of individual traits and environmental situations (e.g., Pervin, 1985). In neutral situations (where there are few constraints on behavior) trait measures have been found to correlate highly with single trait behavior measures (Monson, Hesley, and Chernick, 1982). Measuring trait behaviors over several occasions and several situations also ensures a more reliable trait measure (Epstein \& O'Brien, 1985). The interaction between the individual's propensity for certain behaviors and environmental situations has been modeled in terms of the conditional probability of a certain type of behavior given a certain situation (Wright and Mischel, 1987). For example, a person who scores high in need for achievement, will be more likely to display achievement oriented behavior in an achievement-oriented activity. By integrating traits and situations, researchers have begun to learm how people and situations interact. Research in this area has found, among other things: traits influence behavior only in relevant situations (Monson, Hesley, \& Chernick, 1982), a person's traits can change a situation (Kendrick \& Funder, 1988), traits are more easily expressed in some situations than others (Kendrick \& Funder, 1988; Monson, Hesley, \& Chernick, 1982), and certain people are more responsive to situation specification of behavioral appropriateness (Snyder, 1983). 
Researchers have attempted to organize a taxonomy of personality traits for sixty years (Barrick \& Mount, 1991). A number of models propose various factors as the primary dimensions of personality (e.g., Fiske, 1949; Norman, 1963; McCrae and Costa, 1985; Hogan, 1986; Barrick and Mount, 1991). Factor models have been criticized for several limitations including: the inability to predict specific behavior, the failure to provide causal explanations for behavior, and the disregard of the conditional nature of human experience (McAdams, 1992). Four this study, four specific personality traits that research has shown to be correlated with performance in groups were selected. These four traits are: affiliation, achievement, aggression, and dominance. The following sections present a brief overview of each trait and its affect on group performance.

\section{Afriliation}

Affiliation can be defined as a person's inclination to make friends and accept people (Jackson, 1989). Affiliation is thought to be a function of a generalized expectation concerning people as a source of reward or punishment, and the extent to which situational factors induce stress, or uncertainty (Mehrabian and Ksionzky, 1974). Affiliation has been found to be positively correlated with group performance (Bither, 1971). Some research indicates that affiliative individuals should have increased influence. Sorrentino and Field (1986) found emergent leadership and affiliation were positively related. They found affiliation had a significant, positive relationship with a group member's perceived influence, competence, confidence, and task leadership. The tendency for leaders to have the most influence in a group suggests that affiliative individuals should have more actual influence. In addition, affiliative individuals can increase their influence through their relationships with other group members (Schuler, 1982). However, other research tends to support a negative relationship between affiliation and influence. For example, researchers have found mixed results for the relationship between conformity and affiliation. Several researchers have found a positive correlation between affiliation and conformity (e.g., see McGhee and Teevan, 1967). Others (e.g., Kaplan, 1961) found no significant relationship between affiliation and conformity. Several researchers have found the desire to affiliate increases with increases in the uncertainty of the siruation (e.g., Radloff, 1968).

\section{Achievement}

Achievement can be defined as the desire to excel and strive to accomplish difficult things, to do things as rapidly as possible, and to surpass others. Individuals with high achievement tend to aspire to accomplish difficult tasks and maintain high standards (Jackson, 1984). They respond positively to competition and are willing to work toward distant goals. The strength of an individual's achievement motive can be thought of as the sum of two tendencies: the tend to approach success and the tendency to avoid failure (Atkinson's, 1957). Spence (1983) operationalized the construct of 
achievement motivation as a cluster of interacting factors that involve mastery, work, and competitiveness. Her research has demonstrated that the structure of achievement motivation is similar fo: both genders.

Schneider and Delaney (1972) found support for the view that achievement is a latent disposition. In this view, a person who had a high level of achievement motivation would only manifest prototypical achievement behaviors in achievement-oriented activities. These are activities in which the individual feels responsible for the outcome, knows performance is evaluated, and feels some degree of uncertainty as to success or failure (Sorrentino, 1973). High achievers tend to use different and more successful strategies than people who are driven solely by extrinsic rewards (Spence, 1983). They tend to emerge as leaders (Bither, 1971; Sorrentino \& Field, 1986). Groups composed of high achievers have been found to be more efficient than low achievement groups. For example, contrary to their original hypothesis, Schneider and Delanney (1971) found groups with high achievers solved complex tasks faster than low achiever groups. Sorrentino and Field (1986) found achievement to be significantly, positively related to the group member's perceived influence, competence, confidence, and motivation.

\section{Dominance}

Dominance can be defined as the inclination of individuals to try to control their environment and to influence others (Jackson, 1989). Dominance has both positive and negative connotations, and can be aggressive or nonaggressive (Ray, 1987). Non aggressive dominance or assertiveness is the expression of one's feelings, needs, preferences, or opinions in a non-threatening, non-punitive manner (Hollandsworth, 1977). More aggressive dominance implies the use of commands to attempt to get absolute obedience from others. The disposition for dominance can be described in terms of frequency of prototypically dominant acts (Buss \& Craik, 1980). In describing dominance, Jackson (1989) lists adjectives which include: commanding, controlling, directing, and influential.

The dominance trait affects a group's interaction through various observable behaviors, such as using commands, talking loudly, interrupting and expressing opinions. Many researchers have found dominant individuals influence the group's interaction. Dominant individuals are less likely to yield to normative pressure, and can therefore critically evaluate alternatives without conforming to the group's opinion (Callaway, Marriott, \& Esser, 1985). Research has shown dominant individuals generally: emerge as leaders, use more negative social-emotional remarks, participate in group activities, and influence group decisions (Shaw, 1976; Cattell, 1960; Haythorn, 1953). Status differences based on gender have been found to be influenced by prior task interactions, affecting women's dominance expression in mixed groups (Davis \& Gilbert, 1989). Hoskins (1986) found a curvilinear relationship between dominance and successful interpersonal relations, that may apply to group situations. For example, dominant 
individuals can help the group interaction by explaining their opinions, but they can hurt the interaction by trying to influence too much.

\section{Aggression}

Jackson (1984) defines dispositional aggression as a person's inclination to be combative and argumentative. Hollandsworth (1984) defines aggressive behavior as the use of threats, punishment, rejection, ridicule, or other types of coercive power to meet ones' needs. There is a difference between aggressive and dominant behavior. Ridgeway and Diekema (1989) define dominant behaviors as "behaviors directed toward the control of another through threat." While they interpreted dominance as including the following behaviors: interrupting; dismissing other suggestions in a contemptuous way; maintaining a tense, forward-leaning posture; pointing; speaking with a raised or commanding voice; and asking interrogating questions; other researchers would use aggression to describe these behaviors. Hollandsworth (1984) differentiates between dominance and aggression by stating that aggressive behaviors rely on coercive power. Jackson's definition of aggression also includes behaviors which intimidate other group members. In describing the aggression trait, Jackson lists adjectives that include: argumentative, attacking, threatening, hot-tempered, and hostile. The definition of aggressive behavior used here includes behaviors which use threats, fear, and intimidation to influence.

Using the aggression scale from the Personality Research Form, Brenner and Tomkiewicz (1980) found aggression was positively correlated with group task accomplishment and overall managerial effectiveness; however, it wasn't significantly correlated with the ability to lead or motivate subordinates. Hollandsworth and Cooley (1987) found subjects complied less readily to aggressive behavior than to assertive behavior. In Hull's and Schroeder's (1979) study, they found that aggression elicits responses of anger, hurt, and humiliation; however, aggression does allow group members to reach their goal. Although aggressive individuals can influence the group, they can decrease decision quality and group consensus.

\section{Subjects and Design}

\section{METHODOLOGY}

An experiment was designed to evaluate the effect, on decision quality and consensus, of including an individual with expert knowledge in a small group decision task. Three hundred and eight undergraduate students from a large land grant university were randomly placed into 77 four-person groups. Each group consisted of three members and one expert. There were 231 subjects recruited from an introductory psychology class who served as the group members. The 77 experts were recruited from the sociology pool. The 231 psychology students received three points toward their final class grade for participation; sociology students received \$20. Performance in the task 
had no bearing on either reward. The experimental study focused on group level effects of the experts and is discussed in another paper (Polk, Carolan \& Koball, 1994). The relational study presented in this paper focused on the individual unit of analysis. Measures of initial task knowledge and of the strength of four personality traits were taken for each of the 231 group members. The experts were not included in this study and no personality trait measures were taken of the experts. Of the 231 group members, 93 were male, 133 were female, and 5 omitted too many responses to be included in the analysis. Figure 1 illustrates the hypothesized path model of the relationships between initial knowledge, four personality traits, actual influence, perceived opportunity for influence and individual consensus.

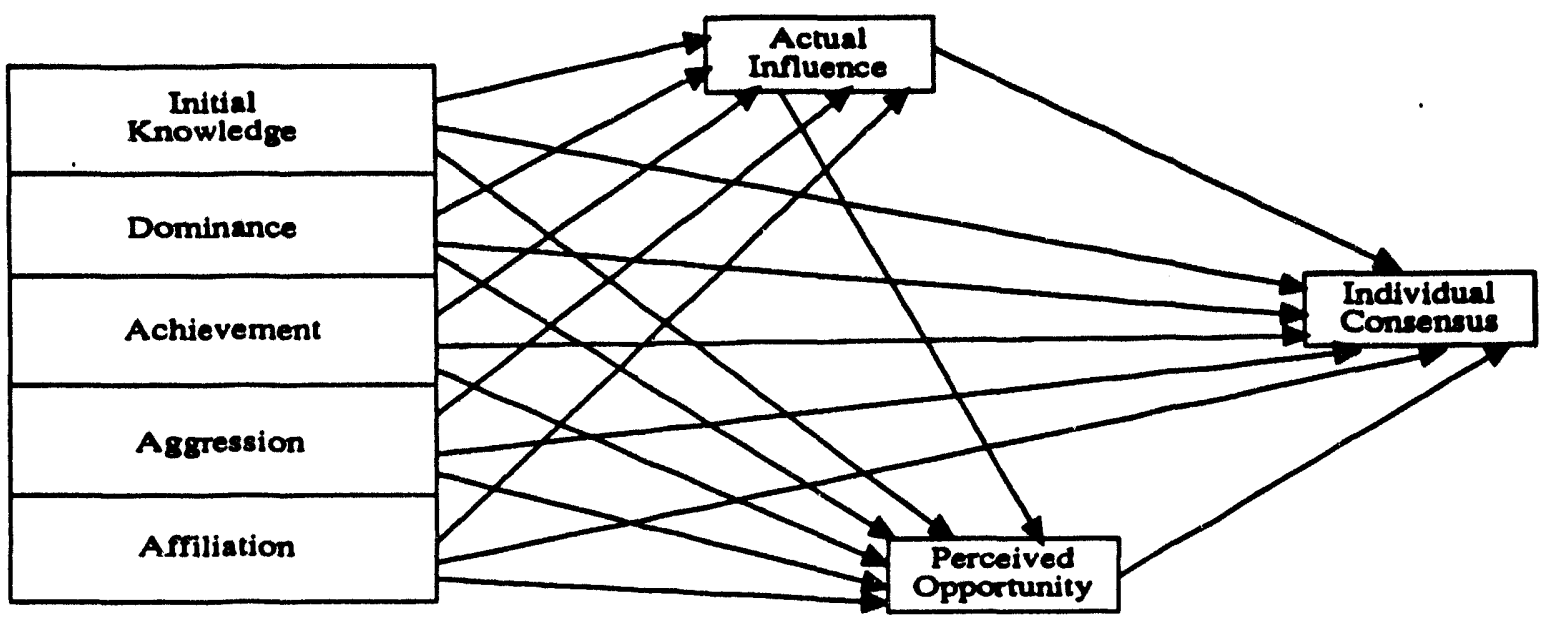

Figure 1: Hypothesized path model.

\section{Task and Procedures}

Subjects completed the Lost on the Moon task. Lost on the Moon is a decision making task developed to study consensus groups (Hall \& Watson, 1971). Subjects are told they have just crash landed on the moon and are stranded. They are asked to individually rank order fifteen items according to their importance for survival. The task has a correct or best answer determined by NASA experts. A quality score can therefore be computed for each individual and for each group. A high quality score implies that specific knowledge about the moon was available to the group and that the group was able to access and use that knowledge. The task is therefore an appropriate task on which to investigate factors which mediate the convergence of individual knowledge on a consensus decision. Each subject completed the ranking three times: initial individual ranking, group ranking, and final individual ranking. 
The experimenter explained the task instructions and told the subjects their goal was to reach the best decision possible. Participants signed a consent form and were given ten minutes to perform the individual-ranking step. They were asked to work silently prior to the group-discussion step. Groups were formed with one expert (trained or untrained) and three randomly placed participants. Before performing the task, group members introduced themselves by giving their name, major, and any classes or hobbies they believe would help them solve the problem. A proctor collected the individual ranking and explained the task to the group. Groups were allowed 30 minutes to interact and rank items. All groups reached consensus within 30 minutes. Participants were then given ten minutes to perform a final individual ranking. After the final individual ranking, they filled out the post experiment questionnaires and the personality test. The subjects were asked not to discuss the exercise with anyone since the experiment was conducted over four days.

\section{Measures}

The independent variables of primary interest were the four personality traits: dominance, aggression, affiliation, and achievement. These exogenous variables were measured using Jackson's Personality Research Form, Form E (PRF-E). A personality test was chosen over a specialized trait measure or behavioral measures since measures of four personality traits were necessary. Jackson's Personality Research Form has been extensively validated and has scales for the four personality traits of interest (Jackson, 1989). In the Mental Measurements Yearbook, Hogan (1989) describes the scales of the PRF as having high internal consistency among many groups, minimal overlap, good test-retest reliability for various groups, and minimal item ambiguity. In addition, this test is relatively free from social desirability response bias (Conoley \& Kramer, 1989). To check the validity of the PRF-E, several studies have compared PRF-E's results with peer evaluations and other personality tests especially with college samples (Conoley \& Kramer, 1989; Jackson, 1989). Conoley and Kramer (1989) found substantial data supporting the PRFs validity.

Each subject received a shortened version of Form E. Instead of measuring all 22 traits, the shortened questionnaire only measured the complete scales for dominance, aggression, affiliation, aggression, social desirability, and infrequency. Both social desirability and infrequency serve as checks on the validity of test for individual respondents. The infrequency measure was used to identify persons whose scores should be excluded from the study. This scale was designed to identify subjects who didn't read the questionnaire, didn't answer the questionnaire seriously, or were emotionally unstable. The resulting 96 questions were randomly ordered.

Initial knowledge. The group member's initial individual quality score, the sum of the absolute differences between the group member's individual rankings and the NASA 
expert's rankings, was used as a measure of the initial knowledge the individual brought to the group. A low score indicates high initial knowledge.

Actual Influence. The group members' actual influence on the final decision was measured with an absolute difference score between the group member's individual ranking and the final group ranking (Bottger, 1984). A low score indicates high influence. While this is a common measure of influence, it is limited in that it does not capture influence that is not directly related to an individuals initial preferences. For example, in the lost on the moon task, an individual with little specific knowledge relevent to the task, but strong communication and leadership skills, may, once given access to accurate information, be the person who argues for the importance of that information and influences the group decision. With the measure of influence based on initial preferences the communicator gets no credit for his or her influence while the individual with the knowledge gets credit for influencing the decision.

Perceived opportunity to influence. Perceived opportunity to influence the final decision was measured with a post-interaction questionnaire. Post-interaction questionnaires and observers' ratings provide valid measures of influence (Hopkins, 1964). A perceived influence scale was derived using nine questionnaire items. Through a factor-analytic study using least squares and maximum likelihood estimation and varimax and promax rotations, a 4-item questionnaire was derived. The questions used to measure the construct of influence focused primarily on the individual's perception of opportunity to express views. So while we originally conceived of this variable as perceived influence, it is, more accurately, perceived opportunity to influence.

Individual consensus. This endogenous variable was measured with a 10 -item scale. This questionnaire was derived from the factor analytic study cited above. This scale contains both the items from a consensus scale and from a perceptions of decision quality scale.

\section{RESULTS}

\section{Reliability Analysis}

Reliability was assessed using Cronbach's alpha, a measure of internal consistency. Alphas above .70 are considered acceptable. The reliabilities ranged from .72 to .89 . Table 1 contains the measures of reliability for the six questionnaire based variables in the study. 
Table 1: Cronbach's Alpha for each Scale

\begin{tabular}{ll}
\hline Variable & Reliability \\
\hline Affiliation & 0.7912 \\
Achievement & 0.7760 \\
Aggression & 0.7173 \\
Dominance & 0.8407 \\
Perceived Opportunity & 0.8844 \\
Individual Consensus & 0.8947 \\
\hline
\end{tabular}

\section{Differences Between Men and Women: Descriptive Statistics}

Before proceeding with the path analysis, descriptive statistics were computed for each of the variables in the hypothesized model for men and women separately. Table 2 contains the number of subjects, means and standard deviations for each of the eight variables. Using an independent t-test to check for differences between males and . females on these variables, we found significant differences in initial knowledge, actual influence, individual consensus and affiliation. All differences were at the $P<.01$ level of significance. On the initial knowledge scale, lower scores reflect higher initial task knowledge; therefore, men on average came into the task with greater initial task knowledge than women. Also, on the actual influence scale, lower scores reflect greater influence; therefore, men had more actual influence on the group decision than women. Women on average expressed greater agreement with the decision than men. The mean for men's and women's perceived opportunity to influence the group was not significantly different. Among the four personality traits, only affiliation exhibited gender differences. Women's mean affiliation score was significantly higher than men's.

Table 2: The Mean and Standard Deviation for each Scale for Males and Females

\begin{tabular}{lcccc}
\hline & \multicolumn{2}{c}{ Males $(\mathrm{n}=93)$} & \multicolumn{2}{c}{ Females $(\mathrm{n}=133)$} \\
\hline Variable & Mean & Std Dev & Mean & Std Dev \\
\hline Initial Knowledge & 44.44 & 10.53 & 49.54 & 11.17 \\
Dominance & 10.34 & 3.63 & 9.71 & 4.37 \\
Achievement & 9.80 & 3.21 & 10.25 & 3.68 \\
Aggression & 9.25 & 3.21 & 9.04 & 3.26 \\
Affiliation & 9.69 & 3.53 & 11.54 & 3.13 \\
Actual Influence & 38.70 & 13.50 & 46.24 & 13.67 \\
Perceived Opportunity & 5.69 & 0.82 & 5.74 & 0.69 \\
Individual Consensus & 5.21 & 0.79 & 5.43 & 0.85 \\
\hline
\end{tabular}



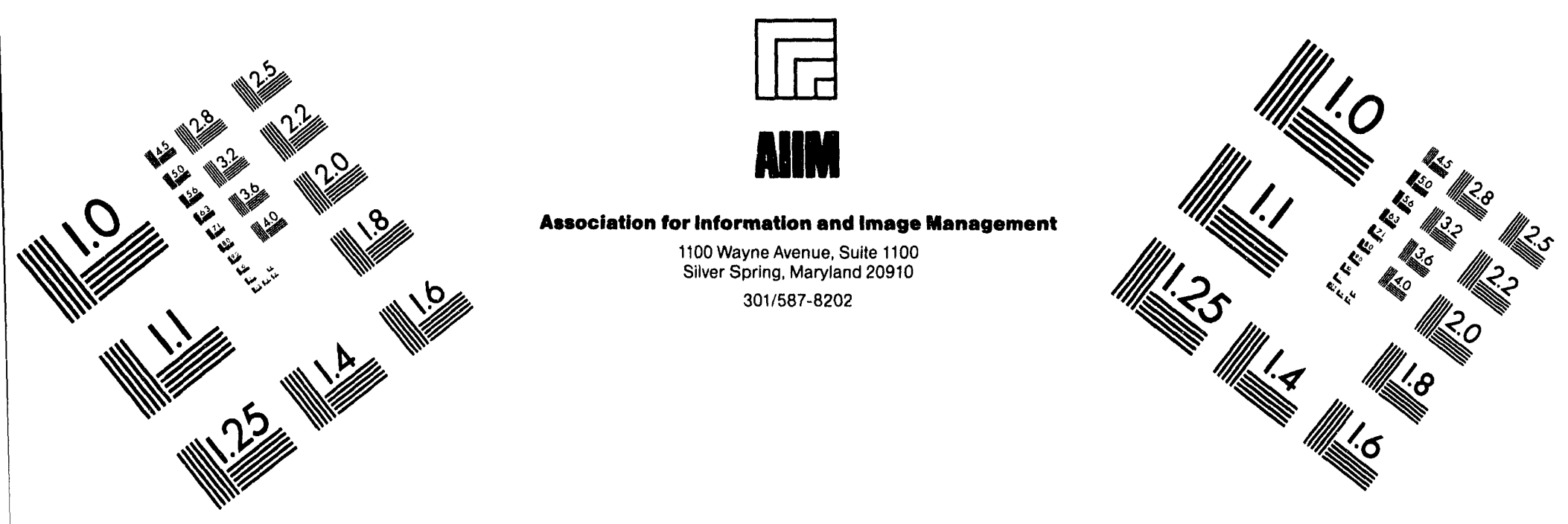

\section{Centimeter}

$\begin{array}{llllllllllllllll}1 & 2 & 3 & 4 & 5 & 6 & 7 & 8 & 9 & 10 & 11 & 12 & 13 & 14 & 15 & \mathrm{~mm}\end{array}$

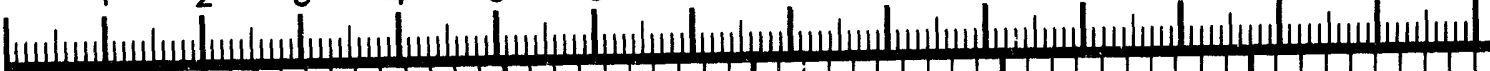

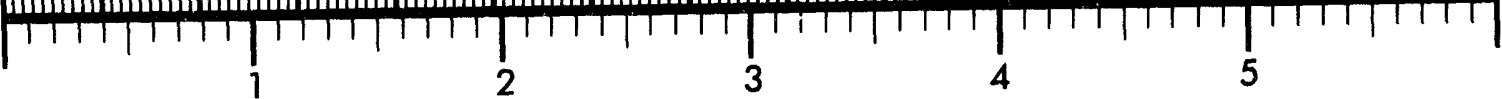
Inches
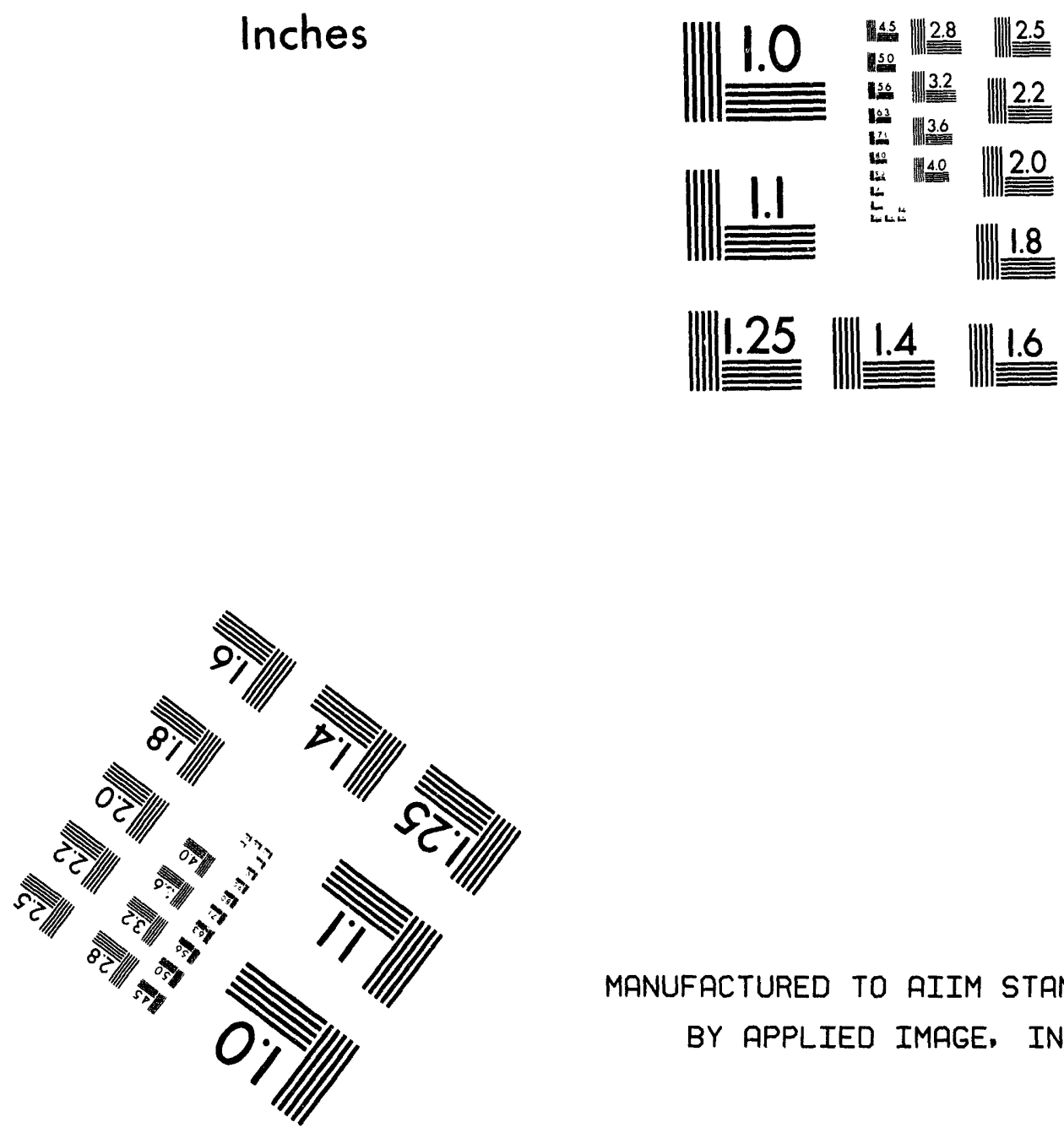

MANUFACTURED TO AIIM STANDARDS BY APPLIED IMAGE, INC.

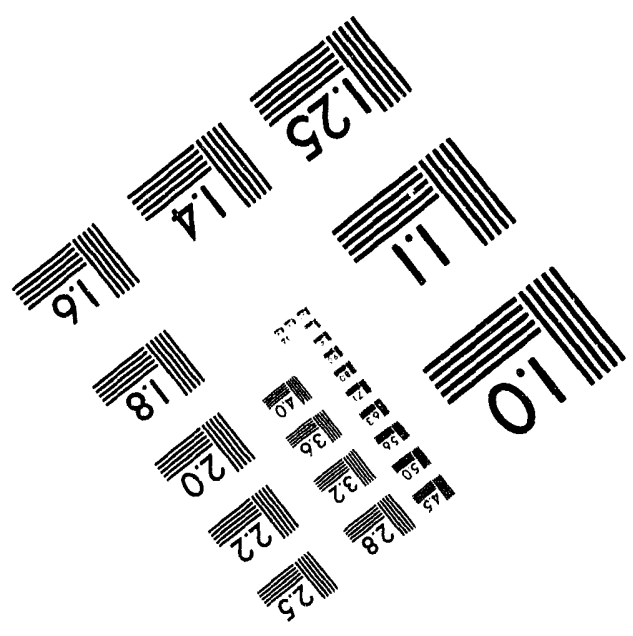



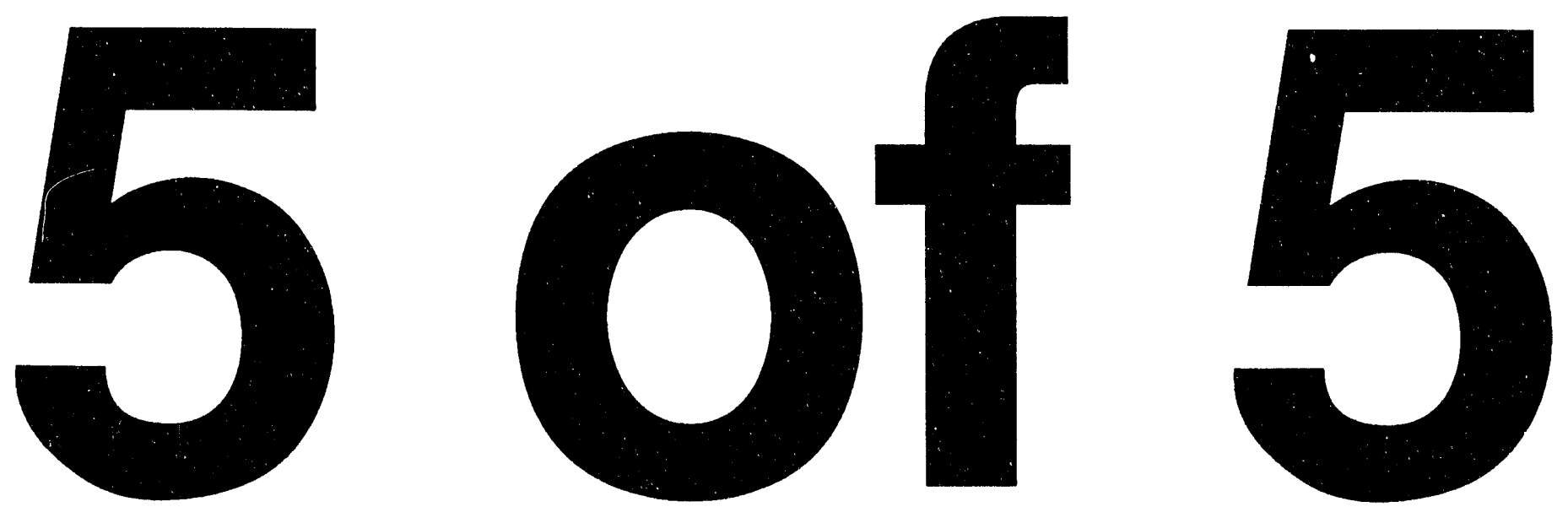


\section{Initial Knowledge, Gender and Influence: Analysis of Variance}

Women scored lower than men on both initial task knowledge and actual influence. As can be seen from an inspection of Table 3, initial task knowledge and actual influence are highly correlated for both men and women. This is, of course, expected since initial task knowledge is a component of actual influence. An analysis of variance was conducted to determine if gender contributed to the variance in actual influence over and above that contributed by differences in initial knowledge. A main effect for initial knowledge on actual influence was confirmed $(F=122.27, P<.0001)$. In addition, when initial knowledge was controlled for, a main effect for gender on actual influence remained ( $F=6.14, P=.01)$. Even when initial knowledge levels were similar, men on the average had more influence on the final decision than women. The significant differences found between the mean scores of men and women on initial knowledge, actual influence, affiliation and individual consensus, suggested that there may be some differences in the way in which men and women influenced the group decision in this study. Separate path analyses were therefore conducted for men and women.

\section{Path Model Analysis}

Using univariate analysis, all variables were tested for deviations from normality. Since none of the variables deviated significantly, Pearson product-moment correlations were used. The correlations between the variables for males and females are listed in Table 3. Since the initial knowledge score and actual influence score were inversely related to the amount of initial knowledge and influence respectively, any correlation or effect with initial knowledge or actual influence are actually in the direction opposite to that indicated in the correlation matrix or path analysis. The relationship between initial knowledge and actual influence is, however, in the correct direction.

Using path model analysis, the direct, indirect, and total effects of each variable relative to ensuing variables were assessed. For men, $\mathrm{R}^{2}$ for the model is .597 . For women, $R^{2}$ for the model is .427 . Figures 2 and 3 illustrate the path models with their direct effects for men and women, respectively.

For the men's model, four significant total effects were found. The total effects for men can be interpreted as:

Affiliation is negatively related to actual influence.

- Initial Knowledge is positively related to actual influence.

- $\quad$ Achievement is positively related to individual consensus.

- Perceived opportunity for influence is positively related to individual consensus. 
Table 3: The Pearson Product-Moment Bivariate Correlation Matrix for the Variables in the Path Model

\begin{tabular}{|c|c|c|c|c|c|c|c|c|}
\hline & $\mathbf{I K}_{\mathbf{a}}$ & DO & $A C$ & $\mathrm{AG}$ & AF & $\mathrm{AI}_{2}$ & $\mathrm{PO}$ & IC \\
\hline \multicolumn{9}{|c|}{ MALESb } \\
\hline $\mathrm{IK}_{\mathbf{a}}$ & & 0.096 & -0.0003 & 0.144 & 0.096 & $0.672^{* *}$ & -0.079 & 0.036 \\
\hline DO & 0.109 & & $0.392 * *$ & $0.207^{*}$ & $0.333^{* *}$ & -0.051 & $0.251^{*}$ & 0.155 \\
\hline AC & 0.006 & $0.308 * *$ & & -0.110 & $0.211^{*}$ & -0.079 & $0.272^{* *}$ & $0.285^{* *}$ \\
\hline AG & 0.094 & $0.343^{* *}$ & -0.058 & & -0.025 & 0.043 & -0.069 & -0.028 \\
\hline $\mathrm{AF}$ & -0.067 & $0.317^{* *}$ & 0.119 & -0.084 & & $0.227^{*}$ & 0.097 & -0.035 \\
\hline $\mathrm{AI}_{\mathrm{a}}$ & $0.545^{* *}$ & -0.035 & -0.042 & -0.012 & -0.083 & & -0.119 & 0.039 \\
\hline PO & -0.062 & -0.002 & $0.281^{* *}$ & -0.001 & 0.111 & -0.237 & & 0.669 \\
\hline IC & -0.094 & 0.078 & 0.155 & -0.080 & $0.269 * *$ & $-0.261 *$ & $0.476^{* *}$ & \\
\hline \multicolumn{9}{|c|}{ FEMALES $b$} \\
\hline Key: & $\begin{array}{l}\text { AI } \\
\text { PO } \\
\text { IC } \\
\text { AF }\end{array}$ & \multicolumn{2}{|c|}{$\begin{array}{l}\text { - actual influence } \\
\text { - perceived opportunity } \\
\text { - individual consensus } \\
\text { - affiliation score }\end{array}$} & $\begin{array}{l}\text { AC } \\
\text { AG } \\
\text { DO } \\
\text { IK }\end{array}$ & \multicolumn{2}{|c|}{$\begin{array}{l}\text { - achievement score } \\
\text { - aggression score } \\
\text { - dominance score } \\
\text { - initial knowledge }\end{array}$} & & \\
\hline $\begin{array}{l}*-p-v \\
*-p-v\end{array}$ & $<.01$ & & & $\ln$ & & & & her \\
\hline 1.8 & . & . & t de the & & & & & \\
\hline
\end{tabular}

For the women's model, six significant total effects were found. The total effects for women can be interpreted as:

- Initial knowledge is positively related to actual influence.

- Achievement is positively related to perceived opportunity for influence.

- Actual influence is positively related to perceived opportunity for influence.

- Affiliation is positively related to individual consensus.

- Actual influence is positively related to individual consensus.

- Perceived opportunity for influence is positively related to individual consensus.

Tables 4 and 5 list the direct, indirect, and total effects for men and women, respectively. For men, the effect between initial knowledge and actual influence, between affiliation and actual influence and between perceived influence and individual consensus were significant at the $p=.01$ level. The effect for achievement on individual consensus was significant at the $p=.05$ level. For women, the effect between perceived influence and individual consensus, between actual influence and perceived opportunity for influence, between achievement and perceived opportunity for influence, and between initial knowledge and actual influence were found to be significant at the .01 level. The other twc effects, affiliation and actual influence on individual consensus, were found to 
be significant at the .05 level. $\mathrm{R}^{2}$, the amount of variance accounted for in the dependent variable by the independent variables, was used to evaluate the model.

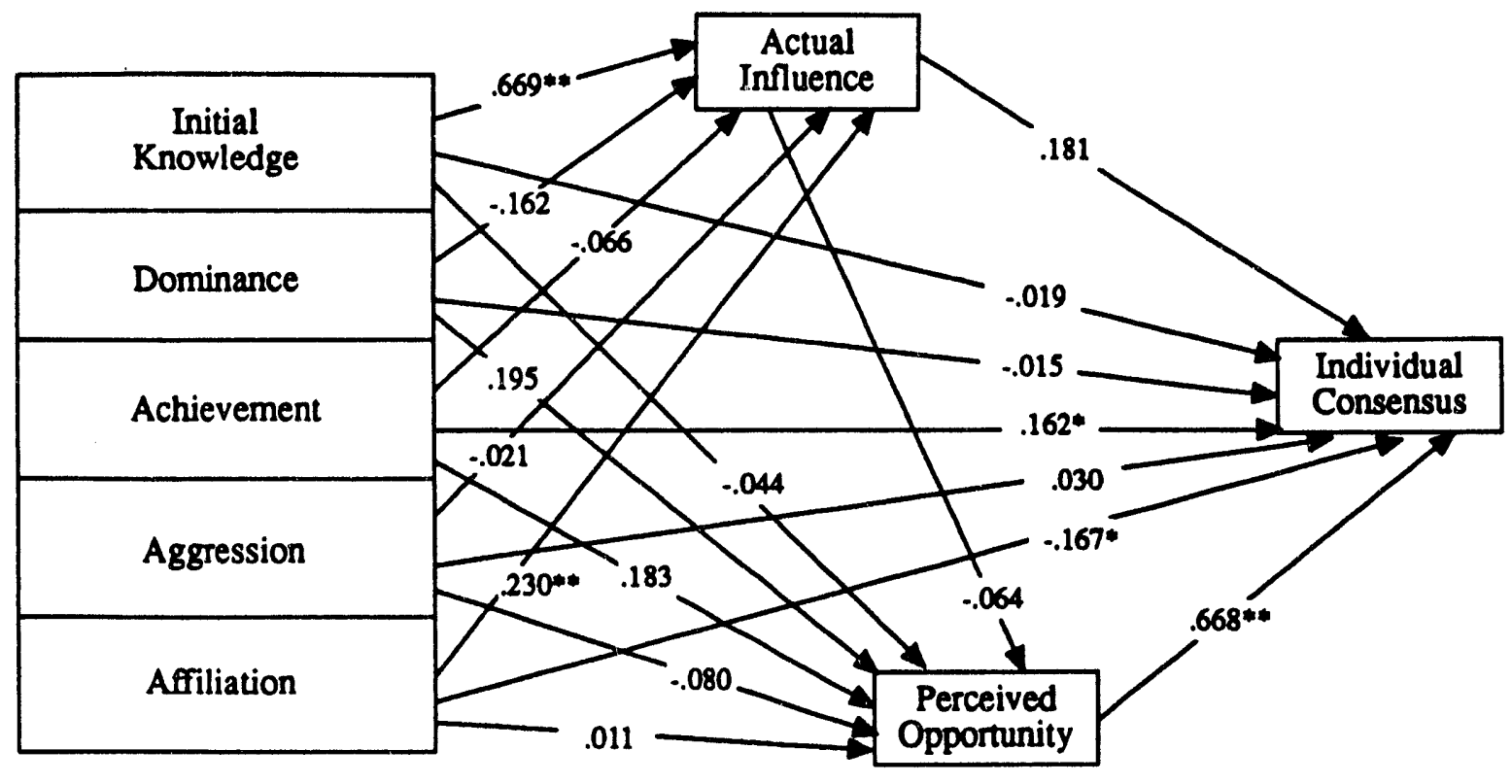

* - p-value $<.05$

** - p-value <.01

Men's model $\cdot \mathbf{R}^{2}=.597$

Figure 2: Path Model for Men with Direct Effect

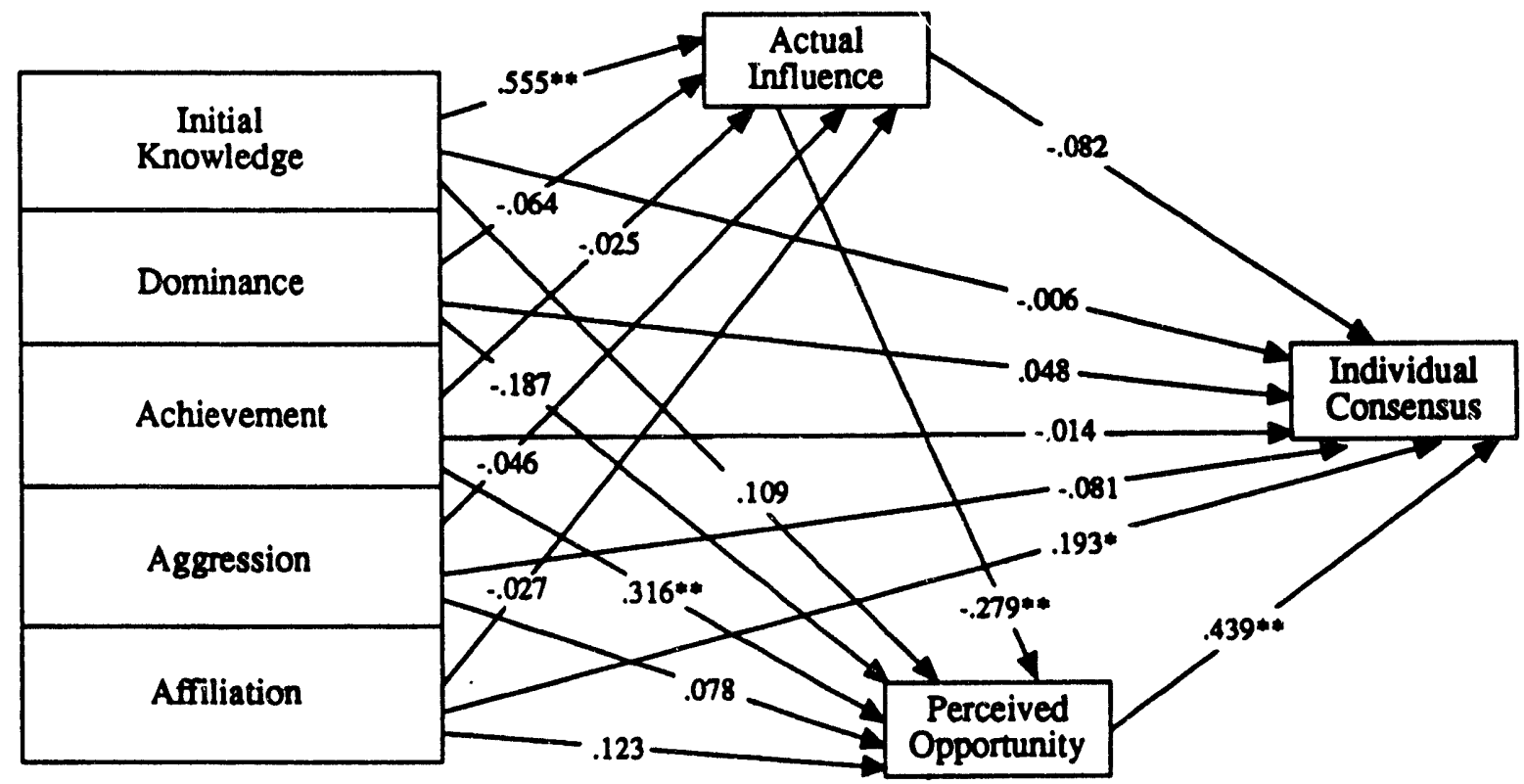

Women's model $-\mathbf{R}^{2}=.427$

- p-value $<.05$

** - p-value $<.01$

Figure 3: Path Model for Women with Direct Effects 
Table 4: Men's Direct, Indirect, and Total Effects for all the Variables in the Path Model

\begin{tabular}{lccc}
\hline Variables & Direct Effects & Indirect Effects & Total Effects \\
\hline Individual consensus & & & \\
IK & $-0.019 \mathrm{a}$ & $0.063 \mathrm{a}$ & $0.044 \mathrm{a}$ \\
DO & -0.015 & 0.107 & 0.092 \\
AC & $0.162^{*}$ & 0.111 & $0.273^{*}$ \\
AG & 0.030 & -0.057 & -0.027 \\
AF & $-0.167^{*}$ & 0.039 & -0.128 \\
AI & $0.181 \mathrm{a}$ & $-0.043 \mathrm{a}$ & $0.138 \mathrm{a}$ \\
PO & $0.668^{* *}$ & $\mathrm{n} / \mathrm{a}$ & $0.668^{* *}$ \\
& & & \\
Perceived opportunity & &. & \\
IK & $-0.044 \mathrm{a}$ & $-0.043 \mathrm{a}$ & $-0.087 \mathrm{a}$ \\
DO & 0.195 & 0.010 & 0.205 \\
AC & 0.183 & 0.004 & 0.187 \\
AG & -0.080 & -0.001 & -0.079 \\
AF & 0.011 & -0.015 & -0.004 \\
AI & $-0.064 \mathrm{a}$ & $\mathrm{n} / \mathrm{a}$ & $-0.064 \mathrm{a}$ \\
& & & \\
Actual influence & & & \\
IK & $0.669 * *$ & $\mathrm{n} / \mathrm{a}$ & $0.669^{* *}$ \\
DO & $-0.162 \mathrm{a}$ & $\mathrm{n} / \mathrm{a}$ & $-0.162 \mathrm{a}$ \\
AC & $-0.066 \mathrm{a}$ & $\mathrm{n} / \mathrm{a}$ & $-0.066 \mathrm{a}$ \\
AG & $0.021 \mathrm{a}$ & $\mathrm{n} / \mathrm{a}$ & $0.021 \mathrm{a}$ \\
AF & $0.230 \mathrm{a} * *$ & $\mathrm{n} / \mathrm{a}$ & $0.230 \mathrm{a} *$ \\
& & & \\
\hline
\end{tabular}

Key:

Al - actual influence

PO - perceived opportunity

IC - individual consensus

AF - affuliation score

- - p-value $<.05$

$*$ - p-value $<.01$
AC - achievement scose

AG - aggression score

DO - dominance score

IK - initial knowledge

a - The initial knowledge and actual influence scores were inversely related to the amount of initial knowledge and influence, respectively. 
Table 5: Women's Direct, Indirect, and Total Effects for all the Variables in the Path Model

\begin{tabular}{llll}
\hline Variables & Direct Effects & Indirect Effects & Total Effects \\
\hline Individual consensus & & & \\
IK & $-0.006 \mathrm{a}$ & $-0.066 \mathrm{a}$ & $-0.072 \mathrm{a}$ \\
DO & 0.048 & -0.070 & -0.022 \\
AC & -0.014 & $0.144^{*}$ & 0.130 \\
AG & -0.081 & 0.043 & -0.037 \\
AF & $0.193^{*}$ & 0.059 & $0.253^{*}$ \\
AI & $-0.082 \mathrm{a}$ & $-0.123 \mathrm{a}^{*}$ & $-0.205 \mathrm{a}^{*}$ \\
PI & $0.439^{* *}$ & $\mathrm{n} / \mathrm{a}$ & $0.439^{* *}$
\end{tabular}

Perceived opportunity

\begin{tabular}{llll} 
IK & $0.109 \mathrm{a}$ & $-0.155 \mathrm{a}^{* *}$ & $-0.046 \mathrm{a}$ \\
DO & -0.187 & -0.017 & -0.170 \\
AC & $0.316^{* *}$ & 0.007 & $0.323^{* *}$ \\
AG & 0.078 & 0.013 & 0.091 \\
AF & 0.123 & 0.008 & 0.131 \\
AI & $-0.279 a^{* *}$ & $n / a$ & $-0.279 a^{* *}$ \\
& & & \\
Actual influence & & & \\
IK & $0.555^{* *}$ & $n / a$ & $0.555^{* *}$ \\
DO & $0.064 a$ & $n / a$ & $0.064 a$ \\
AC & $-0.025 \mathrm{a}$ & $\mathrm{n} / \mathrm{a}$ & $-0.025 \mathrm{a}$ \\
AG & $-0.046 \mathrm{a}$ & $\mathrm{n} / \mathrm{a}$ & $-0.046 \mathrm{a}$ \\
AF & $-0.027 \mathrm{a}$ & $\mathrm{n} / \mathrm{a}$ & $-0.027 \mathrm{a}$ \\
& & & \\
\hline
\end{tabular}

Key:
AI - sctual influence
AC - achievement score
PO - perceived opportunity
AG - aggression score
IC - individual consensus
DO - dominance score
AF - affiliation score
IK - initial knowledge

* p-value <.05

** - p-value <.01

a - The initial knowledge and actual influence scores were inversely related to the amount of initial knowledge influence, respectively. 
Gender differences in the role of affiliation on influence

In order to assess further the difference in the role of affiliation on influence for males and females, an analysis of variance was conducted for the effect of gender and affiliation on influence. Table 6 provides the sample size, mean and standard deviation for each cell. There was a main effect for gender $(F=15.89, p=.0001)$ and an interaction between gender and affiliation relative to actual influence $(F=4.65, p<.05)$. Figure 4 provides an illustration of the interaction. For males, high affiliation appears to have a negative effect on their ability to influence the final decision whereas for females there was not evidence of an effect of affiliation level on influence.

Table 6: Sample Size, Means, and Standard Deviations for the Gender by Afriliation Analysis.

\begin{tabular}{lllll}
\hline Level of & & & & \\
Affiliation & Gender & $\mathrm{N}$ & Mean & SD \\
\hline Low & Male & 50 & 35.52 & 14.62 \\
Low & Female & 40 & 47.15 & 12.82 \\
High & Male & 43 & 42.40 & 11.13 \\
High & Female & 93 & 45.86 & 14.07 \\
\hline
\end{tabular}

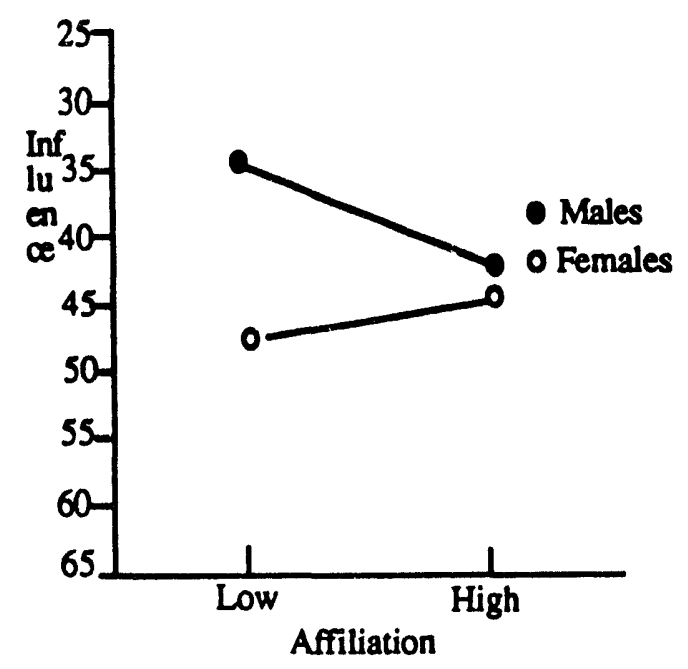

Figure 4. Mean influence level as a function of affiliation and gender. 


\section{DISCUSSION}

For both men and women, as expected the task knowledge they bring to the group is the primary factor that accounts for the degree of influence they have on the final decision. The task is designed to access individual knowledge. And the actual influence measure has the quality of the individual's task knowledge as its primary component; it's based on how much of an individuals initial knowledge remains in the final group decision.

The real question in this task is: why isn't more of the influence accounted for by the initial knowledge of the group members. Or said another way, what is limiting the quality of the group decision. What factors prevent initial knowledge from getting into the group decision? The experimental design provides a partial answer. The contribution of the confederates who served as "experts" in the experimental portion of the study also influenced the decision. One third of this "expert" group ( 25 individuals) were trainned up to a criterion level of task specific knowledge. Had the data from these individuals been included in the analysis, the relationship between initial knowledge and actual influence would have been greater. The path analyses (see Figure 8) suggest two additional factors: gender and affiliation.
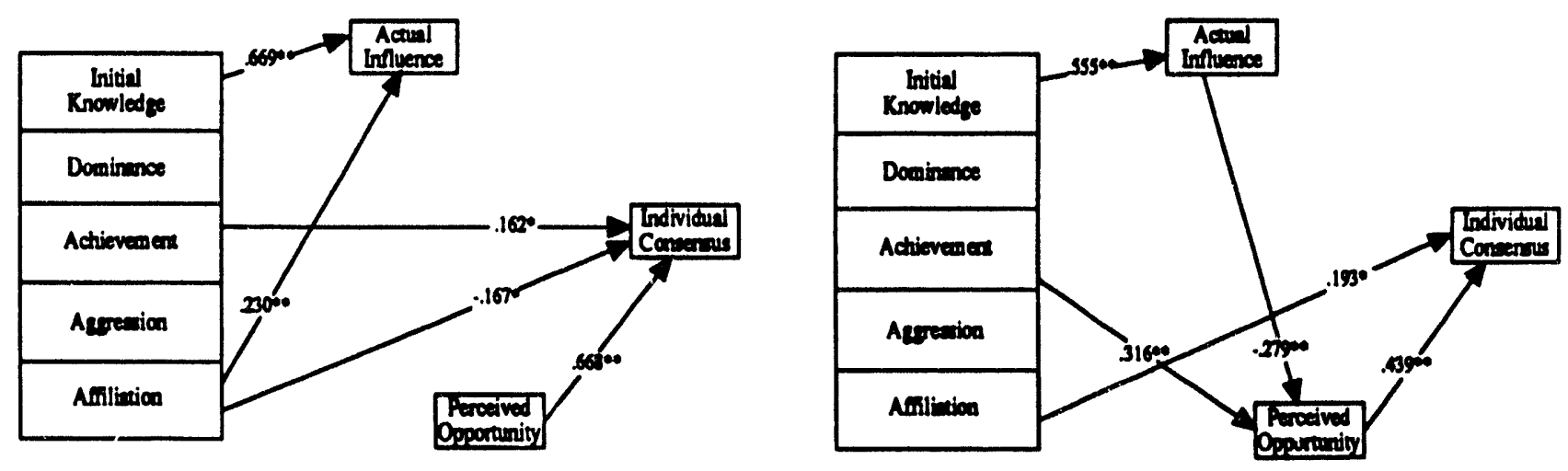

Figure 8: Final Path Models for Males and Females.

Men had more actual influence on the group decision. Most of that difference can be accounted for by the differences in initial knowledge. Men on average came into the task with greater initial task knowledge than women. And initial knowledge accounts for most of the differences in influence in this task. However, even when initial knowledge was statistically controlled for, men on the average still had more influence on the final decision than women. So what accounts for the advantage males have in converting their knowledge to influence? 
The only other contribution to influence from the path model comes from affiliation. And only affiliation exhibited gender differences on the Personality Research Form. The women's mean affiliation score was significantly higher than the men's. Differences in affiliation only contributed to differences in influence for males and the relationship was negative. Since males were found to be less disposed towards affiliation than females and a disposition towards high affiliation served to limit the influence that males had on the decision, one possible interpretation is that the more afffiliation oriented individuals, of which there were significantly more females than males, were more disposed to compromise for the sake of reaching a decision, even when, based on the quality of their individual rankings, they had knowledge to suggest the decision was not the best possible decision. That is perhaps the final decision was, for these individuals, a false consensus.

The path analysis provides some additional support for this interpretation in the direct effects of affiliation on individual consensus. In the men's path model affiliation had a negative effect on individual consensus. That is for males, individuals with higher tendencies for affiliation tend to measure lower on individual consensus. This seems a contrary finding, affiliation is usually found to be positively related to consensus building and conformity. This finding makes sense, however, in the context of a false consensus. The individual consensus measure captures not only the degree to which the decision was thought to be a good compromise but the degree to which the decision was thought to be the best quality decision. While the more affiliative males may have publicly compromised for the sake of a decision, privately they may not have agreed with the decision. While affiliation orientation may help tc explain why men's influence isn't even greater in this study, it does not provide any insight into the source of the observed influence gap between men and women.

For women affiliation orientation affects individual consensus in the opposite direction. Women with high affiliation orientation are more likely to be higher on the individual consensus scale. For women, influence on the decision did not differ as a function of affiliation orientation. This suggests that for some proportion of females affiliation orientation may serve as a means to influence the decision. This gender difference in the relationship among affiliation orientation, influence and individual consensus points to the possiblity of gender differences in the way in which dispositional characteristics interact with situational variables to produce behavior. This combined with the unaccounted for gender influence gap motivated further analysis of the experimental and relational data collected in this research. Two hypotheses guide this further exploration of the data. First, the source of the gender influence gap in this study 
can only be in one of two places; either males with low initial quality scores have more influence than their quality scores would warrant or females with high quality scores have less influence than their quality scores would warrant. Second, if the same measured dispositional trait values are employed differently or perceived by others differently in the same situation depending on gender, then those differences should be more salient for individuals at either extreme of the trait. Analyses are in progress.

\section{Individual Consensus and Perceived Opportunity for Influence}

Perceived opportunity for influence was found to be positively related to individual consensus for both men and women. This positive correlation between perceived influence and individual consensus is consistent with other research findingd (e.g., Effmeyer and Lane, 1984). Perceived influence increases individual consensus. Actual influence, however, was not related to individual consensus in men and only indirectly related through perceived influence in women.

Perception of opportunity to express views and thereby influence the group decision was not significantly different for men and women. Again however, the pattern of effects suggests gender differences in the basis that perception. For the men, differences in perceived opportunity to influence were not related to differences in actual influence, initial knowledge or any of the four personality traits. The implication is that in this study men were in general more likely to see themselves as having been listened to regardless of wether their contribution was directly incorporated into the decision. For, women on the other hand, differences in perception of opportunity to influence the decision were more closely related to the actual influence they had on the decision. Note that for women, perception of opportunity to influence was not a function of initial knowledge but to the extent the knowledge was used in the group decision. The implication is that among those individuals who felt they had less opportunity to express their views, is a significant cadre of women with high quality initial scores that did not get represented in the group decision.

More achievement oriented women were more likely to see themselves as having had the opportunity to contribute to the group than less achievement oriented women. For men perception of opportunity to influence the decision was not related to their achievement orientation. We can interpret this relationship as suggesting that, in this study, men, even when they were not achievement oriented perceived themselves as having been influential in the group process. Women, on the other hand, can be differentiated based on achievement orientation. Women who were less achievement oriented did not see themselves as contributing as tauch to the group process. A positive relationship between achievement orientation and perceived influence is consistent with other research findings (e.g., Sorrentino and Field,1986). 
In conclusion, the results of this study suggests that while task relevant knowledge is the most important factor an individual brings to an information centered group decision making task, at least one dispositional characteristic, their affiliation-orientation may affect the way they influence group decisions. From a management perspective, the results of this study: a) do not support selection of group members on the basis of personality characteristics, except perhaps to assure a mix that includes affiliationoriented individuals and $b$ ) assure that the compromise decisions are not a result of normative influence at the expense of informative influence. 


\section{References}

Allport, G. W. (1937). Personality A psychological interpretation. New York: Henry Holt and Company.

Atkinson, J. W. (1957). Motivational determinants of risk-taking behavior. Psychological Review, 64, 359-372.

Barrick, M. R. \& Mcunt, M. K. (1991). The big five personality dimensions and job performance: A meta-analysis. Personnel Psychology, 44, 1-26.

Bither, S. W. (1971). Personality as a factor in management team decision making. State College, PA: Pennsylvania State University.

Bottger, P. C. (1984). Expertise and air time as bases of actual and perceived influence in problem-solving groups. Joumal of Applied Psychology, 62, 214-221.

Brenner, O. C., \& Tomkiewicz, J. (1980). The relationship between aggression and managerial effectiveness. Psychological Reports, 47, 271-274.

Buss, D. M., \& Craik, K. H. (1980). The frequency concept of disposition: Dominance and prototypically dominant acts. Journal of Personality, 48, 379-392.

Callaway, M. R., Martiott, R. G., \& Esser, J. K. (1985). Effects of dominance on group decision making: Toward a stress reduction explanation of groupthink. Joumal of Personality and Social Psychology, 49(4), 949-952.

Cattell, R. B. \& Stice, G. F. (1960). The dimensions of groups and their relations to the behavior of members. Champaign, IL: Institute for Personality and Ability Testing.

Conoley, J. C., \& Kramer, J. J. (Eds.). (1989). The tenth mental measurements yearbook. Lincoln, Nebraska: University of Nebraska Press.

Corfman, K. P. (1991). Perceptions of relative influence: Formation and measurement. Joumal of Marketing Research, 28(2), 126-136.

Davis, B. M., \& Gilbert, L. A. (1989). Effect of dispositional and situational influences on women's dominance expression in mixed-sex dyads. Joumal of Personality and Social Psychology, 57, 294-300. 
Deutsch, M., \& Gerard, H. B. (1955). A study of normative and informational social influences upon individual judgment. Journal of Abnormal and Social Psychology, $52,629-636$.

Effmeyer, R. C., \& Lane, I. M. (1984, December). Quality and acceptance of an evaluative task: The effects of four group decision-making formats. Grokip and Organizational Studies, 4(9), 509-529.

Epstein, S., \& O'Brien, E. J. (1985). The person-situation debate in historical and current perspective. Psychological Bulletin, 28, 513-537.

Hall, J., \& Watson, H. (1971). The effects of a normative intervention on group decisionmaking performance. Human Relations. 23(4), 299-317.

Hare, A. P. (1980). Consens s versus majority vote: A laboratory experiment. Small Group Behavior, 21, 131-143.

Haythorn, W. (1953). The influence of individual members on the characteristies of small groups. Journal of Abnormal and Social Psychology, 48, 276-284.

Hirokawa, R.Y. (1984). Does consensus really result in higher quality group decisions? In G.M. Phillips \& J.T. Wood (Eds.), Emergent issues in Human decision making. (pp. 40-49). Carbondale, IL: Southern Ilinois University Press.

Hoffman, L.R. (1959). Homegenity of member personality and its effect on group problem-solving. Journal of Abnormal and Social Psychology, 58, 27-32.

Hoffman, L., \& Maier, N. R. F. (1961). Quality and acceptance of problem solutions by members of homogeneous and heterogeneous groups. Abnormal and Social Psycholcgy, 62(2), 401 - 407.

Hogan, R. (1991). Personality and personality measurement. In M. D. Dunnette, \& L. M. Hough (Eds.), Handbook of Industrial and Organizational Psychology (pp. 873919). Palo Alto, CA: Consulting Psychologists Press, Inc.

Hogan (1989)

Hogan, R. (1986). Manual for the Hogan Personality Inventory. Minneapolis, MN: National Computer Systems. 
Hogan, R. (1982). A socioanalyic theory of personality. In M. Page (Ed.), Nebraska symposium on motivation. (Vol. 30, pp.56-89). Lincoln: University of Nebraska Press.

Hollandsworth, J. G., \& Cooley, M. L. (1978). Provoking anger and gaining compliance with assertive versus aggressive responses. Behavior Therapy, 2, 640-646.

Hollandsworth, J. G. (1977). Differentiating assertion and aggression: Some behavioral guidelines. Behavior Therapy, 8, 347-352.

Hopkins, T. D. (1964). The Exercise of Influence in Small Groups. Totowa, NJ: The Bedminster Press.

Hoskins, C. N. (1986). Measuring perceived dominance-accomodation: Development of a scale. Psychological Reports, 58, 627-642.

Hull, D. B., \& Schroeder, H. E. (1979). Some interpersonal effects of assertion, nonassertion, and aggression. Behavior Therapy, 10, 20-28.

Jackson, J. (1975). Normative power and conflict potential. Sociological Methods \& Research, 4(2), 237 - 263.

Jackson, J. (1989). Personality research form manual. Port Huron, MI: Sigma Assessment Systems, Inc.

Kaplan, B. (1961). Studying personality cross-culturally Evanston, Ill: Row, Peterson.

Kaplan, M.F., \& Miller, C.E. (1987). Group decision making and normative versus informational influence: Effects of type of issue and assigned decision rule. Jourmal of Personality and Social Psychology 53(2), 306-313.

Kendrick, D. T.; \& Funder, D. C. (1988). Profiting from controversy: Lessons from the person-situation debate. American Psychologist, 4(1), 23 - 34.

Kipnis, D., Schmidt, S. M., \& Wilkinson, I. (1980). Intraorganizational influence tactics: Explorations in getting one's way. Joumal of Applied Psychology 65, 440-452.

Kirchler, E. \& Davis, J.D. (1986). The influence of member status differences and task type on group consensus and member position change. Loumal of Personality and Social Psychology, 51, 83-91. 
Latane, B. (1981). The psychology of social impact. American Psychologist, 36, 343356.

Laughlin, P. R. (1980). Social combination processes of cooperative problem-solving groups on verbal intellective tasks. In M. Fishbein (Ed.), Progress in social psychology (Vol 1, pp. 127-156). Hillsdale New Jersey: Lawrence Erlbaum Associates.

Maass, A., \& Clark, R. D. (1984). Hidden impact of minorities: Fifteen years of minority influence research. Psychological Bulletin, 25, 428-450.

Marquart, D.I. (1955). Group problem solving. Joumal of Social Psychology, 41, 103113.

McAdams, D. P. (1992). The five-factor model in personality: A critical appraisal. Journal of Personality, 60, 329-361.

McCrae, R. R., \& Costa, P. T. (1985). Updating Norman's adequate taxonomy: Intelligence and personality dimensions in natural language and in questionnaires. Journal of Personality and Social Psychology, 49, 710-721.

Mehrabian, A., \& Ksiozky, S. (1974). A theory of affiliation. Lexington, MS: Lexington Books.

Miner, F.C. (1979). A comparative analysis of three diverse group decision making approaches. Academy of Management Journal, 22(1), 81-93.

Mischel, W. (1968). Personality and assessment. New York: Wiley.

Monson, T. C., Hesley, J. W., \& Chernick, L. (1982). Specifying when personality traits can and cannot predict behavior. An alternative to abandoning the attempt to predict single-act criteria. Joumal of Personality and Social Psychology, 43(2), 385-399.

Moscovici, S., \& Lage, E. (1976). Studies in social influence III: Majority versus minority influence in a group. European Journal of Social Psychology, 6, 149-174.

Paulus, P. B. (Ed.). (1989). Psychology of group influence. Hillsdale, NJ: Lawrence Erlbaum Associates. 
Pervin, L. A. (1985). Personality: Current controversies, issues, and directions. Annual Review of Psychology, 36, 83-114.

Polk, J. W. (1991). The effects of an expert on the small-group consensus process. Unpublished master's thesis, Virginia Polytechnic Institute and State University, Blacksburg.

Polk, J., W., Carolan, T. F. and Koball, E. (1994). The effect of expert knowledge on performance in a small group decision making task. Technical report.

Ray, J. J. (1987). Assertiveness as authoritarianism and dominance. The Journal of Social Psychology, 126(6), 809-10.

Ridgeway, C., \& Diekema, D. (1989, February). Dominance and collective hierarchy formation in male and female task groups. American Sociological Review, 54, 7993.

Radloff, R. (1968). Groups under stress, Appleton-Century-Crofts.

Schneider, F. W., \& Delaney, J. G. (1971). Effect of individual achievement motivation on group problem-solving efficiency. The Joumal of Social Psychology 86, 291298.

Schuler, H. (1982). Liking and influence in group decision making: A test in four different experimental settings. In H. Brandstatter, J. H. Davis, \& G. StockerKreichgauer (Eds.), Group Decision Making (pp. 257-277). New York: Academic Press.

Shaw, M. I. (1976). Group dynamics: The psychology of small proup behavior. New York: McGraw-Hill Book Company.

Silk, A.\& Kalwani, M. O. (1982). Measuring influence in organizational purchase decisions. Journal of Marketing Research, 19(2), 165-181.

Sistrunk, R., and McDavid, J. W. (1965). Achievement motivation, affiliation motivation, and task difficulty as determinants of social conformity. Ioumal of Social Psychology, 66, 41-50. 
Snyder, M. (1983). The influence of individuals on situation: Implications for understanding the links between personality and social behavior. Journal of Personality, 51(3), 497-516.

Sorrentino, R. M., \& Field, N. (1986). Emergent leadership over time: The functional value of positive motivation. Joumal of Personality and Social Psychology, 50(6), 1091-10991.

Sorrentino, R. M. (1973). An extension of theory of achievement motivation to the study of emergent leadership. Joumal of Personality and Social Psychology, 26, 353-368.

Spence, J. T. (Ed.). (1983). Achievement and achievement motives. San Francisco, CA: W. H. Freeman and Company.

Stasser, G., Kerr, N.L., \& Davis, J.H. (1989).Influence processes and consensus models in decision-making groups. In P. Paulus (Ed.) Psychclogy of group influence (2nd ed., pp. 279-326).

Steiner, I.D. (1972). Group process and productivity. New York: Academic Press.

Steiner, I.D. (1976). Task-performing groups. In J.W. Thibaut, J.T. Spence, \& R.C. Carson (Eds), Contemporary topics in social psychology (pp. 393-422). Morristown, NJ: General Learning Press.

Tanford, S., \& Penrod, S. (1984) Social Influence Model: A formal integrativin of research on majority and minority influence processes. Psychological Bulletin, 25. 189-225.

Turner, J.C.\& Oakes, P.J. (1986). The significance of the social identity concept for social psychology with reference to individualism, interactionism, and social influence. British Joumal of Social Psychology, 25, 237-252.

Wood, J. T. (1984). Alternative methods of group decision making. In G. M. Phillips, \& J. T. Wood (Eds.), Emergent issues in human decision making (pp. 3-18). Carbondale, IL: Southern Illinois University Press.

Wright, J. C.; Mischel, W. (1987). A conditional approach to dispositional constructs: The local predictability of social behavior. Joumal of Personality and Social Psychology, 53(6), 1159 - 1177. 
Yukl, G., \& Tracy, J. B. (1991). Consequences of influence tactics used with subordinates, peers, and the boss. Joumal of Applied Psychology. 77.(4), 525-535. 


\title{
Environmental Benchmarking for Performance Excellence
}

\author{
by Brian M. Kleiner, Ph.D.
}

\section{Executive Overview}

Benchmarking is a viable management technique that can help improve an organization's environmental performance. Benchmarking is not a cookbook solution but a systematic process involving searching and finding the organization that is the best at a given process ("best-in-class") and continually adopting or adapting new processes to accelerate improvem $n$ nt. As a continual process including planning, analysis, integration, action, and maturity phases, benchmarking is easily integrated into the planning stage of the management system. Many benefits, such as teamwork and job satisfaction, accompany benchmarking but the greatest advantage is being more competitive, even when the organization resides in the public sector.

Finding a suitable partner is crucial to the success of the benchmarking process. I've included examples of where benchmarking partners may be found. The partner approach to benchmarking shows the traditional "one-on-one" approach is the most efficient and effective because it saves time and money and allows organizations to share and learn from each other. I've provided the rules of partnering to show good and bad benchmarking practices.

Lessons learned from benchmarking research and experience should be included in a benchmarking process. I've presented several success indicators, several commonly made mistakes, and the necessity of a strong, visible management commitment in the benchmarking process.

After reading about the potential achievements of a benchmarking process, many ask how to start the process in their own organization. I offer advice on where and how to start a benchmarking process for the best results: exploring the places to find information and focusing on processes.

\section{What is Environmental Benchmarking?}

The U.S. General Accounting Office defines benchmarking as "measuring performance against that of best-in-class installations or companies, determining how the best-in-class achieve those performance levels, and using the information as the basis for your own installation's strategies and implementation (1992)." At a more practical level, it is "the practice of being humble enough to admit someone else is better at something and being wise enough to try to learn how to match and even surpass them at it (International Benchmarking Clearinghouse, 1993)." Arguments abound on how this new technique is like other modern innovations-some attempt to trace benchmarking to the Egyptian pyramids. But nevertheless, it is a technique which has proven viable in manufacturing domains and offers great opportunity to service and environmental industries. 


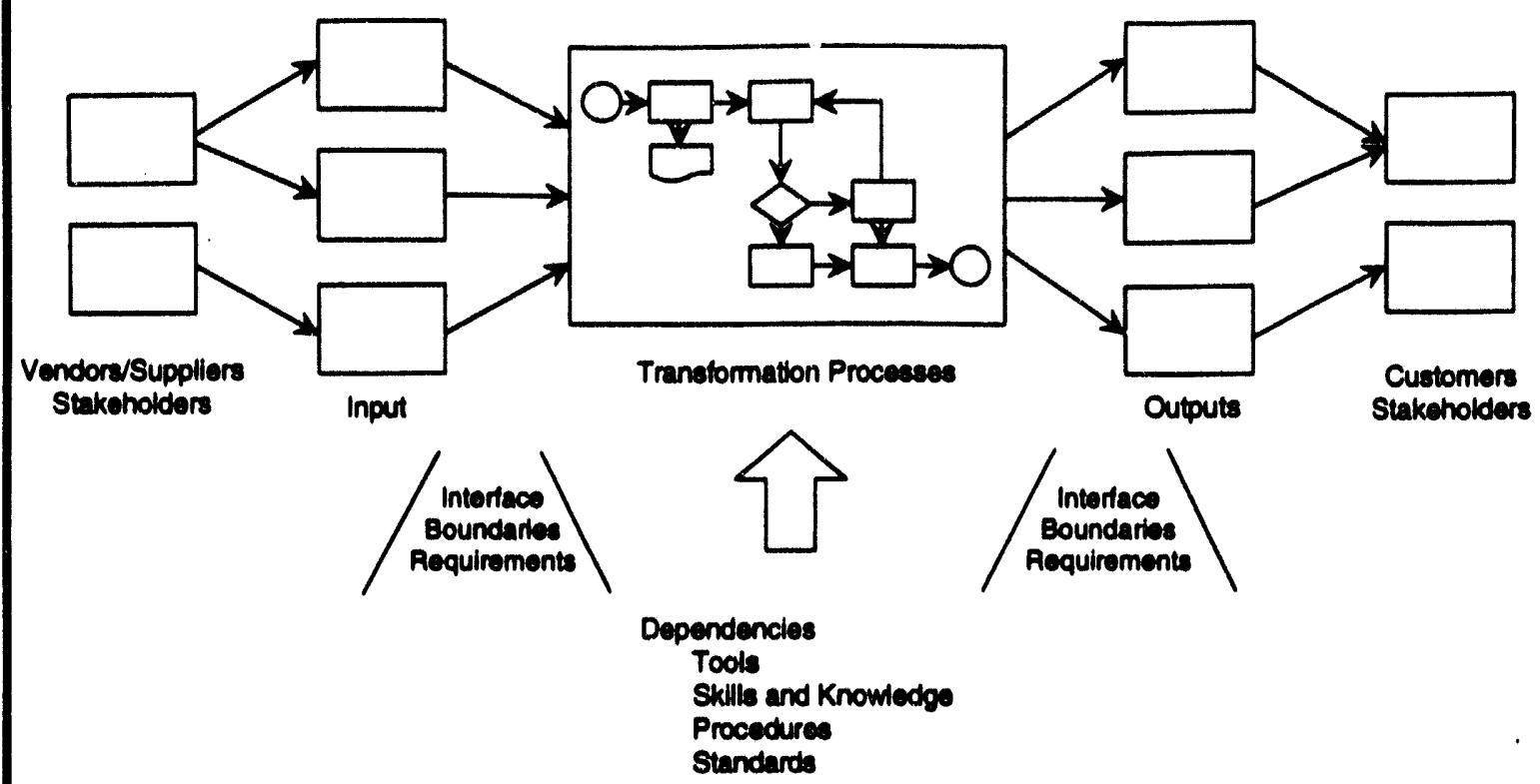

Figure 1

The "system" in which processes function.

To understand benchmarking you need to understand processes. All work gets done through processes which change inputs from suppliers to outputs (i.e., products or services) for customers. For example, waste tank monitoring is a process which converts the information of waste type, level, etc., to the output or service of increased health and safety to workers and others in the local community. Figure 1 illustrates the "system" in which processes function.

In contrasting what benchmarking is to what it is not, the technique promotes a continuous process of performance improvement, not a "program" which yields panacea solutions. It is a proactive planning technique, not a reactive "band-aid" approach.

Benchmarking works because it is consistent with the desire of many organizations to learn and discover new ways to improve performance. It seeks incremented improvements which improve morale and positively affect the bottom line, whether the organization is for-profit or not-for-profit. As seen in Figure 2, those who benchmark find the greatest benefit is being more competitive. In these times of budget reductions and associated programs of cost reduction, even public sector and not-for-profit organizations have to adopt a more commercial, competitive culture.

Benchmarking benefits the public sector by:

- Improving performance of the benchmarked function,

- Meeting stakeholder requirements more adequately,

- Professionally developing employees,

- Breaking down resistance to change,

- Enabling technological breakthroughs,

- Increasing employee motivation and satisfaction,

- Increasing teamwork, and

- Removing subjectivity from goal setting and decision making.

(adapted from Camp, 1989) 


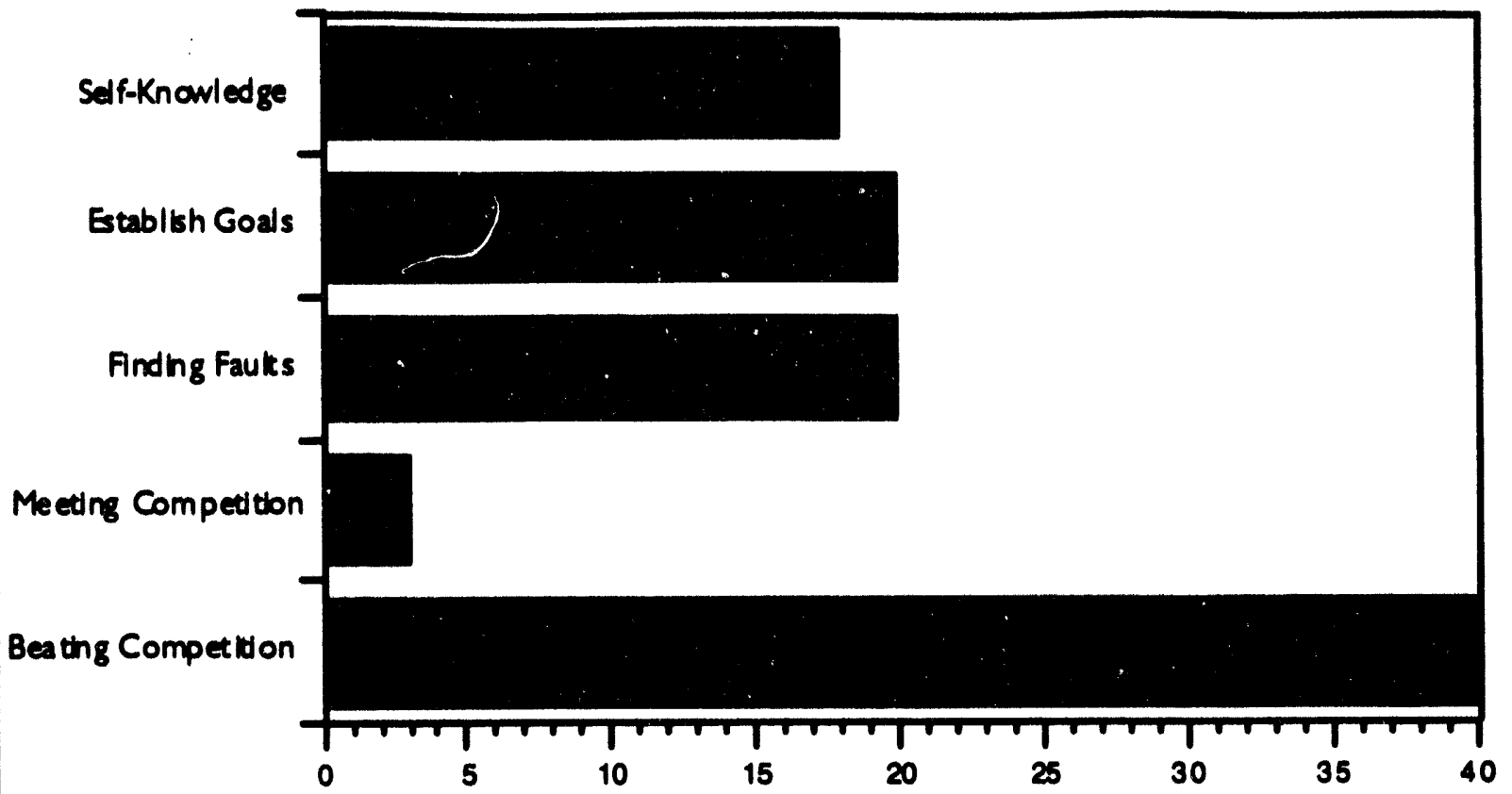

Figure 2

Benefits of Benchmarking

\section{Why Benchmark? Where}

Does Benchmarking Fit in the Management System?

As Williams (1992) illustrates, driving forces such as regulatory issues, increased enforcement activity, liability concerns, investor concerns, competitive pressures, and public perception create a demand for benchmarking. Broadly speaking, benchmarking is a performance management technique which complies with the principles of quality management and continuous improvement. Within a management system, it initially fits best within a planning framework. In a traditional SWOT analysis for example, the organization assesses its strengths, weaknesses, opportunities, and threats. Benchmarking sharpens such an analysis by finding the best-in-class organization for a given process and by assessing why they are the best. Then, the best practices identified are either adopted or adapted. Most existing processes are a projection of past practices into the future, or, "the way we've always done things"; benchmarking offers a projection of best practices into the future. It is an effective way to establish strategic goals based on best performance and then, to tactically pursue implementation by developing a productive partnership with another organization. 


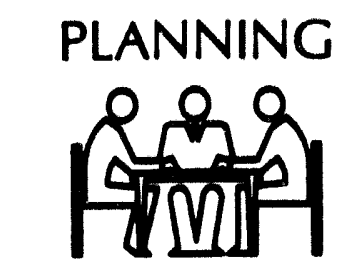

1. Identify what is to be benchmarked.

2. Identify key performance variables to measure.

3. Identify benchmarking partners.

4. Determine data collection method.

5. Collect data.

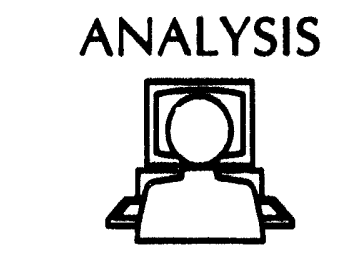

INTEGRATION

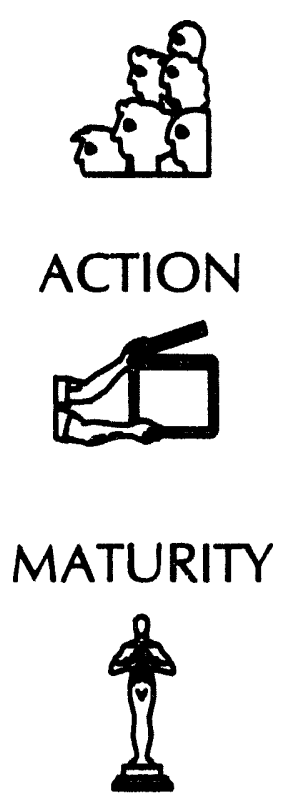

8. Communicate benchmarking findings and gain acceptance.

9. Establish functional goals and operational principles.

6. Determine and understand current performance gap.

7. Predict future performance levels.

10. Implement specific actions and monitor progress.

11. Measure results against stakeholder needs.

12. Recalibrate benchmarks.

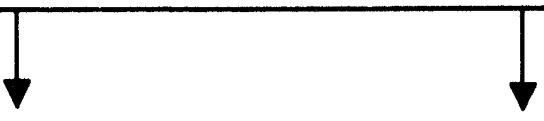

- Leadership position attained.

- Practices are fully integrated into processes.

(groupings adapted from Camp, 1989, 1992)

Figure 3

The Benchmarking Process

\section{The Benchmarking Process}

The generic process as adapted from

Camp (1989) is illustrated in Figure 3.

The planning phase includes deciding what to benchmark. Several questions should be addressed in this phase:

- What "products" or "services" are provided to "customers"?
- What processes or products are most critical to success?

-What areas are causing the most trouble?

- What are the deliverables for the troubled areas?

- What factors are responsible for customer satisfaction?

- Are there other stakeholders who should be considered? 
Selecting a process to benchmark usually is done based on some predetermined need. This need can be translated into performance variables which require improvement. Next, benchmarking partners should be identified. Selecting partners should be based on performance related to the targeted process and not necessarily based on whether the partner is in the same industry. Depending on the process, well-known performers can be identified, such as L.L. Bean for customer service, American Express for purchasing, or Mary Kay for warehousing and distribution. For the broadly defined industry of environmental management, Altany (1990) suggests $3 \mathrm{M}$ and Ben and Jerry's as best performers.

The data collection method used should correspond to the information required depending on the objective of the benchmarking study. As illustrated in Figure 4, benchmarking objectives range from establishing achievable performance targets to developing a "world class" vision for a given function.

\begin{tabular}{|c|c|c|c|c|c|c|c|c|c|c|}
\hline \multirow[b]{3}{*}{$\begin{array}{l}\text { Identify improvement } \\
\text { opportunitles. } \\
\text { Pinpoint improvemen } \\
\text { actions/ressource } \\
\text { allocation. }\end{array}$} & \multicolumn{2}{|c|}{$\begin{array}{c}\text { Type of } \\
\text { Information }\end{array}$} & \multicolumn{2}{|c|}{$\begin{array}{l}\text { Organization } \\
\text { Scope }\end{array}$} & \multicolumn{2}{|c|}{$\begin{array}{l}\text { Functional } \\
\text { Dispersion }\end{array}$} & \multicolumn{2}{|c|}{$\begin{array}{c}\text { Work Level } \\
\text { of Detail }\end{array}$} & \multicolumn{2}{|c|}{$\begin{array}{c}\text { Internal/ } \\
\text { External } \\
\text { Threats }\end{array}$} \\
\hline & 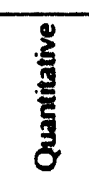 & 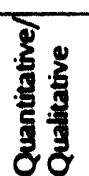 & 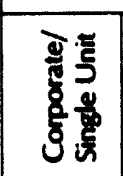 & $\begin{array}{l}5 \\
\frac{5}{3} \\
\frac{9}{8} \\
3\end{array}$ & $\tilde{\xi}$ & 88 & $\frac{8}{2}$ & 总 & 更 & त्व \\
\hline & 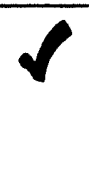 & & 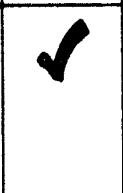 & & & & & & & \\
\hline $\begin{array}{l}\text { Reduce costs/improv } \\
\text { efficiency. }\end{array}$ & & & & & & & & & & \\
\hline $\begin{array}{l}\text { Develop "world-class' } \\
\text { vision for function. }\end{array}$ & & $\checkmark$ & $\checkmark$ & & & & & & & \\
\hline $\begin{array}{l}\text { Establish compettuve } \\
\text { performance targes. }\end{array}$ & & & & & & & & & & \\
\hline $\begin{array}{l}\text { Establish achievable } \\
\text { performance targets. }\end{array}$ & 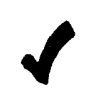 & & & & & & & & & \\
\hline
\end{tabular}

(adapted from Cecil and Ferraro, 1992)

Figure 4

Benchmarking Objectives 
Data is collected from both organizations in the partnership. Every attempt should be made in the planning phase to agree on comparable data. As Kurstedt's (1991) model implies in Figure 5, data is worthless unless it conveys meaningful information.

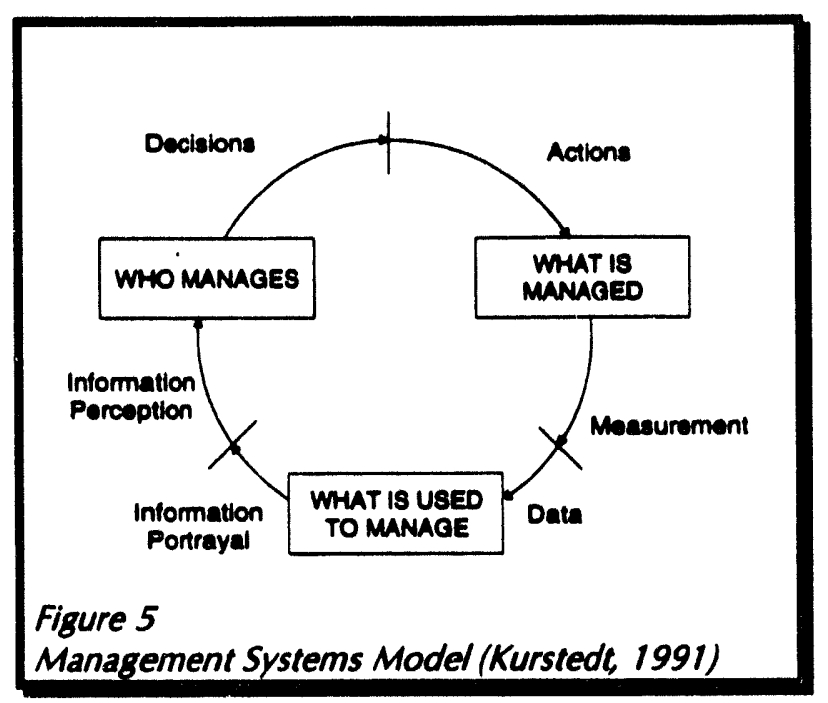

A comparative analysis is performed to arrive at meaningful information. Is the partner better? Why are they better? By how much? What best practices are being used to yield superior performance? How can the partner's practices be adopted and/or adapted? These are just some of the questions to be answered in a properly performed competitive analysis. Graphical portrayals can assist in illustrating potential gaps, as illustrated in Figure 6 . In addition to determining the size of the current gap, every attempt should be made to predict future performance levels as well.

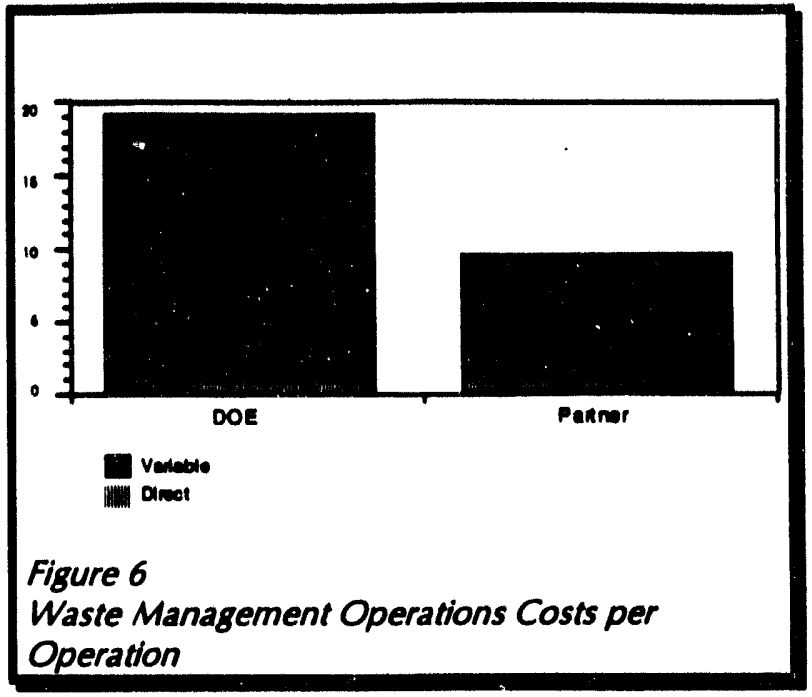

Communication, which is a very important step, should happen during the integration phase. Typically, those who perform benchmarking studies must communicate and gain approval from those with more organizational authority. While each audience must be individually assessed to determine appropriate media and presentation formats, some simple graphics have proven useful as illustrated in Figures 7 and 8 and Table 1. Based on benchmarking findings, operational goals and plans should be established.

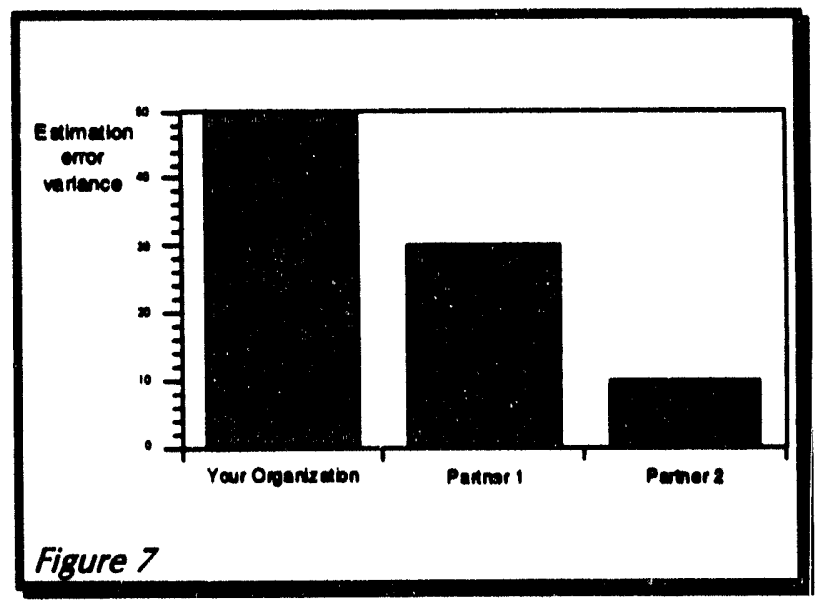




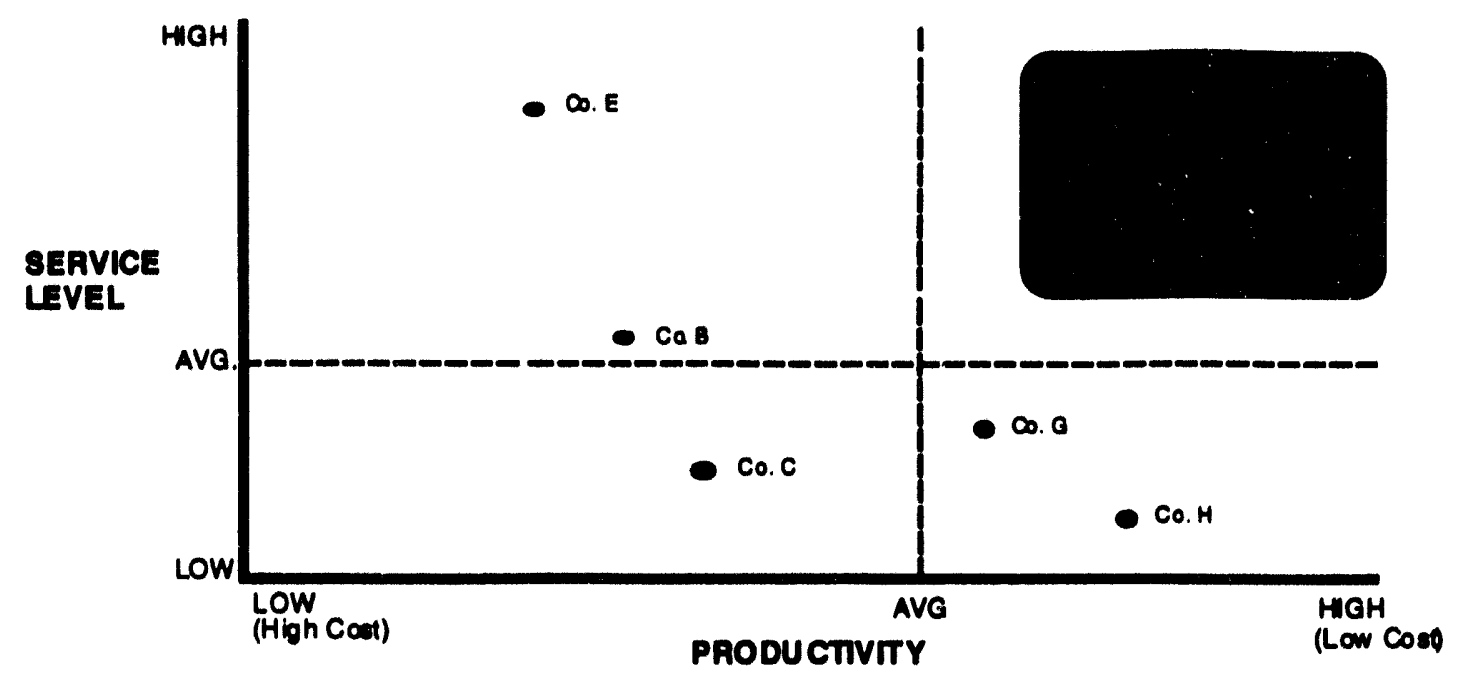

Figure 8

Functional Performances (Productivity vs. Service Level)

Table 1

Hazardous Waste Drum Storage Cost Performances Rating System

\begin{tabular}{|c|c|c|c|c|c|}
\hline $\begin{array}{l}\text { Cost Performance } \\
\text { (cost per drum) }\end{array}$ & $\$ 19$ & $\$ 26$ & $\$ 38$ & $\$ 57$ & $\$ 76$ \\
\hline Operation Rating & 5 & 4 & 3 & 2 & 1 \\
\hline $\begin{array}{l}\text { Waste Management } \\
\text { Operation Type }\end{array}$ & $\begin{array}{l}\text { World } \\
\text { Class }\end{array}$ & Superior & Par & Sub-par & $\begin{array}{l}\text { Needs } \\
\text { improve- } \\
\text { ment }\end{array}$ \\
\hline
\end{tabular}

The action phase starts with implementing actions designed to adopt or adapt the best practices identified by the study. Good principles of project management should be applied, including measuring progress especially as it relates to closing the performance gap. Stakeholder needs should have been converted into measures during the planring phase and progress should be regularly communicated. Benchmarking is a dynamic technique, operating under the framework of quality management and continuous improvement. Therefore, the targets need to be periodically recalibrated, much as world records in 
track and field constantly improve. A given benchmarking process can be considered complete when the best practices are fully integrated and institutionalized into the system.

\section{Partnering}

Benchmarking through the partnering approach has traditionally been "one-onone $e^{n}$ one organization partnering with another organization with similar approaches, principles, values, and measures. They commit to each other by sharing and learning from one another. Partnering with one organization allows more time to be spent delving into more details of the processes and building a solid, trusting relationship. Another benefit of the one-on-one approach is reducing the preparation time and costs that benchmarking with many organizations would require.

Public sector organizations often think it will be nearly impossible to find partners for their processes because of their unique missions. However, public sector organizations perform many of the same basic processes that other public as well as private organizations perform such as purchasing, public outreach, data collection, etc. The partner organization might not be an exact match but has enough comparable data and measures to make the partner-ship beneficial to both organizations.

Benchmarking partners are available in many places. Figure 9 illustrates the variety of ways benchmarking partners have found each other.

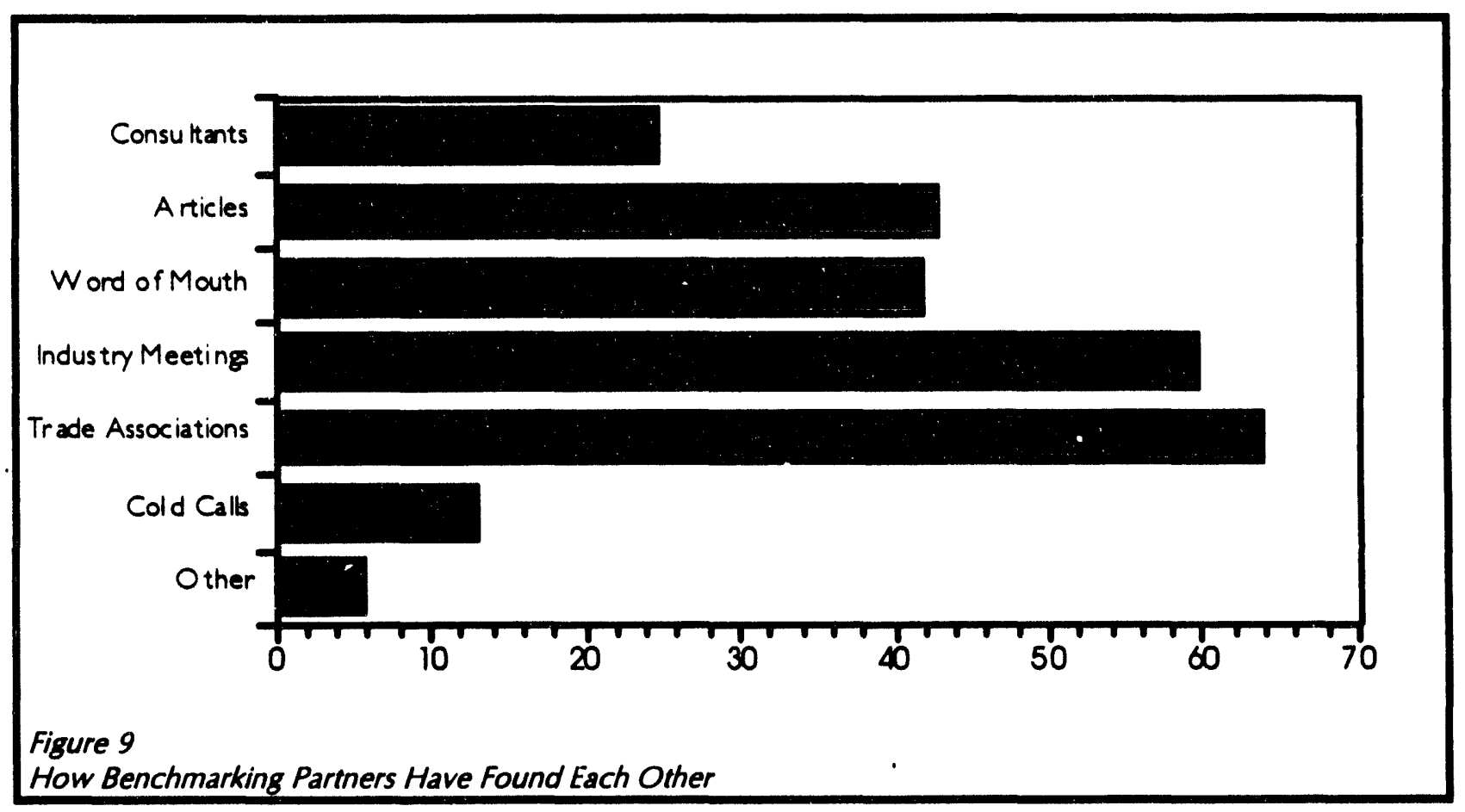


When beginning to partner for a targeted process, the "rules" of benchmarking below should be followed.

\section{Good Practices}

- Using secondary research to find public documents about a target organization

- Observing competitive processes in the open market and performing "reverse engineering"

- Conducting market research and stakeholder-satisfaction surveys

- Collecting information at trade shows

- Asking other organizations to share information about their processes

- Tapping employees to build a "whoknows-what" database about other organizations

- Being honest

\section{Bad Practices}

- Trespassing on another organization's property to collect information

- Bribing people to be informants

- Bugging or eavesdropping on another organization's privileged or private communications

- Learning something, even inadvertently, about a competitor's pricing considerations

- Requiring suppliers to participate in a benchmarking study as a concition of keeping or getting your business

- Trading a company's stock after learning about material information that hasn't been publicly disclosed

Although the above practices are common sense, some practices are ${ }^{. "}$ questionable depending on the relationship between the two partnering organizations and each organization's standards of conduct. Even then, the practices should be considered carefully.
- Recruiting employees from competing organizations to get information

- Asking questions at a technical meeting without identifying your name and organization

- Subscribing to a competitor's technical journals or attending user conferences as a private individual without disclosing your organizational affiliation

In addition, for the best benchmarking results through the partnering approach, these potential problems should be avoided.

- Talking about information you got from one organization with another organization

- Giving information in a public forum about a benchmarking partner without the partner's permission

- Asking for information you wouldn't give yourself

- Asking for information from a benchmarking partner without first understanding your own process

- During a site visit, asking for lastminute changes or additions to the agenda (or bypassing the benchmarking partner's designated host) to get more access to the company

\section{Status of Environmental Benchmarking}

Most environmental organizations do not actively benchmark for many reasons. Some don't understand the benefits of benchmarking or feel they don't have the expertise to begin a benchmarking effort. Others don't know who to partner with or how to partner. But the most common reason the environmental industry does not benchmark is benchmarking is a new technique that does not rank high enough 
on their priority list to get the needed time and resource allocations. As a technique of quality management/ continuous improvement, benchmarking is usually not pursued until several years into the program. (See Figure 10.)

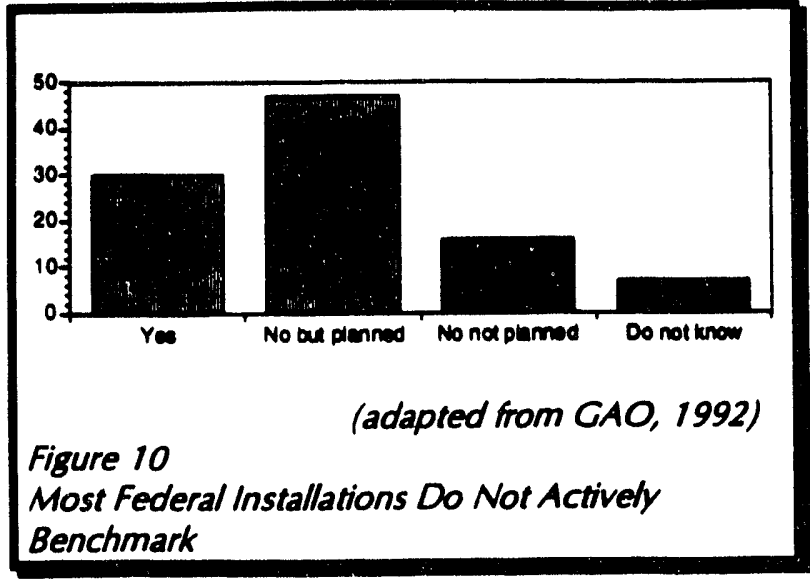

\section{Lessons Learned}

Based on benchmarking research and experience the following lessons learned should be incorporated into the environmental benchmarking process:

Benchmarking is a continual process. As mentioned before, benchmarking is not a panacea or a one-time, cure-all program.

Benchmarking is a line function (i.e., includes all levels of the organization). Benchmarking may start with management but the employees actually doing the process being benchmarked must integrate and institutionalize the new process.

Share all you have and borrow from the best. In a good partnering relationship, you will have chosen a partner who has the best process you need and feels totally comfortable sharing all the information needed to make the process work for you. The partnering should be a "give and take" relationship with both partners sharing openly and improving continually.

Give benchmarking a high priority. Benchmarking is not a technique that can be done partially and still show its potential results. The organization, particularly management, must be willing to allocate the necessary time and resources to make the process work.

Credibility and value of the program depends on your method.

Choose your benchmarking partners carefully. The well-chosen partner will help you reach the performance excellence you're after. A poorly chosen partner will be a waste of efforts and resources for both your organization and the partner organization.

Benchmark more than cost. The real benefit of benchmarking is focusing on processes. By improving processes, results, including cost improvement, will come.

There is no "right" way. Benchmarking, like any other process, must be adopted or adapted to suit your organization's unique needs. In other words, what works for one organization may not work for another until it has been analyzed and integrated. For the best results, the organization should know itself throughout and have processes well documented before partnering with other organizations and trying to implement their processes.

\section{Ten Common Benchmarking Mistakes}

1. Internal processes go unexamined.

2. Site visits feel good but don't elicit data or ideas.

3. Questions and goals are vague.

4. The effort is too broad or has too many parameters. 
5. The focus is not on processes.

6. The team is not committed to the effort.

7. Homework and/or advanced research isn't assigned.

8. The wrong "benchmarkee" (i.e., partner) is selected.

9. The effort fails to look outside the industry.

10. No follow-up action is taken.

\section{Ten Benchmarking Success Indicators}

1. Active management commitment

2. A willingness to change and adapt

3. Proactive behaviors

4. A willingness to define information with benchmark partners

5. A focus on best practices first, performance metrics result

6. A focus on functionally best operations

7. Following a structure process

8. Openness to new ideas and innovation

9. Continuous benchmarking effort

10. Benchmarking becoming institutionalized

(adapted from Camp, 1989)

\section{Management's Role}

Because of the unlimited potential for improvement and overall scope of the benchmarking process, management must take not only an active role in benchmarking but the lead facilitating role. As a part of planning in the management system, benchmarking, to be most effective, is an organization-wide, continual process. Management must oversee the whole effort and play an integral part in keeping the process in action. Management's interest, mentoring, and support will motivate employees throughout the organization to be innovative and creative. Here are some management behaviors that lead to effective benchmarking:
- Provide leadership to plan and organize.

- Agree on the benefits, the partners, the approach, each person's role, and the barriers.

- Communicate that benchmarking is the more effective way.

- Make sure findings are understood and accepted.

- Make sure performance is projected and recalibrated based on findings.

- Have a communication process to inform the organization of progress.

- Integrate findings with the organization's objective setting, performance appraisals, and operating plan process.

- Find successful case histories.

\section{How to Get Started}

After reading or hearing about the significant benefits that can be achieved, many times people ask how they can start a benchmarking process in their organization. The first step is deciding what to benchmark or what area to focus on first to accelerate improvement. After deciding where to begin, the next step is gathering information in a general way and for the specific function targeted.

\section{Information sources - General}

- Focus on an area/element that needs to be pursued (e.g., cost estimating, schedule estimating).

- Contact a business library or academic institution:

- to request a search of information produced in the last three to five years for your topic of interest and

- to have the library identify articles/sources from external reports, public magazines, industry journals, and annual reports. 
- Contact internal experts:

- contractors, other offices, other public offices, functional experts, survey internal reports/surveys, special studies, and surveys.

\section{Information Sources -}

Specific/Functional

- Read trade periodicals.

- Contact professional associations:

- newsletters, seminars (especially speakers/tours), bibliographies, special libraries.

- Contact service agencies/bureaus in the business function:

- to ask if they can share anonymous experiences from their client companies concerning industry and state-ofthe-art methods/practices.

- Contact consulting firms:

- functional experts, ask if they are aware of particular breakthroughs/practices in a specific area.

- Contact industry experts:

- department heads of noncompetitors, teachers/professors at schools/universities.

- Contact systems software development and hardware vendors: to ask about their experiences working in the functional area.

Another early step in benchmarking is planning through training. Here are some options to consider.

- Have an awareness workshop:

- to create baseline understanding of the quality management or continuous improvement context and - to focus on current mission, vision, organizational design.

- Facilitate definition of system: - to define functions, processes, products, practices; to define customers and suppliers, stakeholders; and to define interdependencies and redundancies.

- Facilitate identification of key functions and processes to be benchmarked.

- Facilitate mapping of current key processes.

- Later, during comparative analysis determine and understand performance and methods gap.

One place to turn when starting a benchmarking process is the International Benchmarking Clearinghouse. Started by Xerox in the 1980s, the International Benchmarking Clearinghouse offers a number of services and provides. information on functions and past studies as well as helps you contact their members for more information. Many organizations such as the U.S. Department of Energy (DOE) or the Environmental Protection Agency are ideal for establishing their own Clearinghouse to provide comparable services to those listed below.

International Benchmarking Clearinghouse Services

Networking Services (e.g., meetings)

Information Services (e.g., searches and research)

Benchmarking Services (e.g., partner identifications)

Benchmarking Training

Electronic Network

Database of Past Studies

Here is a partial list of International Benchmarking Clearinghouse members:

Commercial

Xerox (founder)

Weyerhaeuser

Exxon

Grumman

ITT Defense

Shell

Waste Management Company 


\section{Federal}

U.S. Air Force Reserve

U.S. Bureau of the Census

U.S. Department of the Navy

U.S. Internal Revenue Service

U.S. NASA Johnson Space Center

U.S. OPM. Federal Quality Institute

U.S. Department of Energy

Office of Environmental Restoration and Waste Management

\section{Conclusion}

As illustrated in a recent DOE report, "a multi-method [environmental] benchmarking approach, utilizing several partners, involving a large number of interested parties, and developing mutually beneficial partnerships with other federal agencies and commercial organizations can be performed costeffectively to yield valuable results (DOE Office of Environmental Restoration and Waste Management, 1993)." Benchmarking offers both public and commercial organizations the opportunity to apply the principles of quality management and planning in such a way that performance can be dramatically improved. 


\section{Works Cired}

Altany, David. 1990. "Copycats: once obscure, 'benchmarking' is fast becoming the buzzword of the year." Inctustiy Week (November 5) vol. 239 no. 21 pp. 11.14.

Camp, Robert. 1989. The Search for the Best Practices That Lead to Superior Performance. Milwaukee, WI: The Quality Press, 300p.

Cecil, R. and R. Ferraro. 1992. "IEs Fill Facilitator Role in Benchmarking Operations to Improve Performance." Industrial Engineering (April 1992) vol. 24 no. 4 pp. 30-34.

International Benchmarking Clearinghouse. 1993, brochure.

Kurstedt, H.A., Jr. 1991. "Why the Methods of Management Will Work for You." Blacksburg.

VA: Management Systems Laboratories.

U.S. General Accounting Office. 1992. Quality Management, Survey of Federal Organizations. Briefing Report to the Honorable Donald Ritter, House of Representatives. (October 1992) GAO/GCD93-9BR p. 48.

U.S. Department of Energy, Office of Environmental Restoration and Waste Management. 1993. Benchmarking for Cost Improvement, p.9.

Williams, Marcia. 1992. "Why-How to-Benchmark for Environmental Excellence." Total Quality Environmental Management (Winter 1992-93) pp. 177-185.

\section{Glossary}

best practice

- method used in work processes whose outputs best meet customer requirements and represent what you and others (stakeholders) think is most desirable

functional unit or function - goatoriented organizational unit (e.g., operations support)

measurement

- a quantitative performance indicator of a specific process or step

partner

- another organization with whom you exchange best performance measures and practices

practice

- the method we use to perform an activity

(e.g., activity-based costing)

process

- a series of tasks or steps performed to meet tanget a specific objective (e.g., cost estimating)

- conversion of benchmark to operational metric

Brian Kleiner is a reseanch faculty member at Management Systems Laboratories in the Department of Industrial and Systems Engineering at Virginia Polytechnic Institute and State University. He has worked with federal, state, and commercial organizations to research the potential of environmental benchmarking and has led several successful benchmarking implementation efforts. This paper was prepared to help establish a baseline understanding of benchmarking for managers and practitioners.

The preparation of this paper is funded by the U.S. Department of Energy (DOE) Delivery Order No. DE-FG0288DP48058. Management Systems Laboratories thanks DOE for providing a realworld laboratory for the research development, and testing of state of the art management tools and the framework for understanding how to make these tools successful. 


\section{MANAGING COMMUNICATION SUCCESSFULLY IN YOUR MANAGEMENT SYSTEM}

\section{By Brian M. Kleiner}

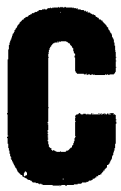

ommunication is overlooked and underrated in terms of its impact and importance in organizations. Bamard instructed that "providing a system of communication is one of three executive functions essential to the survival of an organization." Bennis and Nannus stated "provicing meaning through communication is one of four key leadership strategies." Nevertheless. we generally approach communication passively and reactively: we assume by the lines in our organizational charts and by the arrows on our flow diagrams that communication is truly linking the organization and our processes. We are often perplexed when breakdowns in communication occur. In many organizations, communication problems have become a cliche. It is often cited as the root cause of a problem with no effort direrted at correcting the problem. What we need is a systematic way to understand communication in the management system. a way to assess its effectiveness and methods for continuous performance improvement.

\section{The management system}

Every manager has a domain in which he/she operates. This domain is characterized by a management system that includes people. tools, technology, information, etc. Most managers do not consciously think about their management system. This lack of recognition can lead to a gap between what you do as a manager and what you should do as a manager.

Kurstedt identified a management system as containing three components - "who manages," "what is used to manage" and "what is managed," as illustrated in Figure 1. What is used to manage refers to management tools and what is managed focuses on the organizational system. There are also three interfaces: the measurement-to-data interface between what is being managed and management tools: the information portrayal to information perception interface between tools and who is managing (management tools portray information while the manager perceives the information from the management tools), and the decision-to-action interface between who is managing, and what is being managed. In this interface, the manager yields decisions while the organizational system requires actions. The orga- convert the data to information. Improvement interventions and management team decisions require information about how

\section{The communication process is a glue that holds the management system and its components together and helps achieve balance.}

nizational system yields measures while management tools require data.

\section{A communieations managomont model}

Communication has an important role in your management system, as illustrated in Figure 2. The communication process is a glue that holds the management system and its components together and helps achieve balance. You, the manager, are the sender of information (in the form of decisions and actions) to the organizational system, which is the receiver. The message (decision) must be encoded in a way it can be understood by both sender and receiver. It must be transmitted over a channel that links you with the organizational system. The organizational system must be ready for the message $s 0$ it can be decoded and understood. Noise, including physical noise or other hindrances to communication (e.g. crises, prejudice, etc.), can hinder the process. From this point, the organizational system then becomes the sender of information back to you, because you need feedback on the implementation of the action taken.

At this juncture, your role is to be a receiver, and communication tools should enable this. The communication process facilitates the interfaces as well. First, as manager, your decisions must be converted into actions. You will successfully communicate decisions into actions if you use appropriate communication principles and techniques. There also needs to be a match between the measurement of the organizational system and the data fed into management tools that

Brian M. Klelnor, Ph.D., is director of the Organizational Systems Laboratory at Management Systems Laboratories in the department of industrial and systems engineering, Virginia Polytechnic Institute and State University. well the organization is performing. Tech. niques to collect, process and communicate data are important. Design of management tools will consider the daca required, and if designed concurrently, the organizational system measurement techniques and the measures themselves will be compatible with the requirements of the user-centered tools. As a manager. you want to internalize the information portrayed from your manage. ment tools through information perception. In this regard. you are acting as a receiver of communication and a user of a management tool. User-centered design principles should consider the communication process. In addition, your learning preferences should be considered when selecting or designing management communication tools. Research has shown different people prefer different modes of communication. This preference coupled with situational considerations and design principles will yield an effective information portrayal to information perception interface. In addition to the communication process, there are principles applicable to continuous performance improvement of the three major components of the management system.

\section{Who maneres?}

As a sender and receiver, you should be concemed with your personal communication skills. These skills help maximize the effectiveness of the "who manages" component of the system. Effective communication requires both the sender and the receiver to agree on the intent as well as the content 
of the message. First. you should decide on the best mode of communication for a given situation. Written communication promotes uniformity and provides records for legal and administrative purposes. The masses can be reached through this vehicle as well. The disadvantages of writ. ten communication include the lack of immediate feed. back and the tendency to

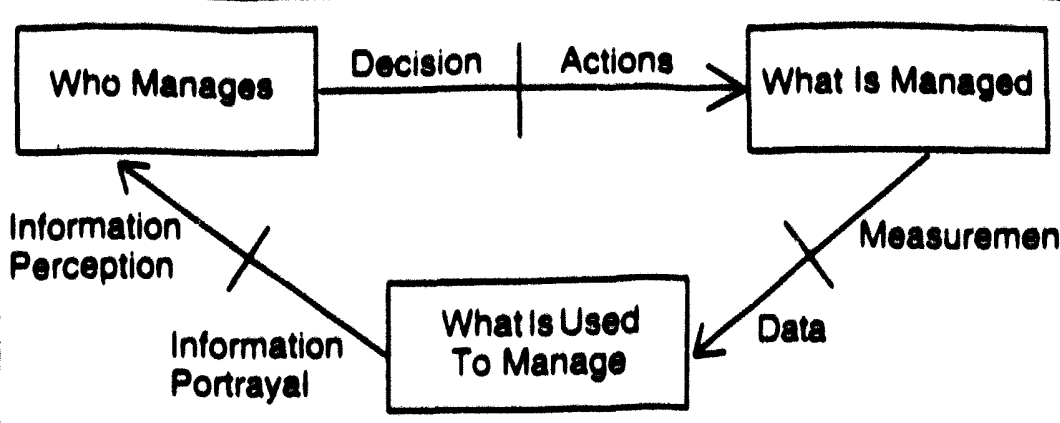

Pigure 1 and boosts morale.

You can recielve reed. back on messages ient and decisions implemented. Problems that may be un. comfortable to deal with are. nevertheless. brought to the surtace where you can act upon them. As manager. you need to know about drug use. processes out of control. dys. functional equipment or sexual harassment at lower levels in your organization. create a paper factory. Oral communication is fast and allow s immediate feedback. How. ever. oral communication does not necessarily save time and therefore reduce cost. Ineffective meetings are an example of this. Finally. oral communication can be helped or hindered by non-verbal communication. It is the job of management to know the right type of communication given a particular situation and to skillfully execute the communication. Various management tools can help you communicate effectively.

\section{What is used to manage?}

Communication tools can be both hard and soft. Hard communication tools include computers. fax machines and telephones. while soft tools include active listening techniques. Active listening techniques assure you both the intent and content of your messages are being received by the receiver. Paraphrasing validates expectations by you stating in your own way what the sender in the organizational system is trying to say. Perception checking is describing to the sender what you perceive their inner state to be at the time of communication. For example. the statement "This decision seems to be troubling you" can lead to further explanation and clarification. Describing the sender's behavior allows you to share with the sender their overt behavior. For example. the statement "you are pacing" allows the sender to share whether they are nervous or angry. giving more context and definition to the communication. Describing your feelings relative to the communication allows the sender to clarify the message. "You really hurt me" or "this new information will change everything I' $m$ working on" can lead to an appropriate modification or recantation of the message. Finally. the Johari Window is an analytical tool that identifies information available for use in communication. By representing information along two dimensions, information known and unknown by yourself and information known and unknown by others. you can set the goal of "open self" and focus on communicating in an arena where information is known by both parties.

\section{What is managed?}

As described by Zaremba (from a management standpoint), managers should create. cultivate and nourish communication networks in the organizational system. Just as the FAA approves certain traffic routes for aircraft travel. certain communication networks should be created and managed. It is instructive to understand the difference between what is used to manage (management tools) and what is managed (the organizational system). The airplanes themselves. whether state-of-the-art technology or not. are the tools that rely on the approved routes for passage. The routes or passages are the networks or channels that need to be managed. The memo is the communication tool, and the path it takes to its destination is the network. There are three basic communication network systems that operate within organizations: external/intemal; formal/informal: and upward/downward/horizontal. Extemal networks, such as public relations. carry information from inside the organization to outside and vice versa. Internal networks are both inter and intradepartmental in nature and channel information inside the organization. From a management perspec. tive, poor internal networks can affect the external network. Formal networks correspond to the organization chart and represent the prescibed channels for communication. Informal networks correspond to the informal organization structure and carry information on routes not prescribed by the organization.

Back to our air traffic metaphor, to the naked eye, any position in the sky should do for travel. However, there are formal. prescribed channels for air travel. The penalty for using the unofficial air traffic networks can be significant, as evidenced by the Korean airliner Flight 007 disaster or the 1987 small plane landing in Red Square. In addition, your job as manager is to ensure the viability of formal routes and to adopt a proactive position with respect to the informal network. You need upward information in order to manage effectively. This helps employees feel like a part of the organization
Finally. as evidenced at such firms as Toyota. employee suggestions are valuable. The problem in the U.S. is we have tended to focus more on evaluating suggestions rather than soliciting suggestions. Of course. you may resist upward communication as many managers have in the past. Though the potential for criticism and bad news do exist. proper management of the organizational system cannot be effective without bottom. up information.

Downward communication is much more likely to be formalized. especially in functionally organized hierarchies. These networks tend not to involve feedback. and therefore. you. as the sender, never know the quality of the received message.

As Zaremba indicates. horizontal com. munication, or the movement of informa. tion along the same strata of the organization. is rarely formalized below the top-most levels of the organization. In managing the organizational system. you should consider creating formal networks for horizontal information. Without such networks. information is likely to get lost, distorted or arrive late.

\section{Communieation manacoment oyotoms}

Communication is also a process and a function that can and should be assessed in part of your management system and can help you continuously improve your system. Part of this improvement process is knowing how you are doing. Many organizations have a communication function or department, but even if not formalized as a department, the process of communication and the success of communication networks should be evaluated and continuously improved. As Sink points out, the criteria used in the measurement process should focus on efficiency, effectiveness, quality, productivity, profitability/budgetability, quality of worklife and innovation.

Survey instruments are effective tools to elicit feedback from customers. Since customers can be internal or external. customer satisfaction instruments can be applied to all types of communication netyour organization. Communication is a vital 


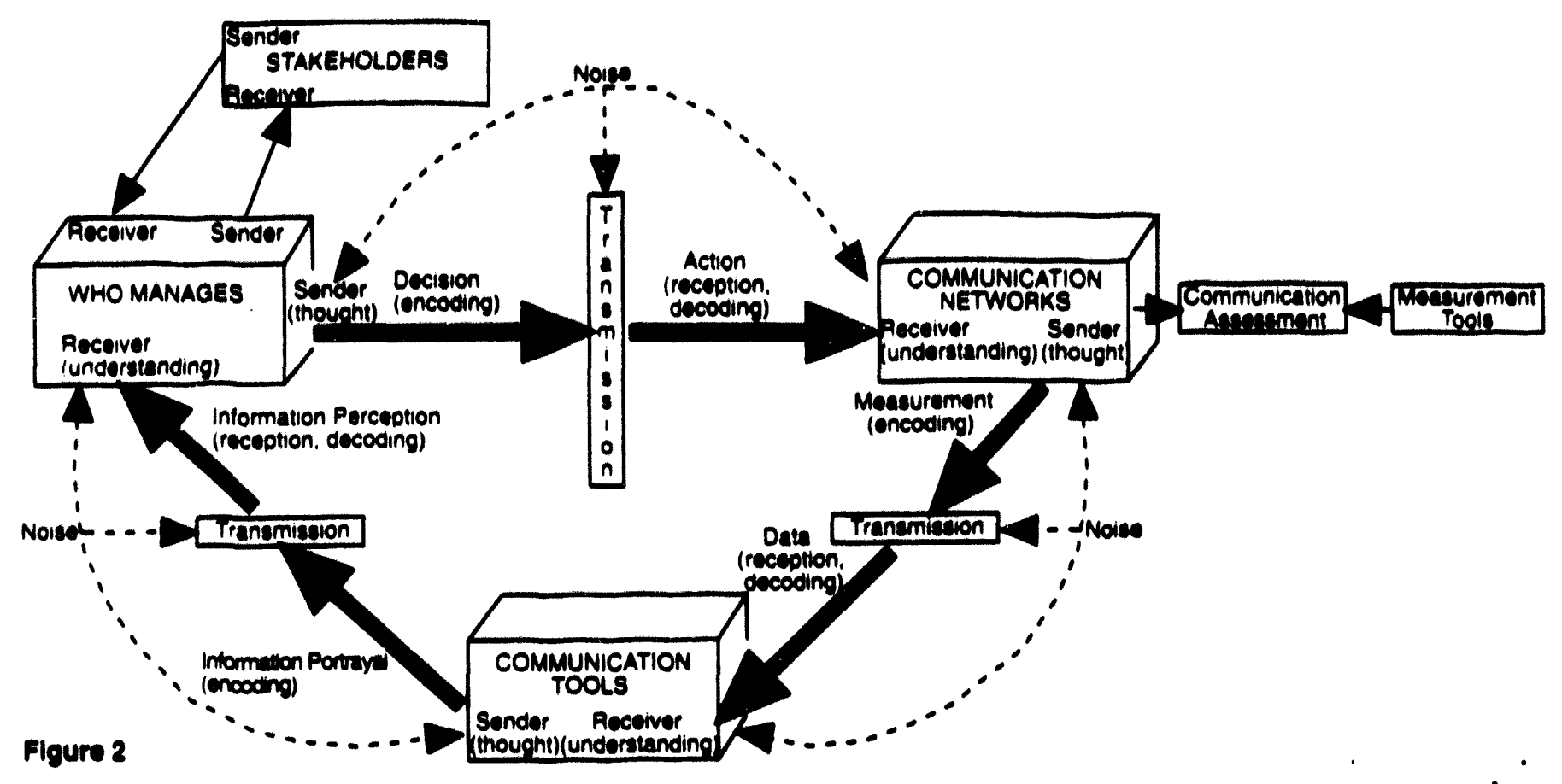

works. An organization's benchmarking process (or procedure for comparing its own performance against the best in the industry or best at a particular function), can be assessed to measure how well the external network allows such important information to enter and be used by the organization. An input output analysis is a procedure whereby your system or subsystem is defined by its upstream and downstream systems. customers. suppliers. stakeholders and transformation processes. The efficiency and effectiveness of communication can be evaluated for internal. external. formal. informal and horizontal networks using this method. The transformation process itself can be assessed in detail using a flow diagram of the formal communication process. It is also beneficial to tlowchan the informal process and to address the gap between the two. Observational analysis can be used for all communication networks. Effective communication networks are frequently transparent. but ineffective networks can be most conspicuous. For example. an unartended. persistently ringing telephone, arguments in the hallway and customer complaints are all indicators of potentially ineffective communication networks and can be documented through observational techniques. Actively walking around. statistically thinking about what you observe as being either specialcause or common-cause related will help you to devise appropriate intervention strategies.

Protocol analysis is a technique that allows the systematic investigation of verbal reports or transcripts. This approach can be useful in analyzing the differences between the informal and formal networks and the underlying causes of those differences. A vertical slice analysis is where an internal. neutral party or an outside consultant conducts structured interviews at each level in the organizational hierarchy. This allows a systematic evaluation of communication at each level of management and reveals if there is a breakdown or barrier at a particular level. This is a most effective method for evaluating upward and downward communication and reveals much about the intemal and formal networks.

A scatter plot can be used to analyze the relationship between the internal and external networks. A given variable in the internal network such as efficiency of design-tomanufacture communication can be plotted against an external network variable such as number of new customer inquiries to see whether internal management is affecting the external network.

An Ishikawa or wishbone diagram can be used to document the potential causes of a communication problem. This tool can be applied to most types of networks. A Pareto Diagram is a tool that allows the identification of the most significant communication problems or factors.

Quality Function Deployment (QFD) is a technique used for converting customer requirements into design specifications. As such, it can be used to assess the extent to which the external customer's voice is being heard in the network and whether it is being translated appropriately in the internal network.

Though taken for granted, communication is a vital process and function in your management system. By having a systematic way to understand communication. a way to assess its effectiveness and methods for continuous improvement. your organizational system and you will be more successful.

\section{For furtuer reading}

Bamine. The Functions of the Executive. Cambindge. Mass.: Hervard University Press.

Bennis and Nannus. Leaders: The Sirategies for Taking Charge. New York: Haper 4 Row.

Mallat. L.A. and Kursiedt. H.A.. "MIS success in a fedend govermment organization." Fall Industrial Engincering Conference Proceedings.

Sink. D.S. and Tutde. T.C.. Planning and Measurement in Your Organization of the Future. Norcross. Georgia: Industrid Engineering and Management Press.

Zarember A. "Management in a new key: Communication Networks." Indusinal Management. Septenv. ber/October 1989. 
Environmental Benchmarking as a Tool for Continuous Performance Improvement

Brian M. Kleiner, Ph.D.

\author{
Management Systems Laboratories \\ Department of Industrial and Systems Engineering \\ Vizginia Polytechnic Institute and State University \\ $1900 \mathrm{Kraft}$ Drive
}

Blacksburg, Virginia 24060

Acknowledgement

The preparation of this paper is funded by the U.S. Department of Energy

(DOE) Delivery Order No. DE-FG0288DP48058A. Management Systems Laboratories thanks DOE for providing a real-world laboratory for the research development, and testing of state of the are management tools and the frame-

work for understanding how to make these tools successful.

\title{
Keywords
}

tenchmarking, environment, waste management, planning, commercial 


\section{Executive Overview}

Benchmarking is a viable management technique that can help improve an organization's environmental performance on a number of dimensions. Benchmarking is not a cookbook solution but a systematic process involving searching and finding the organization that is the best at a given process ("best-in-class") and continually adopting or adapting new processes to accelerate improvement. As a continual process including planning, analysis, integration, action, and maturity phases, benchmarking is easily integrated into the planning stage of the management system. Once processes are strategically identified, they can be improved within the company's total quality management (TQM) program. Many benefits, such as teamwork and job satisfaction, accompany benchmarking but the greatest advantage is becoming more competitive.

Finding a suitable partner is crucial to the success of the benchmarking process. I've included examples of where benchmarking partners may be found. The partner approach to benchmarking shows the traditional "one-on-one" approsch is the most efficient and effective because it saves time and money as well as allows organizations to share and learn from each other. The rules of partnering illustrate good and bad benchmarking practices. The danger of suboptimizing systems through benchmarking is discussed and preventive measures are presented.

I've analyzed lessons learned from benchmarking research and experience. Additionally, I've presented several success indicators in the benchmarking process are presented, including the necessity of a strong, visible tanagement commitment. Since the ultimate intent of benchmarking is positive change or improvement, I discuss the difference between reengineering and continuous performance improvement and the role benchmarking plays in each.

After reading about a benchmarking program and its unlimited potential many people ask how to get a program started. Therefore I have provided difection as to where and how to start a benchmarking process for the best results: exploring the places to find information and focusing on processes. 
Environmental Benchmadjing, as a I'ool of TOM and Continuous Performance Improvements Benchmarking is "the practice of being humble enough to admit someone else is better at something and being wise enough to try to learn how to match and even surpass them at it (International Benchmarking Clearinghouse 1993)." Arguments abound on whether the technique is new-some even attempt to trace benchmarking to the Egyptian pyramids. But nevertheless, it is a technique which has proven viable in the manufacturing sector and offers great opportunity to service and environmental functions both within and outside the manufacturing arena.

To understand benchmarking one needs to understand processes and the context for the understanding of processes is Total Quality Management. Total Quality Management is "...a people-focused management system that aims at continual increase of customer satisfaction at continually lower real cost. Total Quality is a total system approach, and an integral part of high level strategy; it works horizontally across functions and department, involves all employees, top to bottom, and extends backwards and forwards to include the supply chain and the customer chain. Total Quality stresses learning and adaptation to continual change as keys to organizational success."(The Total Quality Leadership Steering Committee and Working Councils, 1992) Essentially, TQM is a way of thinking whereby employees are empowered to identify, analyze, and continuously improve the organization's processes. We define processes as any series of activities performed in an attempt to support or contribute to the mission of the organization. All work gets done through processes which change inputs from suppliers to outputs (i.e., products or services) for customers. For example, waste tank monitoring is a process which converts the information of waste type, level, etc., to the output or service of increased health and safety to workers and others in the local community.

\section{[NSERT EXHIBIT ONE ABOUT HERE]}

A special kind of customer is the "consumer" of a good or service. Consumer identification is the first step towards continuous performance improvement. If the process of 
interest is waste management, then who is the direct recipient of this service or product? The consumer is the user and should play a critical role in defining the wants and needs of the service or product. Once defined, customers who are not direct users can be identified as can suppliers and other stakeholders. These constituencies and the process(es) they have a vested interest in, together form a system illustrated in Exhibit 1.

Benchmarking is therefore a tool of TQM for continuous performance improvement. Within the context of the system, it is very important to identify, understand, and recognize environmental processes and the value they offer to customers. In contrasting what benchmarking is to what it is not, the technique promotes a continuous process of performance improvement, not a "program" which yields panacea solutions. It is a proactive planning technique, not a reactive "band-aid" approach. Benchmarking works because it is consistent with the desire of many organizations to learn and discover new ways to improve performance. It seeks incremental improvements which improve morale and positively affect the bottom line, whether the organization seeks a profit or is not-for-profit. In Exhibit 2, those who benchmark find the greatest benefit is becoming more competitive.

\section{[INSERT EXHIBIT TWO ABOUT HERE]}

\section{Why Benchmark? Where in the Manarement System Does Benchmarking Fit?}

As Williams (1992) illustrates, driving forces such as regulatory issues, increased enforcement activity, liability concerns, investor concerns, competitive pressures, and public perception create a demand for benchmarking. Broadly speaking, benchmarking is a performance management technique which complies with the principles of quality management and continuous improvement. Within a management system, initially its context is a planning framework. In a traditional SWOT analysis for example, the organization assesses its strengths, weaknesses, opportunities, and threats. Benchmarking sharpens such an analysis by finding the best-in-class organization for a given process and by assessing why they are the best. Then, the best practices 
identified are either adopted or adapted. Since most existing processes are a projection of past practices into the future, or, "the way we've always done things;" benchmarking offers a projection of best practices into the future. It is an effective way to establish strategic goals based on best performance and then, to tactically pursue implementation by developing a productive partnership with another organization.

\section{The Benchmarking Process}

The generic benchmarking process as adapted from Camp (1989) is illustrated in Exhibit 3.

\section{[INSERT EXHIBIT THREE ABOUT HERE]}

The planning phase includes deciding what to benchmark. Several questions should be addressed in this phase:

- What "products" or "services" are provided to "customers"?

- What processes or products are most critical to success?

- What areas are causing the most trouble?

- What are the deliverables for the troubled areas?

- What factors are responsible for customer satisfaction?

- Are there other stakeholders who should be considered?

Selecting a process to benchmark usually is done based on some predetermined need. This need can be translated into performance variables which require improvement. Next, benchmarking partners should be identified. Selecting partners should be based on performance related to the targeted process and not necessarily based on whether the partner is in the same industry. Depending on the process, well-known performers can be identified such as L.L. Bean for customer service, American Express for purchasing, or Mary Kay for warehousing and distribution. In the broadly defined industry of environmental management, Altany (1990) suggests $3 \mathrm{M}$ and Ben and Jerry's as best performers. 
The data collection method used should be a function of the information required which depends on the objective of the benchmarking study. As illustrated in Exhibit 4, benchmarking objectives range from establishing achievable performance targets to developing a "world class" vision for a given function.

\section{[INSERT EXHIBIT FOUR ABOUT HERE]}

Data is collected from both partnering organizations. Every attempt should be made in the planning phase to agree on comparable data. As Kurstedt's (1991) model implies in Exhibit 5 , data is worthless unless it conveys meaningful information.

\section{[INSERT EXHIBIT FIVE ABOUT HERE]}

A comparative analysis is performed to arrive at meaningful information. Is the partner better? Why are they better? By how much? What best practices are being used to yield superior performance? How can the partner's practices be adopted and/or adapted? These are just some of the questions to be answered in a properly performed competitive analysis. Graphical portrayals can assist in illustrating potential gaps, as illustrated in Exhibit 6. In addition to determining the size of the current gap, every attempt should be made to predict future performance levels as well.

\section{[INSERT EXHIBIT SIX ABOUT HERE]}

Communication, which is a very important step, should occur during the integr . ion phase. Typically, those who perform benchmarking studies must communicate and gain approval from those with more organizational authority. While each audience must be individually assessed to determine appropriate media and presentation formats, some simple 
graphics have proven useful as illustrated in Figures 7, 8, and 9. Based on benchmarking findings, operational goals and plans should be established.

\section{[INSERT EXHIBITS SEVEN, EIGHT, AND NINE ABOUT HERE]}

The action phase starts with implementing specific actions designed to adopt or adapt the best practices identified by the study. Good principles of project management should be applied, including measuring progress, especially as it relates to closing the performance gap.

Stakeholder needs should have been converted into measures during the planning phase and progress should be regularly communicated. Operating under the framework of quality management and continuous improvement, benchmarking is a dynamic technique. Therefore, the targets need to be periodically recalibrated, much as world records in track and field constantly improve. A given benchmarking process can be considered complete when the best practices are fully integrated and institutionalized into the system.

\section{Partnering}

Benchmarking through the partnering approach has been traditionally "one-on-one", one organization partnering with another organization with similar approaches principles, values, and measures. They commit to each other by sharing and learning from one another. Partnering with one organization allows more time to be spent delving into more details of the processes and building a solid, trusting relationship. Another benefit of the one-on-one approach is reducing the preparation time and costs that benchmarking with many organizations would require. Benchmarking partners are available in many places. Exhibit 10 illustrates the variety of ways benchmarking partners have found each other.

\section{[INSERT EXHIBIT 10 ABOUT HERE]}


When beginning the partner approach for a targeted process, the "rules" of benchmarking below should be followed (adapted from Camp, 1989).

\section{Good Practices}

- Using secondary research to find public documents about a target organization

- Observing competitive processes in the open market and performing "reverse engineering"

- Conducting market research and stakeholder-satisfaction surveys

- Collecting information at trade shows

- Asking other organizations to share information about their processes

- Tapping employees to build a "who-knows-what" database about other organizations

- Being honest

\section{Bad Practices}

- Trespassing on another organization's property to collect information

- Bribing people to be informants

- Bugging or eavesdropping on another organization's privileged or private communications

- Learning something, even inadvertently, about a competitor's pricing considerations

- Requiring suppliers to participate in a benchmarking study as a condition of keeping or getting your business

- Trading a company's stock after learning about material information that hasn't been publicly disclosed

Although the above practices are common sense, some practices are questionable depending on the relationship between the two partnering organizations and each organization's standards of conduct. Even then, they should be considered carefully:

- Recruiting employees from competing organizations to get information 
- Asking questions at a technical meeting without identifying your name and organization

- Subscribing to a competitor's technical journals or attending user conferences as a private individual without disclosing your organizational affiliation

In addition, for the best benchmarking results through the partnering approach, these potential problems should be avoided:

- Talking about information you got from one organization with another organization

- Giving information in a public forum about a benchmarking partner without the partner's permission

- Asking for information you wouldn't give yourself

- Asking for information from a benchmarking partner without first understanding your own process

- During a site visit, asking for last-minute changes or additions to the agenda (or bypassing the benchmarking partner's designated host) to get more access to the company

\section{Status of Environmental Benchmarking}

Most environmental organizations do not actively benchmark for many reasons. Some don't understand the benefits of benchmarking or feel they don't have the expertise to begin a benchmarking effort. Others don't know who to partner with or how to partner. But the most common reason the environmental industry does not benchmark is benchmarking is a new technique that does not rank high enough on their priority list to get the needed time and resource allocations. As a technique of total quality management/continuous improvement, benchmarking is usually not pursued until several years into the improvement program.

\section{Optimization of Systems}

As previously illustrated, organizations can be viewed as systems; at its core, the organization transforms inputs into outputs (products or services) for customers. In organizations managed according to total quality principles and practices, we're very concerned about the optimization of 
systems. For a system to be optimized, the aim of the total system should drive decisions. Benchmarking focuses on the process and seeks to find the best-in-class at performing a process and then promotes adapting or adopting the relevant best practices. In adapting or adopting someone else's best practices, it is quite easy to inadvertently optimize a process and therefore compromise the system in which it operates. For example, if benchmarking the process of waste tank monitoring, a best performer may be located who uses state-of-the-art monitoring equipment. The obvious thing to do is provide justification for a major purchase with the company, gain approval and purchase similar equipment. However, it is quite possible the funds now allocated to waste tank monitoring should have been allocated elsewhere to best serve the total system. A better approach would be to strategically decide which processes to focus improvement efforts on and allow the strategic goals to drive the improvement efforts and allocation decisions accordingly.

\section{Lessons Leamed from Benchmarking Studies}

Based on benchmarking research and experience the following lessons learned should be incorporated into the environmental benchmarking process:

Benchmarking should promote continuous improvement. As mentioned before, benchmarking is not a panacea or a one-time, cure-all program. The partnership is the vehicle for continuous improvement.

Benchmarking is a line function. Benchmarking may start with management but the employees are actually involved with the process being benchmarked must integrate and institutionalize the new process.

Promote open and honest communication. In a good partnering relationship, you will have chosen a partner who feels totally comfortable sharing all the information needed to make their best process work for you and they will have the best process that you need. The partnering should be a "give and take" relationship with both partners sharing openly and improving continually. 
Prioritize. Benchmarking is not technique that can be done partially and still show its potential results. The organization, particularly management, must be willing to allocate the necessary time and resources to make the process work.

Success of the program depends on your method. As Deming (1992) says, "by what method"; with a properly designed, systematic method, results will be successful.

Choose your partmers carefully. The well-chosen partner will help you reach the performance excellence you're after. A poorly chosen partner will be a waste of efforts and resources for both your organization and the partner organization.

Benchmark a wide number of performance indicators. The real benefit of benchmarking is to focus on processes. By improving processes, results, including cost improvement, will come. In addition, cost is not the only important indicator, especially in the environmental domain. Time, safety, productivity, etc. are all important performance indicators, as will be discussed in the next section.

There is no single way. Benchmarking, like any other process, must be adopted or adapted to suit your organization's unique needs. In other words, what works for one organization may not for another until it has been analyzed and integrated. For the best results, the organization should know itself throughout and have processes well documented before partnering with other organizations and trying to implement their processes. 


\section{Ten Benchmarking Success Indicators}

1. Active management commitment

2. A willingness to change and adapt

3. Proactive behaviors

4. A willingness to define information with benchmark partners

5. Focus on best practices first, performance metrics result

6. Focus on functionally best operations

7. Following a structure process

8. Openness to new ideas and innovation

9. Continuous benchmarking effort

10. Benchmarking becomes institutionalized

(adapted from Camp 1989)

\section{Continuous Performance Improvement vs. Reengincering}

When attempting to improve processes, one or more of seven types of performance improvements are desired. According to Sink and Tuttle (1989), performance can be categorized as productivity, efficiency, effectiveness, profitability, innovation, quality of worklife, and quality. In our experience though, competitive firms are most concerned about cost, timeliness. and quality. To achieve these results, one has to answer the strategic questions, "how quickly and at to what extent are improvements needed?". Process reengineering is popular and is used when quick, drastic change is required or desired. Typically this involves eliminating a process, completely redesigning it, or adopting an entirely new process. On the other hand, continuous performance improvement is the goal of quality management. The TQM focus in contrast is long-term, often slowly appearing improvement to existing processes. In either case, benchmarking is an excellent tool to use in order to a) identify a performance goal for improvement, b) identify partners who have accomplished these goals, and c) identify applicable practices to incorporate into a redesign effort. The PLAN-DO-STUDY-ACT cycle, so popular in 
the quality management arena, is an ideal organizing structure for these long term improvements (Deming, 1992).

\section{Manarement's Role}

Because of the unlimited potential for improvement and overall scope of the benchmarking process, management must take not only an active role in benchmarking but the lead facilitating role. As a part of planning in the management system, benchmarking, to be most effective, is an organization-wide, continual process. Management must oversee the whole effort and play an integral part of keeping the process in action. Management's interest, mentoring, and support will motivate employees throughout the organization to be innovative and creative. The following are some management behaviors that lead to effective benchmarking:

- Provide planning leadership.

- Agree on the benefits, the partners, the approach, each member's role, and the barriers.

- Make sure findings are understood and accepted.

- Make sure performance is projected and recalibrated based on findings.

- Have a communication process to inform the organization of progress.

- Integrate findings with the organization's other key business processes.

- Find success stories.

\section{How to Get Started}

Many times people ask how they can start a benchmarking process in their organization after reading or hearing about the significant benefits that can be achieved. The first step is deciding what to benchmark or what area to focus on first to accelerate improvement. After deciding where to begin, the next step is gathering information both in a general way and for the specific function targeted. The following should help (adapted from Camp, 1989): 


\section{Informatlon sources - General}

- Focus on an area/element that needs to be pursued (examples: cost estimating, schedule estimating).

- Contact a business library or academic institution:

- request a search of information produced in the last three to five years for your topic of interest and

- have the library identify articles/sources from external reports, public magazines, industry journals, and annual reports.

- Contact internal experts:

- contractors, other offices, other public offices, functional experts, survey internal reports/surveys, special studies, surveys.

\section{Information Sources - Spectic/Functional}

- Trade periodicals

- Professional associations

- Newsletters, Seminars (especially speakers/tours), bibliographiss, special libraries

- Service agencies/bureaus in the business function

- ask if they can share anonymous experiences from their client companies concerning industry and state-of-the-art methods/practices.

- Consulting firms

- functional experts, ask if they are aware of particular breakthroughs/practices in a specific area.

- Industry Experts

- department heads of noncompetitors, teachers/professors at schools/universities

- Systems software development and hardware vendors -ask about their experiences working in the functional area 
Another early step in benchmarking is planning through training. Here are some options to consider:

- Have an awareness workshop to create a baseline understanding.

- quality management or continuous improvement context

- focus on current mission, vision, organizational design

- Facilitate definition of system:

- define functions; define processes, products, practices; define customers and suppliers, stakeholders; define inter-dependencies, redundancies.

- Facilitate identification of key functions and processes to be benchmarked.

- Facilitate mapping of current key processes.

- Later, during comparative analysis determine and understand performance and methods gap.

One place to turn when starting a comprehensive benchmarking program is the International Benchmarking Clearinghouse. Started by Xerox in the 198Cs, the International Benchmarking Clearinghouse offers a number of services and provides information on functions, past studies as well as help in contacting their members for more information.

\section{International Benchmarking Clearinghouse Services}

Networking Services (e.g., meetings)

Information Services (e.g., searches and research)

Benchmarking Services (e.g., partner identifications)

Benchmarking Training

Electronic Network

Database of Past Studies 
Here is a partial list, applicable to environmental benchmarking, of International Benchmarking Clearinghouse members:

\section{Commercial}

Xerox (founder)

Weyerhaeuser

\section{Exxon}

\section{Grumman}

ITT Defense

Shell

Waste Management Company

\section{Conclusion}

If used correctly, with the right support, benchmarking is an excellent tool for continuous performance improvement. If used inappropriately, benchmarking can easily suboptimize a system, improving areas which strategically require relatively less attention than others.

Benchmarking is, therefore, most successful when it is driven be a strategic decision process. That is, from a planning perspective, benchmarking is most successfull when it is strategically determined that a given process needs to be improved. With respect to implementation, benchmarking is most successful when it is applied within the context of total quality management. It is a technique of TQM and therefore would be a part of the TQM training program and a part of the activities of process improvement or quality improvement teams. Benchmarking offers the commercial firm the opportunity to apply the principles of quality planning and management in a way that performance can be dramatically, continually, and measurably improved. 


\section{Llerature Clied}

Attany, David. "Copycats: once obscure, 'benchmarking' is fast becoming the buzzword of the year." Industry Week (1990) 239(21): 11-14.

Camp, Robert. 1989. The Search for the Best Practices That Lead to Superior Performance. Milwaukee, WI: The Quality Press, 1989.

Cecil, R. and R. Ferraro. "Es Fill Facilitator Role in Benchmarking Operations to Improve Performance." Industrial Engineering (1992) 24(4): 30-34.

Deming, W. Edwards. Out of the Crisis. Cambridge Massachusetts: Massachusetts Institute of Technology, 1992.

Intermational Benchmarking Clearinghouse. Brochure, no date.

Kurstedt, H.A. Jr. Why the Methods of Management Will Work for You. Blecksburg, VA: Management Systems Laboratories, 1991.

Sink, D.S. and T.C. Tuttle. Planning and Measurement in Your Organization of the Future. Norcross, Georgia: Industrial Engineering and Management Press, 1989.

U.S. General Accounting Office. Quality Management, Survey of Federal Organizations. Briefing Report to the Honorable Donald Ritter, House of Representatives. October 1992. GAO/GGD-93-9BR p. 48.

U.S. Department of Energy, Office of Environmental Restoration and Waste Management. Benchmarking for Cost Improvement, September 1993.

Williams, Marcia. (1992). "Why_How to-Benchmark for Environmental Excellence." Total Quality Environmental Management, Winter 1992-93. pp. 177-185. 
Brian M. Kleiner

Environmental Benchmarking

List of Exhibit Captions

Exhibit 1

The "system" in which processes function.

Exhibit 2

Benefits of Benchmarking

Exhibit 3

The Benchmarking Process

Exhibit 4

Benchmarking Objectives

Exhibit 5

Management Systems Model (Kurstedt 1991)

Exhibit 6

Waste Management Operations Costs per Operation (in thousands of dollars)

Exhibit 7

Estimation error variance

Exhibit 8

Functional Performance (Productivity vs. Service Lovel)

Exhibit 9

Hazardous Waste Drum Storage Cost Performances Rating System

Exhibit 10

How to Find Partners? 


\section{Brian M. Kleiner}

\section{Environmental Benchmarking}

\section{Exhibit 1}

The "system" in which processes function.

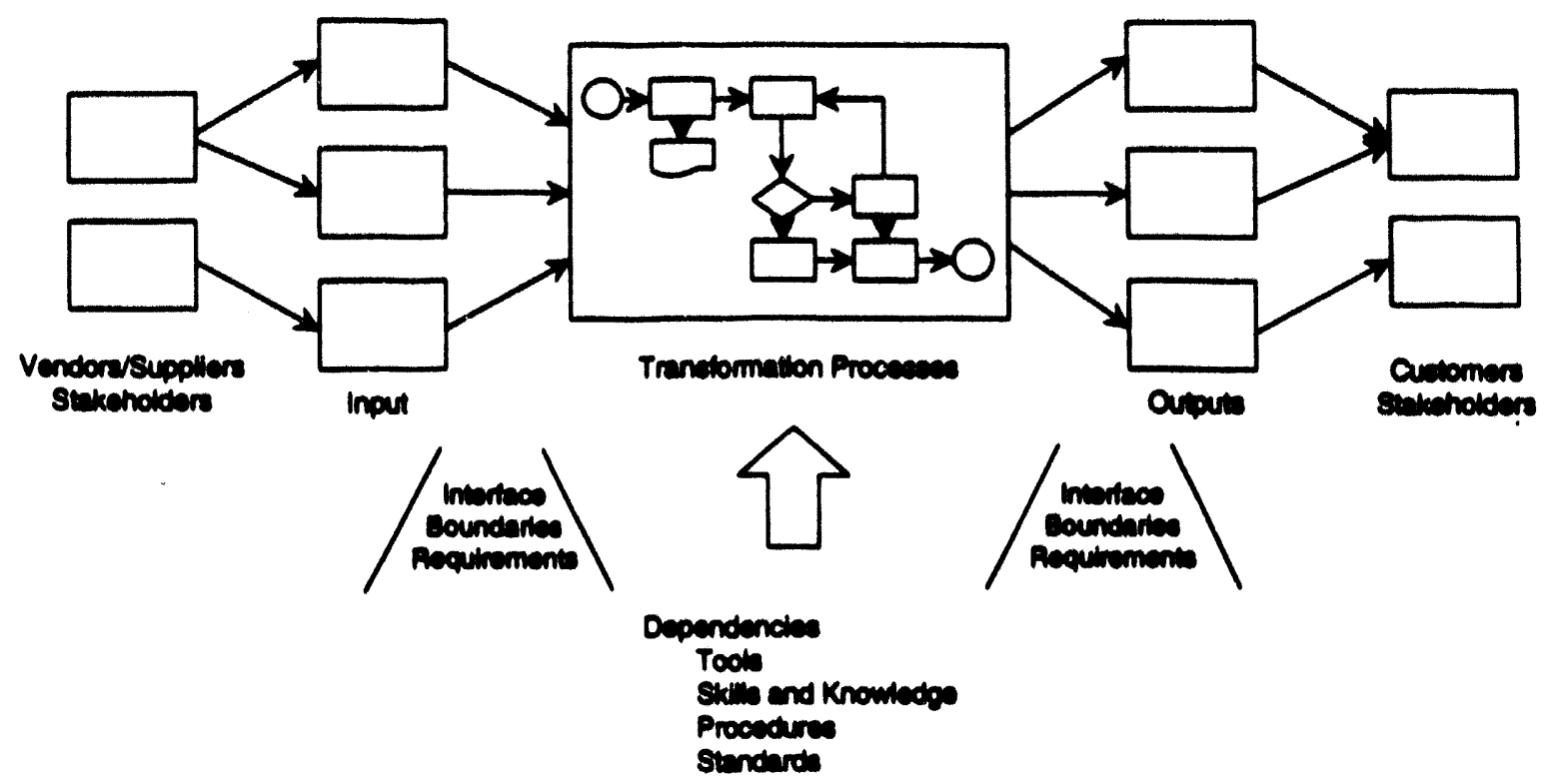


Brian M. Kleiner

Environmental Benchmarking

Exhibit 2

Benefits of Benchmarking

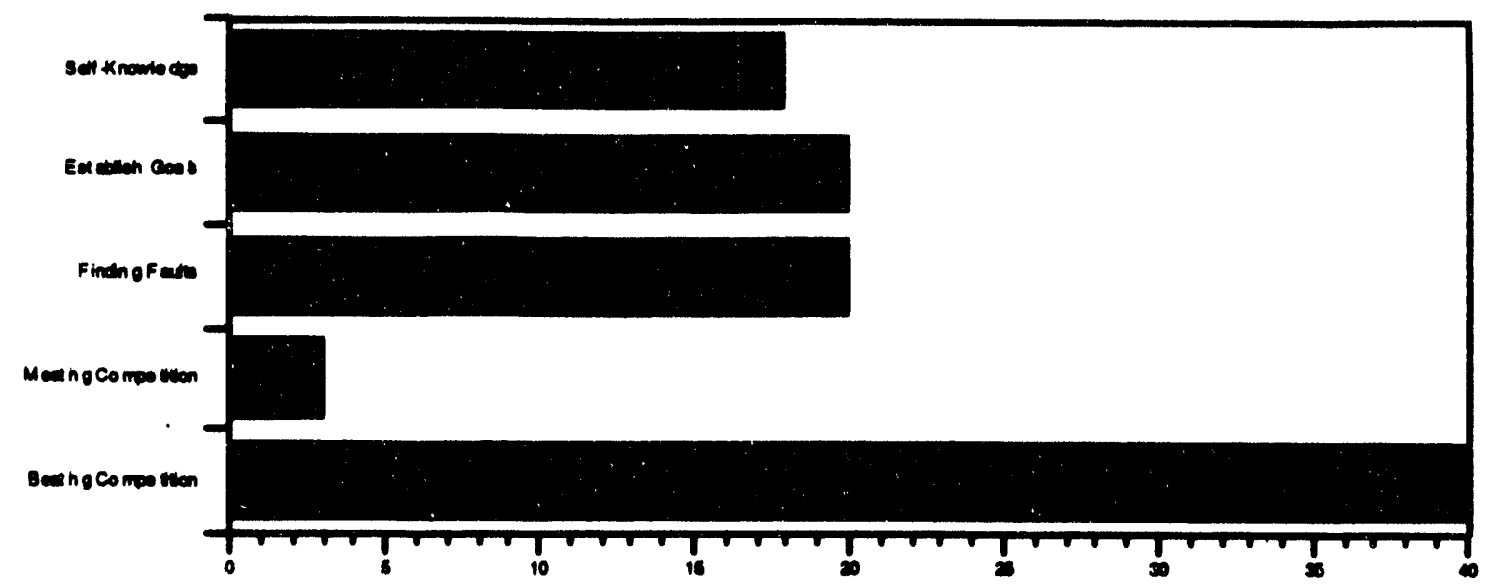


Brian M. Kleiner

Environmental Benchmarking

Exhibit 3

The Benchmarking Process

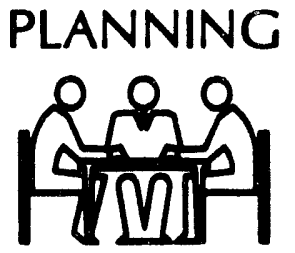

1. Identify what is to be benchmarked.

2. Identify key performance variables to measure.

3. Identify benchmarking partners.

4. Determine data collection method.

5. Collect data.

ANALYSIS

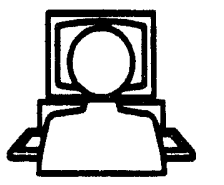

INTEGRATION

6. Determine and understand current performance gap.

7. Predict future performance levels.

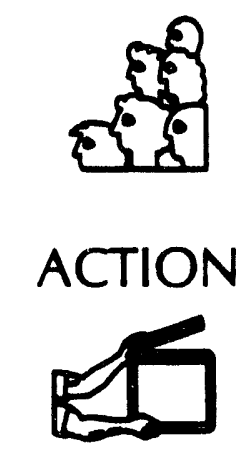

MATURITY

8. Communicate benchmarking findings and gain acceptance.

9. Establish functional goals and operational principles.

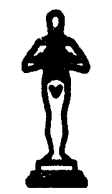

- Leadership position attained.

- Practices are fully integrated into processes.

(groupings adapred from Camp, 1989, 1992)

Figure 3

The Benchmarking Process

10. Implement specific actions and monitor progress.

11. Measure results against stakeholder needs.

12. Recalibrate benchmarks.

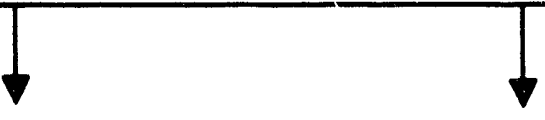


Brian M. Kleiner

Environmental Benchmarking

\section{Exhibit 4}

Benchmarking Objectives

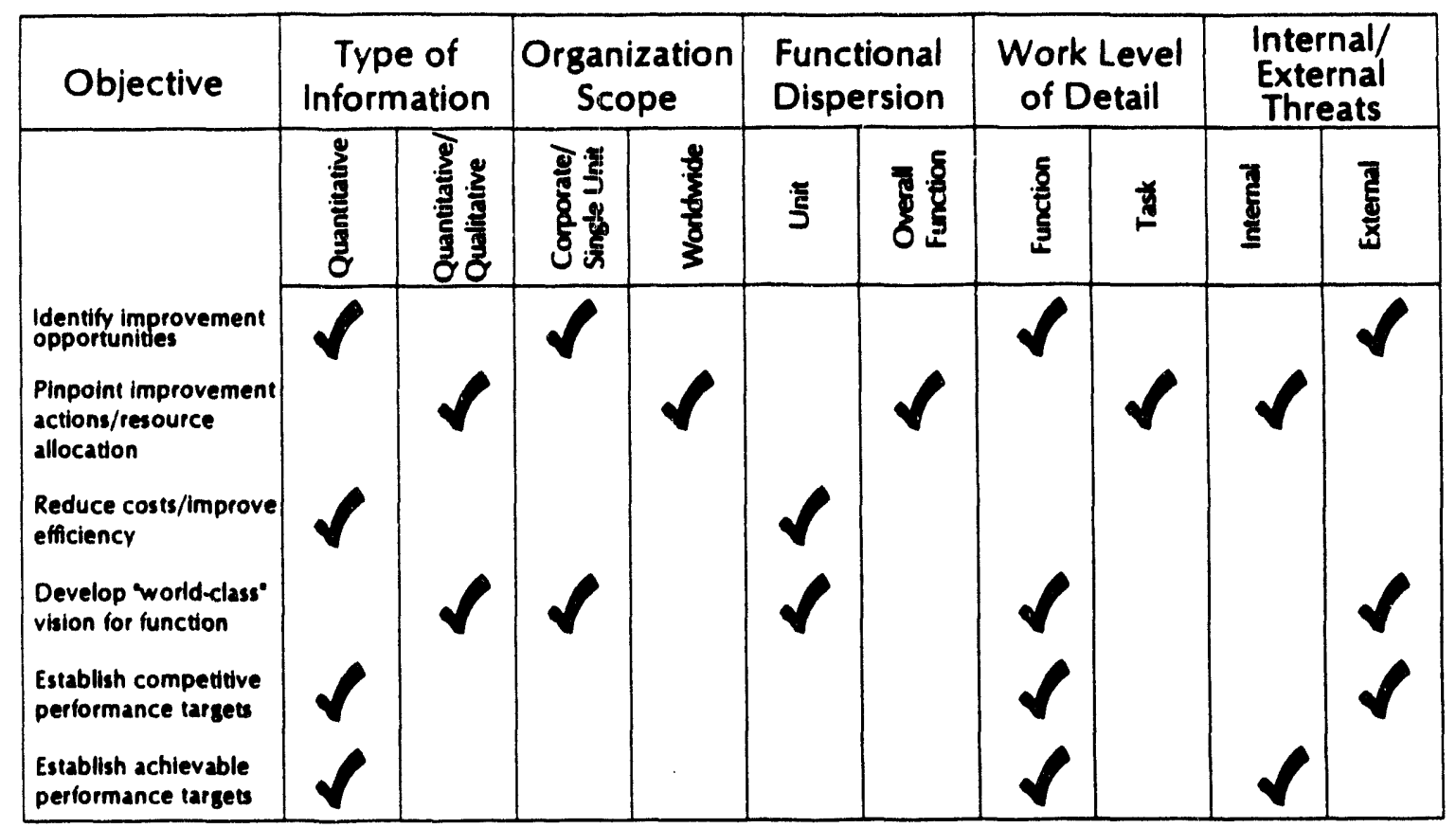

(adapted from Cecll and Ferraro, 1992) 
Brian M. Kleiner

Environmental Benchmarking

Exhibit 5

Management Systems Model (Kurstedt 1991)

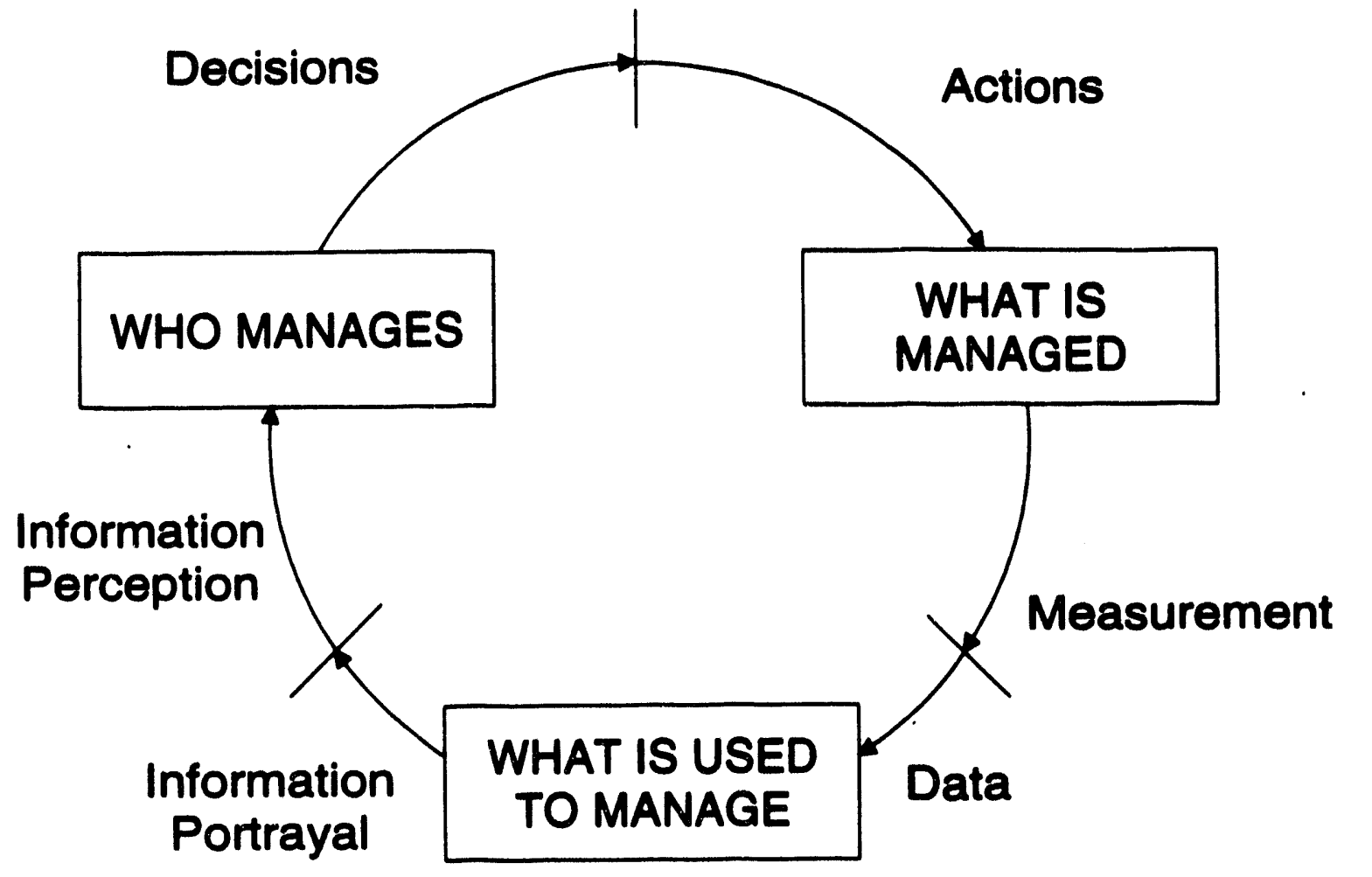


Brian M. Kleiner

Environmental Benchmarking

Exhibit 6

Waste Management Operations Costs per Operation (in thousands of dollars)

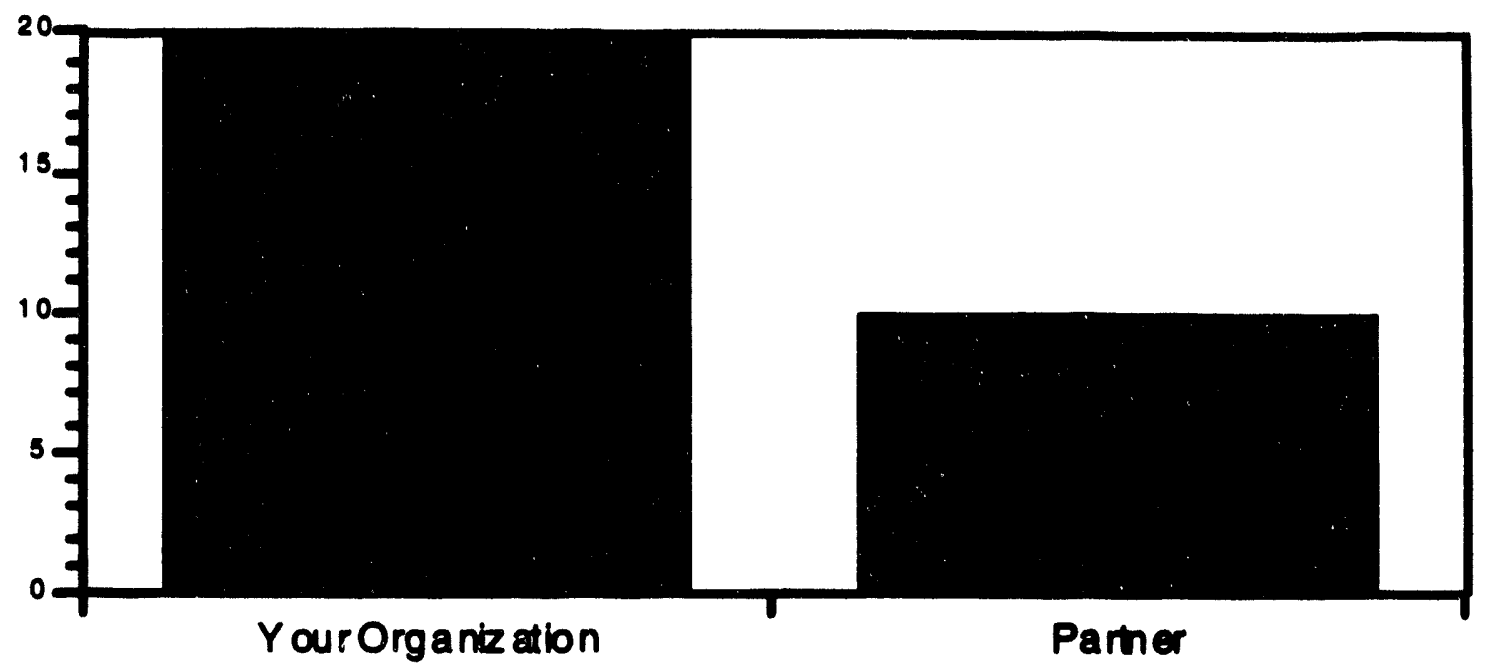

Vartable

Drea 
Brian M. Kleiner

Environmental Benchmarking

Exhibit 7

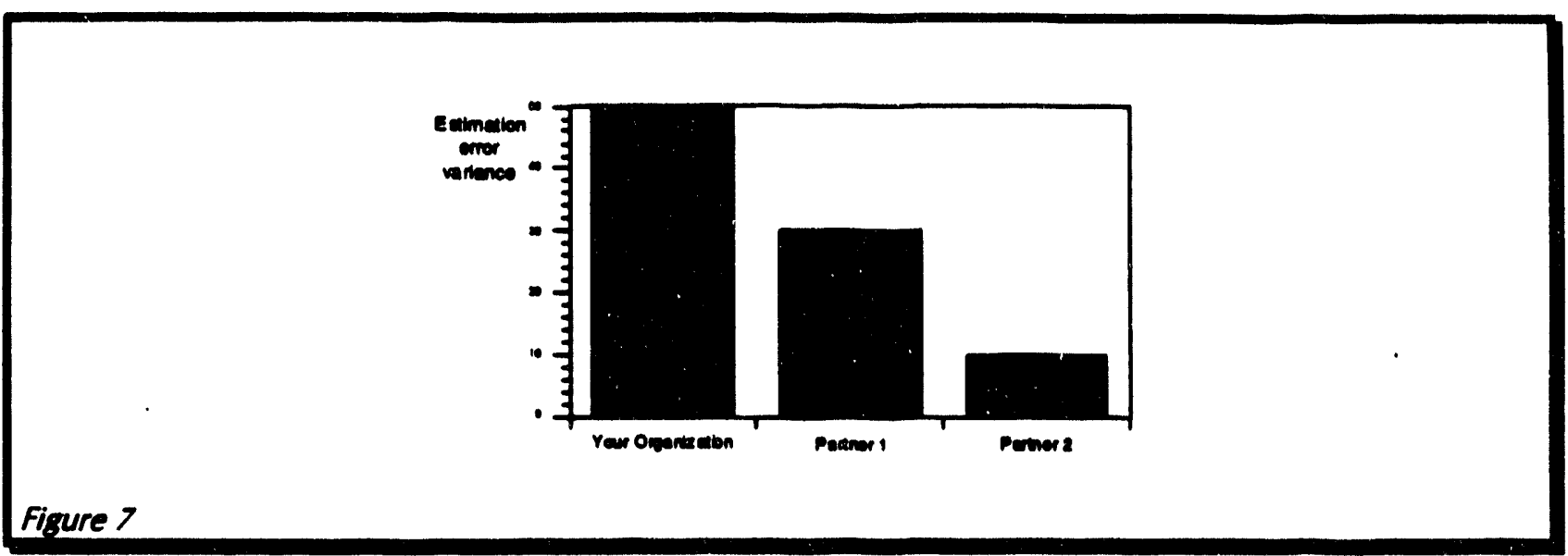


Brian M. Kleiner

Environmental Benchmarking

Exhibit 8

Functional Performances (Productivity vs. Service Level)

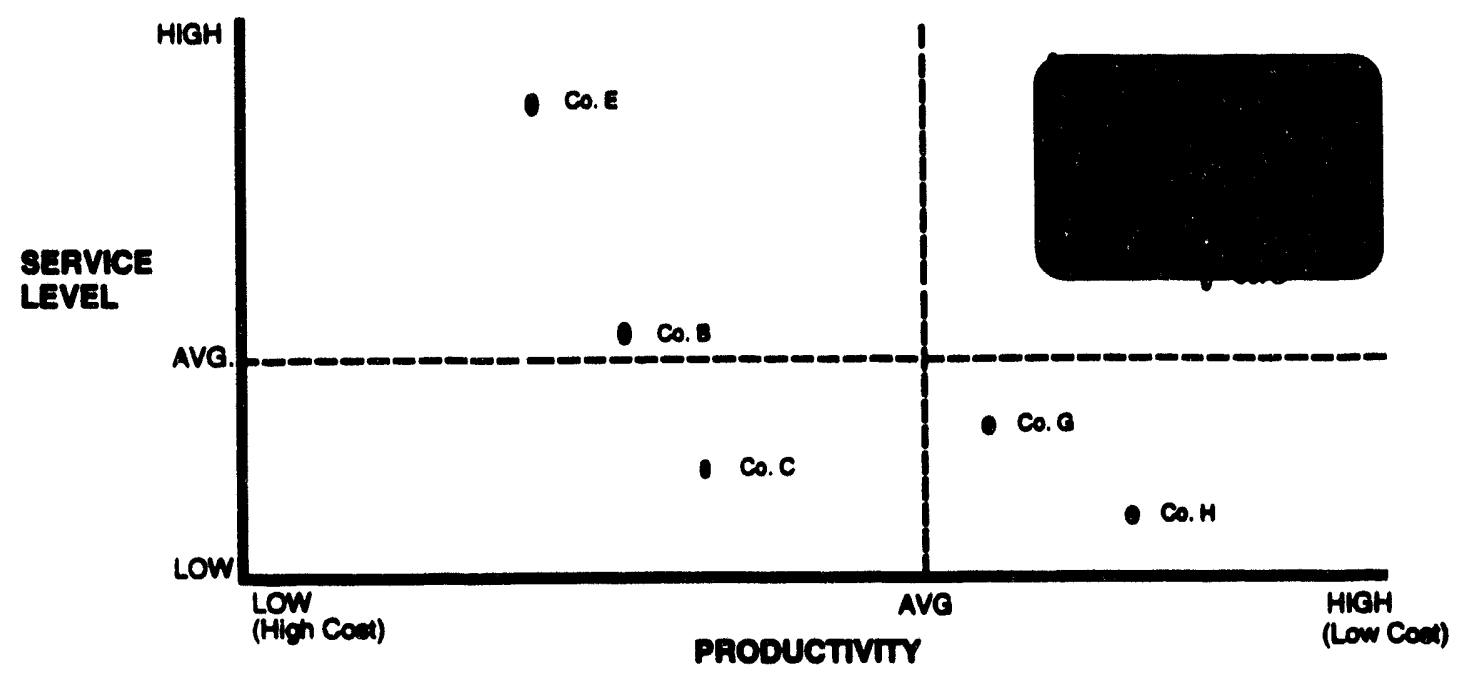


Brian M. Kleiner

Environmental Benchmarking

Exhibit 9

Hazardous Waste Drum Storage Cost Performances Rating System

\begin{tabular}{|l|c|c|c|c|c|}
\hline $\begin{array}{l}\text { Cost Performance } \\
\text { (cost per drum) }\end{array}$ & $\$ 19$ & $\$ 26$ & $\$ 38$ & $\$ 57$ & $\$ 76$ \\
\hline Operation Rating & 5 & 4 & 3 & 2 & 1 \\
\hline $\begin{array}{l}\text { Waste Management } \\
\text { Operation Type }\end{array}$ & $\begin{array}{c}\text { Word } \\
\text { Class }\end{array}$ & superbr & Par & sub-par & $\begin{array}{c}\text { mpode } \\
\text { move- }\end{array}$ \\
\hline
\end{tabular}


Brian M. Kleiner

Environmental Benchmarking

Exhibit 10

How To Find Partners?

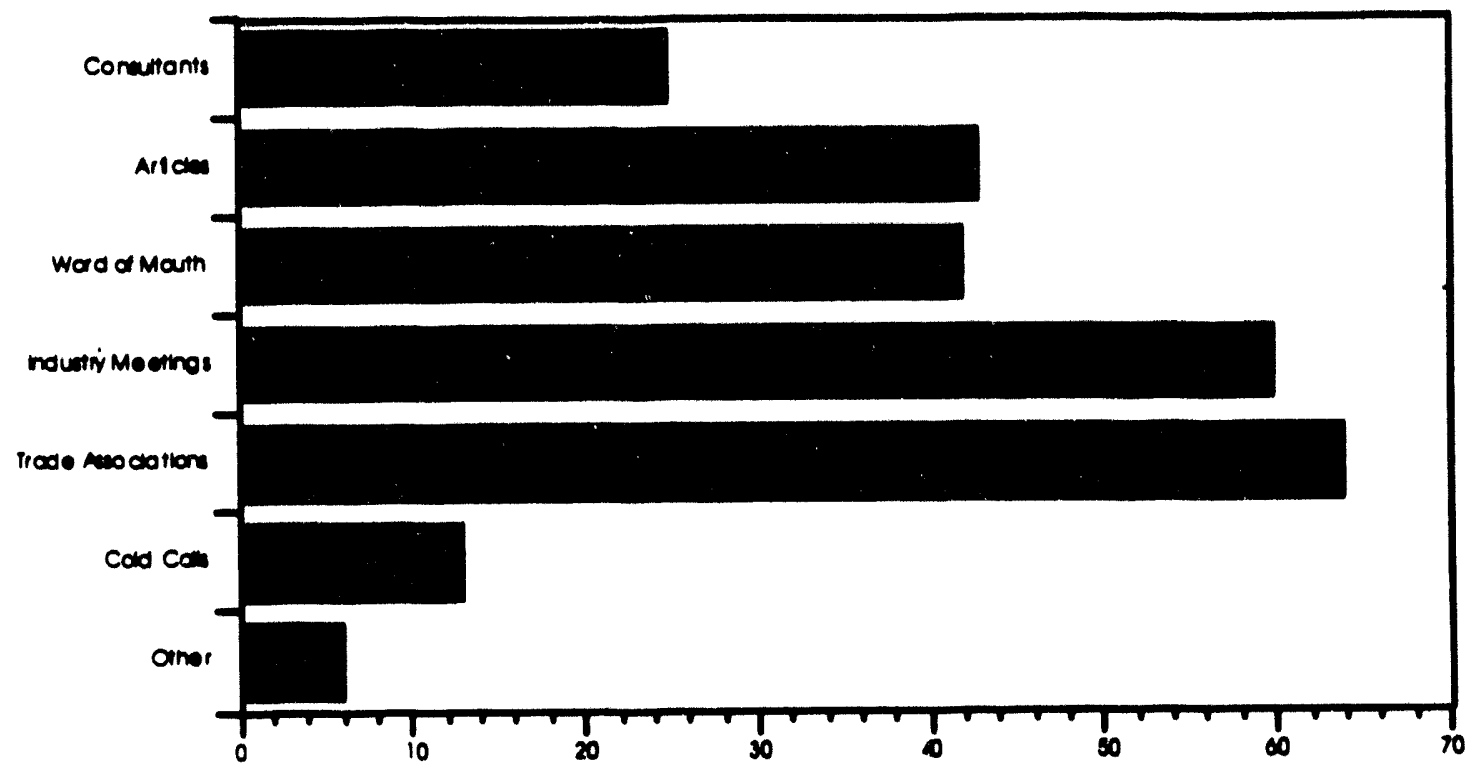




\section{Gloseary}

benchmarking - the practice of being humble enough to admit someone else is better at something and being wise enough to try and learn bow to match and even surpass them at it (International Benchmarking. Clearinghouse, 1993)

best practice - practices or combinations of practices that contribute the "best" measurement values and/or represents what you and others (stakeholders) think are most desirable

functional unit or function - goal-oriented organizational unit (e.8., operations support) measurements - a quantitative performance indicator of a specific process or step optimization of a system - meeting the needs of the total system partner - another organization with whom you exchange best practices practice or methods - the way we perform an activity (e.g., ABC costing) process - a series of tasks or steps performed to meet a specific objective (e.g., cost estimating)

reengineering - mass change to existing business processes or creation of entirely new process

target $\cdot$ conversion of benchmark to operational metric 


\section{Bionongy}

Brian M. Kleiner is a research faculty member a Management Systems Laboratories within the Department of Industrial and Systems Engineering at Virginia Polytechnic Institute and State University. He has worked with federal, state, and commercial organizations to research the potential of environmental benchmarking and has led several successful benchmarking implementation efforts. He has been involved in the total quality field since the early 1980 's. This paper was prepared to belp establish a baseline understanding of benchmarking for environmental managers and practitioners. 

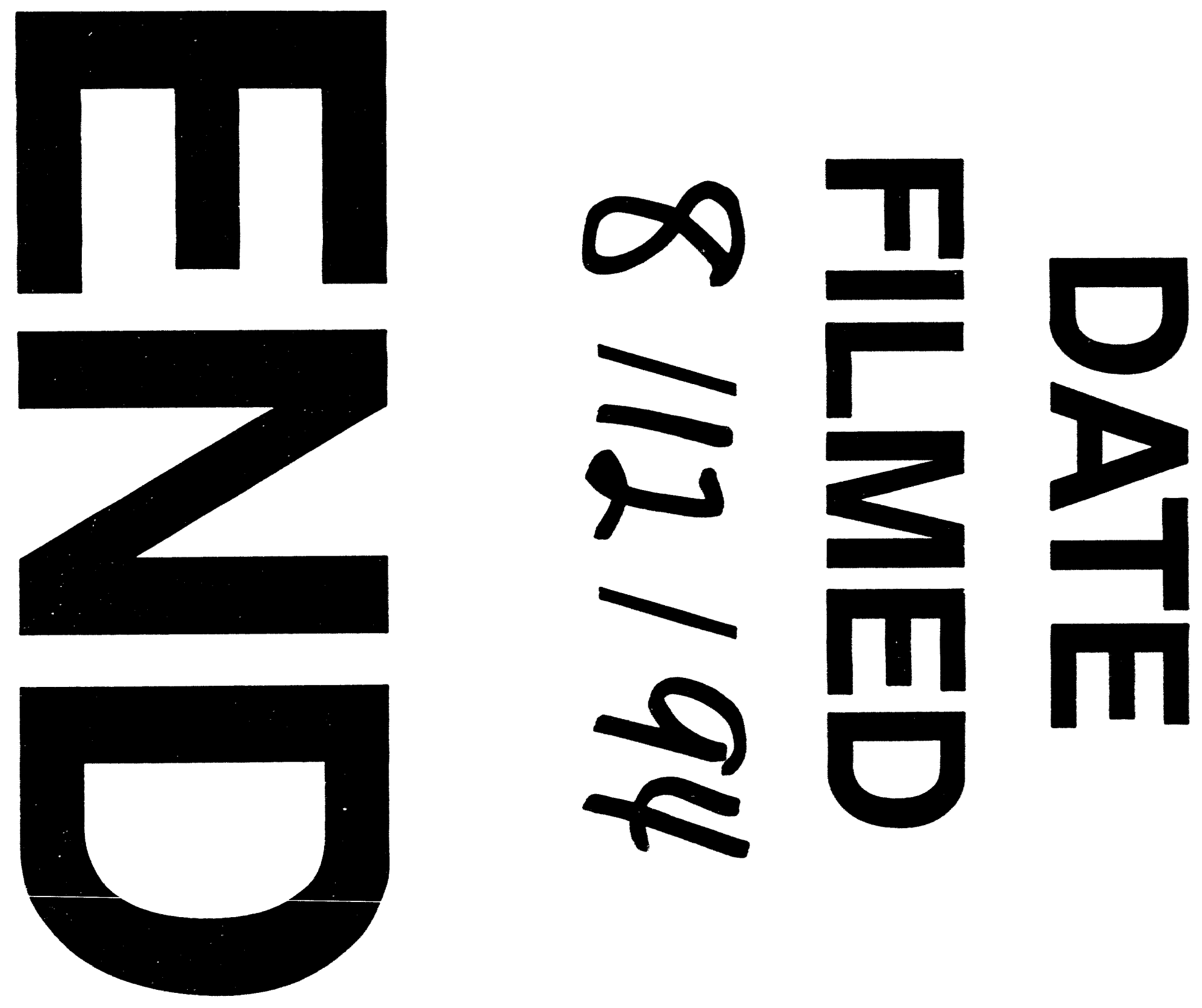
